\title{
DETECTION OF LARGE HOLOCENE EARTHQUAKES IN THE SEDIMENTARY RECORD OF WELLINGTON, NEW ZEALAND, USING DIATOM ANALYSIS
}

\author{
Ursula Alyson Cochran
}

A thesis submitted to Victoria University of Wellington in fulfilment of the requirements for the degree of Doctor of Philosophy in Geology

Victoria University of Wellington 2002 


\section{A B S T R A C T}

New Zealand is situated on the boundary between the Pacific and Australian tectonic plates. The Wellington region lies near the southern end of the Hikurangi subduction zone and within a zone of major, active strike-slip faults. Wellington's paleoseismic and historic records indicate that large surface rupture earthquakes have occurred on these faults in the past. Development of a complete record of past large earthquakes is a high priority for the region because of the risk posed by occurrence of large earthquakes in the future. The existing paleoseismic record has been derived predominantly from studies of fault trench stratigraphy, raised beach ridges and offset river terraces. The sedimentary record of lakes and coastal waterbodies is a source of information that has not been used specifically for paleoseismic purposes in the region. Therefore investigation of Wellington's sedimentary record is used in this thesis to make a contribution to the paleoseismic record.

Holocene sedimentary sequences are studied from three small, low elevation, coastal waterbodies: Taupo Swamp, Okupe Lagoon and Lake Kohangapiripiri. Sequences of between 200 and $650 \mathrm{~cm}$ depth were collected using a handoperated coring device. Sedimentology and diatom microfossil content were analysed and interpreted to enable reconstruction of paleoenvironment at each site. Radiocarbon dating was used to provide chronologies for the sequences that are aged between 5000 and 7500 calibrated years before present (cal. years BP).

Diatom analysis is the main tool used to reconstruct paleoenvironment and detect evidence for occurrence of past large earthquakes. To aid reconstruction of sedimentary sequences used in this project, as well as coastal sequences in New Zealand in general, a coastal diatom calibration set was constructed using 50 sites around New Zealand. Modern diatom distribution and abundance, and associated environmental variables are analysed using ordination and weighted averaging techniques. Detrended correspondence analysis arranges species according to salinity preferences and divides sites clearly into waterbody types along a coastal gradient. This analysis enables reconstruction of waterbody type from fossil samples by passive placement onto ordination diagrams. Weighted averaging regression of calibration set samples results in a high correlation $\left(r^{2}\right.$ jack $\left.=0.84\right)$ between observed and diatom inferred salinity, and enables salinity preferences and tolerances to be derived for 100 species. This confirms for the first time that species' preferences derived in the Northern Hemisphere are generally applicable to diatoms living in the coastal zone of New Zealand. Weighted averaging calibration and the modern analogue technique are used to generate quantitative estimates of paleosalinity for fossil samples. 
Paleoenvironmental reconstructions of Taupo Swamp, Okupe Lagoon and Lake Kohangapiripiri indicate that each waterbody has been isolated from the sea during the late Holocene. Isolation has been achieved through interplay of sediment accumulation causing growth of barrier beaches, and coseismic uplift. Ten distinct transitions between different paleoenvironments are recognised from the three sequences. These transitions involve changes in relative sea level or water table level often in association with catchment disturbance or marine influx events. All transitions occur suddenly and are laterally extensive and synchronous within each waterbody. Quantitative estimates of paleosalinity and waterbody type are used to differentiate between large and small magnitude changes in paleoenvironment. Five transitions involve large amounts of paleoenvironmental change and provide evidence for earthquakes occurring at $\sim 5200, \sim 3200$, and $\sim 2300$ cal. years BP. Five other transitions are consistent with the effects of large earthquakes occurring at $\sim 6800,2200, \sim 1000, \sim 500$ cal. years BP and $1855 \mathrm{AD}$ but do not provide independent evidence of the events.

Environmental transitions at Lake Kohangapiripiri clarify the timing of rupture of the Wairarapa Fault by bracketing incompatible age estimates derived from two different sites on the fault. The oldest environmental transitions recognised at Taupo Swamp and Okupe Lagoon both occur at $\sim 3200$ cal. years BP indicating that western Wellington was uplifted at this time. Environmental transitions are recorded at all three study sites at $\sim 2300$ cal. years BP indicating that the entire western and central Wellington region experienced coseismic uplift at this time. Because of the distance between sites this apparent synchroneity implies that several faults in the region ruptured at a similar time. Investigation of sedimentary sequences contributes to the existing paleoseismic record by providing additional estimates of timing for past large earthquakes, enabling estimation of the areal extent of the effects of past earthquakes, and by highlighting periods of fault rupture activity in the late Holocene. 


\section{A C K N O W L E D G E M E N T S}

\section{Supervision}

Mike Hannah has been an unfaltering source of support and common sense advice throughout the research process. His comments on drafts of the thesis have improved the final result. Thank you for sowing the $\mathrm{PhD}$ seed and seeing me through it.

Margaret Harper has taught me much of what I know about diatoms. Thank you for being patient while I grappled with the concept of a species, for being so willing to discuss queries, for proof reading everything, and for encouraging me to go a step further than I had planned.

James Goff provided supervision in the initial stages of the work and instigated the work on Okupe Lagoon. Thank you for your boundless enthusiasm and encouragement in the world of Earth Science research.

\section{Advice and Discussion}

The following people provided numerous pieces of information and advice. Thank you all for the willingness with which you contributed to my thesis.

Victoria University: James Shulmeister, Warren Dickinson, John Carter, Bill McLea, Jo Anderson, Mathew Prebble, Corinne Watts, Vanessa Thorn, Rodney Grapes, Dick Walcott, Neil Hartstein, Nigel Hill, Venus Sutherland, Brian Dawkins, Peter Barrett, Euan Smith, John Paterson, Mike Crozier, Les Singh. Institute of Geological and Nuclear Sciences: John Begg, Brent Alloway, Kelvin Berryman, Alan Hull, Catherine Chague-Goff.

The University of Auckland: Bruce Hayward.

Overseas Institutions: Steve Juggins, Rick Battarbee, Laurence Cavalho, John Birks, Peter Gell, Nina Bate, Ben Horton, John Anderson, Eileen Hemphill-Haley.

\section{Field Assistance}

Thank you to all my field assistants for being willing to get cold and muddy for the sake of a good sediment sample.

Coring: Alex Pyne, Cliff Atkins, Edward Butler, Jo Anderson, James Goff, Catherine Chague-Goff, Mathew Paterson, Mathew Prebble, Michael Hannah, Neil Hartstein, Illona Keenan, Margaret Cochran, Chris Cochran, Walter Rushbrook, Vanessa Thorn.

Modern Sampling: Katie Abbott, Margaret Cochran, Walter Rushbrook. Surveying: Walter Rushbrook. 


\section{Technical Assistance}

Thank you to everyone who helped in very practical ways.

Alex Pyne (field equipment and procedure), John Carter (laboratory procedure), Stephen Eager (macrofossil and ostracod identification), John Collen (foraminifera identification), Ding Ren (loss on ignition analyses), Jo Anderson (laboratory procedure), Frank Drost (modern sampling equipment), Salli Rowe (graphic design), Andrew Sutton (computers), Eric Broughton (inventions), Val Hibbert (financial transactions), Dave Winchester (surveying equipment), Nancy Bertler (German translations), Alyson Barr (company and accommodation in London), The Holdens HQ, VN and VR (transportation).

The following people and organisations are thanked for providing access to land and permission to take cores.

Lake Kohangapiripiri: Department of Conservation and David Hargest of Hutt City Council.

Taupo Swamp: Queen Elizabeth II National Trust, Wellington Regional Council, and Ross McEwan.

Okupe Lagoon: Kapiti Island Marine Reserve Committee, Commissioner for Crown Lands, and Ati Awa Ki Whakarongotai Inc.

Marc Schallenberg, Alison Berry (Wellington Regional Council), Julie Edwards (Canterbury Regional Council) are thanked for providing additional water chemistry data for some of the modern calibration set sites.

\section{Financial Support}

Thank you to all the organisations that made this research possible.

The Council, Victoria University of Wellington, provided a postgraduate scholarship.

The Science Faculty Leave and Grants Committee, Victoria University of Wellington, provided yearly research grants.

The Earthquake Commission Research Foundation provided a research grant that enabled radiocarbon dates to be obtained.

The New Zealand Vice-Chancellors' Committee awarded a Claude McCarthy Fellowship that made it possible to attend postgraduate courses in London. The Wellington Branch, Federation of University Women, paid course fees. The Royal Society of New Zealand contributed to international presentation of this research.

The School of Earth Sciences, Victoria University of Wellington, subsidised printing, copying and internet use.

Chris and Margaret Cochran generously made up the difference so that I emerge as a rare species: a student-loan-free student. 


\section{Personal Support}

Walter Rushbrook has been my window to the outside world. Thank you for being my social calendar, being such good company, and taking me on excellent holidays. Thank you for your complete faith in my ability, for your sanity, patience, humour, continual encouragement and most importantly, the incentive to finish that you gave me.

Chris and Margaret Cochran have far exceeded the role of 'parent' in the $\mathrm{PhD}$ process. Thank you for contributing in so many ways: everything from fieldwork, advice, bringing meals to university, proof reading, scanning (!) and graphic design. Thank you for always listening and always being so interested.

The students of the School of Earth Sciences have provided great company and have helped to keep the thesis in perspective. In particular I would like to thank Vanessa, Jeremy, Cliff, Nancy, Phil, Ed, Jo, Pi and Maureen.

Finally I would like to acknowledge all staff of the Geology Group, Victoria University for cultivating my interest in Earth Science and for providing such an excellent and enjoyable undergraduate and postgraduate education. 


\section{O N T E N T S}

$\begin{array}{lc}\text { Abstract } & \text { ii } \\ \text { Acknowledgements } & \text { iv } \\ \text { List of Figures } & \text { xi } \\ \text { List of Tables } & x v\end{array}$

ONE Introduction 1

1.1 Aims 1

1.2 Purpose of Research 1

1.3 Setting 2

1.3.1 Tectonic Setting of New Zealand 2

1.3.2 Tectonic Setting of Wellington 4

1.3.3 Wellington's Paleoseismic Record 6

1.3.4 Study Sites 6

1.4 Background to Approach $\quad 7$

1.4.1 Earthquakes in the Sedimentary Record 7

Introduction - Pacific Northwest of America

and Canada. New Zealand

$\begin{array}{ll}\text { 1.4.2 Paleoecology } & 10\end{array}$

1.4.3 Diatom Analysis $\quad 13$

$\begin{array}{ll}\text { 1.5 Summary of Approach } & 15\end{array}$

TWO Paleoecological Methods $\quad 18$

$\begin{array}{ll}2.1 \text { Scope of Chapter } & 18\end{array}$

$\begin{array}{ll}2.2 \text { Field Techniques } & 18\end{array}$

2.2.1 Site Selection 18

$\begin{array}{ll}2.2 .2 \text { Coring } & 19\end{array}$

2.2.3 Surveying $\quad 20$

2.3 Laboratory Techniques 20

2.3.1 Core Description and Sampling $\quad 20$

2.3.2 Radiocarbon Dating 21

2.3.3 Grain Size Analysis $\quad 23$

2.3.4 Diatom Processing 23

2.3.5 Other Microfossils $\quad 25$

2.3.6 Macrofossils 25

2.4 Diatom Analysis $\quad 26$

2.4.1 Identification and Taxonomy 26

2.4.2 Counting 26

2.4.3 Concentration 29 
2.4.4 Ecological Preferences

Introduction $\cdot$ Habitat $\cdot$ Salinity

2.4.5 Data Presentation $\quad 32$

2.5 Detection of Earthquakes $\quad 32$

2.5.1 Introduction $\quad 32$

2.5.2 Suddenness of Change 33

2.5.3 Amount of Change 33

2.5.4 Lateral extent $\quad 36$

2.5.5 Synchroneity 36

2.5.6 Coincidence with Tsunami Deposits 36

2.5.7 Coincidence with Evidence for Catchment Disturbance $\quad 37$

THREE Diatom assemblages of New Zealand coastal waterbodies: a calibration set for quantitative paleoenvironmental reconstruction $\quad 38$

$\begin{array}{ll}3.1 \text { Introduction } & 38\end{array}$

3.1.1 Outline of chapter $\quad 38$

3.1.2 Why collect a calibration set? 38

3.1.3 Quantitative Paleoenvironmental Reconstruction $\quad 40$

3.1.4 Mathematical Methods 42

3.2 A New Zealand Coastal Diatom Calibration Set 44

3.2.1 Aims 44

3.2.2 Design 45

Introduction - Sites $\cdot$ Environmental Variables.

Diatom Samples • Diatom Species

$\begin{array}{ll}\text { 3.2.3 Methods } & 50\end{array}$

Measurement of Environmental Variables $\cdot$ Sample

Collection • Sample Processing · Representativity and

Allochthonous Component of Death Assemblages .

Mathematical Procedures

3.3 Description of the Calibration Set 55

3.3.1 Introduction $\quad 55$

3.3.2 Ordination 64

3.3.3 Canonical Ordination $\quad 66$

3.4 A Diatom-Salinity Transfer Function 73

3.4.1 Fitness for Quantitative Reconstruction 73

$\begin{array}{ll}\text { 3.4.2 Methods } & 75\end{array}$

3.4.3 Weighted Averaging Regression 76

3.4.4 Modern Analogue Technique $\quad 84$

$\begin{array}{ll}3.5 \text { Conclusions } & 85\end{array}$ 
FOUR Taupo Swamp: Evidence for Sudden

Environmental Change from Reconstructed

Waterbody Type and Paleosalinity 87

Summary

4.1 Introduction $\quad 88$

$\begin{array}{lr}4.2 \text { Sedimentary Sequences } & 90\end{array}$

4.2.1 Description of Sequences 90

4.2.2 Interpretation of Sequences 93

4.3 Diatom Analysis 94

4.3.1 Introduction 94

4.3.2 Definition of Diatom Assemblage Zones 94

4.3.3 Interpretation of Diatom Assemblage Zones 95

4.3.4 Reconstruction of Waterbody Type 99

4.3.5 Application of Salinity Transfer Function 101

4.4 Paleoenvironmental Reconstruction 103

$\begin{array}{ll}4.5 \text { Environmental Transitions } & 107\end{array}$

$\begin{array}{ll}\text { 4.5.1 Introduction } & 107\end{array}$

4.5.2 Description of Transitions 109

4.5.3 Change in Elevation $\quad 110$

4.5.4 Age of Transitions 112

4.5.5 The Playing Field Core: Uplift in 1855 AD? 112

4.6 Conclusions 114

FIVE Okupe Lagoon: A History of Punctuated Isolation $\begin{array}{ll}\text { from the Sea } & 115\end{array}$

$\begin{array}{ll}\text { Summary } & 116\end{array}$

$\begin{array}{ll}5.1 \text { Introduction } & 116\end{array}$

$\begin{array}{ll}5.2 \text { Sedimentary Sequences } & 118\end{array}$

5.2.1 Description of Sequences 118

$\begin{array}{ll}5.2 .2 \text { Interpretation of Sequences } & 121\end{array}$

5.3 Diatom Analysis 123

$\begin{array}{ll}\text { 5.3.1 Introduction } & 123\end{array}$

5.3.2 Definition of Diatom Assemblage Zones 123

5.3.3 Interpretation of Diatom Assemblage Zones $\quad 124$

5.3.4 Reconstruction of Waterbody Type 127

5.3.5 Application of Salinity Transfer Function $\quad 129$

5.4 Paleoenvironmental Reconstruction 131

5.5 Environmental Transitions 136

5.5.1 Introduction 136

5.5.2 Description of Transitions 137

5.5.3 Change in Elevation 141

5.5.4 Age of Transitions 143

5.6 Conclusions 143 
SIX Lake Kohangapiripiri: Catchment Disturbance and Water Depth Change over 7500 Years 145

Summary

6.1 Introduction 146

6.2 Sedimentary Sequence 148

6.2.1 Description of Sequence 148

6.2.2 Interpretation of Sequence $\quad 151$

6.3 Diatom Analysis 151

6.3.1 Introduction $\quad 151$

6.3.2 Definition of Diatom Assemblage Zones 151

6.3.3 Interpretation of Diatom Assemblage Zones $\quad 152$

6.3.4 Reconstruction of Waterbody Type 154

6.3.5 Application of Salinity Transfer Function 156

6.4 Paleoenvironmental Reconstruction 158

6.5 Environmental Transitions 161

6.5.1 Introduction 161

6.5.2 Description of Transitions 162

6.5.3 Change in Elevation 165

6.5.4 Age of Transitions 165

6.5.5 Effects of the 1855 AD Earthquake at Lake Kohangapiripiri 167

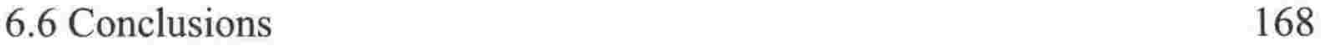

SEVEN A Paleoecological Contribution to Wellington's Paleosesimic Record $\quad 169$

7.1 Scope of Chapter 169

$\begin{array}{ll}7.2 \text { Introduction } & 169\end{array}$

7.3 Wellington's Paleoseismic Record 171

$\begin{array}{ll}\text { 7.3.1 Previous Work } & 171\end{array}$

Wairarapa Fault • Wellington Fault • Ohariu Fault •

Shepherds Gully - Pukerua Fault • Wairau Fault

(Northern Extension) - Other Earthquake Studies .

The Subduction Interface

7.3.2 Evidence of Earthquakes in the Sedimentary Record $\quad 178$

Introduction - Summary of Environmental Transitions .

Comparison of Ages with Previously Recognised Events

7.4 Conclusions 186

7.4.1 Summary of Results 186

7.4.2 Comments for Future Work 187

$\begin{array}{lr}\text { EIGHT Conclusions } & 193\end{array}$

$\begin{array}{ll}\text { Bibliography } & 197\end{array}$ 
APPENDICES $\quad \mathbf{2 1 0}$

I Core Logs 210

II Diatom Species List 235

III Environmental Preferences of Diatom Species 253

IV Plates of Diatom Species 267

V Environmental Variables for Calibration Set Sites 292

Appendices on Compact Disk in Back Pocket

VI Diatom Counts for Sedimentary Sequences

VII Diatom Counts for Calibration Set Sites 


\section{I S T OF F I G UR E}

1.1 Tectonic features of New Zealand.

1.2 Map of the Wellington Region showing major active faults and study sites.

1.3 Model illustrating use of a calibration set for paleoenvironmental reconstruction.

1.4 Siliceous components of a diatom cell wall.

1.5 Effects on a coastal waterbody of an earthquake involving vertical movement of base level.

2.1 The Russian sampler used to collect cores.

2.2 Plot showing increase in diatom species with increasing count totals.

2.3 Plot showing change in percentage of total assemblage for abundant species versus valve count.

2.4 Plot showing change in percentage of total assemblage for less abundant species versus valve count.

2.5 Idealised scheme for diatom assemblage shifts occurring as a result of relative sea level rise.

3.1 Datasets and mathematical steps required for quantitative paleoenvironmental reconstruction.

3.2 Idealised species response models.

3.3 Idealised uplifted coastal waterbody gradient.

3.4 Map showing location of calibration set sites around the coast of New Zealand.

3.5 Calibration set sites arranged according to waterbody type.

3.6 Frequency histogram of diatom valve concentrations for death assemblage samples in the calibration set.

3.7 Concentration of diatom valves in samples along the coastal gradient.

3.8 Species richness of diatom samples along the coastal gradient.

3.9 Plot showing species and proportions of counts in death assemblage samples made on species not present in associated living samples.

3.10 Detrended Correspondence Analysis species plot. 65

3.11 Detrended Correspondence Analysis site plot.

3.12 Detrended Canonical Correspondence Analysis sites, species and environmental variables.

3.13 Principal Components Analysis of environmental variables. 
3.13 Environmental variable plot from a Canonical Correspondence $\begin{array}{ll}\text { Analysis. } & 70\end{array}$

3.15 Partitioning of variance in the diatom data. 73

3.16 Spatial coastal waterbody gradient for the calibration set. 74

3.17 Observed salinity versus diatom inferred salinity. 77

3.18 Plots of residuals for Weighted Averaging (WA) and WA-tol. 78

3.19 WA salinity optima of species in the calibration set plotted $\begin{array}{ll}\text { against their salinity tolerances. } & 79\end{array}$

3.20 Scatter plots showing species abundance along the measured salinity gradient.

3.21 Jackknifed estimates of salinity optima and tolerances for species in the calibration set with N2 $>3$.

4.1 Oblique aerial view of Taupo Swamp looking north. 87

4.2 Map of Taupo Swamp. $\quad 89$

4.3 Core logs of TS97-1 and TS98-2 from Taupo Swamp. 91

4.4 Sediment accumulation rate curves for TS97-1 and TS98-2. 92

4.5 Diatom assemblages of core TS97-1. 96

4.6 Diatom assemblages of core TS98-2. 97

4.7 DCA of calibration set sites with fossil samples from Taupo Swamp incorporated passively.

4.8 Salinity reconstructions for TS97-1 and TS98-2.

4.9 Paleoenvironments that have existed in Taupo Valley over the last 5500 years.

4.10 Composite plot showing summary of paleoenvironmental evidence for environmental transitions at Taupo Swamp.

4.11 Inferred tidal elevation of the sedimentary sequences at Taupo Swamp.

5.1 Oblique aerial view of Okupe Lagoon looking northwest. 115

5.2 Map of Okupe Lagoon. 117

5.3 Core logs of OL97-5 and OL97-3 from Okupe Lagoon. 119

5.4 Sediment accumulation rate curves for cores OL97-5 and OL97-3 based on calibrated radiocarbon age estimates. 120

5.5 Some macrofossils and inclusions removed from core OL97-5. 122

5.6 Diatom assemblages of core OL97-5. 125

5.7 DCA of calibration set sites with fossil samples from OL97-5 incorporated passively into the analysis. 128

5.8 Salinity reconstructions for OL97-5. 130

5.9 Reconstruction of paleoenvironments that have existed at the Okupe Lagoon site over the last 5000 years. 134

5.10 Raised wave-cut platform northwest of Okupe Lagoon. 138 
5.11 Composite plot showing summary of paleoenvironmental evidence for environmental transitions at Okupe Lagoon.

5.12 Inferred tidal elevation for units in the sedimentary sequences at Okupe Lagoon.

6.1 Oblique aerial view of Lake Kohangapiripiri looking east with Lake Kohangatera in the background.

6.2 Map of Lake Kohangapiripiri. 147

6.3 Core log of KP99-1\&2 from Lake Kohangapiripiri. 149

6.4 Sediment accumulation rate curves for KP99-1\&2. 150

6.5 Diatom assemblages of core KP99-1\&2. 153

6.6 Detrended correspondence analysis of calibration set sites with fossil samples from Lake Kohangapiripiri incorporated passively. 155

6.7 Salinity reconstructions for KP99-1\&2. 157

6.8 Reconstruction of paleoenvironments that have existed at the Lake Kohangapiripiri site over the last 7500 years. $\quad 160$

6.9 Composite plot showing summary of paleoenvironmental evidence for environmental transitions at Lake Kohangapiripiri. $\quad 163$

6.10 Elevation of the sedimentary sequence at Lake Kohangapiripiri relative to present mean sea level.

7.1 Maps showing major active faults and paleoseismic localities mentioned in text.

7.2 Ages of earthquakes in the Wellington region identified in previous studies.

7.3 Ages of environmental transitions recognised in this study compared with ages of previously recognised earthquake events in the Wellington region.

7.4 Inferred earthquake event in the sedimentary sequence of Taupo Swamp.

7.5 Temporal coastal waterbody gradient for the Holocene sedimentary sequences. 


\section{I S T O F T A B E S}

2.1 Radiocarbon age results.

2.2 Definitions of sedimentary units according to percentages of sand and organic matter.

2.3 Habitat categories into which diatom species are grouped.

2.4 Salinity categories into which diatom species are grouped.

3.1 Calibration set sites tabulated according to waterbody type and salinity.

3.2 List of environmental variables measured for the calibration dataset.

3.3 Comparison of eigenvalues and gradient lengths for Detrended Correspondence Analysis and Detrended Canonical Correspondence Analysis of the calibration set data.

3.4 Percentage variance of the species data explained by each environmental variable.

3.5 Comparison of Canonical Correspondence Analysis results. $\quad 72$

3.6 Weighted averaging regression statistics for the calibration set. 76

3.7 Modern analogue technique statistics for the calibration set. $\quad 85$

4.1 Definition of diatom assemblage zones for cores TS97-1 and TS98-2.

4.2 Summary of paleoenvironmental evidence for Taupo Swamp with inferred depositional environments.

5.1 Definition of diatom assemblage zones for cores

OL97-5 and OL97-3.

5.2 Summary of paleoenvironmental evidence for Okupe Lagoon with inferred depositional environments.

6.1 Definition of diatom assemblage zones for core KP99-1\&2.

6.2 Summary of paleoenvironmental evidence for Lake Kohangapiripiri with inferred depositional environments.

7.1 Summary evidence for the nature of change occurring at each environmental transition.

7.2 Summary of the seven intervals of time in which surface rupture earthquakes have occurred in the Wellington region. 


\section{CHAPTER ONE}

\section{Introduction}

\subsection{AIMS}

There are two main aims to this thesis:

1. To develop a modern diatom calibration set to aid paleoenvironmental reconstruction of coastal sequences in New Zealand.

2. To contribute to Wellington's paleoseismic record through detection of evidence for large Holocene earthquakes in the sedimentary record.

\subsection{PURPOSE OF RESEARCH}

New Zealand is situated on the boundary between the Pacific and Australian tectonic plates that are moving relative to each other. One result of this movement is that the country is crossed by a number of major active faults, many of which are known to move coseismically, that is during large earthquakes. The devastating effect of large earthquakes on populated areas is well known from a few examples in New Zealand and many examples internationally.

Paleoseismology, or the study of past earthquakes, is able to provide estimates of the timing, location and magnitude of past large earthquakes. This information is crucial to the development of hazard plans aimed at reducing damage caused by earthquakes. Paleoseismic studies are especially important in New Zealand because the historic record of earthquakes is only about 160 years long. Van Dissen and Berryman (1996) show that estimates of earthquake hazard for the Wellington region based solely on the historic record, underestimate the intensity of all events with return times of greater than 50 years. Geological studies of subsurface fault ruptures, offset terraces and raised beaches provide a history of some large earthquakes that have occurred in New Zealand over the last 10,000 years. However, sizeable gaps in the record still exist for most active faults in the country. 
The Wellington region probably represents the greatest seismogenic risk in New Zealand. The capital city and a large population base are situated in close proximity to five major active faults. There is a fairly extensive record of surface rupture earthquakes for the last 1000 years in the Wellington region. However this represents only the last one or two earthquakes on each fault, and for several faults that are considered active, no events have been identified. On faults for which longer records exist, ages of events do not correlate well between different localities. Therefore developing a more comprehensive paleoseismic history is a high priority.

One source of information with potential to expand this history is the sedimentary record, an area where little work has been undertaken in this country. Northern Hemisphere studies have successfully used the sedimentary record to identify earthquakes through recognition of distinctive sedimentary layers caused by seismic shaking or, in coastal areas, the sudden relative sea level change resulting from vertical coseismic movement. The first aim of this research is to investigate the Holocene sedimentary record in Wellington for evidence of earthquakes and to determine how this evidence can contribute to the existing paleoseismic record.

High-resolution paleoenvironmental reconstructions are obtained through analysis of diatom microfossils in the sediment. However there is a lack of ecological diatom work in the coastal environment and in New Zealand in general. This lack of information precipitated the second major part of this research, which is an ecological survey of modern diatoms and associated environmental variables in coastal waterbodies around New Zealand. The dataset is used to enable quantification of aspects of the above sedimentary sequences, to contribute to literature on preferences of diatoms in the coastal zone, and to initiate transfer function style reconstruction in New Zealand.

\subsection{SETTING}

\subsubsection{Tectonic Setting of New Zealand}

New Zealand is a young, tectonically active landmass that exists above sea level largely because of collision between the Pacific and Australian tectonic plates (Fig. 1.1). The Pacific-Australian plate boundary trends in a northeast-southwest direction along the length of the country and involves abutment of oceanic and continental crust in various arrangements. In the North Island of New Zealand oblique plate convergence is accommodated offshore by subduction of oceanic Pacific Plate beneath the Australian Plate in the Hikurangi subduction zone, and onshore by movement along large strike-slip faults. In the central South Island both plate margins consist of continental crust. Motion parallel to the plate 


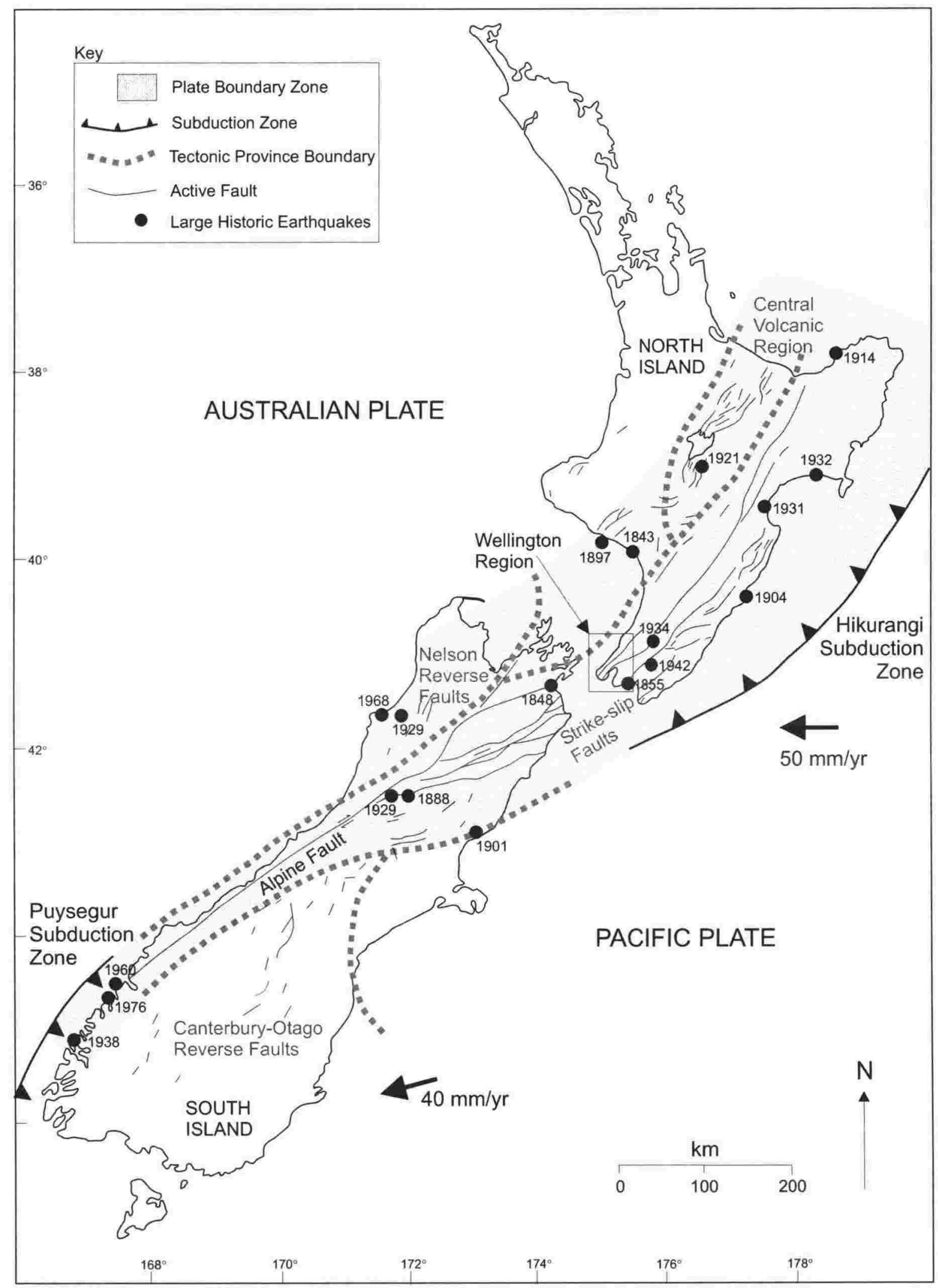

Fig. 1.1. Tectonic features of New Zealand. The Plate Boundary Zone is a zone of deformation resulting from collision of the Pacific and Australian Plates. Relative plate motions and large historic earthquakes are from Walcott (1981). Active faults are grouped into tectonic provinces of similar faulting style (Berryman and Beanland, 1991). 
boundary occurs along the Alpine Fault, a $>500 \mathrm{~km}$ long dextral strike-slip fault. Motion perpendicular to the boundary is achieved by crustal thickening with uplift of the Southern Alps at rates of up to $11 \mathrm{~mm} / \mathrm{yr}$ (Walcott, 1998). Beneath Fiordland, oceanic crust of the Australian Plate is subducted beneath the Pacific Plate in the Puysegur Subduction Zone.

Relative plate motion causes seismic and aseismic deformation of the New Zealand landmass throughout the plate boundary zone (Fig. 1.1), a zone about 400 $\mathrm{km}$ wide in the North Island and $100 \mathrm{~km}$ wide in the South Island (Walcott, 1981). The Pacific Plate moves westward relative to the Australian Plate at rates ranging from $40 \mathrm{~mm} / \mathrm{yr}$ to $60 \mathrm{~mm} / \mathrm{yr}$ and a significant proportion of this movement is thought to accumulate as elastic strain and be released as earthquakes (Walcott, 1981). There are numerous seismogenic sources in New Zealand including the subduction interface itself, reverse faults of the North Island East Coast, short normal faults of the Central Volcanic Region, major strike-slip faults of central New Zealand and reverse faults of Northwest Nelson and Central Otago (Berryman and Beanland, 1991). The largest historic earthquakes occur within the Axial Tectonic Belt, a $100 \mathrm{~km}$ wide zone of high strain rates that extends from the southern South Island across southern and eastern North Island (Walcott, 1981). Historically the central Alpine Fault is an area of low seismicity so movement is either occurring aseismically or strain is accumulating to be released in a future large earthquake (Walcott, 1998).

\subsubsection{Tectonic Setting of Wellington}

Wellington is located near the southern end of the Hikurangi subduction zone and within a zone of major active faults. The North Island Dextral Fault Belt comprises two main fault strands and many associated faults all of which show evidence for repeated movement during the Quaternary (Beanland, 1995). In the Wellington region the fault belt consists of five major active faults, the Wairarapa, Wellington, Ohariu, Shepherds Gully - Pukerua and Wairau Faults (Fig. 1.2). Fault movement is predominantly dextral strike-slip, accommodating $60-90 \%$ of boundary-parallel plate motion (Van Dissen and Berryman, 1996). A smaller component of vertical movement also occurs (Begg and Mazengarb, 1996) and heights of Last Interglacial marine terraces indicate that net uplift is occurring over the region (Pillans, 1990). No evidence of creep has been found on any of Wellington's faults (Van Dissen and Berryman, 1996), so it is likely that much of the movement is achieved coseismically.

Earthquakes associated with the subducting Pacific Plate are frequent in Wellington but most are relatively small. The most frequent damaging earthquakes appear to result from surface rupture of one of the major faults in the region. Only one such earthquake has occurred in historic times, in 1855 on the Wairarapa Fault. Historical accounts of this earthquake have recently been studied in detail because there were limited means by which to measure the earthquake 


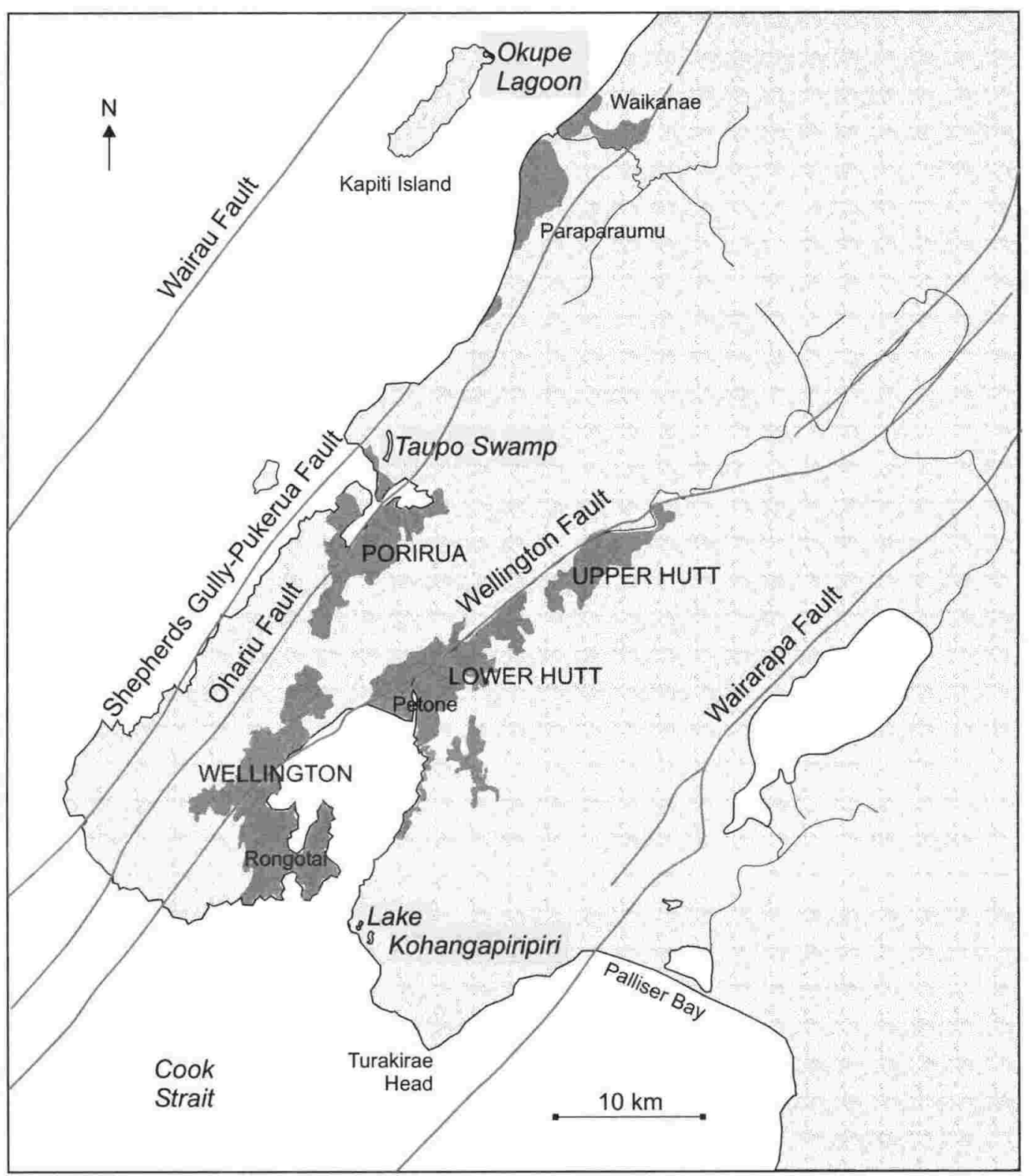

Fig. 1.2. Map of the Wellington Region showing major active faults and study sites (highlighted). 
in 1855 and there is valuable information to be gained from the event (Grapes and Downes, 1997). The earthquake was felt over most of New Zealand with a maximum intensity of MM IX-X in the lower North Island. Severe damage occurred throughout central New Zealand but because of the small population and predominance of wooden buildings at the time, there were only 5-10 deaths. Magnitude is estimated to have been M 8-8.2 +/- 0.5 and the earthquake resulted in uplift and tilting of $5000 \mathrm{~km}^{2}$ of land (Grapes and Downes, 1997). Recent mapping of the 1855 scarp indicates a rupture length of at least $148 \mathrm{~km}$, a dextral horizontal offset of 11-13 m and uplift to the northwest of $6.5 \mathrm{~m}$ near Turakirae Head decreasing northwards (Grapes, 1999).

\subsubsection{Wellington's Paleoseismic Record}

In the Wellington region there are numerous examples of laterally offset streams, ridges and terraces indicating recent movement along faults. Paleoseismic work has focused on these features, trench studies of major faults, and on interpretation of raised Holocene beach ridge sequences at Turakirae Head, Rongotai and Petone. These studies indicate that large surface rupture earthquakes such as the 1855 Wairarapa Fault event have been a feature of Wellington's history throughout the late Quaternary. On the Wairarapa Fault there is geomorphic evidence for at least seven earthquakes of similar magnitude in the late Quaternary (Grapes, 1999). On the Wellington Fault there is evidence of five large surface rupture events in the last 14,000 years and several much older events (Berryman, 1990). Trenches across the Ohariu Fault show evidence for at least five surface rupture earthquakes but timing is only known for the last event (Heron et al., 1998). No earthquakes have been dated on the Shepherds Gully Pukerua Fault or the northern section of the Wairau Fault but both are known to be active. The Wairau Fault is a major active strike-slip fault in the South Island so a surface rupture earthquake on the northern section has serious implications for Wellington. A full description of previous paleoseismic work is presented in Chapter Seven.

\subsubsection{Study Sites}

Around the Wellington coastline are several waterbodies that are thought from geomorphological studies to have been uplifted above mean sea level in large earthquakes during the Holocene. Three sites were selected to examine for evidence of past large earthquakes. Sites are located on different fault bounded blocks and each is at a different stage of isolation from the sea (Fig. 1.2). Taupo Swamp is a fresh water wetland about four metres above sea level lying between the Shepherds Gully - Pukerua and Ohariu Faults. Okupe Lagoon is a brackish lagoon about two metres above mean sea level that is surrounded by raised gravel barriers. It is situated on the northeastern end of Kapiti Island between the northern extension of the Wairau Fault and the Shepherds Gully - Pukerua Fault. Lake Kohangapiripiri is a fresh water lagoon on Wellington's south coast almost 
half way between the Wellington and Wairarapa Faults. Small waterbodies with small catchments were chosen to increase the possibility that they would contain continuous Holocene sedimentary sequences. In large estuarine systems, floods or tidal currents can continuously deposit and erode packages of sediment whereas smaller lagoonal environments are likely to behave more like enclosed lakes.

\subsection{BACKGROUND TO APPROACH}

\subsubsection{Earthquakes in the Sedimentary Record}

\section{Introduction}

In the mid to late nineteenth century geologists started describing surface ruptures along fault lines resulting from contemporary earthquakes. By the early twentieth century seismographs were being used to record earthquakes. These could provide records of present day seismicity, and historical evidence provided records of large earthquakes for a few centuries or even millennia back in time but usually without reference to earthquake source. Only geological studies had the potential to provide records of earthquakes on timescales long enough to enable characterisation of individual faults. However it was not until the mid-twentieth century that paleoseismology started to be regularly used (Yeats and Prentice, 1996). Development of paleoseismology was much enhanced with the availability of radiocarbon dating, enabling quantitative estimates of slip rates, recurrence intervals, or timing of individual earthquakes to be calculated. Radiocarbon dating is still commonly used in paleoseismology, but a higher precision technique is required to differentiate sequences of earthquakes occurring on the same fault over a few decades (Yeats and Prentice, 1996).

Paleoseismology is based around the identification of linear or planer geomorphic features that have been offset by an active fault, beach ridges or terraces that have been raised or lowered relative to sea level, and the relationship between fault strands and local stratigraphy in excavations across fault scarps. The sedimentary record of lakes and coastal waterbodies can also be utilised as a source of information about past large earthquakes. Sims (1975) showed that deformational structures preserved in sand and silt layers within lake sediments can be attributed to liquefaction during earthquakes under certain circumstances. Doig (1990) and Karlin and Abella (1996) documented silty layers in organic-rich lake sediments that are attributed to turbidite deposition resulting from earthquake-induced landslides. Anomalously coarse-grained layers in lake sediments have also been attributed, in the coastal zone, to deposition by earthquake-induced tsunami (Hutchinson et al., 2000). Development of high-resolution paleoenvironmental reconstruction techniques in sea level studies, has opened another avenue for paleoseismology, that is the recognition of earthquake induced uplift and subsidence in sedimentary sequences of coastal environments. 


\section{Pacific Northwest of America and Canada}

In Washington, on the west coast of North America, Atwater (1987) proposed that six couplets of peat overlain by intertidal mud represented burial of lowland soils in repeated coseismic subsidence events. On three occasions burial was associated with deposition of sand sheets possibly caused by tsunami. Fossil trees showing normal growth until the year of death illustrated suddenness of submergence, as did preservation of leaves and stems that were buried before decomposition could take place (Atwater and Yamaguchi 1991). At some sites evidence of strong ground shaking was present in the form of sand intrusions and vented sand volcanoes (Atwater, 1992). Similar evidence for coseismic subsidence has since been documented from British Columbia (Clague and Bobrowsky, 1994; Mathewes and Clague, 1994), Washington (as above), and Oregon (Darienzo and Peterson, 1990; Darienzo et al., 1994; Nelson et al., 1996a). Geological evidence suggests that magnitude eight or greater earthquakes occur on the boundary between the subducting Juan de Fuca plate and the overriding North American plate roughly every 500 years but with a range of between 200 and 1300 years (Clague, 1997).

Long and Shennan (1994) raised the point that organic-inorganic sediment couplets superficially very similar to those attributed to repeated coseismic subsidence in the Pacific Northwest, exist on passive margin coasts where they are attributed to aseismic coastal processes. They called for rigorous testing of the 'earthquake deformation cycle' through application of litho- and biostratigraphic techniques to document in detail the sea level changes taking place in these sediments. In particular they recommended use of pollen and diatom microfossil analysis to assess sea level tendency (proximity of marine conditions) within and across sedimentary units.

Ecological studies of estuarine environments in the Pacific Northwest have been undertaken to improve reconstruction of past changes in such environments (eg, Nelson and Kashima, 1993; Hemphill-Haley, 1995a). Various studies successfully used microfossils (Clague and Bobrowsky, 1994; Hemphill-Haley, 1995b; Nelson et al., 1996a) and traditional sea level techniques (Shennan et al., $1996 ; 1998)$ to identify evidence of rapid sea level rise and confirm the coseismic nature of these changes. Hemphill-Haley (1996) also used diatom microfossils to confirm a seaward source for units inferred to have been deposited by tsunami. The advantage of using microfossils for interpreting coastal sequences was highlighted by Mathewes and Clague (1994) who showed that microfossil analysis could detect small amounts of coseismic movement that left little or no trace in lithology.

Nelson et al. (1996b) provide five criteria by which to assess sedimentary sequences that may have a coseismic origin: suddenness and amount of 
submergence, lateral extent of submerged wetland soils, coincidence of submergence with tsunami deposits and synchroneity of submergence at different sites. The more of these criteria that are met at increasing numbers of sites, the greater the likelihood of a coseismic cause of change. Although designed in the Pacific Northwest, these criteria should be applicable, with minor modifications, to assessment of coastal sedimentary sequences in any seismically active part of the world.

\section{New Zealand}

Paleoseismological work in New Zealand has concentrated on measurement of physical features such as displacements along faults and raised beach ridges. The sedimentary record has been used in fault trench studies where the relationship between local stratigraphy and fault splays provides evidence about recent fault movement. It has also been used in a paleoenvironmental context to determine overall rates of uplift or subsidence (eg, Hull, 1986; Ota et al., 1988, 1989 and 1995). However it is only very recently that workers have started to use the sedimentary record to identify individual coseismic events using highresolution paleoenvironmental reconstruction techniques.

Evidence of a known historical earthquake, the 1855 Wairarapa earthquake, was detected in the sedimentary record of Wellington Harbour using grain size analysis (Dunbar et al., 1997). Chague-Goff et al. (2001) and Zachariasen et al. (2000) used multidisciplinary approaches involving foraminifera, spores, pollen, diatoms and geochemistry to identify possible subsidence events and a tsunami deposit on the east coast of the North Island. Carter et al. (2000) used modern analogue work with benthic foraminifera to define sudden relative sea level changes likely to be the result of coseismic movement at several sites around the North Island. Hill (in Benson et al., 2001) identified earthquake events using diatom analysis of core sequences from a pull-apart basin lake on the Awatere Fault in the South Island. Coseismic events were identified as sudden changes in water depth and sediment input, and most were correlated with events recognised in a trench across the fault 500 metres away.

Several workers have attempted to identify tsunami in the sedimentary record and Goff et al. (2001) outline characteristics used to recognise such deposits. The occurrence of tsunami in New Zealand is not directly related to New Zealand's paleoseismic history because tsunami can be triggered by events other than earthquakes, or by earthquakes elsewhere in the world. However tsunami represent a risk to this country because of the long length of inhabited coastline. Goff and McFadgen (2001) consider that tsunami and associated earthquake events led to abandonment of the Palliser Bay coast by early Maori communities. Identification of tsunami in the fossil record is desirable to start to quantify this risk and for the additional information it can provide to the paleoseismic record. Goff et al. (1998), and Goff and Chague-Goff (1999) outline sedimentological 
evidence for tsunami triggered by the 1855 Wairarapa earthquake and three earlier earthquakes on the Wellington and Wairarapa Faults.

\subsubsection{Paleoecology}

Paleoecology provides the underlying philosophy on which this thesis is based. It is the study and description of past environments using geological and paleontological evidence. Seven principles of paleoecology as outlined by Birks and Birks (1980) are summarised below:

1. Paleoecology is a descriptive, historical science, it depends on inductive inferences and reasoning - one observation leads to another and extrapolations are made in an attempt to present generalisations about nature.

2. The method of multiple working hypotheses is applicable - one must consider as many reasonable explanations as possible, this will encourage seeking of new evidence, and lead to rejection of (hopefully) all but one working hypothesis.

3. Simplicity - let the simplest explanation suffice until more evidence is available that requires a more complex explanation.

4. A sound taxonomy and appreciation of evolutionary processes is essential.

5. The language of paleoecology is primarily that of biology and geology.

6. The data of paleoecology is often multivariate requiring multivariate mathematical methods to simplify and synthesise it prior to interpretation.

7. Uniformitarianism - the present is the key to the past.

Paleoecological evidence consists of physical and chemical characteristics of sediment and the type and preferences of fossils preserved in a sedimentary sequence. This information is collected and analysed to provide a description of the environment most likely to have produced the particular sedimentary deposit and fossil assemblage encountered. The paleoenvironmental reconstruction is tied to a chronology, often using radiocarbon dates, to enable the age and duration of the past environment to be determined. Certain assumptions are made in the reconstruction process, for example, unless evidence exists to the contrary, it is assumed that the fossil assemblage is representative of the initial living community. Processes of transport, dissolution, and redeposition have to be considered when interpreting a fossil deposit. Features such as concentration and preservation of fossils can be used to judge whether the assemblage is a fair representation.

Limitations of the fossil record mean that paleoecology is not directly comparable to modern ecology. To carry out a typical ecological study of species-environment 
relationships at some time in the past, independent evidence as to the nature of the past environment would be required. This is not usually extensive enough in the fossil record and all information, including organisms present, has to be used to deduce the nature of the environment. Quaternary paleoecology has the strongest links to modern ecology because many organisms are still extant and environmental preferences are unlikely to have changed dramatically. This enables modern ecological studies of the environmental preferences and tolerances of species to be utilised directly in paleoenvironmental reconstruction from a fossil assemblage.

Use of modern ecological datasets enables quantification of reconstructions in comparison with traditional paleoecological techniques which usually result in qualitative or descriptive reconstructions such as 'a brackish lagoon of shallow water depth existed in a climate warmer than that of today'. The need to quantify reconstructions has arisen recently in several sub-disciplines of paleoecology. For example the acid rain debate of the 1980 s required quantification of recent lake acidification and the ability to objectively test hypotheses about the cause of acidification (Birks, 1998). Quantification is also required in paleoclimate studies because of their increasing use for predicting future climate change and because of the high resolution of data required for effective decision-making. These demands on paleoecological research, as well as developments in multivariate statistics and computer technology, have resulted in the development of procedures for achieving such reconstructions (Birks, 1998).

Modern ecological datasets specifically designed to contribute to paleoenvironmental reconstruction are called 'training' or 'calibration' sets. Diatom assemblages and environmental variables collected from a large number of waterbodies are used to summarise relationships between species and the environment. Species-environment relations derived in the present are applied to fossil diatom assemblages to estimate characteristics of past environment (Fig. 1.3). In this context, the ecological response of species to their environment is referred to as a 'transfer function'- derived from modern data using regression analysis and applied to fossil data using calibration. Calibration sets are being used increasingly in Quaternary reconstruction work for several reasons:

1. They can be designed to aid reconstruction of a particular environmental variable of interest.

2. Multivariate statistical methods are now available that enable quantitative estimates of past environment to be derived.

3. Modern samples are collected from locations similar to that of the fossil environment so application of modern preferences to fossil species is more robust than using preferences from remote and varied sources. 

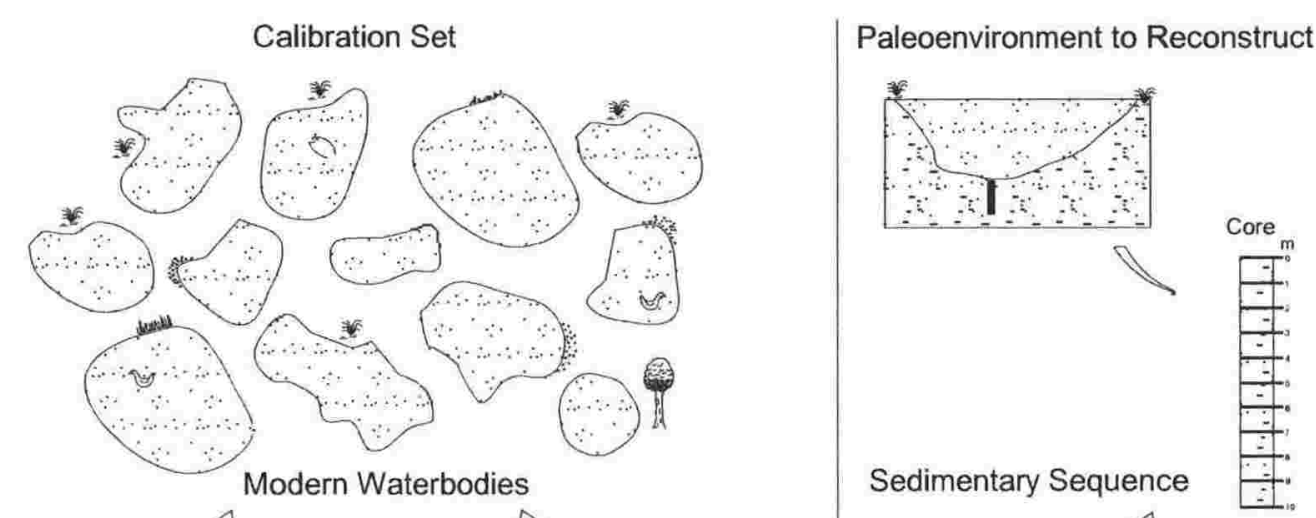

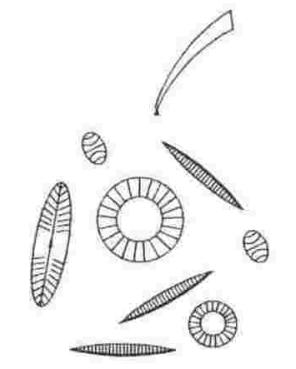

Modern Diatoms
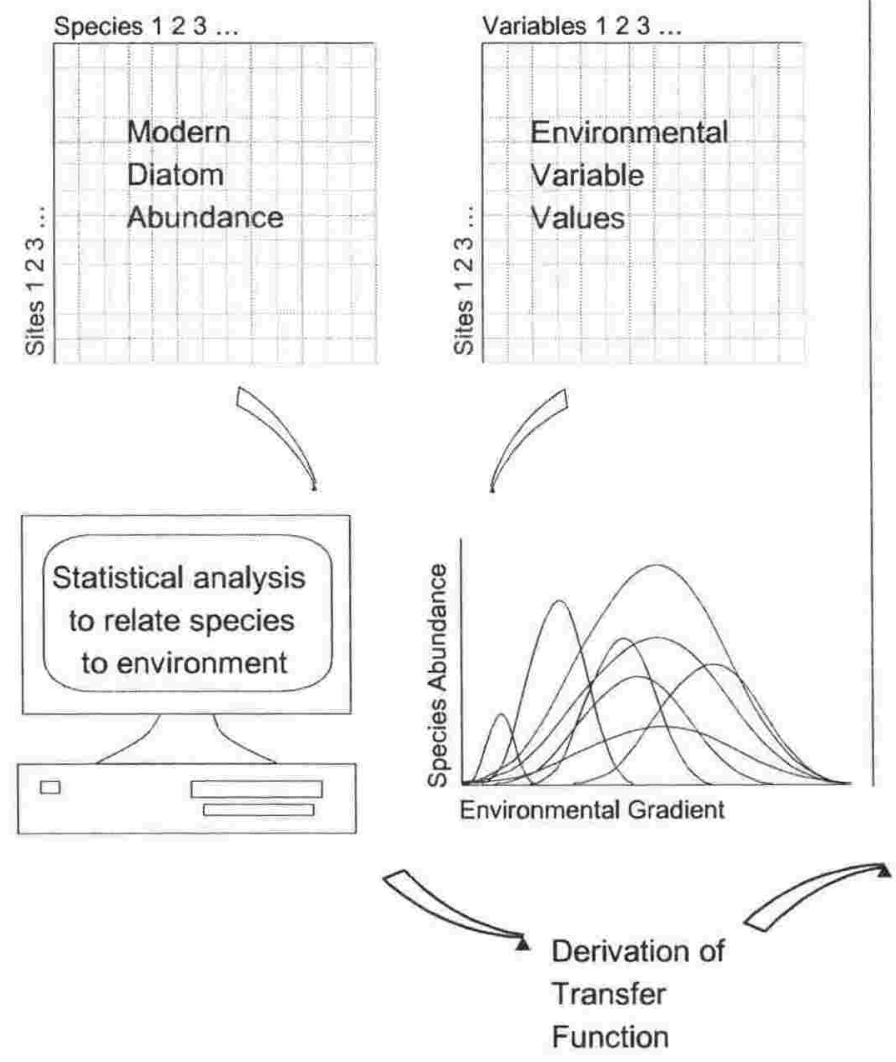

Environmental Variables
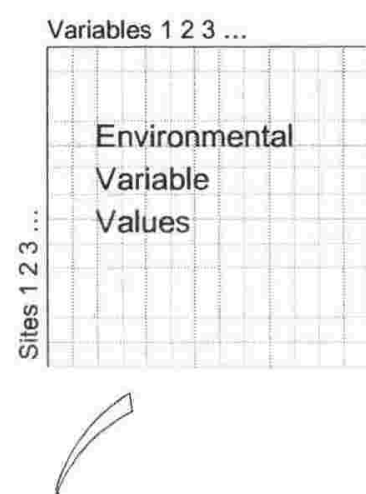

Application of Transfer Function to fossil diatoms to derive estimates of past environment

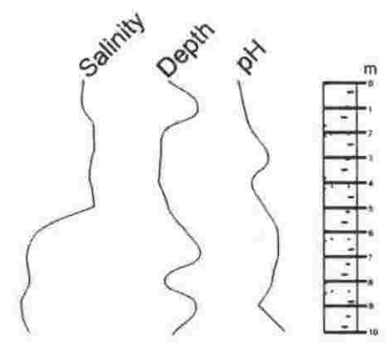

Fig. 1.3. Model illustrating use of a calibration set for paleoenvironmental reconstruction. 


\subsubsection{Diatom Analysis}

Diatoms are single-celled, microscopic algae that live in aquatic environments either as free cells, colonies, chains, or attached to a substrate. Apart from a few rare species that can live heterotrophically in the dark, diatoms photosynthesize so they need light and some moisture to live. Within these restrictions they can live almost anywhere, in fresh and salt water, from the open ocean through to damp soil. Most diatoms are between 0.005 and $0.1 \mathrm{~mm}$ in size so they can only be seen with the naked eye when growing in large numbers where they form the brown slime seen in puddles, on rocks in riverbeds, or in shallow water around lake margins. The most distinctive feature of diatoms is the cell wall (frustule), which is a fairly rigid structure made predominantly of silica with a thin organic coating. Cell contents are effectively enclosed by a glass box with cell growth occurring by movement apart of the base of the box and its lid (valves) and addition of further siliceous components (girdle bands) to the walls of the box (Fig 1.4). Each diatom species has a uniquely structured frustule enabling identification to species level under a microscope.

Diatoms are important for many reasons, not least for the possibility that they account for a fifth of the world's carbon production, more than that of all the world's tropical rainforests (Mann, 1999). Diatomite, the sedimentary rock formed by deposition of concentrated diatom frustules, has numerous and diverse commercial applications such as beer and wine filtration, building materials, pesticides and as an anti-caking agent (Harwood, 1999). In the environmental and earth sciences, applications result from five main attributes of diatoms:

1. Frustules are taxonomically diagnostic

2. Frustules are often preserved in large numbers after death

3. Diatoms are present in a wide range of environments

4. Species are sensitive to a wide range of environmental factors

5. Diatoms respond rapidly to environmental change

Living diatoms are used extensively to monitor water quality. Fossil diatoms are used as biostratigraphic markers in many geological applications because they have been present in the fossil record since the Cretaceous. Fossil diatoms of the Quaternary are widely used for reconstructing past environments. Diatom species in a fossil assemblage are identified, counted, and their preferences analysed to enable reconstruction of particular environmental variables.

A very early application of diatom analysis to a paleoenvironmental problem was tracking isostatic rebound in Scandinavia by identifying marine to freshwater transitions in coastal lakes. This work continues today with increasing numbers of studies continually refining sea level curves for different areas (eg, Stabell, 1980; Kjemperud, 1981 and 1986; Solem and Solem, 1997). More recently, extensive use has been made of diatom analysis in the acid rain debate in Europe. The sensitivity of diatoms to lake water $\mathrm{pH}$ enabled them to be used to determine preindustrialisation acidity levels and thereby ascertain whether acidification was a 


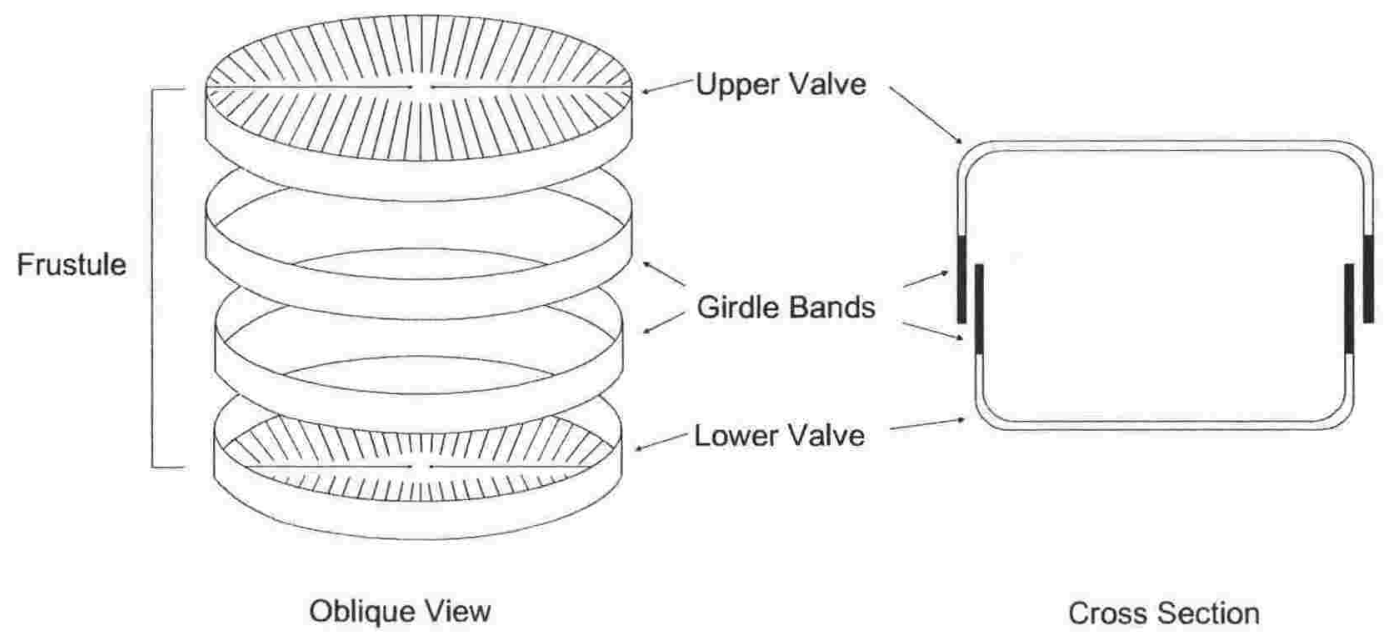

Fig. 1.4. Siliceous components of a diatom cell wall. 
natural process or a result of increasing pollution (Battarbee, 1994). Diatom

analysis has only very recently started to be used in the field of paleoseismology, but has already provided independent evidence for coseismic submergence events and tsunami on the North American west coast (Hemphill-Haley, 1995a) as well as being a useful addition to many paleoseismological studies.

In New Zealand diatoms have been used in water quality studies, biostratigraphic work, and paleoenvironmental reconstruction. A number of surveys of fresh water diatoms exist (Foged, 1979; Cassie, 1989) and a checklist of New Zealand diatoms is currently being updated (Cassie, 1984). However, apart from siterelated studies such as those of Porirua Harbour (Stidolph, 1980) and Kapiti Island (Stidolph, 1985), there have been few surveys of brackish and marine diatoms and there is a general lack of ecological work on species preferences. The calibration set collected as part of this research will provide a measure of how transferable preferences developed in the Northern Hemisphere are to species living in New Zealand. It will also contribute to the use of diatoms for reconstruction of coastal sedimentary sequences.

\subsection{SUMMARY OF APPROACH}

This study attempts to detect the vertical component of movement during an earthquake as recorded by relative sea level and water depth changes in coastal waterbodies (Fig. 1.5). One of the greatest effects of a large earthquake on a coastal waterbody is likely to be a sudden relative sea level change. This would lead to changes to the connection with the sea, energy regime, sediment supply, water depth and water chemistry. These physical and chemical changes would result in large and immediate changes to flora and fauna. Strong shaking in a large earthquake could also cause deformation of sediments, liquefaction, and landslides in the catchment. Associated triggering of a tsunami could result in inundation of the waterbody with marine water, sediment, and allochthonous flora and fauna.

Some evidence of such catastrophic changes is likely to be preserved in the sedimentary record. Past environments are reconstructed from several sites and environmental transitions are investigated to determine the history of such occurrences. Paleoecological techniques (Chapter Two) used to reconstruct past environment include sedimentology for physical features and diatom analysis for ecological aspects. Modern diatom assemblages and associated environmental variables are collected from 50 coastal sites around New Zealand to provide ecological information about diatoms living in the coastal zone at present (Chapter Three). Emphasis is placed on use of this dataset for paleoenvironmental 


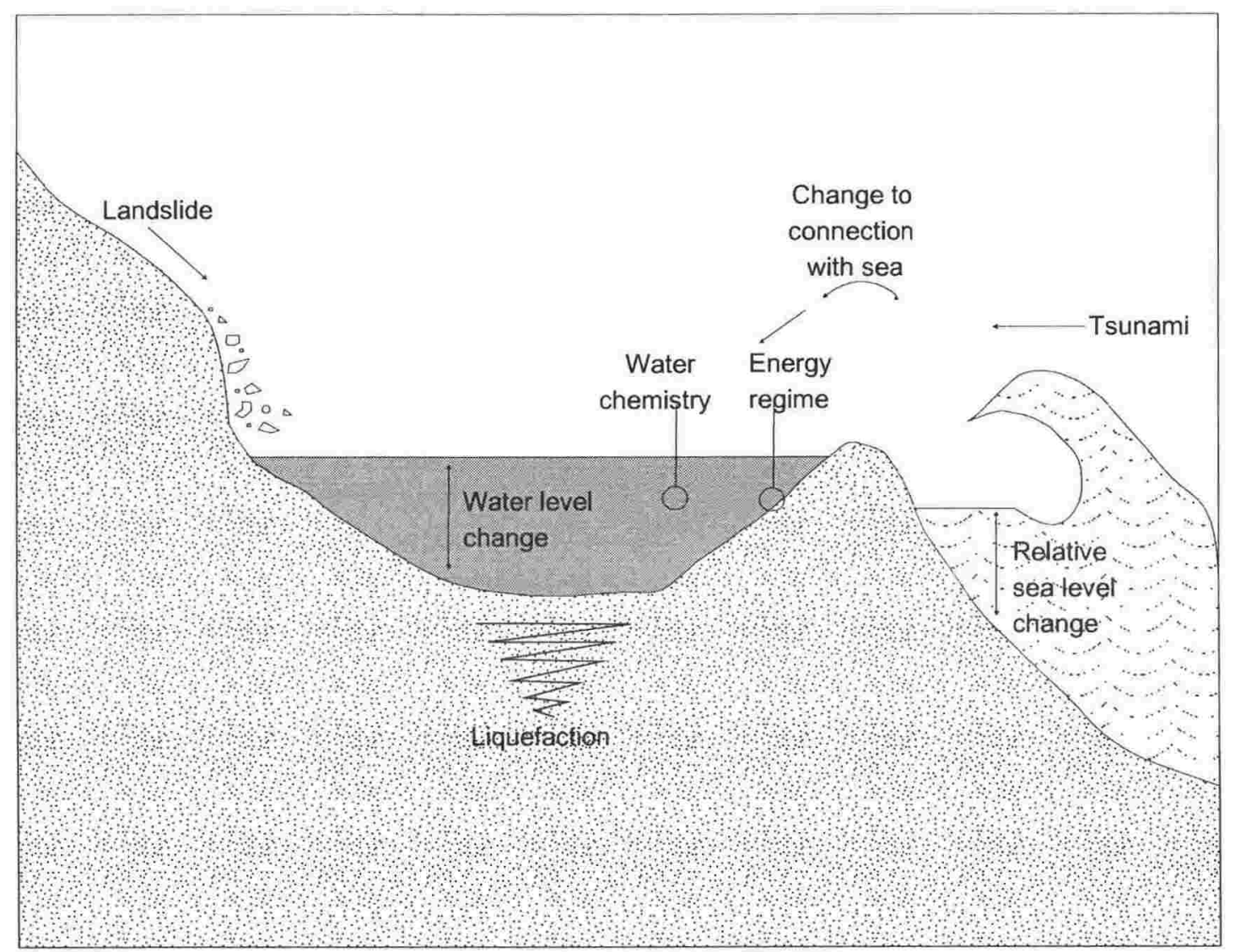

Fig. 1.5. Effects on a coastal waterbody of an earthquake involving vertical movement of base level. Evidence of such changes may be preserved in the sedimentary record. 
reconstruction. Multivariate statistical analysis is used to determine which variables strongly influence diatom species distribution and how this information can be used to aid reconstruction. Species preferences and images are presented (Appendix III and IV) to enable future workers to expand the modern dataset or use it for reconstruction.

Holocene paleoenvironmental histories are established for Taupo Swamp, Okupe Lagoon and Lake Kohangapiripiri (Chapters Four, Five and Six). Transitions in depositional environment are investigated to determine likely causes of change. Criteria used to differentiate between aseismic and coseismic causes of change include suddenness, amount, lateral extent, synchroneity and coincidence with tsunami deposits (Nelson et al., 1996b). Age estimates for each transition are provided by radiocarbon dating. Quantitative estimates of change in waterbody type and paleosalinity are derived through comparison of fossil samples with modern samples and through application of a transfer function derived from the modern dataset. Quantification of variables is considered useful because it provides numerical estimates of amount of environmental change, a key characteristic for determining whether change is coseismic in origin. Paleoseismic evidence at each site is compared with Wellington's existing paleoseismic record to determine what contribution such coastal sequences can provide (Chapter Seven). Conclusions of the thesis are provided in Chapter Eight. 


\section{CHA P T E R T W O}

\section{Paleoecological Methods}

\subsection{SCOPE OF CHAPTER}

This chapter covers paleoecological methods used to reconstruct past environment from Holocene sedimentary sequences. Many of the laboratory and diatom analysis techniques are common to samples in the modern calibration set, for example grain size, diatom processing, identification and counting. However most techniques used in construction of the modern calibration set are outlined in Chapter Three. In this chapter, material is subdivided into field techniques (section 2.2), laboratory techniques (section 2.3), diatom analysis (section 2.4) and methods of detecting earthquakes in the sedimentary record (2.5).

\subsection{FIELD TECHNIQUES}

\subsubsection{Site Selection}

Three broad criteria were used to select sites that were likely to consist of continuous Holocene sedimentary sequences containing evidence of large paleoearthquakes.

1. Coastal sites under ten metres in elevation were selected to enable marine-terrestrial transitional sequences to be studied.

2. Small, low energy waterbodies with small catchments were chosen to lessen the possibility of sequences being affected by movement of sediment within the waterbody or large scale erosional / depositional events from the catchment.

3. Sites where geomorphological or historic studies indicated that vertical coseismic movement had occurred were favoured over those without such evidence.

All waterbodies fitting these criteria in the wider Wellington region (from Levin in the west to Riversdale in the east) were probed or had preliminary cores taken from them. Wetlands and coastal lakes on the west coast north of Paekakariki are numerous but were discounted because dominant controls on their history are ponding by sand dunes and aggradation (Cotton, 1918) rather than tectonic influences. Waterbodies on the east coast are less numerous and contain only very short sequences. The three sites selected, Taupo Swamp, Okupe Lagoon and Lake 
Kohangapiripiri, have sequences that extend back 5000-7500 years (Table 2.1, this Chapter) in addition to the above criteria.

\subsubsection{Coring}

Sediment cores were taken using a Russian sampler (Jowsey, 1966). The sampler has a semi-cylindrical chamber of $5.5 \mathrm{~cm}$ diameter and $50 \mathrm{~cm}$ length (Fig. 2.1). It samples sediment by rotation of this chamber into an undisturbed section of sediment. The addition of extension rods between the sampler and a T-bar handle enables successive depths to be collected. Although the Russian sampler only collects $50 \mathrm{~cm}$ lengths at a time, continuous sampling can be achieved through careful measurement of depth of penetration at each stage. Design of the auger head and fin flange has been improved from the Hiller sampler to limit contamination by fibrous plant remains (Aaby and Digerfeldt, 1986). A compressed-air vibracorer and piston corer were tested at Taupo Swamp and Lake Kohangapiripiri but these corers were unable to penetrate sediment as deeply as the Russian sampler.

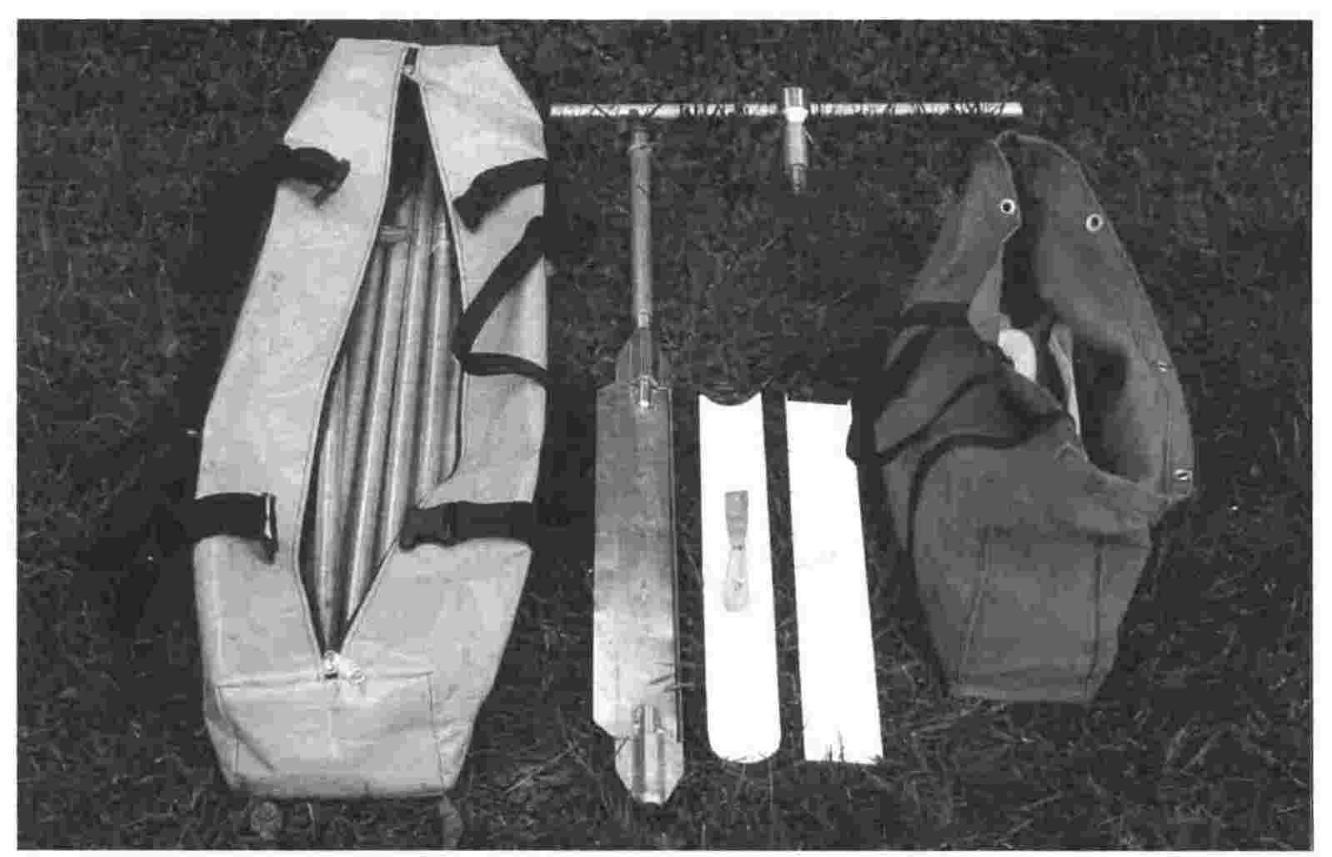

Fig. 2.1. The Russian sampler used to collect cores. The D-shaped core barrel, T-bar handle, extension rods and plastic pipe used to transport core material are pictured.

The Russian sampler was operated by two to four people working from a wooden platform on a muddy surface or in shallow water. At each site coring proceeded until the compactness of sediment or an increase in grain size prohibited deeper penetration. Basement rock was not reached at any site. Lengths of cores collected ranged from $200 \mathrm{~cm}$ at Okupe Lagoon to $650 \mathrm{~cm}$ at Lake Kohangapiripiri. Basic logs of core sections were recorded in the field; cores were sealed in plastic, 
labelled and placed in split plastic downpipe tubing for transport and storage. They were stored at two degrees Celsius in the School of Earth Sciences' cold room.

\subsubsection{Surveying}

Core positions were determined using aerial photos and NZMS 260 1:50,000 maps. Surveying was used solely to measure heights above mean sea level. A SOKKIA electronic distance meter (EDM) was used to measure elevation difference between core sites and a local point of known height. At Taupo Swamp an order-one benchmark (geodetic code: AC09) was used to calculate heights of all cores. At Lake Kohangapiripiri an order-five lighthouse (BOXV) was used and checked against a spot height in the vicinity. At Okupe Lagoon a trig station was used and checked against tidal measurements over a week. In each case the EDM was set up in a stable position where both the core sites and points of known height could be seen. Prisms were set at the same height as the EDM to simplify reduction of levels.

Errors taken into account included benchmark height errors (which are related to the square root of distance from the original datum), measurement errors and instrument errors. The low precision of benchmarks available at Okupe Lagoon and Lake Kohangapiripiri were factors that introduced the greatest errors.

\subsection{LABORATORY TECHNIQUES}

\subsubsection{Core Description and Sampling}

Cores were described and photographed in the sedimentology laboratory at Victoria University soon after collection (Appendix I). The convex side of each semi-cylindrical section of core was used for description because it was uncontaminated by fluids or mud that entered the core hole during sampling. Sedimentary units were defined for each core sequence using grain size, organic content, carbonate and visual core descriptions. Colour was described using Munsell Soil Charts. Contacts, sedimentary features, inclusions and macrofossils were also described. Core logs were summarised onto single pages to illustrate sedimentary units, contacts between units, and additional features such as macrofossils and mottles. These summary logs were checked against original cores to ensure no information had been lost or exaggerated in the process of combining all data and summarising logs.

Samples were removed for grain size analysis, diatom analysis and radiocarbon dating. At each sample position surface sediment was removed from the core with a spatula. Grain size samples of about $20 \mathrm{~g}$ of sediment were taken approximately every $50 \mathrm{~cm}$ or where there were obvious changes. Diatom samples of one gram 
of sediment were taken every ten centimetres and later at five centimetre intervals when significant horizons had been located. Large pieces of wood and shell, and occasionally intervals of organic sediment, were removed for radiocarbon dating. All samples were placed in weighed glass beakers, oven-dried overnight, then left for four hours to cool. They were weighed again and initial beaker weights were subtracted to provide dry sample weights.

\subsubsection{Radiocarbon Dating}

Radiocarbon dating of wood, shell and organic sediment samples was carried out to provide a chronology for the sedimentary sequences. Samples were washed in distilled water to remove extraneous material, oven-dried overnight, weighed and submitted to a radiocarbon dating laboratory for full pretreatment and analysis. An initial batch of samples from near the base of each core was submitted to determine the age of sequences being analysed. A final batch was submitted for dating once significant horizons had been identified. Most samples were dated by standard methods at the Waikato Radiocarbon Dating Laboratory. Samples of small volume or low carbon content were dated by Accelerator Mass Spectrometry at the Rafter Radiocarbon Laboratory, Lower Hutt.

A table of details of all 19 radiocarbon-dated samples is provided (Table 2.1). All dates occur in chronostratigraphic order in each core sequence except in two cases. There are two samples likely to have produced these results, and because of their questionable reliability they have been omitted from further use. Sample NZA 11828 was an organic sand sample, which is not a good material to radiocarbon date because the high-energy environment required to deposit sand is likely to deposit reworked older fragments of organic material as well. The risk of submitting this sample was taken because it was the only material available near one of the major environmental transitions at Okupe Lagoon. Sample Wk 8096, although a reliable dating material, was a long thin twig that could have been dragged to a greater depth during coring giving an age too young for the surrounding sediments. Sample Wk 8353 is in chronological order but it was also an organic sand so is treated with some caution.

Ages are presented in the text as calibrated ages at the 2 -sigma (95\%) confidence interval. Accumulation rates are calculated using end-member values of the 2-sigma age brackets of each date to estimate a maximum and minimum rate. Horizons of interest are either directly dated or ages are estimated by interpolation between the two nearest ages using both the maximum and minimum accumulation rates. 


\begin{tabular}{|c|c|c|c|c|c|c|c|c|}
\hline $\begin{array}{l}\text { Location } \\
\text { \& Core } \\
\text { Number }\end{array}$ & $\begin{array}{l}\text { Sample } \\
\text { Depth } \\
\text { (cm) }\end{array}$ & $\begin{array}{l}\text { Sample } \\
\text { Material }\end{array}$ & $\begin{array}{c}\text { Dating } \\
\text { Technique }\end{array}$ & ${ }^{1}{ }_{(\%)}^{\mathrm{C}}$ & $\begin{array}{c}\text { Radiocarbon } \\
\text { Age } \\
\text { (radiocarbon } \\
\text { years BP) }\end{array}$ & $\begin{array}{c}\text { Calibrated } \\
\text { Age } \\
1 \text { sigma } \\
\text { (cal. years BP) }\end{array}$ & $\begin{array}{l}\text { Calibrated } \\
\text { Age } \\
2 \text { sigma } \\
\text { (cal. years BP) }\end{array}$ & $\begin{array}{c}\text { Lab } \\
\text { Number }^{c}\end{array}$ \\
\hline \multicolumn{9}{|l|}{$\begin{array}{l}\text { TAUPO } \\
\text { SWAMP }\end{array}$} \\
\hline TS97-1 & $238-240$ & $\begin{array}{l}\text { Organic } \\
\text { Mud }\end{array}$ & Standard & -30.5 & $2460+1-80$ & $2720-2340$ & $2750-2330$ & Wk 8095 \\
\hline TS97-1 & $250-258$ & Wood & Standard & -25.9 & $1830+/-160$ & $\begin{array}{c}1930-1910 \text { plus } \\
1900-1530\end{array}$ & $2150-1300$ & Wk $8096^{\star}$ \\
\hline TS97-1 & $536-538$ & Wood & AMS & -26.1 & $4984+1-56$ & $5847-5833$ plus & $5894-5598$ & NZA 9275 \\
\hline TS98-2 & $158-162$ & $\begin{array}{l}\text { Wood } \\
\text { \& peat }\end{array}$ & Standard & -30.7 & $1450+/-140$ & $\begin{array}{c}1520-1220 \text { plus } \\
1210-1180\end{array}$ & $1700-950$ & Wk 8351 \\
\hline TS98-2 & $\begin{array}{l}222.5- \\
227.5\end{array}$ & $\begin{array}{l}\text { Organic } \\
\text { Mud }\end{array}$ & AMS & -29.5 & $2470+1-60$ & $\begin{array}{c}2710-2630 \text { plus } \\
2620-2590 \text { plus } \\
2540-2530 \text { plus } \\
2500-2350\end{array}$ & $2720-2350$ & $\begin{array}{l}\text { Wk } 8352 \text { I } \\
\text { NZA } 11853\end{array}$ \\
\hline TS98-2 & $286-294$ & $\begin{array}{c}\text { Organic } \\
\text { Sand }\end{array}$ & Standard & -29.5 & $3010+/-230$ & $3450-2850$ & $3850-2450$ & Wk 8353 \\
\hline TS98-2 & $493-500$ & Shell & AMS & -1.4 & $4940+1-60$ & $5401-5264$ & $5457-5192$ & NZA 10568 \\
\hline \multicolumn{9}{|l|}{$\begin{array}{l}\text { OKUPE } \\
\text { LAGOON }\end{array}$} \\
\hline OL97-5 & 38 & Wood & Standard & -27.6 & $580+/-130$ & $654-499$ & $716-306$ & Wk $5697^{\dagger}$ \\
\hline OL97-5 & 80 & Wood & Standard & -27.9 & $1210+/-80$ & $1175-978$ & $1280-933$ & Wk $5698^{\dagger}$ \\
\hline OL97-5 & $109-112$ & $\begin{array}{c}\text { Organic } \\
\text { Sand }\end{array}$ & AMS & -28.5 & $4530+1-60$ & $5300-5040$ & $\begin{array}{c}5320-4960 \text { plus } \\
4900-4870\end{array}$ & $\begin{array}{l}\text { Wk } 8349 \text { I } \\
\text { NZA } 11828^{*}\end{array}$ \\
\hline OL97-5 & $128-135$ & Shell & Standard & -0.3 & $3360+/-140$ & $3386-3051$ & $3562-2852$ & Wk $6353^{\dagger}$ \\
\hline OL97-5 & $180-195$ & Shell & Standard & 1.0 & $4780+/-150$ & $5286-4842$ & $5458-4691$ & Wk $5699^{\dagger}$ \\
\hline OL97-3 & $25-27$ & Wood & Standard & -29.3 & $1100+1-80$ & $\begin{array}{l}1170-1160 \text { plus } \\
1120-1110 \text { plus }\end{array}$ & $1180-790$ & Wk $6350^{\dagger}$ \\
\hline OL97-3 & $145-150$ & Shell & Standard & -1.8 & $4010+/-100$ & $4185-3891$ & $4342-3766$ & Wk $6351^{\dagger}$ \\
\hline OL97-3 & $167-180$ & Shell & Standard & -1.5 & $4510+/-140$ & $4857-4517$ & $5049-4357$ & Wk $6352^{\dagger}$ \\
\hline OL97-2 & $166-170$ & Peat & AMS & -22 & $1961+/-60$ & $\begin{array}{c}1985-1861 \text { plus } \\
1842-1833\end{array}$ & $2044-1738$ & NZA 10617 \\
\hline \multicolumn{9}{|l|}{ KOHANGA- } \\
\hline KP99-1 & $46-49$ & $\begin{array}{l}\text { Organic } \\
\text { Mud }\end{array}$ & AMS & -25.4 & $1559+/-55$ & $1524-1386$ & $1553-1322$ & NZA 11750 \\
\hline KP99-2 & 450 & Wood & AMS & -25.4 & $4606+1-70$ & $\begin{array}{c}5450-5385 \text { plus } \\
5329-5291\end{array}$ & $\begin{array}{c}5574-5541 \text { plus } \\
5475-5047\end{array}$ & NZA 10613 \\
\hline KP99-2 & $573-577$ & $\begin{array}{l}\text { Organic } \\
\text { Mud }\end{array}$ & Standard & -27.5 & $5970+/-190$ & $\begin{array}{c}7010-6530 \text { plus } \\
6520-6500\end{array}$ & $7250-6300$ & Wk 8355 \\
\hline
\end{tabular}

Table 2.1. Radiocarbon age results

${ }^{\text {a }}$ Conventional radiocarbon age before present (1950 AD) after Stuiver and Polach, 1977, Radiocarbon 19: 355-363. This is based on the Libby half-life of 5568 years with correction for isotopic fractionation applied.

${ }^{\text {b }}$ Calibrated age in calendar years after Stuiver et al., 1998, Radiocarbon 40(3): 1041-1083.

1 sigma range ( $68 \%$ probability) and 2 sigma range ( $95 \%$ probability) reported.

${ }^{\mathrm{c}}$ Wk: The University of Waikato Radiocarbon Dating Laboratory; NZA: Institute of Geological and Nuclear Sciences Rafter Radiocarbon Laboratory

*Samples omitted from further use, see text.

${ }^{\dagger}$ Dates previously cited in Goff et al., (2000). 


\subsubsection{Grain Size Analysis}

Grain size analysis was carried out to define different sedimentary units in the cores and thereby aid description of past depositional environments. Analysis involved determining proportions of organic matter, carbonate, sand and mud following the procedure of Barrett and Brooker (1989). Proportions were measured in dry weight so samples were dried and weighed at the beginning and after each stage of processing. Samples were digested in $27 \%$ hydrogen peroxide to remove organic matter and heated in $32 \%$ hydrochloric acid to remove carbonate. Sediment was then sieved through a 60 -micron mesh to divide it into a fine fraction (proportion of mud) and a coarse fraction (proportion of sand). Sedimentary units were defined according to percentage of sand (Table 2.2). Sediment consisting of greater than $50 \%$ organic matter was defined as peat and sediments were described as organic if they contained greater than $10 \%$ organic matter.

\begin{tabular}{|lr|}
\hline Sedimentary Units \\
\hline Peat & $>50 \%$ organic matter \\
Mud & $0 \%$ sand \\
Sand-poor Mud & $<20 \%$ sand \\
Sand-rich Mud & $20-50 \%$ sand \\
Muddy Sand & $50-70 \%$ sand \\
Sand & $>70 \%$ sand \\
\hline
\end{tabular}

Table 2.2. Definitions of sedimentary units according to percentages of sand and organic matter.

\subsubsection{Diatom Processing}

Sediment samples were chemically and physically processed to concentrate diatom frustules and facilitate diatom identification and counting. The procedure used is similar to that outlined by Battarbee (1986) and Hinchey and Green (1994) with modifications made by Harper (pers. comms., 1997) and the author (see below). Care was taken to ensure the laboratory, glassware and all equipment was clean. Measures were also taken to avoid contamination between samples. Processing took place in batches of eight samples to match centrifuge capacity.

Chemical treatment was carried out in $100 \mathrm{ml}$ glass beakers in a fume cupboard using hydrogen peroxide to digest organic material and hydrochloric acid to remove carbonates. Approximately $20 \mathrm{ml}$ of $27 \%$ hydrogen peroxide was added slowly to each sample. Highly reactive samples were left overnight before heating; others were heated after about ten minutes. Heating was carried out on a hot plate with samples kept below boiling point to avoid damage to diatom valves and contamination by splattering. Samples were heated for half to two hours until no visible organic material remained and the residue became whitish. About 
$10 \mathrm{ml}$ of $32 \%$ hydrochloric acid was added slowly to the remaining solution of each sample. This was gently heated until visible reactions ceased. A few of drops of $\mathrm{HCl}$ were added to check that no calcareous material remained. Samples were transferred into $45 \mathrm{ml}$ plastic centrifuge tubes and rinsed with filtered water four times, being spun in the centrifuge each time at 3000 revolutions per minute (rpm) for five minutes.

Physical processing of samples involved removing sand grains (generally larger than diatoms) and clay particles (generally smaller than diatoms). To remove sand each sample was suspended in $40 \mathrm{ml}$ of filtered water in a centrifuge tube with a lid, each tube was shaken for 15 seconds, allowed to settle for 45 seconds, and the solution was poured off into a new tube. Sediment remaining in the original tube was discarded, and the shaking and settling process was repeated so samples ended up in their original tubes. The timing of this process meant it was most efficiently carried out in batches of four. For very sandy samples the sand was resuspended before being discarded to collect as much of the fines as possible. Clay was removed by resuspending samples in filtered water with six drops of dilute sodium hexa-metaphosphate (calgon) and centrifuging at $1800 \mathrm{rpm}$ for three minutes. Cloudy solution was discarded, the tubes refilled with filtered water, centrifuged and the process repeated until the solution became clear. This took between two and ten washes. An additional wash with centrifuging at 3000 rpm for five minutes was done at the end to ensure the sample was clean.

Processed samples were diluted to a measured volume, shaken vigorously and a measured aliquot of suspension was pipetted into a new tube. This was diluted with filtered water to $30 \mathrm{ml}$ and poured into a Battarbee tray containing four coverslips. Volumes of dilution and aliquots were experimented with to get the right concentration of valves on the slip for counting. In general, every $0.5 \mathrm{ml}$ of processed sample was diluted to $10 \mathrm{ml}$ and a $0.5 \mathrm{ml}$ aliquot was taken. The suspension in the tray was left to settle in a stable, dust-free environment. After eight hours liquid was removed from the surface of the trays by gentle pipetting to speed up evaporation and minimise the precipitation of salts onto the coverslips. When coverslips had dried completely they were mounted onto labelled microscope slides on a hot plate using naphrax diatom mountant. The naphrax was allowed to bubble gently between the coverslip and slide for a few seconds then the slides were removed from the hot plate and left to cool. Coverslips were tapped with a toothpick to remove bubbles if they were present. These permanent mounts are stored in the paleontology section of the School of Earth Sciences, Victoria University of Wellington. Sample residues are also being stored in five millilitre phials with dilute formalin solution. 
Checks were made at several steps of the above procedure to determine whether excessive or preferential loss of diatom valves occurred at any stage. This involved collecting discarded material at four stages:

1. Suspension poured off after settling for eight hours in beakers

2. Suspension poured off after centrifuging at $3000 \mathrm{rpm}$ for five minutes

3. Discarded sand fraction

4. Suspension poured off after centrifuging at $1800 \mathrm{rpm}$ for three minutes Waste material from eight samples was collected, reduced by gentle heating, mounted onto coverslips and scanned under the microscope. Numbers of diatoms were considered too small to enable precise estimates of loss to be calculated but surveys were sufficient to satisfy the author that there was no excessive loss of diatoms during processing. Relative numbers indicate that twice as many valves are lost during decanting by hand and in the sand removal step than are lost when the centrifuge is used. This acted as a reminder that care must be taken during sand removal with an extra resuspension step used for very sandy samples, and decanting by hand was avoided. Preferential loss of small diatoms of a variety of shapes occurred in steps one, two and four. However, diatoms as small as 2-3 microns long were numerous in fully processed samples, so although proportions of small diatoms may be underestimated, they are not completely removed during processing.

\subsubsection{Other Microfossils}

Foraminifera and pollen grains were used to answer specific questions. Samples were obtained from the stored cores and processed according to standard techniques. To concentrate foraminifera from estuarine mud, water and sodium carbonate were added to the sample and left for an hour. This mixture was sieved through a 75-micron mesh sieve until the water ran clear. Samples were dried and examined on a black tray under a binocular microscope. Samples for pollen analysis were treated with $\mathrm{HCl}$ to remove carbonates, $\mathrm{HF}$ to remove siliceous material, acetolysis to remove cellulose, and were mounted on microscope slides in glycerol jelly (Moore and Webb, 1983).

\subsubsection{Macrofossils}

Macrofossils encountered in the cores during sampling were removed and washed for identification. Wood and seeds were usually described then used for radiocarbon dating. Most shells encountered were Austrovenus stutchburyi, others were identified by Stephen Eagar and their environmental preferences were taken from Beu and Maxwell (1990). 


\subsection{DIATOM ANALYSIS}

\subsubsection{Identification and Taxonomy}

Diatoms were examined at $\times 1600$ magnification under an oil immersion objective on a Leitz microscope with differential interference contrast. Each species or morphological form was measured using an eyepiece micrometer, drawn, described and photographed. These details were catalogued on index cards to provide a permanent record and aid consistency of identification. Species were identified with reference to standard diatom floras including Krammer and LangeBertalot (1986-1991), Hartley (1996), Hendey (1964), Hustedt (1985), John (1983), Lange-Bertalot (1993), Patrick and Reimer (1966 and 1975), Foged (1979) and Witkowski et al. (2000).

Species' names generally follow those presented in Krammer and Lange-Bertalot (1986-1991). Where a different flora had to be used to initially identify a species, the name presented in that flora is used (see Appendix II). This is generally the case for brackish and marine species for which Witkowski et al. (2000) was used most extensively. Although many species have been placed in new or different genera since the compilation of "Süsswasserflora von Mitteleuropa" (eg, see Round et al., 1990), this remains one of the best floras for identifying species under light microscope. The consistency gained from following one flora predominantly will make updating of taxonomy simpler when a light microscope identification guide using new genera is available. Species that did not fit any existing description were placed in genera and given numbers. Some of these were examined under a Scanning Electron Microscope (SEM) to get more information about their structure, but the abundance of many was too low for them to be found under the SEM.

\subsubsection{Counting}

Counts were made along randomly selected transects, with four transects, one from each of four coverslips, used for each sample. For core and death assemblage samples at least 400 diatom valves were counted. This figure is between the generally accepted totals for such studies of 300 to 600 valves (Battarbee, 1986). Trial counts were made on five samples from the base of Taupo Swamp to investigate the relationship between counts, species and percentage abundances. One thousand valves were counted and the number of new species per 100 valves was recorded (Fig. 2.2). These samples have the most diverse diatom assemblages encountered in the fossil sequences and new species are still occurring after a count of 900 valves. However Patterson and Fishbein (1989) show that number of species present in an assemblage has no bearing on number of counts required to accurately measure percentage abundances, and the main interest for this study is percentage abundance of species rather than total diversity of the assemblage. Percentage abundance of moderately abundant 
species (8-35\% of total assemblage) appears to reach a plateau once 400 valves have been counted (Fig. 2.3). Greater counts (600-800 valves) are required to accurately measure percentage abundance of species making up less than about $3 \%$ of the total assemblage (Fig. 2.4). However no use is made of rare species as indicators in this work so extra counts could not be justified.

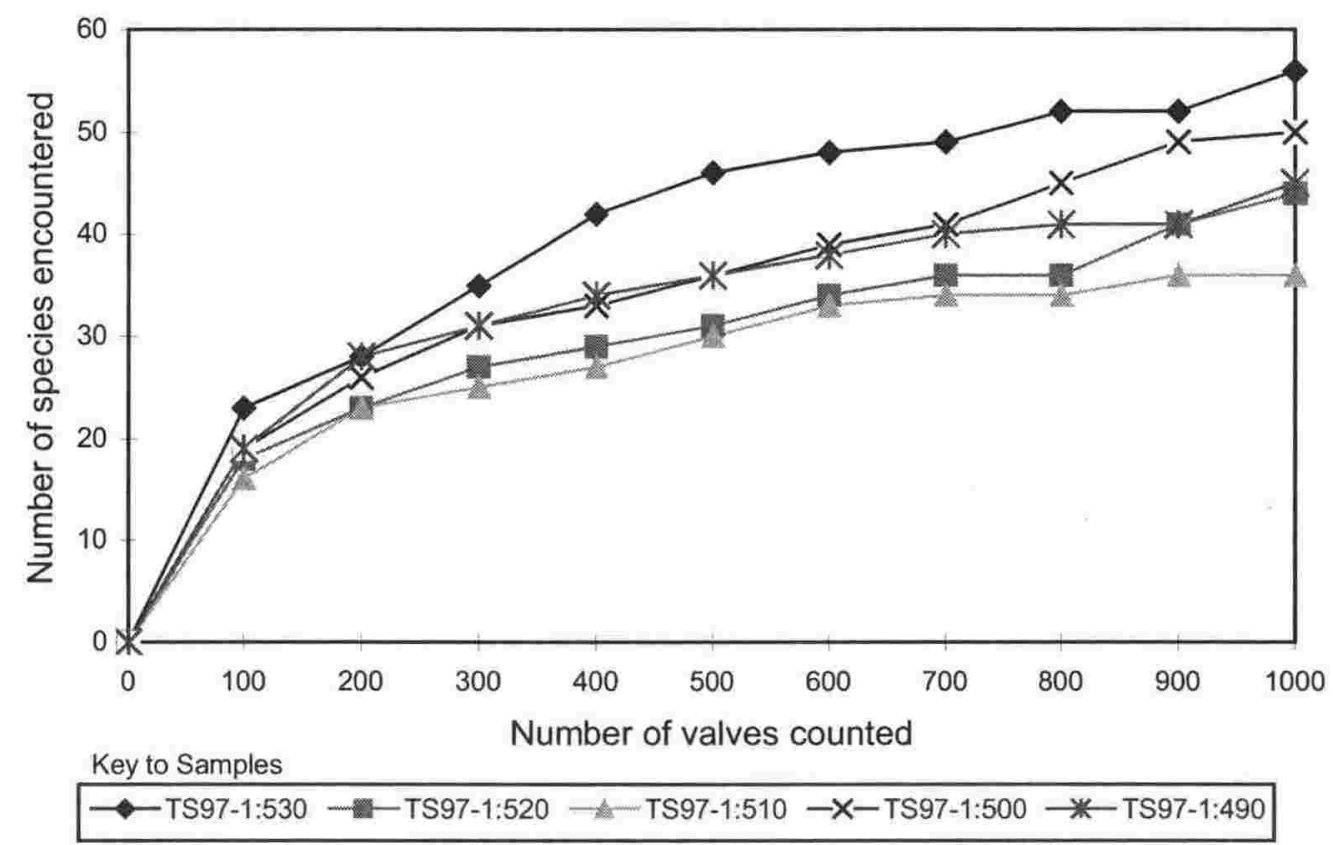

Fig. 2.2. Plot showing the increase in diatom species with increasing count totals for five samples from the base of the Taupo Swamp sequence.

Where samples were poorly preserved or had very low concentrations of diatoms, scans would be made of the slides and as many valves as practicable would be counted, usually over 100 but occasionally less. Such counts were sufficient for assessing whether samples belonged to the same assemblage as the surrounding samples or not. In most cases past environment of poorly preserved samples could be inferred from proximal well preserved samples but where they differed, little weight was given them. Where a sample was dominated by one species, for example Fragilaria pinnata, considerably more than the standard number would be counted, often excluding the common species after a proportion of the count, in order to determine what other species were present in the assemblage.

Fragments of diatoms were counted as one if at least half the valve was present in the field of view and if the whole valve, when complete, would have either been entirely in the field of view or off to the left side. Fragments were distinguished from whole valves so that proportions of broken valves could be used as an environmental indicator if appropriate. Fractions of diatom valves (whole valves that are only partially visible in a given field of view) were counted as one if at least half the valve was in the field of view on the left side. Fractions occurring 


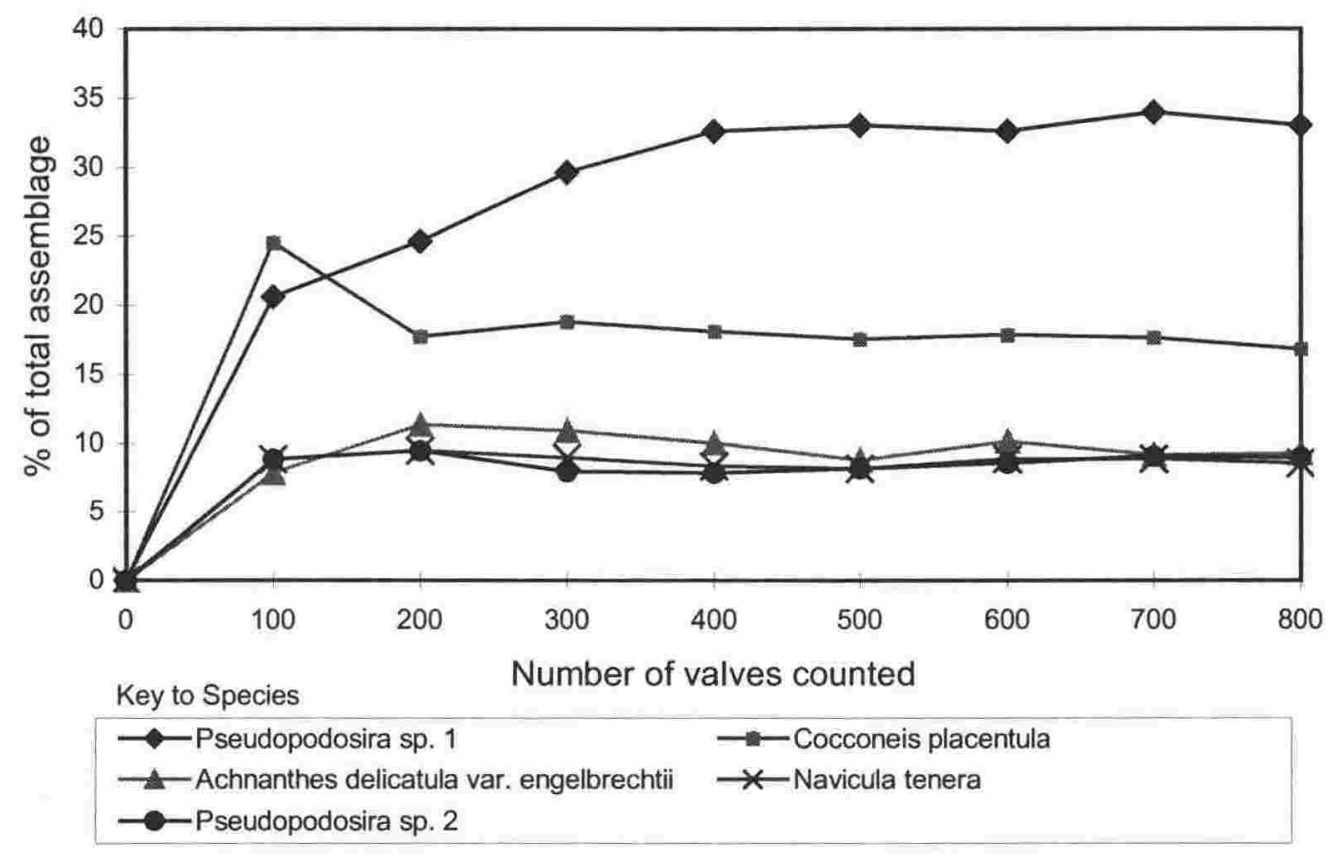

Fig. 2.3. Plot showing change in percentage of total assemblage for each of five abundant species in sample TS97-1: 510, with increasing count totals.

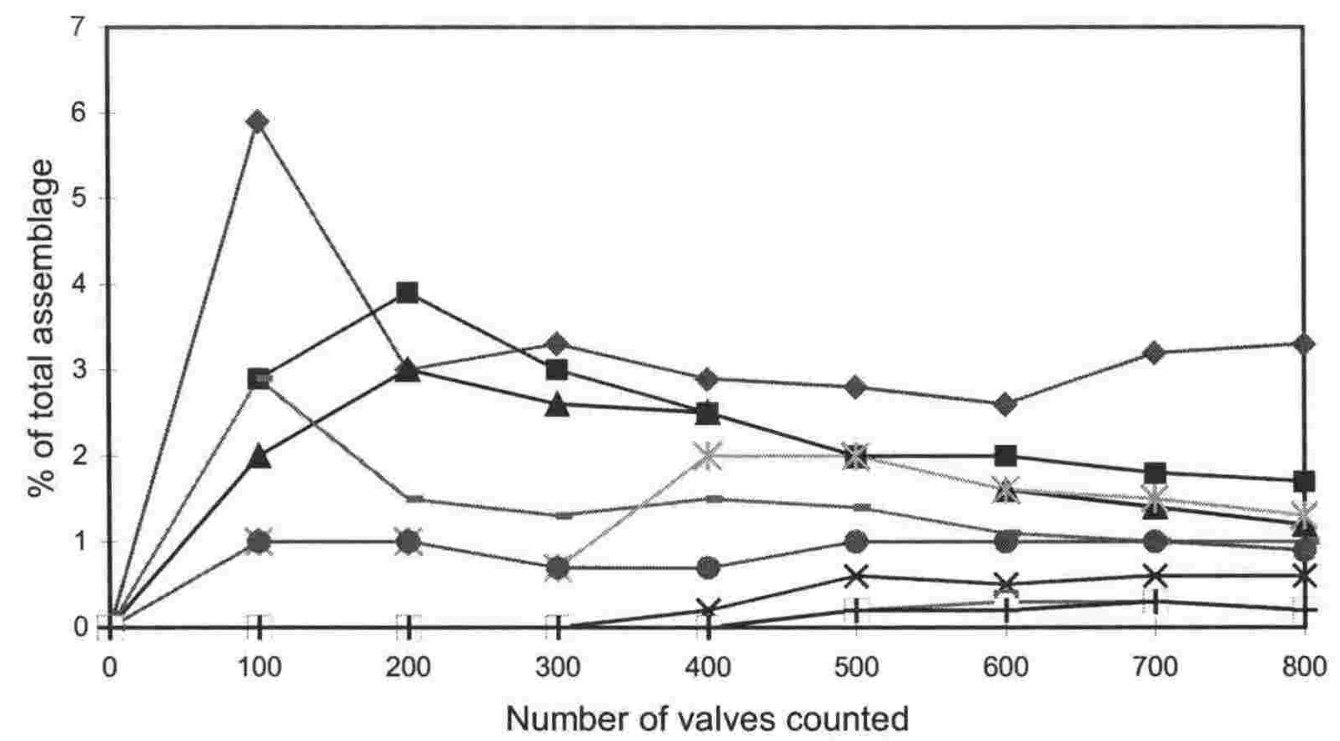

Key to Species

\begin{tabular}{|c|c|c|}
\hline$\multimap$ Fragilaria construens & $\rightarrow-$ Achnanthes reversa & $\longrightarrow$-Opephora olsenii \\
\hline -Achnanthes ploenensis & $\rightarrow$-Cyclotella meneghiniana & - - Navicula cryptotenella \\
\hline$\star$ Paralia sulcata & $\rightarrow-$ Hyalodiscus lentiginosus & -Actinocyclus octonarius \\
\hline
\end{tabular}

Fig. 2.4. Plot showing change in percentage of total assemblage for each of nine less abundant species in sample TS97-1: 510, with increasing count totals. 
on the right side of the field of view were disregarded to reduce the bias towards large valves that counting fractions causes (Laws, 1983).

\subsubsection{Concentration}

Estimates of diatom valve concentration in the sediment were calculated for two main reasons. Diatom concentration can be used directly as an environmental indicator because diatom productivity varies markedly across a coastal gradient. Diatom concentration was also required to construct absolute diatom abundance plots, which were considered necessary in addition to percentage abundance plots because of the limitations of the latter and the potentially large range in concentration of environments being reconstructed. The problem with percentage plots is that values for each species are not independent of each other and each sample sums to $100 \%$ despite the possibility of very few valves being present in some samples (see Birks and Birks, 1980).

The difficulty of calculating precise and accurate diatom concentrations has been demonstrated (Wolfe, 1997). The main requirement of this research is the ability to compare fossil and modern samples and between cores within the project rather than requiring correct absolute numbers. For this reason care was taken to ensure the same slide-making techniques, and methods of calculating concentration, were used for all samples. In such a case concentration measurements should be fairly reproducible (Wolfe, 1997).

Two commonly used methods for determining diatom concentration are the addition of known quantities of external markers such as microspheres or pollen and the use of evaporation trays (Battarbee, 1973). The evaporation tray method was chosen because this results in valves being randomly and evenly distributed over the coverslip so concentration can be reliably calculated from a specified area, also the likelihood of counting a good representation of the initial diatom assemblage is greater, and errors resulting from size sorting across the slip are reduced.

Battarbee trays were constructed from aluminium in Victoria University of Wellington's mechanical workshop according to the design of Battarbee (1986). Samples were evaporated and made into slides as described above. Transect length and width of field of view were recorded during counting so that the area counted could be determined. An estimate of diatom concentration was calculated for core and death assemblage samples using the following equation:

Total valves $=$ valves counted $\mathrm{x}$ area of evaporation tray $\mathrm{x}$ sample volume area of coverslip counted aliquot volume

Concentrations are expressed as valves per gram of sediment after dividing the total valves by the dry weight of initial sample used. 
The preservation of diatom assemblages was estimated qualitatively through notes about evidence of dissolution made during counting and the proportion of broken valves present. A simple indication of the diversity of diatom assemblages was provided by calculating the number of species present (species richness) as a proportion of the total number of valves counted.

\subsubsection{Ecological Preferences}

\section{Introduction}

Scientists have recognised, at least since the mid to late nineteenth century, that diatoms have a variety of life forms and specific ecological preferences. In the early twentieth century the potential of these preferences for paleoenvironmental reconstruction was recognised and since then efforts have been made to determine ecological distributions of individual species. Documentation of species preferences has occurred through specifically designed autecological and synecological studies as well as from many isolated observations. Although ecological knowledge and understanding of individual diatom species is far from complete, there is enough published information to enable paleoecologists to group species according to habitat, salinity, $\mathrm{pH}$, nutrient content, water depth / moisture, oxygen requirements and a number of other environmental factors.

Habitat and salinity were chosen as the two environmental variables likely to be of most use in the reconstruction of coastal waterbody types. An ecological survey of diatoms living in coastal environments in New Zealand was carried out as part of this project and species' preferences for salinity are derived (Chapter 3). However prior to development of the modern calibration set, traditional techniques of ecological classification were used to aid initial interpretation of the sedimentary sequences. Various studies and references were used to place individual species into ecological categories (eg, Van Dam et al., 1994; Round et al., 1990 and the above floras, see Appendix III).

\section{Habitat}

Within a waterbody there are a number of habitats that diatoms can occupy including the water column, the sediment surface, and surfaces of plants, rocks, other algae and even animals. Species have favoured habitats, which enable them to be grouped into habitat categories and utilised in the description of past environments. Many species can live in more than one type of habitat so inferences about past environment are restricted to being fairly general. However, relative proportions of planktonic and benthic forms can provide information about water depth and sand-, mud- and plant-dwelling forms can give indications about factors such as energy regime and macrophyte growth. 
The main distinction in habitat classification is between phytoplankton and phytobenthos (or periphyton). Phytoplankton live in the water column and can be further classified according to size or persistence in the water column. For example some species live their whole life cycle in the water column while others have resting stages in the sediment. In this work only tychoplankton (or pseudoplankton) are differentiated from plankton. Tychoplankton come from other habitats but are often found in the plankton in shallow or turbulent water environments. Phytobenthos are subdivided into haptobenthos, which live attached to surfaces either directly or via a stalk, and herpobenthos, which are unattached and can move freely through sediments. 'Aerophilic' is the term used for diatom species that can live out of waterbodies on soil or the surfaces of trees, rocks or mosses. The categories used in Chapters Four, Five and Six are listed below (Table 2.3).

\begin{tabular}{|l|l|}
\hline Category & Habitat \\
\hline $\begin{array}{c}\text { Phytoplankton } \\
\text { Plankton }\end{array}$ & Water column \\
Tychoplankton & Water column of shallow / turbulent water \\
Phytobenthos & Attached to surfaces \\
Haptobenthos: & Attached to plants \\
Epiphyton & Attached to stones \\
Epilithon & Attached to sand grains \\
Epipsammon & Free-ranging on sediments \\
Herpobenthos: & Muddy sediment surface \\
Epipelon & Out of waterbodies on moist or dry surfaces \\
Aerophilic & \\
\hline
\end{tabular}

Table 2.3. Habitat categories into which diatom species are grouped.

\section{Salinity}

Salinity is a major factor controlling diatom species distributions (Denys and de Wolf, 1999). Each species has an ecological optimum in fresh, brackish or marine waters. Species also have ecological tolerances for salinity with some able to tolerate wide ranges of salinity and others only a very narrow range. The combination of diatoms being sensitive to salinity, diatoms being present across the whole salinity gradient (unlike many microfossil groups), and salinity being a useful indicator of marine influence in the coastal zone, has meant that diatoms are indispensable for reconstruction of paleoenvironments that traverse the marine-fresh water boundary.

Numerous classification systems have been developed for diatom salinity preferences (see summary in Juggins, 1992). Early 'halobian' systems were designed for particular regions but these have been modified for general application. Most systems are based around salinity preferences but some also take tolerance into account. Diatoms were classified in this project into one of eight categories (Table 2.4) based on the system devised by Werff (as cited in Juggins, 1992). Some category names have been changed for additional clarity 
and two indifferent categories are included to cover species with particularly wide tolerances. The marine $\left(>30 \mathrm{~g} \mathrm{l}^{-1}\right)$ category was not required because no open marine environments were being reconstructed. One reason this system is useful is because a key source of ecological information on diatoms (Van Dam et al., 1994) uses the same four categories at the fresh end of the scale to classify 948 taxa found in the Netherlands. Information about preferences of taxa is taken from numerous references (Appendix III).

\begin{tabular}{|lcr|}
\hline Category & Salinity $\left(\mathrm{g}^{-1}\right)$ & Range \\
\hline Salt intolerant & $<0.2$ & Narrow \\
Fresh & 0.2 to 1.0 & Medium \\
Fresh brackish & 1.0 to 2.0 & Narrow \\
Brackish & 2 to 10 & Medium \\
Brackish marine & 10 to 20 & Medium \\
Marine littoral & 20 to 30 & Medium \\
Fresh indifferent & 0 to 10 & Wide \\
Brackish indifferent & 0.2 to 25 & Wide \\
\hline
\end{tabular}

Table 2.4. Salinity categories into which diatom species are grouped. Salinity is quoted in grams per litre $\left(\mathrm{g} \mathrm{l}^{-1}\right)$.

\subsubsection{Data Presentation}

Raw diatom counts were entered into an EXCEL spreadsheet, which consisted of a species versus depth matrix and formulae for calculating proportions and concentrations of each species at each depth (Appendix VI). Species were then grouped into categories of habitat and salinity preference (as defined above) and new spreadsheets of these groupings were constructed. Percentage abundance and concentration plots against depth and time were produced using the plotting programme PSIMPOLL. Percentage abundance plots were found to be useful for comparing proportions of ecological categories and deducing likely characteristics of past environments, whereas concentration plots provided an indication of relative productivity / preservation of different environments. Zones were defined for each sequence by grouping intervals of similar diatom concentration and composition. Zone boundaries were placed at the mid-point between two samples unless a sediment boundary occurred within the interval, in which case it was placed at the sediment boundary.

\subsection{DETECTION OF EARTHQUAKES}

\subsubsection{Introduction}

Paleoenvironmental histories constructed using high resolution sedimentology and diatom analysis are used as the basic information from which to detect evidence of earthquakes in the sedimentary record of coastal waterbodies. A comprehensive approach for such work has been developed and described from the Pacific Northwest of America and Canada (eg, Long and Shennan, 1994, 1998; Darienzo 
et al., 1994; Mathewes and Clague, 1994; Nelson et al., 1996a, 1996b; Shennan et al., 1996, 1998). Although designed for a specific tectonic setting dominated by repeated coseismic subsidence and interseismic uplift (Clague, 1997), the general characteristics of the approach are applicable to assessment of coastal sequences in New Zealand. Changes in paleoenvironment are identified and transition horizons are assessed according to criteria designed to differentiate between coseismic and aseismic causes of change. Presence of a single criterion cannot be used to infer an earthquake because aseismic processes can usually explain each feature in isolation. Even recognition of several criteria at a single site does not denote the occurrence of an earthquake but the likelihood of a coseismic cause of change becomes greater as more criteria are met at more sites (Nelson et al., 1996b). Criteria are discussed fully by Nelson et al. (1996b) so only a brief introduction and considerations specific to this project are outlined below.

\subsubsection{Suddenness of Change}

Macrofossils such as trees or shells preserved in life position can be a good indication of suddenness of change (Atwater and Yamaguchi, 1991) but these are rarely encountered in core sequences. Therefore factors used to assess the suddenness of change in this study include the nature of the sedimentary contact and the nature of the biostratigraphic contact. Where there is no evidence for erosion at the contact, the distance over which diatom assemblages change completely is used to assess suddenness. Gradual relative sea level change results in gradual shifts in species composition of diatom assemblages, for example with brackish species gradually replacing fresh species and then being replaced by marine species (Fig. 2.5). When a sudden change occurs, the distance in the sedimentary sequence over which such shifts occur is small.

\subsubsection{Amount of Change}

Many processes acting at scales ranging from global to local can cause apparent changes in elevation in coastal waterbodies for example, sea level change, sediment accumulation or erosion and tectonic movement of base level. At sites open to marine influence, traditional sea level techniques can be used to recognise relative sea level changes and estimate the amount of change in elevation.

Traditional sea level techniques are based on the identification of sea level index points which are coastal features of known elevation and age (van de Plassche, 1986). The indicative meaning of a sea level index point is the vertical distance between an index point and a former reference water level (eg, mean high water, highest astronomical tide etc) and the indicative range is the uncertainty related to this distance (Nelson et al., 1996b). The tendency of a sea level index point describes whether the point reflects a movement of marine water toward or away from a site (not necessarily indicating a rise or fall in sea level). 
Once such features have been identified in a sequence, the challenge remains to separate the various influences causing inferred relative sea level changes.

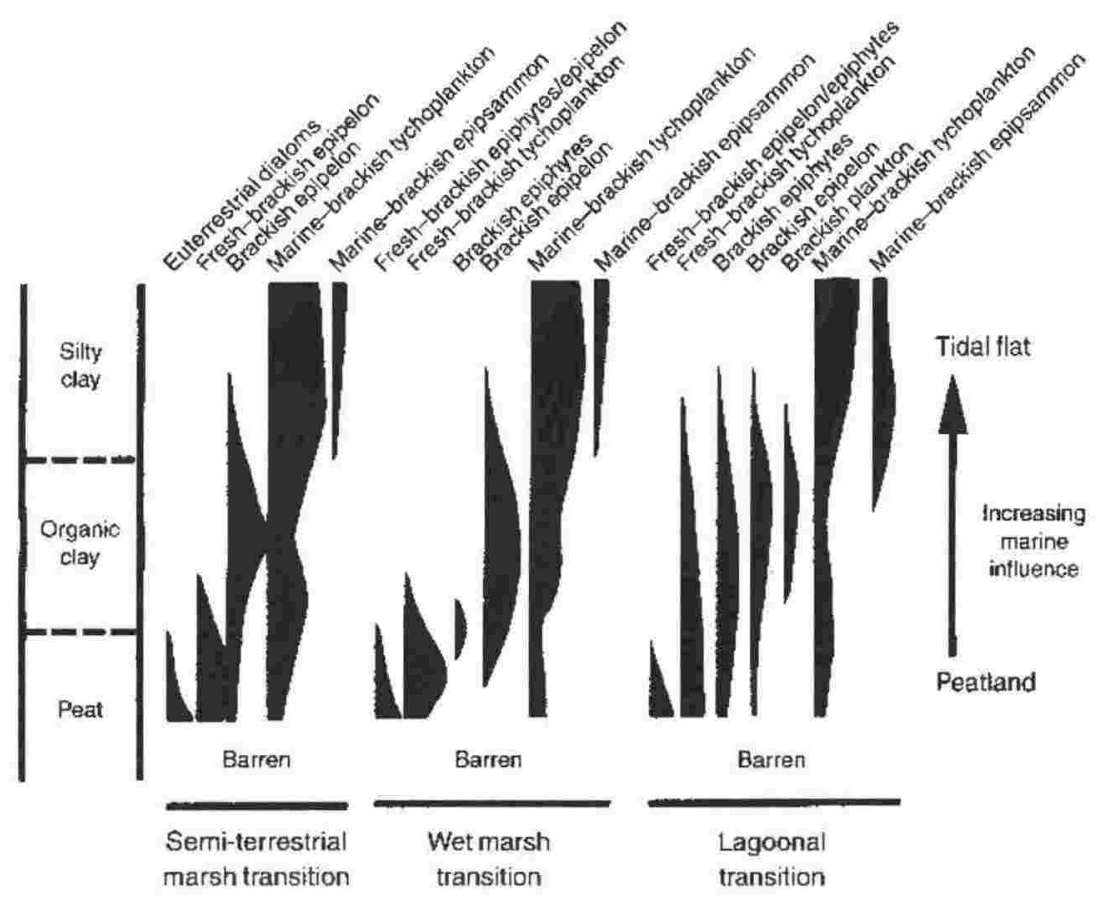

Fig. 2.5 Idealised scheme for diatom assemblage shifts occurring as a result of relative sea level rise (from Denys and de Wolf, 1999). Note the gradational nature of changes to diatom assemblage composition.

Net changes in relative sea level at a particular site can be obtained by determining the height of a sea level indicator above or below the level at which a similar feature is forming at present. However studies involving detection of earthquakes in the sedimentary record are more concerned with amount of change in paleo-elevation across horizons thought to be coseismic in origin than with net changes over time. Nelson et al. (1996b) suggest an inferred change of greater than one metre is more likely to be coseismic in origin than changes involving less than half a metre. This is because many small-scale coastal processes can affect elevation by half a metre or less but would rarely affect it by more than this. Identification of transitions that are coseismic in origin but involved less than a metre of vertical movement would rely on other criteria.

Sediments can be used to provide a broad indication of former sea levels but the most reliable sea level indicators are organic remains in growth position for which relationships between sea level or water table level can be established (Kidson, 1986). Diatoms have been used extensively in sea level studies but emphasis has been on reconstructing salinity of paleoenvironments rather than elevation with respect to a reference water level. Palmer and Abbott (1986) refer to diatom-based sea level index points as the elevation and age of a salinity change inferred from 
diatom assemblages relative to a reference water level. Recently there have been a number of ecological surveys relating diatom assemblages directly to tidal elevations (rather than using salinity as a proxy). Most studies indicate that three zones related to tidal elevation can be clearly defined by diatom assemblages (Nelson and Kashima, 1993; Hemphill-Haley, 1995b; Zong and Horton, 1998). However the vertical range of each zone can be large and the elevation of zone boundaries is likely to vary across regions because of differing hydrological regimes. Additional microfossil groups such as foraminifera and pollen can be used to improve the zonation and refine estimates of past tidal elevation (eg, Shennan et al., 1996).

At sites completely isolated from marine influence, vertical movement of base level is likely to manifest itself as a change in relative water table level. Relative water table level may also change in response to hydrological changes or sediment accumulation or erosion. Diatoms can be used to infer changes to water table level through the effects that water depth has on diatom assemblage composition. Benthic, sediment-dwelling diatoms dominate in shallow water and planktonic species dominate in deeper environments (Campeau et al., 1999).

A sea level curve for the New Zealand region indicates that sea level has remained fairly stable over the last 6500 years with a minimum level of $-40 \mathrm{~cm}$ at 4500 years BP and a maximum of about $+50 \mathrm{~cm}$ at 3500 years BP (Gibb, 1986). Therefore any relative change in sea level greater than this or occurring at a different time is likely to be the result of other factors such as sediment transport or tectonic movement of base level. Gravel barriers exist between the waterbody and sea at all three sites investigated in this project so it is acknowledged that sediment transport and deposition have had an impact on the history of these waterbodies. Barrier closure and breaching are considered as potential causes of environmental transitions recognised at the study sites.

A major limitation in the estimation of amount of change is the lack of information regarding the distribution of diatom species in relation to sea level in New Zealand. Because of the general paucity of ecological information on diatoms in the coastal zone of New Zealand, a broad survey of different waterbody types was considered to be a greater priority than a high resolution tidal elevation survey. For this reason detailed estimates of change in elevation are not attempted at this stage. In this study evidence for relative changes in sea level or water table level is sought using diatom analysis but instead of attempting to quantify change in elevation, quantitative estimates of the amount of paleoenvironmental change are used to assess the magnitude of change. These estimates of change in waterbody type and paleosalinity are derived using the coastal diatom calibration set (Chapter Three) and are thought to act as reliable proxies for elevation. 
A coseismic cause of change is only inferred where a large magnitude change in paleoenvironment is associated with qualitative evidence for vertical movement of base level.

\subsubsection{Lateral Extent}

The lateral extent of contacts formed by environmental transitions within a waterbody can be indicative of the cause of change. For example contacts formed by a sudden coseismic movement of base level will be of lagoon-wide extent whereas those formed as a result of sediment accumulation or erosion in a flood or storm event are more likely to be localised. Multiple cores within a waterbody are required to differentiate between localised aseismic contacts and more extensive, potentially coseismic, contacts. Lateral extent of coseismic contacts can also be used to help determine earthquake source - contacts recognised at a few closely spaced waterbodies are likely to be the result of movement on a small surface fault and those recognised over a large region suggest movement on a major fault such as a subduction interface (Nelson et al., 1996b).

\subsubsection{Synchroneity}

Environmental transitions formed by coseismic movement of base level are synchronous both within waterbodies and across the region affected by the earthquake. Many aseismic coastal processes resulting in sediment accumulation or erosion are time-transgressive within a waterbody, and where aseismic processes result in synchronous changes within a waterbody (eg, barrier closure or breaching), they are unlikely to result in synchronous changes across different waterbodies. Use of this criterion is limited by the resolution of available dating techniques but synchroneity within the bounds of the dating technique should be established to support a coseismic cause of change.

\subsubsection{Coincidence with Tsunami Deposits}

Tsunami triggered by large earthquakes can lead to marine inundation of large areas of coastal lowland. In the Pacific Northwest of America and Canada evidence for inundation of tidal wetland systems generally takes the form of sand beds that thin and fine inland. Sand is considered to represent an unusual depositional event in environments dominated by mud and peat. Microfossils within sand units are derived from marine or subtidal estuarine environments (Hemphill-Haley, 1996). Tsunami deposits have been recognised in New Zealand but many diagnostic characteristics, often involving multidisciplinary approaches, are required to provide a definitive identification (Goff et al., 2001). Tsunami deposits are regarded as an additional criterion because tsunami will only leave a sedimentary deposit if there is an available source of sediment and favourable wave characteristics, and the usually thin deposits will only be preserved if left undisturbed by sediment mixing and burrowing (Nelson et al., 1996b). 


\subsubsection{Coincidence with Evidence for Catchment Disturbance}

Strong ground shaking in an earthquake frequently causes landslides in areas of hill country. Evidence for catchment disturbance is not included as a criterion in the work of Nelson et al. (1996b) because of the restricted distribution of landslide deposits in tidal wetland environments. However in small coastal waterbodies where hillslopes are in close proximity to sedimentary sequences being investigated, the potential exists for useful information to be collected. Unusually coarse-grained deposits consisting of angular clasts and entirely terrestrial material such as wood fragments are likely to be the result of catchment disturbance. Occurrence of such deposits at horizons involving large changes in paleoenvironment strengthens the case for a catastrophic cause of change. 


\section{CHAPTER THREE}

\section{Diatom Assemblages of New Zealand Coastal Waterbodies: A Calibration Set for Quantitative Paleoenvironmental Reconstruction}

\subsection{INTRODUCTION}

\subsubsection{Outline of Chapter}

The chapter begins with reasons for collecting a modern calibration set and some background to quantitative paleoenvironmental reconstruction (this section). Aims of the New Zealand coastal diatom calibration set are outlined in section 3.2, and design and methods are discussed. The calibration set is described in section 3.3 using simple exploratory methods and ordination techniques. A diatom-salinity transfer function is derived in section 3.4 using weighted averaging regression. Salinity optima and tolerances are presented for calibration set species and compared with other studies. The modern analogue technique is also presented in this section because it is used to check the reliability of the transfer function. Reconstruction of past environments using results from ordination, the transfer function and the modern analogue technique is carried out in Chapters Four, Five and Six.

\subsubsection{Why Collect a Calibration Set?}

A calibration set is a modern ecological dataset specifically designed to aid paleoenvironmental reconstruction. A calibration set generally consists of many samples of the organism of interest (eg, diatoms, foraminifera, ostracods, chironomids) collected across an environmental gradient of interest (eg, salinity, temperature, nutrients) and associated with other environmental variables measured from each sample location. Statistical methods are then used to determine relationships between organisms and measured environmental variables and these relationships are used to estimate environmental parameters from fossil assemblages. Diatoms have been used in calibration sets for the reconstruction of lake water acidity (Battarbee, 1994), climate change (Gasse et al., 1995; Lotter et al., 1997; Gaiser et al., 1998; Reed, 1998), nutrient enrichment (Bennion, 1994; 
Lotter et al., 1998; Reavie and Smol, 2001), and change in the coastal zone (Juggins, 1992; Campeau et al., 1999).

It makes intuitive sense that comparison of fossil assemblages with modern assemblages from known environments will improve a paleoenvironmental reconstruction for Holocene and late Quaternary sedimentary sequences. For example, incorporation of a calibration set into a paleoecological study enables species' preferences to be derived directly from sites in the same region and of the same type as that being reconstructed. Without a calibration set, species preferences are usually gathered from various published sources and species are classified into broad ecological categories. This can be a subjective process and there is a lack of reliable information for allocating species to categories (Vos and de Wolf, 1993).

More information can be gained about past environments using a calibration set compared with traditional techniques because numerous statistical methods exist with which to relate modern and fossil datasets. Fossil samples can be plotted passively onto ordinations of modern data, similarity measures can be used to determine closest modern analogues, and models of modern species-environment relations (transfer functions) can be used to derive quantitative estimates of past environment. For these general reasons and the three specific reasons outlined below, a calibration set was included as part of this research.

There has been little ecological diatom work in New Zealand so when using traditional paleoecological procedures, Northern Hemisphere species preferences are used and the assumption has to be made that they are relevant globally. Development of a calibration set in New Zealand will provide a check as to whether species living here conform to Northern Hemisphere preferences. It is also hoped the calibration set will aid reconstruction of coastal sequences in New Zealand by initiating transfer function style reconstruction in this country.

In comparison with inland waters, there has been little work on diatoms from the coastal zone. Hendey (1964) was almost the sole reference along with individual papers and monographs until publication of "Diatom Flora of Marine Coasts I" (Witkowski et al., 2000). There are also few calibration sets that cover the range of waterbodies present in the coastal environment. This has meant many species found in coastal sedimentary sequences cannot be identified or utilised in reconstruction. Existence of an appropriate calibration set enables all species in a fossil dataset to be used in reconstruction as long as the same taxa occur in reasonable numbers in the modern dataset. 
The use of diatom analysis in a paleoseismic context is fairly new so a calibration set is used to investigate new ways that diatoms can be useful in this field. It is hoped analysis of the calibration set will provide techniques for characterisation of waterbody types. Also quantification of important variables will provide an additional, less biased, assessment of amount and suddenness of change in past environment at horizons thought to be coseismic in origin.

\subsubsection{Quantitative Paleoenvironmental Reconstruction}

Imbrie and Kipp (1971) first presented quantitative paleoenvironmental reconstructions using relationships between modern foraminifera and sea surface parameters to estimate past sea surface characteristics from fossil foraminifera. The major assumption in their work, which applies to paleoecology in general, is that the ecosystem of interest has not changed significantly since the time of the fossil organisms. They describe their approach as taking this assumption to its 'logical limit: writing equations relating portions of the biological side of this ecosystem to selected physical parameters of the oceans - and then using those equations on samples from cores to make fully quantitative estimates of past marine climates' (p.71, Imbrie and Kipp, 1971). In the thirty years since Imbrie and Kipp's study, the general approach has remained valid but there has been an improvement in mathematical techniques for modelling species-environment relationships and for assessing the performance of calibration sets. For a full description of theory and approaches to quantitative paleoenvironmental reconstruction refer to Birks (1995).

The basic situation (Fig. 3.1) is that a matrix of fossil data $\mathbf{Y}_{\mathbf{f}}$ exists that consists of abundances of taxa in a certain number of samples. We want to use this fossil data to reconstruct $\mathbf{X}_{\mathbf{f}}$, the unknown environmental conditions of the past. To do this quantitatively, we collect $\mathbf{Y}_{\mathrm{m}}$ a dataset of modern species from a large number of sites, and $\mathbf{X}_{\mathrm{m}}$ a set of associated environmental variables. The relationships between the modern taxa and environmental variables are modelled statistically to derive $\mathbf{R}$. Relationships between fossil species and their environment are assumed to have been similar to the modern relationships and $\mathbf{R}$ is used to transform the fossil data into quantitative estimates of the past environment. The two main steps to quantitative reconstruction require two different types of mathematical problem (Fig. 3.1). The first stage, in which the relationship between modern species and their environment is modelled, is a regression problem. The ecological response function or 'transfer function' $\mathbf{R}$ is a regression coefficient. The second stage, in which the transfer function is used to estimate past environment from fossil assemblages, is a calibration problem. 


\section{Regression $Y_{m}=R X_{m}$ (solve for $R$ )}

MODERN DATASET
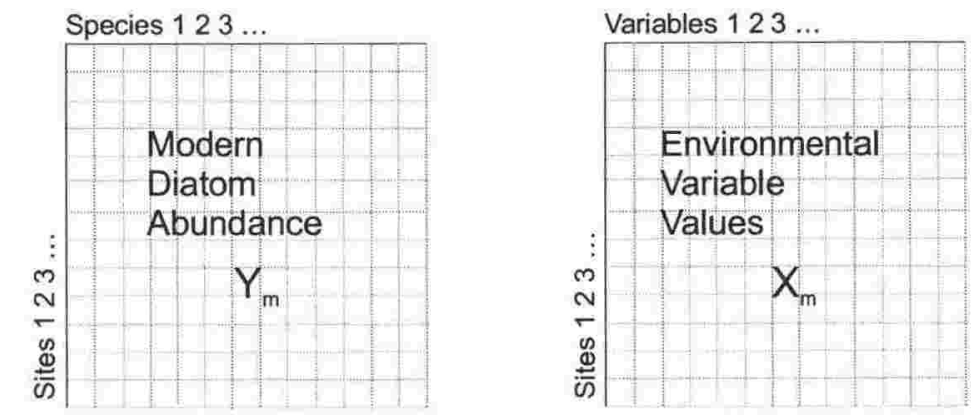

2. Calibration $X_{f}=Y_{f} R^{-1}$

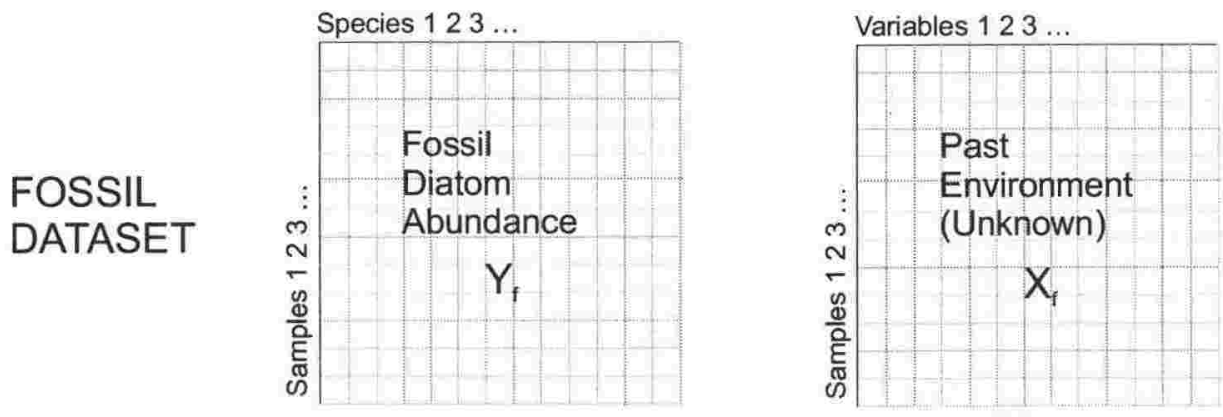

Fig. 3.1 Datasets (modern and fossil) and mathematical steps (regression and calibration) required for quantitative paleoenvironmental reconstruction (modified from Birks, 1995).

There are five major assumptions made in the quantitative reconstruction process and outlined by Birks (1995):

1. Taxa in the calibration set are systematically related to the environment in which they live.

2. The environmental variables to be reconstructed are, or are linearly related to, an ecologically important variable in the system of interest.

3. Taxa in the calibration set are the same biological entities as those in the fossil dataset and their ecological responses to the variables of interest have not changed significantly since deposition of the fossil sample.

4. Mathematical methods used in regression and calibration adequately model biological responses to the environmental variables of interest.

5. Environmental variables other than the one of interest have negligible influence or their joint distribution with the variable of interest in the fossil set is the same as in the calibration set.

These assumptions are discussed in relation to this study in section 3.4. Juggins (1992) lists some additional assumptions that are considered relevant to the 
calibration set constructed in this study because they were derived for quantitative reconstructions in the estuarine environment:

1. The relationship between the environmental variable of interest and the processes of erosion, transport and deposition has not changed over the relevant timespan.

2. The modern samples are representative of the surface sediment diatom assemblages at that point in time and space.

3. Post-burial dissolution has not significantly altered the composition of the fossil diatom assemblages.

\subsubsection{Mathematical Methods}

A basic concern in the statistical analysis of ecological and paleoecological data is the response of species to their environment. Species' abundance is considered to be a response variable and measured environmental variables are treated as explanatory variables. Most statistical methods used in ecology assume some type of species response model, i.e. an estimate of how species in the modern dataset respond to environmental variables in the ecosystem of interest. Two of the most commonly used models are the linear response model and the unimodal response model (Fig. 3.2). One type of unimodal response model is the normal or Gaussian curve where curve parameters equal species preferences and values on the $\mathrm{x}$-axis are expressed in standard deviation units (SD). Hill and Gauch (1980) state that a species can be expected to increase in abundance, reach a maximum and decrease over an interval of about 4 SD. Similarly, a full turnover in species composition of samples can be expected in 4 SD. Choice of response model depends on the length of the environmental gradient. If the measured gradient is $<2$ SD species are unlikely to have optima located within the sampled gradient so linear methods are appropriate. If the gradient length is over 2 SD some species will have optima located within the gradient and a unimodal model is appropriate (Birks, 1995).

LINEAR

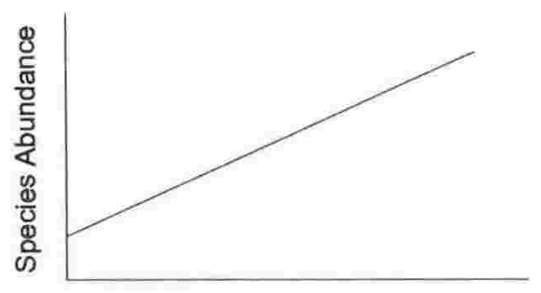

Environmental Gradient
UNIMODAL

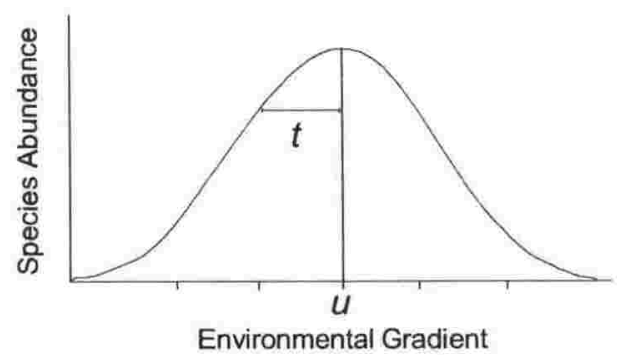

Environmental Gradient

Fig. 3.2. Idealised species response models. The mean and standard deviation of the Gaussian-type unimodal model are equal to the ecological optimum $(u)$ and tolerance $(t)$ of the species. 
Ecological and paleoecological data are multivariate - they contain large numbers of taxa and samples, often with environmental data associated with each sample. Multivariate data analysis is required to simplify or reduce the dimensionality of the data so that major patterns can be visualised and interpreted. In this chapter ordination (indirect gradient analysis) is used to explore and describe the calibration set, and regression analysis (direct gradient analysis) is used to determine modern species preferences. The particular methods and models used in this thesis are discussed in more detail in section 3.3.3, but a brief introduction is given below.

Ordination is a method of summarising multivariate data visually as twodimensional scatter plots which can then be interpreted using external environmental data. This is termed indirect gradient analysis because environmental data is used externally rather than incorporated into the analysis. Samples are arranged along axes on the basis of species composition so that similar samples occur close together and dissimilar samples are plotted further apart (ter Braak, 1995). Ordination can be considered a method of recovering underlying structure from the species data. Axes are constructed that best explain the measured species occurrences and these are thought of as 'theoretical' environmental variables. Canonical ordination is a method of multivariate direct gradient analysis that aims to relate species to measured environmental variables (ter Braak, 1995). In canonical ordination axes are also constructed so as to best explain the species data but they are constrained to being linear combinations of measured environmental variables.

In ordination and canonical ordination, when using a unimodal response model, the 'best explanation' of the species data is one that maximises the separation or dispersion of species response curves. Wide separation of response curves (and so of optima also) is an indication of an important axis / environmental variable or one that explains much variation in the species data.

Regression analysis aims to describe species' abundance as a function of one or more environmental variables. This is achieved by fitting a response model to the data that consists of a systematic part describing expected species' response to explanatory variables, and an error part describing how the observed response differs from the expected response (ter Braak and Looman, 1995). There are numerous regression techniques using both linear and unimodal response models. In this study weighted averaging regression and calibration are used for quantitative paleoenvironmental reconstruction. Weighted averaging (WA) assumes a unimodal response model but is based on a simpler and ecologically reasonable estimate of species' optima that does not involve fitting curves to the species data. The basic concept behind WA is that the most abundant species at a site will tend to be those whose optima is close to the value of the environmental variable at that site (Birks, 1995). Optima are obtained by averaging values of the 
environmental variable at sites where the species is present. The calculation is weighted according to species' abundance. In the reverse situation (calibration) the value of an environmental variable for a fossil sample is estimated by averaging optima of all species that occur in that sample. Calculations are also weighted according to species abundance.

The modern analogue technique (MAT) is an example of a quantitative reconstruction technique that does not have an underlying statistical model. It relies on calculation of similarity or dissimilarity coefficients to compare modern and fossil samples according to their species assemblages (Birks, 1995). Past environment can be inferred for each fossil sample using measured environmental variables of the closest modern analogues. The technique can also be used to determine whether a fossil sample has any good analogues in the modern dataset and so provides an indication of the reliability of a reconstruction.

The most thorough method of evaluating a quantitative paleoenvironmental reconstruction is through comparison with historical measurements of the variable over the most recent period of the sedimentary record. Such comparisons have been made (eg, Bennion et al., 1995) and provide validation of the procedure in general, but lack of comparative data in most cases prohibits use of this technique for routine evaluations. In this study several reconstructions are carried out using different statistical methods and these are compared to provide an indication of the reliability of reconstruction (see Chapters Four, Five and Six).

\subsection{A NEW ZEALAND COASTAL DIATOM CALIBRATION SET}

\subsubsection{Aims}

Benefits of using a calibration set to aid paleoenvironmental reconstruction and the importance of using one in the coastal zone of New Zealand, are outlined in the introduction to this chapter. Specific objectives for the calibration set are derived through consideration of the nature of the sedimentary sequences being reconstructed and the purpose of reconstruction.

The coastline of the Wellington region is steep and actively uplifting with a very narrow coastal plain. The three sites being investigated are small, shallow coastal waterbodies with small, fresh water inflows and protection from the sea in the form of gravel barriers. Geomorphic evidence at the sites, and initial investigation of the sedimentary sequences, suggests that each site has changed from a sheltered marine setting to a dominantly fresh water setting during the Holocene. It is envisaged that sedimentation took place over time in a series of discrete waterbodies each a little shallower and a little more isolated from the sea than the previous waterbody. These initial indications led to the development of an idealised coastal gradient for the uplifted waterbody (Fig. 3.3). 
For reconstruction of gradients of this kind it is obviously useful to be able to document the isolation process. From a paleoseismic viewpoint, it would be beneficial to be able to identify discrete phases of different waterbody types and the nature and amount of environmental change that occurred between them. Therefore one of the aims here is to attempt to characterise different waterbody types in the calibration set according to their diatom assemblages. In this way past waterbody types can be recognised through statistical comparison of modern and fossil assemblages. The second aim is based on the idea that a potentially useful method for estimating magnitude of environmental change is through the quantitative reconstruction of an important environmental variable in the system. Salinity is chosen as a variable that is important in determining diatom distribution in the coastal zone and that is likely to be useful in characterising environmental change. In summary, the aims around which the calibration set is designed are:

1. To characterise small, shallow waterbody types across a coastal gradient using diatom assemblages.

2. To determine salinity preferences for diatom species living in the coastal zone of New Zealand.

\subsubsection{Design}

\section{Introduction}

The aims listed above and the scope of the thesis as a whole, provide constraints within which the calibration set is designed. There are several major features that differ from other calibration sets because location within the coastal zone presents a number of challenges not encountered in open marine or terrestrial settings (these are discussed below). The Holocene sites are referred to repeatedly because the underlying purpose of the calibration set is to aid their reconstruction. However the calibration set is considered relevant for reconstruction of any small, shallow, coastal waterbody consistent with the gradient defined in Fig. 3.3.

\section{Sites}

There are several general considerations to address when choosing sites for a calibration set:

1. Use of sites that are likely to contain the same taxa as are present in the fossil assemblages will reduce problems resulting from lack of modern analogues.

2. Use of sites that cover a spectrum of values for the environmental variable of interest that is wider than that preserved in the past environment will reduce problems arising from truncation of species response curves (illustrated in section 3.4). 


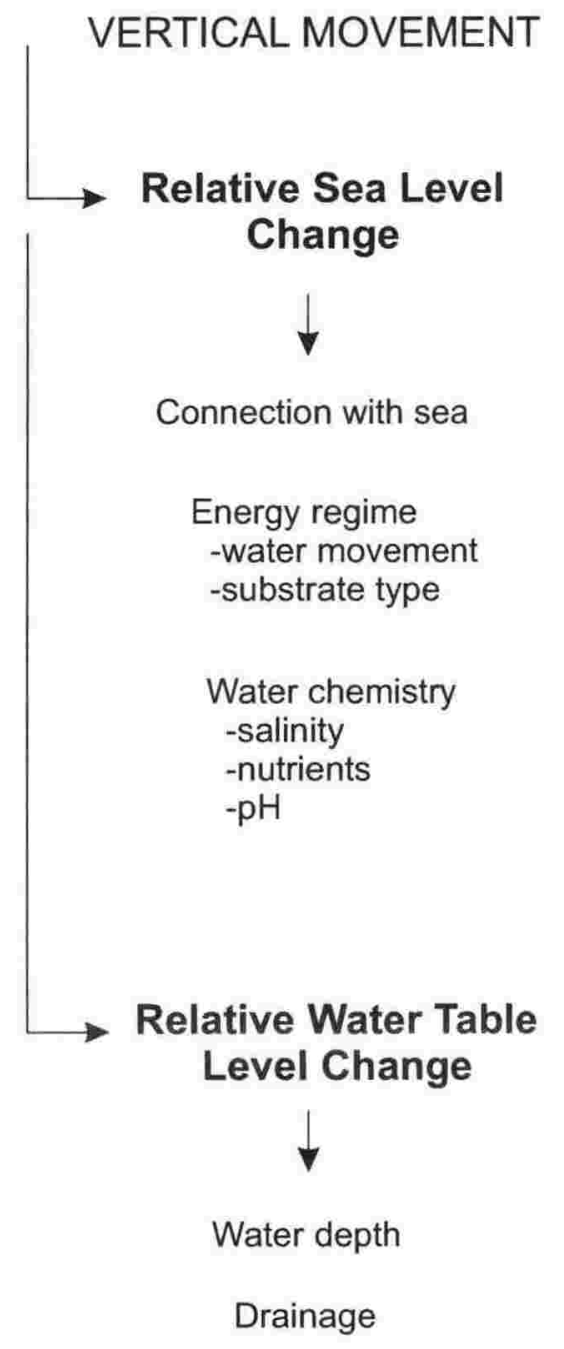

Fig. 3.3. Idealised coastal waterbody gradient showing changes likely to result from vertical movement of base level.

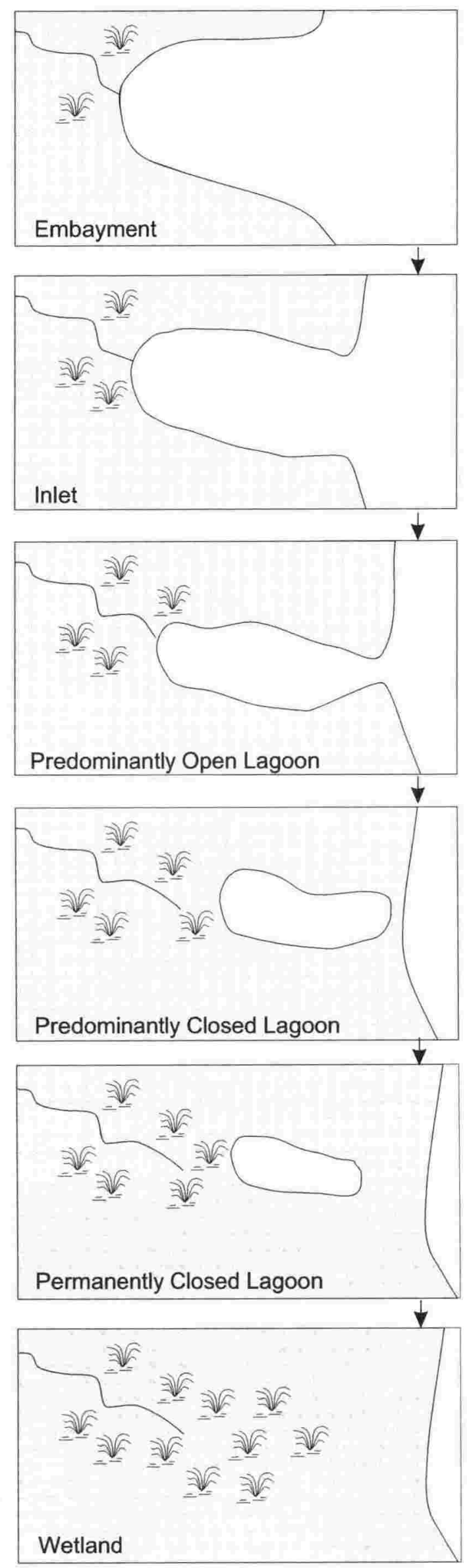


3. Use of sites likely to have the same relationship between the environmental variables of interest and other environmental variables as occurred in the past environment should be attempted to satisfy assumption five of quantitative paleoenvironmental reconstruction (section 3.1.3).

4. Use of sites likely to have the same relationship between the environmental variables of interest and the processes of erosion, transport and deposition as occurred in the past environment should be attempted to satisfy assumption one for the estuarine context (section 3.1.3).

These requirements are usually met by selecting sites in the same geographic region and of the same depositional environment as the fossil sites but across a wider ecological gradient. In most calibration sets a large range in values of the environmental variable of interest can be measured from a set of sites with constant depositional environment. For example $\mathrm{pH}$ can be measured in lakes, sea surface temperature at open marine sites, and even athalassic salinity can be measured using inland saline lakes.

A different approach is required here because one of the aims is to characterise different waterbody types along a gradient from marine to terrestrial environments. The waterbodies of interest include inlets, lagoons, coastal lakes and wetlands so it is not possible to sample across such a coastal gradient and keep depositional environment constant. Therefore the aim here is to select sites that will define a spatial gradient of waterbody types as equivalent as possible to the temporal gradient of environments represented by the sedimentary sequences. The only difference in design should be that the modern sampled gradient is longer than the fossil one in order to satisfy point two above.

The uplifted coastal waterbody model (Fig. 3.3) was developed from initial investigations of past sequences so it provides a starting point for definition of the gradient of interest. Waterbodies that belong to this gradient are small, shallow, low energy and have small catchments. Depositional environment is determined predominantly by connection with the sea because fresh water inflow has negligible influence. In all other respects the gradient is assumed to be an idealised gradient - with environmental variables and processes interacting in an intuitive and predictable way. For example, as connection with the sea becomes restricted, the energy regime changes with decreasing flow speed and grainsize, water chemistry changes with decreasing salinity and $\mathrm{pH}$, water depth is also likely to decrease. Modern sites are selected to conform to this idealised uplifted coastal gradient and to represent as many waterbody types from the gradient as possible. If the gradient is a standard coastal gradient that existed in the past then points three and four above are more likely to be satisfied. 
Calibration sets can vary in size from a minimum of about 30 sites to hundreds of sites. Large calibration sets perform better than smaller ones because there are fewer errors resulting from lack of modern analogues or truncation of species response curves (eg, Wilson et al., 1994). However, collection of a high quality calibration set is time consuming, so size has to be limited according to the scope of the project. In this study the calibration set is part of a wider study so 50 sites was considered a maximum.

\section{Environmental Variables}

There are a large number of chemical and physical environmental variables that diatoms have been shown to respond to. It would be an impossible task to measure everything affecting assemblage composition of diatoms in a waterbody at any particular time. A general aim for a calibration set is to measure 'important' variables, i.e. those explaining a large amount of variation in diatom distribution over the environmental gradient of interest. The priority for this study was to measure variables that would be useful in characterisation of different waterbody types. Because of the large range in physical conditions across a coastal gradient, emphasis was placed on measuring physical variables.

An important consideration in the measurement of environmental variables is the representativity of measurements. The ultimate aim is to obtain an estimate of the environmental conditions that existed at the time the diatom death assemblage was living. In practice, this is hard to obtain because the time period represented by the death assemblage is unknown and many environmental factors are naturally variable. The approach used in this study was to carry out repeat measurements for salinity, conductivity, $\mathrm{pH}$, water temperature and water depth that would provide reasonable estimates of values that the living diatoms were responding to at that point in space and time. Living assemblages were collected as well as death assemblages and comparison between the two was used to judge whether environmental variables had changed significantly between time of measurement and time that death assemblage species were living.

The number of measurements taken at each site was adjusted according to variability of water chemistry. For example sites completely isolated from the sea have stable water chemistry compared with those open to the sea. For closed sites at least two readings were taken with a minimum separation of one hour. For most tidally influenced sites measurements were taken at each of four states of the tide - high, low, incoming, and outgoing. Where possible, these measurements were repeated in summer and winter. Physical variables such as distance from the sea, elevation, grainsize and aquatic plant growth were assumed constant at each site because of the short time period represented by living diatoms in comparison with the time period over which physical changes take place. Care was taken to avoid collection of sediment deposited rapidly by a single catastrophic event such as a storm or flood. 


\section{Diatom Samples}

Samples were located in the littoral zone of each waterbody for several reasons. Firstly, cores for the Holocene sedimentary sequences were taken from the littoral zone of each waterbody because changes in water depth and sea level are likely to be more clearly reflected in this zone than in deeper water sites. Secondly, the littoral zone has a high diversity of benthic diatom species. Deeper water, central locations are often dominated by planktonic species and these are less useful for the calibration set because of their potential for transport. A typical aim for calibration set samples is to collect a sample that will represent the waterbody as a whole in which case collection from the deepest, most central part of a waterbody is sensible (Battarbee, 1986). However, in this calibration set, because of the great variety of conditions that occur over the area of most waterbodies, modern samples were collected to represent sub-sites within a waterbody type not the waterbody as a whole. For example collection of a sample consisting of an average assemblage for an inlet would be an unrealistic task, whereas collection of a salt marsh assemblage at the head of an inlet or a tidal channel assemblage at the inlet mouth is more achievable.

Death assemblage samples (those collected from beneath the sediment surface) are used in this calibration set because they generally contain a time-averaged amalgamation of diatoms from all habitats and so are direct analogues for fossil assemblage samples. The depth below the surface that diatom assemblages were taken varied according to energy regime of the sites. At high energy, tidal sites death assemblage samples were collected deeper below the sediment surface because of the effects of wave action and the likelihood of live diatoms being incorporated lower into the sediment. At tidal sites samples were collected below low tide to minimise effects of daily exposure on diatom preservation and assemblage composition. Areas of fresh water or marine inflow to a waterbody were avoided because of problems resulting from highly fluctuating water chemistry, transport of diatom valves and storm or flood deposits.

\section{Diatom Species}

The main requirements for samples in a calibration set in terms of diatom content are firstly that they contain well preserved organisms in high numbers, and secondly that they are representative of diatom assemblages at the collection site at that point in time (assumption two of reconstruction in the estuarine context). The first requirement is easy to satisfy because diatoms are numerous in surface sediment samples of all types of coastal waterbodies (Denys and de Wolf, 1999). Preservation and abundance depend on many factors but these will generally be acceptable as long as sites with extreme chemical characteristics or of extreme high energy are avoided. Sixty samples were initially collected and this allowed samples with the poor assemblages to be removed from the calibration set. 
The second requirement is harder to satisfy because of the likelihood that allochthonous valves have been incorporated into the sampled assemblage. Allochthonous valves, those that have been transported away from their life position, do not represent a response to environmental variables measured for that sample and are not easy to differentiate from autochthonous valves (Beyens and Denys, 1982). Diatom samples from low energy sites are not likely to contain large proportions of allochthonous diatoms but in tidal environments allochthoneity can be a problem (Vos and de Wolf, 1988).

One measure taken in this calibration set to assess whether allochthonous valves have been incorporated into death assemblage samples, is the collection of living diatoms in addition to death assemblages. Living diatoms can be assumed to be autochthonous because they would die if transported too far from their source environment. Therefore comparison of living and death assemblage samples can provide an indication of the allochthonous component of the death assemblage. For example Juggins (1992) defined 'life ranges' for species collected in modern samples in the Thames Estuary and used these to downweight species in death assemblage samples that occurred outside their life range.

Consistent, high quality taxonomy is a basic requirement for accurate paleoenviromental reconstruction (Birks, 1994). Species were identified to the lowest taxonomic level, varieties were counted separately in case they represented different environmental conditions, and all taxa were documented. This documentation provided a reference system for checking valves and maintaining consistency within the calibration set and between the modern and fossil samples.

\subsubsection{Methods}

\section{Measurement of Environmental Variables}

Salinity, conductivity, $\mathrm{pH}$ and temperature were measured at the site with portable conductivity and $\mathrm{pH}$ meters. Repeat measurements were taken as described in section 3.2.2. Shading, aquatic plant growth, flow, and exposure to air were all described qualitatively at the sites and allocated into set categories (Table 3.2). Sediment samples were collected for grainsize analysis in order to determine proportions of sand, mud, organic matter and carbonate. Characteristics such as area of water, catchment area, catchment relief and distance from sea were measured from 1:50,000 topographic maps. Catchment cover, catchment bedrock, exposure to fresh and salt water, elevation and water depth were described in the field and checked against topographic and geologic maps. Distances and depths were recorded as continuous variables, others were divided into ordinal or nominal categories. Water clarity was measured using a Secchi disk. Annual precipitation and air temperature were taken from the National Institute of Water and Atmosphere's climate database using proximal climate stations for each site. 


\section{Sample Collection}

Samples for grain size and diatom death assemblages were collected beneath the upper $0.5 \mathrm{~cm}$ of surface sediment which was removed with a spatula. At high energy, tidal sites $1.5 \mathrm{~cm}$ of surface sediment was removed to account for live diatoms being incorporated lower into the sediment by wave action. About $20 \mathrm{~g}$ was collected for grainsize analysis. Diatom death assemblages were collected using a plastic syringe inserted into the sediment. At each site at least three $1.5 \mathrm{~cm}^{2}$ samples were taken and amalgamated to avoid effects of within-site variability (Juggins, 1992).

Diatoms living on the sediment surface (epipsammon and epipelon) were collected by suction through a glass tube as described by Round (1953). A one metre length of one centimetre diameter glass tubing was sealed with a thumb at one end, lowered through water to the sediment surface, drawn over the sediment while slowly releasing the thumb, and a mix of surface sediment, algae and water was collected. To retrieve the sample the tube was again sealed with a thumb, removed from the water, and the contents transferred to a $250 \mathrm{ml}$ collection pot. To ensure a representative diatom assemblage was collected, about five such scrapings were taken at each sample site. Diatoms living on aquatic plants (epiphyton) were collected by clipping leaves and stems off plants living either fully or partially submerged in the immediate vicinity of the sample site. A handful of plant material was collected, consisting of the dominant species present at the site, and placed in a plastic pot. Diatoms living on rock surfaces (epilithon) were sampled by collecting a $250 \mathrm{ml}$ pot full of any submerged pebbles present in the vicinity of the sample site.

There are various methods of ensuring that modern diatoms collected are indeed alive. However these methods are time consuming and are either biased towards motile species or result in poor slides that prohibit identification of taxa (Juggins, 1992). Instead it was assumed that the majority of species in the modern samples were alive when collected or, if dead, were likely to have been autochthonous to the site anyway. This is considered a reasonable assumption because of the careful collection methods used for modern samples and because in a similar environmental context Juggins (1992), using staining, concluded that over $95 \%$ of valves in modern samples had intact chloroplasts. Hemphill-Haley (1995b) also stained diatom samples from Willapa Bay, Washington and concluded that autochthonous benthic species usually greatly exceed any allochthonous taxa. 


\section{Sample Processing}

Modern grain size, organic carbon and diatom death assemblage samples were processed exactly as described for fossil samples in Chapter Two. Living diatom samples required slightly different preparation before processing. Surface sediment samples were shaken thoroughly in their collection pots and a wellmixed aliquot of sample was poured off and retained. Hydrogen peroxide was added to the plants and pebbles of the epiphytic and epilithic samples in their collection pots to detach diatoms from the surfaces. The following day plant material and pebbles were picked out with tweezers, rinsed with filtered water and disposed of. In all three cases sediment was left to settle for at least eight hours, excess water was poured off and samples were transferred to $100 \mathrm{ml}$ glass beakers. The clean nature of the epiphytic and epilithic samples meant that shorter treatments and fewer washes were required.

\section{Representativity and Allochthonous Component of Death Assemblages}

Diatom assemblages of death assemblage samples were compared with assemblages from corresponding modern samples to provide a check on the fitness of each death assemblage sample for inclusion in the calibration set. Assemblages of modern samples are not directly comparable with death assemblages because they were collected from individual habitats and the death assemblage represents an amalgamation of these habitats. However the death assemblages should contain similar species to the modern samples unless: environmental conditions have changed since the time the death assemblage species were alive; allochthonous valves were transported to the site and incorporated into the death assemblage; or species have dissolved or been transported away from the site since the time the death assemblage species were alive.

All of these situations are undesirable because of the errors that can be introduced into the calibration set and resulting paleoenvironmental reconstructions. Death assemblages that contained many species or large proportions of species that were not present in modern samples from the same site were identified as potential problem samples. Original count and environmental data were revisited in attempts to determine reasons for differences and in some cases samples were eliminated from further analysis. 


\section{Mathematical Procedures}

Species and environmental data were entered into and stored in EXCEL

worksheets. Data were converted to Cornell condensed format using WINTRAN 1.01 (Juggins, 1998). Ordination analyses were carried out using CANOCO 3.12 (ter Braak, 1991) and plotted in CANODRAW LITE 3.0 (Smilauer, 1992).

CALIBRATE 0.85 (Juggins and ter Braak, 1997-99) was used for weighted averaging regression and calibration and various plots. MAT98 version 1.2 (Juggins, 2000) was used to determine closest modern analogues for fossil samples and to reconstruct salinity using the modern analogue technique.

Simple exploratory analysis was used to provide an indication of how individual species and environmental variables changed along the coastal gradient. For example species abundances were plotted in PSIMPOLL with sites arranged according to waterbody type along the coastal gradient. Environmental variables were plotted in EXCEL with sites arranged as above.

Frequency histograms were plotted for each environmental variable to look at their distribution in the calibration set. Continuous variables that show a skewed or log-normal distribution can benefit from transformation to a normal distribution (Jongman et al., 1995). A log-normal distribution was assumed for variables that had two of the following three characteristics: a skewed frequency histogram, a standard deviation larger than the mean and a maximum value twenty times larger than the minimum value (Jongman et al., 1995). Five variables (distance from the sea, proximity to fresh water inflow, mud, organics and carbonate) were log transformed using CALIBRATE and the transformations were retained for all further analyses.

Because a coastal gradient that extends from marine waters to fresh waters consists of a wide variety of waterbody types or depositional environments it is hard to define exactly which sites will conform to characteristics of the gradient as 
of nominate variables that had an unrealistic influence on ordination solutions because they only had one occurrence in the calibration set.

Initially a DCA was run using the complete species dataset to determine whether linear or unimodal statistical methods were appropriate for further analysis. The resulting first axis had a gradient length over seven standard deviation units indicating that unimodal methods were appropriate. Indirect gradient analysis was carried out using correspondence analysis (CA) and DCA to examine structure in the species data. Direct gradient analysis was carried out using CCA and detrended canonical correspondence analysis (DCCA) to examine which environmental variables were important in explaining variation in the diatom data. DCCA site and species plots were compared with DCA plots to provide an indication of how well the measured environmental variables explained the diatom data.

Various methods were used to examine the dataset of environmental variables and reduce it to a smaller set of statistically significant variables that could explain variation in the species data almost as well as the full set. A series of CCAs was carried out with each environmental variable as the sole constraining variable in the analysis to estimate the amount of variation in the diatom data that individual variables could account for. An associated Monte Carlo permutation test (99 unrestricted permutations) was carried out on the first axis of each analysis to determine the statistical significance of each variable. PCA with environmental variables entered as species, data centred and standardised, and scores scaled for a correlation biplot, was used to examine correlations between measured environmental variables. These were compared with an environmental variable plot and correlation matrix resulting from CCA. This enabled four sets of highly correlated variables to be defined and used for further analysis.

Forward selection with associated Monte Carlo permutation tests ( 99 unrestricted permutations) was carried out on the environmental dataset as part of a CCA. Variables were chosen so there would be at least one representative from each of the four sets defined previously, and salinity was chosen first over waterbody type and exposure to salt because, as a continuous variable, it is preferred over the former variables for reconstruction purposes. Seven environmental variables were found to be statistically significant and explain the species data well. These were used to represent the four sets and partial CCAs were carried out to determine the percentage of variation in the diatom data that each was explaining. In each case variables of one set were entered as environmental variables and the remaining three sets were entered as covariables. Partial CCAs were also used to determine what percentage variance could be accounted for by interactions between sets. 


\subsection{DESCRIPTION OF THE CALIBRATION SET}

\subsubsection{Introduction}

The calibration set consists of 50 waterbody sites from around the coastline of New Zealand (Fig 3.4). Over half the sites are located in the Wellington region because of the similar geologic and climatic setting to the Holocene sites. Most of the remaining sites are in the South Island of New Zealand. Exact locations and general site characteristics are listed in Appendix $\mathrm{V}$ and all other characteristics are used as explanatory environmental variables. Sites consist of bays, lagoons, lakes and wetlands, and range from marine to fresh water conditions (Fig. 3.5). The structure of Table 3.1 indicates that calibration set sites represent a coastal gradient with samples from progressively isolated sites having progressively lower salinities.

Ten waterbody types are used in the calibration set and are defined largely by their connection with the sea. Inlets are defined as being a tidal arm of the sea with a small fresh water inflow as opposed to estuaries that are generally considered to be the lower parts of rivers open to the sea (Boggs, 1995). Lagoons are shallow areas of brackish water near or connected with the sea and with little fresh water input (Boggs, 1995). Lagoons are divided into three types depending on the frequency of influx of brackish water: predominantly open, predominantly closed and permanently closed. Permanently closed lagoons, coastal lakes, and lakes, are differentiated by their distance from the sea being hundreds of metres, $\sim 1 \mathrm{~km}$, and $>5 \mathrm{~km}$ respectively. Wetlands are defined as having $<0.1 \mathrm{~m}$ water depth and abundant macrophytes. Coastal wetlands are potential outliers in this dataset because wetlands exist at a wide range of salinity values and a large dataset would be required to characterise them. A few were sampled in this study to provide analogues for fossil samples that may have been wetlands rather than open water environments. Other exceptions to the diagonal structure include samples from Okupe Lagoon which have much higher salinities than all other permanently closed lagoons in the calibration set. It was noted in the field that the lagoon must have groundwater access to the sea, or salts must be concentrated by evaporation, because there is no open connection with the sea and salinity is high. 


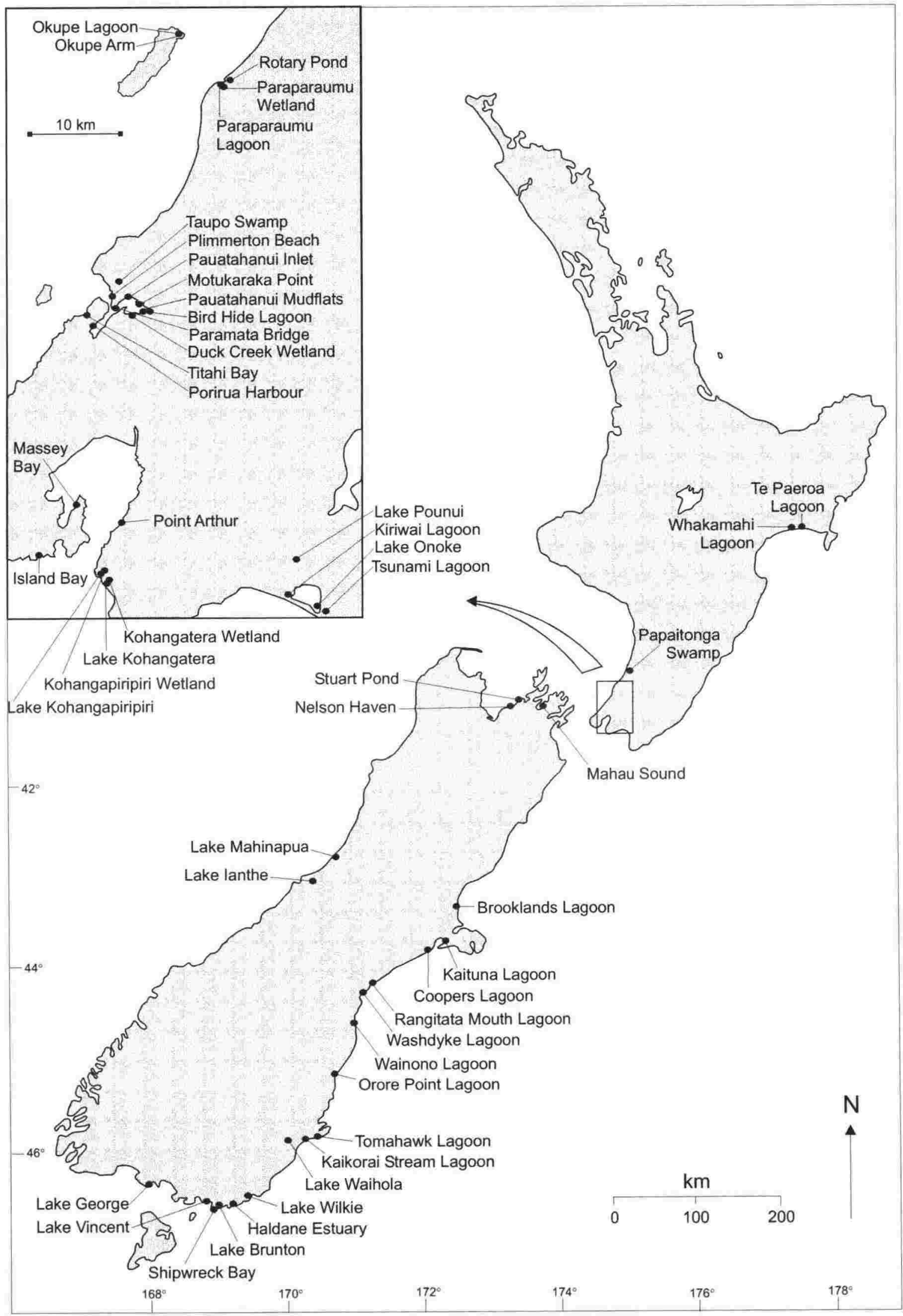

Fig. 3.4 Map showing location of calibration set sites around the coast of New Zealand and in the Wellington region (inset). 

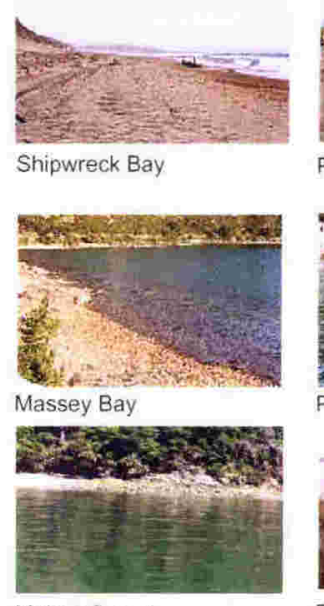

Mahau Sound

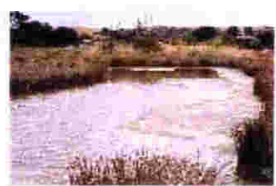

Bird Hide Lagoon

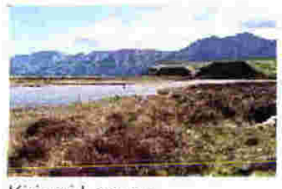

Kiriwai Lagoon

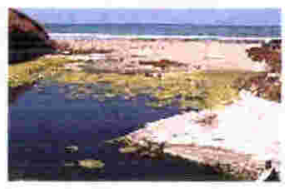

Orore Point
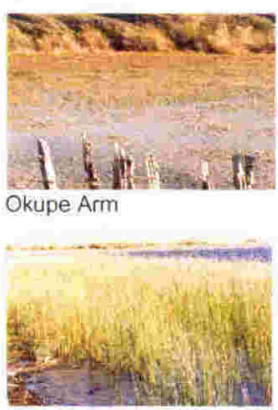

Lake Kohangapiripiri
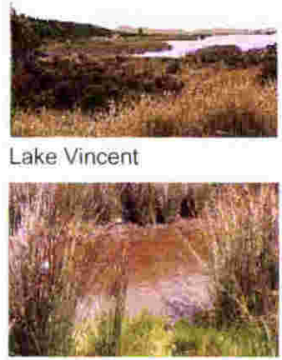

Paraparaumu Wetlan

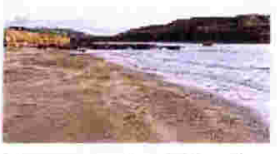

Plimmerton South Beach

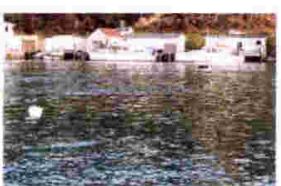

Paremata Bridge

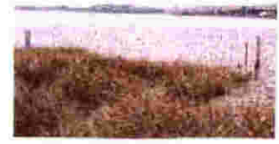

Pauatahanui Mudflats

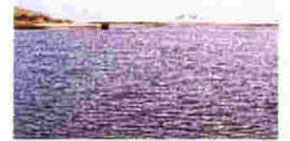

Kaikorai Lagoon

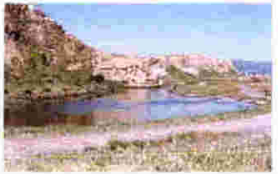

Tsunami Lagoon

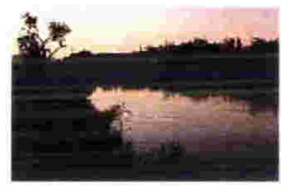

Rotary Pond

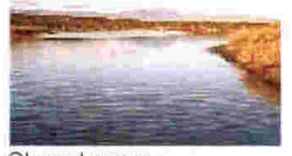

Okupe Lagoon

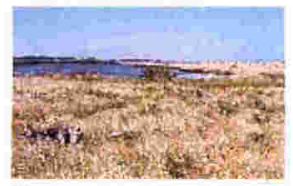

Coopers Lagoon

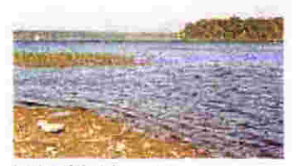

Lake Mahinapua

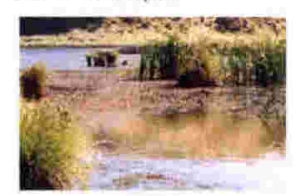

Kohangapiripiri. Wetland

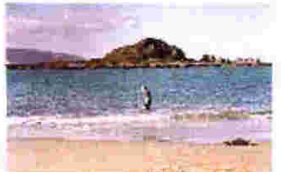

Island Bay

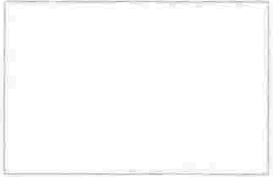

Pauatahanui Inlet

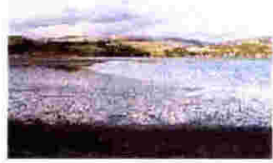

Motukaraka Point

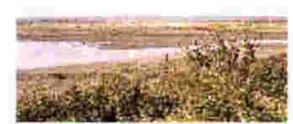

Whakamahi Lagoon

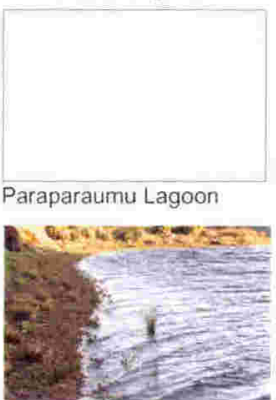

Lake Kohangatera

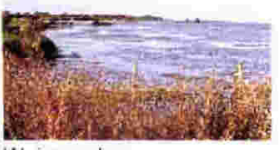

Wainono Lagoon

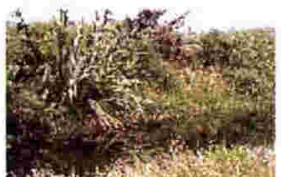

Rangitata Mouth

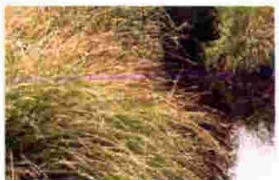

Lake Pounui

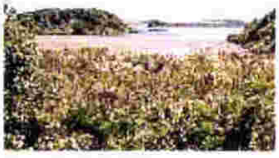

Papaitonga Swamp

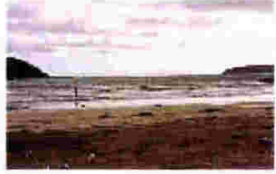

Titahi Bay

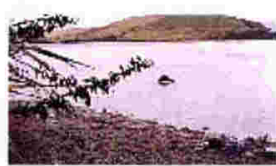

Haldane Estuary

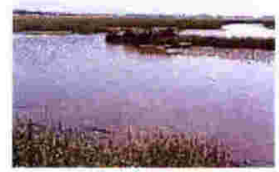

Brooklands Lagoon

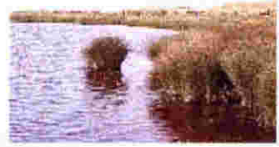

Lake Brunton

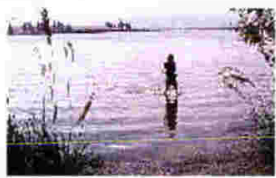

Lake Waihola

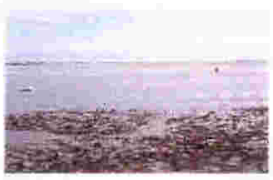

Kaituna Lagoon

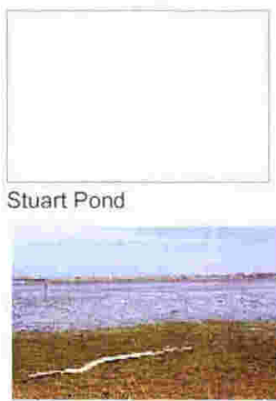

Waitarakao Lagoon

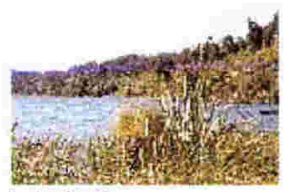

Lake lanthe

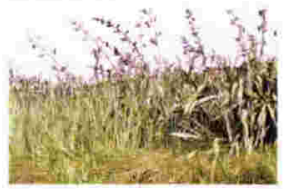

Taupo Swamp

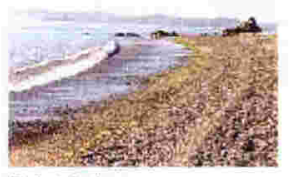

Point Arthur

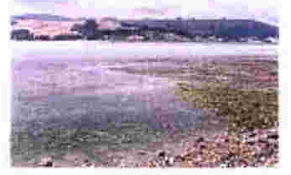

Porirua Harbour

Nelson Haven

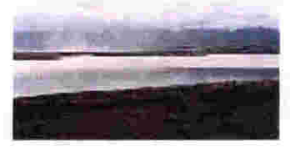

Lake Onoke

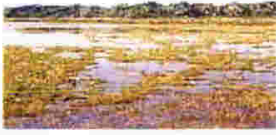

Te Paeroa Lagoon

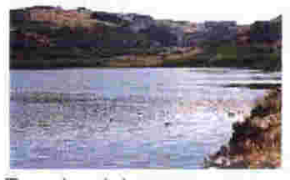

Tomahawk Lagoon

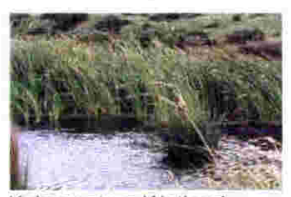

Kohangatera Wetland

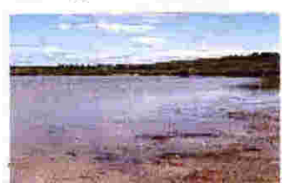

Lake George

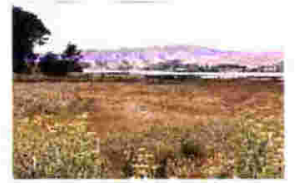

Duck Creek Wetland

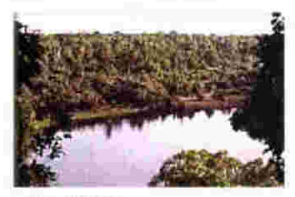

Lake Wilkie

Fig. 3.5. Calibration set sites arranged according to waterbody type with open bays at the top and fresh water wetlands at the base. Images illustrate the range of waterbodies sampled along the coastal gradient. 


\begin{tabular}{|c|c|c|c|c|}
\hline & $\begin{array}{l}\text { MARINE LITTORAL } \\
20-35 \mathrm{gl}^{-1}\end{array}$ & $\begin{array}{c}\text { BRACKISH MARINE } \\
10-20 \mathrm{gl}^{-1}\end{array}$ & $\begin{array}{c}\text { BRACKISH FRESH } \\
1-10 \mathrm{gl}^{-1}\end{array}$ & $\begin{array}{l}\text { FRESH } \\
<1 \mathrm{gl}^{-1}\end{array}$ \\
\hline OPEN BAY & $\begin{array}{l}\text { Shipwreck Bay } \\
\text { Plimmerton Beach } \\
\text { Island Bay } \\
\text { Titahi Bay }\end{array}$ & & & \\
\hline $\begin{array}{l}\text { SHELTERED } \\
\text { EMBAYMENT }\end{array}$ & $\begin{array}{l}\text { Point Arthur } \\
\text { Massey Bay } \\
\text { Paramata Bridge }\end{array}$ & & & \\
\hline INLET & $\begin{array}{l}\text { Pauatahanui Inlet } \\
\text { Haldane Estuary } \\
\text { Porirua Harbour } \\
\text { Mahau Sound } \\
\text { Motukaraka Point } \\
\text { Pauatahanui Mudflats } \\
\text { Brooklands Lagoon }\end{array}$ & Nelson Haven & & \\
\hline $\begin{array}{l}\text { PREDOMINANTLY } \\
\text { OPEN LAGOON }\end{array}$ & & $\begin{array}{l}\text { Kaikorai Stream } \\
\text { Bird Hide Lagoon } \\
\text { Whakamahi Lagoon }\end{array}$ & $\begin{array}{l}\text { Lake Brunton } \\
\text { Lake Onoke }\end{array}$ & \\
\hline $\begin{array}{l}\text { PREDOMINANTLY } \\
\text { CLOSED LAGOON }\end{array}$ & & & $\begin{array}{l}\text { Tsunami Lagoon } \\
\text { Paraparaumu Lagoon } \\
\text { Kiriwai Lagoon } \\
\text { Te Paeroa Lagoon } \\
\text { Orore Point Lagoon }\end{array}$ & $\begin{array}{l}\text { Rotary Pond } \\
\text { Kaituna Lagoon } \\
\text { Tomahawk Lagoon } \\
\text { Lake Kohangatera }\end{array}$ \\
\hline $\begin{array}{l}\text { PERMANENTLY } \\
\text { CLOSED LAGOON }\end{array}$ & $\begin{array}{l}\text { Okupe Lagoon } \\
\text { Okupe Arm }\end{array}$ & & Wainono Lagoon & $\begin{array}{l}\text { Stuart Pond } \\
\text { Kohangatera Wetland } \\
\text { Lake Kohangapiripiri } \\
\text { Waitarakao Lagoon } \\
\text { Coopers Lagoon } \\
\text { Rangitata Mouth }\end{array}$ \\
\hline COASTAL LAKE & & & & $\begin{array}{l}\text { Lake Vincent } \\
\text { Lake George } \\
\text { Lake Mahinapua }\end{array}$ \\
\hline LAKE & & & & $\begin{array}{l}\text { Lake Pounui } \\
\text { Lake lanthe }\end{array}$ \\
\hline $\begin{array}{l}\text { COASTAL } \\
\text { WETLAND }\end{array}$ & & Duck Creek Wetland & Paraparaumu Wetland & \\
\hline WETLAND & & & & $\begin{array}{l}\text { Kohangapiri Wetland } \\
\text { Taupo Swamp } \\
\text { Papaitonga Swamp } \\
\text { Lake Wilkie }\end{array}$ \\
\hline
\end{tabular}

Table 3.1 Calibration set sites tabulated according to waterbody type and salinity. Salinity categories are listed in grams per litre $\left(\mathrm{g} \mathrm{l}^{-1}\right)$. Letters in red are those used as site codes in statistical analysis. 
Twenty-six physical and chemical environmental variables were measured and 25 of these were used as explanatory variables in attempts to explain the diatom data (Table 3.2). Secchi depth was measured at each site but because of shallow water depths, was not a limiting factor at any site, so was removed from further analysis. Most variables consist of continuous, quantitative measurements; nine variables are measured on an ordinal scale and three on a nominal scale. Nominal variables were separated into individual classes for analysis in CANOCO. Values of environmental variables for each site are listed in Appendix V.

\begin{tabular}{|c|c|c|}
\hline ENVIRONMENTAL VARIABLE & CODE & UNIT / CATEGORY \\
\hline \multicolumn{3}{|l|}{ Continuous variables: } \\
\hline Distance from sea (via opening if present) & DIST SEA & Metres \\
\hline Proximity to nearest fresh water inflow & PROX FI & Metres \\
\hline Water depth (where sample collected) & DEPTH & Metres \\
\hline Water Temperature & TEMP & Degrees Celsius \\
\hline Salinity & SAL & Grams per litre \\
\hline Conductivity & COND & Millisiemens per centimetre \\
\hline $\mathrm{pH}$ & $\mathrm{pH}$ & $\mathrm{pH}$ units \\
\hline Sand (> 60 micronns) & SAND & Percent \\
\hline Mud $(<60$ microns) & MUD & Percent \\
\hline Organic matter & ORGAN & Percent \\
\hline Carbonate & CARB & Percent \\
\hline Air Temperature (annual for 1998) & AIR TEMP & Degrees celcius \\
\hline Precipitation (annual for 1998) & PRECIP & Millimetres \\
\hline Secchi depth & & Metres \\
\hline \multicolumn{3}{|l|}{ Nominal variables: } \\
\hline \multirow[t]{5}{*}{ Catchment cover (dominant type) } & BUSH & Native Bush \\
\hline & FOREST & Exotic forest \\
\hline & SCRUB & Scrub \\
\hline & PASTURE & Pasture \\
\hline & URBAN & Urban \\
\hline \multirow[t]{7}{*}{ Catchment bedrock (dominant type) } & FELSIC & Felsic intrusives \\
\hline & MAFIC & Mafic intrusives \\
\hline & MAFINT & Mafic / intermediate volcanics \\
\hline & SCHIST & Quartzo-feldspathic schist \\
\hline & TERT & Tertiary \& Mesozoic sandstone, mudstone, conglomerate \\
\hline & QUAT & Quaternary sands, gravels, tills \\
\hline & HOLO & Holocene coastal sediments \\
\hline \multirow[t]{2}{*}{ Rock surfaces / Pebbles } & PEBB & Present \\
\hline & & Absent \\
\hline
\end{tabular}

Table 3.2. List of environmental variables measured for the calibration dataset. Part A: Continuous and nominal variables. 


\begin{tabular}{|c|c|c|c|}
\hline ENVIRONMENTAL VARIABLE & CODE & \multicolumn{2}{|l|}{ CATEGORY } \\
\hline \multicolumn{4}{|l|}{ Ordinal variables: } \\
\hline \multirow[t]{10}{*}{ Water body type } & \multirow[t]{10}{*}{$\mathrm{H} 2 \mathrm{O} \mathrm{BOD}$} & \multicolumn{2}{|l|}{ Open bay } \\
\hline & & \multirow{2}{*}{\multicolumn{2}{|c|}{$\begin{array}{l}\text { Sheltered embayment } \\
\text { Inlet }\end{array}$}} \\
\hline & & & \\
\hline & & \multicolumn{2}{|c|}{ Predominantly open lagoon } \\
\hline & & \multicolumn{2}{|c|}{ Predominantly closed lagoon } \\
\hline & & \multicolumn{2}{|c|}{ Permanently closed lagoon } \\
\hline & & \multicolumn{2}{|c|}{ Coastal lake } \\
\hline & & \multicolumn{2}{|l|}{ Lake } \\
\hline & & \multicolumn{2}{|l|}{ Coastal wetland } \\
\hline & & \multicolumn{2}{|l|}{ Wetland } \\
\hline \multirow[t]{9}{*}{ Exposure to salt } & \multirow[t]{9}{*}{ EXPOS } & \multicolumn{2}{|c|}{ Continuous open connection with sea } \\
\hline & & \multicolumn{2}{|c|}{ Daily connection with sea (tidal) } \\
\hline & & \multicolumn{2}{|c|}{ Daily connection with inlet (tidal) } \\
\hline & & \multicolumn{2}{|c|}{ Seasonal / annual influx from sea } \\
\hline & & \multicolumn{2}{|c|}{ Seasonal / annual influx from inlet / lagoon } \\
\hline & & \multicolumn{2}{|c|}{ Seepage through barrier/groundwater } \\
\hline & & \multicolumn{2}{|c|}{ Windblown only - frequent } \\
\hline & & Windblown only - occa & \\
\hline & & No exposure & \\
\hline Size of nearest fresh water inflow & SIZE FI & River & \\
\hline & & Stream & \\
\hline & & Creek & \\
\hline & & Runoff only & \\
\hline Salinity Range ( $\max$ - min of measurements) & RANGE & $<0.1 \mathrm{gl}^{-1}$ & \\
\hline & & $0.1-1 \mathrm{gl}^{-1}$ & \\
\hline & & $1-5 \mathrm{gl}^{-1}$ & \\
\hline & & $5-10 \mathrm{gl}^{-1}$ & \\
\hline & & $10-15 \mathrm{gl}^{-1}$ & \\
\hline & & $15-20 \mathrm{gl}^{-1}$ & \\
\hline & & $20-25 \mathrm{gl}^{-1}$ & \\
\hline Height above mean sea level & ELEVTN & $<0$ metres above mea & level \\
\hline & & $0-2.5 \mathrm{~m}$ & \\
\hline & & $2.5-5 \mathrm{~m}$ & \\
\hline & & $5-10 \mathrm{~m}$ & \\
\hline & & $10-15 m$ & \\
\hline & & $>15 \mathrm{~m}$ & \\
\hline Shading & SHADE & Shaded for most of das & \\
\hline & & Shaded part of day by & nd plants \\
\hline & & Shaded part of day by & plants \\
\hline & & Exposed throughout de & \\
\hline Aquatic plant growth & PLANT & Abundant & Present \\
\hline & & Frequent & Occasional \\
\hline Flow & FLOW & Continuously fast & Periodic \\
\hline & & Periodically fast & Slow \\
\hline & & Continuous & None \\
\hline Exposure to air & EXPO & Daily & \\
\hline & & Monthly & Occasionally \\
\hline & & Seasonally / Annually & Never \\
\hline
\end{tabular}

Table 3.2. List of environmental variables measured for the calibration dataset. Part B: Ordinal variables. 
Diatoms were numerous and well preserved in most death assemblage samples collected. Ten sites were removed from the calibration set and are not listed above because of low concentrations or poor preservation of diatoms. In the remaining 50 death assemblage samples counted, 305 taxa were identified representing 64 genera. Species counts are presented in Appendix VII. Estimated concentrations of diatom valves ranged from a few tens of thousands of valves per gram of dry sediment to a few hundred million, with most samples having concentrations in the tens of millions (Fig. 3.6).

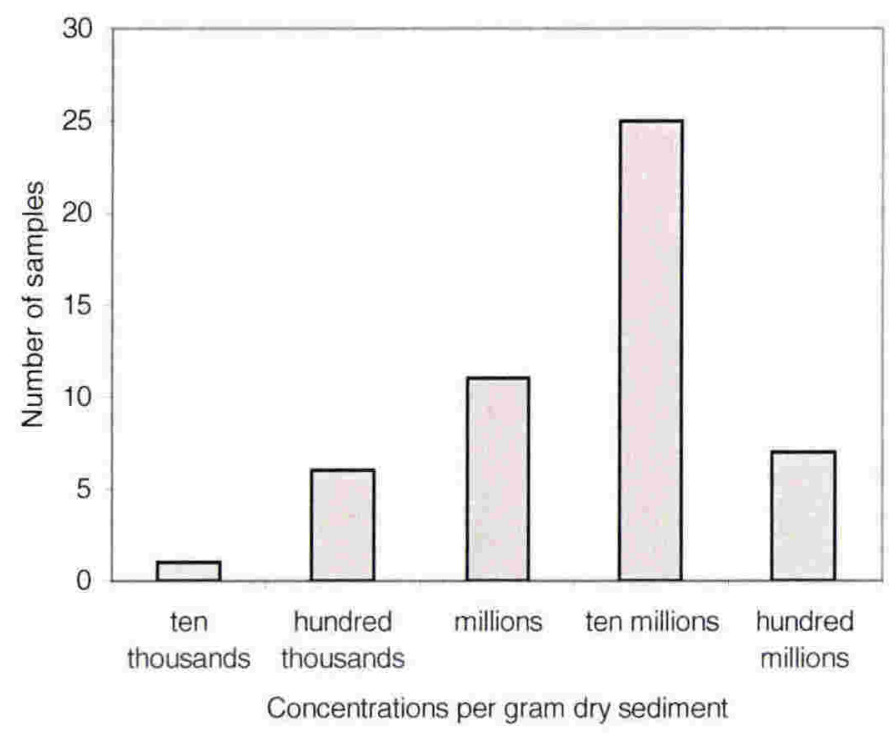

Fig. 3.6. Frequency histogram of diatom valve concentrations for death assemblage samples in the calibration set. Most samples had tens of millions of diatom valves per gram of sediment.

Although there is a large range in concentration of diatom valves between samples, there is only a weak trend of decreasing concentration towards the high energy, open marine sites along the coastal gradient (Fig. 3.7). Many of the low concentration samples were from sandy sampling sites and high concentration samples appear to be from muddy sites. However this may not be an ecological feature but a result of concentration being measured relative to sediment weight. In hindsight it seems that volume would have been a more appropriate standard to use when comparing concentrations from such a diverse range of sediment types. Another factor affecting concentration values is the occurrence of numerous small Fragilaria species. Most of the very high concentration samples have large numbers of these very small valves so they may have similar diatom biomass values to other samples, but they have much higher concentrations.

The number of species encountered in any one sample (species richness) ranged between 12 and 47 . The number of species is presented as a proportion of the total number of valves counted in each sample so comparisons can be made between 


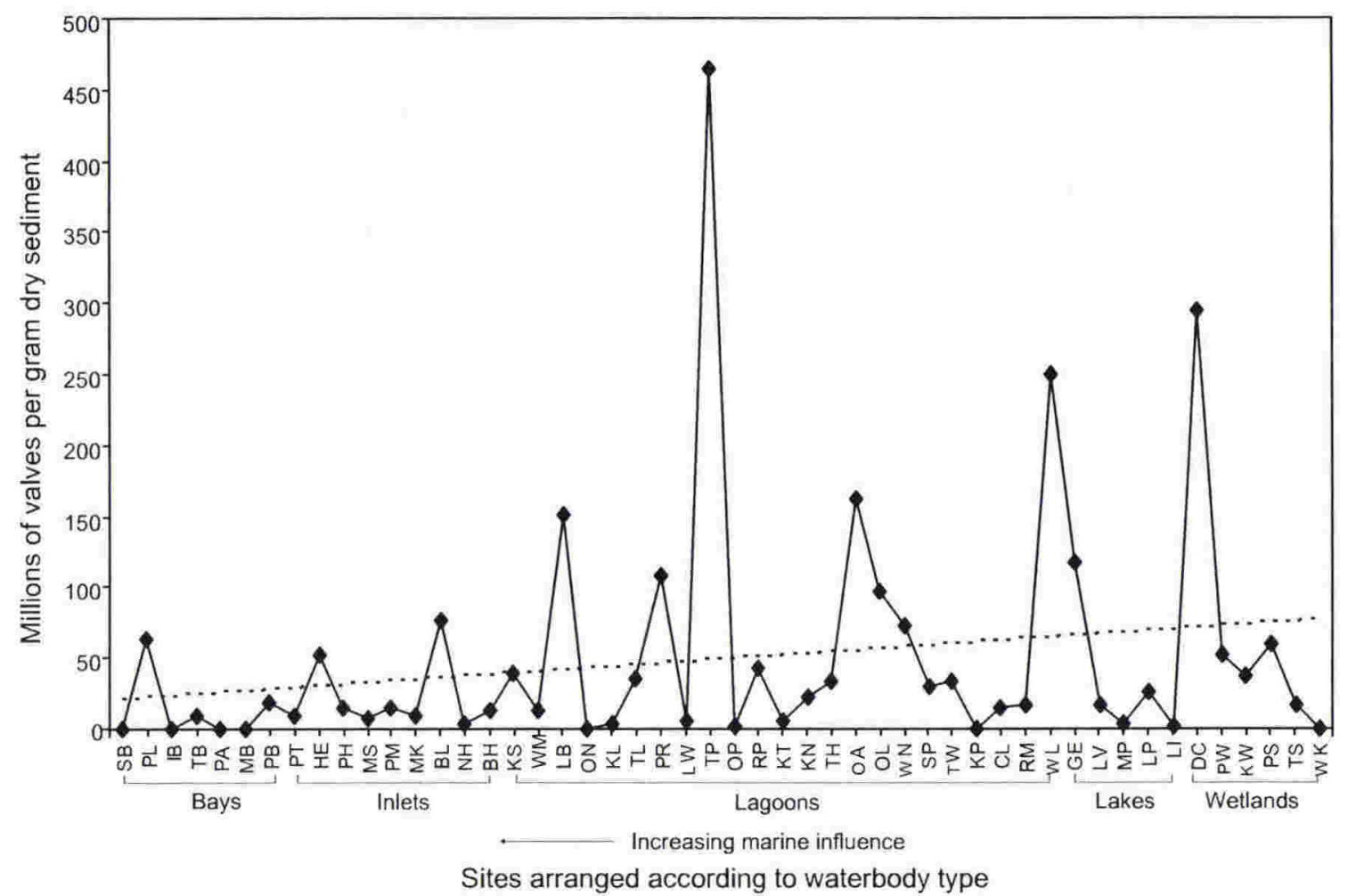

Fig. 3.7. Concentration of diatom valves in samples along the coastal gradient.

Concentration is variable with a slight trend of decreasing concentration with increasing marine influence. Very high concentrations are recorded for samples with high proportions of Fragilaria species.

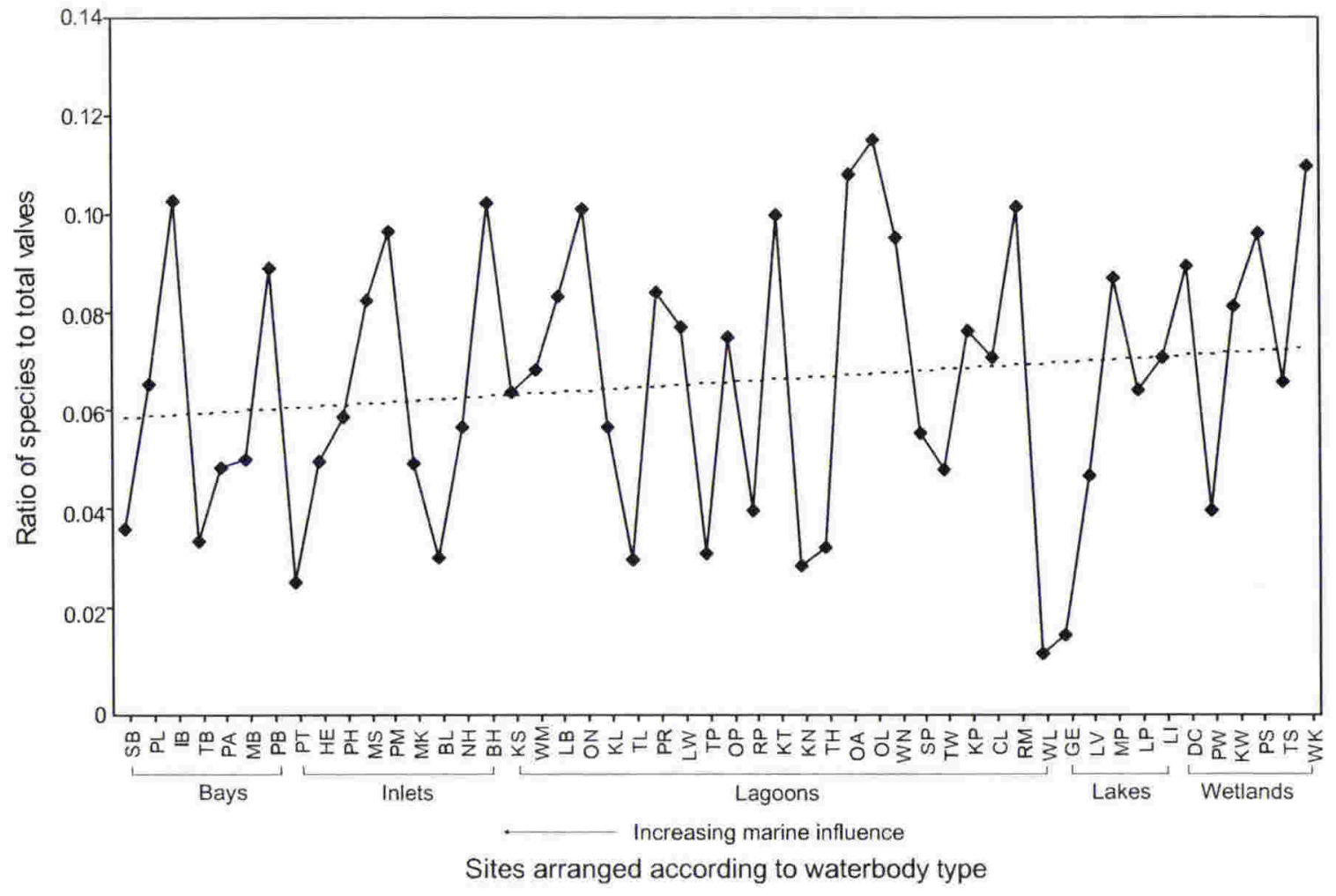

Fig. 3.8. Species richness of diatom samples along the coastal gradient. Species richness is variable with a slight trend of decreasing richness with increasing marine influence. 
samples (Fig 3.8). Although there is a range in species richness in the calibration set samples, there is only a weak trend of decreasing richness towards open marine sites along the coastal gradient. What is more notable is the variability along the gradient suggesting that waterbody type, marine influence and other factors associated with the coastal gradient have little influence on species richness.

Each death assemblage sample was checked for the number of species present that were not present in any of the modern samples from the same site and for the proportion of counts that these 'missing species' represented (Fig. 3.9). There was only one site where all the death assemblage species were present in at least one of the modern samples (Haldane Estuary), but for most sites the percentage of counts representing 'missing species' was under $10 \%$. Reasons for higher percentages included poor modern analogues (for example not all three types of modern samples were collected) or blooms of single species in either the modern or death assemblage samples. Blooms in modern samples not present in the death assemblage could well be a response to changed environmental factors. Blooms in death assemblages may have been an actual bloom in response to the environment or may represent transport of diatoms to the site. Five samples with high proportions were considered potentially problematic so their affect on subsequent analyses was assessed. One sample was removed from further analysis

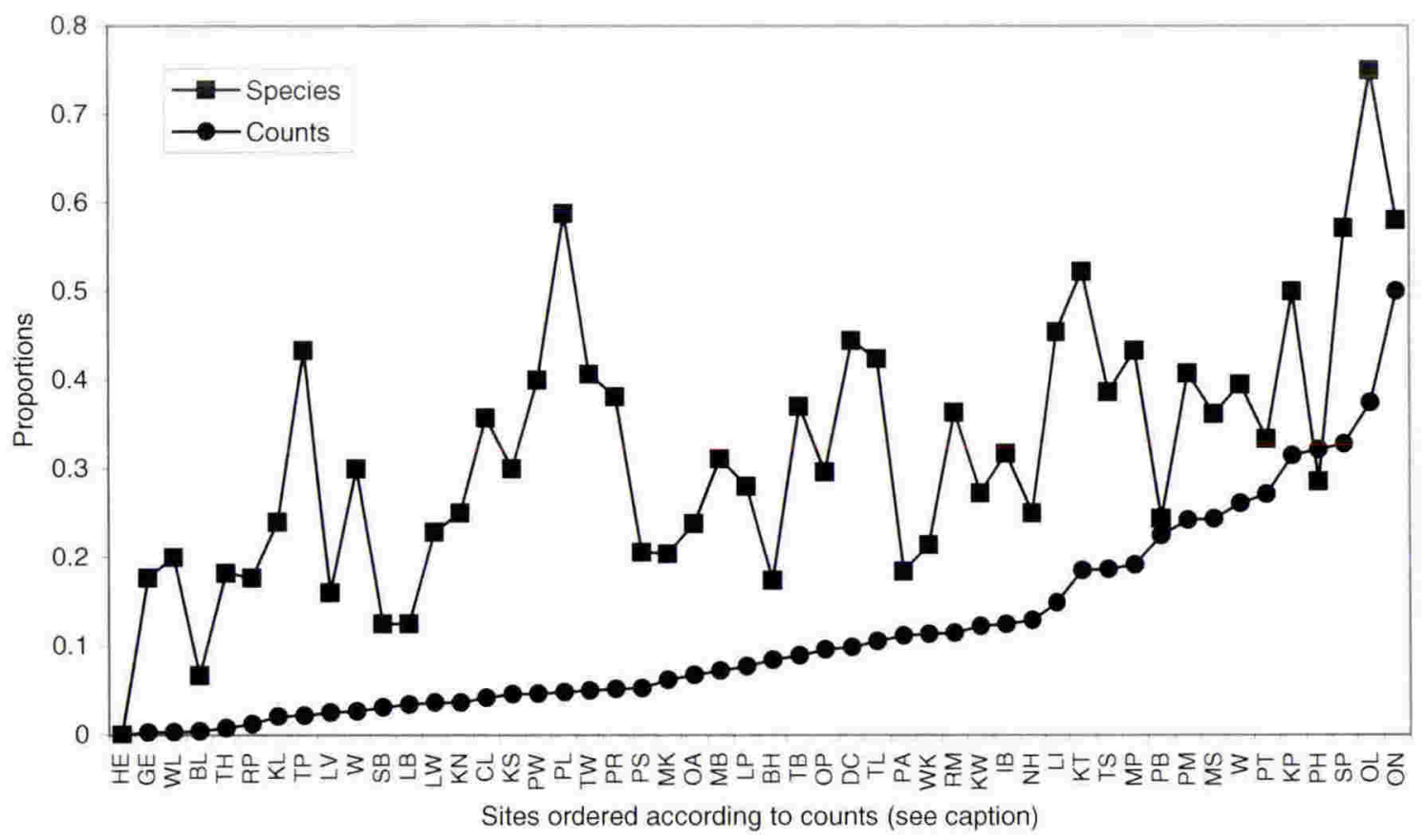

Fig. 3.9. Plot showing the proportion of counts made of species that were present in the death assemblage and not in the associated modern samples (circles) and the proportion of species present in death assemblages that were not present in modern samples (squares). 
(Shipwreck Bay) because fresh water diatoms that would be considered allochthonous to the death assemblage of an open marine bay also occurred in the modern samples. This could be the result of large-scale transport of fresh water diatoms to all habitats sampled or it may be a case of post-sampling contamination.

\subsubsection{Ordination}

Correspondence analysis (CA) of the species dataset resulted in high eigenvalues for the first four axes but plots showed a prominent arch effect. The arch effect is a mathematical artefact that arises when the simplest solution for the second axis, with greatest dispersion of species curves, is to fold the first axis in half (ter Braak, 1995). The second axis becomes a quadratic function of the first axis and not a reflection of real structure in the original data. Hill and Gauch (1980) proposed detrended correspondence analysis (DCA) as a modification of CA that solves several problems including the arch effect. DCA is used here to overcome the interpretation difficulties that arise when the arch effect occurs in CA.

DCA of the calibration set samples yields an important first axis with a long gradient length (Table 3.3). The high eigenvalue of the first axis indicates that species and sites are well spread out along this axis and the underlying 'theoretical' gradient explains the data well. The long gradient length (measured in standard deviation units) indicates that at least one complete turnover in taxonomic composition of samples occurs in the dataset. This is not surprising considering the large range of waterbody types that form the calibration set.

The first axis of the DCA separates species according to salinity preferences (Fig. 3.10) with marine and brackish species on the right of the plot, indifferent species in the centre, and fresh water species on the left. It is clear that the first axis explains much of the variation in the distribution of marine and brackish species because they plot in a narrow band parallel with axis one. Indifferent and fresh water species are well separated along axis one but they also spread out along axis two indicating there is another gradient important in explaining aspects of their distribution. At the extreme fresh water end of the first axis, species are separated into two discrete groups on the second axis. This may be the result of a sampling gap in the gradient of axis two because of the small size of the calibration set. However one of the negative features of DCA is that it can excessively polarise species at the variable end of a gradient that is crossed, at one end only, by another strong gradient (Hill and Gauch, 1980) so it is likely that the extent of separation has been exaggerated. Environmental factors likely to be explaining variation along the second axis are more easily estimated from the DCA site plot. 


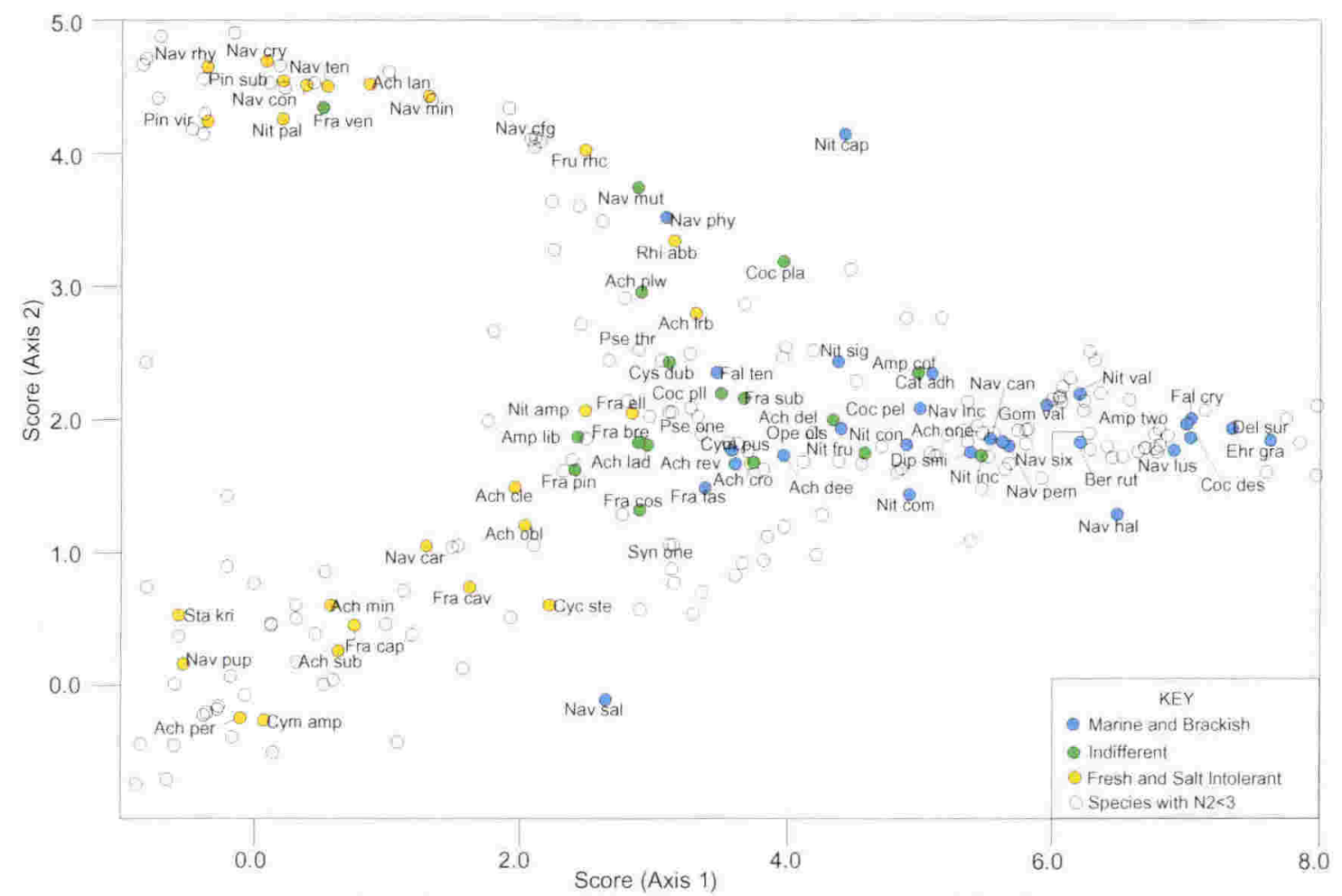

Fig. 3.10. DCA species plot. Species with more than three effective occurrences (N2) are labelled with code names as listed in Appendix II and colour-coded according to traditional salinity preferences.

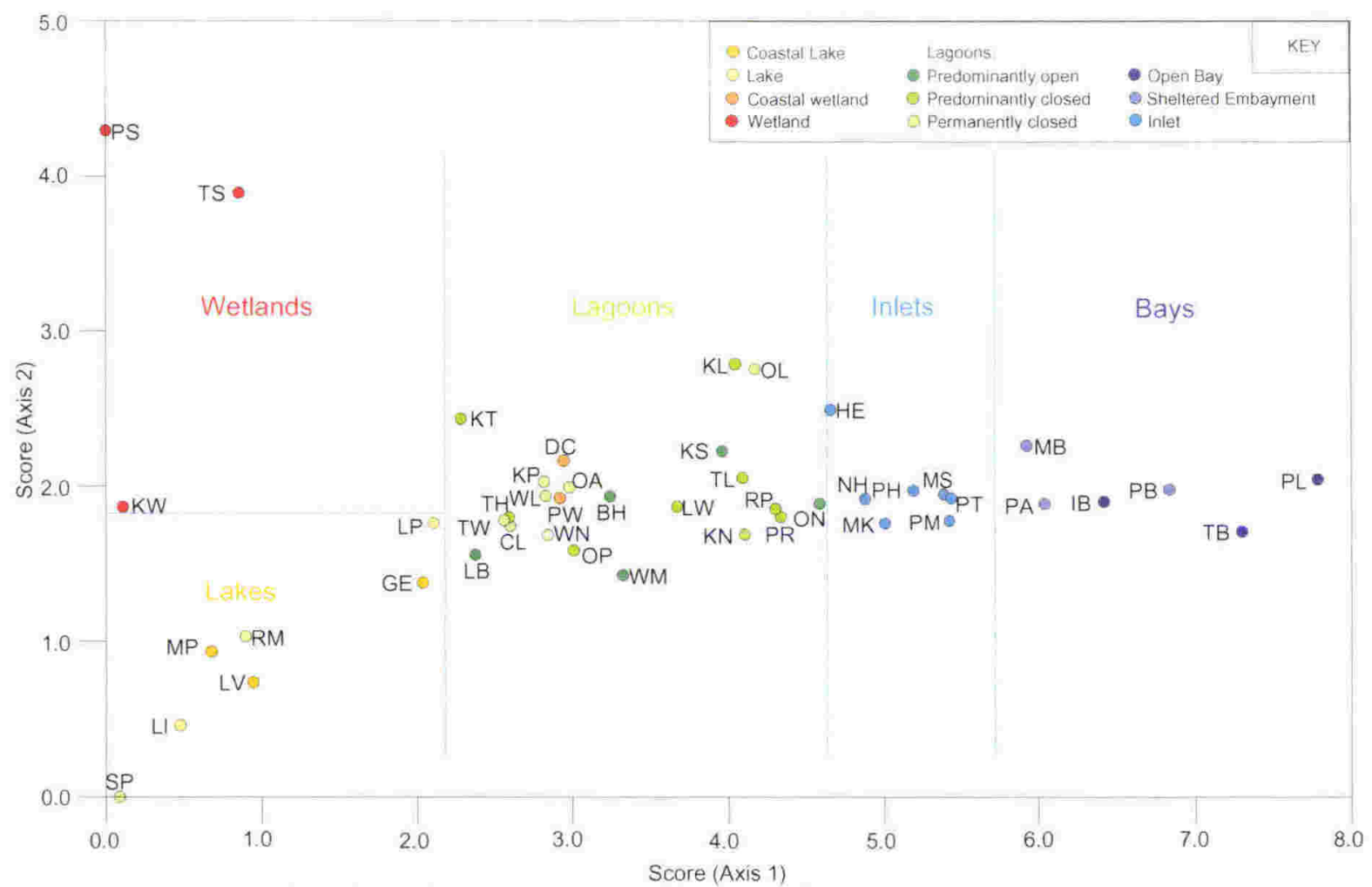

Fig. 3.11. DCA site plot with site codes as listed in Table 3.1. Sites are colour-coded to illustrate their distribution along axes according to waterbody type. Dashed lines show corresponding divisions of ordination space. 
The site plot of the same DCA (Fig. 3.11) shows clearly that the first axis can be taken to represent a coastal gradient because sites are arranged according to their waterbody type. The large amount of variation in the diatom data of this dataset means that sites are well spread out in ordination space facilitating division of axes according to site attributes. The first axis, for example, can be divided according to waterbody type into sections representing bays, inlets, lagoons and lakes. The diatom assemblages of the different lagoon types are obviously not distinct enough to enable division into lagoon type possibly because this dataset is not large enough. The second axis represents a gradient that explains variation at the fresh water end of the first axis only. Although there are not many sites with which to characterise the second axis, it clearly divides sites into lakes at the base of the axis and wetlands at the top.

Prior to addition of measured environmental variables to the ordination analyses, it can be seen from the above DCA plots that the main variation in the diatom data (first axis) can be explained by change along a coastal gradient. Diatom assemblages along this gradient are distinct enough to enable division of ordination space according to waterbody type. This division is used to reconstruct waterbody type for fossil samples by plotting fossil diatom assemblages passively onto the DCA site plot (refer to Chapters Four, Five and Six). Secondary variation in the diatom data (second axis) is related to the difference between wetland and lake environments at the fresh water end of the first gradient.

\subsubsection{Canonical ordination}

Axes in indirect gradient analysis as in Figs 3.10 and 3.11 above, represent theoretical gradients and are extracted in such a way as to maximise dispersion in the species data. Axes in direct gradient analysis also aim to maximise dispersion in the species data but they are constrained to being linear combinations of measured environmental variables (ter Braak, 1995). Therefore comparison of the DCA and DCCA species and site plots can provide an indication of how well the measured environmental variables explain the species data (ter Braak, 1986). Both species and sites of the DCCA (Fig. 3.12) are generally less tightly clustered than in the DCA plots, but the order and position of species and sites along the first axis is very similar. Fresh water sites and species plot on the left of the figure and there is a continuum through to marine sites and species on the right. The order and position of species and sites on the second axis is also very similar with only one site (SP), and associated species, having moved from low second axis to high second axis in the DCCA. Eigenvalues and gradient lengths are also very similar between the two types of analysis (Table 3.3) so it can be concluded that the measured environmental variables account for the main variation in the diatom data. 


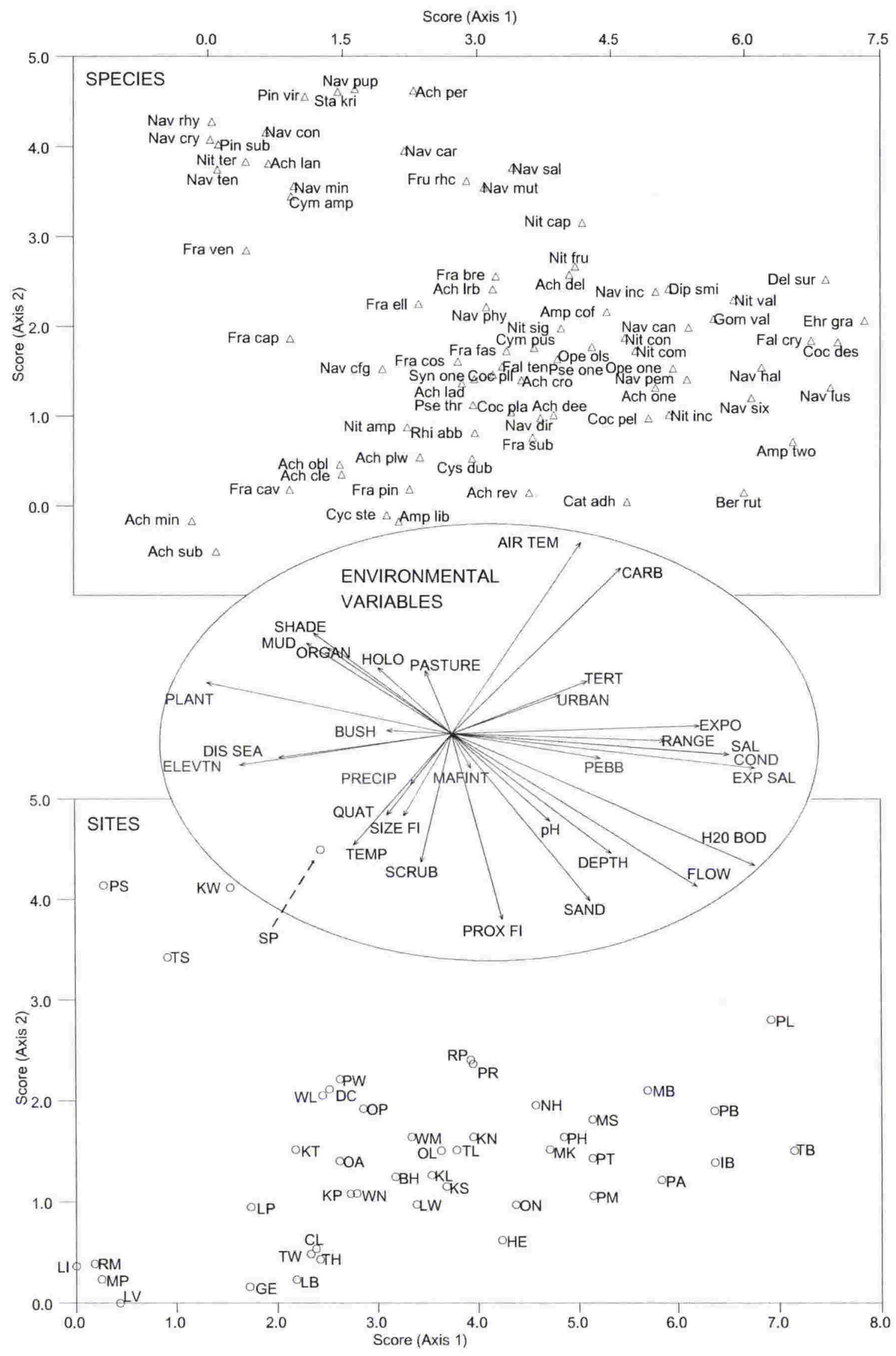

Fig. 3.12. Species, site and environmental variable plot for a DCCA of calibration set data. Only species with more than three effective occurrences (N2) are shown. Codes for species are listed in Appendix II, for sites in Table 3.1, and for environmental variables in Table 3.2 . 


\begin{tabular}{|l|llll|}
\hline & Axis 1 & Axis 2 & Axis 3 & Axis 4 \\
\hline DCA & & & & \\
Eigenvalues & 0.83 & 0.51 & 0.43 & 0.36 \\
Gradient Length & 7.78 & 4.29 & 4.17 & 3.25 \\
DCCA & & & & \\
Eigenvalues & 0.81 & 0.56 & 0.37 & 0.29 \\
Gradient Length & 7.27 & 4.16 & 3.62 & 2.86 \\
\hline
\end{tabular}

Table 3.3. Comparison of eigenvalues and gradient lengths for DCA and DCCA of the calibration set data.

Examination of environmental variables in the initial DCCA (Fig. 3.12) indicates that the first axis is indeed a coastal gradient with variables such as exposure to salt, salinity, conductivity, exposure to air, distance from the sea and elevation all highly correlated with axis one. There are no variables highly correlated with axis two although air temperature and proximity to fresh water inflow appear to be explaining some of the variation on this axis. Full interpretation of environmental variables is not made using this DCCA because it contains all measured environmental variables and variance inflation factors indicate there are some highly correlated variables that should be removed. Inclusion of highly correlated variables in an analysis causes canonical coefficients to be unstable and prevents separation of the effects of different variables (ter Braak, 1986). It is also likely there are variables in the dataset that explain negligible amounts of variation. Further analysis is carried out below to derive a minimal set of environmental variables that can explain the species data almost as well as the full set.

The individual explanatory power and statistical significance of each environmental variable was assessed by running a series of CCAs in which each variable was entered as the sole constraining variable in the analysis (Table 3.4). Twenty-three variables explained more than $3 \%$ of the total variance in the diatom data and 23 were statistically significant at the $95 \%$ confidence level. Twelve variables were eliminated from further analysis because their individual contribution as explanatory variables was not statistically significant. These were mainly nominate variable categories but water temperature and proximity to fresh water inflow were also not significant. 


\begin{tabular}{|c|c|c|}
\hline $\begin{array}{l}\text { ENVIRONMENTAL } \\
\text { VARIABLE }\end{array}$ & $\begin{array}{l}\text { PERCENTAGE } \\
\text { VARIANCE }\end{array}$ & P-VALUE \\
\hline Waterbody type & 5.85 & $0.01 \star$ \\
\hline Exposure to salt & 5.83 & $0.01 \star$ \\
\hline Salinity & 5.46 & $0.01 \star$ \\
\hline Conductivity & 5.42 & $0.01 \star$ \\
\hline Flow & 5.13 & $0.01 *$ \\
\hline Distance from sea & 4.88 & $0.01 \star$ \\
\hline Felsic intrusive bedrock & 4.82 & $0.02 \star$ \\
\hline Exposure to air & 4.76 & $0.01 \star$ \\
\hline Exotic forest & 4.32 & 0.12 \\
\hline Elevation & 4.08 & $0.02 \star$ \\
\hline Aquatic plant growth & 4.01 & $0.01 \star$ \\
\hline Salinity Range & 3.82 & $0.01 \star$ \\
\hline Carbonate & 3.8 & $0.01 \star$ \\
\hline Depth & 3.71 & $0.01 \star$ \\
\hline Pebbles & 3.51 & $0.01 \star$ \\
\hline $\mathrm{pH}$ & 3.48 & $0.01 \star$ \\
\hline Annual precipitation & 3.39 & $0.05 \star$ \\
\hline Tertiary sedimentary bedrock & 3.21 & $0.01 \star$ \\
\hline Annual air temperature & 3.21 & $0.02 \star$ \\
\hline Shade & 3.14 & $0.01 \star$ \\
\hline Sand & 3.12 & $0.03 \star$ \\
\hline Organic matter & 3.04 & $0.02 \star$ \\
\hline Size fresh water inflow & 3.01 & $0.02 \star$ \\
\hline Native bush & 2.92 & 0.1 \\
\hline Mud & 2.89 & $0.05 *$ \\
\hline Proximity to fresh water inflow & 2.78 & 0.08 \\
\hline Urban development & 2.67 & 0.21 \\
\hline Water temperature & 2.42 & 0.27 \\
\hline Pasture & 2.36 & 0.27 \\
\hline Holocene coastal sediments & 2.36 & 0.4 \\
\hline Quaternary sands, gravels, tills & 2.22 & 0.4 \\
\hline Scrub & 2.13 & 0.54 \\
\hline Mafic intrusives & 1.92 & 0.45 \\
\hline Mafic / intermediate volcanics & 1.41 & 0.93 \\
\hline Quartzo-feldspathic schist & 1.16 & 0.8 \\
\hline
\end{tabular}

$\star=$ Statistically significant variable at the $95 \%$ confidence level.

Table 3.4. Percentage variance of the species data explained by each environmental variable when it was the sole constraining variable in a CCA and results of a Monte Carlo permutation test on the resulting first axis.

A principal components analysis (PCA) with environmental variables entered as species illustrates correlations between environmental variables (Fig. 3.13). Variables with arrows that plot close together are highly correlated, for example, salinity, conductivity, exposure to air and exposure to salt are all highly correlated. Environmental variables from a CCA (Fig. 3.14) reflect structure in the species data as well as correlations between variables. Correlations between environmental variables are similar to those in the PCA but there are some key differences. Environmental variables associated with the coastal gradient are even more highly correlated with each other and with axis one than in the PCA. The importance of $\mathrm{pH}$ as a variable correlated with axis two is greater in the CCA reflecting its importance as an explanatory variable for diatom distribution. 


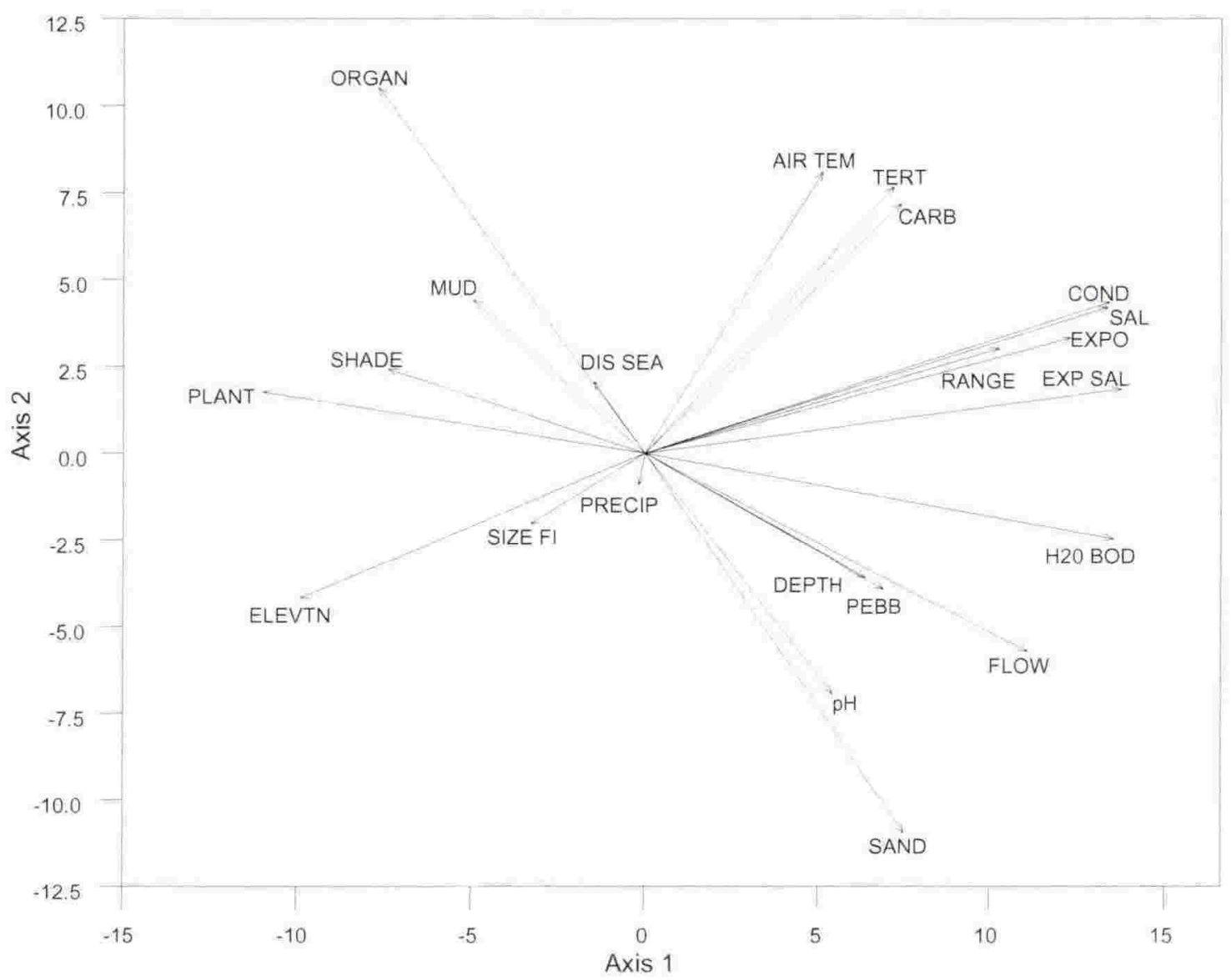

Fig. 3.13. PCA with environmental variables entered as species in the analysis. This illustrates correlations between variables based on the environmental variable data.

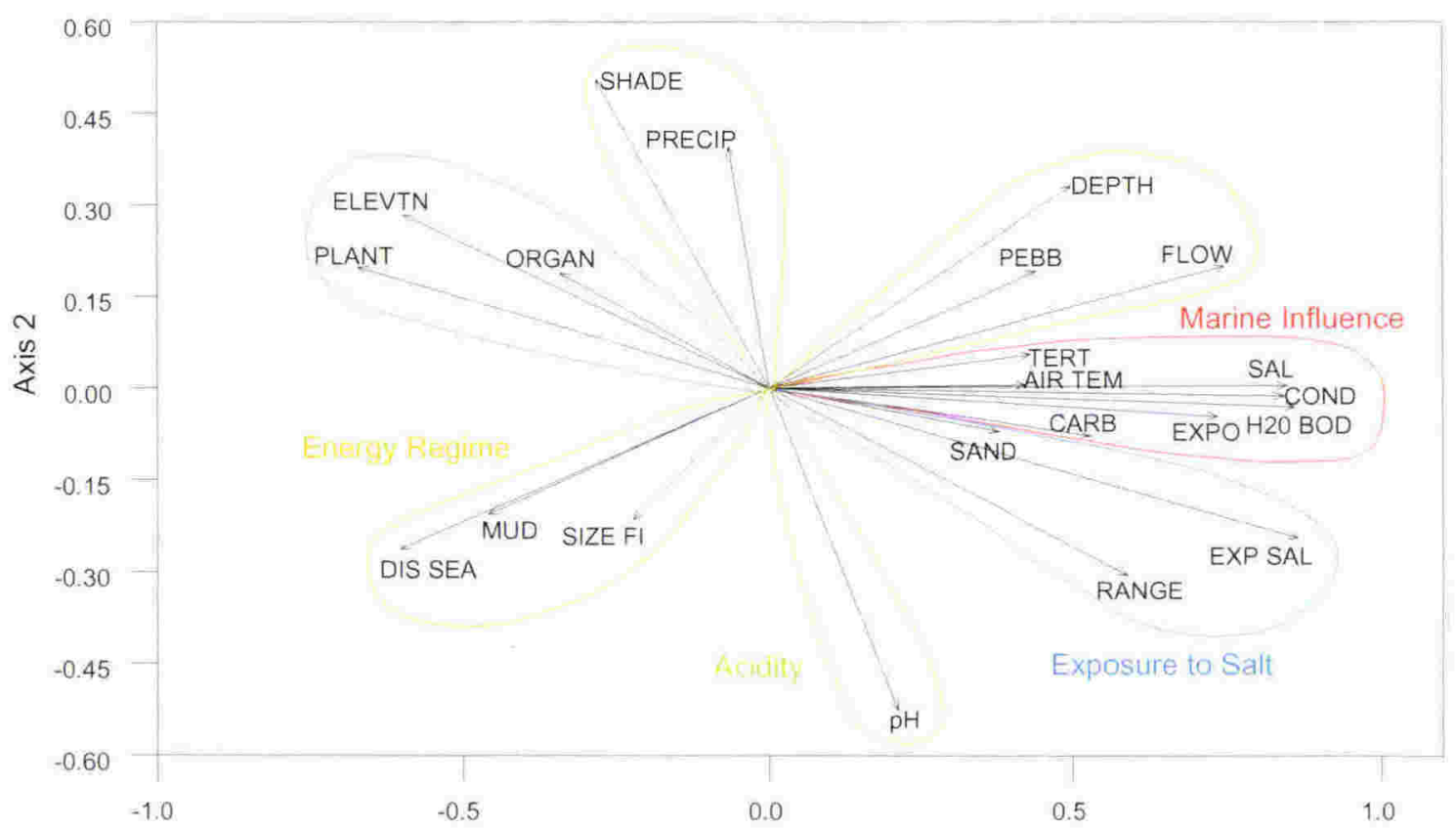

Axis 1

Fig. 3.14. Environmental variable plot from a CCA. Variables are grouped into highly correlated sets of variables which define four gradients in the dataset. 
Four groups of correlated variables are present in the CCA. These can be thought of as environmental gradients so are named in accordance with the types of variables they contain. Marine influence, energy regime and exposure to salt are all correlated with axis one and acidity is correlated with axis two.

It was concluded from the DCA and DCCA that the first axes are successful in maximising dispersion in the species data (eigenvalues of 0.83 and 0.81 respectively) and that they represent a coastal gradient. The PCA and CCA plots and associated correlation matrices confirm this conclusion and further indicate that the generalised coastal gradient is made up of three contributing gradients of marine influence, energy regime and exposure to salt. Variation along the second axis can be explained by an acidity gradient. There are no variables highly correlated with axis three or four.

Ordering of species and sites along the first axis remained constant throughout the process of removing superfluous environmental variables. However all CCAs were dominated by the arch effect. Generally the arch effect can be removed from a CCA by eliminating superfluous environmental variables (ter Braak, 1995). Forward selection of environmental variables was carried out in an attempt to define a minimal set of variables that would explain the calibration set almost as well as the full set and rid the analyses of the arch effect. However an arch was still present in the CCA using only seven forward selected environmental variables. The arch effect is thought to arise when the first axis fully explains the species data (ter Braak, 1995) or when a short gradient is dominated by a long gradient (ter Braak, 1986). The statistics of the DCA and DCCA suggest that both these characteristics could explain why the arch effect occurs in this calibration set. Eigenvalues for the first axes are much higher than those for the second axes indicating that although the first axis doesn't explain the species data completely, it explains a lot more than the second axis. Gradient lengths also indicate that the second axis is much shorter than the first axis.

Although use of a forward-selected, minimal set of environmental variables did not succeed in removing an arch structure from the resulting CCA, forward selection did result in definition of a much smaller set of environmental variables that describe the species data almost as well as the full set of variables.

Eigenvalues for a CCA using the forward selected environmental variables are only slightly lower than for a CCA using all 23 individually significant variables (Table 3.5). This means that an appreciable amount of variation in the species data is explained by these seven environmental variables and they can be used to represent the full set in further analysis. This simplified dataset is used to investigate the partitioning of variance and interactions between the four major environmental gradients noted above. In the reduced set of environmental variables, salinity and carbonate represent the marine influence gradient; 
distance from the sea and flow represent the energy regime gradient; exposure to salt represents the gradient of the same name; and $\mathrm{pH}$ and precipitation represent the acidity gradient.

\begin{tabular}{|c|c|c|c|c|c|}
\hline & Axis 1 & Axis 2 & Axis 3 & Axis 4 & $\begin{array}{l}\text { Total } \\
\text { Inertia }\end{array}$ \\
\hline CCA with all significant variables & & & & & 11.638 \\
\hline Eigenvalues & 0.80 & 0.68 & 0.58 & 0.48 & \\
\hline Species-environment correlations & 0.99 & 0.98 & 0.95 & 0.91 & \\
\hline Cumulative $\%$ variance: of species data & 6.8 & 12.6 & 17.6 & 21.7 & \\
\hline of species-environ. relation & 12.6 & 23.3 & 32.4 & 39.9 & \\
\hline Sum of all unconstrained eigenvalues & & & & & 11.638 \\
\hline Sum of all canonical eigenvalues & & & & & 6.318 \\
\hline CCA with forward selected variables & & & & & 11.638 \\
\hline Eigenvalues & 0.77 & 0.56 & 0.47 & 0.37 & \\
\hline Species-environment correlations & 0.97 & 0.90 & 0.91 & 0.92 & \\
\hline Cumulative $\%$ variance: of species data & 6.6 & 11.4 & 15.4 & 18.6 & \\
\hline of species-environ. relation & 26.6 & 45.9 & 62.2 & 74.9 & \\
\hline Sum of all unconstrained eigenvalues & & & & & 11.638 \\
\hline Sum of all canonical eigenvalues & & & & & 2.886 \\
\hline
\end{tabular}

Table 3.5. Comparison of CCA results for analyses using all significant environmental variables and using seven forward selected environmental variables.

A series of partial CCAs was carried out to determine statistically how much variation in the data each of the four main environmental gradients are explaining (Fig. 3.15). The total variation explained by the four gradients (as represented by the seven forward selected environmental variables) is $24.8 \%$. This is less than half that explained by the full set of environmental variables (54.3\%), but is fairly typical of similar studies involving noisy species data (eg, Gasse et al., 1995; Jones and Juggins, 1995; Reed, 1998). All four gradients account for significant amounts of variation in the data. Interactions between pairs of gradients only account for $2.2 \%$ and interactions between more than two gradients make up only $1.4 \%$ (other). The greatest interactions are between marine influence and exposure to salt indicating that these gradients cannot be considered independent of each other. This makes intuitive sense because the marine influence and exposure to salt gradients plot close together in the CCA environmental variable plot (Fig. 3.14) suggesting they are correlated. 


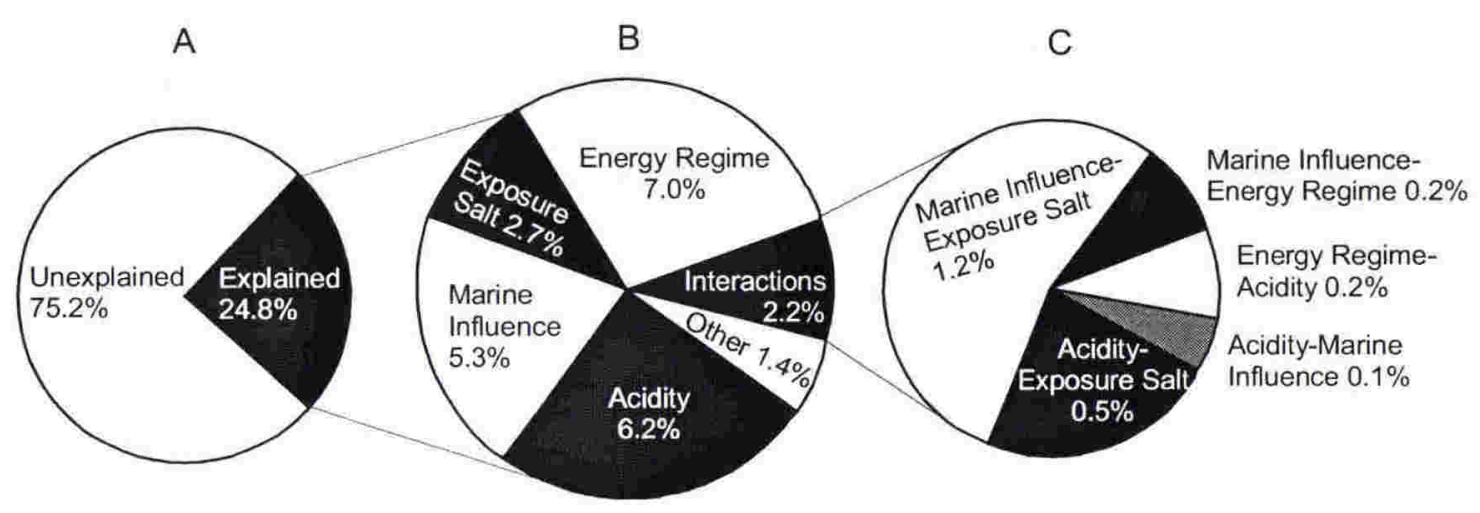

Fig. 3.15. Partitioning of variance in the diatom data using a minimum set of environmental variables representing four gradients in the dataset. A: Total variation partitioned into explained and unexplained portions. B: Explained variation partitioned into the unique contribution of each gradient and interactions between gradient pairs. C: Interactions partitioned into gradient pairs for which there are interactions.

Ordination and canonical ordination of the calibration set enable a more detailed definition of the idealised uplifted coastal waterbody gradient (introduced in section 3.2.1) to be presented. This revised gradient is a spatial coastal gradient derived using environmental variables that are important in explaining diatom distribution in this calibration set (Fig. 3.16). The assumption made in section 3.2.2 about an idealised coastal gradient across which environmental variables and processes interact in an intuitive and predictable way, can be seen to hold true for the calibration set. Accepting that a 'standard' coastal gradient for small, shallow waterbodies is defined, and considering the calibration set was initially designed around what was known of the Holocene sites, it is suggested that relationships between variables characteristic of this gradient can be assumed to have been similar for the fossil sequences.

\subsection{A DIATOM-SALINITY TRANSFER FUNCTION}

\subsubsection{Fitness for Quantitative Reconstruction}

Direct gradient analysis enables some assessment of assumptions one, two and five of quantitative paleoenvironment reconstruction (as outlined in section 3.1.3) to be made in relation to this calibration set. The similarity between DCA and DCCA indicates there is a strong relationship between species distributions and measured environmental variables in the calibration set. Although this does not constitute proof that environmental variables are causing species to respond, it indicates it is fair to assume that taxa in the calibration set are systematically related to their environment (assumption one). CCA enabled statistical assessment 

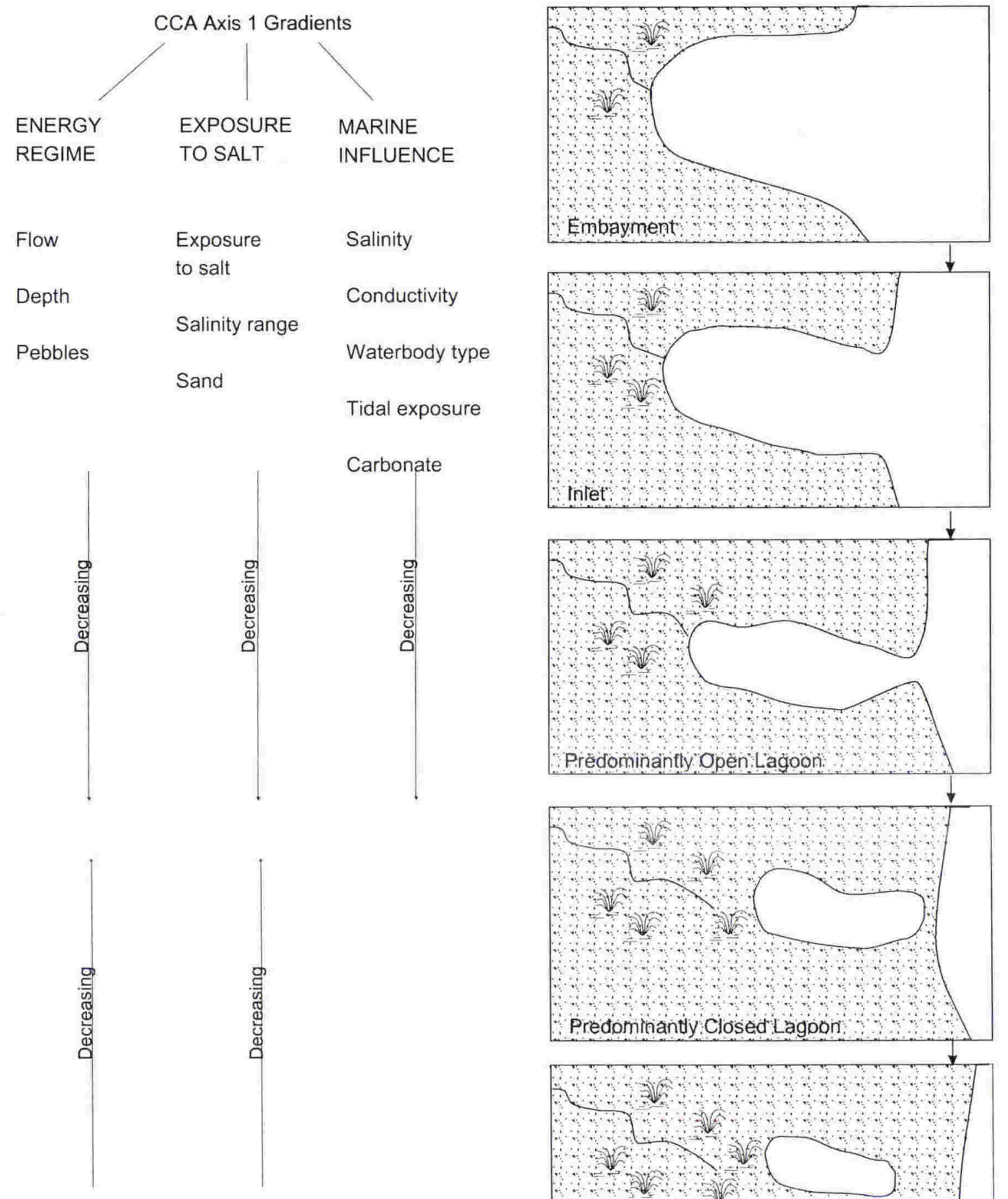

$\begin{array}{ll}\text { Distance from sea } & \text { Elevation } \\ \text { Size fresh inflow } & \text { Organics }\end{array}$

Mud Aquatic Plants

Fig. 3.16. Spatial coastal waterbody gradient for the calibration set. Defined using statistically significant variables associated with three gradients explaining variation in

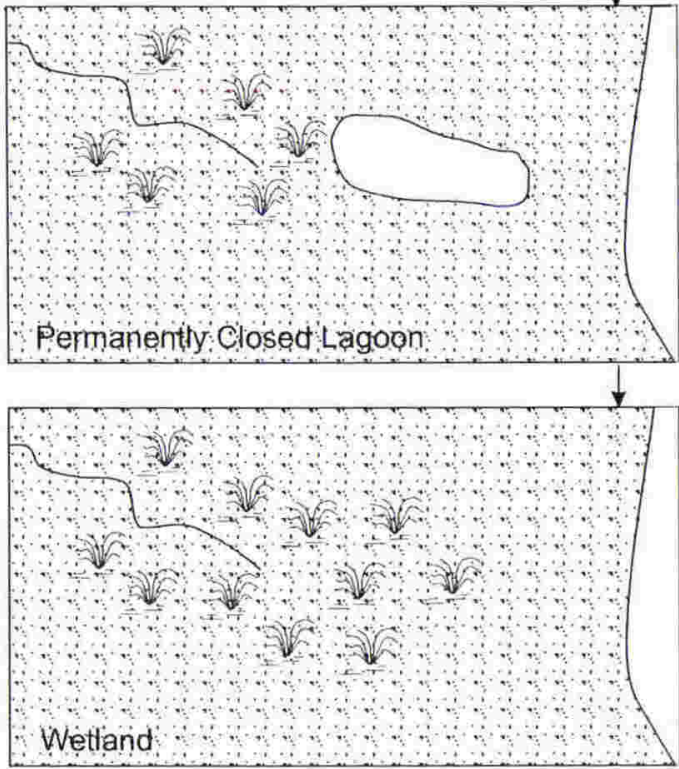
diatom data along axis one of a CCA. 
of the importance of environmental variables in terms of explaining variation in diatom assemblages. It can be assumed that salinity is an important variable in the system because it individually explained more than $5 \%$ of variance in the diatom data and was statistically significant at the $99 \%$ confidence level (assumption two). Salinity was chosen over four other variables (waterbody type, exposure to salt, flow and conductivity) because it is a continuous, quantitative variable and it explained slightly more variation than conductivity.

Variance partitioning of a minimal set of environmental variables indicated that salinity, although accounting for a significant amount of variation on its own, was also interacting with other variables to explain the diatom data. In this calibration set it cannot be assumed that variables other than salinity have negligible influence. However, as suggested above, it is likely that interactions between variables define a standard coastal gradient that would be found for other datasets of modern and fossil sites of the same waterbody type. Therefore it is assumed that the joint distribution of salinity and other important environmental variables was the same in the fossil set as it is in the calibration set (assumption five). Salinity is selected as a proxy to represent the coastal gradient and interpretation of results should take into account that reconstructed values of salinity are indicative of position on the gradient as well as actual values of salinity.

\subsubsection{Methods}

A DCCA with salinity as the sole constraining variable was run to determine the gradient length of salinity in the dataset. The gradient length was greater than two standard deviation units and the constrained axis was significant so unimodal regression techniques were considered appropriate for development of a salinity transfer function (Birks, 1995). Weighted averaging (WA) and weighted averaging with tolerance downweighting (WA-tol) were used, as implemented by the program CALIBRATE 0.85 (Juggins and ter Braak, 1997-99). Weighted averaging partial least squares was tried on the dataset but it offered no improvement over WA because best results were obtained with one component (ter Braak and Juggins, 1993). Inverse deshrinking and cross validation by jackknifing were used for all analyses. WA models were assessed for effects of different data transformations. Square root transformation of species data (which results in downweighting of dominant species) and $\log (\mathrm{x}+1)$ transformation of salinity produced the most favourable model statistics so were retained for analysis of the calibration set.

Outliers are model dependant so it was not assumed that outliers identified in ordination analyses would also be outliers in weighted averaging regression. An initial set of outliers was defined by running a weighted averaging regression on the complete calibration set and noting those sites that had a residual (inferred value-observed value) greater than the standard deviation of the environmental variable of interest in the calibration set (Jones and Juggins, 1995). Death 
assemblage samples with poor relations to their modern equivalents (as identified above) were also considered potential outliers. Twelve outliers were identified and a series of WA analyses were carried out to investigate their effects. Initially all outliers were removed from the model then each outlier was added back into the model separately to determine their individual effects. The effect of highly abundant species (maximum abundance $>50 \%$ ) was assessed in the same way. The best model, as assessed by model statistics, involved removal of nine outlying sites and one abundant species, and represented a $20-30 \%$ improvement over a model with no outliers removed.

\subsubsection{Weighted Averaging Regression}

Weighted averaging regression was used to estimate salinity optima and tolerances for diatom species in the calibration set. As a way of assessing the validity of these estimates, a calibration step is performed on the modern sites and diatom-inferred estimates of salinity are compared with measured salinity values (ter Braak and Juggins, 1993). Statistics generated by CALIBRATE include the correlation between diatom-inferred and measured salinity $\left(\mathrm{r}^{2}\right)$ in the calibration set and the root mean squared error (RMSE). Weighted averaging with tolerance downweighting produced the highest correlation and lowest error (Table 3.6). However, cross validated statistics (X-val) such as those obtained through jackknifing, are a more reliable indication of the predictive ability of the calibration set (Jones and Juggins, 1995). Jackknifing provides an estimate of bias and standard error through computer-intensive, 'leave-one-out' recalculations of the statistic (Dixon, 1993). Of the jackknifed models, WA performs slightly better than WA-tol but performance is similar so both are used for weighted averaging calibration.

\begin{tabular}{|l|l|l|l|l|}
\hline & WA & WA-tol & X-val WA & X-val WA-tol \\
\hline $\mathbf{R}^{2}$ & 0.8933 & 0.9560 & 0.8364 & 0.8315 \\
\hline RMSE & 0.1929 & 0.1238 & 0.2397 & 0.2481 \\
\hline
\end{tabular}

Table 3.6 Weighted averaging regression statistics for the calibration set.

A strong, linear relationship exists between measured salinity and diatom-inferred salinity of the modern samples (Fig. 3.17) indicating that the calibration set can be used successfully for reconstruction of salinity. In comparison with the ideal 1:1 relationship, WA shows a fair amount of scatter at the ends of the gradient whereas in WA-tol sites are tightly clustered at the ends. Both models show scatter in the brackish section of the gradient. These trends are highlighted in plots of residuals (inferred minus observed salinity values) (Fig. 3.18). 

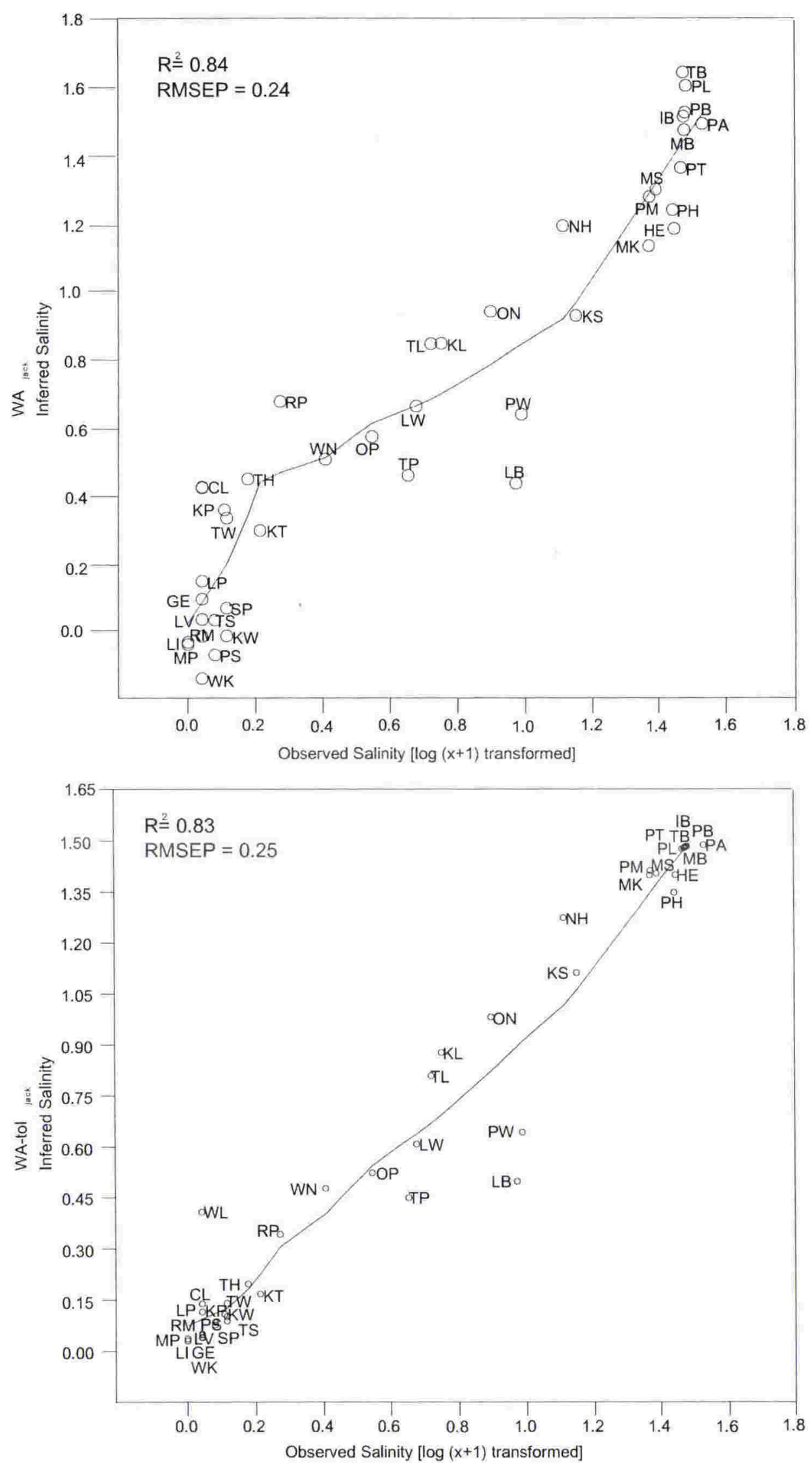

Fig. 3.17. Observed salinity versus diatom inferred salinity using jackknifed WA (top) and jackknifed WA with tolerance downweighting (bottom). Regression lines are LOWESS scatterplot smoothers as implemented by CALIBRATE. 

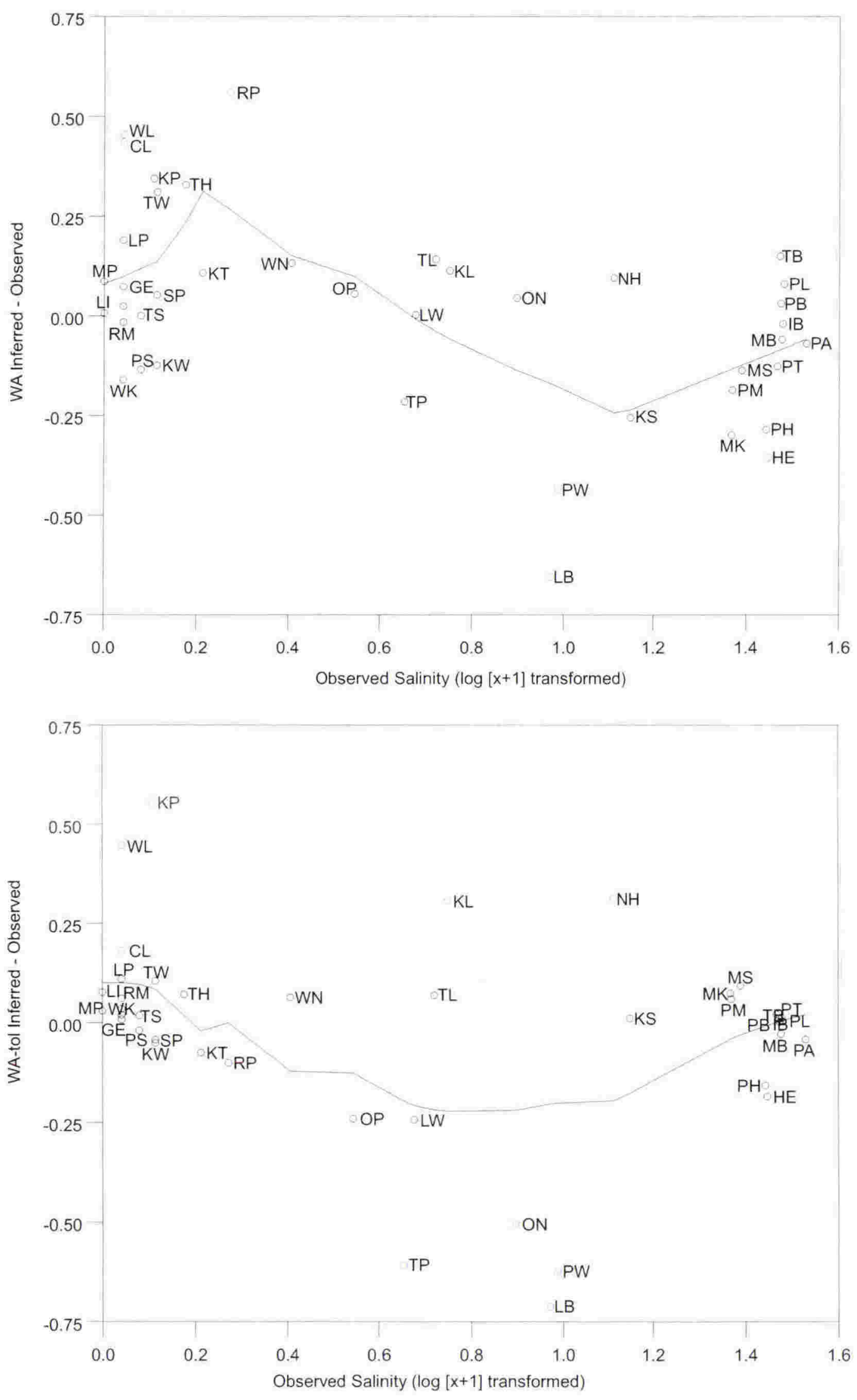

Fig. 3.18. Plots of residuals for WA (top) and WA-tol (bottom). Lines are LOWESS smoothers as implemented by CALIBRATE. 
If estimates of salinity using diatoms were perfect, sites would plot in a horizontal line at zero. In WA, sites at the fresh end of the gradient tend to be overestimated, and sites at the marine end are generally underestimated. There is a large amount of scatter in the brackish region but no trend towards over- or under-estimation. In WA-tol, sites at each end of the gradient are fairly well estimated but there is scatter in the middle of the gradient with a tendency towards under-estimation. Retaining both models for comparative calibration is considered useful in that each may perform better in different sections of the gradient.

The reason for high amounts of scatter in the brackish section of the gradient is likely to be the result of errors related to the inherently variable nature of brackish environments. Water chemistry at these sites is dependant on fresh water influx and influx from the sea - a balance that is continuously changing in many lagoonal environments. Difficulties in characterising environmental variables that the sampled diatom assemblages have responded to, is likely to have contributed to greater errors in this part of the gradient than at the ends. Another source of error is that many diatom species inhabiting brackish water environments have wide tolerances and it is likely this calibration set is not large enough to properly characterise distributions of tolerant species. Wide tolerance of species in the centre of the gradient is certainly a feature of this calibration set (Fig. 3.19) and explains the differing behaviour of WA and WA-tol in different parts of the gradient.

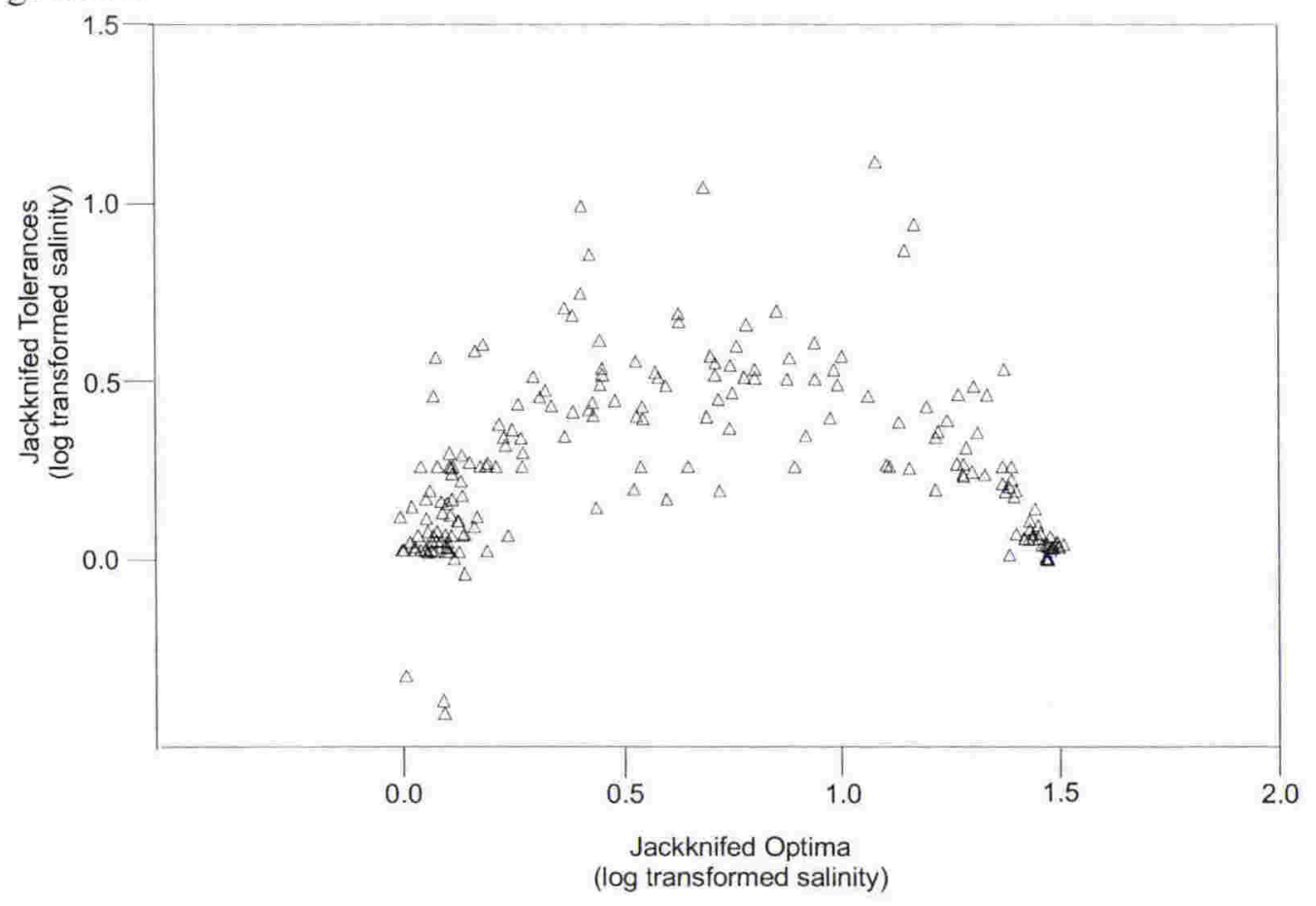

Fig. 3.19. WA salinity optima of species in the calibration set plotted against their salinity tolerances. Species with fresh and marine optima have low tolerances and species with brackish optima have high tolerances. 
The correlation between diatom-inferred and observed salinity values for this calibration set is similar to correlations derived in other diatom transfer function studies. Wilson et al. (1994) list statistics for various WA and WA-tol models based on different subsets of sites and chemical datasets from their study of Canadian saline lakes. Their bootstrapped $r^{2}$ values range between 0.72 and 0.84 . Gasse et al. (1995) construct a diatom-conductivity transfer function for African saline lakes with jackknifed $\mathrm{r}^{2}$ values of 0.81 and 0.80 for WA and WA-tol respectively. Reed (1998) reports high apparent $r^{2}$ values for conductivity of Spanish salt lakes but jackknifed statistics are much lower at 0.57 and 0.48 for WA and WA-tol respectively. Although not aimed at reconstructing salinity or conductivity, the study of Campeau et al. (1999) is used as an example of a transfer function constructed in the coastal zone. They derive a coastal water depth transfer function with bootstrapped $r^{2}$ values of 0.87 and 0.89 . Errors are harder to compare between calibration sets because units depend on transformations and variables being reconstructed.

Plots of individual species abundance along the measured salinity gradient (Fig. 3.20) enable visual assessment of the unimodal species response model used in WA. Although some species show sigmoidally increasing (Cocconeis discrepans, Diploneis vacillans) or sigmoidally decreasing (Achnanthes minutissima, Epithemia sorex) responses because their optima occur near the ends of the measured gradient, most responses to salinity are well summarised by a unimodal curve. Species shown in Fig. 3.20 are a selection of the 61 species that reached an effective number of occurrences (N2) of greater than 5 in the calibration set. They are ordered according to their optima from marine to fresh so it can be seen again that marine and fresh species tend to have narrow tolerances (eg, Navicula lusoria, Nitzschia valdestriata, Navicula pupula, Navicula rhyncocephala) and brackish species have wide tolerances (eg, Nitzschia frustulum, Achnanthes delicatula, Cocconeis placentula).

All species were included in WA (except those eliminated by removal of outlying sites) because lowest errors occur in both WA regression and calibration when all taxa are included in the analyses (Birks, 1994). However preference estimates for species with low occurrence in the calibration set are likely to be less reliable than those with many occurrences and because of the small size of the calibration set, lower N2 values have to be tolerated in comparison with those used in large calibration sets. Salinity optima and tolerances are presented for 100 species with N2 $>3$ in the calibration set (Fig. 3.21). Preferences are plotted in units of logtransformed salinity because use of a linear scale enables a visual appreciation of tolerances to be gained more easily than when using a log scale. Backtransformed optima and tolerances are presented for all species in Appendix III. Salinity optima range from 29.75 to $0.13 \mathrm{~g} \mathrm{l}^{-1}$ in a fairly continuous way. 

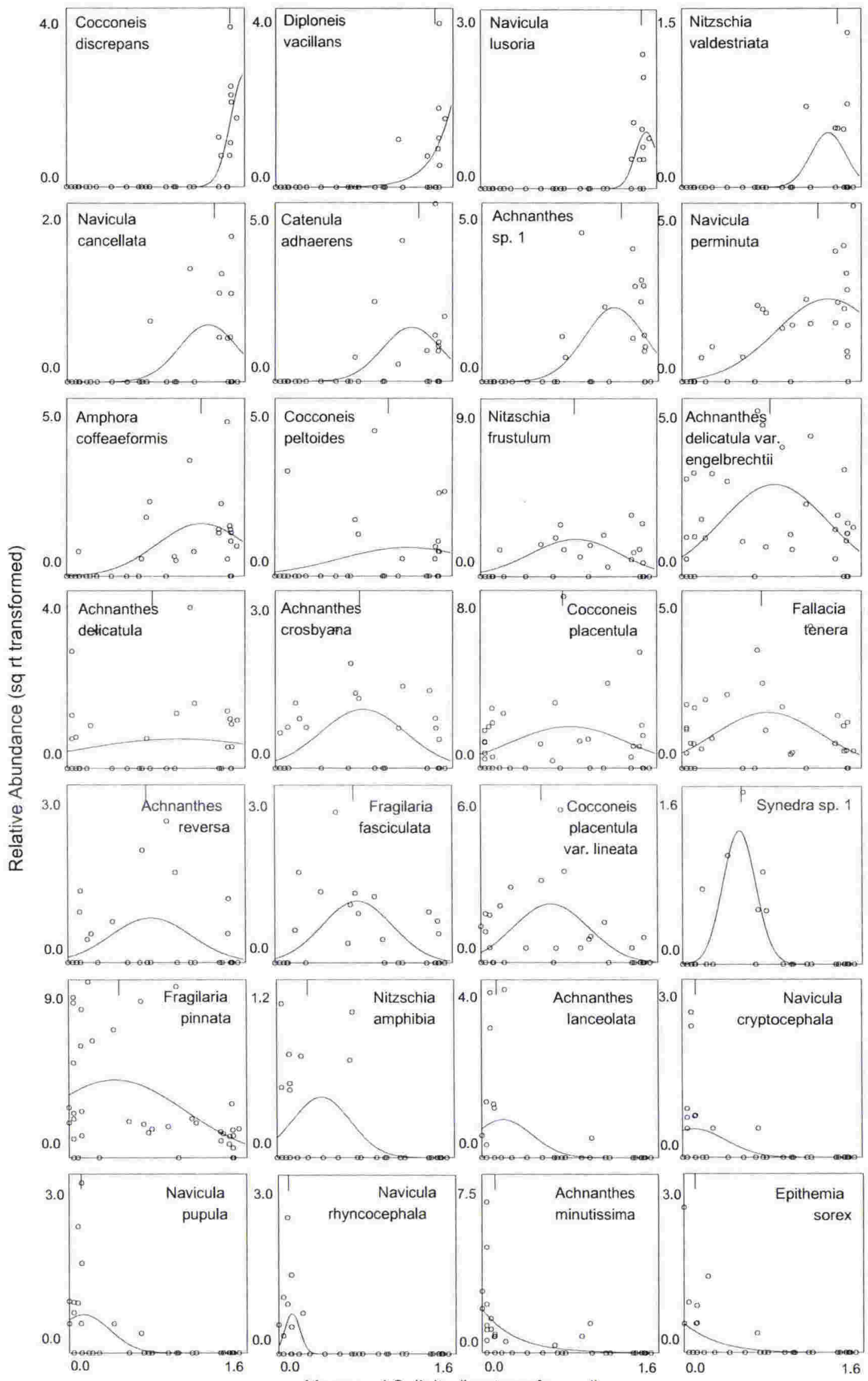

Measured Salinity (log transformed)

Fig. 3.20. Scatter plots showing species abundance along the measured salinity gradient. Curves are Gaussian logistic regression curves fitted in CALIBRATE. Tick marks indicate position of the estimated WA optimum for each species. 
Achnanthes hyperboreoides

Ehrenbergia granulosa

Amphora sp. 2

Fallacia sp. 1

Nitzschia persuadens

Rhaphoneis amphiceros

Cocconeis discrepas

Navicula lusoria

Fallacia cryptolyra

Dephineis c. f. surirella

Diploneis vacillans var. vacillans

Nitzschia liebetruthii

Amphora aff. Profusa

Berkeleya rutilans

Nitzschia valdestriata

Navicula sp. 6

Paralia sulcata

Gomphonema valentinica

Cocconeis scutellum var. parva

Navicula cancellata

Navicula halophila

Opephora guenter-grassii

Catenula adhaerens

Opephora sp. 1

Di ploneis smithii var. smithii

Nitzschia compressa var. compressa

Amphora sirigosa

Achnanthes sp. 1

Nitzschia inconspicua

Navicula perminuta

Armphora cofleaeformis

Navicula incertata

Cocconeis c. f. peltoides

Opephora olsenii

Fallacia florinae

Fallacia clepsidroides

Nitzschia sigma

Fragilaria subsalina

Nitzschia constricta

Nitzschia capitellata

Nitzschia frustulum

Achnanthes delicatula var.

engelbrechlii

Achnanthes delicatula ssp. delicatula

\begin{tabular}{llll}
1.5 & 1 & 0.5 & 0 \\
\hline
\end{tabular}

Fig. 3.21. Jackknifed estimates of salinity optima and tolerances for species in the calibration set with $\mathrm{N} 2>3$.

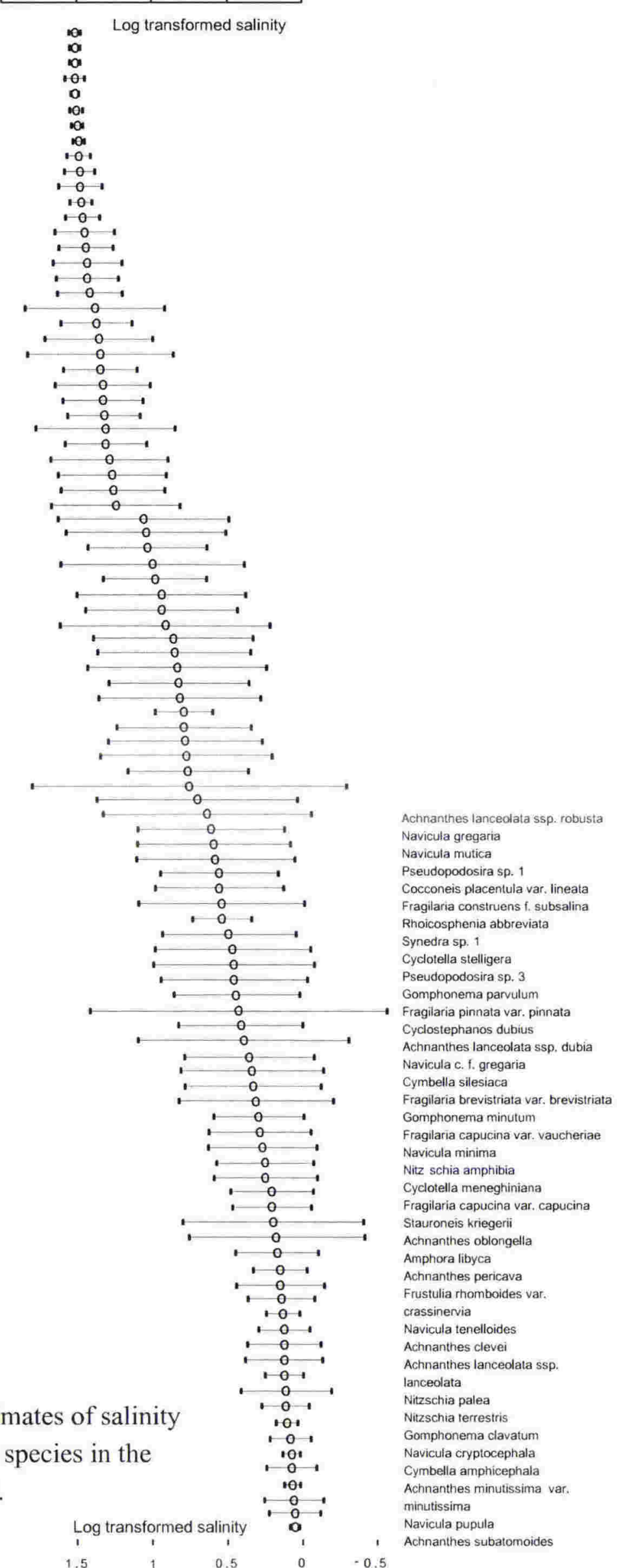

Log transformed salinity

$-0$.

101

(

Navicula mutica

sp. dubia

Fragilaria brevistriata var, brevistriata Gomphonema minutum

Nitz schia amphibia

Cyclotella meneghinian

Achnanthes lanceolata ssp.

Achnanthes subatomoides 
There is one conspicuous gap in the gradient between 10 and $14 \mathrm{~g} \mathrm{l}^{-1}$ where, although there are sites of this salinity, there are no species' optima.

Species' salinity optima derived from the calibration set are compared with published preferences in Appendix III. To enable comparison between qualitative salinity categories and quantitative salinity optima, the optima were placed into the first six categories of the classification listed in Table 2.4 (Chapter Two). Of the 267 species for which there are both published salinity preferences and optima derived from the calibration set, 244 preferences match in a broad sense (fresh, brackish, marine) and approximately half of these match exactly according to the more detailed classification (salt intolerant, fresh, fresh brackish, brackish, brackish marine, marine littoral). The preferences of 23 species do not match at all. Of these species, 18 have an effective number of occurrences in the calibration set of $<3$ so the derived optima cannot be considered reliable. In four cases (Achnanthes lanceolata ssp. robusta, Cyclotella stelligera, Cymbella minuta and Rhoicosphenia abbreviata) species predominantly occur at fresh water sites but also have occurrences at marine sites that result in their derived optima being brackish instead of fresh. Because these species occur at high and low salinity sites rather than across a continuum of sites from fresh to marine, their 'fresh' published preferences are preferred, and their marine occurrences are considered to be a result of transport. In one case where a species occurs at opposite ends of the coastal gradient (Nitzschia dissipata var. dissipata), it is thought that a different salt-water species was not differentiated from the fresh water taxa at the identification stage.

Species' salinity tolerances are not compared with published preferences because many classification schemes do not take tolerance into account. Tolerances derived from the calibration set are likely to have been affected by allochthonous occurrences of valves in a similar way to the optima described above. For example species mentioned above have very wide tolerances (Fig. 3.21) resulting from the occasional presence at marine sites of predominantly fresh water species. There are several other species that have one-off occurrences at extreme ends of the gradient - these may be allochthonous occurrences outside the species' life range and so have resulted in wider tolerances (eg, Achnanthes lanceolata ssp. dubia, Cymbella silesiaca, Frustulia rhomboides var. crassinervia and Nitzschia capitellata).

For classification into broad categories such as marine, brackish and fresh, there is a $91 \%$ agreement between published preferences and those derived from the calibration set. This indicates that published salinity preferences, derived predominantly from Northern Hemisphere studies, are generally applicable to diatoms living in the coastal zone of New Zealand. However for classification at a more detailed level, there is only $48 \%$ agreement between the two sources used here. There are several main reasons why this is likely to be the case. Firstly, there 
are only a few ecological salinity classifications presented in international literature that use more than the broadest of categories so there is not always a high level of detail available. Secondly, results from this study suggest that the general lack of work in the coastal zone worldwide has meant that many species are classified as fresh water when they are also widespread in brackish environments. To list a few examples Fragilaria capucina var. vaucheriae, Fragilaria ulna, Gomphonema minutum, Gomphonema parvulum and Hantzschia amphioxys are classified as fresh water species but are commonly found at brackish sites in the calibration set. Fresh indifferent or fresh brackish species such as the varieties of Cocconeis placentula, Fragilaria construens f. subsalina, Fragilaria pinnata var. pinnata, Fragilaria subsalina, Nitzschia inconspicua and Nitzschia frustulum are also thought to have higher salinity optima than their published classifications imply. The small size of this calibration set inhibits detailed descriptions of species distributions so the suggested bias of international literature toward the fresh water end of salinity classifications can only be regarded as a preliminary observation.

\subsubsection{Modern Analogue Technique}

The modern analogue technique (MAT) is used to provide an indication of which fossil samples have good or fair modern analogues in the calibration set. Salinity reconstructions are also produced using MAT as a means of assessing the reliability of WA reconstructions (Birks, 1995).

Squared chord distance was used as the dissimilarity coefficient. Percentiles were calculated for the modern calibration set to provide a guide as to what might be the cut-off for a good analogue (Birks, 1995). The fifth percentile was used as the cut-off for a 'good' analogue and the tenth percentile was used to indicate a 'fair' analogue. The weighted mean of the six closest analogues produced the best statistics for the calibration set (Table 3.7) but a weighted mean of three closest analogues was used for calibration because of the small size of the dataset. Use of six closest analogues for fossil samples would result in quite dissimilar samples being used in the estimates.

Apparent RMSE and $r^{2}$ are both worse than those derived from WA. However the relationship between observed and inferred values is adequate enough to warrant use of MAT for comparative purposes. Salinity reconstructions for each of the Holocene sedimentary sequences using MAT are presented in Chapters Four, Five and Six. Samples without good or fair modern analogues are highlighted and reconstructions of these samples are treated with caution. 


\begin{tabular}{|c|l|l|}
\hline N closest matches & RMSE & $\mathbf{R}^{2}$ \\
\hline 1 & 10.1050 & 0.3860 \\
\hline 2 & 8.9162 & 0.4720 \\
\hline 3 & $\mathbf{8 . 2 1 0 3}$ & $\mathbf{0 . 5 3 6 1}$ \\
\hline 4 & 7.9810 & 0.5509 \\
\hline 5 & 7.9469 & 0.5509 \\
\hline 6 & 7.8001 & $\mathbf{0 . 5 6 6 2}$ \\
\hline 7 & 7.9621 & 0.5445 \\
\hline 8 & 7.9952 & 0.5450 \\
\hline 9 & 7.8089 & 0.5668 \\
\hline 10 & 7.8148 & 0.5696 \\
\hline
\end{tabular}

Table 3.7. Modern analogue technique statistics for the calibration set.

\subsection{CONCLUSIONS}

The New Zealand coastal diatom calibration set presented in this chapter consists of 50 sites, 305 diatom taxa and 23 statistically significant environmental variables. Detrended correspondence analysis yields an important first axis $(\lambda=0.83)$ that separates species according to salinity preference and sites according to waterbody type. The environmental gradient that best explains variation in the diatom data is a coastal gradient defined according to waterbody type and salinity. Comparisons between indirect and direct gradient analysis indicate that measured environmental variables explain the species data well. A minimal set of environmental variables (exposure to salt, salinity, distance from the sea, flow, carbonate, $\mathrm{pH}$ and precipitation) explain the data almost as well as the full set of variables, and indicate the main coastal gradient (axis one) is made up of three correlated gradients related to marine influence, exposure to salt and energy regime. A secondary gradient in the data (axis two) can be partially explained as an acidity gradient. Gradients and variables associated with axis one interact in a predictable way and constitute a spatial coastal gradient considered to be equivalent to the temporal coastal gradient present in the Holocene sedimentary sequences.

Weighted averaging regression of the calibration set resulted in a high correlation $\left(r^{2}\right.$ jack $\left.=0.84\right)$ between observed and diatom inferred salinity values for the modern sites and a relatively low root mean squared error of prediction. Greatest prediction error occurs in the centre of the salinity gradient as a result of the inherent variability of brackish water environments. Nevertheless regression statistics and comparison with other diatom-salinity transfer functions indicate the predictive ability of the calibration set is powerful enough to reconstruct salinity from past environments. Salinity optima and tolerances are presented for 100 species. In terms of three broad salinity categories (fresh, brackish, marine) these optima are consistent with published preferences providing confirmation that 
preferences derived predominantly in the Northern Hemisphere are applicable to diatom species in the coastal zone of New Zealand. Differences between published preferences and those derived from the calibration set arise when a more detailed classification system is used. This is thought to be a result of the broadness of many classifications presented in international literature and the lack of work in the coastal zone. The calibration set is considered just large enough to enable use of the modern analogue technique to check reconstructions made using weighted averaging. 


\section{CHAPTER FOUR}

\section{Taupo Swamp: Evidence for Sudden Environmental Change from Reconstructed Waterbody Type and Paleosalinity}

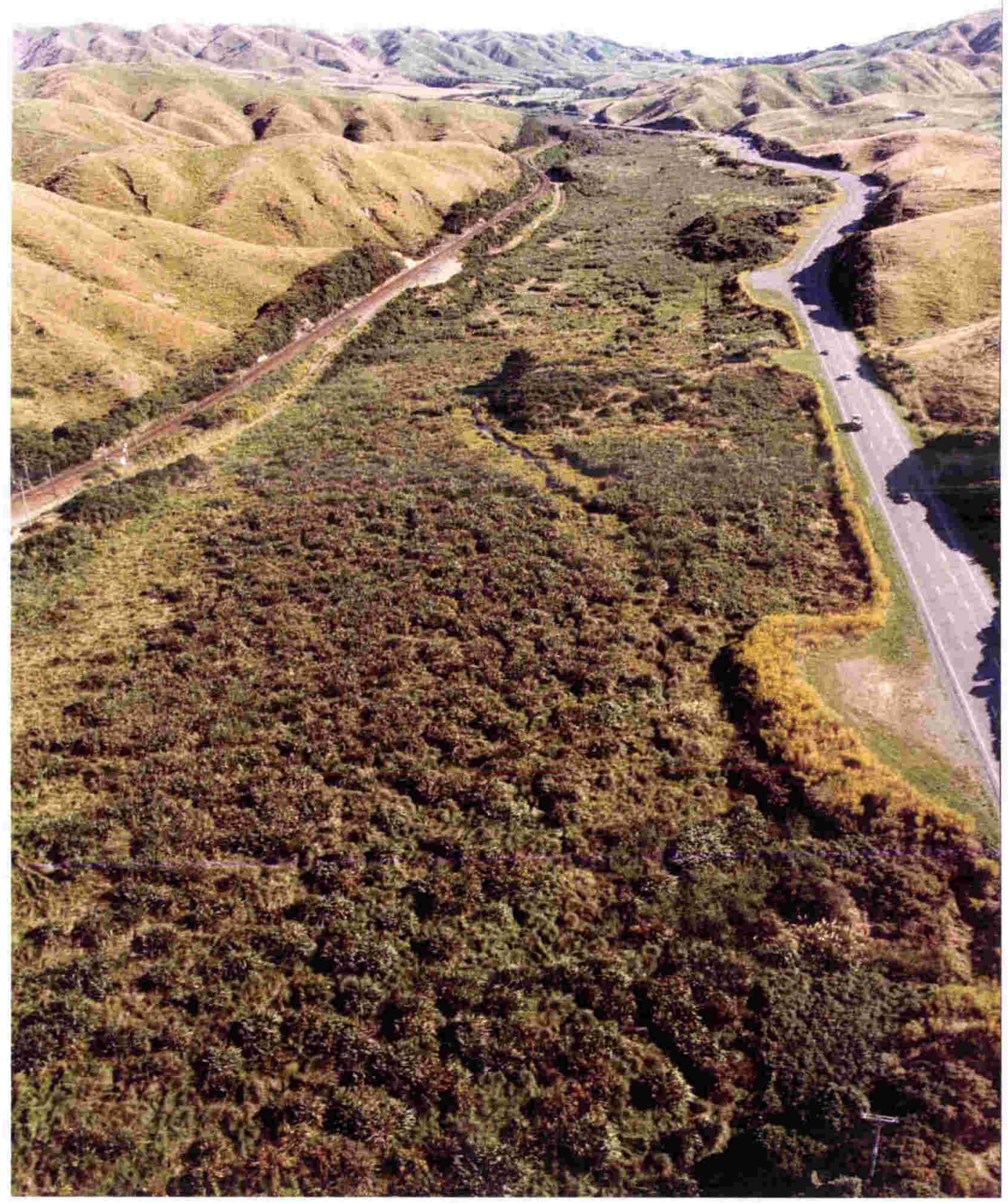

Fig. 4.1. Oblique aerial view of Taupo Swamp looking north. Photo by Lloyd Homer. 
High resolution paleoenvironmental evidence is presented in this chapter for the transformation of central Taupo Valley from an inlet of the sea to a fresh water wetland. Sedimentological, macrofossil and diatom analysis of two $\sim 500 \mathrm{~cm}$ long cores indicates that three different depositional environments have existed in the central part of the valley in the last 5500 years. A predominantly open lagoon existed between 5500 and about 3100 cal. years BP (calibrated years before present). A very shallow fresh brackish pond existed for a short time before the fresh water wetland environment of the present day became established at about 2400 cal. years BP. Environmental transitions happened suddenly, involved large magnitude changes to the environment and occurred synchronously across the valley so are consistent with the effects of past large earthquakes. In the lower valley immediately north of Plimmerton Township a very shallow, fresh brackish lagoon existed until at least $1850 \mathrm{AD}$, and may well have disappeared as a result of a small amount of uplift in the 1855 AD Wairarapa earthquake. The likelihood that transitions were caused by coseismic movement of base level is discussed further in Chapter Seven where transitions are compared with those described from other sites.

\subsection{INTRODUCTION}

Taupo Swamp is a narrow two kilometre long fresh water swamp (Fig 4.1) occupying a north trending valley that drains into the outer Harbour of Porirua. The swamp is situated in an area of Triassic sedimentary basement rock that is closely bounded by the northeasterly trending Pukerua-Shepherds Gully Fault in the west and the Ohariu Fault in the east (Fig. 4.2). The swamp is fed by Taupo Stream that drains an 820 ha catchment consisting predominantly of pasture. Taupo Swamp was classified by Moar (1949) as a topogenous mire because a topographic barrier at the southern end prevented free drainage. The swamp used to rise from two metres above mean sea level (amsl) behind this barrier (a beach ridge now occupied by Plimmerton township), to $20 \mathrm{~m}$ amsl at its northern end. However the southern end of the swamp has been drained and developed for farming and industrial use (Fig. 4.2). Various attempts have been made to infill or drain other parts of the swamp, and the stream has been channelised for much of its length, but the central and northern sections are now protected by the Queen Elizabeth II Trust. Taupo Swamp is one of few topogenous mires with predominantly indigenous vegetation remaining in New Zealand and is a highly visible example because it lies between State Highway One and the Main Trunk Railway. 


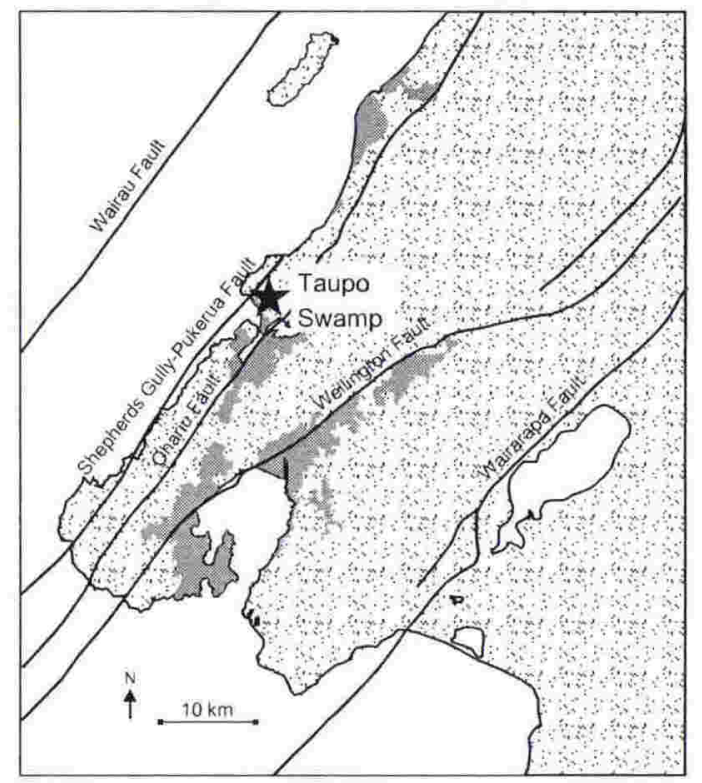

A

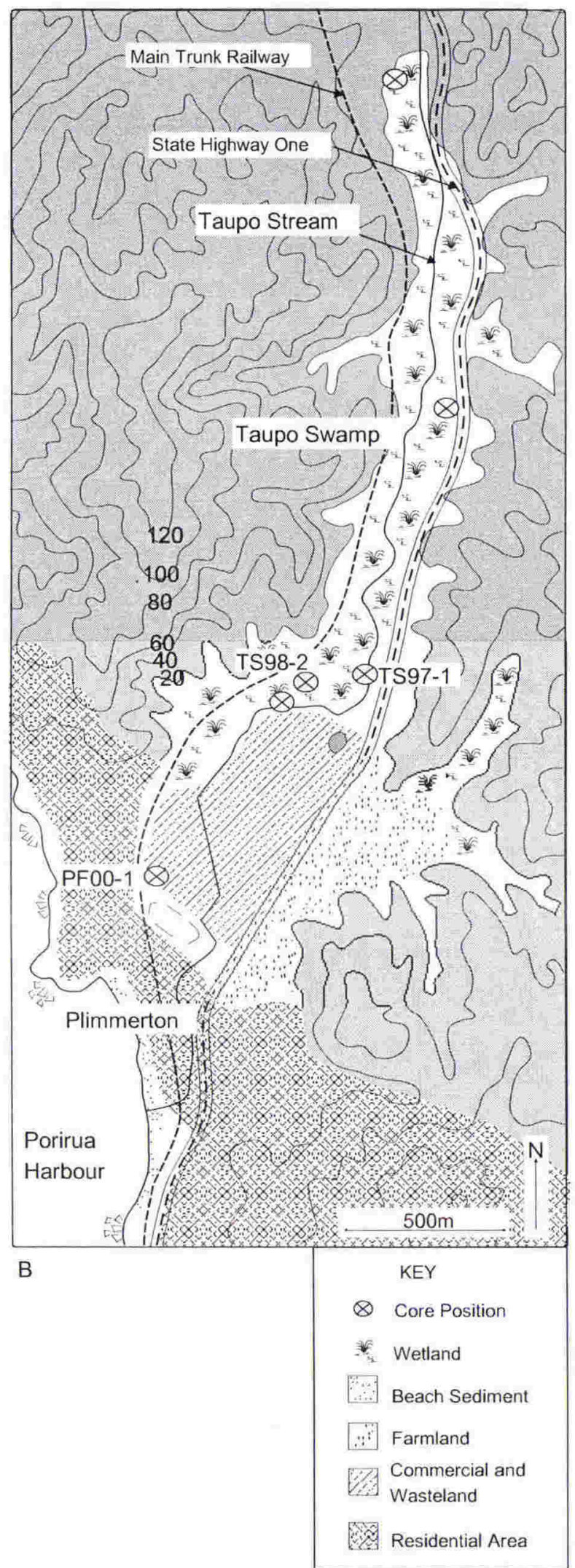

Fig. 4.2. Map of Taupo Swamp. A: Location in the Wellington region. B: Site map showing core positions. Only cores discussed in this chapter are labelled. 
The main vegetation types in the swamp are sedgeland and tussockland with New Zealand flax (Phormium tenax) being one of the major species (Bagnall and Ogle, 1981). Despite past attempts at draining the swamp, the water table is generally maintained at the swamp surface and is preventing exotic weeds from becoming more dominant (Bagnall \& Ogle, 1981). Moar (1949) noted a small area of Leptocarpus rushland considered to be a remnant of saline vegetation that would have inhabited the site when the valley was open to the sea. However by the time of the survey by Bagnall and Ogle (1981) it had been fully replaced by Carex sedgeland.

Adkin (1921) in a geomorphological study of Porirua Harbour described Taupo Valley as a drowned valley into which, at some stage, the sea penetrated about one kilometre further than the present coastline. Later movement of gravel across the valley mouth caused a lagoon to form and finally the valley to be completely isolated from the sea. Aggradation then filled the waterbody to form the wetland environment present at the site today. Adkin (1921) attributes the raised gravel beach ridge and shore platform of the Plimmerton coast to uplift in the 1855 earthquake on the Wairarapa Fault. Eiby (1990) discusses much of the historical and geological evidence for the Porirua area and concludes that no uplift took place in 1855 . He argues that remnants of an uplifted wave-cut platform in outer Porirua Harbour provide evidence for about one metre of uplift in a prehistoric event or several events on the Ohariu Fault. This study of the depositional and paleoseismic history of Taupo Swamp provides some insight into these discussions.

\subsection{SEDIMENTARY SEQUENCES}

\subsubsection{Description of Sequences}

Six cores were taken at various sites throughout Taupo Swamp in an attempt to develop a generalised stratigraphy for the swamp (Fig. 4.2). However only two cores penetrated deeper than $250 \mathrm{~cm}$ so these two were studied in detail. It is thought that the presence of pieces of wood and tough, fibrous organic material inhibited hand-operated coring in the swamp. TS97-1 is a $550 \mathrm{~cm}$ long sequence from the margin of the valley near the present stream course, it has an age near the base of 5598-5894 cal. years BP (Fig. 4.3). TS98-2 is a $500 \mathrm{~cm}$ sequence from the centre of the valley that is $5192-5457$ cal. years BP at the base. The cores are 4.8 and $4.2 \mathrm{~m}$ amsl respectively. A short core immediately north of the playing field at the southern end of the valley was also analysed to provide insight into the history of this reclaimed part of the swamp. This core is discussed separately in section 4.5.5. Core logs presented below are summaries of full core logs presented in Appendix I. 


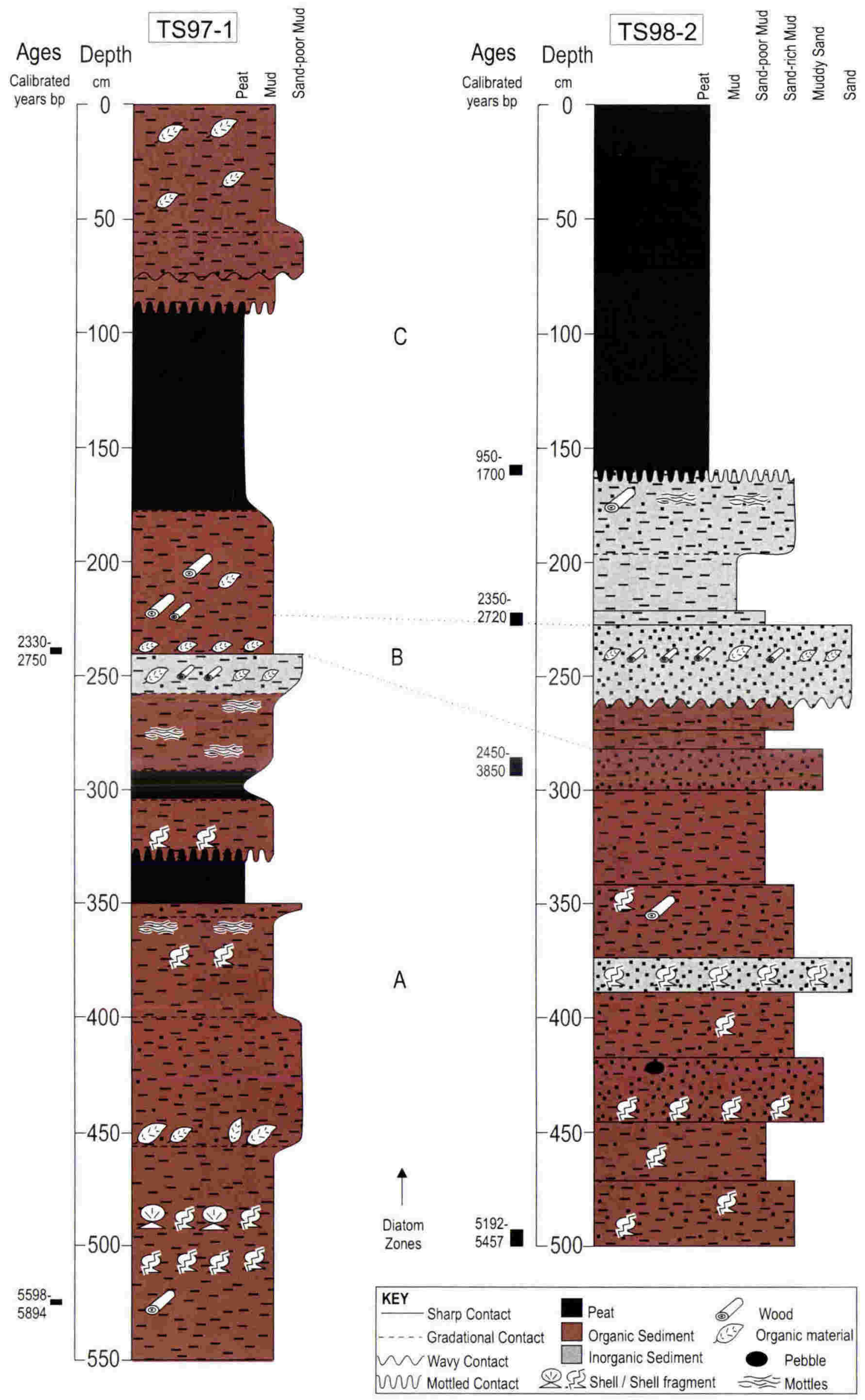

Fig. 4.3. Core logs of TS97-1 and TS98-2 from Taupo Swamp. 
TS97-1 consists predominantly of brownish black, organic mud and peat. Units of sand-poor mud occur occasionally throughout the sequence. Contacts between units are generally gradational. Small pieces of wood and fibrous plant material are scattered throughout the core. In the lower half of the sequence the bivalve Austrovenus stutchburyi is present and is most common in the lowest $50 \mathrm{~cm}$. Small gastropods and the mussel Hyridella menziesi occur in several layers between 550 and $324 \mathrm{~cm}$ depth. Foraminifera and ostracods are also present. There are no shells in the upper half of the core and sediments are generally finergrained and more organic.

TS98-2 is a coarser-grained sequence than TS97-1, consisting of alternating sands and sand-rich muds between 500 and $160 \mathrm{~cm}$ depth. Most of this sediment is rich in organic material with pieces of wood and fibrous plant material occurring throughout. A thick unit of peat exists from $160 \mathrm{~cm}$ to the top of the core. Contacts between units are generally sharp. An assemblage of shells, foraminifera and ostracods similar to that found in TS97-1 occurs in distinct layers between 500 and $374 \mathrm{~cm}$ depth.

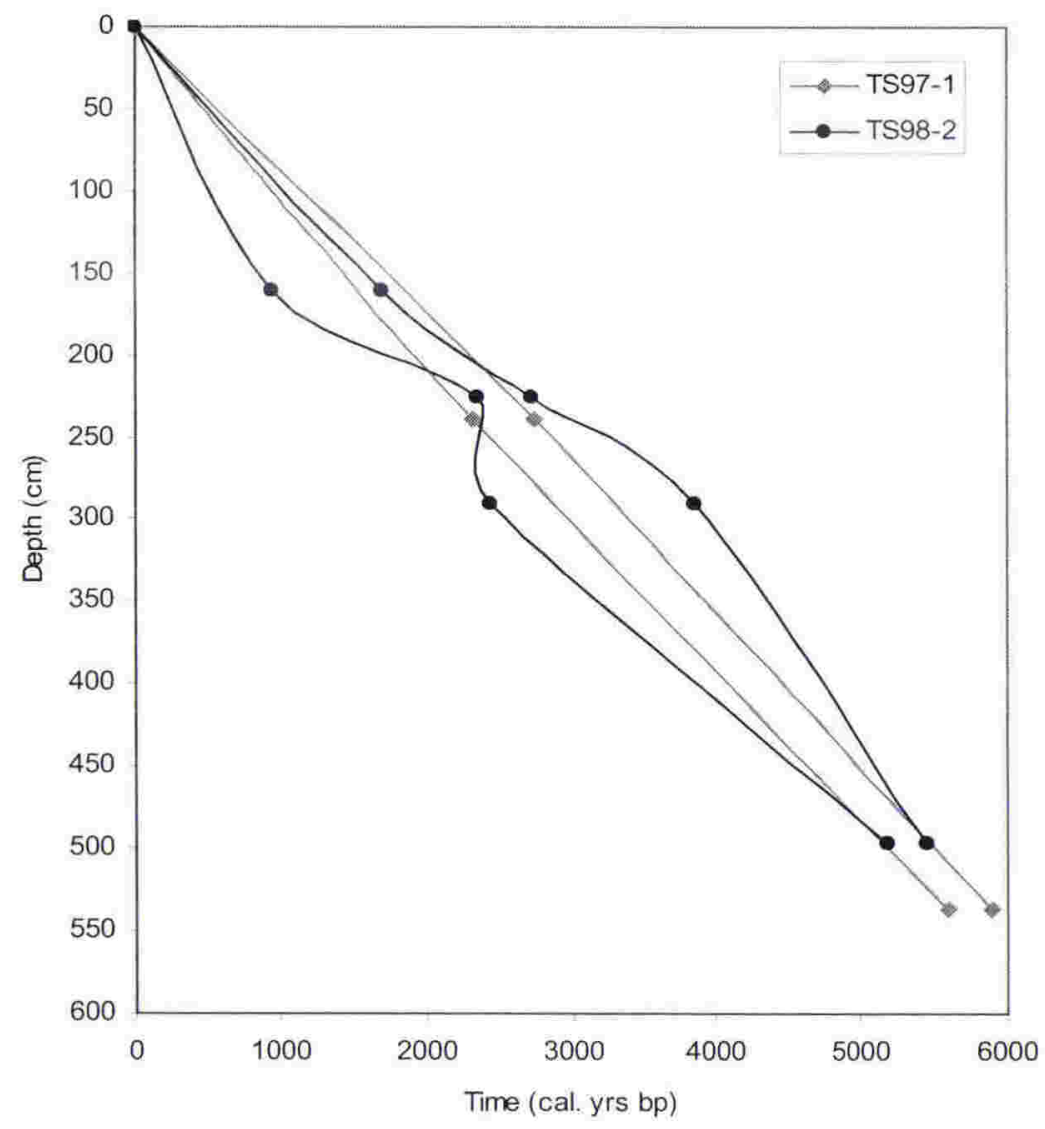

Fig. 4.4. Sediment accumulation rate curves for TS97-1 and TS98-2 based on calibrated radiocarbon age estimates. Maximum and minimum curves for each core are based on end-member values of the 2-sigma age range. 
Broad estimates of sediment accumulation rates are calculated for cores TS97-1 and TS98-2 using available radiocarbon ages (Fig. 4.4). Rates average about one millimetre per year ( $\mathrm{mm} / \mathrm{yr}$ ) for TS97-1. In the lower half of TS98-2 rates are likely to have been between 0.7 and $1.5 \mathrm{~mm} / \mathrm{yr}$ and slightly higher for the peat unit at the top of the core between about 0.9 and $1.7 \mathrm{~mm} / \mathrm{yr}$. In the central part of TS98-2 estimates range between 0.36 and $6.5 \mathrm{~mm} / \mathrm{yr}$ which is partly a result of age overlap between adjacent dates when using the 2 -sigma range but may also indicate widely varying rates of deposition in this interval.

\subsubsection{Interpretation of Sequences}

The generally high organic content and fine grain size of TS97-1 indicates the sequence was deposited in a low energy environment and TS98-2 was deposited in a slightly higher energy regime. The lower halves of both cores were deposited in an environment subject to varying conditions as represented by the alternating units of different grain sizes and organic content. The presence of the estuarine bivalve Austrovenus stutchburyi below $300 \mathrm{~cm}$ indicates the environment was saline. However the small size of the valves $(\sim 17 \mathrm{~mm}$ length) suggests the site was a brackish environment at the head of an estuary (Beu and Maxwell, 1990) rather than an open marine site. Small brackish and freshwater gastropods and the freshwater mussel Hyridella menziesi also occur in this interval supporting the interpretation that the environment was brackish with fresh water inflows rather than fully marine. The foraminiferal assemblage was dominated by Ammonia parkinsoniana (John Collen, pers. comm., 1999) which is widespread in brackish and slightly brackish environments and often dominant in intertidal and subtidal zones of estuaries (Hayward et al., 1999). Ostracod species present are also typical of brackish water conditions (Steven Eager, pers. comm., 1999).

The upper halves of the sequences represent a more stable environment with a major proximal source of organic material and little input of siliciclastic sediment. The units of peat in particular are likely to have been deposited in a marsh or wetland environment. The position of the transition between the brackish environment and fresh wetland that exists at the site today is not obvious from the sedimentology alone. In TS97-1 between 238 and $241 \mathrm{~cm}$ depth there is a particularly organic-rich layer that contains many small rootlets. This may represent initiation of soil / wetland conditions at the site. In TS98-2 there is a unit of sand between 227 and $262 \mathrm{~cm}$ depth that appears non-marine because it is banded by high concentrations of organic material. This may represent movement of the stream bed over the core site. 


\subsection{DIATOM ANALYSIS}

\subsubsection{Introduction}

About 200 diatom species were identified in TS97-1 and TS98-2 (full species counts are presented in Appendix VI). Both cores have very similar diatom assemblages with differences usually reflecting the differing locations of the core sites within the valley. Diatom samples were generally analysed at $10 \mathrm{~cm}$ spacing, with $20 \mathrm{~cm}$ spacing used over a stable interval in TS97-1 and $5 \mathrm{~cm}$ spacing used over transitional intervals. In the lower halves of both cores counts always consisted of at least 400 diatom valves. In the upper halves of the cores counts were made of at least 100 valves and occasionally less because of difficulties with diatom concentration. Concentrations calculated for these upper samples were moderate but even when made using concentrated solution there appeared very few diatoms on the microscope slides. This discrepancy is thought to be a result of using sediment weight in the concentration calculations. For example a peat sample with low concentrations of diatoms will have a relatively higher concentration calculated for it in comparison with a sand sample because of its light weight.

Three distinct assemblages divide the sedimentary sequence at Taupo Swamp into three zones (section 4.3.2). Paleoenvironmental interpretations of zones (section 4.3.3) are made using traditional paleoecological procedures of allocating species into salinity and habitat types based on published autecological information (Appendix III). In section 4.3.4 fossil diatom assemblages are plotted passively onto a detrended correspondence analysis of calibration set samples to determine past waterbody type. Salinity reconstructions are presented in section 4.3.5 using the diatom-salinity transfer function derived in Chapter Three.

\subsubsection{Definition of Diatom Assemblage Zones}

Three diatom assemblage zones are defined for the sedimentary sequence at Taupo Swamp according to species composition, concentration, species richness and preservation (Table 4.1). Key species of each zone are those that are abundant in the assemblage and / or unique to the zone. Zone $\mathrm{A}$ at the base of the sequence consists of a diverse assemblage of diatoms that occur in high concentrations in the sediment and are well preserved. There are ten key species with a further five that occur in greater abundance or solely in TS98-2. Pseudopodosira species are fairly abundant but do not fit existing taxa descriptions in reference floras consulted in this study. Zone B has a peak of high concentration at the base of the zone and then is of moderate concentration and moderate species richness. It is defined by the abundant presence of small Fragilaria species. Zone C contains moderate concentrations of diatoms with high species richness. The fluctuation in diatom concentration that occurs between samples in zone $\mathrm{C}$ (particularly in TS98-2) suggests that dissolution may have affected the assemblages. The zone is characterised by the occurrence of seven key species. 


\begin{tabular}{|c|c|c|c|c|}
\hline Zones (cm) & Key Species & Concentration & $\begin{array}{l}\text { Species } \\
\text { Richness }\end{array}$ & Preservation \\
\hline $\begin{array}{l}\text { C } \\
\text { TS97-1: 222.5-0 } \\
\text { TS98-2: 227.5-0 }\end{array}$ & $\begin{array}{l}\text { Aulacoseira crenulata } \\
\text { Navicula contenta } \\
\text { Achnanthes oblongella } \\
\text { Achnanthes lanceolata } \\
\text { Fragilaria exigua } \\
\text { Pinnularia subcapitata } \\
\text { Navicula angusta } \\
\text { Aulacoseira } \\
\quad \text { crassipunctata } \\
\text { Fragilaria pinnata } \\
\text { Fragilaria brevistriata }\end{array}$ & Moderate & Very high & $\begin{array}{l}\text { Some } \\
\text { evidence of } \\
\text { dissolution }\end{array}$ \\
\hline $\begin{array}{l}\text { B } \\
\text { TS97-1: } 241-222.5 \\
\text { TS98-2: } 282.5- \\
227.5\end{array}$ & $\begin{array}{l}\text { Fragilaria pinnata } \\
\text { Fragilaria brevistriata } \\
\text { Fragilaria construens } \quad \text { f. venter } \\
\text { Fragilaria exigua }^{T S-1} \\
\text { Achnanthes ploenensis } \\
\text { Achnanthes delicatula } \\
\quad \text { var. engelbrechtii }^{T S-2} \\
\text { Cocconeis placentula }\end{array}$ & $\begin{array}{l}\text { High in peak at } \\
\text { base then } \\
\text { moderate }\end{array}$ & Moderate & Good \\
\hline $\begin{array}{l}\text { A } \\
\text { TS97-1: } 540-241 \\
\text { TS98-2: } 500-282.5\end{array}$ & $\begin{array}{l}\text { Pseudopodosira sp. } 1 \text { \& } 2 \\
\text { Cocconeis placentula } \\
\text { Fragilaria pinnata } \\
\text { Achnanthes delicatula } \\
\quad \text { var. engelbrechtii } \\
\text { Paralia sulcata } \\
\text { Achnanthes reversa } \\
\text { Fallacia tenera } \\
\text { Gomphonema valentinica } \\
\text { Achnanthes ploenensis } \\
\text { Cocconeis c. f. } \\
\text { peltoides } \\
\text { Catenula adhaerens } \\
\text { Opephora olsenii } \\
\text { Fr-2 } \\
\text { Fragilaria subsalina } \\
\text { Navicula perminuta } \\
\text { TS-2 }\end{array}$ & High & Very high & Good \\
\hline
\end{tabular}

Table 4.1. Definition of diatom assemblage zones for cores TS97-1 and TS98-2. Species with numbers in superscript occur only or more abundantly in the corresponding core.

\subsubsection{Interpretation of Diatom Assemblage Zones}

The three diatom assemblage zones of Taupo Swamp represent three different depositional environments. Diatom species have been grouped according to their salinity and habitat preferences to enable description of these past depositional environments (Figs 4.5 and 4.6). In zone A brackish marine, brackish and fresh indifferent species all occur in fairly high proportions. The 'unknown' category 

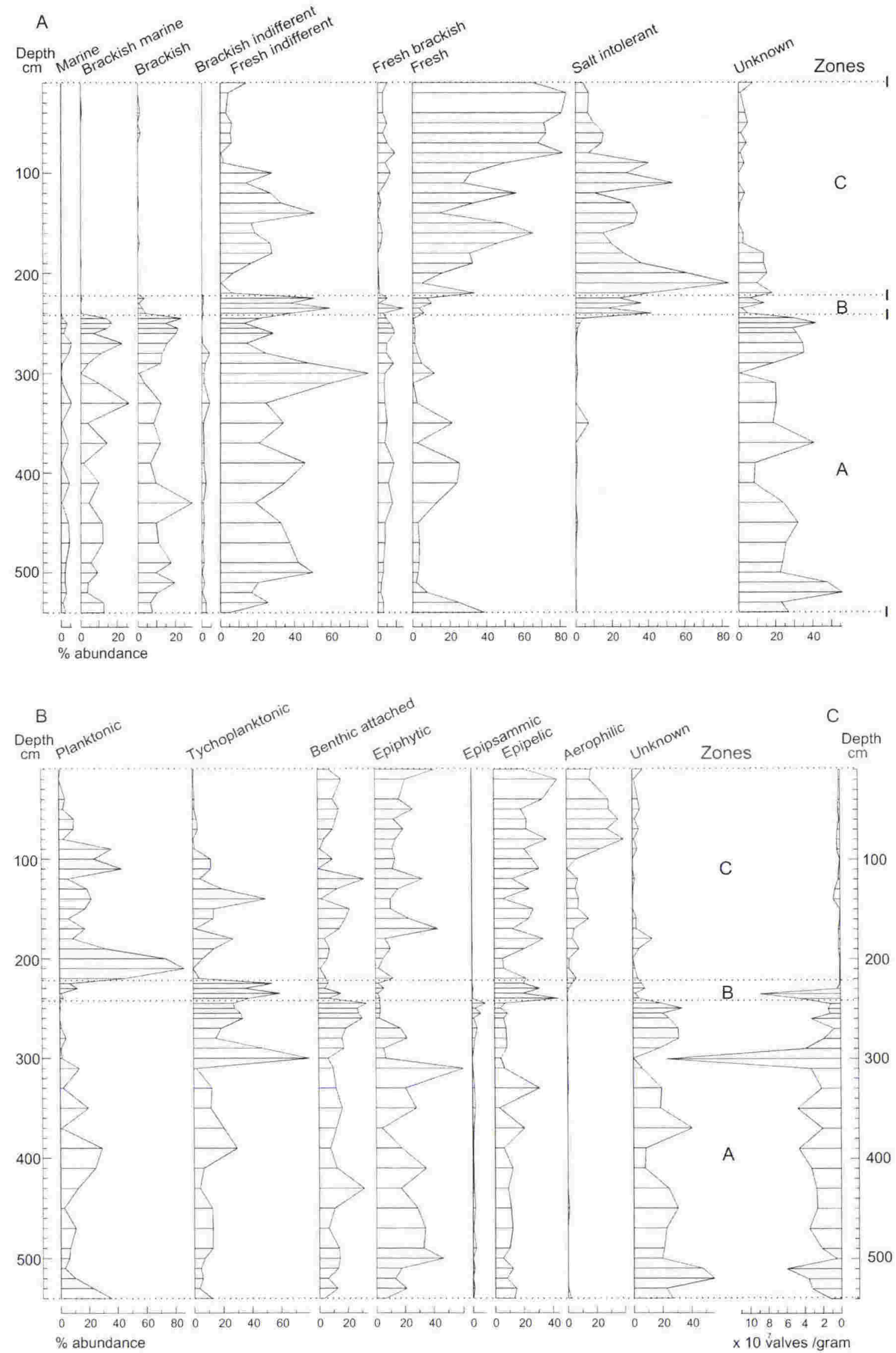

Fig. 4.5. Diatom assemblages of core TS97-1. A: Diatoms grouped according to salinity preferences. B: Habitat preferences. C: Concentration of diatom valves in the sediment. 

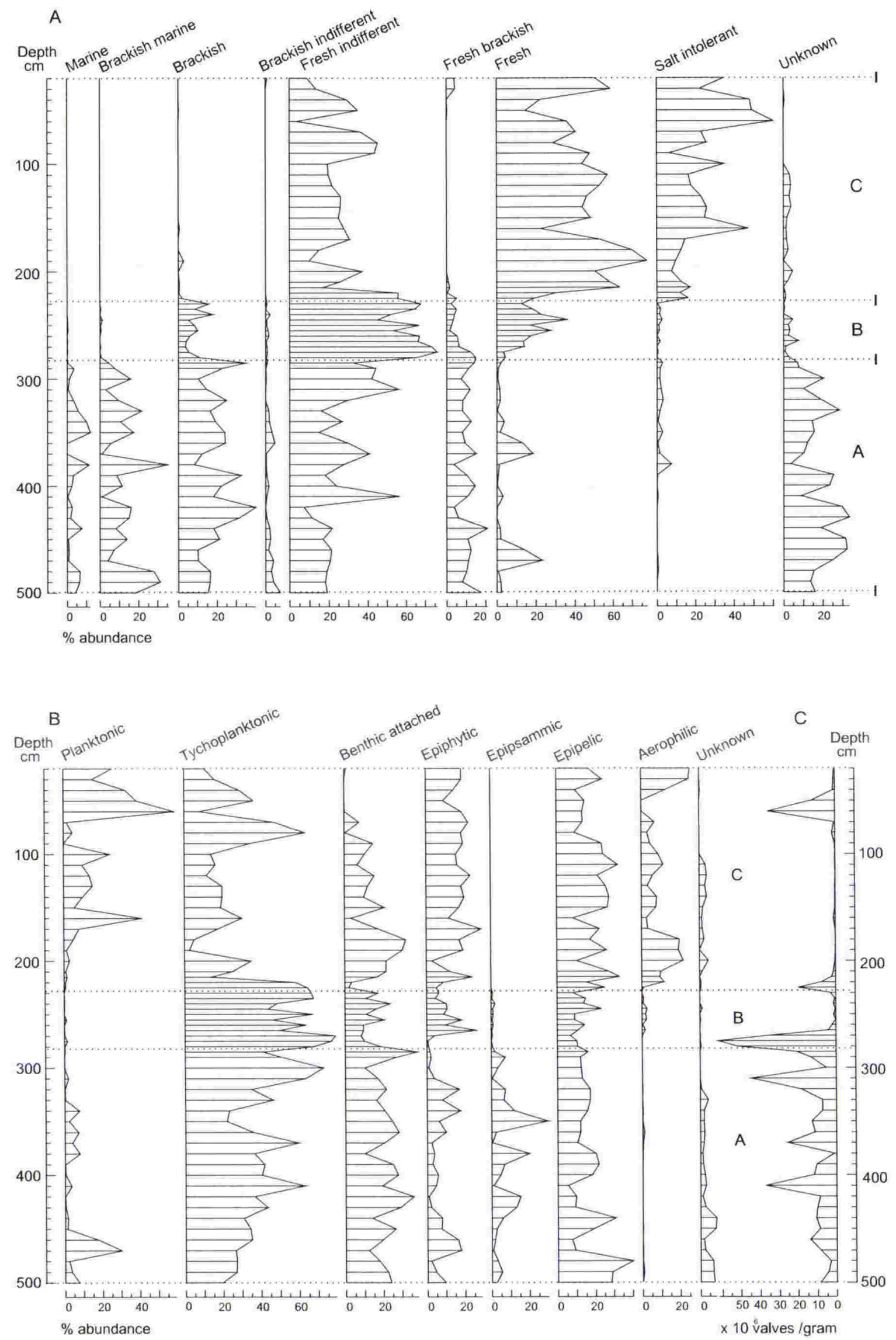

Fig. 4.6. Diatom assemblages of core TS98-2. A: Diatoms grouped according to salinity preferences. B: Habitat preferences. C: Concentration of diatom valves in the sediment. 
includes high proportions of Pseudopodosira species that are likely to have brackish salinity preferences but they have not been identified to species level so are not placed in a salinity category. A brackish environment is inferred with salinities high enough to support brackish marine diatoms throughout this interval. Small numbers of marine species suggest the waterbody was open to the sea but the connection was probably some distance from the core sites. Fresh brackish species make up a small but consistent proportion of the assemblage, possibly inhabiting slightly less saline waters towards the landward edge of the waterbody. Fresh water diatoms are likely to have been transported to the waterbody from a fresh water source or they inhabited a fresher surface layer of water. Transport is supported by peaks in fresh water diatoms being made up of single species (eg, Cyclotella stelligera) and these species being from easily transportable habitats such as planktonic and tychoplanktonic habitats. In TS97-1 a short-lived fresh water event occurred at $300 \mathrm{~cm}$ depth because the assemblage of this single sample consists entirely of fresh indifferent and fresh water species.

The habitats of species in zone A are predominantly benthic and tychoplanktonic. This indicates that the waterbody was shallow. The planktonic species present are fresh and fresh indifferent species that were probably transported into the waterbody as noted above. Epipelic and epiphytic species are both present in high proportions so a muddy substrate with macrophytes growing is inferred. The proportion of epiphytics is higher in core TS97-1 suggesting that macrophytes were more numerous or more proximal at the valley margin site than the central valley site of TS98-2. The higher proportion of epipsammic species in TS98-2 also reflects the higher energy regime position of this core in comparison with the valley margin core. Peaks in epipsammic species generally coincide with peaks in marine and brackish marine species and units of coarser sediment in the core (eg, at $380 \mathrm{~cm}$ depth in TS98-2) highlighting periods of greater marine influence at the site. There is a complete lack of aerophilic species in both cores indicating that although the waterbody was shallow, there was permanent open water at the site.

Zone B is dominated by the fresh indifferent, tychoplanktonic species Fragilaria pinnata and Fragilaria brevistriata. Other species present are brackish, fresh brackish or fresh. The complete lack of marine and brackish marine species indicates the environment was isolated from the sea. Water of the past environment appears to have been fresh at the marginal valley site and fresh brackish at the central valley site. This is because salt intolerant species are present in TS97-1 in zone B and very few brackish species whereas TS98-2 contains brackish species and very few salt intolerant species. Tychoplanktonic and benthic species predominate, so the environment was very shallow. Zone $\mathrm{C}$ is dominated by fresh and salt intolerant diatoms indicating that a pure fresh water environment existed in the valley throughout this time. The occurrence of some fresh indifferent and fresh brackish species can be explained by looking at the habitat preferences of these species. They all have tychoplanktonic or aerophilic 
habitat preferences so are probably present because they are competitive in low water settings rather than because the salinity suits them particularly. Habitat analysis indicates very shallow water depths, with high proportions of aerophilic species suggesting that a wetland environment existed at both sites. Although there are high proportions of planktonic species also, the category is made up of one species, Aulacoseira crenulata, which is known from modern sampling to be a shallow water planktonic form (Cochran, unpublished data). Pools of water within the wetland would have allowed Aulacoseira cremulata to exist as part of the assemblage. The presence of acidophilous species such as Pinnularia subcapitata and Navicula angusta indicates waters were slightly acidic which is typical of peat-forming environments.

\subsubsection{Reconstruction of Waterbody Type}

A detrended correspondence analysis (DCA) of 46 calibration set samples in Chapter Three resulted in an ordering of sites along axis one according to waterbody type. This enabled division of ordination space into five waterbody types based on diatom assemblages. Diatom assemblages from Taupo Swamp are incorporated passively into the same DCA to provide an indication of the waterbody types that the fossil diatom assemblages represent (Fig. 4.7). Passive samples have no influence on the extraction of axes but are plotted on an ordination diagram by means of transition formulae (ter Braak, 1988). Samples are positioned according to their species composition so samples with similar diatom assemblages occur close together on the diagram.

Diatom samples from TS97-1 and TS98-2 conform to the coastal gradient defined by the calibration set because they plot along axis one of the DCA in a similar manner to the modern samples (Fig. $4.7 \mathrm{~A}$ ). At the landward end of the gradient they spread along axis two into a field that, although defined by only three modern samples, was identified as a wetland field. Fossil samples from Taupo Swamp plot in the lagoon, lake and wetland fields. Diatom assemblage zones are well separated by the DCA (Fig. 4.7 B and C) and transitions between zones involve long distances (particularly in TS97-1) indicating large changes in waterbody type between zones. Samples of zone A plot centre right in the lagoon field with scatter around a central cluster representing fluctuating conditions. For example the sample at $300 \mathrm{~cm}$ depth in TS97-1 that was identified as a fresh water event plots at the far left of the lagoon field and samples representing greater marine influence plot at the far right (eg, sample 380 in TS98-2). The position of zone B samples reflects the difference between core sites with TS97-1 samples plotting in the lake field and TS98-2 samples at the left of the lagoon field. Zone $\mathrm{C}$ samples from both cores plot in the wetland field. These waterbody types derived through comparison of fossil diatom assemblages with those from modern waterbodies, are consistent with and confirm the validity of paleoenvironmental interpretations made directly from fossil assemblages. 

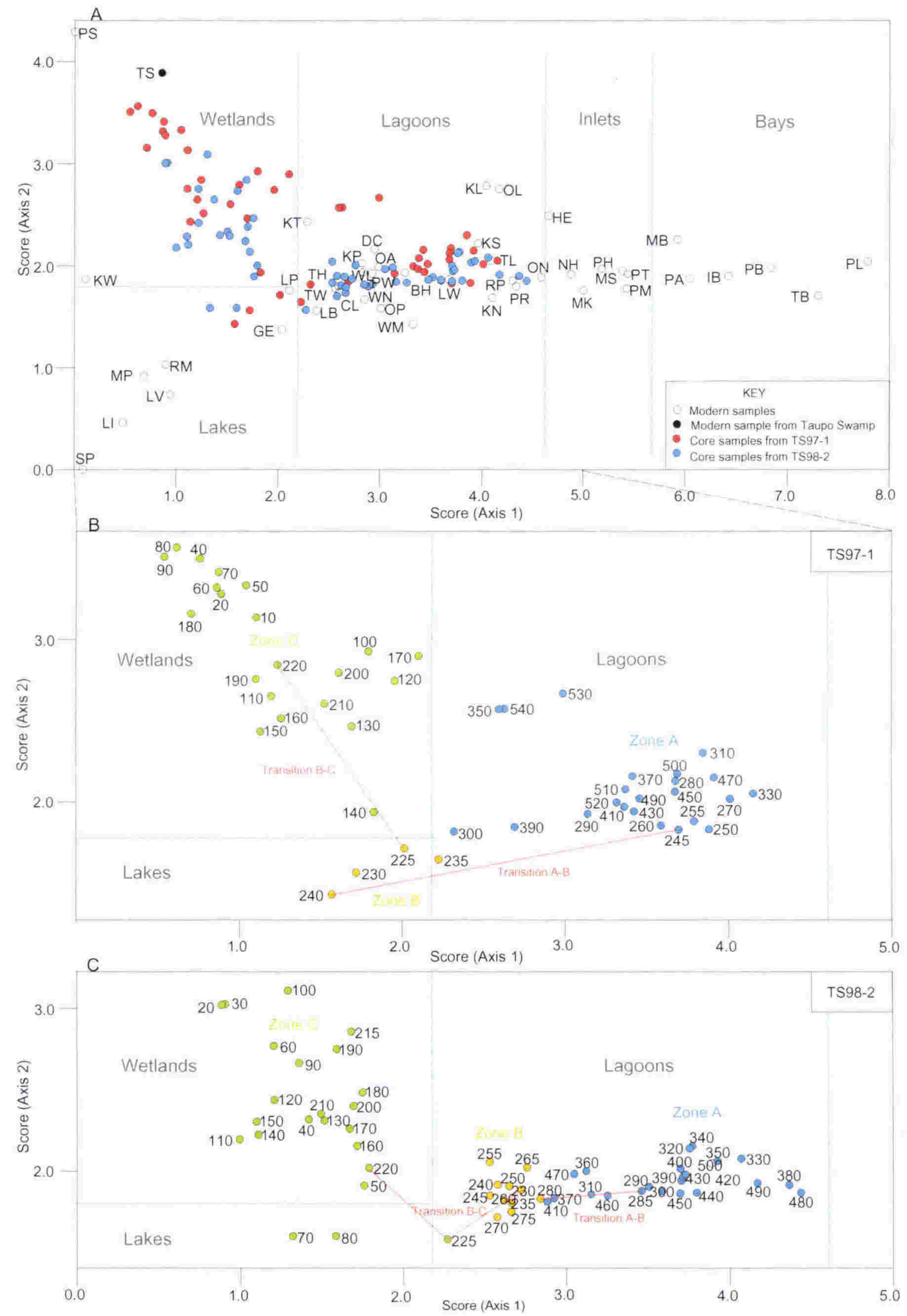

Fig. 4.7. DCA of calibration set sites with fossil samples from Taupo Swamp incorporated passively. A: Distribution of modern sites and division of axes into waterbody types.

Coloured dots show where fossil samples plot in relation to modern sites. B and C: Left side of A enlarged to show samples from TS97-1 and TS98-2 respectively. Samples are labelled according to depth and coloured according to diatom assemblage zones. 


\subsubsection{Application of Salinity Transfer Function}

The DCA (above) illustrates that fossil diatom assemblages from Taupo Swamp are part of a coastal gradient similar in nature to that defined by the calibration set assemblages. Calibration set samples extend further in both the seaward and landward directions along axis one and also in both directions along axis two. This is desirable for reconstruction to avoid truncation of species response curves. However in the wetland field of the DCA, the upper half of axis two, there are very few modern samples so reconstruction is likely to be inhibited by lack of analogues. Poor preservation and low counts of zone $\mathrm{C}$ samples will also inhibit their reconstruction. In terms of species, $76 \%$ of those present in TS97-1 are also present in the calibration set and $82 \%$ of species in TS98-2 are represented. There are species in each zone that are not present in the calibration set but they are generally of low abundance.

Weighted averaging calibration is based on the idea that the value of an environmental variable at a particular site will be close to the optima of those species that are abundant at the site (Birks, 1995). The value of the environmental variable is estimated as the average of the optima of all the species present weighted according to species' abundance. Salinity optima for species in the calibration set derived in Chapter Three are used here to estimate salinity values for the fossil samples. Weighted averaging calibration was carried out using CALIBRATE (Juggins and ter Braak, 1997-99) and MAT98 (Juggins, 2000) was used to apply the modern analogue technique. The computer intensive crossvalidation procedure of bootstrapping is not available in CALIBRATE so a means of assessing results is provided by comparison of three different models / methods used for reconstruction of the same data. Simple weighted averaging, weighted averaging with tolerance downweighting and the modern analogue technique were all used to reconstruct salinity (Fig. 4.8).

The lower halves of both cores show a great amount of variability in salinity values but the three different models are generally consistent in the direction of fluctuation for each sample (Fig. 4.8). For example the fresh water event at 300 $\mathrm{cm}$ in TS97-1 is picked up by all three models, as is the marine event at $380 \mathrm{~cm}$ in TS98-2. In the upper halves of the cores WA and WA-tol are in close agreement whereas the MAT reconstruction differs at several points. This is no doubt a result of the lack of close modern analogues for this upper section. Only three modern samples are fresh water wetlands and three modern samples are used by the technique to estimate past salinity for the fossil sample. This means that where a fossil sample is dissimilar to one of the wetland samples, another sample will become the next closest analogue and result in a higher salinity value for the reconstruction. 

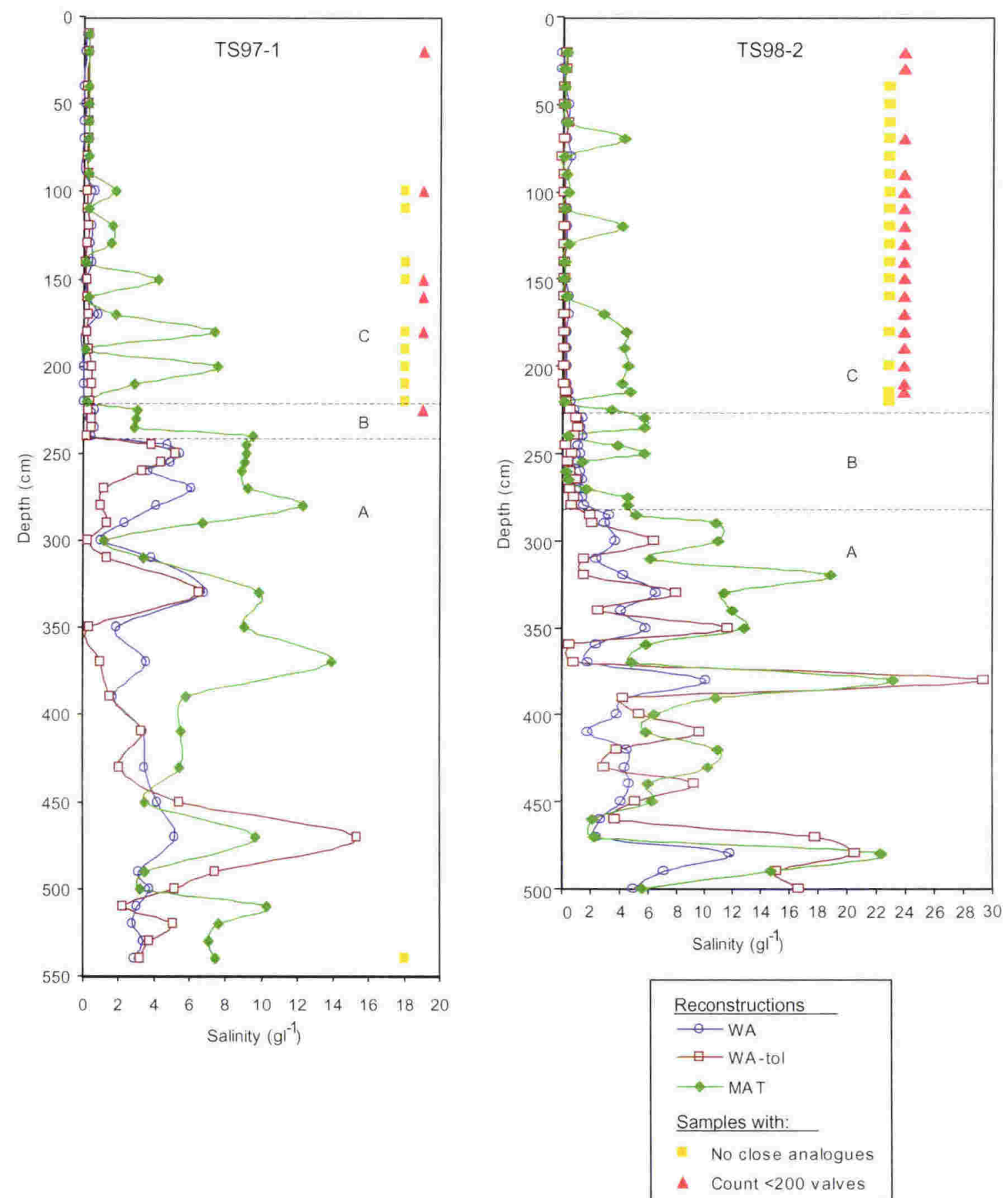

Fig. 4.8. Salinity reconstructions for TS97-1 and TS98-2 using weighted averaging (WA), weighted averaging with tolerance downweighting (WA-tol) and the modern analogue technique (MAT). Dotted lines show diatom zone boundaries. 
Salinities in the lower halves of the cores average about five grams per litre $\left(\mathrm{gl}^{-1}\right)$ in TS97-1 and $7 \mathrm{gl}^{-1}$ in TS98-2. The widely varying salinity values in these reconstructions are probably partly a result of the lower predictive ability of the calibration set in the brackish part of the gradient (Chapter Three) but sedimentology of both sequences confirms the environment was also variable in nature (section 4.5.2 above). WA and WA-tol salinity reconstructions for diatom zone $\mathrm{C}$ in the upper halves of both cores average about $0.2 \mathrm{gl}^{-1}$ which is consistent with present day salinity measurements at the site. As mentioned above MAT is problematic in this zone because of the small size of the calibration set. However towards the top of the zone where fossil samples approach modern samples in terms of assemblage similarity, MAT reconstructions coincide with those of WA and WA-tol. Zone B is differentiated from zone $\mathrm{C}$ by higher salinities in both the WA and WA-tol reconstructions. At the TS97-1 site salinity of zone B is about $0.4 \mathrm{gl}^{-1}$ and at the TS98-2 site it averages $1 \mathrm{gl}^{-1}$. Although MAT indicates lower salinities for zones B and C than those of zone A, zone B is not clearly differentiated from zone $\mathrm{C}$ because of poor reconstructions in the upper part of the sequence.

In terms of position on the generalised coastal gradient defined in Chapter Three, these salinity reconstructions indicate existence of a waterbody open to the sea and movement in a landward direction with decreasing depth up the core sequence to a waterbody completely isolated from the sea and located at the extreme landward end of the gradient. Landward movement along the gradient did not occur continuously through time but appears to have occurred in two main steps. Between these transitional steps environments were stable, i.e. even if they were highly variable over the short term (as in the case of zone A), they did not represent any movement along the gradient in the long term.

\subsection{PALEOENVIRONMENTAL RECONSTRUCTION}

Sedimentology, macrofossils and diatom assemblages provide evidence with which to determine the nature of past depositional environments at Taupo Swamp (Table 4.1). Interpretation of this evidence indicates that three different depositional environments have existed at the Taupo Swamp core sites over the last 5500 years (Fig. 4.9). 


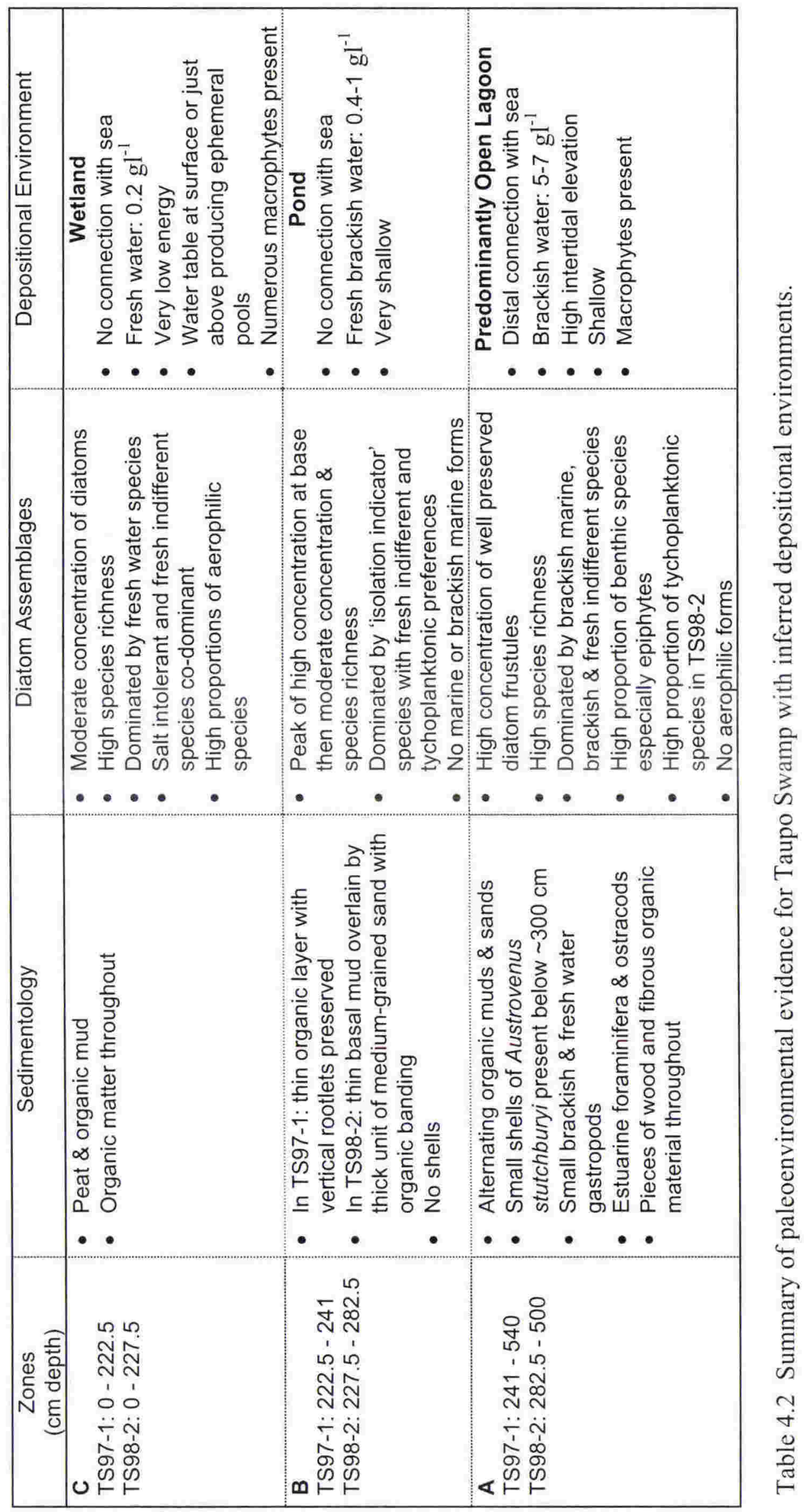




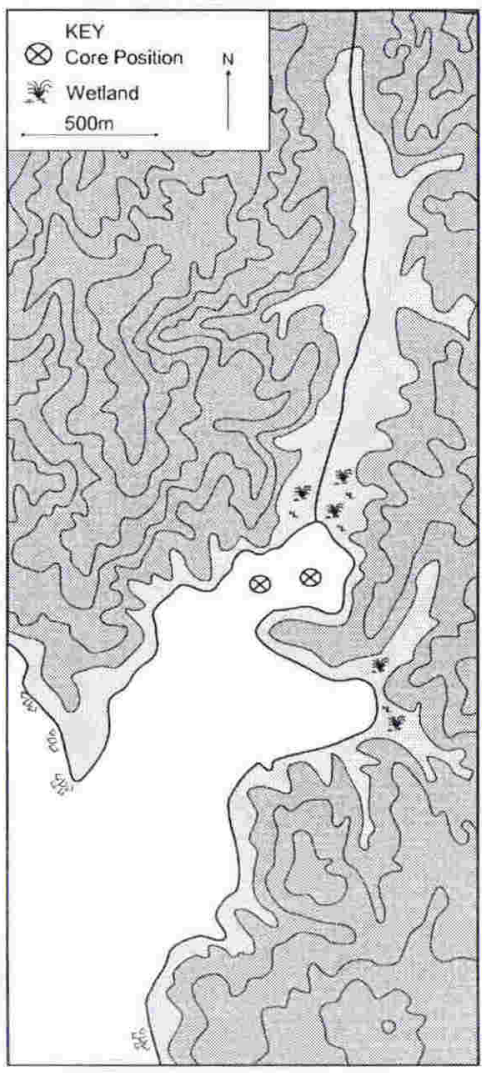

A

Predominantly Open Lagoon $\sim 5500-3100$ cal. years BP

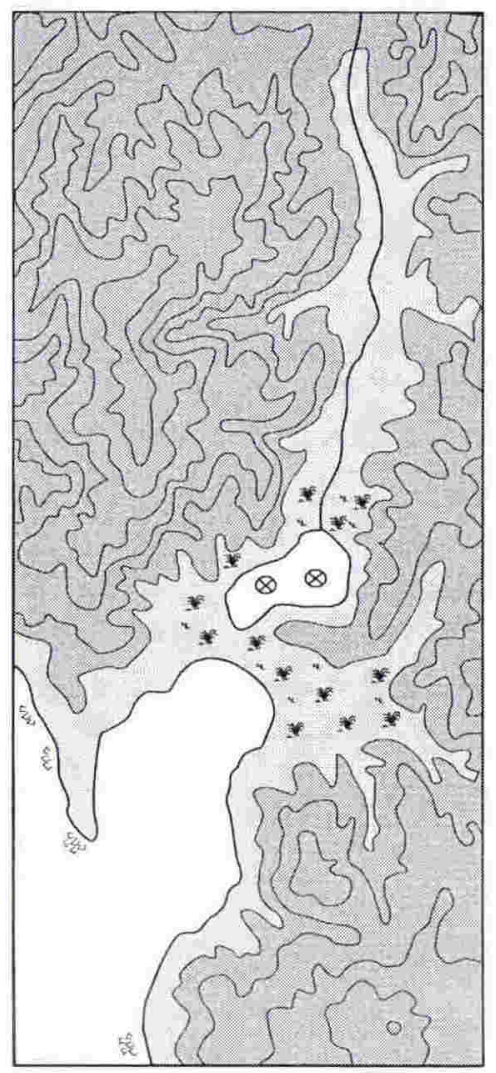

B

Pond

$\sim 3100-2400$ cal. years BP

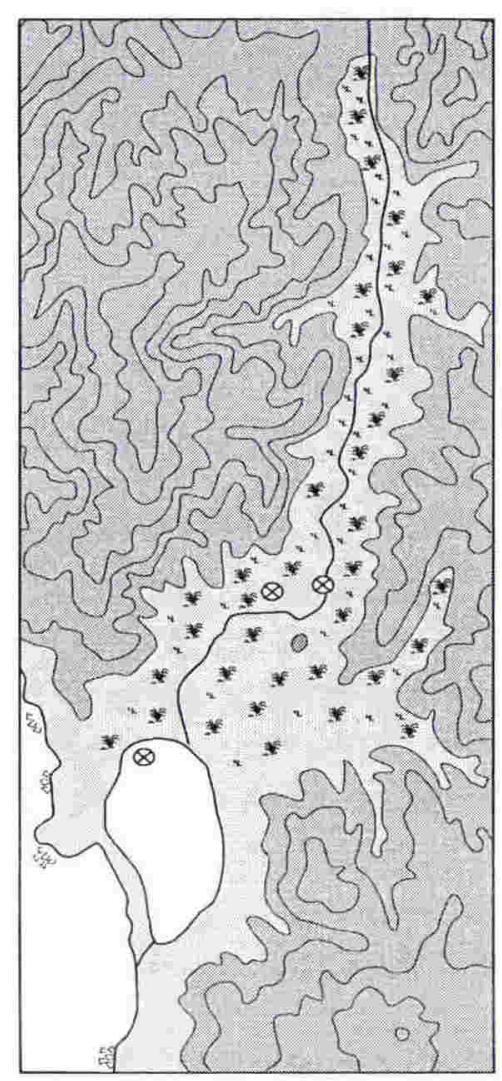

C 2400-0 cal. years BP

Fig. 4.9. Paleoenvironments that have existed in Taupo Valley over the last 5500 years as reconstructed from cores TS97-1 and TS98-2. At core position PF00-1(lower valley) only very recent sediment was sampled. 
The presence of small shells of Austrovenus stutchburyi and estuarine foraminifera and ostracods indicate the environment of zone A was brackish water with a fairly distal connection to the sea. The alternating grain size and organic content of sediments throughout this zone suggest a variable energy regime consistent with a tidally influenced site. Diatom assemblages also denote the existence of a brackish water environment with salinities averaging around $7 \mathrm{gl}^{-1}$ in the centre of the valley and $4 \mathrm{gl}^{-1}$ at the valley margin. An open connection with the sea is suggested by the presence of open marine diatoms. In effect the environment could have been a small sheltered inlet or an open lagoon at the head of such an inlet. The high number of epiphytic diatoms and the organic nature of the sediment are more in line with a sheltered, low energy lagoon than the tidal flat of an inlet. The high proportion of organic material in the sediment also suggests deposition took place at a high intertidal elevation as organic material generally increases from low to high intertidal zones (Nelson et al., 1996b). The site is unlikely to have been a salt marsh because although high proportions of tychoplanktonic species indicate the area was shallow, the complete lack of aerophilic species implies it was permanently ponded. A predominantly open lagoon at the head of an inlet is inferred as the past depositional environment.

Zone B is dominated by small Fragilaria species that have been termed 'isolation indicators' as they are found in high numbers around marine isolation contacts in Norwegian basins and are thought to be associated with rapid changes in water chemistry (Stabell, 1985). In modern sampling in New Zealand high abundances are also associated with very shallow water i.e. $<5 \mathrm{~cm}$ depth (Cochran, unpublished data). These species are fairly indifferent to salinity but the complete lack of marine and brackish marine species in the assemblage indicates the waterbody was isolated from the sea. Brackish and fresh species existed in the central valley but salt intolerant wetland species inhabited the valley margin and only a few slightly brackish species continued to live there. Salinities estimated for the paleoenvironment range from $\mathrm{gl}^{-1}$ in the central valley to $0.4 \mathrm{gl}^{-1}$ at the margin.

Zone B is represented in TS97-1 by a thin unit of organic mud with small vertical rootlets preserved. This suggests the site was shallow enough for plants to become established at this time. However in TS98-2 the sedimentary sequence consists of a thin unit of mud at the base and a medium grained, well sorted sand with organic banding above this. The coarseness of the sand is unusual for deposition in such a shallow, low energy waterbody. Therefore the core site is thought to have been located near the fresh water inflow and the sand deposited relatively rapidly by the stream. Sediment accumulation rates support the possibility of a period of rapid deposition in the central part of TS98-2. Zone B is considered to represent an extremely shallow, isolated, fresh brackish waterbody that was a short-lived, transitional environment between those of zones A and C. In comparison with modern samples (Fig. 4.7) zone B is defined as a lake 
(TS97-1) or a permanently closed lagoon (TS98-2) but because of its very shallow and short-lived nature, 'pond' is adopted as a more appropriate term.

The sedimentary sequences of zone $\mathrm{C}$ consist of peat and organic mud that point toward the existence of a wetland environment as is present at the site today. Fresh and salt intolerant diatoms dominate the assemblages of zone $\mathrm{C}$ indicating that a pure fresh water environment existed in the valley throughout this interval. Diatom-inferred salinity averages about $0.2 \mathrm{gl}^{-1}$. High proportions of aerophilic species are consistent with a wetland environment with the water table being at or near the surface. However greater numbers of the planktonic species Aulacoseira crenulata suggest the wetland was wetter than the site today with the presence of ephemeral pools of water. Numerous macrophytes grew in the valley resulting in high proportions of epiphytic diatoms. Slightly acidic waters are inferred for the interval because of the presence of acidophilous species. An increase in acidity is considered to be the final stage in the progressive decrease in salinity and height of the water table associated with a relative sea level fall (Long and Shennan, 1994).

\subsection{ENVIRONMENTAL TRANSITIONS}

\subsubsection{Introduction}

Paleoenvironmental reconstruction of sedimentary sequences from Taupo Swamp indicates that isolation of TaupoValley from the sea occurred in two discrete steps between $\sim 3000$ and 2000 cal. years BP. The two main transitions in depositional environment (Fig. 4.10) involve a lowering of relative sea level. These are investigated for information about the cause of change in environment. The criteria of Nelson et al. (1996b) are used to help distinguish between coseismic and aseismic causes of change (Chapter Two).

Adkin (1921) cites evidence for transport of gravel along the eastern coast of outer Porirua Harbour and also for the existence of an inner and outer gravel beach ridge. Eiby (1990) ascribes the existence of a remnant rock platform in outer Porirua Harbour to one or more coseismic uplift events on the Ohariu Fault. These studies indicate that both sediment transport and coseismic movement of base level are likely to have played important parts in the history of isolation of Taupo Valley. Sea level change is unlikely to have been a major factor because the main sea level fall around New Zealand in the last 6500 years occurred at a time when no change is inferred from the sequences - about 4500 years BP (Gibb, 1986). 


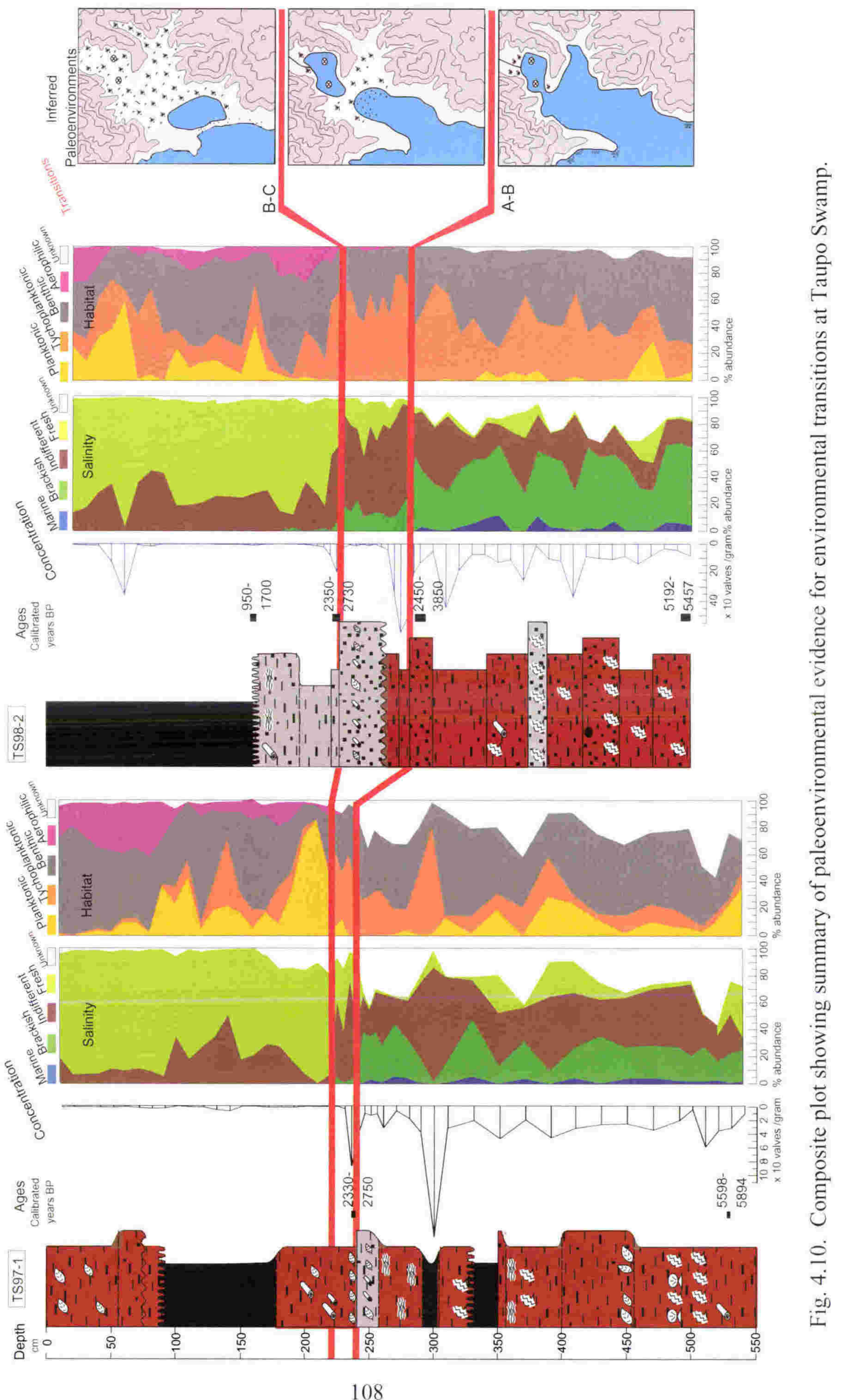




\subsubsection{Description of Transitions}

The change at transition A-B (241 cm depth in TS97-1 and $282.5 \mathrm{~cm}$ depth in TS98-2) is inferred to have happened suddenly because diatom assemblages were traced over the transition horizon using wet mounts between the permanently mounted samples and they change completely over a three centimetre interval. Considering the likely occurrence of some bioturbation this is a very rapid transition. There is a sharp contact in both sedimentary sequences to finer-grained, more organic sediment. In TS97-1 a two centimetre thick organic rootlet layer occurs immediately above the transition indicating that soil-forming conditions initiated at the site. A large scale environmental change is inferred to have occurred across the transition because the paleoenvironment changed from an open brackish lagoon at the head of an inlet to an isolated, fresh brackish, very shallow pond. Only six diatom species overlap into zone B out of an assemblage of at least 80 species in zone A. Diatom inferred salinity decreases by an order of magnitude from brackish to fresh at the valley margin site and from brackish to fresh brackish in the valley centre.

A barrier closing across the valley mouth could cause a large change in salinity quite suddenly but would not account for the shallowing and draining of the site that occurs in this case. The contacts are unlikely to be erosional from channel cutting or a flood event because both cores show the same transition occurring synchronously in different parts of the valley. If the change is coseismic in origin then inundation of the valley by tsunami at this time is a possibility. There are relatively coarse grained and inorganic units immediately below the environmental transitions in each core that may represent a higher energy influx to the sites. However there is no evidence to suggest an open marine source for the units and in TS97-1 the lower contact appears gradational. The core sites are over a kilometre from the open coast so any indication of such an event is likely to be minor.

Changes to diatom assemblages at the B-C transition (222.5 $\mathrm{cm}$ depth in TS97-1 and $227.5 \mathrm{~cm}$ in TS98-2) include the complete disappearance of brackish species and substantial increases in the proportions of salt intolerant and aerophilic diatoms. These changes are not the result of gradual decline or increase throughout zone $\mathrm{B}$ but occur suddenly at the B-C horizon suggesting that the environmental change also occurred suddenly. The change is not distinguishable in the sedimentary sequence of TS97-1 but involves a sharp contact to finer grained sediment in TS98-2. This probably relates to movement of the stream channel away from the core site. Transition B-C consists of a change in paleoenvironment from a fresh brackish shallow pond to a completely fresh water wetland environment. The final stage of isolation at the core sites is denoted by decline of reconstructed salinity values and inferred water depth across the zone boundary. The transition may represent a longer-term adjustment to changes that occurred at the A-B transition, for example final drainage of the site due to water 
table adjustment. However if the waterbody of zone B existed for more than a few decades then change is more likely to be the result of an additional event.

An extensive impediment to drainage in Taupo Valley must also be invoked to explain the persistence of the wetland environment through to the present day. Although closure of a barrier across the valley mouth could explain development of a wetland, the present day extent of the wetland for two kilometres up the valley suggests that tectonic tilting may have contributed to the process.

\subsubsection{Change in Elevation}

A relative change in elevation is inferred at the A-B transition because the paleoenvironment changes from a high intertidal elevation to a supratidal elevation. At the B-C transition, change in elevation is inferred from a change in water table level - from below the surface in the open water pond environment of zone $\mathrm{B}$ to at the surface in the wetland environment of zone $\mathrm{C}$. However there are no ideal sea level index points preserved in the sedimentary sequences of Taupo Swamp because environments of zones A and B are unlikely to have been naturally adjacent if sudden barrier closure and / or coseismic uplift is the cause of transition between them. There are also no modern equivalent index points in the valley from which to measure an elevation difference because the environment has changed so completely over the time period investigated.

If it is assumed that the environments of zone A and B were naturally adjacent, and that tidal range has remained the same over the last few thousand years, a minimum estimate of net vertical movement can be calculated. Zone A was a tidal environment with an elevation around the high intertidal level. The environment of zone B was supratidal so the A-B transition is assumed to have been in the vicinity of paleo mean high water springs. The current spring tidal range for Wellington is $1.02 \mathrm{~m}$ (Hydrographic Information 2001, Land Information New Zealand) so mean high water springs is $0.51 \mathrm{~m}$ amsl. When the cores are placed according to their position above present mean sea level (Fig. 4.11) estimates of net uplift of $190 \mathrm{~cm}$ for TS97-1 and $90 \mathrm{~cm}$ for TS98-2 are calculated.

The difference in estimates between cores indicates that paleo mean high water springs was probably further up the valley from the TS98-2 core site at this time. This makes sense considering TS98-2 is located in the valley centre at a lower elevation than the valley margin core and highlights why sudden environmental transitions cannot be used as reliable sea level index points. The difference in measured net uplift between the cores and remnant rock platform suggests there is a higher platform that has not been identified or preserved. It is possible that platforms have not been distinguished or heights measured accurately considering the only remains of the $\sim$ one metre platform are isolated smooth-topped stacks (Eiby, 1990). 


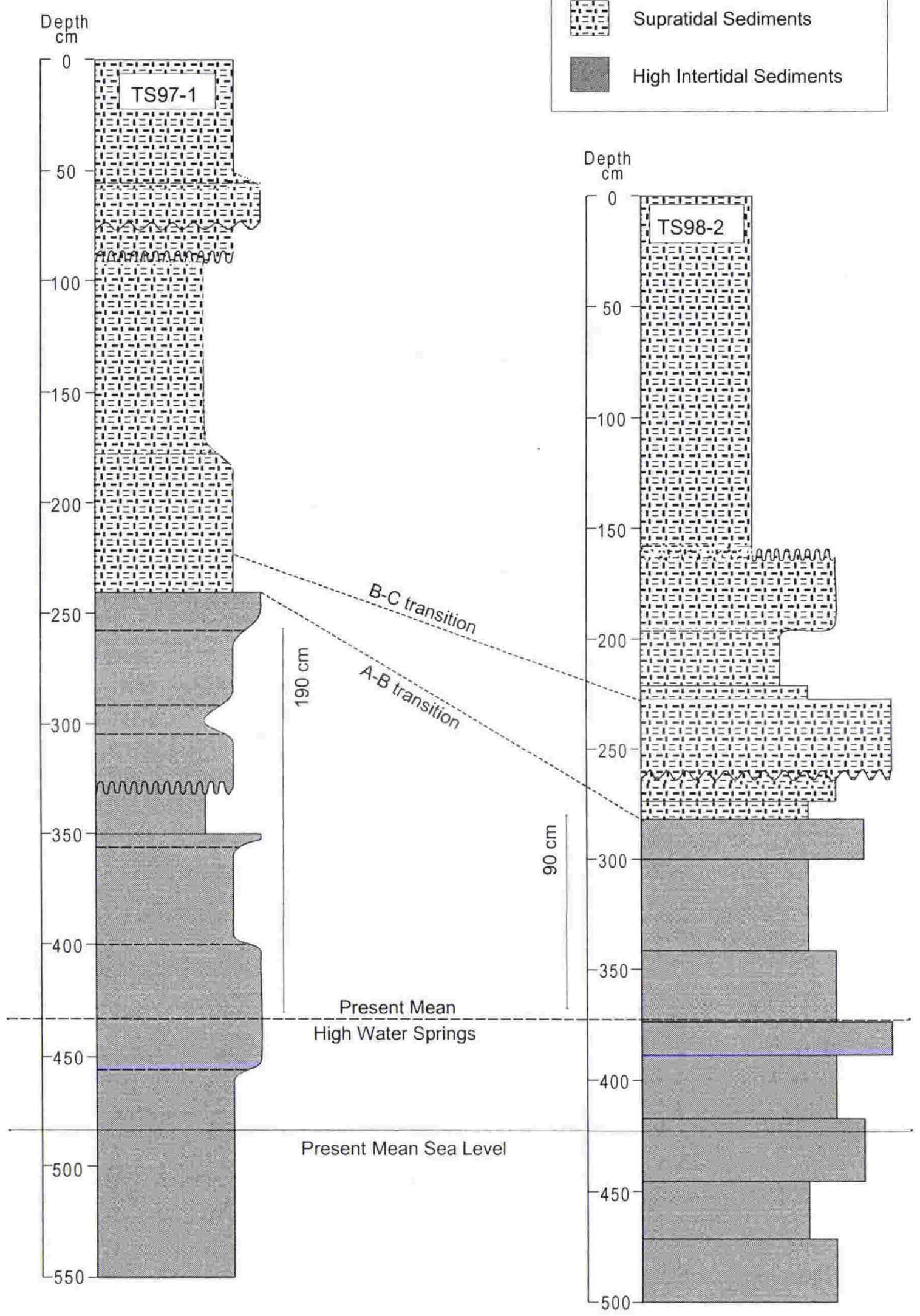

Fig. 4.11. Inferred tidal elevation of the sedimentary sequences at Taupo Swamp and their position relative to present mean sea level. If the A-B transition is taken to be in the vicinity of paleo mean high water springs, estimates of net vertical movement of $190 \mathrm{~cm}$ and $90 \mathrm{~cm}$ are obtained. However transition A-B is unlikely to be a good sea level index point (see text). 


\subsubsection{Age of Transitions}

The A-B and B-C transitions at Taupo Swamp are considered to be closely spaced in time because of the thickness of sediment deposited in zone B. In TS97-1 only $19 \mathrm{~cm}$ of sediment is present in zone B and at the average sediment accumulation rate for this core that represents less than 200 years. In TS98-2 there is $20 \mathrm{~cm}$ of fine-grained sediment and a unit of sand that, as discussed above, was probably deposited relatively rapidly by the stream so 200 years is a reasonable estimate for the time span of zone B in this core as well. Three bulk sediment radiocarbon dates will not provide the resolution required to clearly differentiate the age of two such closely spaced events. However there is clear evidence from diatom assemblages that two events did occur in this interval and the dates provide a bracket for timing of these events.

In TS97-1 there is a radiocarbon dated sample from the organic rootlet layer immediately above the A-B transition so it provides a minimum age for the transition of 2750-2330 cal. years BP. In TS98-2 there is a date below the A-B transition but it was an organic sand sample likely to have had older organic material incorporated into it so at 3850-2450 cal. years BP, it is treated as a maximum age. Therefore the age range of 3850 and 2330 cal. years BP brackets the A-B transition. The B-C transition in TS97-1 is estimated to have occurred between 2600 and 2100 cal. years BP using maximum and minimum sediment accumulation rates (and rounding to hundreds enclosing the greatest age range). A dated sample immediately above the B-C transition in TS98-2 provides a minimum age of 2720-2350 cal, years BP for the B-C transition. Therefore an age range of 2600-2350 cal. years BP can be inferred for the B-C transition. Estimated ranges for the A-B and B-C transitions overlap to some extent and support the idea that transitions were closely spaced in time. The dates are also consistent with events being synchronous in both cores.

\subsubsection{The Playing Field Core: Uplift in 1855 AD?}

There is definite evidence of coseismic uplift in outer Porirua Harbour in the form of a raised beach ridge and wave-cut platform. Diatom assemblages in sedimentary sequences from Taupo Valley indicate that this uplift is more likely to have occurred several thousand years ago than in the 1855 Wairarapa earthquake as proposed by Adkin (1921). Historical evidence for the occurrence of vertical movement in the Porirua area as a result of the 1855 earthquake is contradictory. Eiby (1990) analyses geological and historical evidence and concludes there was no uplift. Grapes and Downes (1997) uncover numerous primary and secondary historical reports of the earthquake and descriptions of effects in the Porirua area include uplift, no effect, subsidence and tsunami inundation. The report considered most reliable (that of Edward Roberts of the Royal Engineers) suggests uplift occurred as far north as Paekakariki (13 km north of the mouth of Taupo Valley) where uplift at the coast was very slight 
(Grapes and Downes, 1997). Reports of subsidence are thought to have referred to differential subsidence caused by compaction of soft sediments rather than movement of base level (Grapes and Downes, 1997). A short core (PF00-1) was collected at the southern end of the valley $2.2 \mathrm{~m}$ amsl in an attempt provide geological evidence for effects of the 1855 earthquake.

The core consists of $31 \mathrm{~cm}$ of sediment that is predominantly organic sand-poor mud. Between 7.5 and $9 \mathrm{~cm}$ depth a dark brown thin organic rootlet layer is preserved and the top one centimetre is dark brown organic soil material. Five samples analysed from the upper $15 \mathrm{~cm}$ have high concentrations of well preserved diatoms with concentration decreasing by an order of magnitude above the lower soil layer. Samples are dominated by fresh indifferent, tychoplanktonic Fragilaria species but there is a diverse assemblage of species from marine through to fresh water preferences. The domination by Fragilaria species was probably the result of extreme shallowness of the waterbody. It appears to have been a fresh brackish environment with inputs from both fresh water and marine sources.

Pollen analysis was carried out in an attempt to date the sediment using the arrival of exotic plant species. For example Pinus radiata was first recorded in Wellington in $1865 \mathrm{AD}$ and is considered to have its first appearance as pollen in sedimentary sequences about 1890 AD (Dunbar et al., 1997). Unfortunately pollen assemblages were not well preserved and no exotic plant species were encountered. This could be interpreted to mean the all the sediment is older than late 1800 s or that pollen grains have been transported out of the system and / or not preserved. Instead it is assumed that no sediment is missing from the sequence and in that case diatom assemblages indicate a waterbody existed at the site in the very recent past. This is supported by the presence of a lagoon at the lower end of Taupo Valley on the earliest Admiralty chart of Porirua Harbour (Stokes, 1858) which was surveyed in 1850 .

Diatom assemblages and sedimentology are consistent with occurrence of a small amount of uplift in the lower Taupo Valley in the 1855 Wairarapa earthquake because they indicate the site changed from a shallow waterbody to soil-forming conditions at some time in the very recent past. Disappearance of the waterbody in the last 150 years could also be the result of infilling by sediment accumulation or anthropogenic drainage. Samples from deeper in the sequence and further cores would be required to distinguish between these causes by documenting whether the change took place gradually or suddenly, whether there is evidence of tsunami inundation and whether the change is valley-wide. However support for a coseismic cause of disappearance of the waterbody comes from Maori history of the area. It is recorded that Taupo $\mathrm{Pa}$, situated at Plimmerton between the lagoon and Porirua Harbour, no longer had the defence of water on both sides after 1855 
because uplift raised the lagoon bed above water level (Pomare in: Anon., 1989). The amount of vertical movement could have been very minor considering the waterbody was very shallow (in the order of a few centimetres or decimetres) which explains the contradictory historical reports and lack of more definitive geological evidence in the form of uplifted coastal features. Evidence of this uplift would not be expected in diatom assemblages of the central valley cores because the amount of vertical movement would only result in small hydrological changes to the wetland.

\subsection{CONCLUSIONS}

Sedimentology and diatom analysis of two $\sim 500 \mathrm{~cm}$ sedimentary sequences from Taupo Valley enable past environment to be reconstructed for the late Holocene. Six radiocarbon dated samples provide a chronology for the sequences. Diatom assemblages are interpreted using traditional salinity and habitat preferences and through comparison and calibration with a modern coastal diatom calibration set. There is good agreement between these diatom-based reconstructions and with grain size and macrofossil data resulting in reconstructions of waterbody type, salinity and energy regime.

Paleoenvironmental reconstructions indicate the site has changed from a tidally influenced brackish lagoon to a shallow fresh brackish pond and finally to a fresh water, slightly acidic wetland over the last 5500 years. Both environmental transitions occurred suddenly around 3100 and 2400 cal. years BP and involved large magnitude changes in environment. The first transition involved shallowing and isolation of the waterbody from the sea. The second transition involved freshening and shallowing of the waterbody to the point where the water table was at the sediment surface. Transitions are consistent with coseismic uplift and a minimum amount of vertical movement of between 90 and $190 \mathrm{~cm}$ uplift is estimated from the sequences.

A short core from the lower Taupo Valley indicates that a fresh brackish lagoon existed immediately behind Plimmerton in the very recent past. Transformation of the area from a waterbody to a soil-forming environment could well have occurred as the result of a few centimetres to decimetres of uplift in the 1855 


\section{CHA PTER F IVE}

\section{Okupe Lagoon: A History of Punctuated Isolation from the Sea}

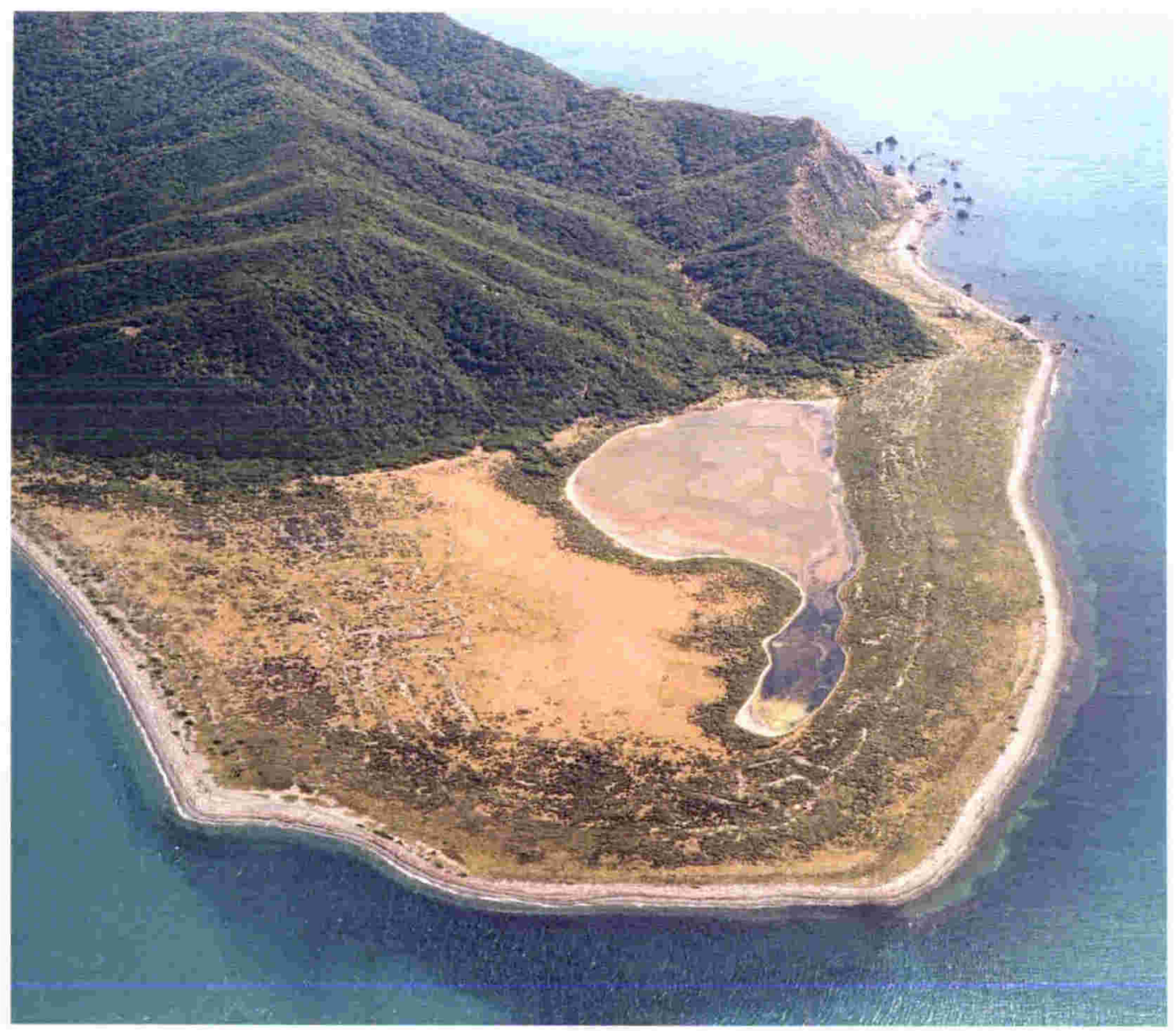

Fig. 5.1. Oblique aerial view of Okupe Lagoon looking northwest. Photo by Lloyd Homer. 
A record of isolation from the sea of a site close to sea level is presented in this chapter. Sediment transport and accumulation have played a large part in the history of Okupe Lagoon but coseismic movements of base level also appear to have been important causal factors in the isolation process. Sedimentology, macrofossils and diatom assemblages of cores taken from the lagoon indicate that five different depositional environments have existed at the site over the last 5000 years. Between about 5000 and 3200 cal. years BP (calibrated years before present) a sheltered inlet existed on the coast - probably protected by an earlier form of the boulder bank that lies to the north of the waterbody today. A shallower, more sheltered environment, such as a predominantly open lagoon, is inferred to have existed from 3200 until 2300 cal. years BP. At 2300 cal. years BP the environment changed to a predominantly isolated marsh environment with little or no open water at the site. The present configuration of an open water lagoon completely isolated from the sea initiated about 1000 cal. years BP. The lagoon became more favourable for diatom habitation around $500 \mathrm{cal}$. years BP possibly as a result of increased nutrient input into the waterbody. Transitions between depositional environments appear to have occurred suddenly, consisted of large magnitude changes in diatom assemblages, water depth changes, catchment disturbance and possibly influx from the sea.

\subsection{INTRODUCTION}

Okupe Lagoon (Fig. 5.1) is located on Kapiti Island which is a bird sanctuary and nature reserve that lies $5.5 \mathrm{~km}$ off the west coast of the southern North Island of New Zealand (Fig. 5.2). The indurated sedimentary basement rock of Kapiti Island is considered to be part of Wellington region Torlesse Terrane rather than Pelorus Group of the Marlborough Sounds region to the south (Moore and Francis, 1988). The seaward extension of the Wairau Fault runs west of Kapiti Island (Carter et al., 1988). A zone of phyllonite along the eastern side of the Island suggests it is also bounded by a fault in the east (Fleming and Hutton, 1949 ) but evidence for Quaternary movement on this fault is lacking (Moore and Francis, 1988). Minor deposits of Holocene sediments exist around the coast of the Island. Okupe Lagoon exists on a lobe of such sediments at the northeastern end of Kapiti Island (Fig. 5.2). The water surface of the lagoon is only two metres above mean sea level (amsl) and water depth is about $50 \mathrm{~cm}$. The lagoon is completely isolated from the sea by boulder and gravel storm beach ridges. However it probably has groundwater connection with the sea because water in the lagoon has salinity values of over 20 grams per litre $\left(\mathrm{g} \mathrm{l}^{-1}\right)$. The catchment consists of one small valley, the streambed of which is dry for most of the year. Fresh water input is assumed to be rainfall and some groundwater from the catchment. 


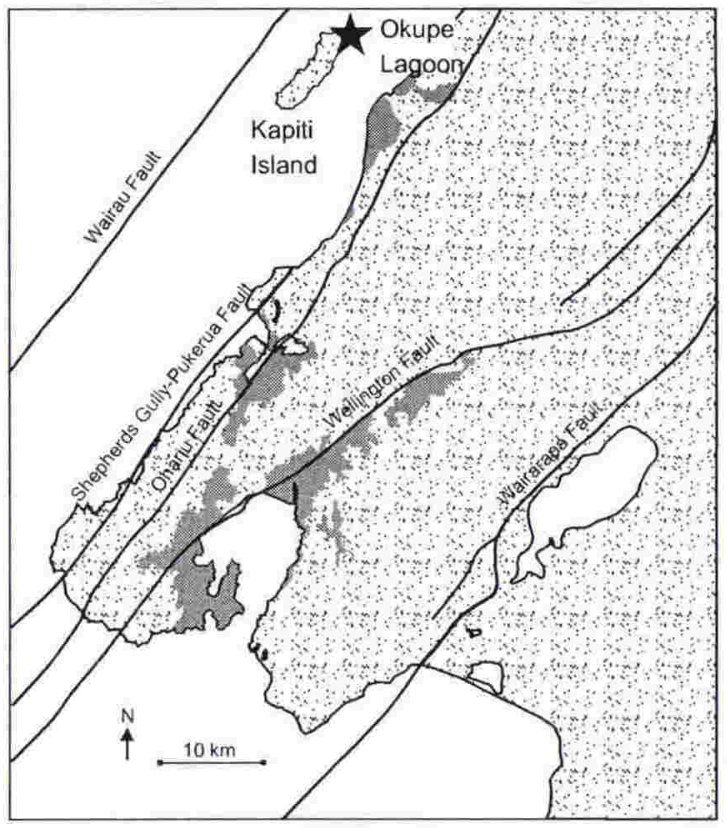

A

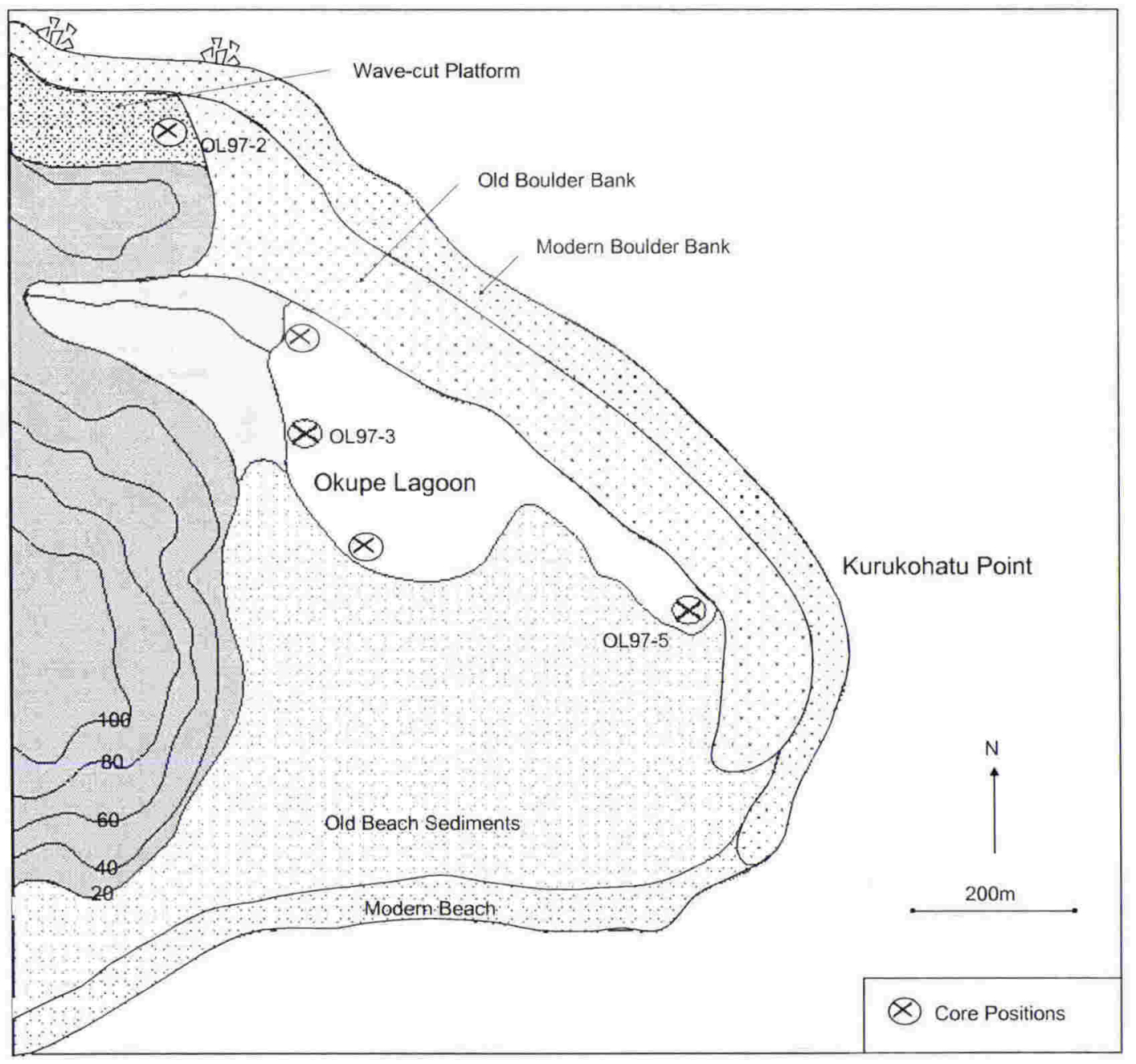

Fig. 5.2. Map of Okupe Lagoon. A: Location in the Wellington region. B: Site map showing core positions. Only cores discussed in this chapter are labelled. 
Several geological surveys of Kapiti Island note the presence of raised beaches, boulder-banks and benches indicating recent uplift of the island (Ferrar, 1928; Fleming and Hutton, 1949; Moore and Francis, 1988). Moore and Francis (1988) describe remnants of old beaches, wave-cut platforms, sea caves and stranded stacks ranging from 1.5 to 4 metres above high water mark along the east coast of Kapiti Island. At Kurukohatu Point they note the existence of two distinct beach ridges, the lower one is attributed to the modern storm beach and the higher one is an older beach thought to have been uplifted by about two metres. Features were not directly measured or dated by Moore and Francis (1988) but were considered to be a result of 1.5 - $2 \mathrm{~m}$ of uplift in the 'c. $1460 \mathrm{AD}$ earthquake' or the combined effects of a number of late Holocene earthquakes.

The sedimentology and diatom analysis presented here is part of a wider study designed to describe the storm ridges at Kurukohatu Point and determine the depositional history of Okupe Lagoon. The wider study included surveying profiles of the storm ridges and analysing fossil foraminifera and pollen in cores taken from the lagoon. The results of these parts of the work are reported elsewhere (Goff et al., 2000) but some details are used in this chapter to support the diatom evidence. In particular Goff et al., (2000) provide revised estimates of height differences between the two beach ridges and foraminiferal data provide useful information regarding tidal elevation of sediments.

\subsection{SEDIMENTARY SEQUENCES}

\subsubsection{Description of Sequences}

Four cores were taken from Okupe Lagoon and one from a rock pool on the raised wave-cut platform to the northwest of the lagoon (Fig 5.2). Cores OL97-3 and OL97-5 were chosen for detailed analysis because they appeared to contain the most complete sedimentary records. Core OL97-3 was taken from the western edge of the lagoon and is $180 \mathrm{~cm}$ long. Core OL97-5 was collected from the far eastern edge and is $195 \mathrm{~cm}$ long. Both sequences are about 5000 years old at the base (Fig 5.3). Core OL97-2 was also analysed to provide insight into the history of the raised wave-cut platform. Core logs and descriptions presented below are summaries of full core logs presented in Appendix I.

The lower half of core OL97-5 consists of grey sand with whole shells and fragments of Austrovenus stutchburyi and occasional rounded greywacke pebbles. At $134 \mathrm{~cm}$ depth there is the last occurrence of shell fragments and the size of shells at this level is significantly smaller than those at the base of the core. At $113.5 \mathrm{~cm}$ there are several rounded greywacke pebbles and pieces of wood and above this point the sediment has a much higher organic content. The interval from this point up to $36 \mathrm{~cm}$ is a dark brown organic sand with occasional mottles. 


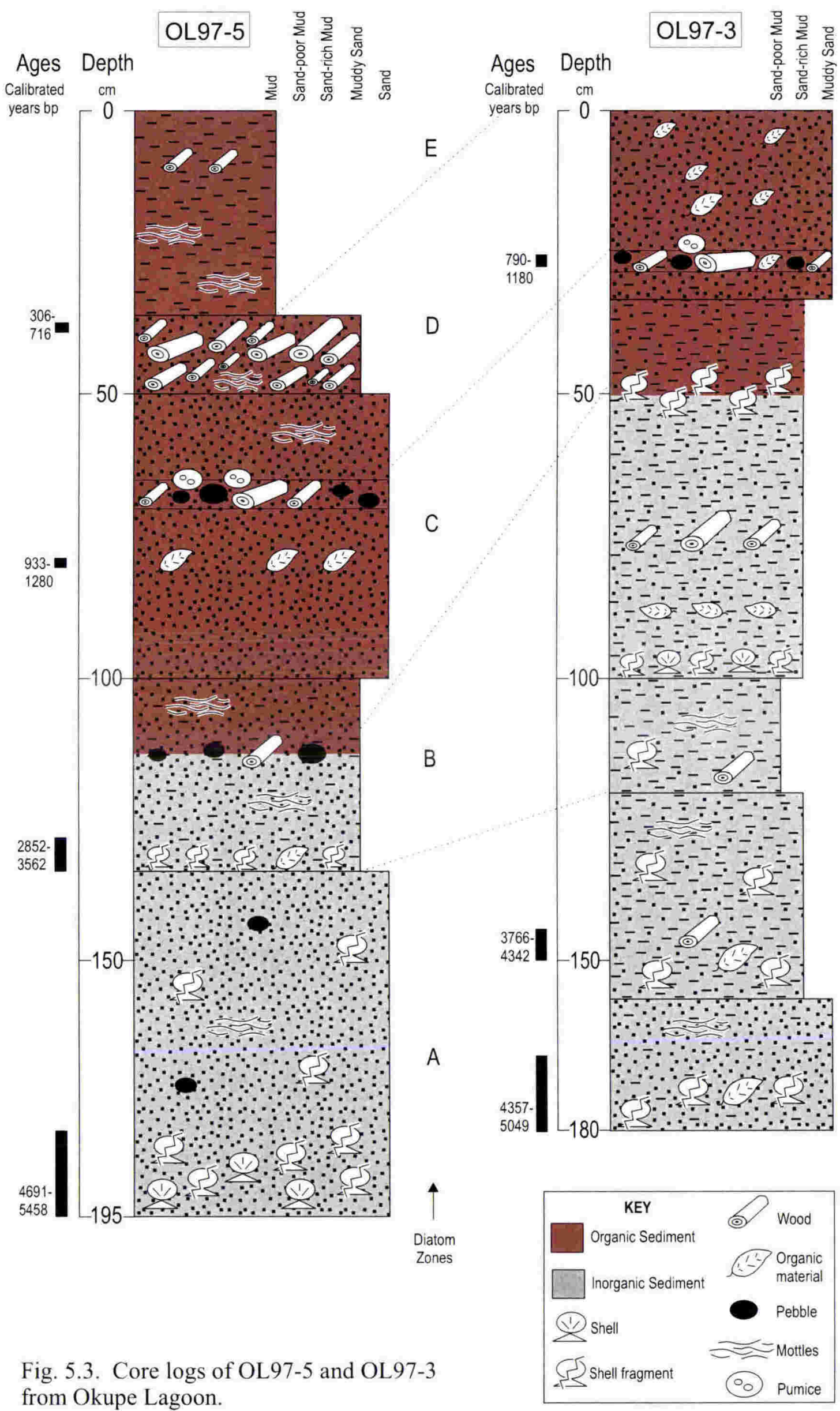


There are concentrations of organic material, wood and rounded greywacke pebbles at several points. A deposit of large wood fragments in a matrix of muddy sand occurs between 50 and $36 \mathrm{~cm}$. From $36 \mathrm{~cm}$ to the surface the sequence consists of organic mud. It is brown and heavily mottled between 36 and $20 \mathrm{~cm}$ and grey with occasional small pieces of wood above this.

Core OL97-3 is generally finer grained than the sediment of OL97-5. Grey muddy sand and sand-rich mud make up most of the sequence. Shell fragments occur scattered and in layers from the base of the core up to $50 \mathrm{~cm}$ depth and layers of organic material and wood occur throughout the core. Above $50 \mathrm{~cm}$ the organic content of the sediment is higher giving it an olive-black colour. The most distinctive sedimentary feature that can be correlated between cores is a $3-4 \mathrm{~cm}$ thick layer of large pebbles and pieces of wood that occurs between $65-69 \mathrm{~cm}$ depth in OL97-5 and between 24-27 cm in OL97-3. In both cases pumice is found immediately above the pebble and wood layer. The layer is dated at $1180-790$ years BP in OL97-3 which is consistent with interpolation between dates either side of the pebble layer in OL97-5. Other correlations are made on the basis of diatom assemblages.

Broad estimates of sediment accumulation rates are calculated for cores OL97-5 and OL97-3 using available radiocarbon ages and are shown in Fig. 5.4. Rates are low in the lower halves of both cores ranging between 0.2 and $0.5 \mathrm{~mm} / \mathrm{yr}$. In OL97-5 the rate increases in the upper half of the sequence to between 0.5 and 2 $\mathrm{mm} / \mathrm{yr}$ but in OL97-3 rates remain low throughout the sequence.

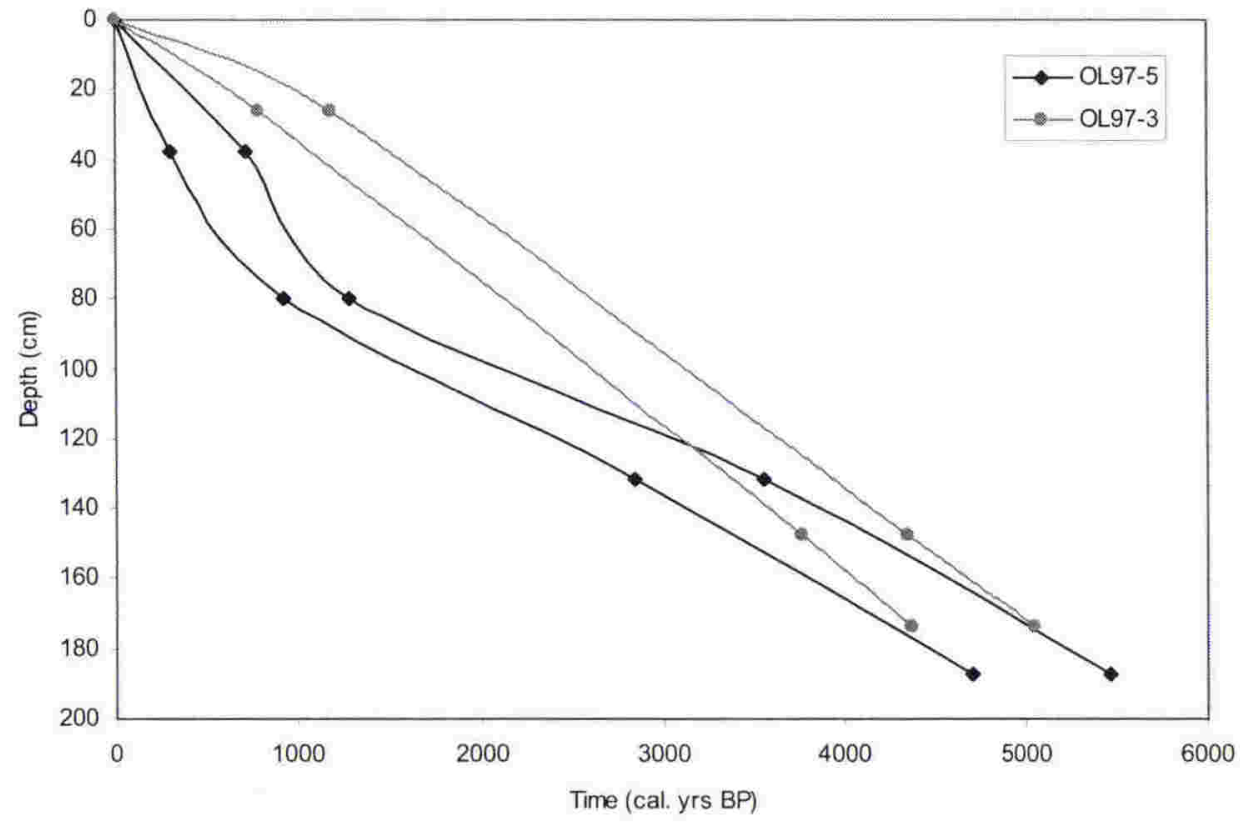

Fig. 5.4. Sediment accumulation rate curves for cores OL97-5 and OL97-3 based on calibrated radiocarbon age estimates. Maximum and minimum curves for each core are based on end-member values of the 2-sigma age range. 
Core OL97-2 is a $170 \mathrm{~cm}$ long sequence taken from a rockpool on the raised wave-cut platform to the northwest of Okupe Lagoon. It consists entirely of brownish black peat. The initiation of peat deposition within the rockpool must have occurred after isolation from the sea. Therefore the age of 2045-1738 years BP from the base of this core provides a minimum age for the time of uplift of the platform. This single date indicates a sediment accumulation rate for OL97-2 of about $0.9 \mathrm{~mm} / \mathrm{yr}$.

\subsubsection{Interpretation of Sequences}

The sandy inorganic nature of the sediment in the lower halves of cores OL97-5 and OL97-3 is consistent with deposition in a high energy, wave-dominated environment. Austrovenus stutchburyi lives in the low-tide zone of estuaries and enclosed bays and shell size decreases with the low salinities of inland parts of such coastal waterbodies (Beu and Maxwell, 1990). The size of $A$. stutchburyi shells at the base of these sequences suggests they were living towards the outer part of a bay or estuary with fairly high salinities. The smaller shell size at $134 \mathrm{~cm}$ depth in OL97-5 indicates the environment freshened in this interval. Low sediment accumulation rates are consistent with the lack of accommodation space in shallow marine environments under stable sea level.

The change to organic sedimentation higher up the sequences, incorporation of wood fragments and lack of shell material are indicative of transformation from an open marine site to a terrestrial site isolated from the sea. Considering present day morphology, this is likely to have occurred through closure of a barrier around the core sites. Concentrated layers of wood fragments, rounded pebbles and pieces of pumice in both cores represent influxes of material from the catchment and barrier. Large wood fragments are likely to have been sourced from the catchment whereas the pebbles, because of their roundness, are likely to have come from the barrier. The large size of these inclusions indicates that unusual depositional events were responsible for their incorporation into the lagoonal sediments. Some macrofossil fragments and other inclusions were removed from OL97-5 (Fig. 5.5) and these illustrate the evidence for isolation from the sea and the coarse nature of influxes to the waterbody. The increase in accumulation rate in the upper part of OL97-5 may be a result of the large influx of wood near the top of the sequence rather than an increase in background sedimentation rate. There is no similar layer in OL97-3 hence the lower accumulation rate. 


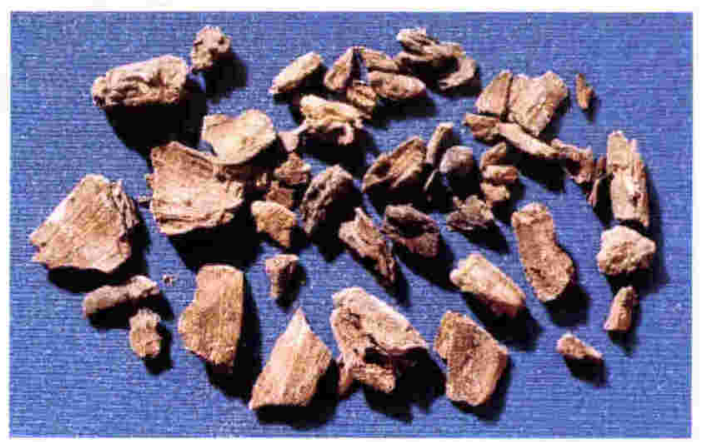

Depth and Environmental Transitions

(introduced later in chapter)

$36-50 \mathrm{~cm}$

(D-E transition)

$65-69 \mathrm{~cm}$

(C-D transition)

$113.5 \mathrm{~cm}$

(B-C transition)

$134 \mathrm{~cm}$

(A-B transition)
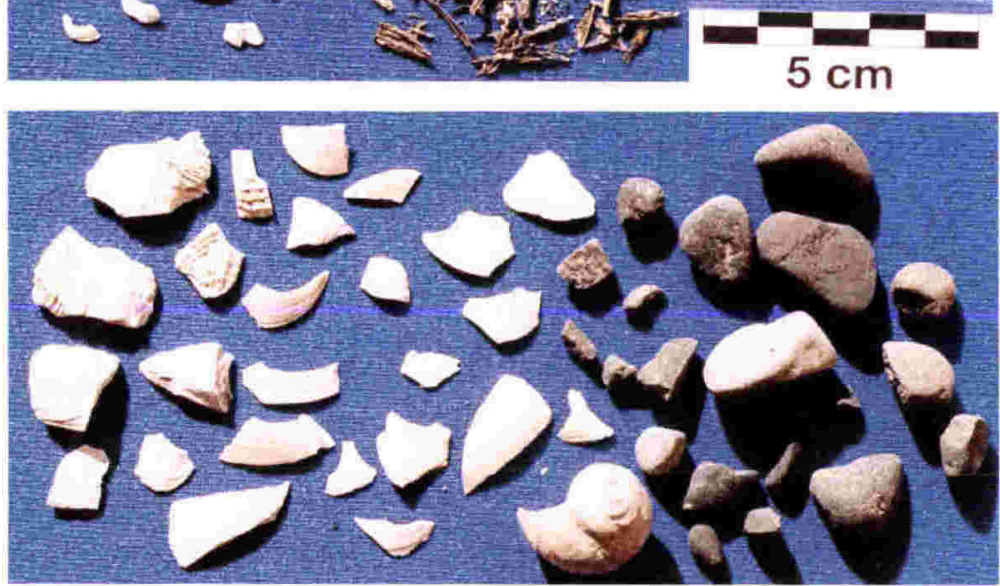

$134-186 \mathrm{~cm}$

Fig. 5.5. Some macrofossils and inclusions removed from core OL97-5 illustrating the change from a predominantly marine environment at the base of the core to a terrestrial one at the top. Most large pebbles and wood fragments occur in concentrated layers at transitional boundaries that are introduced later in the chapter. 


\subsection{DIATOM ANALYSIS}

\subsubsection{Introduction}

Diatom assemblages are described from core OL97-5 because of superior preservation of diatom valves in this core. Samples were analysed at five centimetre intervals and full counts, or at least over 200 valves, were achieved for most samples. The lateral extent of assemblages was investigated by making low counts from OL97-3 at ten centimetre intervals. Although diatoms are very poorly preserved in OL97-3, the same assemblages are recognised so it is assumed that they are of lagoon-wide extent. Diatom assemblages at Okupe Lagoon divide the sedimentary sequence into five zones (section 5.3.2). Paleoenvironmental interpretations of these zones (section 5.3.3) are made though classification of species according to their published salinity and habitat preferences (Appendix III). Quantitative reconstructions of past waterbody type and salinity are carried out using the calibration set (Chapter Three) and are presented in sections 5.3.4 and 5.3.5. Full species counts are presented in Appendix VI.

\subsubsection{Definition of Diatom Assemblage Zones}

A total of 72 diatom taxa were identified in the sequence. Zones were defined according to species composition, valve concentration, species richness and preservation. Key species of each zone (Table 5.1) are those that are abundant in the assemblage and / or unique to the zone. Zone A at the base of the sequence has low concentrations of diatoms and many frustules are broken indicating the assemblage is not well preserved. There is a moderate diversity of species but the zone is defined by five main species including a Fallacia species that does not fit any taxa descriptions in reference floras consulted for this work. Zone B has low concentrations of diatoms, many frustules are broken, and there is some evidence of dissolution indicating poor preservation. The assemblage is dominated almost entirely by Paralia sulcata. Zone $\mathrm{C}$ has low concentrations of diatoms with moderate species richness. It is dominated by small Fragilaria species and Paralia sulcata but is differentiated from other zones by the presence of Navicula contenta, Navicula mutica and Hantzschia amphioxys. The assemblage of zone D is characterised by moderate concentrations and species richness and the presence of six key species. Zone E is missing from the top of core OL97-3 so it is defined solely from OL97-5. Moderate-high concentrations of diatoms define zone E which is similar in assemblage composition to zone D but nine key species are present. 


\begin{tabular}{|c|c|c|c|c|}
\hline Zones (cm) & Key Species & Concentration & $\begin{array}{l}\text { Species } \\
\text { Richness }\end{array}$ & Preservation \\
\hline $\begin{array}{l}\text { E } \\
\text { OL97-5: 36-0 }\end{array}$ & \begin{tabular}{l} 
Fragilaria pinnata \\
Fragilaria elliptica \\
Amphora coffeaeformis \\
Cocconeis placentula \\
Stauroneis salina \\
Navicula incertata \\
Achnanthes brevipes \\
$\quad$ var. intermedia \\
Gyrosigma balticum \\
Fragilaria construens \\
\multicolumn{1}{c}{ f. subsalina }
\end{tabular} & Moderate-High & Moderate & Good \\
\hline $\begin{array}{l}\text { D } \\
\text { OL97-5: } 62.5-36 \\
\text { OL97-3: } 24-0\end{array}$ & $\begin{array}{l}\text { Fragilaria pinnata } \\
\text { Amphora coffeaeformis } \\
\text { Cocconeis placentula } \\
\text { Fragilaria elliptica } \\
\text { Stauroneis salina } \\
\text { Navicula incertata }\end{array}$ & Moderate & Moderate & Good \\
\hline $\begin{array}{l}\text { C } \\
\text { OL97-5: 107.5-62.5 } \\
\text { OL97-3: 50-24 }\end{array}$ & $\begin{array}{l}\text { Fragilaria pinnata } \\
\text { Fragilaria construens } \\
\quad \text { f. subsalina } \\
\text { Fragilaria elliptica } \\
\text { Paralia sulcata } \\
\text { Achnanthes hauckiana } \\
\text { Stauroneis salina } \\
\text { Amphora coffeaeformis } \\
\text { Cocconeis placentula } \\
\text { Navicula contenta } \\
\text { Navicula mutica } \\
\text { Hantzschia amphioxys }\end{array}$ & Low & Moderate & Good \\
\hline $\begin{array}{l}\text { B } \\
\text { OL97-5: } 134-107.5 \\
\text { OL97-3: } 120-50\end{array}$ & Paralia sulcata & Low & Very low & $\begin{array}{l}\text { Many valves } \\
\text { broken } \\
\text { Evidence of } \\
\text { dissolution }\end{array}$ \\
\hline $\begin{array}{l}\text { A } \\
\text { OL97-5: 195-134 } \\
\text { OL97-3: 180-120 }\end{array}$ & $\begin{array}{l}\text { Paralia sulcata } \\
\text { Fallacia sp. } 1 \\
\text { Dimeregramma minor } \\
\quad \text { var. nana } \\
\text { Hyalodiscus } \\
\text { lentiginosus } \\
\text { Thalassiosira eccentrica }\end{array}$ & Low & Moderate & $\begin{array}{l}\text { Many valves } \\
\text { broken }\end{array}$ \\
\hline
\end{tabular}

Table 5.1. Definition of diatom assemblage zones for cores OL97-5 and OL97-3.

\subsubsection{Interpretation of Diatom Assemblage Zones}

Diatom species were grouped according to their salinity and habitat preferences to enable inferences to be made about the depositional environment of each zone (Fig. 5.6). Most species in zone A are brackish marine tychoplanktonic forms indicating a shallow water marginal marine environment. The highest proportion of open marine species occurs in this zone suggesting that transport to the waterbody from the open sea was less restricted here than in all subsequent environments. The fresh and brackish water component of the assemblage was 
A

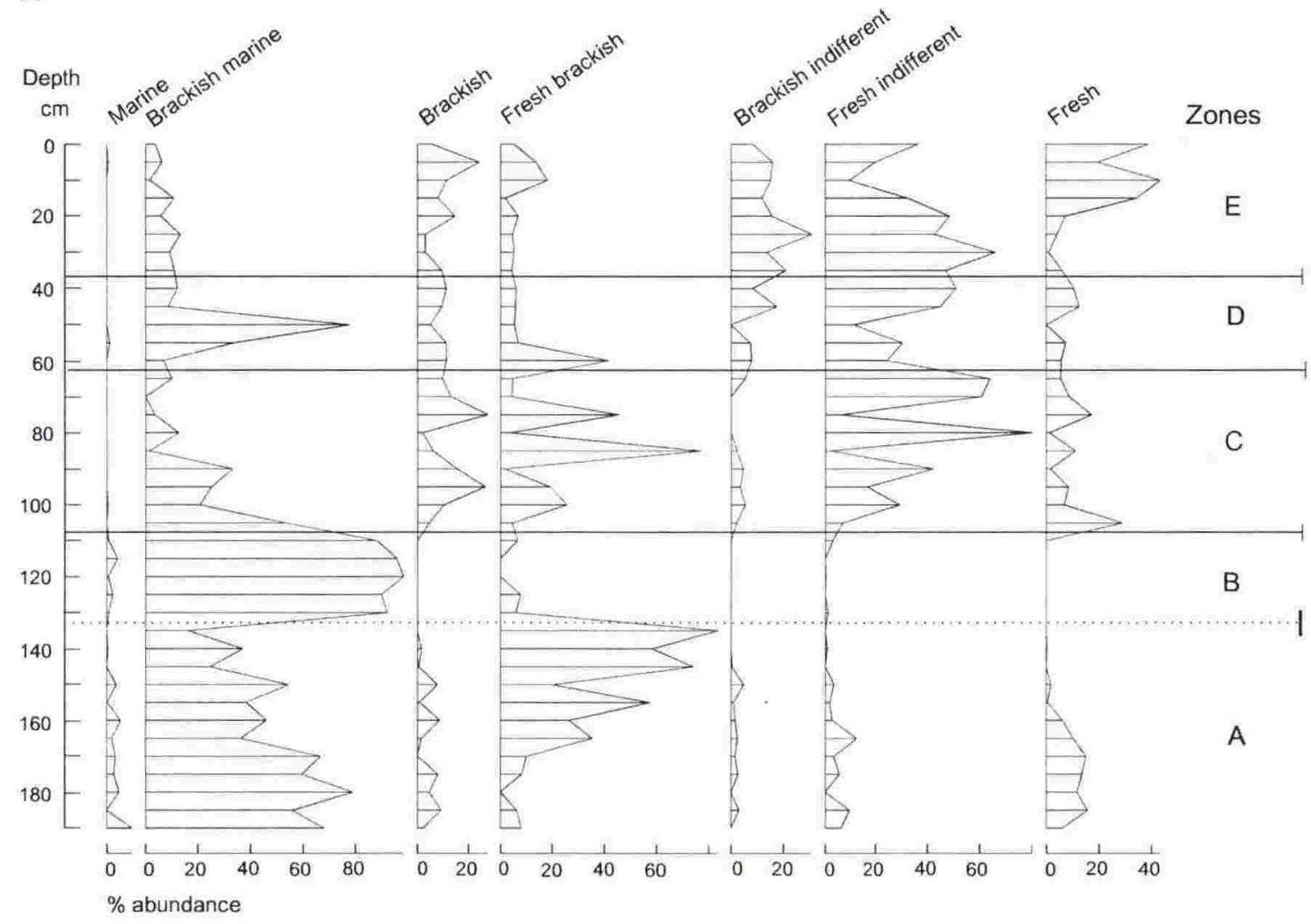

B

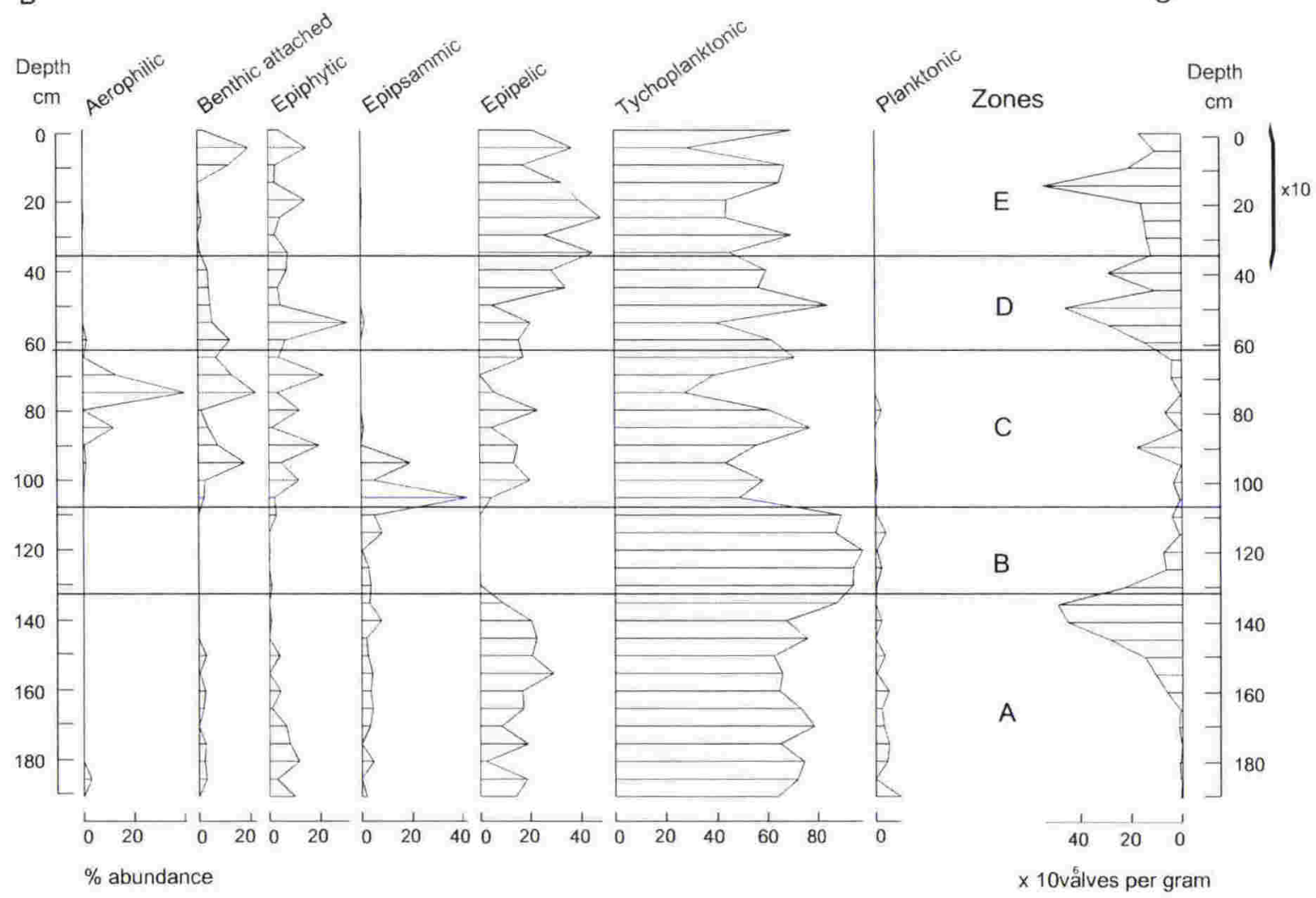

Fig. 5.6. Diatom assemblages of core OL97-5. A: Diatoms grouped according to salinity preferences. B: Habitat preferences. C: Concentration of diatom valves in the sediment. 
probably transported from adjacent fresh and brackish water environments. Low concentrations of diatom valves in the sediment probably partially reflect naturally low numbers of diatoms living in the original environment. Much lower levels of primary productivity have been recorded for diatoms living on active beach sands than for assemblages on silt and clay in more sheltered settings (McIntire and Moore, 1977). However poor preservation is likely to have affected concentration values because many valves are broken. Preservation problems are well documented for such active environments (Denys and de Wolf, 1999). Above $160 \mathrm{~cm}$ diatom concentration increases and there is a gradual decline in brackish marine and marine species, and an increase in fresh brackish species. This suggests the salinity of the environment decreased and the site became slightly more sheltered from the sea.

The assemblage of zone B is dominated by Paralia sulcata, a brackish marine tychoplanktonic species. There are two main reasons why this may be so. Paralia sulcata is known to be more competitive than other species under conditions of widely varying salinity, low light, and with substrates of fine-grained, organicrich sediment (Zong, 1997). Paralia sulcata is also often the only diatom remaining in high intertidal sediments, its heavy silicification allowing it to survive transport and dissolution that smaller, finer diatoms do not (Denys and de Wolf, 1999). Therefore the assemblage is either autochthonous and represents a fairly extreme environment unfavourable to most species, or the assemblage has been damaged by transport and / or dissolution. The high number of broken valves and evidence of dissolution favour the latter explanation. Although detailed interpretation is inhibited, the past environment of zone B is likely to have been a shallow marginal marine setting open to the sea.

In Zone $\mathrm{C}$ there are fairly equal proportions of brackish marine, brackish, fresh indifferent and fresh diatoms suggesting it was a brackish water environment. Brackish marine diatoms consist of low numbers of Paralia sulcata throughout the zone and peaks of single species near the base of the zone. For example there are peaks in the epipsammic diatom Opephora olsenii at $105 \mathrm{~cm}$ and a peak in Gomphonema valentinica at $90 \mathrm{~cm}$. These may be the result of sporadic marine influxes into the waterbody. Species are predominantly tychoplanktonic and epipelic in their habitat preferences and there are no planktonics so it was a shallow environment. Aerophilic species consist of up to $40 \%$ of the assemblage in the upper part of the zone suggesting that the waterbody may have been very shallow to non-existent at times. The site being too dry or ephemeral to support a productive diatom community at this time would explain the low concentrations of diatoms observed in the zone. A marsh environment above high tide could produce the mixture of salinity preferences seen here along with aerophilic species and low concentrations resulting from only occasional inundation by water. 
The occurrence of species with a range of preferences from brackish marine to fresh, including fairly high proportions of indifferent species, suggests that zone D was a brackish environment. Higher concentrations of diatoms in comparison with zone $\mathrm{C}$ and the lack of aerophilic species, indicate a permanently ponded, more favourable waterbody such as a lagoon. The zone has a peak in the brackish marine diatom Gomphonema valentinica at $55 \mathrm{~cm}$ depth and a large peak in Paralia sulcata at $50 \mathrm{~cm}$. This peak consists of one sample in which predominantly whole valves of Paralia sulcata make up $80 \%$ of the assemblage. This either represents a response to a short-lived change in conditions at the site, i.e. a 'bloom' that has been preserved in the record, or an influx to the site from a seaward source. In either case a short-term increase in marine conditions is implied.

Assemblage composition of zone $\mathrm{E}$ is similar to that of zone $\mathrm{D}$ and to that of present day assemblages confirming the existence of a brackish, permanently closed lagoon. The reasonable proportions of brackish marine species indicate the waterbody was quite saline but salinity appears to decline near the top of this zone with an increase in fresh water species. The lack of planktonic species indicates the environment was shallow but with permanent water to support the benthic species. The concentration of diatoms is greater in this zone than that of zone D implying that an environment more favourable to diatom habitation was established by this time.

Nine samples were analysed from core OL97-2 to determine what environment the sequence was deposited in. Samples contain very high concentrations of well preserved diatoms. The sequence is dominated by the fresh tychoplanktonic diatom Fragilaria elliptica. Most other species are fresh water forms with only minor occurrences of brackish and brackish indifferent species. This confirms the peat was deposited in a fresh water pool.

\subsubsection{Reconstruction of Waterbody Type}

As introduced in Chapter Four (section 4.3.4), diatom assemblages were incorporated passively into a DCA of calibration set sites to provide a reconstruction of waterbody type for the fossil samples (Fig. 5.7). Diatom samples from OL97-5 conform to the coastal gradient defined by the calibration set because they plot along axis one of the calibration set DCA in a similar manner to the modern samples. Fossil samples plot in the lagoon and inlet fields with two samples in the bay field (Fig. 5.7 A). Transitions between zones A, B and C involve long distances along axis one (Fig. 5.7 B) indicating there are large changes in waterbody type between zones. Transitions between zones C, D and E are smaller because they all represent lagoonal environments and different lagoon types are not well separated on the DCA. 

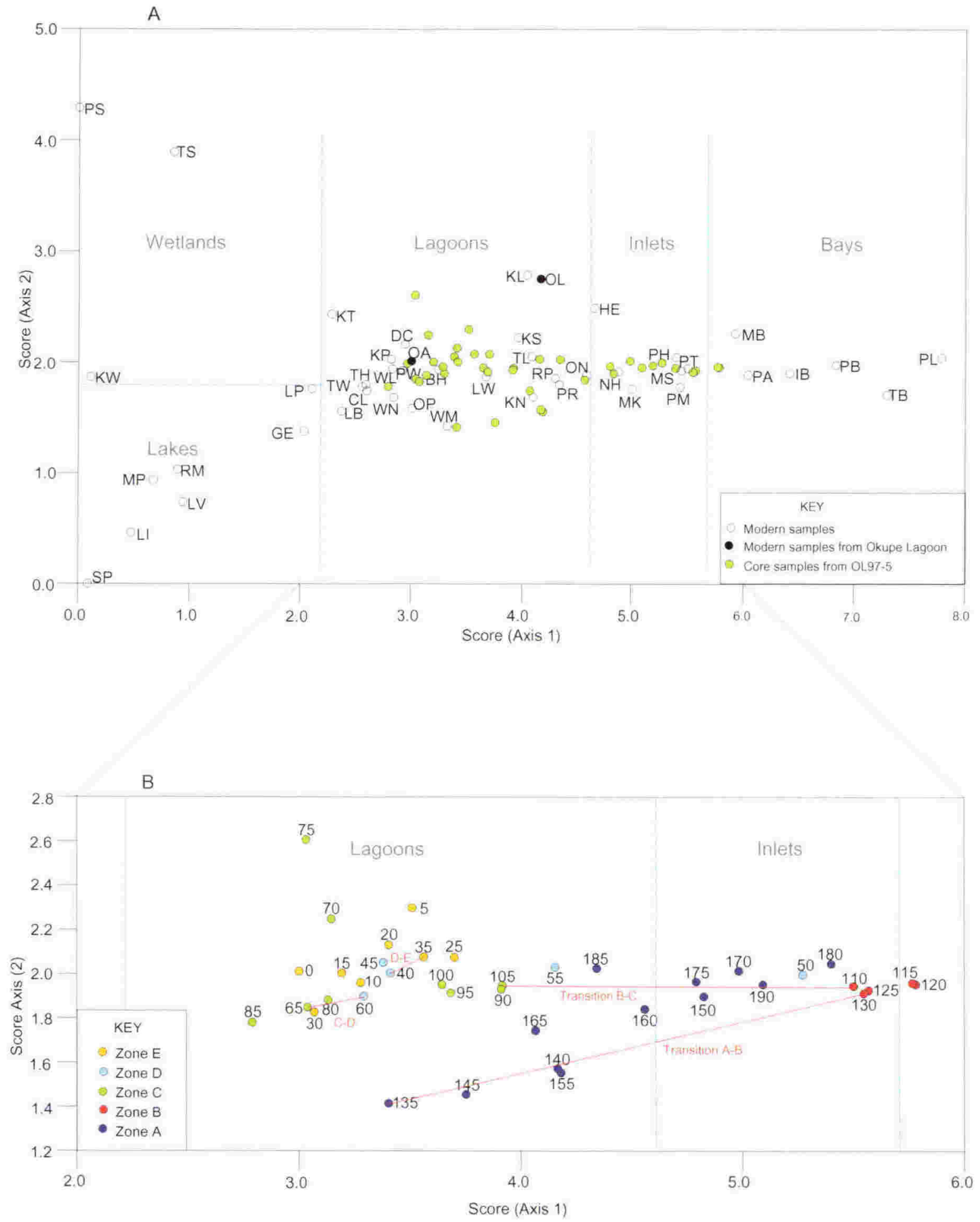

Fig. 5.7. DCA of calibration set sites with fossil samples from OL97-5 incorporated passively. A: Distribution of modern sites and division of axes into waterbody types. Coloured dots show where fossil samples plot in relation to modern sites. B: Central section of A enlarged and showing fossil samples labelled according to depth and coloured according to diatom assemblage zones. 
Assemblages at the base of OL97-5 plot in the inlet field and there is a progression through zone A to samples that plot on the right side of the lagoon field (Fig. 5.7 B). This trend along axis one suggests that connection with the sea became more restricted during the time of zone $\mathrm{A}$ and an inlet progressed to a predominantly open lagoon. The lagoonal environment at the top of zone A is quite different from the lagoonal environments at the top of OL97-5 as indicated by the large distance between them along axis two. Samples of zone B plot in the inlet and bay fields but little emphasis is placed on reconstruction of zone B because of the poor preservation of assemblages as discussed above. Samples in the lower half of zone $\mathrm{C}(105,100,95,90)$ plot in the centre right of the lagoon field and samples in the upper part of this zone $(85,80,75,70,65)$ plot centre left and are well spread out along axis two. Dispersion of these zone $\mathrm{C}$ samples along axis two is in the direction associated with wetland sites supporting the idea that the environment was a brackish marsh. Zone D samples plot in the centre of the lagoon field with the exception of sample 50 and 55. These two samples were dominated by Paralia sulcata and Gomphonema valentinica respectively and are substantially differentiated from other zone D samples on the DCA diagram supporting the suggestion that they represent marine influxes to the site. Samples from zone E plot in the central lagoonal field and the sample at the top of the core plots, as would be expected, in the same position as the modern sample from the core site $(\mathrm{OA})$.

\subsubsection{Application of Salinity Transfer Function}

The DCA of modern sites and fossil samples from Okupe Lagoon (above) illustrates that the calibration set successfully defines a spatial coastal gradient that is of wider ecological amplitude but otherwise very similar to the temporal gradient of OL97-5. This is exactly the situation desired for calibration of fossil samples. Another indication that the calibration set is suited for reconstruction of fossil samples from Okupe Lagoon is that $81 \%$ of taxa in the fossil samples are present in the calibration set. Most of the taxa not present have low abundances in the core sequence. However it must be kept in mind that the calibration set is small - 50 modern samples are used to help reconstruct 39 fossil samples. It is also obvious that fossil assemblages in the lower half of OL97-5 have been altered by transport and / or dissolution so they do not provide complete information about the original depositional environment.

Weighted averaging calibration and the modern analogue technique, as introduced in Chapters Three and Four, were carried out on samples from TS97-1 and TS98-2. There is a fair amount of variability between the three different salinity reconstructions for Okupe Lagoon and the modern analogue technique indicates that most samples in the lower half of the sequence have no close modern analogues in the calibration set (Fig. 5.8). However all three reconstructions 


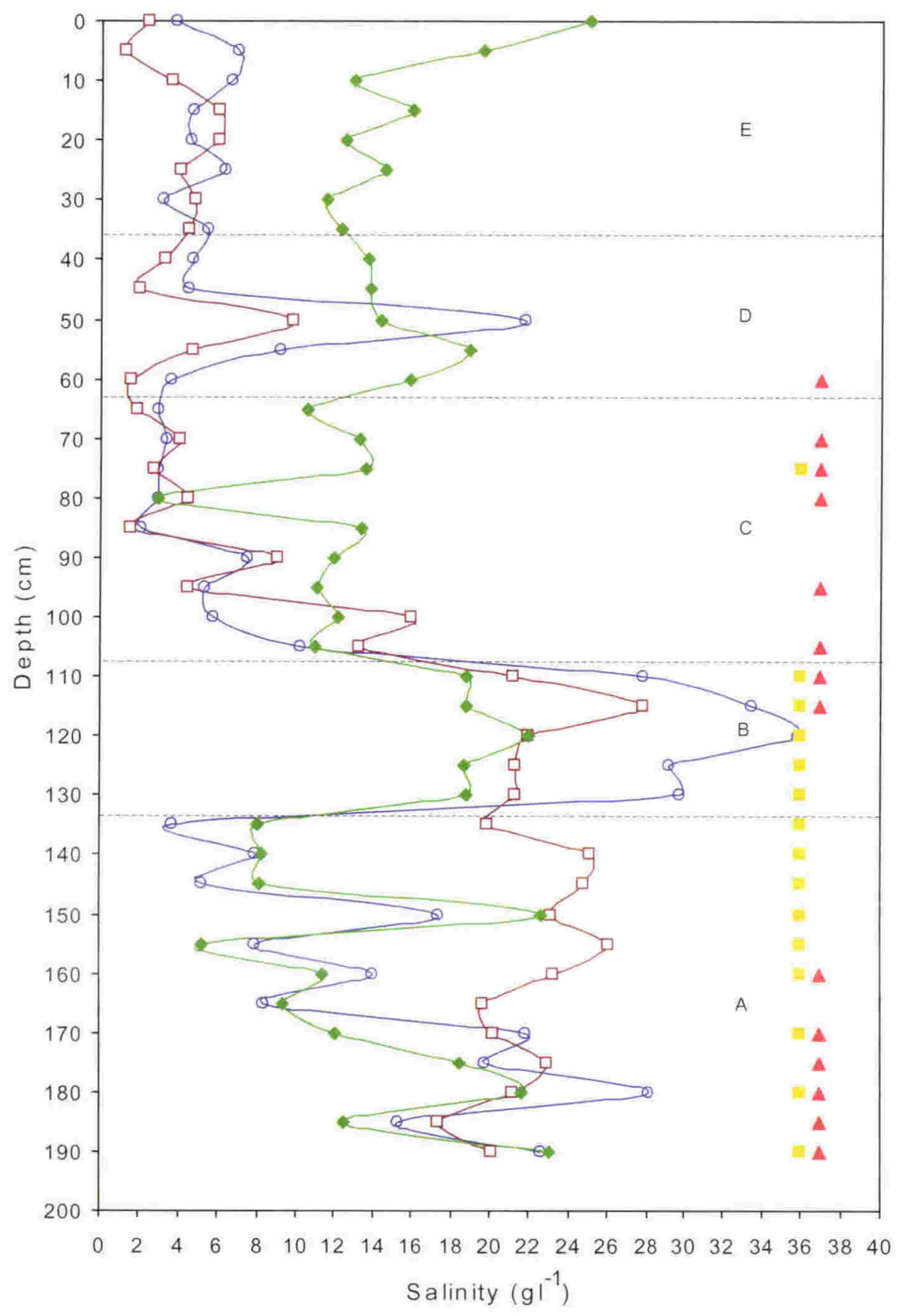

Reconstructions

- $W$ WA

E-WA-tol

$\because$ MAT

Samples with:

- No close analogues

A Count $<200$ valves

Fig. 5.8. Salinity reconstructions for OL97-5 using weighted averaging (WA), weighted averaging with tolerance downweighting (WA-tol) and the modern analogue technique (MAT). Dotted lines show diatom zone boundaries. 
pick out the same main changes in salinity and these are consistent with interpretations made from the diatom assemblages (above).

Salinities in the lower part of zone A average about $20 \mathrm{gl}^{-1}$ indicating sheltered rather than open marine conditions. WA and MAT reconstructions suggest salinity declines towards the top of zone A whereas the WA-tol reconstruction remains fairly constant. Zone B is sharply differentiated by higher salinities in all reconstructions but this can only be attributed to the salinity preference of Paralia sulcata not to the salinity of the past environment. Salinities of zones C, D and E are likely to have been fairly similar. Average estimates range from $4 \mathrm{~g} / \mathrm{l}^{-1}$ for WA and WA-tol and $13 \mathrm{gl}^{-1}$ for the MAT reconstruction. WA and WA-tol pick out the peak in Paralia sulcata at $50 \mathrm{~cm}$ as a high salinity peak. Only MAT estimates the present day salinity accurately probably because modern samples from Okupe Lagoon act as closest modern analogues for predicting salinity for the top few samples in the core. Modern samples from Okupe Lagoon can be considered outliers in the calibration set - because of such high salinities occurring in an enclosed waterbody - which is possibly why WA and WA-tol underestimate recent salinity.

It must be remembered that the reconstructed salinity value for any sample represents position on the coastal gradient as well as an estimate of paleosalinity. This is because of the numerous environmental variables that are correlated with salinity along such a gradient and the effects of which on diatom assemblages are hard to separate. Looking at the reconstructions in this light, and discounting the high salinities of the Paralia zone between 134 and $107.5 \mathrm{~cm}$, WA and WA-tol show an overall trend of movement in a landward direction along the gradient with decreasing depth. MAT shows less of an overall trend which is likely to be a result of the lack of close analogues for the lower part of the sequence.

\subsection{PALEOENVIRONMENTAL RECONSTRUCTION}

Diatom assemblages of cores OL97-5 and OL97-3 at Okupe Lagoon define five zones. These assemblages as well as sedimentological and macrofossil characteristics are interpreted to determine the nature of past depositional environment at Okupe Lagoon (Table 5.2). Foraminiferal assemblages (Goff et al., 2000) also provide useful information. Five main paleoenvironments are inferred to have existed at the site over the last 5000 years (Fig. 5.9).

The environment at the core sites about 5000 years BP was a marine inlet (Fig. 5.9). Coarse-grained, inorganic sediment and numerous shell fragments are indicative of a high energy regime. The presence of large Austrovenus stutchburyi shells and the foraminiferal assemblage (Goff et al., 2000) suggest a low intertidal 


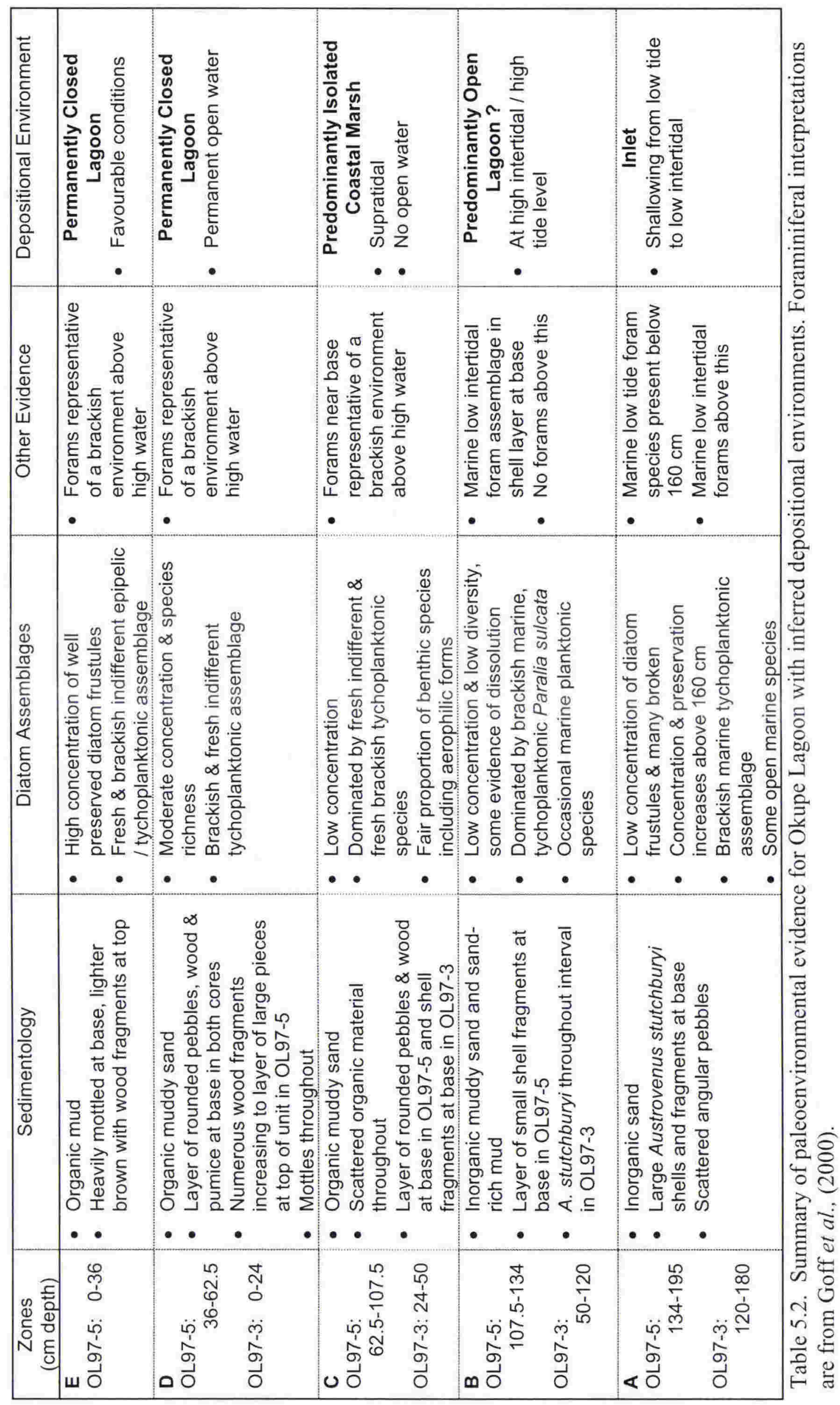




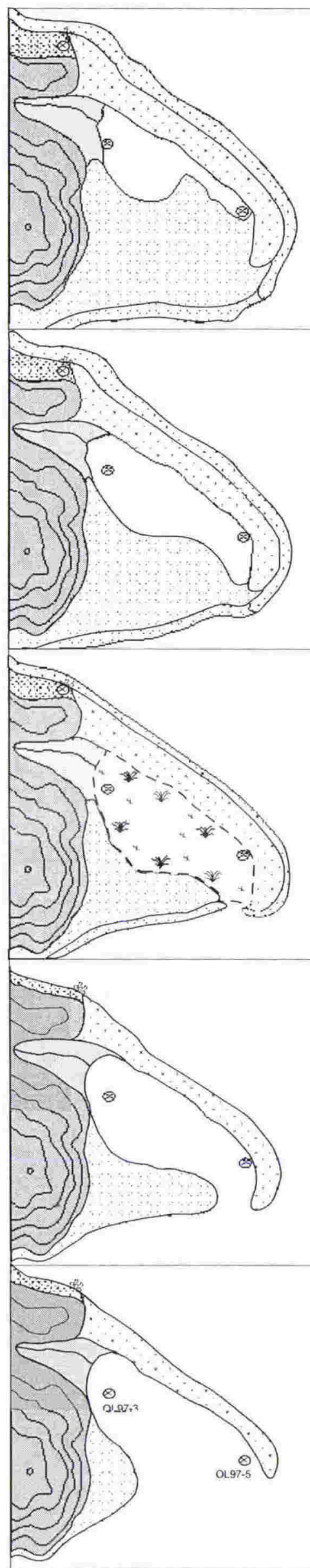

E

Permanently Closed Lagoon

Favourable Phase

$\sim 500$ to present

D

Permanently Closed Lagoon

Permanent Open Water

$\sim 1000-500$ cal. years BP

C

Predominantly Isolated Marsh

Supratidal

2300-1000 cal. years BP

B

Predominantly Open Lagoon? High Intertidal - High Tide 3200-2300 cal. years BP

\section{A}

Inlet

Low Tide - Low Intertidal

$\sim 5000-3200$ cal. years BP

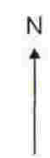

$500 \mathrm{~m}$

KEY

Old Boulder Bank

Modern Boulder Bank

Old Beach Sediments

Modern Beach

Wave-cut Platform

(8) Core Positions

Fig. 5.9. Reconstruction of paleoenvironments that have existed at the Okupe Lagoon site over the last 5000 years. 
elevation. At the OL97-3 core site the environment remained constant throughout zone A but in OL97-5 some change is inferred. Increasing diatom valve concentration and decreasing proportions of brackish marine species indicate the environment freshened through time with estimates of $20 \mathrm{~g} / \mathrm{l}^{-1}$ salinity at the base of zone A to about $14 \mathrm{~g} / \mathrm{l}^{-1}$ at the top. This is supported by a decrease in size of A. stutchburyi shells and a change from low tide foraminiferal assemblages to low intertidal assemblages at about $160 \mathrm{~cm}$ depth (Goff et al., 2000). Comparison with modern samples in a DCA suggests the environment at the top of zone A in OL97-5 was a predominantly open lagoon. As there is no evidence of a largescale change in waterbody type within zone A, this is interpreted to mean that the inlet had become shallower and less saline by this time, i.e. deposition was occurring in the intertidal rather than low tide zone.

The sheltered nature of the environment inherent in an inlet as opposed to open coast suggests the boulder bank at the northeastern end of Kapiti Island was present by 5000 years BP. The difference between core sequences can be explained by their positions relative to this barrier (Fig. 5.9). OL97-5 is situated immediately behind the barrier and so is likely to have been in a more sheltered environment relative to OL97-3. Sediment accumulation probably occurred to a greater extent in the lee of the barrier causing the inlet to shallow to intertidal elevations at OL97-5 and not at OL97-3.

The environment of zone B was open to the sea because of the inorganic nature of the sediment and occurrence of $A$. stutchburyi fragments. However it was more protected from the sea than zone A, as indicated by the finer grainsize and smaller, fewer shell fragments. Again there is a difference between OL97-5 and OL97-3 with shells of $A$. stutchburyi and intertidal foraminifera only occurring in a thin layer at the very base of zone B in OL97-5. The small size of shells suggests it was a last attempt by these organisms to live in an unfavourable environment. In OL97-3 shell fragments occur throughout zone B suggesting it was still at a lower tidal elevation than OL97-5. Poor preservation of diatoms in both core sequences suggests that diatom assemblages of zone B have been damaged by transport and / or dissolution. Paralia sulcata and the occasional marine planktonic diatoms that occur in the zone are either the last remains of the original assemblage or they have been transported to the site and no information about the original assemblage is available. Dominance of an assemblage by Paralia sulcata is known to be a common occurrence in high intertidal coastal environments (Denys and de Wolf, 1999) and because such a setting is consistent with the sedimentological evidence of zone $\mathrm{B}$, it is a reasonable paleoenvironmental estimate to make in this case. A predominantly open lagoon at high intertidal (OL97-3) to high tide level (OL97-5) is inferred for the environment of zone B. 
The initiation of organic sedimentation at the base of zone $\mathrm{C}$ as well as the complete lack of shells and incorporation of wood fragments into the sequence suggests the environment was predominantly isolated from the sea. Foraminifera present near the base of zone C in OL97-5 are indicative of sheltered waters of lowered salinity above mean high water (Goff et al., 2000). Diatom assemblages indicate the environment was brackish and may have had sporadic influxes of salt water especially during the lower part of the zone. Salinities predicted for the zone range between 4 and $13 \mathrm{~g} / \mathrm{l}^{-1}$. High occurrences of aerophilic diatoms in the upper part of the zone imply that the environment was subject to extended periods of dry or moist soil conditions. The site is inferred to have been a coastal wetland above high tide level.

Zone D and E represent permanently closed lagoonal environments. Sediment is organic in both cores with organic material and wood fragments scattered throughout the zones. Sediment of zone D is coarser-grained than zone $\mathrm{E}$ and has much higher concentrations of wood fragments. Foraminifera and diatom assemblages are similar to those living in the lagoon today. Therefore the waterbody can be inferred to have been a shallow lagoon that, despite its high salinities, was completely isolated from the sea. The higher concentrations of diatoms in zone $\mathrm{E}$ are possibly a result of increased nutrient input into the waterbody as a result of the influxes from the catchment that occurred at this time (inferred from the sedimentology).

\subsection{ENVIRONMENTAL TRANSITIONS}

\subsubsection{Introduction}

The history represented by the five different paleoenvironments that have existed at Okupe Lagoon over the last 5000 years is one of isolation from the sea. The four main transitions between different paleoenvironments are investigated for information they may provide about causes of change in environment. Criteria used to differentiate coseismic from aseismic change in depositional environment include the suddenness of the change, amount of change, lateral extent of horizons, synchroneity of change, and coincidence with tsunami deposits (Nelson et al., 1996b).

There is impressive evidence at the site today of large scale movement and deposition of sediment in the form of two boulder and cobble beach ridges that protrude from Kapiti Island for distance of almost one kilometre. These surround the lagoon to the northeast and beach sediments have built up in the south causing the lagoon to be entirely surrounded by sediment (Fig. 5.1). There is equally impressive evidence at the site for occurrence of one or more coseismic uplift events during the Holocene. A landward boulder beach ridge, now out of reach of 
storm waves, is an average $8.06 \mathrm{~m}$ above mean sea level and an average $3.5 \mathrm{~m}$ above its modern equivalent (Goff et al., 2000). There is an associated raised wave-cut platform to the west on which rock pools, stacks and wave-cut notches are all well isolated from the sea (Fig. 5.10). These features indicate a relative sea level lowering of 3-4 m. Considering that a sea level curve for the New Zealand region shows only decimetre-scale fluctuations over the last 6500 years (Gibb, 1986), sediment transport and coseismic movement are likely to have been the major factors causing environmental change at Okupe Lagoon.

\subsubsection{Description of Transitions}

Transition A-B (Fig. 5.11) consists of a sharp contact from sand to muddy sand in OL97-5 (134 cm depth) and from sand-rich mud to sand-poor mud in OL97-3 (120 cm depth). Immediately above this contact in OL97-5 is a thin layer containing the last occurrence of intertidal shells and foraminifera. The change in diatom assemblage takes place between adjacent samples in both cores suggesting that the transition occurred suddenly. There is a large magnitude change in depositional environment from the low intertidal zone of an inlet to what is inferred to be a predominantly open lagoon at high intertidal / high tide level. Continued sediment accumulation and barrier growth could have caused a relative sea level lowering at the site but this transition does not represent a continuous progression from low intertidal to intertidal to high intertidal as would be expected with gradual accumulation. The suddenness and environmental discontinuity observed at this horizon suggest the process was accelerated by a single event such as a large storm or coseismic uplift at this time. The environmental discontinuity (low tide to high tide) could represent an actual change or an apparent change resulting from erosion of sediment. In either case a mechanism is required by which a higher elevation environment became permanently established after the event.

At transition B-C (Fig. 5.11) there is a layer of pebbles and wood in OL97-5 (113.5 cm depth) and a layer of large shell fragments in OL97-3 (50 cm depth). Organic sedimentation begins from this point on in both cores. The change occurs suddenly in the diatom record with no indication of freshening or shallowing within zone B. There is a large change in paleoenvironment from what was a predominantly open lagoon to an isolated brackish marsh environment. This involved a change in relative sea level from about high tide level to above high tide level. The presence of wood, pebbles and shells at the transition horizons suggest that some type of depositional event occurred at this time. The wood and pebbles in core OL97-5 were probably derived from the catchment and barrier and could have been deposited as the result of a storm event. Large shell fragments in core OL97-3 are the last occurrence of shells in this core and because they are all fragmented it is likely they were transported from a seaward source rather than 

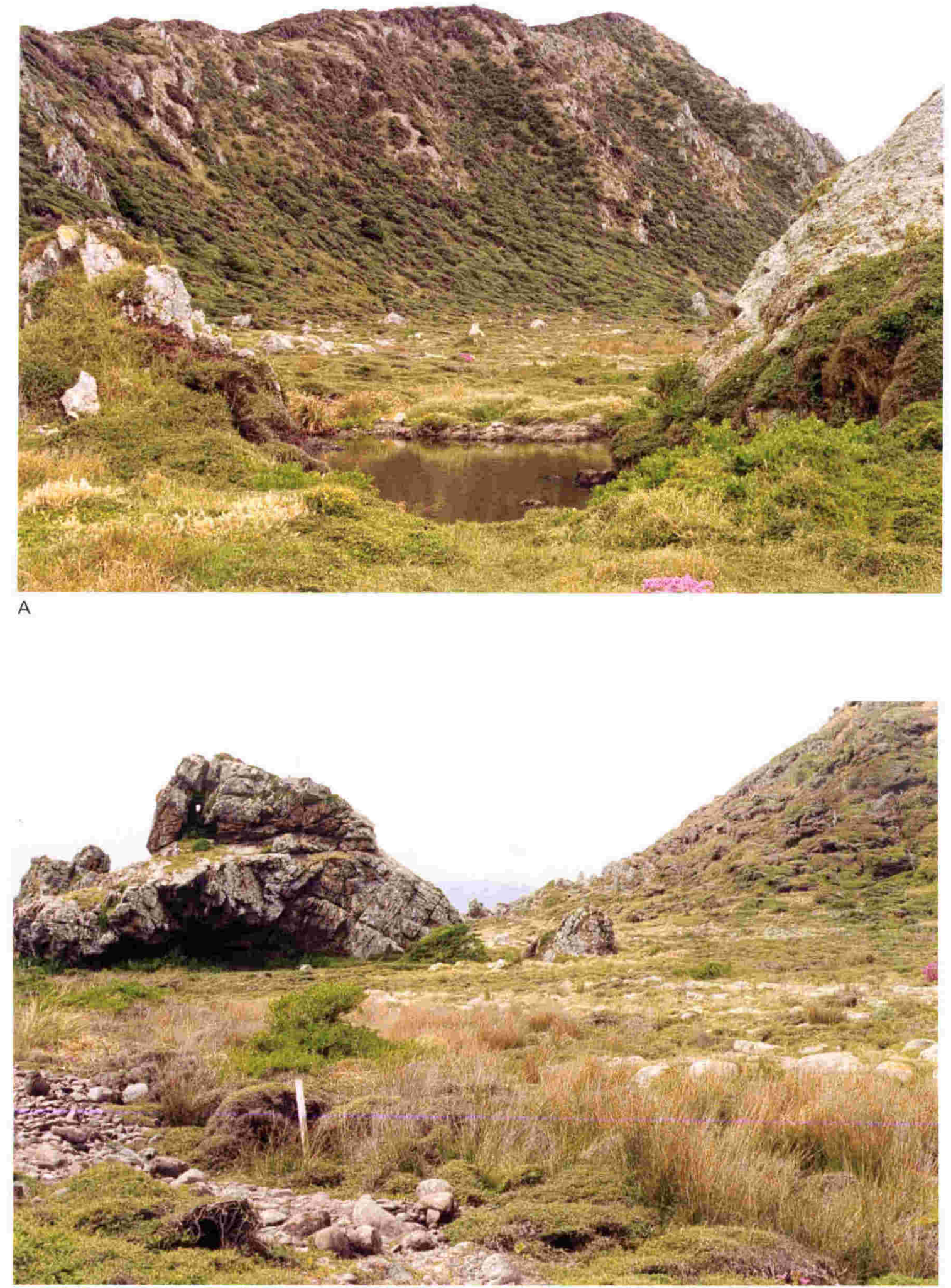

B

Fig. 5.10. Raised wave-cut platform northwest of Okupe Lagoon. A: Large rock pool between rock stacks with platform in background. B: Large stack with sea cave and platform in foreground. Boulders of modern storm beach present lower left corner. 


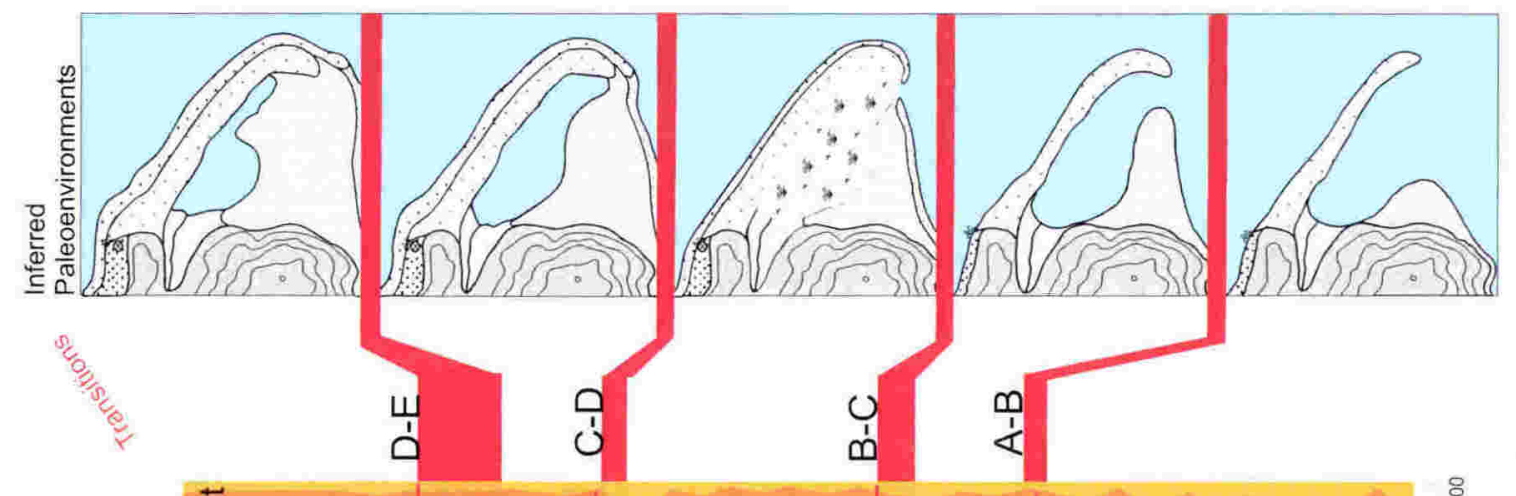

0
0
0
0
0
0
$\vdots$
$\vdots$
$\frac{0}{3}$

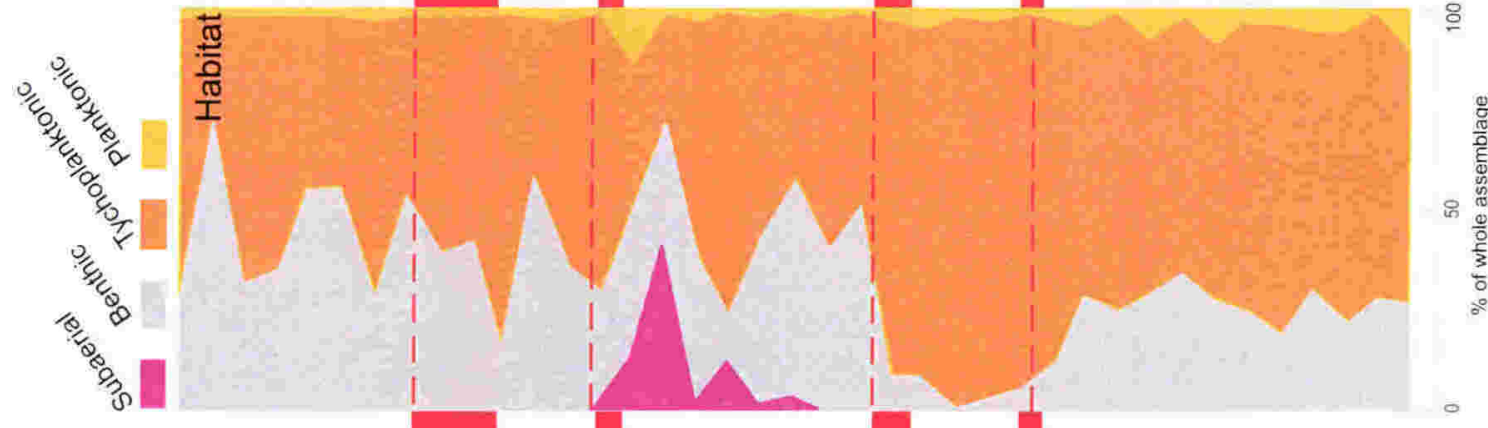

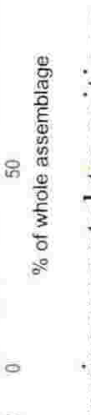

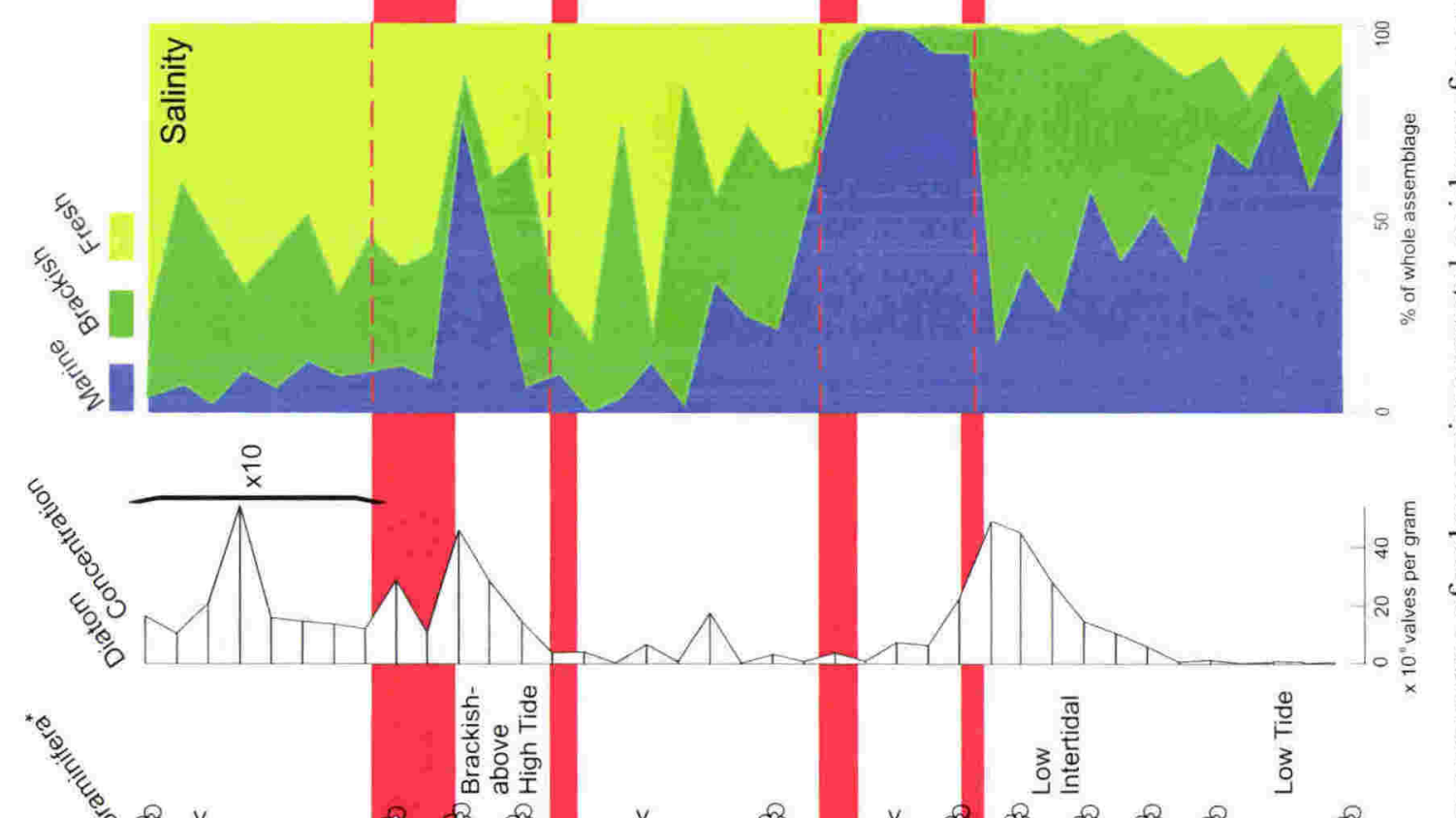

के

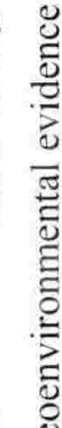

造

空

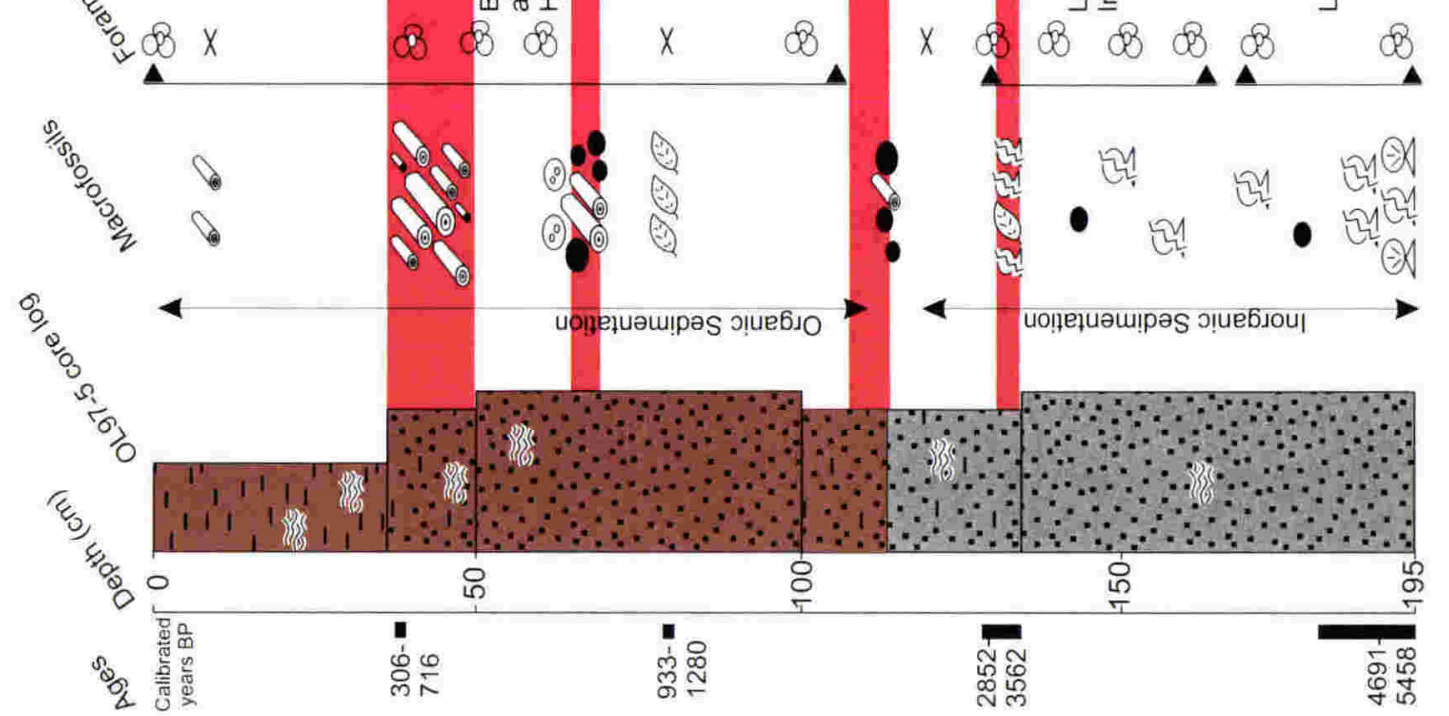

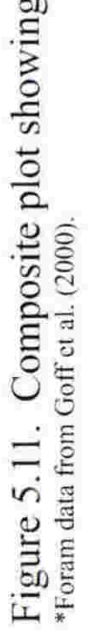


having lived at the site. This could also be achieved by a large storm event. The cause of isolation of the waterbody from the sea at transition B-C is most likely due to the final closing of the barrier around the core sites. Continued sediment transport and deposition could have led to such a closure with a storm event acting as the final trigger. However evidence for substantial shallowing of the waterbody occurring concurrently with isolation adds strength to the possibility that coseismic uplift was the final trigger for barrier closure. In this case seismic shaking and tsunami inundation would explain the influx of material at transition horizons.

At transition C-D there is a distinct 3-4 cm thick layer of large rounded pebbles and wood fragments in both cores with pieces of pumice immediately above each layer (62.5 cm depth in OL97-5, $24 \mathrm{~cm}$ in OL97-3). The transition involves a paleoenvironmental change from a marsh to a lagoonal environment with permanent open water. A large storm event causing movement of material off the boulder bank and out of the catchment could explain the deposition of a lagoonwide pebble and wood layer. However, this explanation does not provide a mechanism for creation of a permanent waterbody. Another possible explanation is that coseismic subsidence, or compaction of sediments through liquefaction, led to lowering of the site below the water table enabling permanent open water to exist. Disturbance of the catchment and barrier by seismic shaking could have led to release of material into the waterbody.

A sharp transition from organic muddy sand to organic mud occurs at the boundary between zone $\mathrm{D}$ and $\mathrm{E}$ at $36 \mathrm{~cm}$ depth in OL97-5. This is a permanent grainsize change implying a long term change to water depth or sediment supply. The transition is immediately preceded by a $14 \mathrm{~cm}$ thick concentration of large wood fragments in the core, and accompanied by a ten-fold increase in diatom concentration. At the base of the wood layer at $50 \mathrm{~cm}$ there is a large peak in the brackish marine diatom Paralia sulcata. There was obviously some disturbance of the catchment at this time causing a large influx of wood into the lagoon.

This could also bring about a renewed source of nutrients causing diatoms to flourish. These two features could be explained by flooding, landsliding, or even early land clearance as human habitation of Kapiti Island began almost 1000 years ago (Maclean, 1999). However the peak in Paralia sulcata is most simply explained by transport to the waterbody from a seaward source and none of the above events explain such an influx to the lagoon. A coseismic cause for the transition is a possibility because the shaking, movement of base level, and tsunami inundation associated with an earthquake could explain all these features. 


\subsubsection{Change in Elevation}

Paleoenvironmental reconstruction of the core sequences provides a broad indication of tidal elevation of the past environments (Fig. 5.12). The lowest two foraminifera samples in OL97-5 suggest the environment may have been just below low tide level (Goff et al., 2000). Otherwise diatoms, foraminifera and macrofossils are consistent with deposition in the low intertidal zone for sediment at the base of the cores (zone A). Deposition in the high intertidal to high tide zone is inferred for the central parts of the cores (zone B) and above this sedimentation was supratidal (zones C, D, E). Changes in relative sea level are inferred across transitions A-B and B-C, and water depth changes are inferred across transitions C-D and D-E. However there are problems estimating the amount of change in elevation across individual transitions because of the lack of good sea level index points and the lack of ecological preference information regarding water depth for diatoms.

A minimum estimate for net vertical movement at the site can be obtained by assuming that mean tide level was in the vicinity of the A-B transition (Fig. 5.12). When the cores are placed relative to present mean sea level (similar in elevation to mean tide level) it can be seen that they indicate a change in elevation of between 60 and $70 \mathrm{~cm}$ over the last 5000 years. This estimate, derived from sediments preserved at the site today, represents net uplift and it is possible that subsidence also occurred at the site. For example transition C-D described above is more consistent with coseismic subsidence than uplift. It can also be expected that a catastrophic event causing an environmental transition in the intertidal zone would result in some erosion of sediment. Erosion of sediment at the A-B transition is a likelihood because transport of material is implied by the Paralia sulcata dominated assemblage of zone B. Erosion of sediment from the waterbody would result in shortening of the sediment column and thereby an underestimation of amount of uplift. Differential subsidence due to liquefaction and associated compaction of soft sediments would also result in shortening so both these factors may explain the difference between net uplift observed in the cores and the amount of uplift inferred from the height difference of the modern and raised storm beach ridges $(3-4 \mathrm{~m})$. 


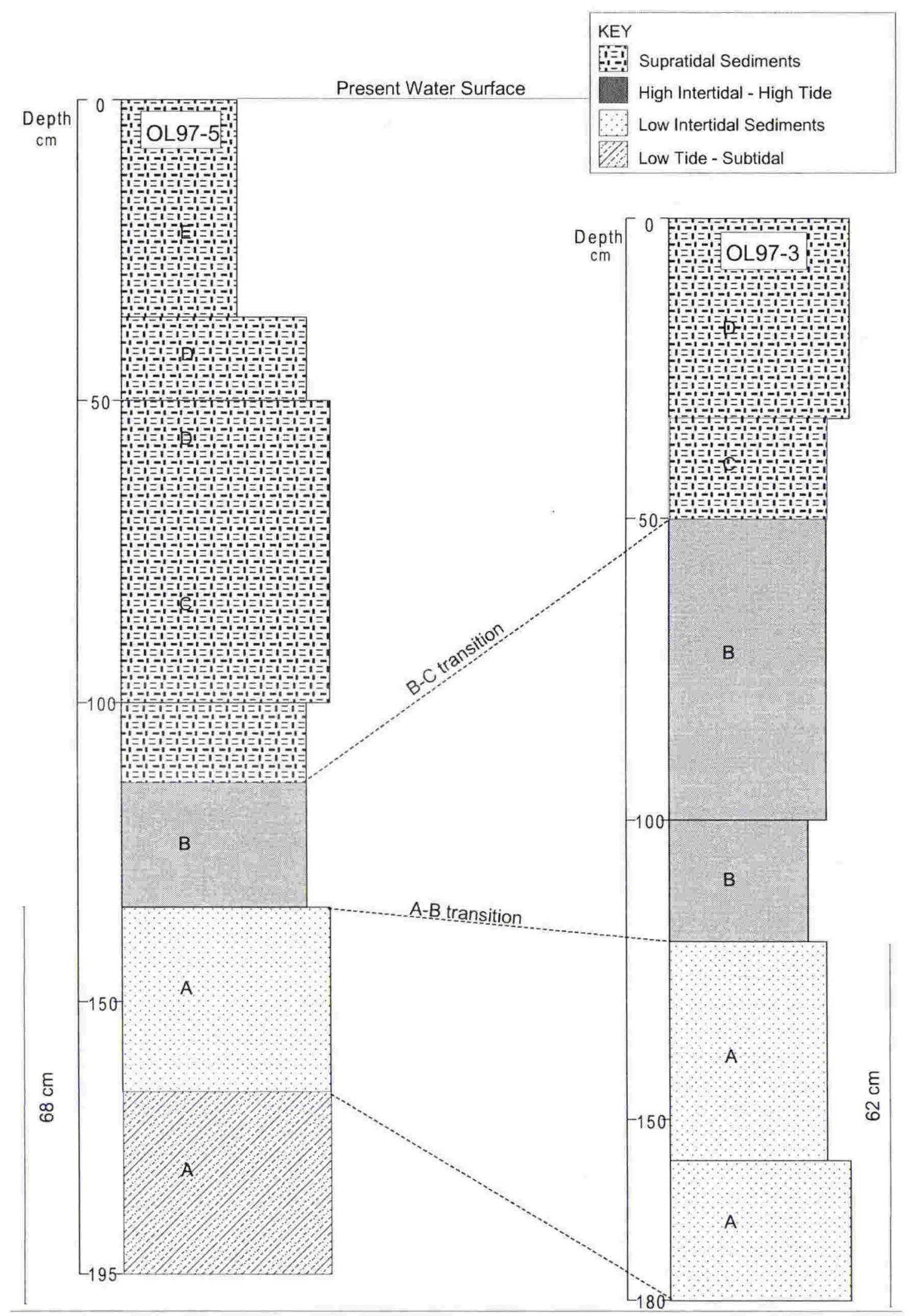

Present Mean Sea Level

Fig. 5.12. Inferred tidal elevation for units in the sedimentary sequences at Okupe Lagoon and their position relative to present mean sea level. If the A-B transition is taken to be in the vicinity of mean tide level, estimates of net vertical movement of $68 \mathrm{~cm}$ and $62 \mathrm{~cm}$ are obtained. However transition A-B is unlikely to be a good sea level indicator (see text). 


\subsubsection{Age of Transitions}

Transition A-B is radiocarbon dated at 3562-2852 cal. years BP by shell material immediately above the transition horizon in OL97-5. An estimate of the age in OL97-3 is calculated using maximum and minimum sediment accumulation rates (calculated using the two sigma range) for the interval between the two dates closest to the A-B transition. The estimated age of 3600-3100 cal. years BP (rounded to hundreds enclosing the greatest range) is consistent with that in OL97-5. The B-C transition is not directly dated in either core so an estimate is calculated from OL97-5 because, unlike core OL97-3, there are dates within zone B and C. Transition B-C is inferred to have occurred between 2400 and 2100 cal. years BP. Transition C-D is dated directly from wood material in OL97-3 at $1180-790$ cal. years BP and is estimated from OL97-5 to be $1000-800$ cal. years BP. Transition D-E is dated at 716-306 cal. years BP from wood near the top of the wood layer associated with this transition. The consistency of age estimates between cores indicates transitions are likely to have occurred synchronously across the waterbody but the resolution of radiocarbon dating is not great enough to prove this.

The age of peat at the base of the large rockpool on the raised intertidal platform immediately north of Okupe Lagoon is $2044-1738$ cal. years BP. Isolation from the sea of the rock platform and associated boulder beach ridge must have occurred prior to this date to enable fresh water peat to start accumulating. Therefore it is likely that coseismic uplift inferred at either or both of transitions $\mathrm{A}-\mathrm{B}$ and $\mathrm{B}-\mathrm{C}$ was responsible for uplift of the inner beach ridge and platform.

\subsection{CONCLUSIONS}

Sedimentology and diatom analysis of two $\sim 200 \mathrm{~cm}$ sedimentary sequences from Okupe Lagoon on Kapiti Island enable past environment to be reconstructed for the late Holocene. Nine radiocarbon dated samples provide a chronology for the sequences. Diatom assemblages are interpreted using traditional salinity and habitat preferences and also through comparison and calibration with a modern coastal diatom calibration set. The combination of grain size, macrofossil and diatom data, has enabled detailed reconstructions of waterbody type, salinity and energy regime to be established.

Paleoenvironmental reconstructions indicate that five different depositional environments have existed at the site over the last 5000 years. These environments include a sheltered inlet, a predominantly open lagoon, a marsh and a permanently closed lagoon indicating that relative sea level lowering and isolation from the sea occurred over this time period. This isolation was not continuous but was punctuated by large and sudden changes in environment. Four main environmental transitions that are laterally extensive and synchronous across 
the waterbody, are recognised in the sedimentary sequences of Okupe Lagoon. Transitions A-B and B-C at about 3200 and 2300 cal. years BP respectively, involve relative movements of base level resulting in large magnitude changes to depositional environment. Transitions C-D and D-E occurred at about 1000 and 500 cal. years BP respectively. These involve smaller magnitude changes but they are accompanied by evidence for catchment disturbance, and in the case of transition D-E, evidence for marine inundation. Transitions are consistent with the occurrence of past large earthquakes and a minimum of $60-70 \mathrm{~cm}$ of uplift is inferred from the sedimentary sequences. The likelihood that coseismic events were the cause of environmental transitions at Okupe Lagoon is considered further in Chapter Seven. 


\section{CHA P T E R S IX}

\section{Lake Kohangapiripiri: Catchment Disturbance and Water Depth Change Over 7500 Years}

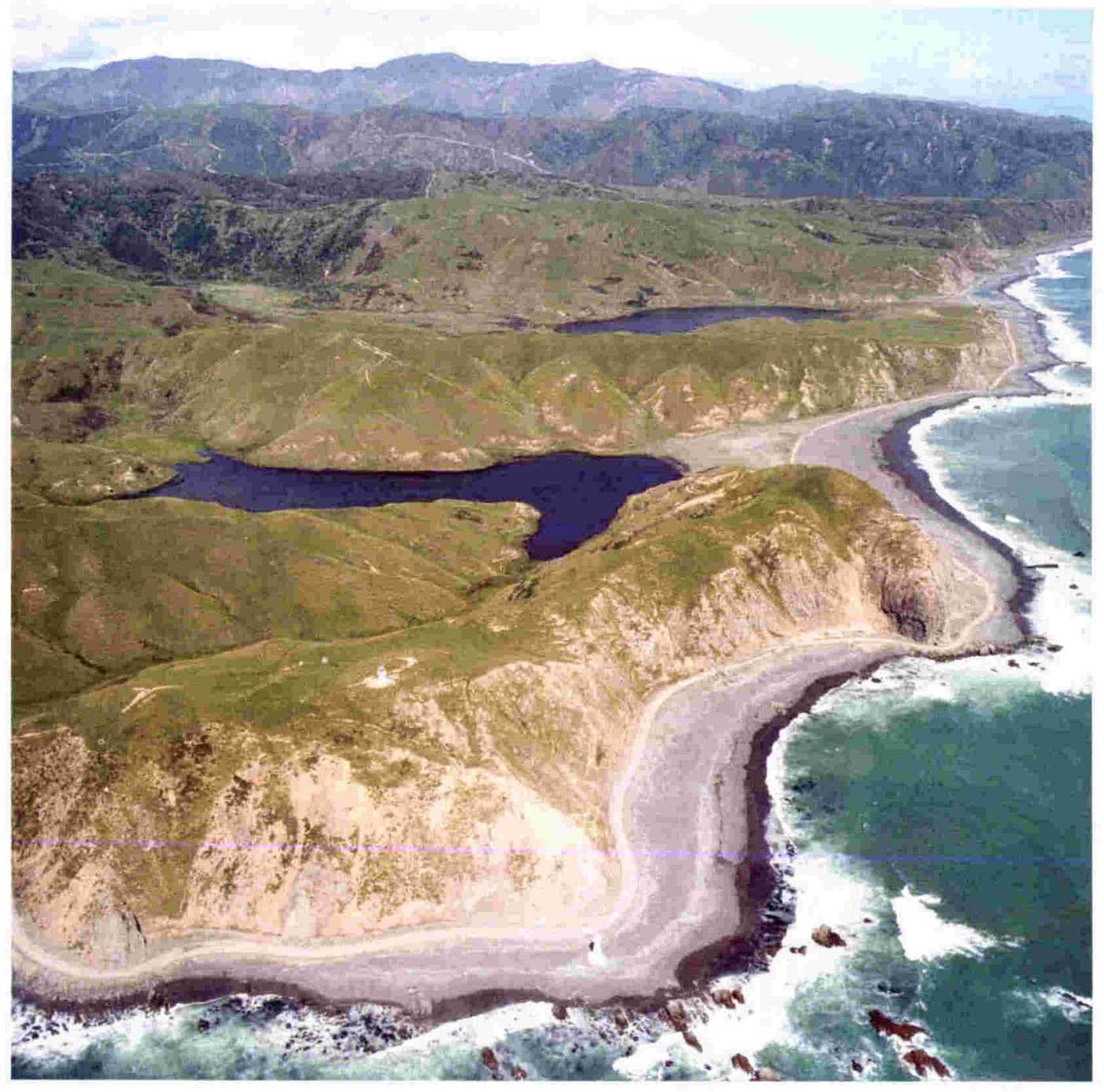

Fig. 6.1. Oblique aerial view of Lake Kohangapiripiri looking east with Lake Kohangatera in the background. Photo by Lloyd Homer. 


\section{SUMMARY}

Lake Kohangapiripiri is a small lagoon that is completely isolated from the sea by a gravel barrier. The barrier consists of a large volume of sediment and several raised beach ridges indicating that sediment transport along the coast and coseismic uplift events are likely to have had an impact on the Holocene history of Lake Kohangapiripiri. A sedimentary sequence recovered from the seaward end of the lagoon is conspicuously uniform apart from the occurrence of several very coarse grained layers that represent high energy influxes into the waterbody. Diatom analysis indicates that four different depositional environments have existed at the site over the last 7500 years. A predominantly closed lagoon is represented at the base of the sequence between about 7500 and 6800 cal. years BP. A permanently closed lagoon existed from this point on but went through a deep phase between about 5200 and $2200 \mathrm{cal}$. years BP, and through a shallow phase from $2200 \mathrm{cal}$. years BP to the present. Lagoon-wide shallowing in the very recent past is inferred from the upper two samples in the sequence. Three main transitions between inferred paleoenvironments and two minor transitions are recognised in the sequence and described in this chapter. The nature of these transitions is consistent with the occurrence of past large earthquakes.

\subsection{INTRODUCTION}

Lake Kohangapiripiri (Fig. 6.1) is one of two freshwater lagoons on Wellington's south coast immediately east of Wellington Harbour entrance (Fig. 6.2). The lagoon is situated on a block of Triassic sedimentary basement rock that is bounded by the Wellington Fault in the west and the Wairarapa Fault in the east. Net vertical tectonic movement over the last 125,000 years has been uplift because Last Interglacial marine benches occur at heights of about $30-120 \mathrm{~m}$ in Fitzroy Bay (Begg and Mazengarb, 1996). However it is possible that subsidence has also occurred at the site. The lagoon surface is only two metres above mean sea level (amsl) but is completely isolated from the sea by a barrier consisting of two raised gravel storm beach ridges. Cameron Creek drains a 343 ha catchment of scrub and pasture and runs into the northern end of the lake. At the southern end there is an outlet channel that has breached the highest raised beach but drains into the back of a younger storm beach where it must seep through the gravel at times of high lake level. The lagoon itself is small (11 ha) and has an average depth of $1.6 \mathrm{~m}$. Wetlands surrounding the lagoon and in Cameron Valley are significant because of the occurrence of regionally endangered plants and some uncommon birds (Clelland, 1984). 


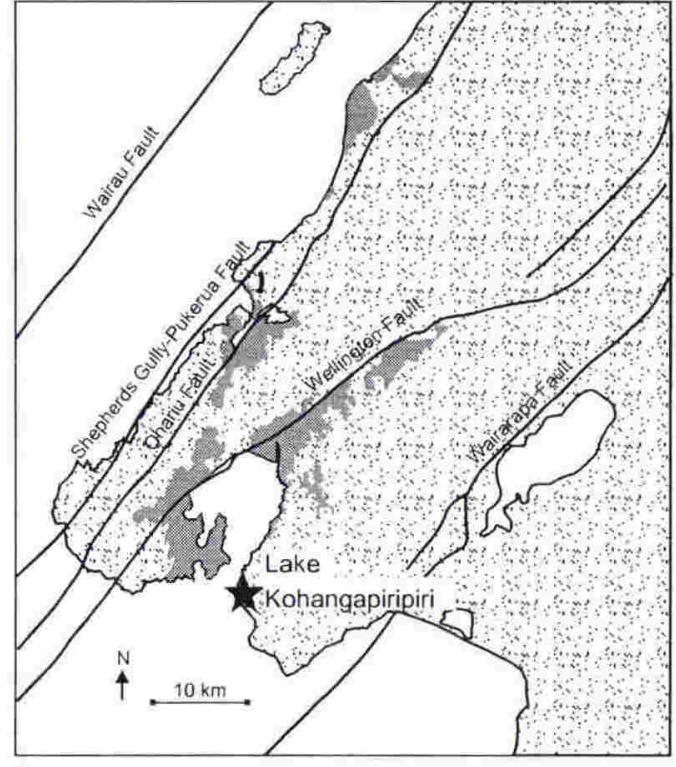

A

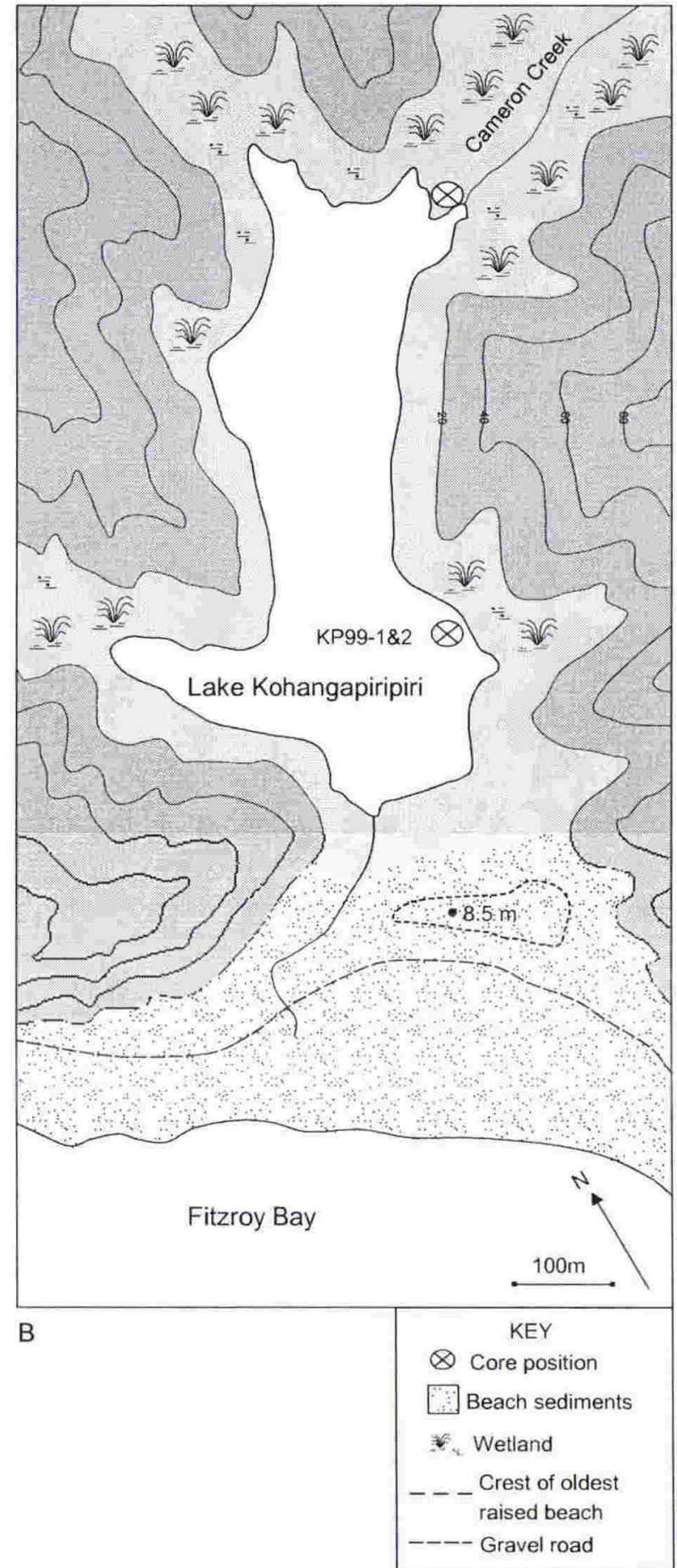

Fig. 6.2. Map of Lake Kohangapiripiri. A: Location in the Wellington region. B: Site map showing core positions. Unlabelled core at Northeastern end of Lake is that of Cochran et al. (1999). 
Cotton (1921a) described the lakes as drowned valleys. Wave-cut cliffs and truncated spurs are evidence that the valleys were once inlets of the sea and the initial submergence of the valleys is ascribed to the same tilting that formed Wellington Harbour. Cotton (1921b) used the development of the lake margins and delta growth as evidence for stability of the land for thousands of years prior to the 1855 earthquake. Adkin (1955) surveyed the beach ridges in front of Lake Kohangapiripiri and as a result of finding more than one raised beach ridge, concluded that the 1855 earthquake was not an isolated event. Stevens (1973) also indicates that the gravel barrier was formed by more than one uplift event.

Cochran et al. (1999) carried out a paleoenvironmental study of Lake Kohangapiripiri and determined that the lake has been fresh water for at least 7000 years. Although a number of environmental changes were interpreted from the lake record, the stability of the environment was the most interesting result. Information presented in this Chapter is an extension of the previous work, with the investigation of a core retrieved from an open water, seaward position as opposed to a landward wetland site as studied previously.

\subsection{SEDIMENTARY SEQUENCE}

\subsubsection{Description of Sequence}

Cores KP99-1 and KP99-2 were collected from the seaward end of Lake Kohangapiripiri off the eastern shore about 150 metres from the barrier (Fig. 6.2). KP99-1 is a piston cored section that penetrated down to $350 \mathrm{~cm}$ and KP99-2 was taken with a Russian-type corer down to $650 \mathrm{~cm}$ depth. These cores were taken less than $50 \mathrm{~cm}$ apart so are considered to represent the same sedimentary sequence and are used as a composite core (KP99-1\&2). The age near the base is about 7500 cal. years BP and the elevation of the lake floor at the core site is $1.75 \mathrm{~m}$. Full core logs are presented in Appendix I.

The sequence consists predominantly of uniform grey organic mud (Fig. 6.3). Throughout this mud there are occasional mottles of organic material, wood fragments and specks of vivianite. In the lowest $40 \mathrm{~cm}$ of core angular, weathered, greywacke clasts are scattered throughout the lake mud and there is a thin layer of similar clasts between 592 and $589 \mathrm{~cm}$ depth. Immediately above this there is a layer of angular, poorly sorted sand and small angular pebbles with specks of vivianite between 585 and $577 \mathrm{~cm}$. Similar sand layers exist between 505 and $500 \mathrm{~cm}$ depth and between 434 and $429 \mathrm{~cm}$. Within the latter sand layer are two distinct bands of wood fragments and for $11 \mathrm{~cm}$ above the sand the sediment is dark brown due to numerous pieces of organic matter and wood fragments. 


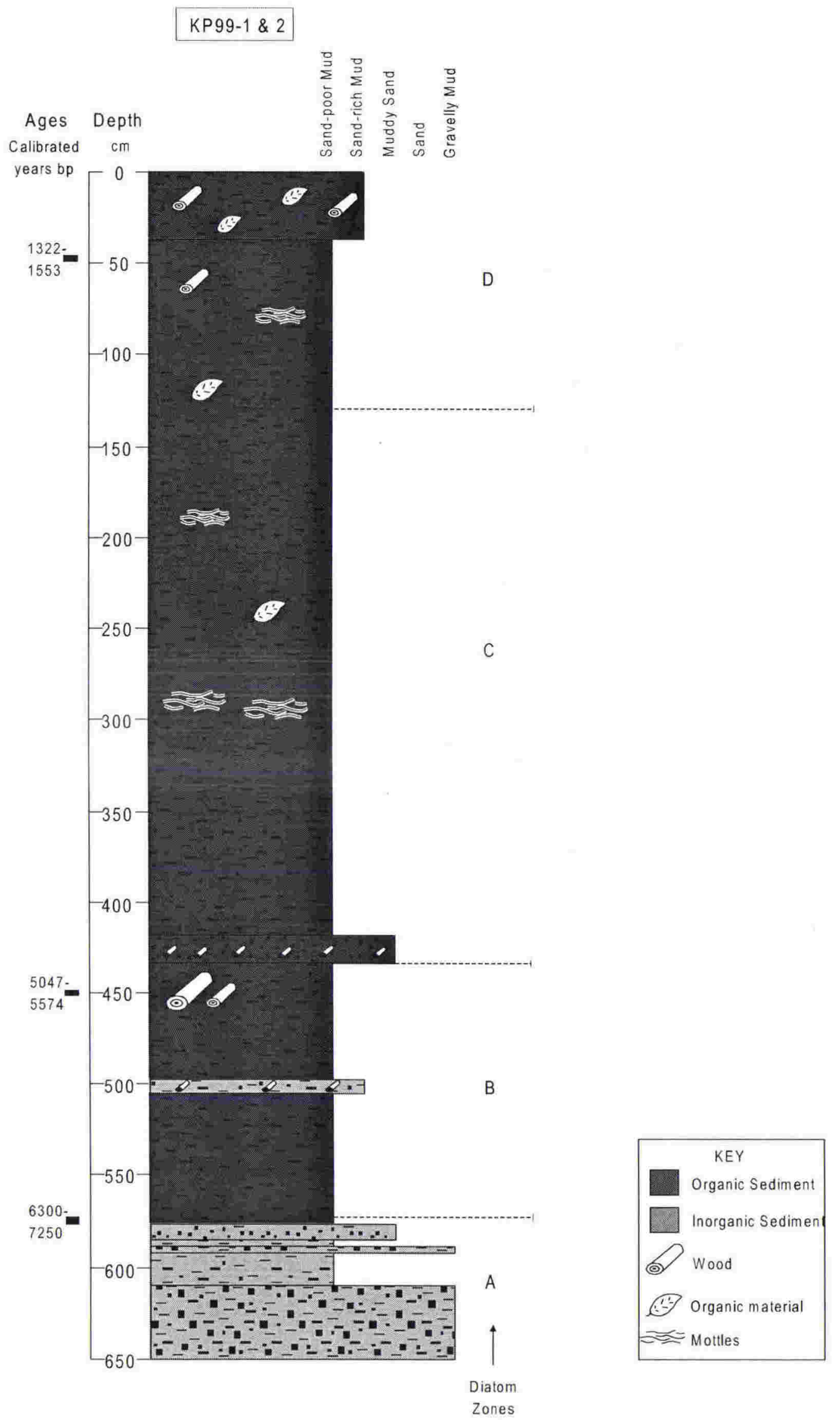

Fig. 6.3. Core log of KP99-1 \& 2 from Lake Kohangapiripiri. 
From $60 \mathrm{~cm}$ to the surface there is a gradational increase in organic content and a colour change from yellowish grey to reddy brown. Near the top of the sequence at $38 \mathrm{~cm}$ there is a sharp texture change to increased sand content in the mud above.

Sediment accumulation rates estimated using the three available radiocarbon ages (Fig. 6.4) indicate that most of the sequence was deposited at rates of about $1 \mathrm{~mm} / \mathrm{yr}$. A lower rate of about $0.3 \mathrm{~mm} / \mathrm{yr}$ is calculated for the upper $50 \mathrm{~cm}$ of the sequence. A similar decrease in accumulation rate is noted for the upper $140 \mathrm{~cm}$ of the wetland core from Lake Kohangapiripiri (Cochran et al., 1999). This slowing of sediment accumulation may be an apparent change resulting from loss of sediment in the upper sections of cores or older radiocarbon ages towards the present. However as the decrease is observed at both ends of the lake it may have been an actual occurrence resulting from the lake-wide shallowing and wetland formation inferred from the diatom record (see below).

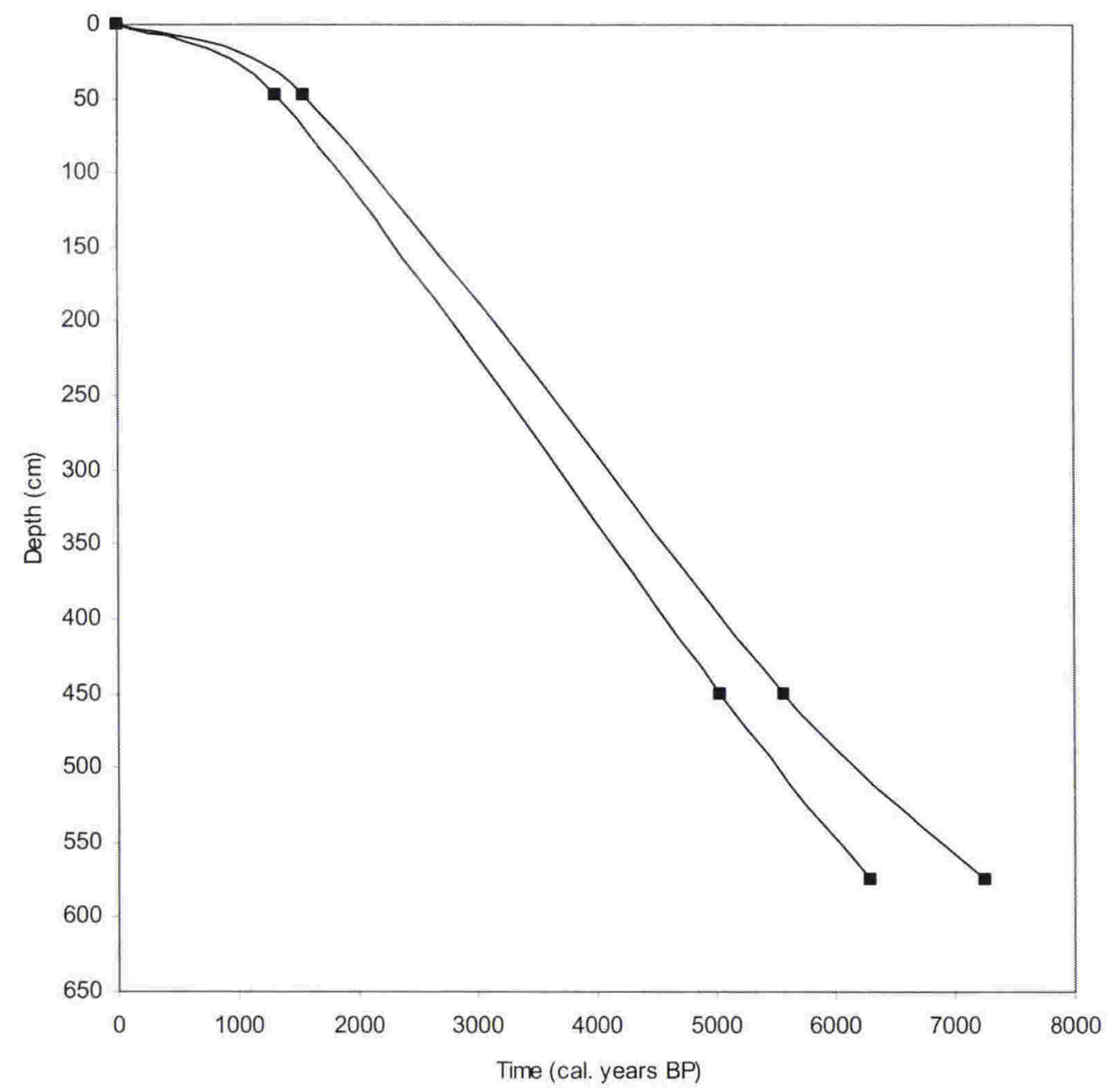

Fig. 6.4. Sediment accumulation rate curves for KP99-1 \&2 based on calibrated radiocarbon age estimates. Maximum and minimum curves are calculated from end-member values of the 2-sigma age range. 


\subsubsection{Interpretation of Sequence}

Most of the sequence is consistent with deposition in a small enclosed lagoon as exists at the site today. However some type of catchment disturbance or highenergy influx is required to explain the incorporation of layers of gravel, sand and wood into the sequence. The angular, poorly sorted, weathered greywacke gravel at the base of the core and in the layer at $592-589 \mathrm{~cm}$ depth appears to be sourced from nearby hillsides and is likely to have entered the waterbody as a result of small landslides. Layers of sand at $\sim 580 \mathrm{~cm}, \sim 500 \mathrm{~cm}$ and $430 \mathrm{~cm}$ depth are also angular, poorly sorted greywacke grains that could be consistent with the fine toe of a landslide off a nearby hillside. Although sand grains in the layer at $430 \mathrm{~cm}$ depth are better rounded than grains in other layers, none of the sand deposits appear well-sorted or rounded enough to be sourced from the beach. Coarsegrained layers are not considered to be flood deposits because of the distance of the fresh water inflow from the core site and the lack of similar deposits in cores from the mouth of Cameron Creek (Cochran et al., 1999). The increase in grain size at the very top of the sequence is likely to be the result of shallowing and higher energy associated with the littoral zone of the lake.

\subsection{DIATOM ANALYSIS}

\subsubsection{Introduction}

Diatom samples were analysed at $20 \mathrm{~cm}$ intervals throughout most of the core and at 10 and $5 \mathrm{~cm}$ intervals at the base and top of the core where most changes take place. At least 400 diatom valves were counted in most samples but in some low concentration samples at the base of the sequence only 100 valves were counted. About 130 species were encountered in the sequence (full species counts are presented in Appendix VI). Diatoms are well preserved throughout the sequence. The sequence at Lake Kohangapiripiri is divided into four main zones based on diatom assemblages (Table 6.1). Very similar assemblages were recognised in a core from the wetland at the landward end of the lake (Cochran, et al., 1999) indicating that zones and the changes they represent are of lagoon-wide extent.

\subsubsection{Definition of Diatom Assemblage Zones}

Zone $\mathrm{A}$ at the base of the sequence has a moderate concentration of valves and moderate species richness. It is defined by the presence of Pseudopodosira sp. 1 and 2, Actinocyclus octonarius, Chaetoceros spores, Hyalodiscus pustulatus and Campylodiscus echeneis. These species occur in small proportions but are unique to this zone. Zone B has moderate to high concentrations of valves and moderate species richness. It is defined by five key species. Zone $\mathrm{C}$ makes up most of the sequence of KP99-1\&2. Concentration is high and species richness is low because the zone is dominated by a few key species. It is defined by high proportions of Cyclostephanos dubius, Aulacoseira granulata and Cyclotella stelligera. 
Zone D at the top of the sequence has high concentrations and moderate to high species richness. It is defined by six key species and much lower proportions of the zone $\mathrm{C}$ species that dominate much of the sequence.

\begin{tabular}{|c|c|c|c|c|}
\hline Zones $(\mathrm{cm})$ & Key Species & Concentration & $\begin{array}{l}\text { Species } \\
\text { Richness }\end{array}$ & Preservation \\
\hline $\begin{array}{l}\text { D } \\
130-0\end{array}$ & $\begin{array}{l}\text { Fragilaria pinnata } \\
\text { Fragilaria construens } \\
\quad \text { f. subsalina } \\
\text { Cocconeis placentula } \\
\text { Achnanthes delicatula } \\
\quad \text { var. engelbrechtii } \\
\text { Achnanthes reversa } \\
\text { Fragilaria tenera }\end{array}$ & High & Mod-High & Good \\
\hline $\begin{array}{l}\text { C } \\
434-130\end{array}$ & $\begin{array}{l}\text { Cyclotella stelligera } \\
\text { Cyclostephanos dubius } \\
\text { Aulacoseira ambigua } \\
\text { Fragilaria pinnata } \\
\text { Cyclotella meneghiniana } \\
\text { Aulacoseira granulata }\end{array}$ & High & Low & Good \\
\hline $\begin{array}{l}\text { B } \\
577-434\end{array}$ & $\begin{array}{l}\text { Fragilaria pinnata } \\
\text { Aulacoseira ambigua } \\
\text { Cyclotella stelligera } \\
\text { Fragilaria construens } \\
\quad \text { f. subsalina } \\
\text { Cyclotella meneghiniana }\end{array}$ & Moderate-High & Moderate & Good \\
\hline $\begin{array}{l}\text { A } \\
650-577\end{array}$ & $\begin{array}{l}\text { Fragilaria pinnata } \\
\text { Aulacoseira ambigua } \\
\text { Cyclotella meneghiniana } \\
\text { Pseudopodosira sp. } 1 \& 2 \\
\text { Actinocyclus octonarius } \\
\text { Chaetoceros spores } \\
\text { Campylodiscus echeneis } \\
\text { Hyalodiscus pustulatus }\end{array}$ & Moderate & Moderate & Good \\
\hline
\end{tabular}

Table 6.1 Definition of diatom assemblage zones for core KP99-1\&2.

\subsubsection{Interpretation of Diatom Assemblage Zones}

Diatom species are grouped according to their salinity and habitat preferences to aid description of the paleoenvironment of each zone (Fig. 6.5). Zone A has high proportions of fresh indifferent and fresh water planktonic and tychoplanktonic species. It is likely to have been a predominantly fresh water, shallow waterbody. However the presence of marine and brackish diatoms indicates there was some connection with the sea that enabled transport of marine diatoms into the waterbody. The 'unknown' category includes a fair proportion of Pseudopodosira species in this zone, these probably have brackish salinity preferences but because they have not been identified to species level they are not placed in a salinity category. The moderate proportions of these taxa as well as other brackish species suggest the connection with the sea led to raised salinity levels in the waterbody. 

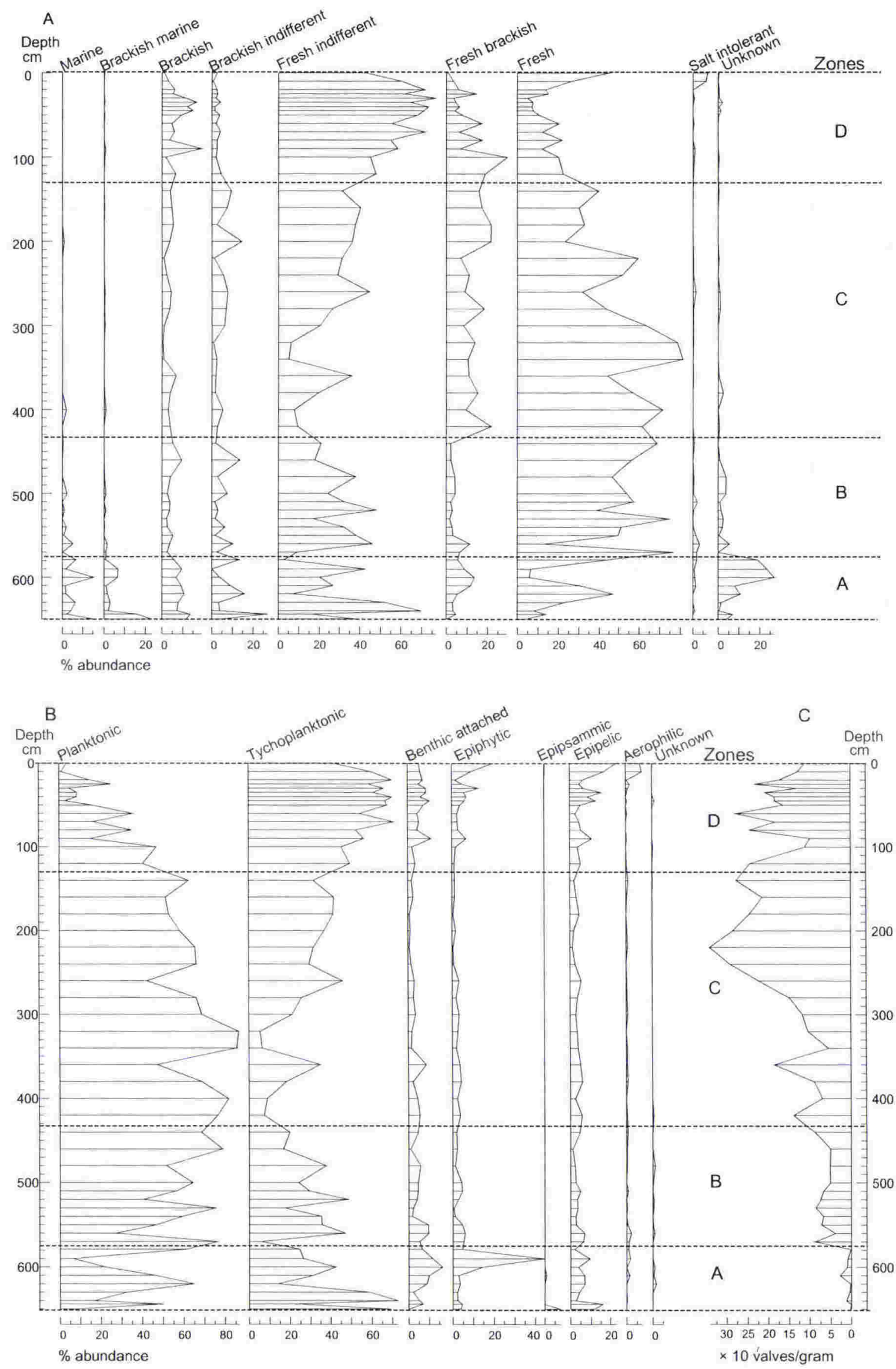

Fig. 6.5. Diatom assemblages of core KP99-1\&2. A: Diatoms grouped according to salinity preferences. B: Habitat preferences. C: Concentration of diatom valves in the sediment. 
However most of the assemblage indicates only a slightly brackish waterbody, so the connection was probably indirect or episodic.

The assemblage of zone B is dominated by fresh and fresh indifferent species of planktonic and tychoplanktonic habitats and so is consistent with a fresh water, moderate depth waterbody. Marine and brackish marine species occur in very minor proportions in the lower part of the zone and are non-existent in the upper part of the zone and brackish species are down to proportions typical of the remainder of the sequence. These factors suggest salinity was lower than that of zone $\mathrm{A}$ and the connection with the sea was no longer present. The small numbers of marine diatoms at the base of the zone were probably blown into the waterbody or washed over the barrier during storms.

Zone $\mathrm{C}$ is dominated by planktonic species with preferences for fresh or fresh brackish water. This domination by planktonic species indicates the waterbody was comparatively deep during this time with little opportunity for benthic species to grow at the site. There is also a fair proportion of fresh indifferent tychoplanktonic species. These were probably transported from shallower habitats and suggest the lake was turbulent and well mixed as it is at present.

Increased proportions of tychoplanktonic and benthic species in zone D and a corresponding decrease of planktonics indicate the environment was shallower than that of zone $\mathrm{C}$. The slight increase in brackish species may represent a response to shallowing rather than an increase in salinity because the planktonic species of zone $\mathrm{C}$ would have inhabited the freshest surface waters of the lake whereas benthic species could be exposed to slightly more saline waters near the sediment surface. The upper two samples in this zone appear to represent a further shallowing with planktonics decreasing to less than $5 \%$ and benthics increasing. Salt intolerant and aerophilic species, typical of marginal wetland sites, also increase in these two samples.

\subsubsection{Reconstruction of Waterbody Type}

Diatom counts from KP99-1\&2 were included passively into a detrended correspondence analysis (DCA) of calibration set samples (described in Chapter Three) to assist with reconstruction of waterbody type (introduced in Chapter Four) for the core samples. Samples from KP99-1\&2 plot at the landward end of the gradient defined by modern samples with most samples either at the left side of the lagoon field or in the lake field (Fig. 6.6 A). Despite their occurrence at one end of the gradient, samples from KP99-1\&2 are conformable with calibration set samples because they follow the pattern identified for calibration set samples at the terrestrial end of the gradient. Lake samples move toward low values on axis two and wetland samples plot at high values on axis two which enables differentiation between these fresh waterbody types. 


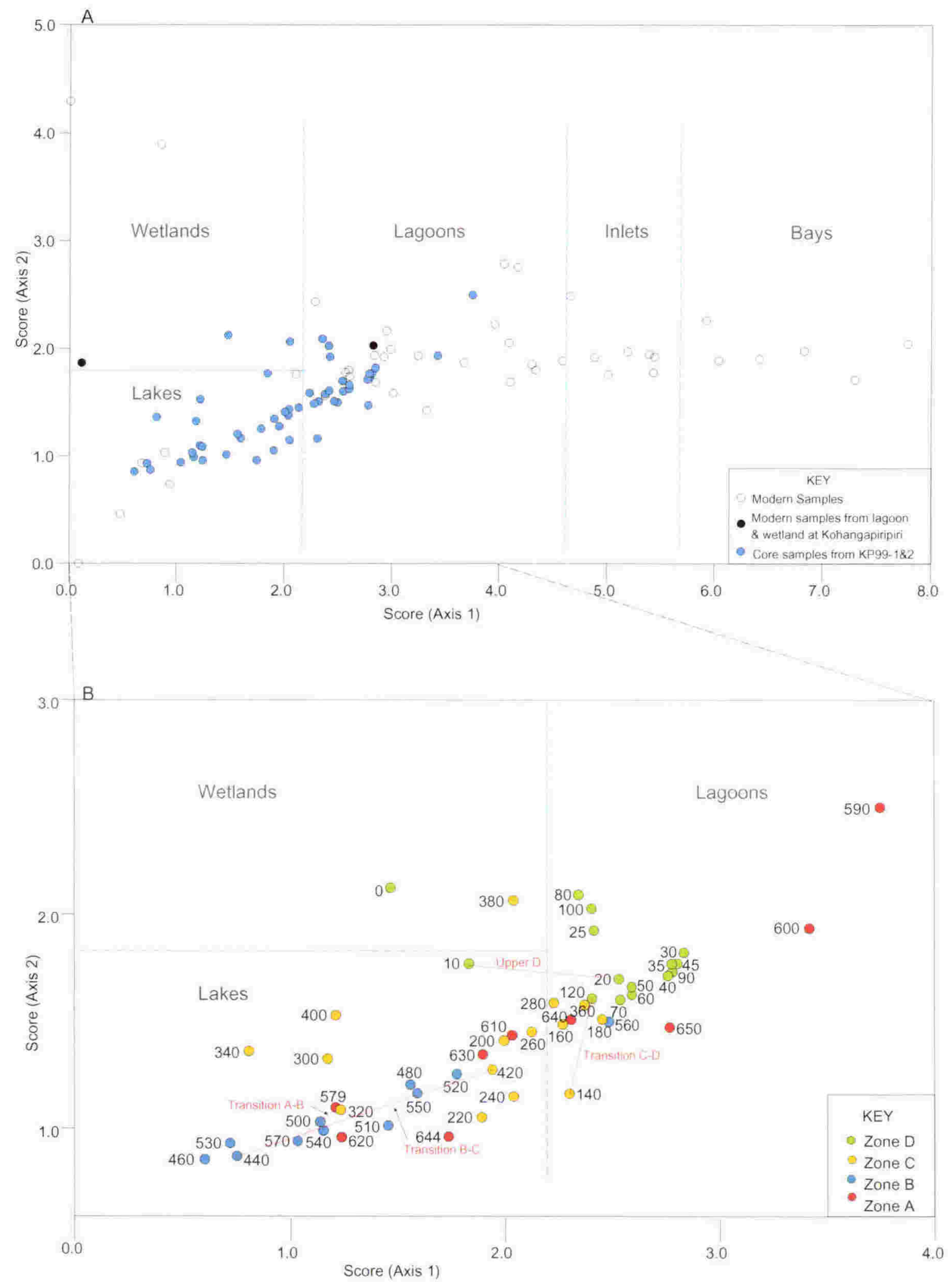

Fig. 6.6. Detrended correspondence analysis of calibration set sites with fossil samples from Lake Kohangapiripiri incorporated passively. A: Distribution of modern sites and division of axes into waterbody types. Coloured dots show where fossil samples plot in relation to modern sites. B: Left side of A enlarged to show samples from KP99-1\&2 labelled according to depth and coloured according to diatom assemblage zones. 
Diatom assemblage zones are fairly well separated by the DCA except for zone A samples that are spread throughout the lagoon and lake fields. This lack of consistency between samples within zone A is probably a result of allochthonous taxa, transported by landslides that occurred at this time, being incorporated into assemblages. For example in the inferred landslide deposit at $590 \mathrm{~cm}$ depth a large peak in Cocconeis placentula differentiates the assemblage at this horizon from the rest of zone A. Zone B samples plot in the lake field which confirms the fresh water nature of the waterbody at this time and suggests there was a depth of water greater than the very shallow depths typical of many of the calibration set lagoon sites. Samples of zone $\mathrm{C}$ also plot predominantly in the lake field and show a progression toward the left side of the lagoon field. This progression is likely to represent the gradual shallowing that occurs through the zone. Zone $\mathrm{C}$ samples are dominated by planktonic species very few of which were encountered in the calibration set so some of the scatter of zone $\mathrm{C}$ samples is attributed to lack of modern analogues. Zone D samples plot in at the left side of the lagoon field reflecting the shallowness and slight brackishness of the waterbody at this time. The upper two samples $(0$ and $10 \mathrm{~cm}$ ) plot in the wetland and upper lake fields respectively confirming the freshening and shallowing observed for these samples.

Distances between zones are moderate indicating the magnitude of environmental change between zones was also moderate. The measurement of distance between zones A and B is hampered by the large spread of samples in zone A. The relatively large distance between the upper two samples of zone D and the remaining samples support the idea that a fairly large-scale change occurred at this time. Although the diatom assemblages of zone $\mathrm{B}$ and $\mathrm{C}$ are most similar to the coastal lake assemblages of modern samples, the corresponding waterbodies are referred to as permanently closed lagoons because of their proximity to the sea (a few hundred metres rather than the $\sim 1 \mathrm{~km}$ defined for coastal lakes in Chapter Three). The fact that they plot in the lake field highlights their relative depth and freshness in comparison with the permanently closed lagoon of zone D.

\subsubsection{Application of Salinity Transfer Function}

Weighted averaging calibration and the modern analogue technique were used to reconstruct salinity of diatom assemblages from KP99-1\&2 (as introduced in Chapters Three and Four). The DCA (Fig. 6.6) shows that the calibration set is adequate for reconstruction of samples from KP99-1\&2 because core samples plot within the gradient defined by the calibration set samples. However there are few calibration set samples landward of the most landward core samples and there are also few calibration set samples in the transitional field between lakes and lagoons. Both these factors are likely to introduce problems for reconstruction of samples from Lake Kohangapiripiri. Species present in the sedimentary sequence 


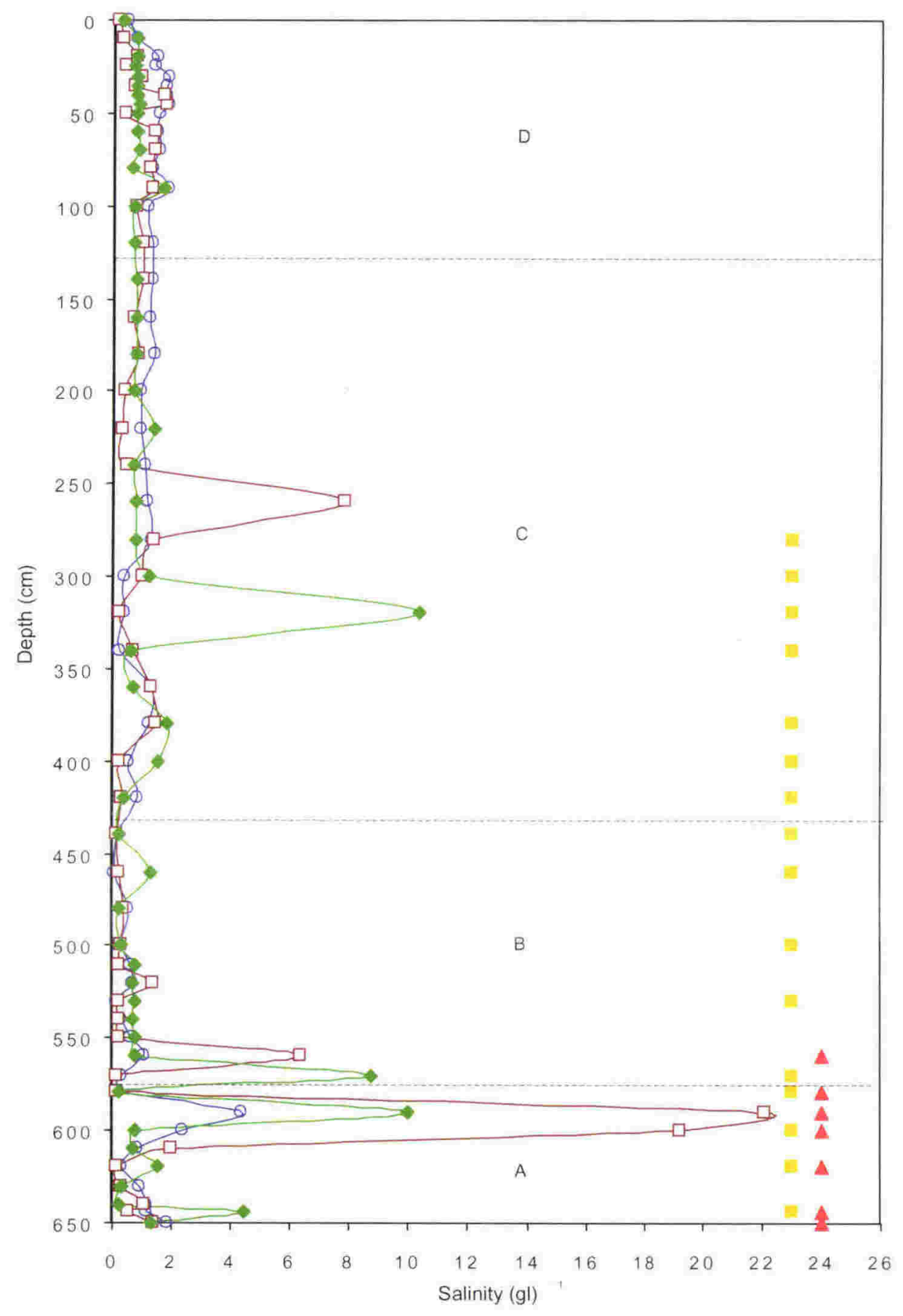

$$
\begin{aligned}
& \frac{\text { Reconstructions }}{- \text { WA }} \\
& \square \text { WA-tol } \\
& \text { Samples with: } \\
& \text { No close analogues } \\
& \text { Count <200 valves }
\end{aligned}
$$

Fig. 6.7. Salinity reconstructions for KP99-1\&2 using weighted averaging (WA), weighted averaging with tolerance downweighting (WA-tol) and the modern analogue technique (MAT). Dotted lines show diatom zone boundaries. 
of Lake Kohangapiripiri are well represented in the calibration set with $84 \%$ of species present.

The three models used for reconstruction, simple weighted averaging (WA), weighted averaging with tolerance downweighting (WA-tol) and the modern analogue technique (MAT) provide similar salinity reconstructions for KP99-1\&2 (Fig. 6.7). Salinity values average $1-2 \mathrm{gl}^{-1}$ in zone A with the high amount of variability probably a result of influxes to the waterbody as described above. Samples in zone B have the lowest salinity values of the sequence at around 0.5 $\mathrm{gl}^{-1}$. In zone $\mathrm{C}$ there are anomalously high values for several samples but these are probably overestimated as a result of the lack of close modern analogues and the remainder of the interval is reconstructed at about $0.9 \mathrm{gl}^{-1}$. Zone D is slightly higher than zone $\mathrm{C}$ at $1.0 \mathrm{gl}^{-1}$ and appears to freshen to present day values $(0.2-0.3$ $\mathrm{gl}^{-1}$ ) in the top two samples.

Salinity values do not change by a great amount across diatom assemblage zone boundaries because zones are defined to a greater extent by habitat preferences of species than by salinity preferences. However there is a decline in salinity at the A-B transition from fresh brackish to fresh water supporting the idea that influxes from the sea could not occur as easily during the interval of zone B. The upper three zones are all fresh water so little movement along the coastal gradient is inferred for the sequence at Lake Kohangapiripiri. This supports the conclusion that the trend apparent in the DCA of Lake Kohangapiripiri samples (Fig. 6.6) is predominantly reflecting water depth change rather than large changes in salinity.

\subsection{PALEOENVIRONMENTAL RECONSTRUCTION}

Sedimentology and diatom assemblages of KP99-1\&2 provide evidence with which to infer the nature of past environments of deposition at Lake Kohangapiripiri (Table 6.2). These depositional environments represent two different waterbody types but differences in water depth enable division of the sequence into four main paleoenvironments that have existed at the site over the last 7500 years (Fig. 6.8).

Between $\sim 7500$ and $\sim 6800$ cal. years BP a waterbody existed on the coast at this site but there is only a small thickness of zone $\mathrm{A}$ at the base of the sequence and for much of this section there is gravel dispersed throughout the mud, together with layers of gravel and sand, indicating that normal sedimentation was interrupted by landslides. The presence of small proportions of marine and brackish marine diatoms and the fresh brackish salinity values suggest the waterbody had an indirect or episodic connection with the sea. Therefore it is likely that a barrier existed across the mouth of the valley at this time and was 


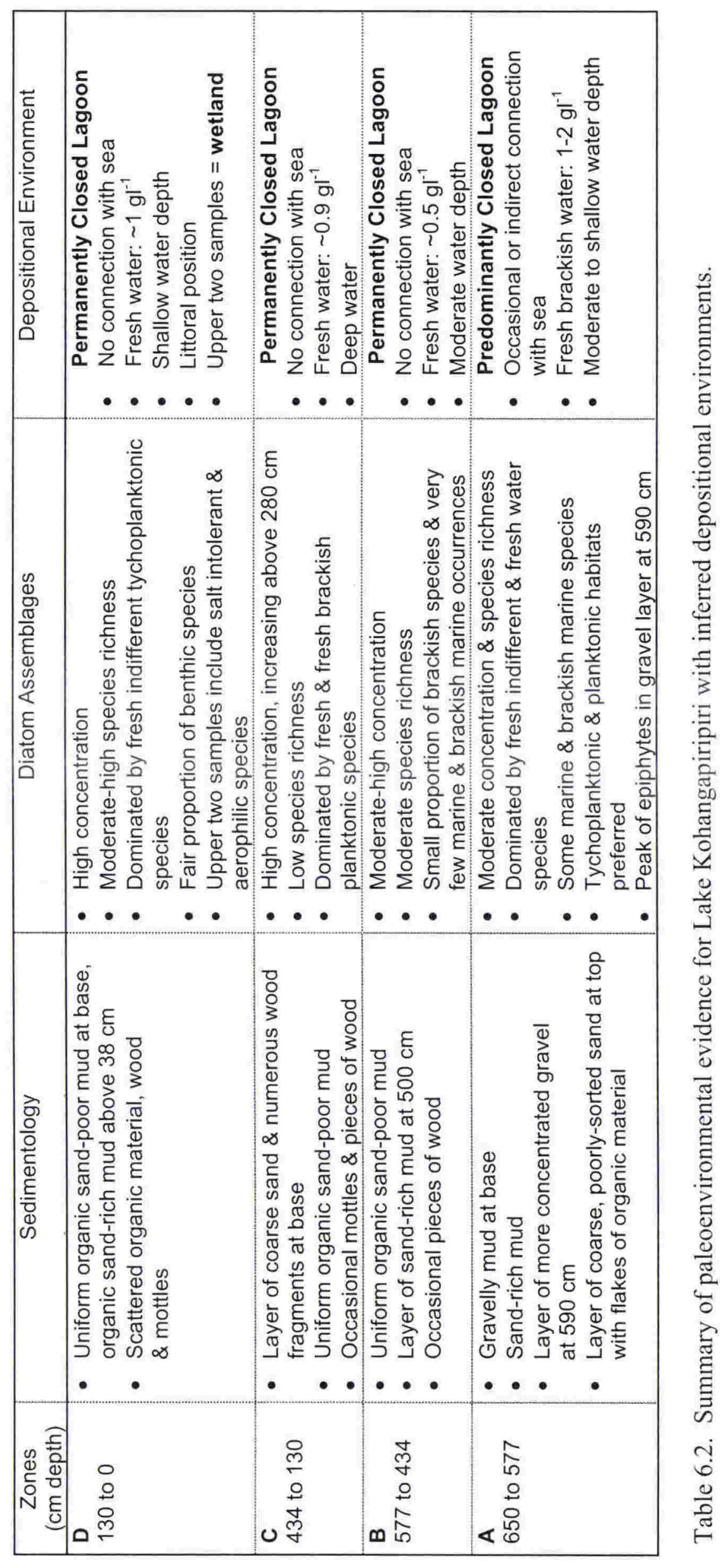



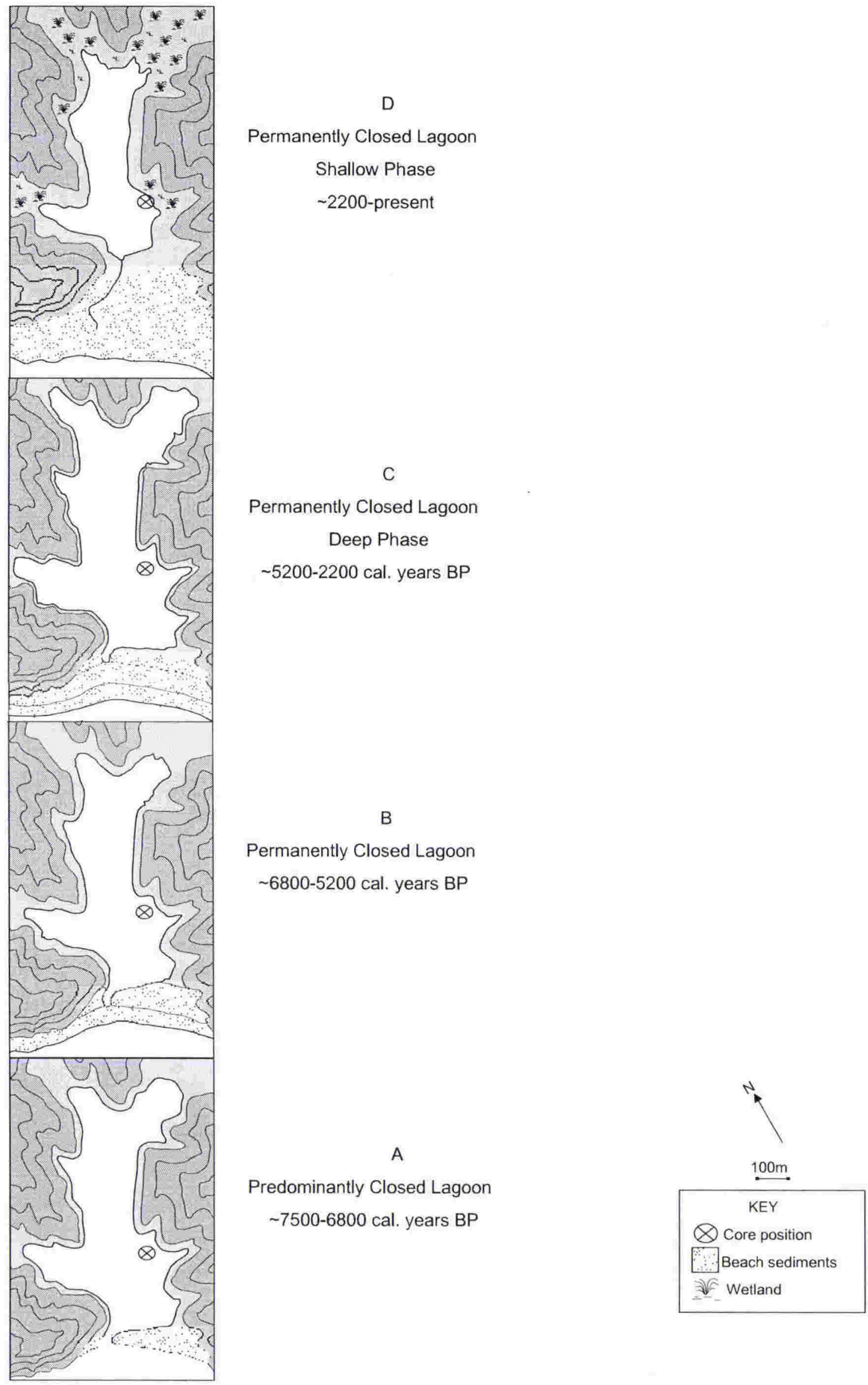

Fig. 6.8. Reconstruction of paleoenvironments that have existed at the Lake Kohangapiripiri site over the last 7500 years. 
only breached during high tides or when high water levels existed in the lagoon. The paleoenvironment is inferred to have been a predominantly closed lagoon of moderate to shallow water depths.

Sedimentation of zone B was more stable with only one coarse-grained layer deposited. The waterbody was almost completely isolated from the sea with reconstructed salinity values being in the fresh water range and marine diatoms only occasionally transported into the system. This implies the barrier had enlarged by the time of zone B. A permanently closed lagoon of moderate water depth is inferred to have existed at the site. The landslide deposit within zone B at $500 \mathrm{~cm}$ depth coincides with the disappearance of marine and brackish marine diatoms from the record. This may indicate the occurrence of an event involving an increase in barrier height and landsliding in the catchment but because of the small proportions of diatoms involved in this observation, higher resolution counting would be required to confirm the occurrence of a significant change at this point.

A deep period in the lagoon's history is inferred from the high proportions of planktonic species throughout zone $\mathrm{C}$. The water was fresh throughout this time indicating that despite a relative rise in water table level the waterbody remained completely isolated from the sea. Gradual shallowing within this deep water interval is inferred from the small increase in tychoplanktonic species relative to planktonic species towards the top of zone C. Diatom assemblages have affinities with modern assemblages from coastal lakes but because of the proximity to the open sea of the waterbodies of both zones B and C they are referred to as permanently closed lagoons.

In zone $\mathrm{D}$ there are high proportions of tychoplanktonic species and an increase in benthic species. The assemblages indicate that zone D was a shallower environment than that of zone $\mathrm{C}$. Coarsening of sediment at the top of the zone is consistent with the higher energy regime of a shallower site. The upper two samples of zone D suggest that a further shallowing and freshening of the waterbody took place in the very recent past. The existence of salt intolerant and aerophilic species in these samples suggests wetland conditions initiated close to the site as occurs in littoral positions around the waterbody today.

\subsection{ENVIRONMENTAL TRANSITIONS}

\subsubsection{Introduction}

The sedimentary sequence at Lake Kohangapiripiri occupies the most landward position on the coastal gradient of all the Holocene sites investigated in this thesis. Isolation from the sea is an important change at the base of the sequence but only the final stage of isolation is preserved. What is more notable about the sequence 
is the length of time it has been isolated from marine influence despite its low elevation and close proximity to the open sea. Instead of relative changes in sea level, the recent Holocene history of Lake Kohangapiripiri has been dominated by relative movement of water table level and influxes to the waterbody from the catchment.

Paleoenvironmental reconstruction of the waterbody indicates that a barrier has existed across the mouth of the valley for the last 7500 years. At one stage the barrier was large enough to completely isolate a relatively deep waterbody from the open sea a few hundred metres away. Therefore sediment transport and deposition in Fitzroy Bay have been important factors in the history of Lake Kohangapiripiri. However the fact that the barrier consists of several raised beach ridges indicates that coseismic uplift is likely to have played a part in the formation and endurance of the barrier. Historical evidence suggests that $2.1 \mathrm{~m}$ of uplift occurred at Pencarrow Head immediately west of the lake in the 1855 Wairarapa earthquake (Grapes and Downes, 1997). Matthews (1980) suggests that rapid aggradation of the beach in front of Lake Kohangapiripiri since 1941 is related to the arrival of a pulse of gravel derived from earthquake-induced landslides of the 1840s and 1850s. This indicates that sediment supply and transport and coseismic events have acted together to build and maintain a barrier between the lagoon and sea.

Coseismic events are also likely to have impacted on the lagoon itself. At Lake Kohangapiripiri there are three main transitions in depositional environment and two minor transitions one of which requires further investigation and the other occurs so recently it is hard to characterise (Fig. 6.9). Although each environmental transition is consistent with a coseismic origin, none of these changes provide much information with which to infer the cause of change.

\subsubsection{Description of Transitions}

Transition A-B at $577 \mathrm{~cm}$ depth involves deposition of a layer of sand and completion of the final stage of isolation of the waterbody from the sea. The cause of change was not necessarily of great magnitude considering the waterbody was already predominantly closed. The shift in diatom assemblage occurs moderately suddenly at this point but without the earlier part of the record it is hard to put this shift in context. The transition appears to occur near the base of the sedimentary sequence from the northeastern wetland of Lake Kohangapiripiri (Cochran et al., 1999) but synchroneity is hard to establish because part of the record is missing near this point. It is likely the transition was caused by final closing of the barrier that was in place between the waterbody and sea at this time. This could have occurred gradually through sediment accumulation or rapidly in a storm event or earthquake uplift. The layer of sand deposited at this time consists of angular, poorly sorted greywacke clasts consistent with the fine toe of a landslide off the nearby hillside. The concurrence of this deposit with the change in diatom 


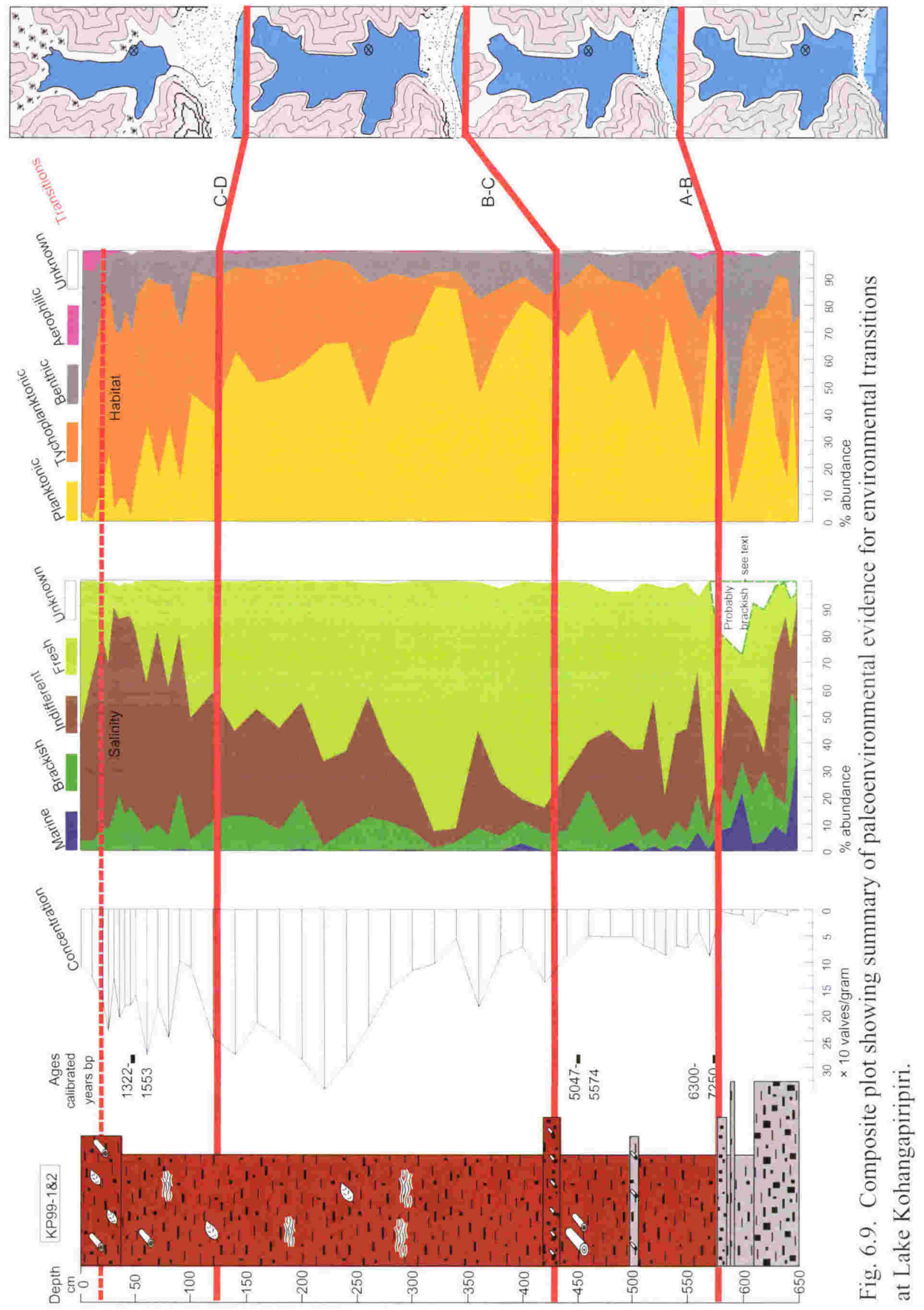


assemblage favours a catastrophic cause rather than continuation of sedimentary processes.

Within zone B deposition of an inferred landslide deposit appears to coincide with the disappearance of marine and brackish marine species. However marine and brackish marine species were already present at very low abundances so no large scale environmental change is required to explain their disappearance. For this reason and because higher resolution diatom counts would be required to check the nature of this change, it is presented as a tentative transition not warranting too much emphasis.

Transition B-C at $434 \mathrm{~cm}$ depth involves deposition of a layer of sand and wood fragments within the lake sediments and initiation of a deep water phase in the waterbody's history. This change is of moderate magnitude as it is estimated to involve at least a metre increase in water depth. It occurs suddenly because the three planktonic species that dominate zone $\mathrm{C}$ all increase noticeably at this depth. The transition is a lake-wide event that occurs synchronously in the wetland core (Cochran et al., 1999). A sudden deepening of the lake basin could be caused by coseismic subsidence, compaction due to liquefaction of soft sediments, or increased ponding behind a higher or less permeable barrier. The incorporation of layers of sand and wood into the record indicates that disturbance of the catchment occurred concurrently.

Transition C-D at $130 \mathrm{~cm}$ depth is not represented by a change in sediment but involves a shallowing of the lake as inferred from the diatom flora. Gradual shallowing occurred throughout the upper section of zone $\mathrm{C}$ but at the C-D transition a larger magnitude and more sudden change in water depth was achieved. A very similar shallowing signal is found in the sedimentary sequence of the wetland. This transition could be the result of sediment accumulation and infilling of the lake basin. A moderately sudden change in the diatom flora may occur when accumulation reaches a point where light levels on the lake floor make habitation of the sediments favourable to diatoms. However this would result in fairly localised changes around the shallow margin of the waterbody. Transition C-D occurs synchronously at both ends of the lake suggesting that a lake-wide cause of shallowing is required.

A more sudden shallowing appears to be represented by the top two samples within zone D. This involves a fairly large change in species and the exact signal occurs synchronously in the wetland core. Because it is only represented by the top two samples in each core, further investigation is needed to confirm the nature of this change. A sudden shallowing of lake-wide extent would be consistent with an earthquake-induced change in water table level. 


\subsubsection{Change in Elevation}

At Lake Kohangapiripiri, core elevations and diatom assemblages could be taken to indicate that substantial subsidence has occurred at the site over the last 7000 years because evidence of marine influence in the sedimentary sequence is nearly five metres below present sea level (Fig. 6.10). However, in this case, the existence of terrestrial sediments below sea level is considered to be the result of a large barrier having been in place at the mouth of the valley throughout this time. Estimates of relative sea level change cannot be made from the sedimentary sequence because of the complete lack of tidal influence at the site over the last 7500 years. Estimates for the amount of deepening and shallowing may provide rough guidelines as to the amount of vertical movement of base level but water depth preferences of diatom species are not known well enough to provide quantitative estimates of change in water depth.

A better indication of the amount of vertical movement of base level is obtained from the raised beach ridges in front of Lake Kohangapiripiri. The height of the oldest raised beach ridge is $8.5 \mathrm{~m}$ amsl, about $5 \mathrm{~m}$ above the modern storm beach. The beach ridge raised in the 1855 earthquake is about $2 \mathrm{~m}$ above the modern storm beach (Grapes and Downes, 1997) so about $3 \mathrm{~m}$ uplift occurred prior to this and since the formation of the oldest storm beach. The number of events responsible for this uplift is not known because storm beach ridges were not necessarily preserved from every uplift event. The easily transportable nature of sediment on the Fitzroy Bay coast (compared with, for example, the boulder beaches of Turakirae Head) and the power of the southerly swell in Cook Strait could well have caused beach ridges to merge or be destroyed if the amount of uplift was not large. Extraction of gravel in recent times has blurred evidence of the 1855 raised beach ridge.

\subsubsection{Age of Transitions}

The age of transition A-B at 6300-7250 cal. years BP was determined by a radiocarbon date on organic mud immediately above the transition horizon. Two radiocarbon dates, one below transition $\mathrm{B}-\mathrm{C}$ and one above transition C-D were used to calculate ages for these transitions using maximum and minimum sediment accumulation rates. Transition B-C occurred between 5400 and 4900 cal. years BP and transition C-D has an age of about 2300-2100 cal. years BP. An estimate for the tentative landslide event within zone B was calculated in the same way to be 6000-5800 cal. years BP. Shallowing in the upper part of zone D occurs at about $15 \mathrm{~cm}$ depth so if the slow accumulation rate for this part of the core is real the change occurred about 50 years ago. If the accumulation rate has been affected by loss of sediment then the rate typical for the rest of the sequence of 1 $\mathrm{mm} / \mathrm{yr}$ suggests the shallowing occurred about 150 years ago. 


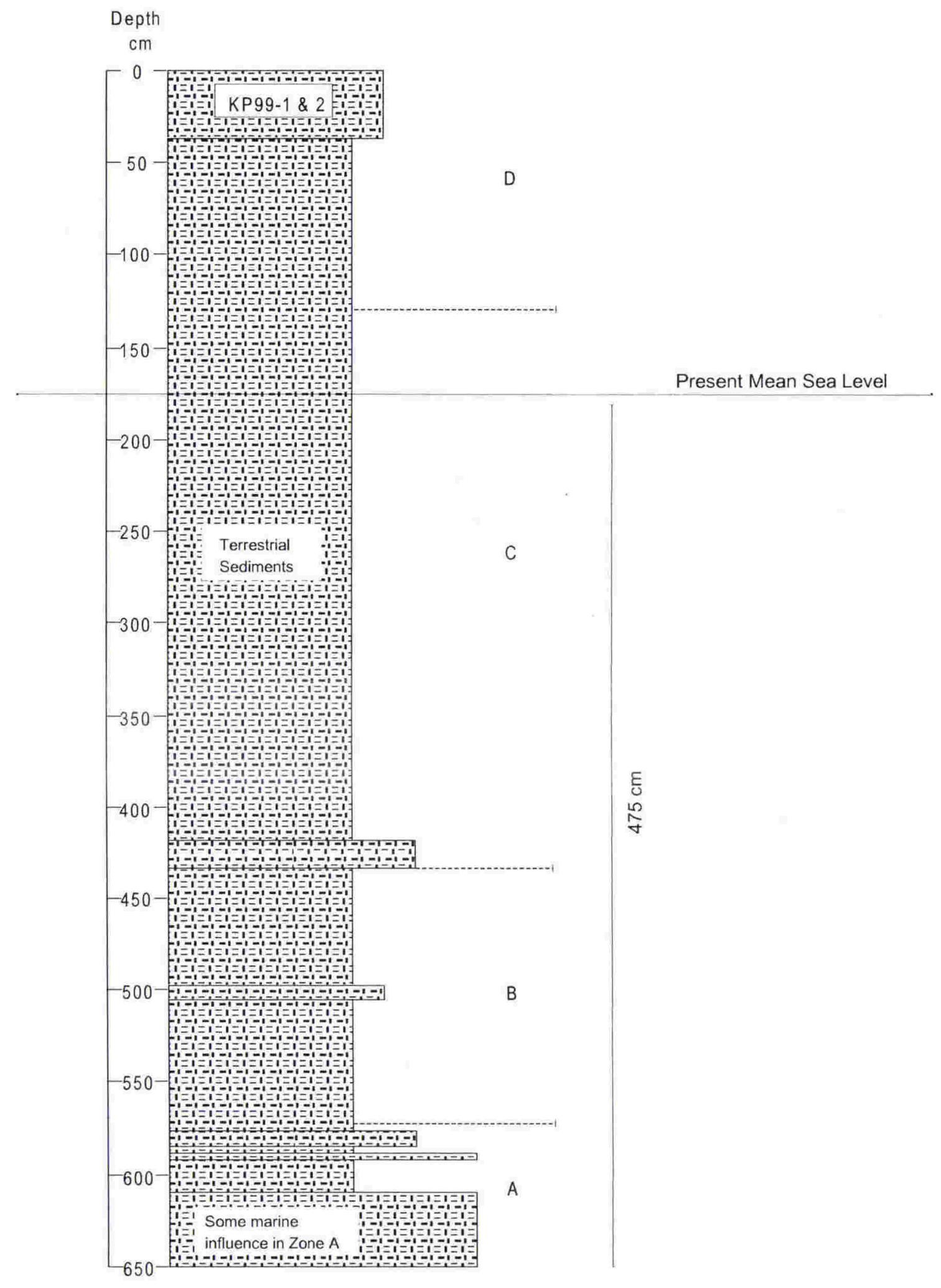

Fig. 6.10. Elevation of the sedimentary sequence at Lake Kohangapiripiri relative to present mean sea level. About $475 \mathrm{~cm}$ of terrestrial sediments exist below present mean sea level. 
As noted above, diatom assemblage zones recognised in KP99-1\&2 can be correlated with very similar zones in a landward core analysed in a previous study. The sedimentary sequence of the landward core was dated with six radiocarbon dates (Cochran et al. 1999) so ages for environmental transitions can be calculated as above. Ages calculated in this way indicate that transitions at each end of the waterbody occurred synchronously (within the resolution of radiocarbon dating).

\subsubsection{Effects of the 1855AD Earthquake at Lake Kohangapiripiri} Lake Kohangapiripiri is the only site of the three Holocene sites studied that is unarguably within the area of land uplifted in the $1855 \mathrm{AD}$ earthquake on the Wairarapa Fault. It is well documented by historical evidence that $2.1 \mathrm{~m}$ of uplift occurred at Pencarrow Head, strong shaking was felt throughout the Wellington region resulting in numerous small slips and several large landslides, and a tsunami inundated the southern coast of the North Island as a result of the earthquake (Grapes and Downes, 1997). The storm beach in Fitzroy Bay was uplifted (Adkin, 1955) but what affect did these occurrences have on the lagoon itself?

There are several 1840s maps of the Fitzroy Bay area that suggest the surface area of Lake Kohangapiripiri was more extensive prior to the earthquake than it is at present and that the outlet extended further towards the sea (Cochran, 1995). If it is assumed that the very recent sedimentary record is complete, then the affect of the earthquake on the lagoon was sudden shallowing of the waterbody and associated shrinking of the margins with further encroachment of wetland conditions. This is recorded in the change to diatom assemblages in the top $15-20 \mathrm{~cm}$ of sedimentary sequence at both ends of the lagoon. There is no indication of change in the sedimentology of the sequence and there is no evidence of shaking or marine inundation at this time. This has implications for detection of earthquake signatures in coastal waterbodies - where waterbodies are well protected from the sea a change in relative water table level may be the only effect preserved in the record. For example small landslides produce localised deposits that will not be present at all core sites and a large tsunami wave would be required to overtop or breach an $8.5 \mathrm{~m}$ high gravel barrier and deposit marinederived material in the back barrier environment.

Association of this sudden shallowing with the 1855 earthquake strengthens the possibility that transition C-D was also caused by coseismic uplift. Transition C-D consists of sudden shallowing without any other features indicative of an earthquake. Characterisation of the changes to diatom assemblages as a result of a water depth change of about two metres is not attempted here because higher resolution sampling and counting would be required to do this adequately. 


\subsection{CONCLUSIONS}

Sedimentology and diatom analysis of a $650 \mathrm{~cm}$ long, 7500 year old sedimentary sequence from the seaward end of Lake Kohangapiripiri were used to reconstruct past environment at the site. Three radiocarbon dates provided sufficient age control to confirm that environmental changes recognised in the seaward sequence were approximately synchronous with changes identified in landward cores from a previous study (Cochran et al., 1999). Therefore environmental transitions are assumed to be of lagoon-wide extent. Diatom assemblages were interpreted using traditional salinity and habitat preferences and also through comparison and calibration with a modern coastal diatom calibration set. Analysis resulted in reconstructions of waterbody type, water depth and salinity.

Lake Kohangapiripiri has been predominantly isolated from the sea by a large gravel barrier for the last 7500 years. Despite much of the sedimentary sequence being below present sea level and only a few hundred metres from the open sea, there is little evidence of marine influence preserved in the sedimentary record. Four main paleoenvironments are recognised at the site and these reflect barrier growth at the base of the sequence and relative changes in water table level. Coarse-grained influxes into the lagoon indicate that catchment disturbance occurred in conjunction with the two oldest environmental transitions at about 6800 and $5200 \mathrm{cal}$. years BP.

This association suggests transitions were caused by catastrophic events rather gradual barrier growth. Another inferred landslide deposit that is only associated with a minor change in depositional environment occurred about 5900 cal. years BP. The third main environmental transition at about 2200 cal. years BP involved lagoon-wide shallowing. Lagoon-wide shallowing also occurred in very recent times and may be the result of uplift in the 1855 Wairarapa earthquake. 


\section{CHAPTER SEVEN}

\section{A Paleoecological Contribution to Wellington's Paleoseismic Record}

\subsection{SCOPE OF CHAPTER}

Holocene histories of three waterbodies in the Wellington region are described in Chapters Four, Five and Six and at each of these sites evidence for sudden environmental change is encountered. In this chapter, a summary of Wellington's existing paleoseismic record, derived predominantly from fault trench studies and raised beach ridge sequences, is presented. This existing history provides a record of past large earthquakes with which to compare the record of environmental transitions detected in this study. The main features of environmental transitions are summarised, and a distinction is made between transitions that are considered to provide evidence for coseismic movement of base level, and those that are consistent with the occurrence of a large earthquake, but do not provide independent evidence for such an event. The timing of previously recognised earthquakes is compared with the timing of environmental transitions in the sedimentary record documented here.

\subsection{INTRODUCTION}

Paleoseismological studies involve detection and interpretation of geological evidence for past movement along and across faults. A general aim of such studies is to characterise the nature of fault movement and earthquake occurrence over an extended period of time, that is geological time rather than historical time. Historical records such as seismicity catalogues are useful to the study of earthquakes because they provide extensive information about events too small to be preserved in the geological record. In some cases written descriptions of large un-seismically recorded earthquakes provide enough information to estimate basic parameters of movement (eg, Grapes and Downes, 1997). However because of the generally long time interval between major earthquakes on a single fault, the generally short period for which seismicity has been recorded in most countries, and the importance of major earthquakes from a hazard perspective, geological investigations are required to supplement historical records of fault movement. 
Paleoseismology is inextricably linked with seismic hazard analysis through the idea that 'the past is the key to the future'. In other words, sufficient knowledge of past earthquake occurrence should provide insight into future earthquake occurrence through development of models that describe fault behaviour. For many seismically active regions the Gutenberg-Richter relationship suggests that earthquakes become about ten times less frequent with every unit increase in magnitude (Sibson, 1989). For individual faults there are a number of models that attempt to describe fault behaviour. Some faults have been shown to follow a time-predictable model in which the time that elapses between earthquakes is related to the amount of movement that occurred in the previous earthquake (Thatcher, 1984). Study of several fault zones with long paleoseismic records led to development of the characteristic earthquake model that suggests individual faults and fault segments frequently generate similar sized earthquakes with a narrow range of magnitudes around the maximum (Schwartz and Coppersmith, 1984). Examples of non-periodic, chaotic recurrence patterns, period recurrence and clustering of earthquakes in time have all been documented from various faults around the world. In New Zealand it appears that different models of fault behaviour are required to explain fault movement in each of the main tectonic provinces (Berryman and Beanland, 1991). At present there is not a large enough paleoseismic dataset to thoroughly assess different models and describe settings in which they are applicable, so the challenge remains to enlarge the dataset and continue model development and assessment.

In the Wellington region of New Zealand the existing paleoseismic record indicates that large surface rupture earthquakes have been a feature of the region's history throughout the late Quaternary. Slip rate estimates have been calculated for most major active faults and much evidence of past earthquakes has been documented and dated. However more information is desirable because on some faults considered active no evidence of past earthquakes has been documented. On other faults only the last couple of events have been dated, and on the one fault where many events have been identified, estimates of timing do not coincide well between different localities. A source of information about past earthquakes that is proving to be useful internationally and has not previously been utilised in Wellington, is the sedimentary record. In this chapter 'sedimentary record' refers specifically to sequences deposited in lakes and coastal waterbodies rather than fault related sequences such as colluvial wedges. One main aim of this thesis is to contribute to Wellington's paleoseismic history by investigating the Holocene sedimentary record for evidence of earthquakes. 


\subsection{WELLINGTON'S PALEOSEISMIC RECORD}

\subsubsection{Previous Work}

Paleoseismic work in the Wellington region has focused predominantly on identifying earthquakes associated with each major fault. Therefore this section is arranged according to major faults from east to west across the region. Studies of raised beach sequences not directly related to a particular fault are outlined at the end of the section. Mention is also made of the subduction interface beneath Wellington although no large magnitude earthquakes are known to have occurred on it.

\section{Wairarapa Fault}

The Wairarapa Fault is situated on the western side of the Wairarapa Valley about $30 \mathrm{~km}$ east of Wellington city (Fig. 7.1). It is an active fault with an estimated Quaternary slip rate of 6-11 mm/yr, which accounts for one third to a half of the boundary parallel component of plate motion (Grapes, 1999). The fault trace is well defined for a distance of $90 \mathrm{~km}$ north of Palliser Bay and it continues south into Cook Strait (Carter, et al., 1988) and north to southern Hawkes Bay as a series of subparallel fault traces (Grapes, 1999). There is much geomorphic evidence along the fault for occurrence of repeated large surface rupture earthquakes and detailed information of prehistoric earthquakes comes from three main sources: raised beach ridges at Turakirae Head, offset river terraces on the Waiohine River, and stratigraphy of a fault trench excavated near Tea Creek Road.

At Turakirae Head on the south coast immediately west of the Wairarapa Fault, a series of raised beach ridges are recognised as being the result of repeated large magnitude coseismic uplift events (eg, Wellman, 1969). Moore (1987) presented numerous radiocarbon dates of shell and organic material in an attempt to date earthquakes responsible for uplift of each beach ridge. Hull and McSaveney (1996) re-assessed existing interpretations in the light of modern material being incorporated into the beach previously considered as that uplifted in 1855 AD. Radiocarbon dating of in-situ shell material supported their hypothesis that the beach was modern, the strength of the southerly swell having been underestimated previously. The current interpretation is that evidence of four earthquake events is preserved at Turakirae Head, of which the 1855 Wairarapa Fault event is the youngest, and all previous events are similar in magnitude. It is assumed that previous uplifts at Turakirae Head were caused by movement on the Wairarapa Fault because of the association of the 1855 uplift and Wairarapa Fault movement, the lack of any likely sources east of the Wairarapa Fault and the consistency of uplift to the northwest for structures west of the fault (Grapes, 1999). 


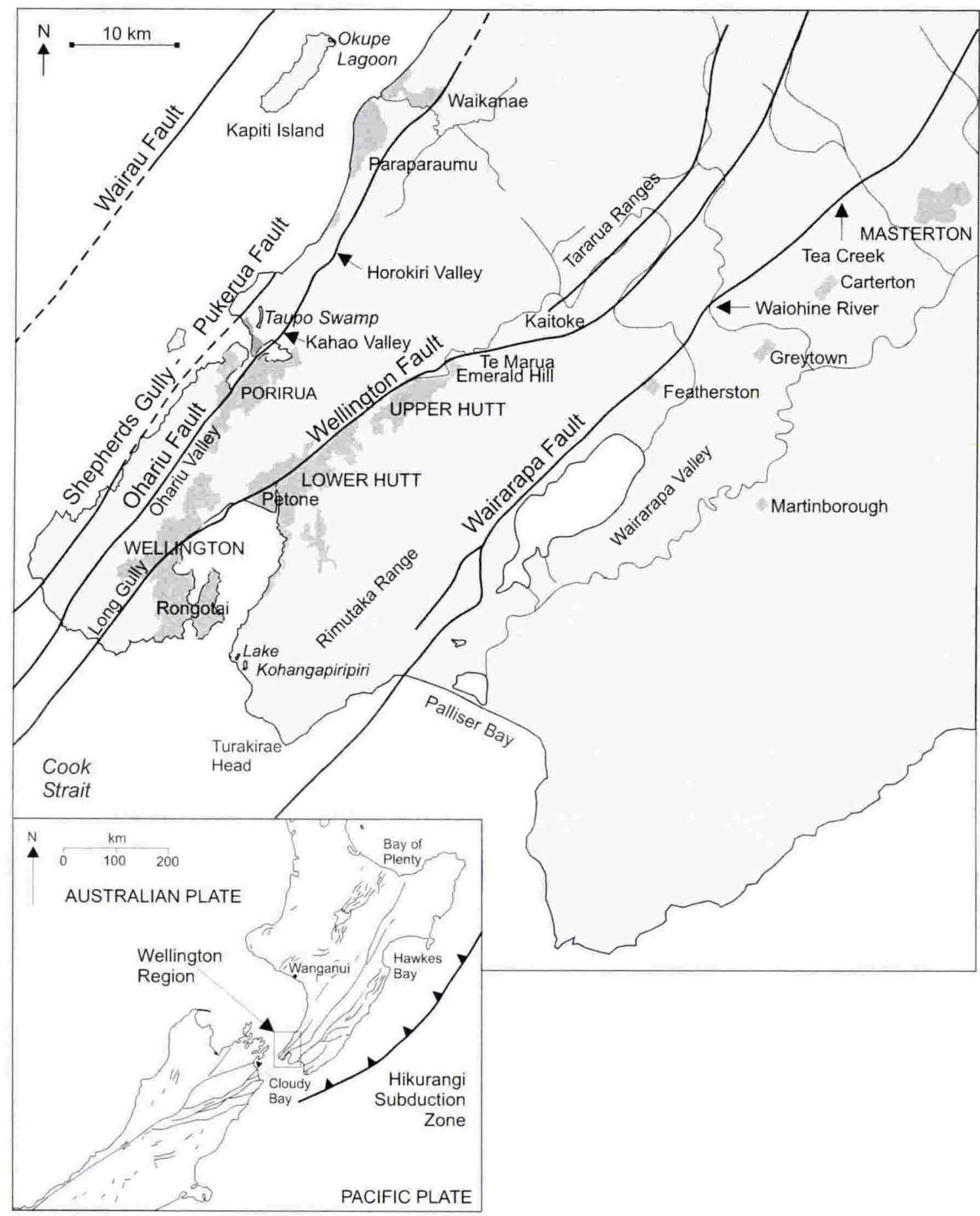

Fig. 7.1. Map of the Wellington Region with an inset of central New Zealand showing major active faults and paleoseismic localities mentioned in text. 
The Wairarapa Fault cuts a series of river terraces on the Waiohine River northwest of Greytown. Lensen and Vella (1971) surveyed eight terraces, measured seven vertical displacements caused by fault movement and five horizontal displacements. They calculated average rates of faulting of between 3.4 and $6 \mathrm{~mm} / \mathrm{yr}$ for the horizontal component and $0.5-0.9 \mathrm{~mm} / \mathrm{yr}$ for the vertical component based on age estimates for the Waiohine Surface (oldest terrace) of 35 , 000 and 20,000 years. Grapes and Wellman (1988) recognised a slightly different arrangement of seven terraces with six vertical and horizontal displacements. Grapes (1999) considers the Waiohine Surface to be about 11,000 years old and thereby calculates a much higher horizontal slip rate of about $11.5 \mathrm{~mm} / \mathrm{yr}$. Terraces have not been directly dated so timing of past earthquakes and slip rates are not tightly constrained. However measurement of 53 dextral displacements additional to those preserved at Waiohine River indicate that at least seven dextral displacement events of the same or greater offset as the 1855 earthquake have occurred along the Wairarapa Fault (Grapes and Wellman, 1988).

At a small drainage channel site near Tea Creek Road a trench was excavated across the Wairarapa Fault to look for evidence of past surface rupture earthquakes (Van Dissen and Berryman, 1996). Trench stratigraphy consisted of four cycles of sedimentation with each cycle composed of units considered to be the result of strong shaking and ponding of drainage due to offset along the fault scarp. Timing of these inferred earthquakes was estimated by radiocarbon dating of bark, twigs and seeds considered to have died as a result of strong shaking in the earthquakes.

Studies outlined above indicate there is much evidence for coseismic movement along the Wairarapa Fault in the late Quaternary. However timing of earthquakes is not well constrained. Most horizontal displacements have not been dated at all and event-related features that have been directly dated do not coincide well. It is generally accepted that the age of uplifted beaches at Turakirae Head should coincide with timing of lateral movement along the Wairarapa Fault (Grapes, 1999). However dating of these two components does not closely agree, for example note the complete offset of Hull and McSaveney's (1996) ages for uplift of Turakirae beach ridges and Van Dissen and Berryman's (1996) estimates of event timing from the Tea Creek trench (Fig. 7.2). It is likely this represents a systematic offset resulting from the type of deposits being dated in each case rather than two sites recording different events, but it means that absolute ages of fault movement are uncertain.

\section{Wellington Fault}

The Wellington Fault is a major active dextral strike-slip fault that runs from Cook Strait in the south, through Wellington City and the cities of Lower and 


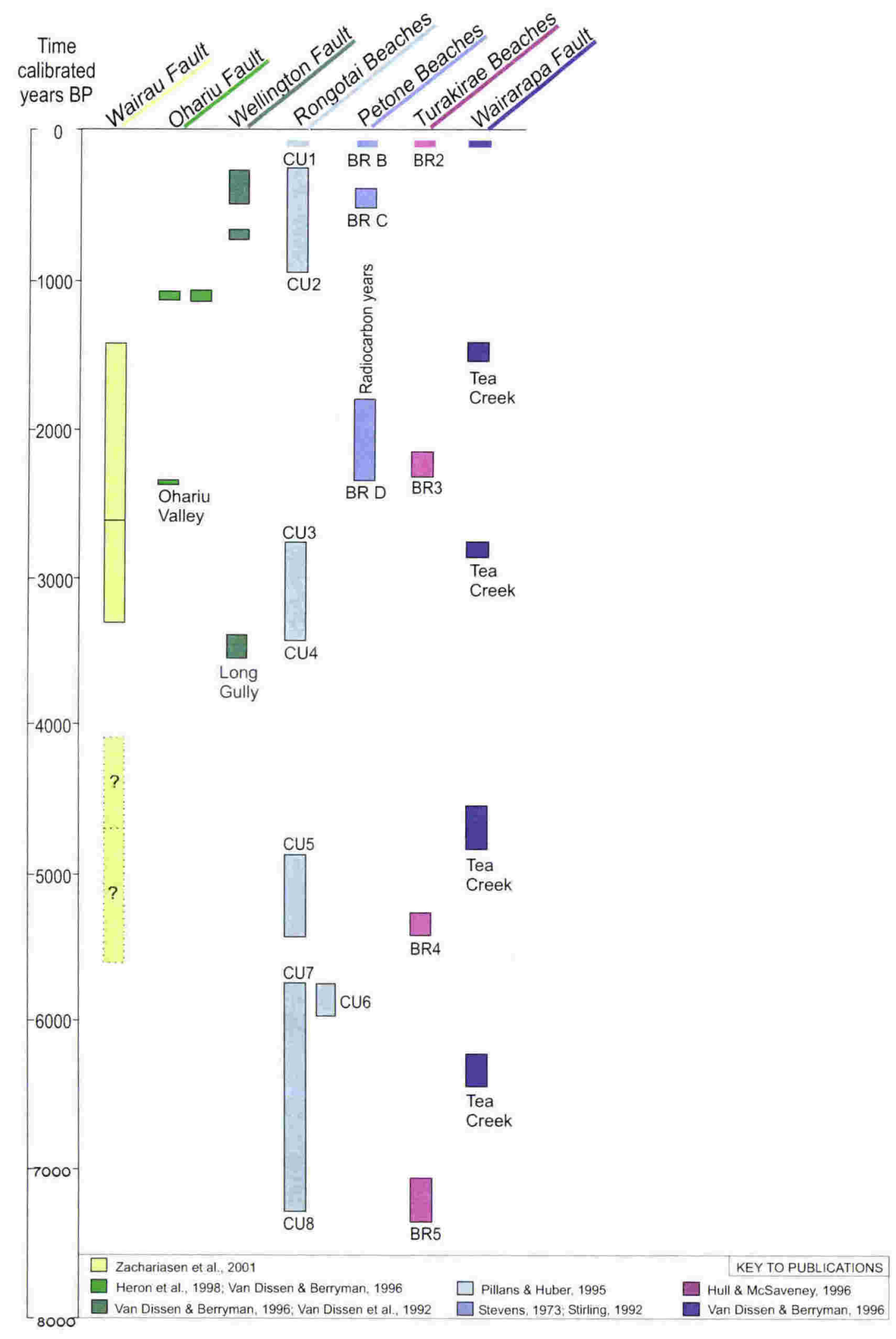

Fig. 7.2. Ages of earthquakes in the Wellington region identified in previous studies. Lengths of colour bars represent one or two sigma ranges of radiocarbon dates following those quoted in each publication. Labels for raised beaches also follow those used in publications. 
Upper Hutt, and north almost to the Bay of Plenty (Lensen, 1958). It has been traced on the continental shelf in Cook Strait heading towards Cloudy Bay where it may merge with the Ohariu Fault (Carter et al., 1988). There are numerous examples of laterally offset streams, ridges and terraces indicating recent movement along the fault, with most displaying a small component of vertical uplift to the northwest (Lensen, 1958). Displacements younger than 14,000 years indicate a change in sense of vertical movement, with uplift generally occurring to the southeast (Berryman, 1990). Paleoseismological studies have focussed on the southern section of the fault because of the great hazard that rupturing in this region presents. Berryman (1990) proposed that the $75 \mathrm{~km}$ long section of the Wellington Fault from Cook Strait to Kaitoke is a single rupture segment, referred to as the Wellington-Hutt Valley Segment. He calculated an average horizontal displacement length of 3.8-4.6 $\mathrm{m}$ from about five displacements across river terraces at Te Marua, and an estimated slip rate of 6-7.6 mm/yr based on displacement of terraces aged between 140,000 and 14,000 years at Emerald Hill. Using these estimates Berryman (1990) suggests that earthquakes of magnitude 7.1-7.8 are likely to occur on the Wellington-Hutt Valley segment every 485-783 years.

Van Dissen et al., (1992) provide ages for three surface rupture events on the Wellington Fault from radiocarbon dates on organic material in trenches excavated across the fault at Long Gully and Kaitoke, and from offset river terraces at Te Marua. In Long Gully the most recent event occurred about 300-450 cal. years BP and the penultimate event occurred at some time between 560-670 and 790-930 cal. years BP. An older event is recorded at 3380-3540 cal. years BP but there may have been younger events of which no evidence is preserved in the trenches. At Te Marua a maximum age for the last surface rupture event of 310-470 cal. years BP, agrees well with the Long Gully age.

A minimum age of 200-300 years is provided by existence of several unfaulted late Holocene terraces that would have required several hundred years to form following the last event. A second age estimate for the penultimate event of between $670-730$ and $770-830 \mathrm{cal}$. years BP comes from a tree in a trench across the fault at Kaitoke and falls within the bracket of time estimated at Long Gully. Although there is evidence of five large surface rupture events in the last 14,000 years at Te Marua and several much older events at Emerald Hill, only the last two events, and possibly a third older event, have been directly dated (Fig. 7.2).

\section{Ohariu Fault}

The Ohariu Fault extends northeast from the continental shelf in Cook Strait (Carter et al., 1988), through Porirua City to Waikanae (Heron et al., 1998). Recently a probable extension of the Ohariu Fault has been confirmed as an active strike-slip fault and named the Northern Ohariu Fault (Van Dissen et al., 1999). The Ohariu Fault is a dextral strike-slip fault with small amounts of uplift occurring to the northwest except at Porirua and Horokiri Stream where uplift 
occurs to the southwest (Heron et al., 1998). The fault has an average horizontal slip rate of 1-2 mm/yr, single event lateral displacements of $3.7 \mathrm{~m}$, a recurrence interval of 2000-5000 years for an estimated earthquake magnitude of 7.1-7.5 (Heron et al., 1998). These estimates are based on the preferred scenario of a single rupture segment from Cook Strait to Waikanae. However at Porirua Harbour there is an inferred $1.5 \mathrm{~km}$ right step that may be a pull-apart basin situated at the boundary between different rupture segments. Timing of 
penultimate events occurring between 1400-2600 and 2600-3300 cal. years BP respectively (Zachariasen et al., 2001). The seismic history of the northern section is unknown.

\section{Other Earthquake Studies}

Raised beach ridges that are not uniquely related to a particular fault also provide records of earthquake events occurring in the region. Pillans and Huber (1995) describe a series of paleoshorelines at Rongotai that provide evidence for the occurrence of eight earthquakes totalling 7-9 $\mathrm{m}$ of uplift in the last 6500 years. Five of these events are attributed to movement on the Wairarapa Fault because of their similar age to raised beaches at Turakirae Head described by Moore. However with reassessment of ages by Hull and McSaveney (1996), only three paleoshorelines correlate well with Turakirae beaches (Fig. 7.2). Small local faults are discounted as causing the remaining events because of the lack of evidence for Holocene movement on such structures. Movement on the Wellington Fault is a possibility because recent events appear to have caused uplift to the southeast despite the typical sense of movement being uplift to the northwest over geologic time (Lewis, 1989; Berryman, 1990).

Stranded beaches also occur at Petone and according to Stevens (1973), the youngest was uplifted in the 1855 earthquake on the Wairarapa Fault, the second was uplifted about 460 years ago and the third beach known as 'the rise' is older than 1800 years and may be as old as 2350 years. Stevens (1973) correlates the second beach with Wellman's (1969) beach ridge C at Turakirae Head and thereby with movement on the Wairarapa Fault, but since re-interpretation of the Turakirae Beaches, this correlation is no longer valid. Stirling (1992) recognises the three beaches described above $(\mathrm{B}, \mathrm{C}$ and $\mathrm{D})$ as well as two older beaches $\mathrm{E}$ and $\mathrm{F}$ that can only be constrained to being younger than 4000 years.

\section{The Subduction Interface}

Another potential earthquake source is the subduction interface that lies about 25 km beneath Wellington city (Begg and Mazengarb, 1996). There have been no large subduction thrust earthquakes in historic times although the $1855 \mathrm{AD}$ Wairarapa earthquake may have involved rupture of part of the subduction interface in addition to the Wairarapa Fault (Darby and Beanland, 1992). Little is known of the seismic behaviour of the subduction interface in this region but Van Dissen and Berryman (1996) derive estimates of certain parameters (earthquake of magnitude 8.2 , recurrence interval of 2000 years) through comparison with similar subduction zones worldwide. They show that such a movement represents a contribution to seismic hazard in the Wellington region in the 100-500 year time period. The position of the locked section of the subduction interface (that thought to slip in large earthquakes) is not known precisely but is estimated to underlie the southern North Island east of the Rimutaka Range (Robinson et al., 1998). 


\subsubsection{Evidence of Earthquakes in the Sedimentary Record}

\section{Introduction}

Environmental transitions that occur in the sedimentary sequences of Taupo Swamp, Okupe Lagoon and Lake Kohangapiripiri were investigated closely to determine possible causes of change (Chapters Four, Five and Six respectively). Characteristics used to look at transitions included the nature of the initial and resulting paleoenvironments, the sedimentary contact, any unusual features immediately preceding or following the transition, and the change in terms of species composition. In this section features of each transition are summarised and an assessment is made as to the likely cause of change using criteria (outlined in section 2.5, Chapter Two) of Nelson et al. (1996b).

Because of difficulties associated with measuring change in tidal elevation and water depth (Chapter Two), the difference in paleoenvironment across each transition is used as an indication of amount of change that occurred. Two factors are used to provide quantitative estimates of this change in order to avoid bias introduced in quantitative descriptions and to enable direct comparison between transitions. The salinity factor (Table 7.1) is the difference in paleosalinity across the transition calculated using an average of both weighted averaging reconstruction models. It is presented in log salinity units so that transitions occurring at high and low salinity values are directly comparable. The DCA scores are the difference in DCA axis scores (for axis one and two) between samples either side of the transition using the DCA analyses of fossil and modern samples presented in Chapters Four, Five and Six. This difference represents movement along the coastal waterbody gradient so is a quantitative measure of change in waterbody type.

\section{Summary of Environmental Transitions}

Ten transitions are identified in total (Table 7.1) and each transition meets the basic requirements of being laterally extensive within each waterbody according to available core samples and synchronous within the bounds of radiocarbon dating. At Okupe Lagoon and Taupo Swamp transitions are thought to have occurred suddenly because diatom assemblages change completely between five centimetre interval samples. At transition A-B in Taupo Swamp wet mounts were used to check between permanently mounted samples and the distance over which assemblages changed completely was three centimetres. At Lake Kohangapiripiri diatom assemblages change over $10 \mathrm{~cm}$ or $20 \mathrm{~cm}$ intervals but transitions were not checked at a higher resolution. 


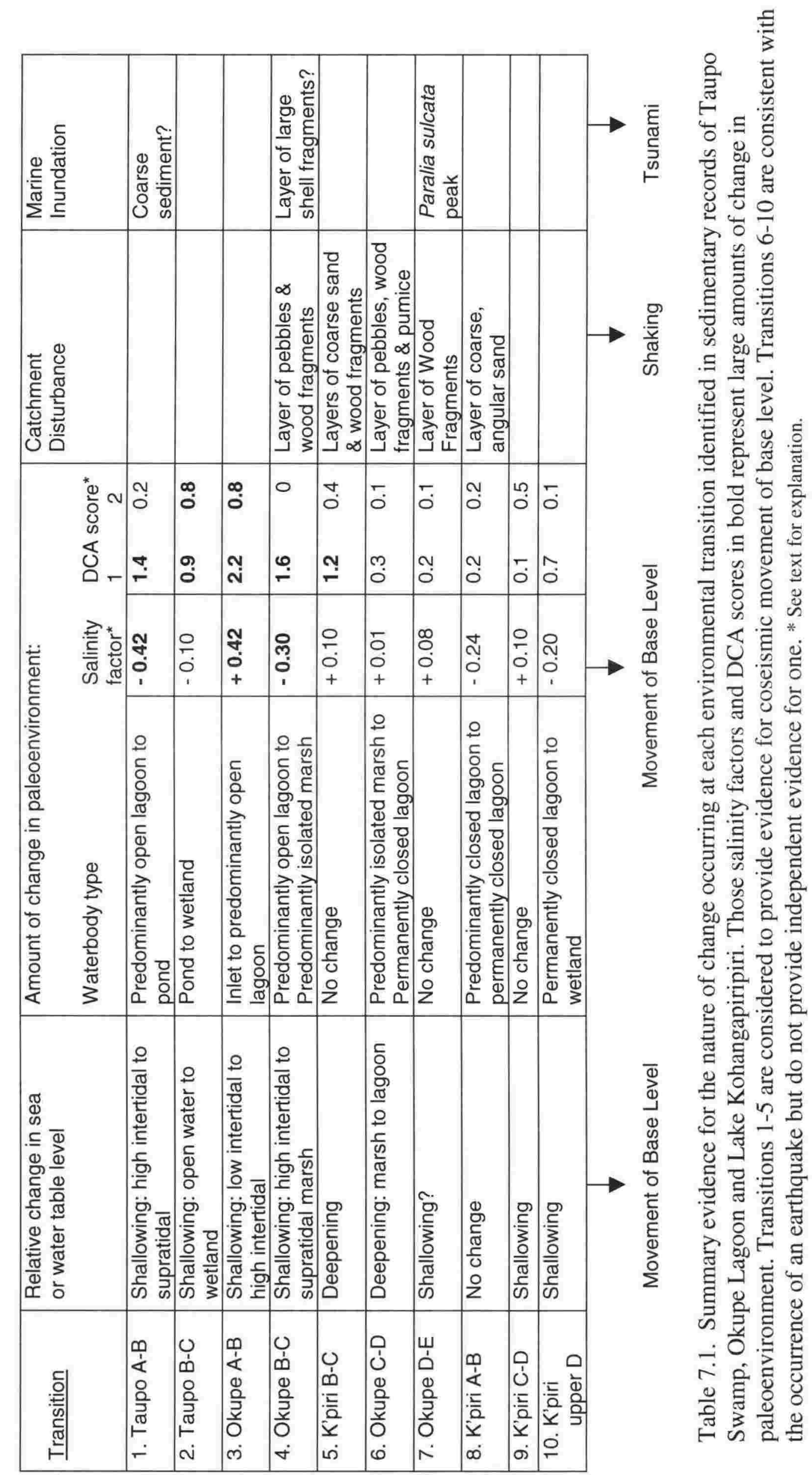


Transitions one to five (Table 7.1) consist of a change in relative sea level or water table level associated with a large amount of change in waterbody type (A-B and B-C at Taupo Swamp and Okupe Lagoon, and B-C at Lake Kohangapiripiri). Because of the good preservation of assemblages, transitions A-B and B-C at Taupo Swamp are the most convincing examples of large magnitude changes in relative sea and water table levels. The inland position of core sequences and their distance from valley sides may explain the lack of evidence for marine inundation or catchment disturbance. Although the amount of change in paleoenvironment at transitions A-B and B-C at Okupe Lagoon is probably overestimated because of the lack of modern analogues for the assemblage of zone $\mathrm{B}$, the transitions do represent permanent changes to relative sea level and are still considered to involve a large amount of change. In addition, transition B-C is accompanied by evidence for catchment disturbance and possible evidence for marine inundation. Unlike most other transitions, transition B-C at Lake Kohangapiripiri involves an increase in water depth as opposed to a decrease. Although the salinity factor is less useful in an isolated waterbody, a large environmental change is inferred from the DCA score. Coincidence of evidence for catchment disturbance at this horizon supports the idea that the change was coseismic in origin.

Transitions six to ten (C-D and D-E at Okupe Lagoon and A-B, C-D and upper D at Lake Kohangapiripiri) consist of little or no evidence for vertical movement and smaller amounts of paleoenvironmental change. Transitions C-D and D-E at Okupe Lagoon include strong evidence for catchment disturbance and for marine inundation at D-E only. The paleoenvironmental change that occurs across the transitions may well have been caused by a catastrophic event and is consistent with the effects of an earthquake but does not provide independent evidence for such an event. At Lake Kohangapiripiri transition A-B could well be due to closing of the barrier without any movement of base level. The coincidence of a landslide deposit at the transition horizon is the only piece of evidence to link the change to an earthquake. Transitions C-D and upper D involve lagoon-wide shallowing but the magnitude of change inferred from DCA scores is not great enough to unequivocally assign a coseismic cause to the transitions especially as there is no associated evidence for catchment disturbance or marine inundation.

At this initial stage, and taking into account all available information, it is proposed that transitions one to five provide independent evidence for coseismic movements of base level and transitions six to ten are consistent with the effects of an earthquake but do not provide independent evidence for such an event. This is the first study of its kind in the region so emphasis is on identification of transitions that fulfil some of the criteria rather than replication of transitions at several sites on each fault-bounded block. Ideally investigation of similar sequences at a greater number of sites would be undertaken to confirm the nature 
of transitions summarised above and strengthen the case for coseismic causes of change.

\section{Comparison of Ages with Previously Recognised Events}

Timing of environmental transitions recognised at Okupe Lagoon, Taupo Swamp and Lake Kohangapiripiri are compared with timing of earthquake events recognised in previous studies (Fig. 7.3) in order to evaluate synchroneity of transitions with events that are uniquely related to particular faults.

Transition A-B at Taupo Swamp was not directly dated but is bracketed by radiocarbon dates before and after the event horizon (Fig. 7.3). The ages for transition A-B at Okupe Lagoon fall near the centre of this bracket so until higher resolution dating proves otherwise, it can be assumed these transitions occurred at a similar time if not synchronously. The time span represented by transitions A-B at Taupo Swamp and A-B at Okupe Lagoon coincides well with the timing of uplift of beaches at Rongotai (CU $3 \& 4$ ). Inferred surface ruptures on faults at this time include one on the Wellington fault near the beginning of the time span. This estimate is the oldest known event on the Wellington Fault at 3540-3380 cal. years BP and comes from a trench in Long Gully (Van Dissen et al., 1992). There is also an event inferred from the Tea Creek Road trench on the Wairarapa Fault that occurs towards the end of the interval and a possible rupture of the Wairau Fault. An event greater than the magnitude $8+$ Wairarapa earthquake of 1855 would be required on the Wairarapa Fault at this time to cause the changes observed at the A-B transitions. This is because the distance of the sites from this fault (Okupe Lagoon is $\sim 45 \mathrm{~km}$ from the Wairarapa Fault) exceeds that over which any significant uplift occurred in 1855 . Therefore movement on the Wairau or Wellington Faults, because of their greater proximity to the sites, is favoured as the cause of transitions A-B over movement on the Wairarapa Fault.

Transition B-C at Taupo Swamp coincides even more closely with transition B-C at Okupe Lagoon than the A-B transitions. Within the resolution of radiocarbon dating these events can be considered synchronous because higher resolution age control would be required to prove that they occurred at different times. The bracket of time within which the B-C transitions occurred is $2720-2100$ cal. years BP. Within this time a shallowing event occurred at Lake Kohangapiripiri (transition C-D), and beaches were raised at Petone (BR D) and Turakirae Head (BR 3). In terms of fault rupture, a possible event on the Wairau Fault is recorded and there is a tentative estimate for the penultimate event on the Ohariu Fault put forward by Heron et al. (1998). Their date of 2360-2350 cal. years BP comes from a root collected from a trench across the Ohariu Fault in the Ohariu Valley. An earthquake on the Ohariu Fault at the time of the B-C transitions is consistent with the evidence at Taupo Swamp and Okupe Lagoon because the large magnitude of environmental change at these sites indicates a proximal source. Taupo Swamp is 


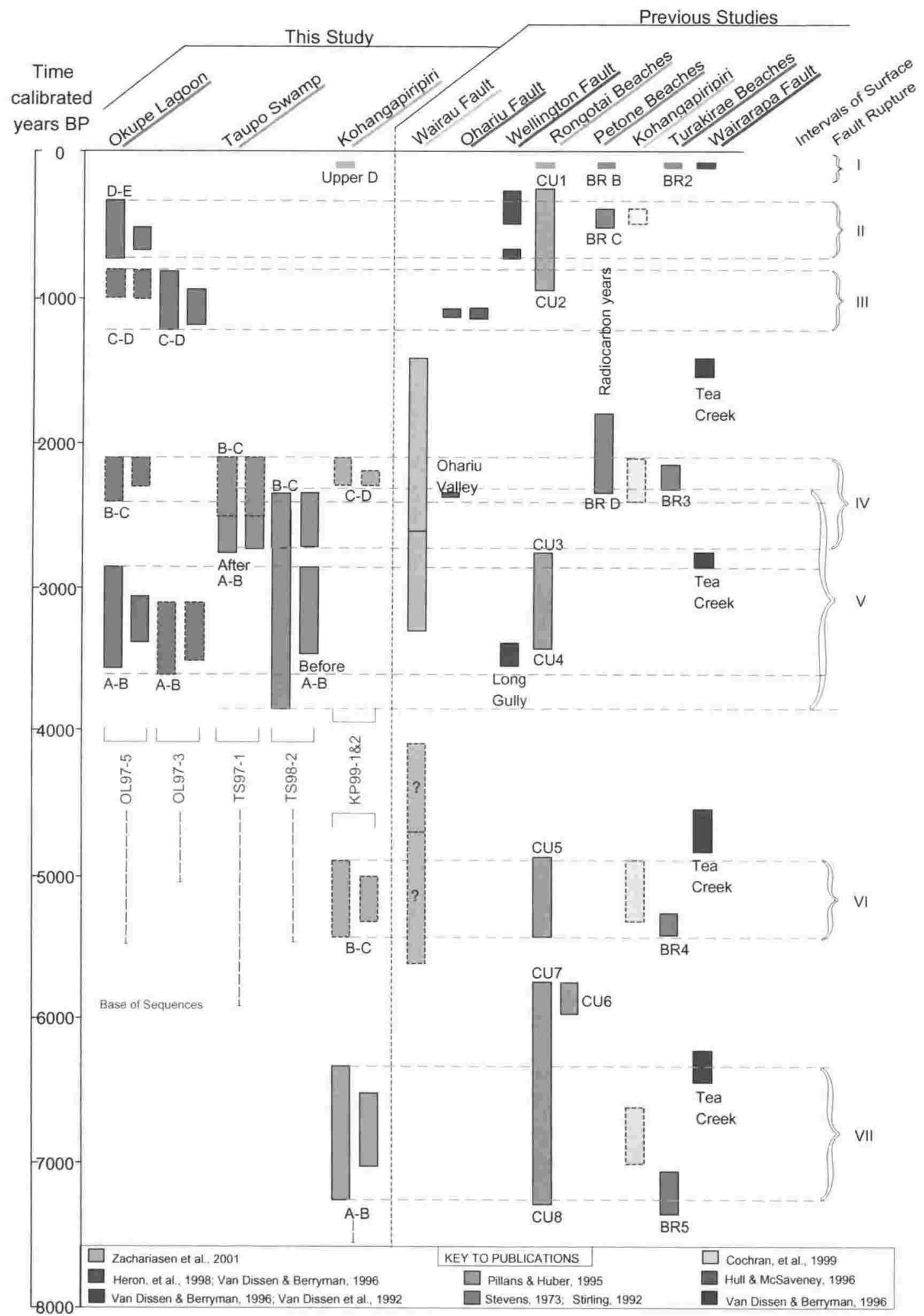

Fig. 7.3. Ages of environmental transitions recognised in this study compared with ages of previously recognised earthquake events in the Wellington region. Brackets highlight intervals of surface fault rupture as described in text and summarised in Table 7.2. Lengths of colour bars: One (right) and two (left) sigma ranges of radiocarbon dates are presented for sites in this study. Bars with dotted outlines are dates estimated from accumulation rates. For previous studies one or two sigma ranges are presented following those quoted in publication. 
about $2 \mathrm{~km}$ northwest of the fault in Kahao Stream and Okupe Lagoon about 12 $\mathrm{km}$ northwest of the fault at Waikanae. The sense of movement implied by the transitions, that of uplift to the northwest, is conformable with geomorphic observations on the Tongue Point-Makara, Makara-Porirua, Kahao Stream and MacKays Crossing sections of the fault (Heron et al., 1998). It is also possible that the B-C transitions were caused by movement on the Wairau Fault.

Coincidence of the A-B transitions at Taupo Swamp and Okupe Lagoon and also of the B-C transitions at these sites provides support for a regional cause of change in environment at each site as opposed to a local cause. Sudden shallowing and isolation from the sea occurring synchronously at sites nearly $30 \mathrm{~km}$ apart is explained more simply by an external mechanism, such as coseismic movement of base level, than by local sedimentary processes acting on different coasts and under different wind and wave climates.

Transition C-D at Okupe Lagoon coincides with estimates for the timing of the last earthquake on the Ohariu Fault. Van Dissen and Berryman's (1996) estimate is the overlap of four dates of the most recent event as represented at four different sites along the fault. Heron et al.'s (1998) estimate is the overlap of eight dates made under the assumption that the fault segments north and south of Porirua Harbour ruptured together. The date from Okupe Lagoon made on the distinct layer of wood, pebbles and pumice in core OL97-3, correlates well with these estimates. This indicates that transition C-D at Okupe Lagoon is likely to be a signature of the last surface rupture earthquake on the Ohariu Fault. Wood, pebbles and pumice deposited in the lagoon at the transition horizon suggest the area experienced strong shaking at this time. The slight rise in relative water table level may have been the result of differential subsidence due to liquefaction of soft sediments during the earthquake.

The age of transition D-E at Okupe Lagoon at the $95 \%$ confidence level coincides with the ages of both the last two earthquake events on the Wellington Fault. The date comes from a layer of large wood fragments deposited by some form of catchment disturbance. If the disturbance was seismic shaking it is likely that wood fragments deposited in the lagoon were already dead. They would give an older radiocarbon age than the event itself so this transition is more likely to be a signature of the last event on the Wellington Fault than the penultimate event. The occurrence of a large peak in brackish marine diatoms at the D-E transition suggests this event triggered a tsunami that inundated the lagoon.

Transitions A-B and B-C at Lake Kohangapiripiri are thought to be signatures of earthquake events on the Wairarapa Fault. They coincide with uplift events of Pillans and Huber (1995) at Rongotai and they appear to bracket dates at Turakirae Head and Tea Creek on the Wairarapa Fault. This clustering of ages may indicate that dates in each cluster represent one earthquake event, one occurring at some 
time around 7250-6300 cal. years BP and the other around 5400-4900 cal. years BP. If this were the case it would support the idea that ages of beach ridge uplift at Turakirae Head and surface rupture in the Tea Creek Road trench are offset for some systematic reason. The difference between ages is similar in each case with Turakirae beach ages consistently $400-600$ years older. If this also applies to the more recent events it suggests there was an event not preserved at Turakirae Head around 3000 cal. years BP or the Tea Creek Road event at 2860-2750 cal. years BP was not the result of an earthquake.

Transition C-D at Lake Kohangapiripiri coincides with the B-C transitions at Okupe Lagoon and Taupo Swamp and also with uplift of beach ridge D at Petone and beach ridge three at Turakirae Head. There is evidence for surface rupture earthquakes on both the Ohariu and Wairau Faults around this time and also on the Wairarapa Fault but the timing for this latter event is not well constrained. Because of the large distance between sites $(\sim 60 \mathrm{~km})$ it is considered likely that effects at each site were caused by different events. For example effects at Okupe Lagoon and Taupo Swamp are more likely to have been caused by movement on the Wairau or Ohariu Faults and effects at Lake Kohangapiripiri by an earthquake on the Wairarapa Fault. In making this assertion it must be remembered that the major active faults in the Wellington region are predominantly strike-slip, steeply dipping structures and any large earthquake involving surface rupture will involve a relatively small component of vertical movement. Therefore the areal extent of vertical movement is expected to be more restricted than for example, a subduction interface event.

The shallowing event at the top of the sequence at Lake Kohangapiripiri is only 15 $\mathrm{cm}$ below the sediment surface and is estimated using sediment accumulation rates to be almost present day. Therefore it is possible that it is a signature of the 1855 $\mathrm{AD}$ surface rupture earthquake on the Wairarapa Fault, or it may have been caused by anthropogenic drainage at the site. In the wetland core from Lake Kohangapiripiri the equivalent event is at $20 \mathrm{~cm}$ depth and is estimated by sediment accumulation rates to have occurred around the time of the last event on the Wellington Fault (Fig. 7.3). Further dating of the recent sedimentary record at Lake Kohangapiripiri would be required to decide which of these two possibilities is most likely.

The timing of all environmental transitions recognised in this work coincides with one or more surface rupture events in the Wellington region. This does not prove that transitions were caused by these same events but it does indicate there were potential seismic sources that could have been responsible for each transition. Another potential cause of coseismic movement of base level is movement along the plate boundary interface itself. In terms of evidence for such an event from the sedimentary record, a synchronous movement of base level would be expected at 
most sites in the region with increasing effects toward the east because of the increasing shallowness of the plate interface in this direction.

There is one interval of time (2720-2100 cal. years BP) in which inferred uplift is recorded in the sedimentary sequences of all three sites. As noted above, if rupturing on surface faults caused this movement then several events are invoked because a very large surface rupture earthquake would be required to cause uplift at sites up to $60 \mathrm{~km}$ apart. It is possible these effects were caused by a single earthquake on the subduction interface - effects would be widespread because it underlies most of the region - or that a subduction earthquake triggered other faults to rupture. This latter explanation is favoured because the study sites are probably too far west to experience uplift in a subduction earthquake - it is generally thought that uplift occurs seaward of the locked section of the subduction interface (Clague, 1997). Consistent evidence at a greater number of sites and higher resolution dating would be required to strengthen the case for occurrence of a subduction earthquake at this time.

Ages of environmental transitions identified in this work highlight, in a broad sense, intervals of time in which fault ruptures occurred in the Wellington region (Table 7.2). Interval VII (7250-6300 cal. years BP) involved a Wairarapa Fault rupture that is recorded at Lake Kohangapiripiri and at several raised beach sites. Interval VI (5400-4900 cal. years BP) was similar with the addition of a Wairau Fault event. There are no events for at least 1000 years after this (except for a poorly constrained one on the Wairau Fault) and then intervals V and IV represent phases of multiple fault rupture. Interval V (3850-2330 cal. years BP) involved rupture of the Wellington, Wairau and Wairarapa Faults with effects recorded at Rongotai, Okupe Lagoon and Taupo Swamp. Uplift is recorded at all three study sites during interval IV (2720-2100 cal. years BP) as well as at Petone and Turakirae, and there are inferred ruptures of the Wairau, Ohariu and Wairarapa Faults. Subsequently there is another interval of time in which no ruptures are recorded (assuming the Tea Creek date relates to the previous Wairarapa Fault event). Around $1200 \mathrm{cal}$. years BP another period of activity began with interval III involving an Ohariu Fault rupture, and interval II involving two Wellington Fault events. Interval I represents the 1855 Wairarapa Fault earthquake.

These periods of activity could well be an artefact of the record because there are no doubt events that have not yet been identified and the long age ranges of most radiocarbon dates extend the apparent intervals in which activity occurred.

However it is also possible that clustering of large magnitude earthquakes in time does occur in the Wellington region. Robinson et al. (1998) use computer models of seismicity to show that when interactions between faults are considered there is an increase in the likelihood of several large magnitude earthquakes occurring within a short time frame. In particular, a large earthquake on the subduction interface is most likely to 'induce' other large earthquakes. The models indicate 
the time interval in which there is an increased likelihood of a large event following a large event is 0-3 years, or at most 0-10 years (Robinson et al., 1998). Currently available age resolution for inferred paleoseismic events would not enable such small inter-event times to be identified and events would probably appear synchronous in the record. Therefore the most likely occurrence of clustering of large earthquakes as a result of one event triggering others identifiable in the record at present is interval IV at 2720-2100 cal. years BP (Table 7.2). In this interval there is evidence of three surface rupture events and uplift is recorded at all three study sites.

\begin{tabular}{|c|c|c|c|c|c|c|c|}
\hline Intervals of Fault Rupture & 1 & II & III & IV & V & $\mathrm{VI}$ & VII \\
\hline Calibrated years BP & $\begin{array}{l}1855 \\
\mathrm{AD}\end{array}$ & $\begin{array}{l}306- \\
716\end{array}$ & $\begin{array}{l}790- \\
1180\end{array}$ & $\begin{array}{l}2100- \\
2720\end{array}$ & $\begin{array}{l}2330- \\
3850\end{array}$ & $\begin{array}{l}4900- \\
5400\end{array}$ & $\begin{array}{l}6300- \\
7250\end{array}$ \\
\hline $\begin{array}{l}\text { Study Sites } \\
\text { Taupo Swamp } \\
\text { Okupe Lagoon } \\
\text { Lake Kohangapiripiri }\end{array}$ & $=$ & 圆 & (1) & 들 & a & ] & 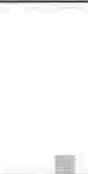 \\
\hline $\begin{array}{l}\text { Major Active Faults } \\
\text { Wairau Fault } \\
\text { Shepherds Gully-Pukerua } \\
\text { Ohariu Fault } \\
\text { Wellington Fault } \\
\text { Wairarapa Fault }\end{array}$ & 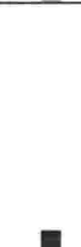 & E & घ & 口 & : & - & 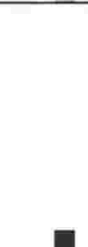 \\
\hline $\begin{array}{l}\text { Raised Beaches } \\
\text { Rongotai Beaches } \\
\text { Petone Beaches } \\
\text { Turakirae Beaches }\end{array}$ & a & घ & E & a & 圆 & = & ㅁ \\
\hline
\end{tabular}

Table 7.2. Summary of the seven intervals of time in which surface rupture earthquakes have occurred in the Wellington region as recognised from study sites

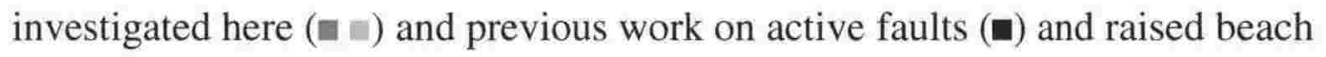
ridges ( $\square$ ) (see Fig. 7.3). Interval I represents the only surface rupture event that has occurred in historic times. Other intervals involve one or several surface ruptures.

- = Environmental transitions 1-5 that provide independent evidence for earthquake occurrence. = Environmental transitions 6-10 that do not provide independent evidence for earthquake occurrence.

\subsection{CONCLUSIONS}

\subsubsection{Summary of Results}

This study illustrates what a dominant role large magnitude earthquakes have played in the history of coastal waterbodies in the Wellington region over the last 7000 years. Within this time frame they are a frequent occurrence and their effects are recorded at widely spaced sites on different fault-bounded blocks.

Environmental transitions recognised at Taupo Swamp, Okupe Lagoon and Lake Kohangapiripiri occurred suddenly, are laterally extensive and synchronous within 
each waterbody. Five transitions consist of large magnitude changes in environment and evidence for movement of base level - these are considered to provide evidence for the occurrence of past large earthquakes. Five other transitions consist of smaller magnitude changes in environment but they are associated with catastrophic depositional events from the catchment or from a seaward source. These are consistent with the effects of past earthquakes but do not provide independent evidence for such events. All transitions coincide with one or more surface rupture earthquakes recognised in the Wellington region in previous studies.

Comparison of timing of transitions with previously recognised events highlights intervals of time in the last 7000 years in which there appear to have been multiple large magnitude earthquakes in the Wellington region. Environmental transitions at Taupo Swamp and Okupe Lagoon indicate that uplift of western Wellington occurred at some time between 3850-2330 cal. years BP. Surface rupture earthquakes occurred in this interval of time on the Wellington, Wairau and Wairarapa Faults. Coincidence of environmental transitions at Taupo Swamp, Okupe Lagoon and Lake Kohangapiripiri between 2720-2100 cal. years BP indicates that uplift occurred across the western and central Wellington region at sites $60 \mathrm{~km}$ apart at this time. There is evidence to suggest that the Wairau, Ohariu and Wairarapa Faults all ruptured in this time interval. Another time interval involving several large earthquakes in the region is the period from 1100 cal. years BP to present.

\subsubsection{Comments for Future Work}

This study is a beginning in terms of investigating what contribution diatom analysis of sedimentary sequences can make to paleoseismic histories in this country. Despite the small number of samples used in this study - both within each waterbody and in the region as a whole - the potential of the technique for application in New Zealand can be seen. There are some specific results from this work but it is also hoped the project has set the scene for similar work to be carried out in New Zealand and that application of techniques used here will enable future studies to cover more sites and longer time periods. A few observations that may be of interest to workers involved in similar studies in the future are noted below.

\section{Detection of Earthquakes in the Sedimentary Record}

The record of pre-historic large earthquake signatures that can be detected in sedimentary sequences of lakes and coastal waterbodies is useful for several main reasons. In regions where seismicity is dominated by movement on a subduction thrust or other sub-surface structures, sedimentary sequences may be the only paleoseismic record accessible at the surface. However this study illustrates that even in regions where surface faulting is responsible for most major earthquakes, the sedimentary record is a useful addition to information from fault trenches and 
sequences of raised beach ridges. Recognition of events in the sedimentary record provides additional estimates of timing for past large earthquakes identified by other means. Often a longer and / or more complete record can be obtained by coring sedimentary sequences than is preserved as colluvial wedges in fault trenches. A better indication of the areal extent of effects of past events is obtained because sites can be spread out across a region rather than associated with a single structure. Rough estimates of the amount of vertical movement can be made and this is likely to be refined with further work on elevational distributions of microfossils. The main disadvantage of using the sedimentary record to detect evidence of past earthquakes is that sequences are not usually uniquely related to particular faults so many sites and good age control are required to determine a seismic source for past events.

Microfossils are essential to such studies because sedimentology does not provide the detail of environmental information required to differentiate between aseismic and coseismic causes of change. Microfossils can be used to pick up transitions not noticeable in sediment. Particularly in fine-grained organic sediment a large change in water chemistry and microfossil assemblages can occur without any change in appearance of the sedimentary sequence. For example transition A-B in TS97-1 at Taupo Swamp involves a change from a brackish open lagoon to a fresh pond as inferred from the diatom record but would not be picked as an important horizon in the sedimentary sequence (Fig. 7.4). Macrofossils are useful in the identification of earthquake events for example through recognition of catastrophic depositional events as at Okupe Lagoon (see Fig. 4.5) or the evidence for suddenness of submergence given by buried plant fossils in growth position in Washington (Atwater and Yamaguchi, 1991). However macrofossils are often too scarce in the record to be of consistent use for all events at all sites (Nelson et al., 1996) particularly where small diameter cores are required to study sequences not exposed in outcrop.

Shennan et al. (1996) illustrate that when several microfossil groups are used in combination the resolution of resulting paleoenvironmental reconstructions is greater than in studies using a single group. This is particularly true for the estimation of past tide level. Where modern distributions of pollen, diatoms and foraminifera enabled identification of three or four distinct elevation-related assemblages, Shennan et al. (1996) show that use of the three groups in combination enabled recognition of seven distinct categories. However at the sampling resolution required for such studies, analysis of three or more fossil groups represents a large commitment of resources and in most cases only one group can be used. For sequences that cover a wide range of environments from marine through to fresh water conditions, diatoms are very useful because they live, and are generally preserved, in all aqueous environments along such a gradient. 


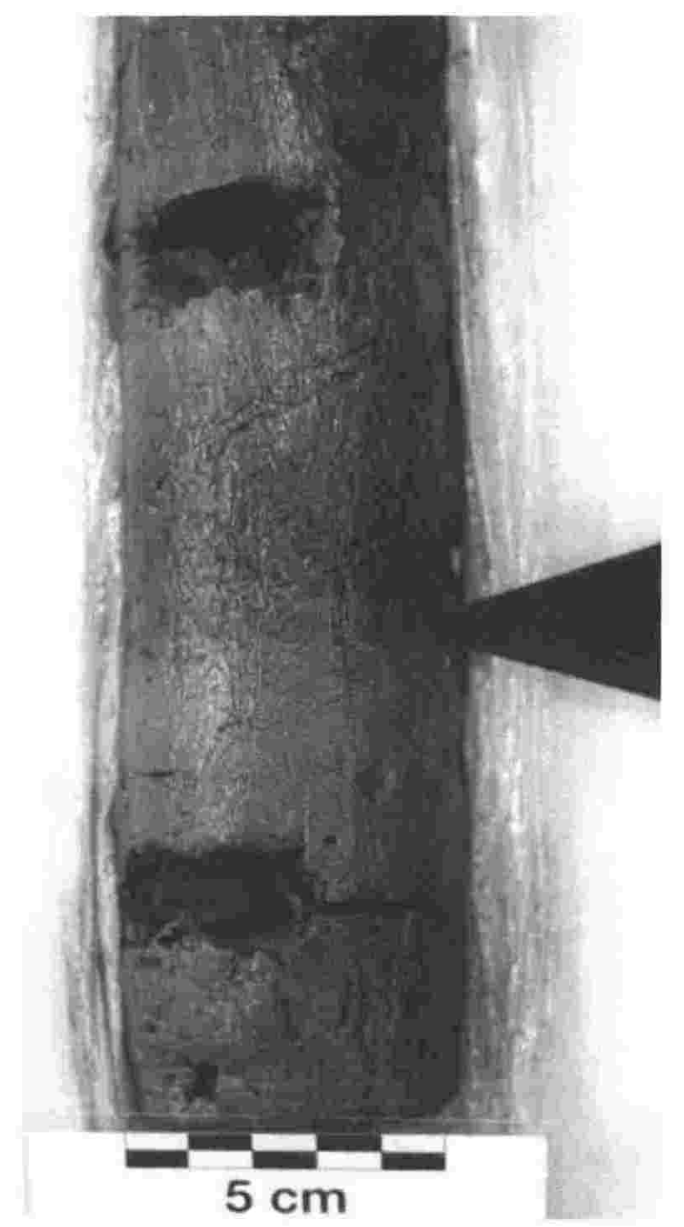

Fig. 7.4. Inferred earthquake event in the sedimentary sequence of Taupo Swamp (TS97-1). Transition A-B occurs at the horizon indicated by the black arrow head (241 cm depth). This photo illustrates the need for microfossil analysis of such sequences - diatoms indicate that this horizon involves a sudden change from a brackish, predominantly open lagoon to a fresh water pond.

\section{Comparison of Setting: Pacific Northwest and Wellington}

Methods for detection of earthquakes in the sedimentary record have been developed predominantly in the Pacific Northwest of America and Canada. Criteria for the assessment of coseismic versus aseismic causes of change have been developed using sequences from passive margin coasts in addition to those from active margins in order to thoroughly test the 'earthquake deformation cycle' identified from sediments of the Pacific Northwest. In theory these methods and criteria should be applicable to any seismically active coast but there are several major differences of Wellington's tectonic setting from that of the Pacific Northwest.

One of the main differences in setting is the dominance of surface faulting in the Wellington region. All previously identified earthquakes are attributed to surface faults and no conclusive evidence for movement on the subduction interface beneath Wellington has been documented. Vertical movement on these 
predominantly strike-slip faults is unlikely to be as widespread as subduction interface earthquakes of the Pacific Northwest. Therefore evidence for earthquakes in the sedimentary record is likely to be restricted to fault-bounded blocks in the Wellington region rather than extending across long lengths of coastline as occurs in the Pacific Northwest. Where evidence is preserved across several fault-bounded blocks the record can be assessed for the likelihood of a subduction interface earthquake but rupturing of several faults in a close interval of time may also cause a widespread record of effects.

Repeated uplift rather than subsidence has been the dominant factor in the history of waterbodies used in this work. It also appears from the sedimentary record that there is little or no interseismic movement of base level as is documented in the Pacific Northwest. One implication of these differences is there are fewer sea level index points preserved in the sequences of the Wellington region. Ideal sea level index points consist of boundaries between different depositional environments for which a tidal elevation is known. Repeated uplift results in a unidirectional change in paleoenvironment from marine to fresh and environmental transitions are often coseismic rather than those resulting from gradual sediment accumulation. In the Pacific Northwest repeated cycles have been documented (Shennan et al., 1996) in which the coast subsides coseismically then sediment gradually accumulates. In this case peat-mud couplets are preserved in the record and transitions are a mix of coseismic contacts and natural contacts that provide good sea level index points.

\section{Study Sites and the Coastal Gradient}

The three study sites from the coast of the Wellington region are all small, low elevation coastal waterbodies with small fresh water inflows. These are found to provide fairly complete and in situ records of paleoenvironmental change in comparison with large, high-energy coastal waterbodies (eg, Zachariasen et al., 2000). Paleoenvironments inferred from the sedimentary sequences of each Holocene site are plotted against the idealised coastal gradient as presented in Chapter Three (Fig. 7.5). In this comparison it can be seen that all sites progress through time in a landward direction along the gradient with Okupe Lagoon occupying the most seaward position and Lake Kohangapiripiri the most landward position.

Relative position on the coastal gradient is found to be an important factor in determining the nature of evidence for earthquakes that may be preserved. In the most seaward position, as exemplified by Okupe Lagoon, relative changes in sea level provide evidence for vertical movements of base level. Where fossil 
Inferred Waterbody Types mid-Holocene to present

$\mathrm{X}=$ core position

$\begin{array}{lll}\text { Okupe } & \text { Taupo } & \text { Lake } \\ \text { Lagoon } & \text { Swamp } & \text { Kohangapiripiri }\end{array}$
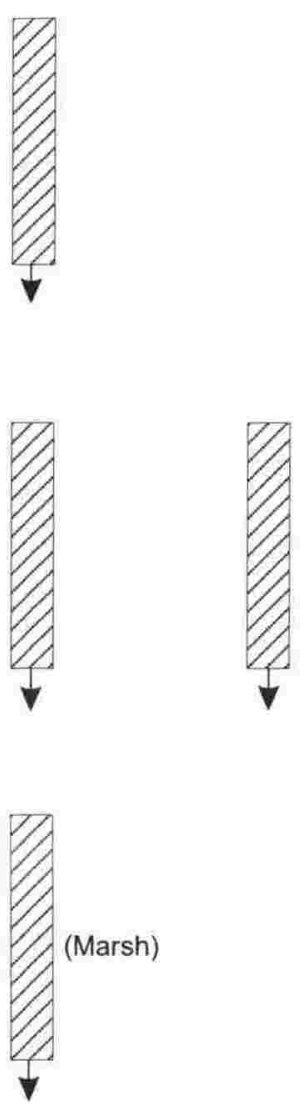

(Pond)
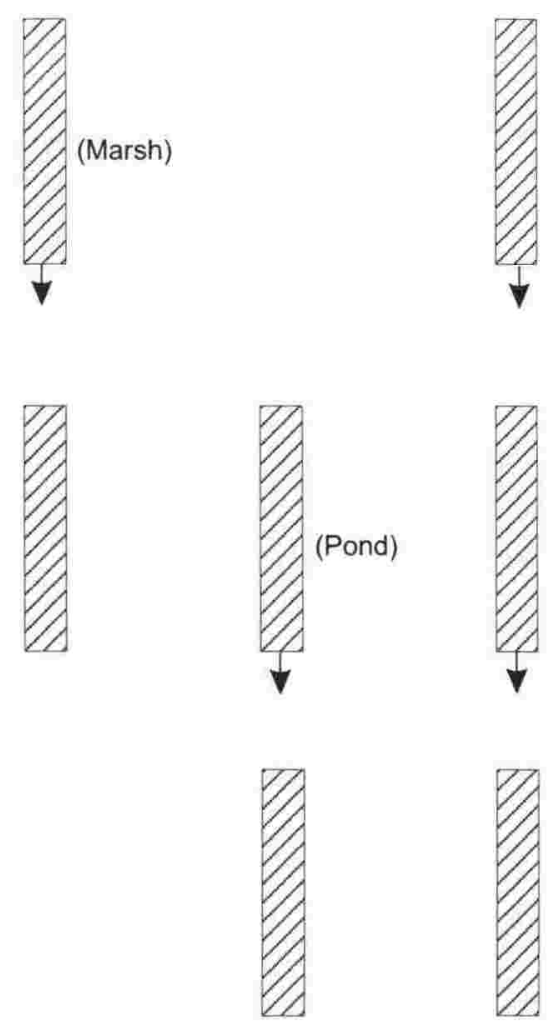
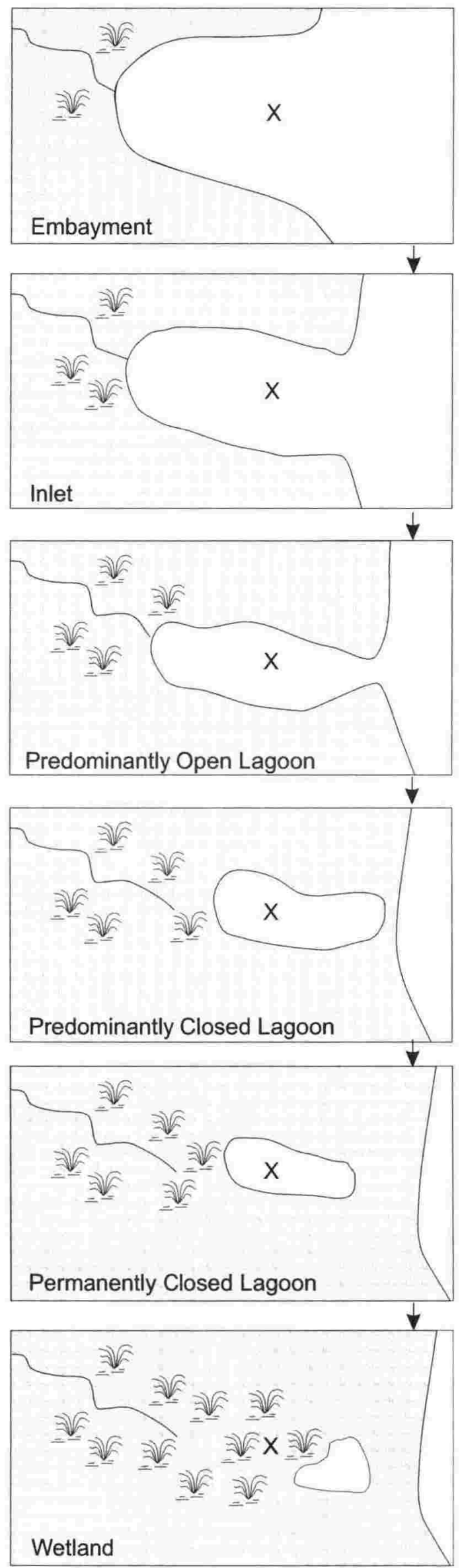

Fig. 7.5. Temporal coastal waterbody gradient for the Holocene sedimentary sequences. Inferred waterbody types illustrate how sedimentation at each site has progressed along the coastal gradient with time. 
assemblages can be related to tide level estimates of the amount of vertical movement can be made. Disadvantages of seaward sites include the increased likelihood of erosion of sediment because of the higher energy environment and the difficulty in differentiating tsunami deposits from surrounding sandy marine sediment.

In the most landward position, as at Lake Kohangapiripiri, vertical movements of base level are likely to be recorded as relative movements of water table level which can be less obvious to identify. In the case where the sequence is entirely terrestrial, estimates for the amount of vertical movement have to be made from inferred water depth changes. The record is likely to be more complete in waterbodies with small inflow and no connection with the sea. Tsunami deposits would be clearly distinct from surrounding sediments at such terrestrial sites but only those large enough to overtop or breach any barrier between sea and waterbody would be recorded.

Small landslides or slips resulting from earthquake-induced shaking are recorded at sites from both ends of the gradient but because of the localised nature of such deposits they cannot be relied on to occur at every core site. The absence of evidence for catchment disturbance at Taupo Swamp may be for this reason or because the source for such deposits (hillsides or barriers) is further from the core sites than it is at Okupe Lagoon or Lake Kohangapiripiri. There are no apparent differences along the coastal gradient in determining the suddenness, lateral extent or synchroneity of potential earthquake events. 


\section{CHAPTER EIGHT}

\section{Conclusions}

The first aim of this thesis was to develop a modern diatom calibration set to aid paleoenvironmental reconstruction of coastal sequences in New Zealand. A calibration set consisting of 50 sites was collected from the coastline of the southern North Island and the South Island of New Zealand. Sites define a coastal gradient from marine through to terrestrial environments consisting of open bays, sheltered embayments, inlets, lagoons, lakes and wetlands. Diatom death assemblage samples were collected from each site and 305 taxa were identified. Twenty-six physical and chemical environmental variables were measured and 23 of these were found to be statistically significant in terms of explaining diatom distribution and abundance.

Multivariate statistical techniques were used to analyse and summarise the calibration set. Detrended correspondence analysis yielded an important first axis that arranged species according to their salinity preferences and sites according to waterbody type. The clear separation of sites enabled division of ordination space into waterbody type and reconstruction of fossil samples by passive placement onto ordination diagrams. The similarity of results obtained from ordination and canonical ordination analyses indicated that measured environmental variables explained the diatom data well. Canonical ordination and forward selection of environmental variables enabled seven variables to be identified that described the diatom data almost as well as all 23 variables. Variation along the dominant first axis was effectively explained by exposure to salt, salinity, distance from the sea, flow and carbonate. Variation along the second axis was explained by $\mathrm{pH}$ and precipitation. These variables were used to define an idealised spatial coastal gradient to aid the reconstruction of temporal coastal gradients of Holocene sedimentary sequences.

A diatom-salinity transfer function was derived using weighted averaging regression of the calibration set. There is a high correlation between observed and inferred salinity values and a relatively low root mean squared error of prediction - indicating that the calibration set can be used to reconstruct salinity from past environments. Salinity optima and tolerances for 100 species are presented and compared with published preferences. The modern analogue technique was used as an alternative method for reconstructing paleosalinity from fossil samples to provide a means of assessing the validity of reconstructions carried out using 
weighted averaging. Paleosalinity reconstructions derived from the different techniques were generally compatible. The deviations that did occur are likely to be the result of lack of modern analogues for some samples - a problem that could be corrected by expansion of the calibration set.

The calibration set aids diatom-based reconstruction of coastal sequences in several ways. In this thesis quantification of estimates for past waterbody type and salinity enable the magnitude of paleoenvironmental change to be assessed objectively. This is an important characteristic for differentiating between coseismic and aseismic causes of change when assessing sedimentary sequences for evidence of past earthquakes. In more general terms, and for future workers on coastal sedimentary sequences in New Zealand, there are two main implications of this work. Firstly, the compatibility of diatom salinity preferences derived from the calibration set and those derived from Northern Hemisphere literature, indicates that traditional paleoecological techniques that rely on qualitative autecological information are generally appropriate for reconstruction in New Zealand. Secondly, the existence of the calibration set provides an alternative option for paleoenvironmental reconstruction of coastal sequences. That option is direct analysis of fossil diatom count data using the calibration set data to derive high resolution, quantitative estimates of past waterbody type and paleosalinity. The procedure of categorising each species according to salinity and habitat preferences can be bypassed depending on the aims and resolution required for each piece of work. Fossil datasets must be compatible with the nature of the coastal gradient defined by the calibration set, basic requirements and assumptions of the procedure must be addressed and ideally the calibration set should be enlarged to improve the quality of reconstruction.

The second aim of the thesis was to contribute to the understanding of Wellington's paleoseismic record through detection of evidence for large Holocene earthquakes in the sedimentary record. This was achieved by developing paleoenvironmental histories for three Holocene coastal waterbodies to act as a basis from which to assess whether evidence of past earthquakes was preserved. The three sites used, Taupo Swamp, Okupe Lagoon, and Lake Kohangapiripiri are all small, low elevation coastal waterbodies that are now completely isolated from the influence of the sea. At each site there is geomorphological evidence in the form of gravel barriers and raised beach ridges to suggest that sediment transport and coseismic uplift events have operated together to isolate each site from the sea. Diatom analysis, sedimentology and radiocarbon dating of sedimentary sequences within each waterbody are used to elucidate the Holocene history of each site and tie paleoenvironmental events to a chronology.

Taupo Swamp was once part of outer Porirua Harbour and cores from central Taupo Valley illustrate that the transformation from a marine to terrestrial environment occurred in two distinct stages closely spaced in time. A 
predominantly open lagoon at high intertidal elevation existed between 5500 and about 3100 cal. years BP. Isolation from the sea occurred about 3100 cal. years BP and a very shallow fresh brackish pond existed at the site for a short time. A fresh water wetland environment became established shortly after isolation at about $2400 \mathrm{cal}$. years BP, and has persisted in the central valley until the present day. In the lower valley a very shallow, fresh brackish lagoon existed until very recently and may well have disappeared as a result of a small amount of uplift in the 1855 AD Wairarapa earthquake.

At Okupe Lagoon on the northeastern end of Kapiti Island five different paleoenvironments have existed at the site over the last 5000 years. Between about 5000 and $3200 \mathrm{cal}$. years BP, deposition took place at low intertidal to intertidal elevations within a sheltered inlet. At 3200 cal. years BP the environment appears to have changed to a predominantly open lagoon at high intertidal-high tide elevations. At $\sim 2300$ cal. years BP a predominantly isolated marsh environment with little or no open water existed at the site. A deepening took place about 1000 cal. years BP that enabled the present configuration of an open water lagoon to become established. This environment was saline but completely isolated from the sea. At around $500 \mathrm{cal}$. years BP a small change in sediment supply or water depth occurred. Evidence of catchment disturbance is preserved at three horizons that coincide with environmental transitions.

At Lake Kohangapiripiri east of the Wellington Harbour entrance a barrier has existed between the lagoon and sea since $7500 \mathrm{cal}$. years BP. At that time the barrier was low and allowed indirect or episodic connection between the lagoon and the sea. From 6800 cal. years BP onwards a permanently closed lagoon has existed at the site and the main paleoenvironmental transitions recorded in the sedimentary sequence involve changes in water depth. In two cases layers of angular gravel accompany environmental transitions suggesting that catchment disturbance occurred at the same time. A deep phase in the lagoon's history initiated about $5200 \mathrm{cal}$. years BP and a shallow phase occurred between $2200 \mathrm{cal}$. years BP and the present. Further shallowing is inferred to have occurred in the very recent past.

Ten transitions between different paleoenvironments were identified from the three Holocene sites and these were examined in detail to determine likely causes of change. Environmental transitions occur suddenly, are laterally extensive within each waterbody, and synchronous within each waterbody within the bounds of radiocarbon dating. Five transitions involve large magnitude changes in paleoenvironment and evidence for movement of base level. These are thought to have been caused by large earthquakes at $\sim 5200, \sim 3200$, and $\sim 2300$ cal. years BP. Five other transitions involve smaller amounts of paleoenvironmental change and evidence for catchment disturbance and / or marine inundation. These do not 
necessarily involve movement of base level but are consistent with the effects of large earthquakes occurring at $\sim 6800, \sim 2200, \sim 1000, \sim 500$ cal. years BP and 1855 AD.

Recognition of these events contributes to the existing paleoseismic record by providing additional estimates of timing and enabling estimation of the areal extent of effects of past large earthquakes. For example, ages of environmental transitions at Lake Kohangapiripiri clarify event history on the Wairarapa Fault by bracketing incompatible age estimates from two different sites along the fault. Transitions at Okupe Lagoon and Taupo Swamp indicate that western Wellington was uplifted coseismically at about 3200 cal. years BP. At about 2300 cal. years $\mathrm{BP}$ uplift is recorded at all three sites suggesting that the entire western and central Wellington region experienced coseismic movement at that time. Because of the distance between study sites this is likely to have been the result of several faults rupturing at a similar time. Two minor paleoenvironmental changes associated with evidence for catchment disturbance at Okupe Lagoon, are thought to indicate strong shaking on Kapiti Island during the last earthquake on the Ohariu Fault and on the Wellington Fault - the latter also appears to have triggered a tsunami that inundated Okupe Lagoon.

These results illustrate that evidence of earthquakes is preserved in the sedimentary record of the Wellington region and that sedimentology and diatom analysis are effective tools with which to detect such evidence. Investigation of more sites and longer records is required to refine the timing of events identified here and extend the paleoseismic record back in time. Use of the approach is likely to be beneficial to paleoseismic records in other parts of the country and in the search for evidence of subduction related earthquakes in New Zealand. 


\section{B I B L I O G R A P H Y}

Anon. (1989). Taupo Swamp, Plimmerton, Management Plan, pp. 20. Queen Elizabeth II National Trust, Wellington.

Aaby, B. and Digerfeldt, G. (1986). Sampling techniques for lakes and bogs. In "Handbook of Holocene Palaeoecology and Palaeohydrology." (B. E. Berglund), pp. 181-194. John Wiley and Sons Ltd, Chichester.

Adkin, G. L. (1921). Porirua Harbour: a study of its shoreline and other physiographic features. Transactions and Proceedings of the New Zealand Institute 53, 144-156.

(1955). Field Notebook No. 41. Held by the Institute of Geological and Nuclear Sciences.

Atwater, B. F. (1987). Evidence for great Holocene earthquakes along the outer coast of Washington State. Science 236, 942-944.

(1992). Geologic evidence for earthquakes during the past 2000 years along the Copalis River, southern coastal Washington. Journal of Geophysical Research 97, 1901-1919.

Atwater, B. F., and Yamaguchi, D. K. (1991). Sudden, probably coseismic submergence of Holocene trees and grass in coastal Washington State. Geology 19, 706-709.

Bagnall, R. G., and Ogle, C. C. (1981). The changing vegetation structure and composition of a lowland mire at Plimmerton, North Island, New Zealand. New Zealand Journal of Botany 19, 371-387.

Barrett, P. J., and Brooker, M. R. (1989). Grainsize analysis at Victoria University of Wellington. School of Earth Sciences, Victoria University of Wellington, Wellington.

Battarbee, R. W. (1973). A new method for the estimation of absolute microfossil numbers, with reference especially to diatoms. Limnology and Oceanography $\mathbf{1 8}$, 647-653.

(1986). Diatom analysis. In "Handbook of Holocene Palaeoecology and Palaeohydrology.” (B. E. Berglund), pp. 527-570. John Wiley and Sons Ltd, Chichester.

(1994). Diatoms, lake acidification and the Surface Water Acidification Programme (SWAP): a review. Hydrobiologia 274, 1-7. 
Beanland, S. (1995). "The North Island Dextral Fault Belt, Hikurangi Subduction Margin, New Zealand." Unpublished PhD thesis, Victoria University of Wellington, Wellington.

Begg, J. G., and Mazengarb, C. (1996). "Geology of the Wellington area, scale 1:50 000. Institute of Geological and Nuclear Sciences geological map 22."

1 sheet +28 p. Institute of Geological and Nuclear Sciences Limited, Lower Hutt.

Bennion, H. (1994). A diatom-phosphorus transfer function for shallow, eutrophic ponds in southeast England. Hydrobiologia 275/276, 391-410.

Bennion, H., Wunsam, S., and Schmidt, R. (1995). The validation of diatomphosphorus transfer functions: an example from Mondsee, Austria. Freshwater Biology 34, 271-283.

Benson, A., Hill, N., Little, T. A., and Van Dissen, R. J. (2001). Paleoseismicity, rates of active deformation, and structure of the Lake Jasper pull-apart basin, Awatere Fault, New Zealand. Earthquake Commission Research Report 97/262. School of Earth Sciences, Victoria University of Wellington, Wellington.

Berryman, K. (1990). Late Quaternary movement on the Wellington Fault in the Upper Hutt area, New Zealand. New Zealand Journal of Geology and Geophysics 33, 257-270.

Berryman, K., and Beanland, S. (1991). Variation in fault behaviour in different tectonic provinces of New Zealand. Journal of Structural Geology 13, 177-189.

Beu, A. G., and Maxwell, P. A. (1990). "Cenozoic Mollusca of New Zealand.” New Zealand Geological Survey, Department of Scientific and Industrial Research, Lower Hutt.

Beyens, L., and Denys, L. (1982). Problems in diatom analysis of deposits: allocthonous valves and fragmentation. Geologie en Mijnbouw 61, 159-162.

Birks, H. J. B. (1994). The importance of pollen and diatom taxanomic precision in quantitative paleoenvironmental reconstruction. Review of Palaeobotany and Palynology 83, 107-117.

(1995). Quantitative Paleoenvironmental Reconstruction. In "Statistical

Modelling of Quaternary Science Data.” (D. Maddy, and J. S. Brew), pp. 161-254. Quaternary Research Association, Cambridge.

(1998). Numerical tools in palaeolimnology - progress potentialities, and problems. Journal of Paleolimnology 20, 307-332.

Birks, H. J. B., and Birks, H. H. (1980). "Quaternary Paleoecology." Edward Arnold Publishers Ltd, London.

Boggs Jr., S. (1995). "Principles of Sedimentology and Stratigraphy." PrenticeHall Incorporated, New Jersey. 
Bukhtiyarova, L., and Round, F. E. (1996). Revision of the genus Achnanthes sensu lato section Marginulatae Bukht. sect. nov. of Achnanthidium Kutz. Diatom Research 11, 1-30.

Campeau, S., Pienitz, R., and Hequette, A. (1999). Diatoms as quantitative paleodepth indicators in coastal areas of the southeastern Beaufort Sea, Arctic Ocean. Palaeogeography, Palaeoclimatology, Palaeoecology 146, 67-97.

Carter, L., Lewis, K. B., and Davey, F. (1988). Faults in Cook Strait and their bearing on the structure of central New Zealand. New Zealand Journal of Geology and Geophysics 31, 431-446.

Carter, R., Hayward, B. W., Grenfell, H. R., and Lipps, J. H. (2000).

Foraminiferal documentation of earthquake-related vertical displacements in Holocene coastal lagoon sediments, Hawke's Bay, New Zealand, pp. 71. Department of Geology, University of Auckland, Auckland.

Cassie, V. (1984). "Checklist of the Freshwater Diatoms of New Zealand." Bibliotheca Diatomologica Band 4. J. Cramer, Vaduz.

- (1989). "A Contribution to the Study of New Zealand Diatoms." Bibliotheca Diatomologica Band 17. J Cramer, Berlin, Stuttgart.

Chague-Goff, C., Dawson, S., Goff, J. R., Zachariasen, J., Berryman, K. R., Garnett, D. L., Waldron, H. M., and Mildenhall, D. C. (2001). A tsunami (c. 6300 years BP) and other Holocene envrionmental changes, northern Hawke's Bay, New Zealand. Sedimentary Geology in press.

Clague, J. J. (1997). Evidence for large earthquakes at the Cascadia subduction zone. Reviews of Geophysics 35, 439-460.

Clague, J. J., and Bobrowsky, P. T. (1994). Evidence for a large earthquake and tsunami 100-400 years ago on western Vancover Island, British Columbia. Quaternary Research 41, 176-184.

Clelland, D. (1984). Unprotected Natural Areas of the Wellington Region: A Survey of Eleven Areas of Biological Significance. Department of Lands and Survey, Wellington.

Cochran, U. (1995). "A paleoenvironmental history of Lake Kohangapiripiri, Fitzroy Bay, Wellington." Unpublished BSc Honours thesis, Victoria University of Wellington.

Cochran, U., Goff, J., Hannah, M., and Hull, A. (1999). Relative stability on a tectonically active coast: paleoenvironment during the last 7000 years at Lake Kohangapiripiri, Wellington, New Zealand. Quaternary International 56, 53-63. 
Cotton, C. A. (1918). The Geomorphology of the Coastal District of Southwestern Wellington. Transactions and Proceedings of the New Zealand Institute 50, 212-222.

(1921a). The warped land-surface on the south-eastern side of the Port Nicholson deression, Wellington, New Zealand. Transactions and proceedings of the New Zealand Institute 53, 131-143.

(1921b). For how long will Wellington escape destruction by earthquake? The New Zealand Journal of Science and Technology 3, 229-231.

Darby, D. J., and Beanland, S. (1992). Possible source models for the 1855 Wairarapa Earthquake, New Zealand. Journal of Geophysical Research 97, $12,375-12,389$.

Darienzo, M. E., and Peterson, C. D. (1990). Episodic tectonic subsidence of late Holocene salt marshes, northern Oregon central Cascadia margin. Tectonics $\mathbf{9}$, $1-22$.

Darienzo, M. E., Peterson, C. D., and Clough, C. (1994). Stratigraphic evidence for great subduction-zone earthquakes at four estuaries in Northern Oregon, U. S. A. Journal of Coastal Research 10, 850-876.

Denys, L., and de Wolf, H. (1999). Diatoms as indicators of coastal paleoenvironments and relative sea-level change. In "The Diatoms: Applications for the Environmental and Earth Sciences." (E. F. Stoermer, and J. P. Smol), pp. 277-297. Cambridge University Press, Cambridge.

Dixon, P. M. (1993). The bootstrap and the jackknife: Describing the precision of ecological indices. In "Design and Analysis of Ecological Experiments."

(S. M. Scheiner, and J. Gurevitch), pp. 290-318. Chapman and Hall, New York.

Doig, R. (1990). 2300 yr history of seismicity from silting events in Lake Tadoussac, Charlevoix, Quebec. Geology 18, 820-823.

Dunbar, G. B., Barrett, P. J., Goff, J. R., Harper, M. A. and Irwin, S. L. (1997). Estimating vertical tectonic movement using sediment texture. The Holocene 7, 213-221.

Eiby, G. (1990). Changes to Porirua Harbour in about 1855: historical tradition and geologic evidence. Journal of the Royal Society of New Zealand 20, 233-248.

Ferrar, H. T. (1928). Geological notes on Kapiti Island. The New Zealand Journal of Science and Technology 9, 312-315.

Fleming, C. A., and Hutton, C. O. (1949). Notes on the Geology of Kapiti Island, Cook Strait, New Zealand. Transactions of the Royal Society of New Zealand 77, 456-468. 
Foged, N. (1979). "Diatoms in New Zealand, the North Island." Bibliotheca Phycologica, Band 47. J. Cramer, Vaduz.

Gaiser, E. E., Philippi, T. E., and Taylor, B. E. (1998). Distribution of diatoms using intermittent ponds on the Atlantic Coastal Plain: development of a model to predict drought periodicity from surface-sediment assemblages. Journal of Paleolimnology 20, 71-90.

Gasse, F., Juggins, S., and Khelifa, L. B. (1995). Diatom-based transfer functions for inferring past hydrochemical characteristics of African lakes.

Palaeogeography, Palaeoclimatology, Palaeoecology 117, 31-54.

Gibb, J. G. (1986). A New Zealand regional holocene eustatic sea-level curve and its application to determination of vertical tectonic movements. Royal Society of New Zealand Bulletin 24, 377-395.

Goff, J. R., Crozier, M., Sutherland, V., Cochran, U., and Shane, P. (1998). Possible tsunami deposits from the 1855 earthquake, North Island, New Zealand. In "Coastal Tectonics." (I. S. Stewart, and C. Vita-Finzi), pp. 353-374. Geological Society, London, Special Publications, London.

Goff, J. R., and Chague-Goff, C. (1999). A late Holocene record of environmental changes from coastal wetlands: Abel Tasman National Park, New Zealand. Quaternary International 56, 39-51.

Goff, J. R., Rouse, H. L., Jones, S. L., Hayward, B. W., Cochran, U., McLea, W., Dickinson, W. W., and Morley, M. S. (2000). Evidence for an earthquake and tsunami about 3100-3400 yr ago, and other catastrophic saltwater inundations recorded in a coastal lagoon, New Zealand. Marine Geology 170, 231-249.

Goff, J., Chague-Goff, C., and Nichol, S. (2001). Palaeotsunami deposits: a New Zealand perspective. Sedimentary Geology 143, 1-6.

Goff, J. R., and McFadgen, B. G. (2001). Catastrophic seismic-related events and their impact on prehistoric human occupation, coastal New Zealand. Antiquity 75, 155-162.

Grapes, R. (1999). Geomorphology of faulting: The Wairarapa Fault, New Zealand. Zeitschrift fur Geomorphologie 115, 191-217.

Grapes, R., and Downes, G. (1997). The 1855 Wairarapa, New Zealand, Earthquake - Analysis of Historical Data. Bulletin of the New Zealand Society for Earthquake Engineering 30, 271-368.

Hartley, B. (1996). “An Atlas of British Diatoms.” Biopress Ltd, Bristol.

Harwood, D. M. (1999). Diatomite. In "The Diatoms: Applications for the Environmental and Earth Sciences.” (E. F. Stoermer and J. P. Smol), pp. 436-446. Cambridge University Press, Cambridge. 
Hayward, B. W., Grenfell, H. R., Reid, C. M., and Hayward, K. A. (1999).

"Recent New Zealand Shallow-Water Benthic Foraminifera: Taxonomy, Ecologic Distribution, Biogeography, and Use in Paleoenvironmental Assessment." pp. 258. Institute of Geological and Nuclear Sciences, Lower Hutt.

Hemphill-Haley, E. (1995a). Diatom evidence for earthquake-induced subsidence and tsunami $300 \mathrm{yr}$ ago in southern coastal Washington. Geological Society of America Bulletin 107, 367-378.

Hemphill-Haley, E. (1995b). Intertidal diatoms from Willapa Bay, Washington: Application to studies of small-scale sea-level changes. Northwest Science 69, $29-45$.

(1996). Diatoms as an aid in identifying late-Holocene tsunami deposits. The Holocene 6, 439-448.

Hendey, N. I. (1964). "An Introductory Account of the Smaller Algae of British Coastal Waters.” Her Majesty's Stationery Office, London.

Heron, D., Van Dissen, R., and Sawa, M. (1998). Late Quaternary movement on the Ohariu Fault, Tongue Point to MacKays Crossing, North Island, New Zealand. New Zealand Journal of Geology and Geophysics 41, 419-439.

Hill, M. O., and Gauch, J. H. G. (1980). Detrended correspondence analysis: an improved ordination technique. Vegetatio 42, 47-58.

Hinchey, J. V., and Green, O. R. (1994). A guide to the extraction of fossil diatoms from lithified or partially lithified sediments. Micropaleontology 40, 638-372.

Hull, A. G. (1986). Pre-A. D. 1931 subsidence of Ahuriri Lagoon, Napier, Hawke's Bay, New Zealand. New Zealand Journal of Geology and Geophysics 29, 75-82.

Hull, A. G., and McSaveney, M. J. (1996). A 7000-year record of great earthquakes at Turakirae Head, Wellington, New Zealand. Institute of Geological and Nuclear Sciences, Lower Hutt.

Hustedt, F. (1985). “The Pennate Diatoms.” Koeltz Scientific Books, Hirschberg.

Hutchinson, I., Guilbault, J.-P., Clague, J. J., and Bobrowsky, P. T. (2000). Tsunamis and tectonic deformation at the northern Cascadia margin: a 3000-year record from Deserted Lake, Vancouver Island, British Columbia, Canada. The Holocene 10, 429-439.

Imbrie, J., and Kipp, N. G. (1971). A new micropaleontological method for quantitative paleoclimatology: application to a late Pleistocene Caribbean core. In "The Late Cenozoic Glacial Ages." (K. K. Turekian), pp. 71-181. Yale University Press, New Haven and London. 
John, J. (1983). "The Diatom Flora of the Swan River Estuary Western Australia." Bibliotheca Phycologica Band 64, J. Cramer, Hirschberg.

Jones, V. J., and Juggins, S. (1995). The construction of a diatom-based chlorophyll a transfer function and its application at three lakes on Signy Island (maritime Antarctic) subject to differing degrees of nutrient enrichment. Freshwater Biology 34, 433-445.

Jongman, R. H. G., ter Braak, C. J. F., and van Tongeren, O. F. R. (eds.). (1995). "Data Analysis in Community and Landscape Ecology." Cambridge University Press, Cambridge.

Jowsey, P. C. (1966). An improved peat sampler. New Phytologist, 65, 245-248.

Juggins, S. (1992). "Diatoms in the Thames Estuary, England. Ecology, paleoecology, and salinity transfer function." Bibliotheca Diatomologica Band 25, J. Cramer, Berlin / Stuttgart.

(1998). WinTran, Computer Program. University of Newcastle, Newcastle upon Tyne.

(2000). Modern Analog Technique, Computer Program. University of Newcastle, Newcastle upon Tyne.

Juggins, S., and ter Braak, C. J. F. (1997-99). Calibrate. A C++ program for analysing and visualising species environment relationships and for predicting environmental variables from species assemblages. University of Newcastle, Newcastle upon Tyne.

Karlin, R. E., and Abella, S. E. B. (1996). A history of Pacific Northwest earthquakes recorded in Holocene sediments from Lake Washington. Journal of Geophysical Research 101, 6137-6150.

Kidson, C. (1986). Sea-level changes in the Holocene. In "Sea-level Research: a manual for the collection and evaluation of data." (O. van de Plassche), pp. 27-67. Geo Books, Norwich.

Kjemperud, A. (1981). Diatom changes in sediments of basins possessing marine/lacustrine transitions in Frosta, Nord-Trondelag, Norway. Boreas 10, $27-38$

Krammer, K. and Lange-Bertalot, H. (1986). "Bacillariophyceae 1:

Naviculaceae.” VEB Gustav Fischer Verlag, Jena.

(1988). "Bacillariophyceae 2: Bacillariophyceae, Epithemiaceae, Surirellaceae." Gustav Fischer Verlag, Stuttgart / New York.

(1991). "Bacillariophyceae 3: Centrales, Fragilariaceae, Eunotiaceae." Gustav Fischer Verlag, Stuttgart / Jena. 
(1991). "Bacillariophyceae 4: Achnanthaceae." Gustav Fischer Verlag, Stuttgart / Jena.

Lange-Bertalot, H. (1993). " 85 New Taxa and much more than 100 taxonomic clarifications supplementary to SuBwasserflora von Mitteleuropa." J. Cramer, Berlin.

Laws, R. A. (1983). Preparing strewn slides for quantitative microscopical analysis: A test using calibrated microspheres. Micropaleontology 29, 60-65.

Lensen, G. J. (1958). The Wellington Fault from Cook Strait to Manawatu Gorge. New Zealand Journal of Geology and Geophysics 1, 178-196.

Lensen, G., and Vella, P. (1971). The Waiohine River faulted terrace sequence. Recent Crustal Movements, Royal Society of New Zealand Bulletin 9, 117-119.

Lewis, K. B. (1989). Reversal of throw and change of trend on the Wellington Fault, Wellington Harbour. New Zealand Journal of Geology and Geophysics 32, 293-298.

Long, A. J., and Shennan, I. (1994). Sea-level changes in Washington and Oregon and the 'Earthquake Deformation Cycle'. Journal of Coastal Research 10, 825-838.

(1998). Models of rapid relative sea-level change in Washington and Oregon, USA. The Holocene 8, 129-142.

Lotter, A. F., Birks, H. J. B., Hofmann, W., and Marchetto, A. (1997). Modern diatom, cladocera, chironomid, and chrysophyte cyst assemblages as quantitative indicators for the reconstruction of past environmental conditions in the Alps. I. Climate. Journal of Paleolimnology 18, 395-420.

(1998). Modern diatom, cladocera, chironomid, and chrysophyte cyst assemblages as quantitative indicators for the reconstruction of past environmental conditions in the Alps. II. Nutrients. Journal of Paleolimnology 19, 443-463.

McIntire, C. D. and Moore, W. W. (1977). Marine littoral diatoms: ecological considerations. In "The Biology of Diatoms." (D. Werner), pp. 333-371. University of California Press, Berkeley.

MacLean, C. (1999).“Kapiti.” Whitcombe Press, Wellington.

Mann, D. G. (1999). The species concept in diatoms. Phycologia 38, 437-495.

Mathewes, R. W., and Clague, J. J. (1994). Detection of large prehistoric earthquakes in the Pacific Northwest by microfossil analysis. Science 264, 688-691. 
Matthews, E. R. (1980). Observations of beach gravel transport, Wellington Harbour entrance, New Zealand. New Zealand Journal of Geology and Geophysics 23, 209-222.

Moar, N. T. (1949). "Study of some mires in the South West Wellington Province." Unpublished Msc thesis, Victoria University of Wellington, Wellington.

Moore, P. D., and Webb, J. A. (1983). "An Illustrated Guide to Pollen Analysis." Hodder and Stoughton Educational, London.

Moore, P. R. (1987). Age of the raised beach ridges at Turakirae Head, Wellington: a reassessment based on new radiocarbon dates. Journal of the Royal Society of New Zealand 17, 313-324.

Moore, P. R., and Francis, D. A. (1988). Geology of Kapiti Island, Central New Zealand, New Zealand Geological Survey, Lower Hutt.

Nelson, A. R., and Kashima, K. (1993). Diatom zonation in Southern Oregon tidal marshes relative to vascular plants, foraminifera, and sea level. Journal of Coastal Research 9, 673-697.

Nelson, A. R., Jennings, A. E., and Kashima, K. (1996a). An earthquake history derived from stratigraphic and microfossil evidence of relative sea-level change at Coos Bay, southern coastal Oregon. Geological Society of America Bulletin 108, 141-154.

Nelson, A. R., Shennan, I., and Long, A. J. (1996b). Identifying coseismic subsidence in tidal-wetland stratigraphic sequences at the Cascadia subduction zone of western North America. Journal of Geophysical Research 101, 6115-6135.

Ota, Y., Berryman, K. R., Hull, A. G., Miyauchi, T., and Iso, N. (1988). Age and height distribution of Holocene transgressive deposits in eastern North Island, New Zealand. Palaeogeography, Palaeoclimatology, Palaeoecology 68, 135-151.

Ota, Y., Berryman, K. R., Brown, L. J., and Kashima, K. (1989). Holocene sediments and vertical tectonic downwarping near Wairoa, Northern Hawke's Bay, New Zealand. New Zealand Journal of Geology and Geophysics 32, 333-341.

Ota, Y., Brown, L. J., Berryman, K. R., Fujimori, T., Miyauchi, T., Beu, A. G., Kashima, K., and Taguchi, K. (1995). Vertical tectonic movement in northeastern Marlborough: stratigraphic, radiocarbon, and paleoecological data from Holocene estuaries. New Zealand Journal of Geology and Geophysics 38, 269-282.

Palmer, A. J. M., and Abbott, W. H. (1986). Diatoms as indicators of sea-level change. In "Sea-Level Research: a manual for the collection and evaluation of data." (O. van de Plassche). Geo Books, Norwich. 
Patrick, R., and Reimer, C. W. (1966). "The Diatoms of the United States." The Academy of Natural Sciences of Philadelphia, Philadelphia.

(1975). "The Diatoms of the United States." The Academy of Natural Sciences of Philadelphia, Philadelphia.

Patterson, R. T., and Fishbein, E. (1989). Re-examination of the statistical methods used to determine the number of point counts needed for micropaleontological quantitative research. Journal of Paleontology 63, 245-248.

Pillans, B. (1990). Pleistocene marine terraces in New Zealand: a review. New Zealand Journal of Geology and Geophysics 33, 219-231.

Pillans, B., and Huber, P. (1995). Interpreting coseismic deformation using Holocene coastal deposits, Wellington, New Zealand. Quaternary International 26, 87-95.

Reavie, E. D., and Smol, J. P. (2001). Diatom-environmental relationships in 64 alkaline southeastern Ontario (Canada) lakes: a diatom-based model for water quality reconstruction. Journal of Paleolimnology 25, 25-42.

Reed, J. M. (1998). A diatom-conductivity transfer function for Spanish salt lakes. Journal of Paleolimnology 19, 399-416.

Robinson, R., Benites, R., and Van Dissen, R. (1998). Evidence for temporal clustering of large earthquakes in the Wellington Region from computer models of seismicity. Bulletin of the New Zealand Society for Earthquake Engineering 31, 24-32.

Round, F. E. (1953). An investigation of two benthic algal communities in Malham Tarn, Yorkshire. Journal of Ecology 41, 174-197.

Round, F. E., Crawford, R. M., and Mann, D. G. (1990). “The Diatoms: Biology and Morphology of the Genera." Cambridge University Press, Cambridge.

Round, F. E., and Bukhtiyarova, L. (1996). Four new genera based on Achnanthes (Achnanthidium) together with a re-definition of Achnanthidium. Diatom Research 11, 345-361.

Schoeman, F. R., and Archibald, R. E. M. (1976). "The Diatom Flora of Southern Africa." Council for Scientific and Industrial Research, Pretoria.

Schwartz, D. P., and Coppersmith, K. J. (1984). Fault behavior and characteristic earthquakes: Examples from the Wasatch and San Andreas Fault Zones. Journal of Geophysical Research 89, 5681-5698.

Shennan, I., Long, A. J., Rutherford, M. M., Green, F. M., Innes, J. B., Lloyd, J. M., Zong, Y., and Walker, K. J. (1996). Tidal marsh stratigraphy, sea-level change and large earthquakes, I: A 5000 year record in Washington, U.S.A. Quaternary Science Reviews 15, 1023-1059. 
Shennan, I., Long, A. J., Rutherford, M. M., Innes, J. B., Green, F. M., and Walker, K. J. (1998). Tidal marsh stratigraphy, sea level change and large earthquakes - II: Submergence events during the last 3500 years at Netarts Bay, Oregon, USA. Quaternary Science Reviews 17, 365-393.

Sibson, R. H. (1989). Earthquake faulting as a structural process. Journal of Structural Geology 11, 1-14.

Sims, J. D. (1975). Determining earthquake recurrence intervals from deformational structures in young lacustrine sediments. Tectonophysics 29, 141-152.

Smilauer, P. 1992. Canodraw Lite 3.0, Computer Program. University College London, London.

Solem, T., and Solem, J. O. (1997). Shoreline displacement on the coast of SorTrondelag and More og Romsdal, central Norway; a botanical and zoological approach. Norsk Geologisk Tidsskrift 77, 193-203.

Stabell, B. (1980). Holocene shorelevel displacement in Telemark, southern Norway. Norsk Geologisk Tidsskrift 60, 71-81.

(1985). The development and succession of taxa with the diatom genus Fragilaria Lyngbye as a response to basin isolation from the sea. Boreas 14, 273-286.

Stevens, G. R. (1973). Late Holocene marine features adjacent to Port Nicholson, Wellington, New Zealand. New Zealand Journal of Geology and Geophysics 16, 455-484.

Stidolph, S. R. (1980). A record of some coastal marine diatoms from Porirua Harbour, North Island, New Zealand. New Zealand Journal of Botany 18, 379-403.

(1985). Some benthic diatoms from Kapiti Island, near Cook Strait, New Zealand. Nova Hedwigia 41, 393-415.

(1993). A light and electron microscopical study of Hyalodiscus pustulatus A. Schmidt (Bacillariophyceae) from New Zealand marine habitats. Botanica Marina 36, 79-86.

Stirling, M. W. (1992). Late Holocene beach ridges displaced by the Wellington Fault in the Lower Hutt area, New Zealand. New Zealand Journal of Geology and Geophysics 35, 447-453.

Stokes, C. J. L. (1858). Porirua Harbour and Mana Island. Hydrographic Office of the Admiralty, London. 
Stuiver, M., and Polach, H. A. (1977). Discussion: Reporting of 14C Data. Radiocarbon 19, 355-363.

Stuiver, M., Reimer, P. J., Bard, E., Beck, J. W., Burr, G. S., Hughen, K. A., Kromer, B., McCormac, G., van der Plicht, J., and Spurk, M. (1998). INTCAL98 radiocarbon age calibration, 24,000-0 cal BP. Radiocarbon 40, 1041-1083.

ter Braak, C. J. F. (1986). Canonical correspondence analysis: a new eigenvector technique for multivariate direct gradient analysis. Ecology 67, 1167-1179.

(1988). Canoco - a fortran program for canonical community ordination. Microcomputer Power, New York.

(1995). Ordination. In "Data Analysis in Community and Landscape Ecology.” (R. H. G. Jongman, C. J. F. ter Braak, and O. F. R. van Tongeren), pp. 91-173. Cambridge University Press, Cambridge.

ter Braak, C. J. F., and Juggins, S. (1993). Weighted averaging partial least squares regression (WA-PLS): an improved method for reconstructing environmental variables from species assemblages. Hydrobiologia 269/270, 485-502.

ter Braak, C. J. F., and Looman, C. W. N. (1995). Regression. In "Data Analysis in Community and Landscape Ecology." (R. H. G. Jongman, C. J. F. ter Braak, and O. F. R. van Tongeren), pp. 29-77. Cambridge University Press, Cambridge.

Thatcher, W. (1984). The earthquake deformation cycle, recurrence, and the timepredictable model. Journal of Geophysical Research 89, 5674-5680.

Van Dam, H., Mertens, A., and Sinkeldam, J. (1994). A coded checklist and ecological indicator values of freshwater diatoms from the Netherlands.

Netherlands Journal of Aquatic Ecology 28, 117-133.

Van de Plassche, O. (ed.). (1986). "Sea-level research: a manual for the collection and evaluation of data." Geo Books, Norwich.

Van Dissen, R. J., Berryman, K. R., Pettinga, J. R., and Hill, N. L. (1992).

Paleoseismicity of the Wellington - Hutt Valley segment of the Wellington Fault, North Island, New Zealand. New Zealand Journal of Geology and Geophysics 35, $165-176$.

Van Dissen, R. J. and Berryman, K. R. (1996). Surface rupture earthquakes over the last $\sim 1000$ years in the Wellington region, New Zealand, and implications for ground shaking hazard. Journal of Geophysical Research 101, 5999-6019.

Van Dissen, R. J., Heron, D., and Palmer, A. (1999). Ohariu and Northern Ohariu Faults: Field Guide to Late Quaternary Active Faulting. In "Geological Society of New Zealand Annual Conference." (A. Palmer), pp. 29-69. Geological Society of New Zealand, Palmerston North. 
Vos, P. C., and de Wolf, H. (1988). Methodological aspects of paleo-ecological diatom research in coastal areas of the Netherlands. Geologie en Mijnbouw 67, $31-40$.

(1993). Diatoms as a tool for reconstructing sedimentary environments in coastal wetlands; methodological aspects. Hydrobiologia 269/270, 285-296.

Walcott, R. I. (1981). “...The gates of stress and strain..." In "Large earthquakes in New Zealand: Anticipation, Precaution, Reconstruction." (M. M. Cresswell), pp. 202. The Royal Society of New Zealand, Napier, New Zealand.

(1998). Modes of oblique compression: Late Cenozoic tectonics of the South Island of New Zealand. Reviews of Geophysics 36, 1-26.

Wellman, H. W. (1969). Tilted marine beach ridges at Cape Turakirae, New Zealand. Tuatara 17, 82-93.

Wilson, S. E., Cumming, B. F., and Smol, J. P. (1994). Diatom-salinity relationships in 111 lakes from the Interior Plateau of British Columbia, Canada: the development of diatom-based models for paleosalinity reconstructions. Journal of Paleolimnology 12, 197-221.

Witkowski, A., Lange-Bertalot, H., and Metzeltin, D. (2000). "Diatom Flora of Marine Coasts I." A. R. G. Gantner Verlag K. G., Ruggell.

Wolfe, A. P. (1997). On diatom concentrations in lake sediments: results from an inter-laboratory comparison and other tests performed on a uniform sample. Journal of Paleolimnology 18, 261-268.

Yeats, R. S., and Prentice, C. S. (1996). Introduction to special section: paleoseismology. Journal of Geophysical Research 101, 5847-5853.

Zachariasen, J., Berryman, K., Chague-Goff, C., Cochran, U., Hollis, C. J., Mildenhall, D. C., and Naish, T. (2000). Late Holocene subsidence at Wairoa, Hawke's Bay, New Zealand: evidence for Hikurangi subduction zone earthquakes? (Abstract). In "Geological Society of New Zealand and New Zealand Geophysical Society Joint Annual Conference.” (T. Little), pp. 176. Geological Society of New Zealand, Wellington.

Zachariasen, J., Berryman, K., Langridge, R., Prentice, C., Rymer, M., Stirling, M., and Villamor, P. (2001). Timing of late Holocene surface rupture of the Wairau Fault, Marlborough, New Zealand. in review.

Zong, Y. (1997). Implications of Paralia sulcata abundance in Scottish isolation basins. Diatom Research 12, 125-150.

Zong, Y., and Horton, B. P. (1998). Diatom zones across intertidal flats and coastal saltmarshes in Britain. Diatom Research 13, 375-394. 


\section{A P P E N D I X I}

\section{Core Logs}

Item 1: Table of Grainsize Data

Abbreviations used:

crse coarse fraction

wet wet sediment

orgs organic material

dry dry sediment

carb carbonate

Item 2: Core Logs

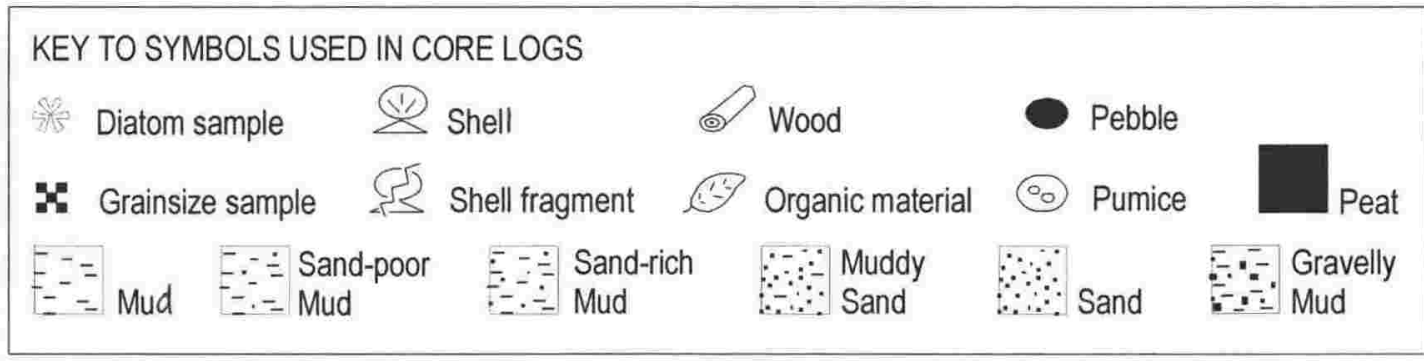

See Table 2.2 (Chapter Two) for definition of sedimentary units.

Core TS97-1. Collected 4/5/97 from Taupo Swamp.

Grid Reference (NZ Map Grid): R26 674129.

Fossil Record Number: R26/f217.

Note: Sampling for pollen took place before cores were

photographed so sample positions visible in the photos do not relate to samples used in this project - see 'samples' column for correct diatom and grainsize sample positions.

Core TS98-2. Collected 19/6/98 from Taupo Swamp.

Grid Reference (NZ Map Grid): R26 673129.

Fossil Record Number: R26/f218.

Core OL97-3. Collected 14/7/97 from Okupe Lagoon.

Grid Reference (NZ Map Grid): R25 741404.

Fossil Record Number: R25/f1.

Note: Base of core was sampled before section was photographed.

Core OL97-5. Collected 15/7/97 from Okupe Lagoon.

Grid Reference (NZ Map Grid): R25 746402.

Fossil Record Number: R25/f2.

Note: This core was extensively sampled by several users before cores were photographed so much of core is missing.

KP99-1\&2. Collected 11/5/99 from Lake Kohangapiripiri.

Grid Reference (NZ Map Grid): R27 654810.

Fossil Record Number: R27/f301. 
Item 1: Table of Grainsize Data

\begin{tabular}{|c|c|c|c|c|c|c|c|c|c|c|c|c|c|}
\hline \multirow{2}{*}{$\begin{array}{l}\text { SAMPLES } \\
\text { cm depth }\end{array}$} & \multicolumn{8}{|c|}{ WEIGHTS (g) } & \multicolumn{5}{|c|}{ PERCENTAGES } \\
\hline & WET & DRY & ORGS & CARB & CRSE & FINE & TOTAL & LOSS & SAND & MUD & ORGS & CARB & LOSS \\
\hline \multicolumn{14}{|l|}{ KP99-1\&2 } \\
\hline 0 & & 14.06 & 2.30 & 0.62 & 2.71 & 8.24 & 13.87 & 0.19 & 19.27 & 58.61 & 16.36 & 4.41 & 1.35 \\
\hline 50 & & 8.42 & 1.07 & 0.53 & 0.65 & 6.08 & 8.33 & 0.09 & 7.72 & 72.21 & 12.71 & 6.29 & 1.07 \\
\hline 100 & & 9.69 & 1.16 & 0.67 & 0.75 & 7.04 & 9.62 & 0.07 & 7.74 & 72.65 & 11.97 & 6.91 & 0.72 \\
\hline 150 & & 7.83 & 0.83 & 0.69 & 0.52 & 5.66 & 7.70 & 0.13 & 6.64 & 72.29 & 10.60 & 8.81 & 1.66 \\
\hline 200 & & 10.85 & 1.16 & 0.78 & 0.93 & 7.84 & 10.71 & 0.14 & 8.57 & 72.26 & 10.69 & 7.19 & 1.29 \\
\hline 250 & & 8.63 & 0.86 & 0.64 & 1.19 & 5.84 & 8.53 & 0.10 & 13.79 & 67.67 & 9.97 & 7.42 & 1.16 \\
\hline 300 & & 8.96 & 0.78 & 0.72 & 1.15 & 6.20 & 8.85 & 0.11 & 12.83 & 69.20 & 8.71 & 8.04 & 1.23 \\
\hline 350 & & 11.71 & 0.78 & 0.73 & 0.70 & 9.36 & 11.57 & 0.14 & 5.98 & 79.93 & 6.66 & 6.23 & 1.20 \\
\hline 400 & & 9.74 & 0.94 & 0.67 & 1.20 & 6.80 & 9.61 & 0.13 & 12.32 & 69.82 & 9.65 & 6.88 & 1.33 \\
\hline 450 & & 7.56 & 1.16 & 0.46 & 0.72 & 5.16 & 7.50 & 0.06 & 9.52 & 68.25 & 15.34 & 6.08 & 0.79 \\
\hline 500 & & 11.38 & 0.84 & 0.74 & 3.66 & 6.08 & 11.32 & 0.06 & 32.16 & 53.43 & 7.38 & 6.50 & 0.53 \\
\hline 550 & & 8.73 & 1.01 & 0.66 & 0.54 & 6.39 & 8.60 & 0.13 & 6.19 & 73.20 & 11.57 & 7.56 & 1.49 \\
\hline 580 & & 24.99 & 0.00 & 1.88 & 16.78 & 6.15 & 24.81 & 0.18 & 67.15 & 24.61 & 0.00 & 7.52 & 0.72 \\
\hline 600 & & 9.93 & 0.78 & 1.02 & 1.12 & 7.08 & 10.00 & -0.07 & 11.28 & 71.30 & 7.85 & 10.27 & -0.70 \\
\hline 620 & & 31.81 & 0.80 & 1.42 & 20.46 & 8.98 & 31.66 & 0.15 & 64.32 & 28.23 & 2.51 & 4.46 & 0.47 \\
\hline 650 & & 28.45 & 0.88 & 1.33 & 17.25 & 8.88 & 28.34 & 0.11 & 60.63 & 31.21 & 3.09 & 4.67 & 0.39 \\
\hline \multicolumn{14}{|l|}{ OL97-5 } \\
\hline 0 & 20.17 & 7.09 & 2.38 & 0.66 & 0.00 & 3.99 & 7.03 & 0.06 & 0.00 & 56.28 & 33.57 & 9.31 & 0.85 \\
\hline 45 & 25.27 & 14.28 & 2.61 & 0.47 & 9.90 & 1.27 & 14.25 & 0.03 & 69.33 & 8.89 & 18.28 & 3.29 & 0.21 \\
\hline 60 & 26.69 & 16.50 & 1.91 & 0.32 & 13.33 & 0.92 & 16.48 & 0.02 & 80.79 & 5.58 & 11.58 & 1.94 & 0.12 \\
\hline 90 & 28.86 & 23.12 & 1.68 & 0.64 & 19.38 & 1.37 & 23.07 & 0.05 & 83.82 & 5.93 & 7.27 & 2.77 & 0.22 \\
\hline 120 & 35.28 & 28.48 & 0.74 & 1.31 & 18.41 & 7.88 & 28.34 & 0.14 & 64.64 & 27.67 & 2.60 & 4.60 & 0.49 \\
\hline 150 & 26.02 & 21.05 & 0.54 & 2.18 & 15.58 & 2.63 & 20.93 & 0.12 & 74.01 & 12.49 & 2.57 & 10.36 & 0.57 \\
\hline 170 & 26.39 & 20.06 & 1.15 & 2.43 & 14.25 & 2.12 & 19.95 & 0.11 & 71.04 & 10.57 & 5.73 & 12.11 & 0.55 \\
\hline 190 & 32.62 & 26.32 & 1.37 & 2.22 & 19.62 & 3.02 & 26.23 & 0.09 & 74.54 & 11.47 & 5.21 & 8.43 & 0.34 \\
\hline \multicolumn{14}{|l|}{ OL-97-3 } \\
\hline 0 & 19.29 & 10.50 & 1.30 & 0.56 & 6.33 & 2.26 & 10.45 & 0.05 & 60.29 & 21.52 & 12.38 & 5.33 & 0.48 \\
\hline 20 & 17.73 & 8.16 & 1.52 & 0.53 & 4.42 & 1.67 & 8.14 & 0.02 & 54.17 & 20.47 & 18.63 & 6.50 & 0.25 \\
\hline 40 & 22.21 & 14.99 & 0.94 & 0.95 & 5.51 & 7.54 & 14.94 & 0.05 & 36.76 & 50.30 & 6.27 & 6.34 & 0.33 \\
\hline 70 & 19.41 & 14.61 & 0.40 & 0.95 & 4.82 & 8.39 & 14.56 & 0.05 & 32.99 & 57.43 & 2.74 & 6.50 & 0.34 \\
\hline 100 & 16.07 & 10.88 & 0.42 & 1.20 & 2.02 & 7.21 & 10.85 & 0.03 & 18.57 & 66.27 & 3.86 & 11.03 & 0.28 \\
\hline 130 & 19.55 & 12.74 & 0.57 & 1.86 & 3.86 & 6.37 & 12.66 & 0.08 & 30.30 & 50.00 & 4.47 & 14.60 & 0.63 \\
\hline 160 & 17.95 & 13.28 & 0.40 & 1.90 & 6.91 & 4.05 & 13.26 & 0.02 & 52.03 & 30.50 & 3.01 & 14.31 & 0.15 \\
\hline 180 & 22.59 & 16.32 & 0.86 & 1.64 & 9.12 & 4.68 & 16.30 & 0.02 & 55.88 & 28.68 & 5.27 & 10.05 & 0.12 \\
\hline \multicolumn{14}{|l|}{ TS98-2 } \\
\hline 60 & & 5.15 & 3.17 & 0.11 & 0.00 & 1.80 & 5.08 & 0.07 & 0.00 & 34.95 & 61.55 & 2.14 & 1.36 \\
\hline 140 & & 2.93 & 2.23 & 0.10 & 0.00 & 0.52 & 2.85 & 0.08 & 0.00 & 17.75 & 76.11 & 3.41 & 2.73 \\
\hline 185 & & 17.21 & 0.53 & 0.45 & 8.08 & 8.00 & 17.06 & 0.15 & 46.95 & 46.48 & 3.08 & 2.61 & 0.87 \\
\hline 200 & & 10.60 & 0.91 & 0.31 & 1.02 & 8.31 & 10.55 & 0.05 & 9.62 & 78.40 & 8.58 & 2.92 & 0.47 \\
\hline 250 & & 22.72 & 2.05 & 0.22 & 18.22 & 2.07 & 22.56 & 0.16 & 80.19 & 9.11 & 9.02 & 0.97 & 0.70 \\
\hline 290 & & 19.88 & 1.78 & 0.38 & 11.62 & 6.02 & 19.80 & 0.08 & 58.45 & 30.28 & 8.95 & 1.91 & 0.40 \\
\hline 320 & & 10.40 & 1.54 & 0.35 & 2.09 & 6.28 & 10.26 & 0.14 & 20.10 & 60.38 & 14.81 & 3.37 & 1.35 \\
\hline 355 & & 13.10 & 2.31 & 0.26 & 4.62 & 5.91 & 13.10 & 0.00 & 35.27 & 45.11 & 17.63 & 1.98 & 0.00 \\
\hline 385 & & 29.29 & 0.85 & 1.80 & 21.02 & 5.48 & 29.15 & 0.14 & 71.77 & 18.71 & 2.90 & 6.15 & 0.48 \\
\hline 400 & & 8.57 & 1.56 & 0.27 & 2.24 & 4.40 & 8.47 & 0.10 & 26.14 & 51.34 & 18.20 & 3.15 & 1.17 \\
\hline 425 & & 12.30 & 1.28 & 0.27 & 7.28 & 3.36 & 12.19 & 0.11 & 59.19 & 27.32 & 10.41 & 2.20 & 0.89 \\
\hline 460 & & 9.12 & 1.60 & 0.74 & 1.22 & 5.55 & 9.11 & 0.01 & 13.38 & 60.86 & 17.54 & 8.11 & 0.11 \\
\hline 475 & & 13.68 & 1.06 & 0.54 & 5.70 & 5.99 & 13.29 & 0.39 & 41.67 & 43.79 & 7.75 & 3.95 & 2.85 \\
\hline 500 & & 9.22 & 1.31 & 0.46 & 1.83 & 5.58 & 9.18 & 0.04 & 19.85 & 60.52 & 14.21 & 4.99 & 0.43 \\
\hline \multicolumn{14}{|l|}{ TS97-1 } \\
\hline 60 & & 7.38 & 1.07 & 0.83 & 1.54 & 3.93 & 7.37 & 0.01 & 20.87 & 53.25 & 14.50 & 11.25 & 0.14 \\
\hline 120 & & 2.11 & 1.40 & 0.13 & 0.00 & 0.58 & 2.11 & 0.00 & 0.00 & 27.49 & 66.35 & 6.16 & 0.00 \\
\hline 190 & & 4.73 & 1.45 & 0.36 & 0.00 & 2.91 & 4.72 & 0.01 & 0.00 & 61.52 & 30.66 & 7.61 & 0.21 \\
\hline 230 & & 5.36 & 1.44 & 0.42 & 0.00 & 3.48 & 5.34 & 0.02 & 0.00 & 64.93 & 26.87 & 7.84 & 0.37 \\
\hline 250 & & 9.92 & 0.80 & 0.30 & 1.83 & 6.78 & 9.71 & 0.21 & 18.45 & 68.35 & 8.06 & 3.02 & 2.12 \\
\hline 350 & & 9.71 & 1.97 & 0.64 & 1.90 & 5.60 & 10.11 & -0.40 & 19.57 & 57.67 & 20.29 & 6.59 & -4.12 \\
\hline 450 & & 7.95 & 2.19 & 0.46 & 0.92 & 4.31 & 7.88 & 0.07 & 11.57 & 54.21 & 27.55 & 5.79 & 0.88 \\
\hline 550 & & 15.03 & 4.37 & 0.99 & 0.42 & 9.22 & 15.00 & 0.03 & 2.79 & 61.34 & 29.08 & 6.59 & 0.20 \\
\hline
\end{tabular}




\section{Core: TS97-1}

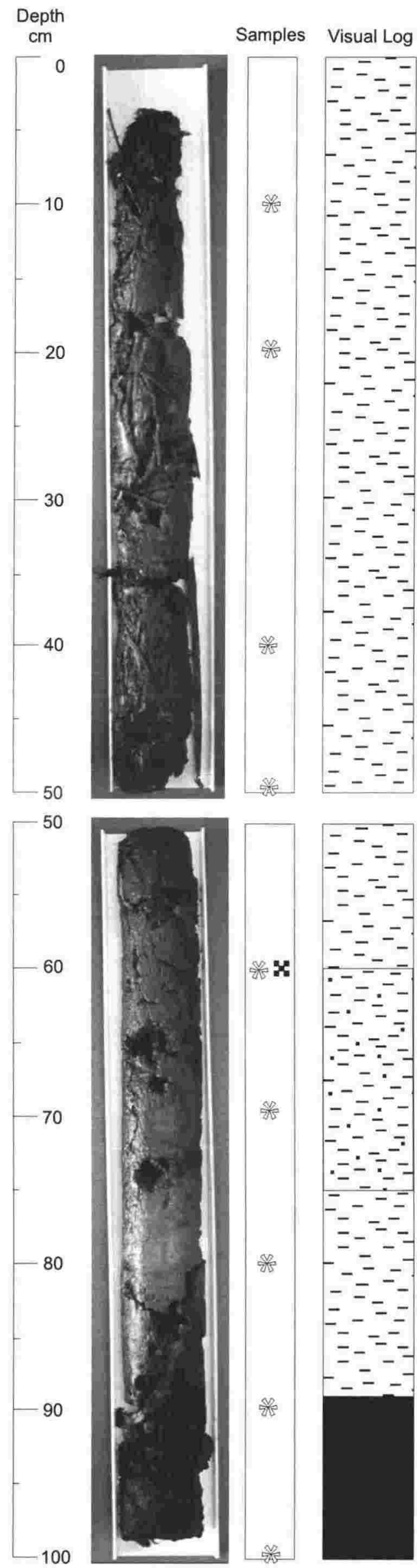

\section{Description}

\section{Organic Mud \\ Dark olive brown $2.5 \mathrm{Y} 3 / 3$. Water-logged and full of living roots and reed material.} Sediment less water-logged and less root material. Layers of fine
black matted organic material.
As above.

Olive black $5 Y 3 / 2$.

Contact gradational (over $\sim 4 \mathrm{~cm}$ ).

Organic sand-poor mud

Dark brown. Gritty texture.

\section{Contact wavy, gradational.}

Organic mud

Bands of dark greyish yellow $2.5 \mathrm{Y} 4 / 2$, brownish black $2.5 \mathrm{Y} 3 / 2$ and black 2.5 Y $2 / 1$.

Contact wavy, gradational.

Peat

Black 2.5Y 2/1. Crumbly texture. 


\section{Core: TS97-1}

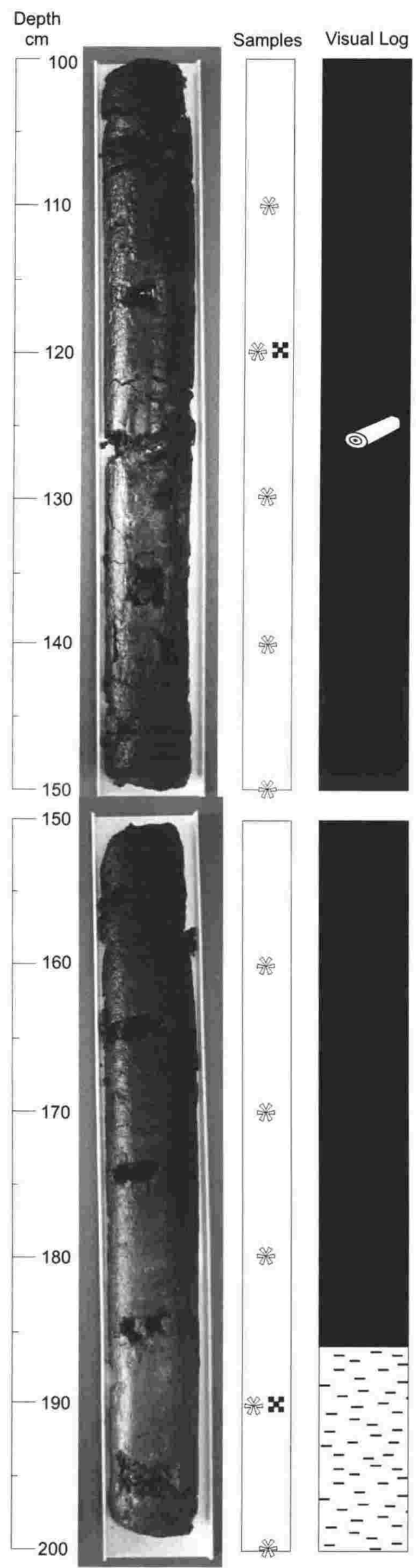

Sharp contact.

Brownish black 2.5Y 3/1. High mud content.

Large piece of wood.

Brownish black 7.5 Y $2 / 2$ with lighter brown mottles.

Gradational, wavy contact.

Black 2.5Y 2/1. Crumbly texture, fine organic matter.

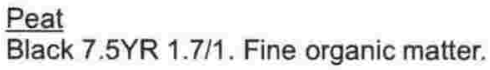

Dark brown 7.5YR 2/1. High mud content.

Gradational change (over $\sim 15 \mathrm{~cm}$ ).

Organic Mud

Brownish black 2.5Y $3 / 2$ 


\section{Core: TS97-1}

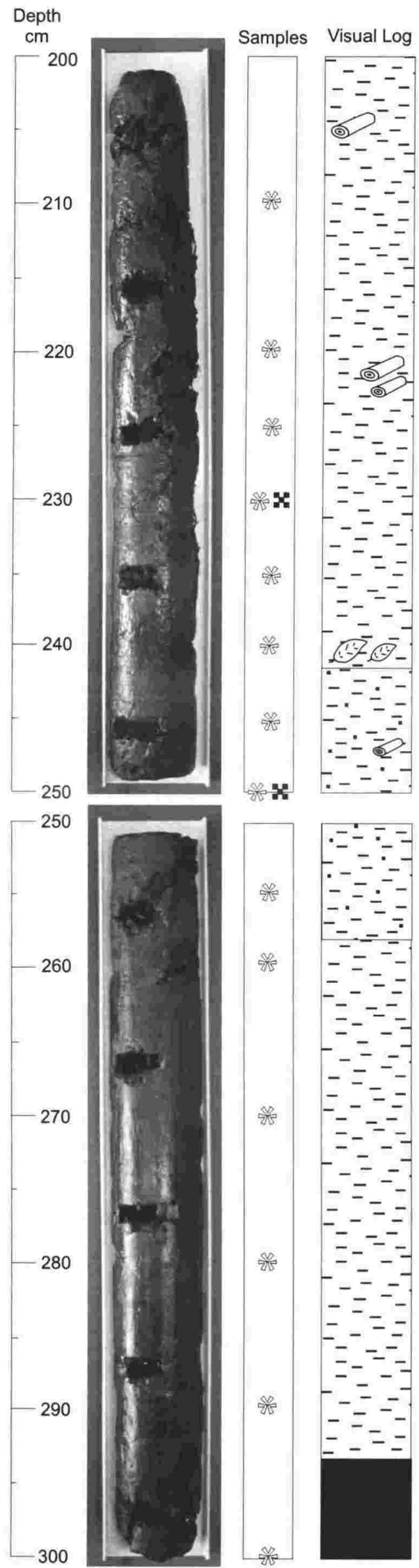

Description

\section{Organic Mud}

Brownish black 10YR 2/2. Organic matter throughout. Gradational change of decreasing silt content and increasing organic content from base to top.

Many small pieces of organic material including small rootlets. Contact sharp.

Sand-poor Mud

Dark olive brown 2.5 Y $3 / 3$

\section{Sand-poor Mud \\ Olive brown 2.5 Y $4 / 3$.}

Contact gradational.

\section{Organic Mud}

Gradational change of decreasing silt content and increasing organic material toward base.

Brownish black $2.5 Y 3 / 1$

\section{Contact gradational.}

Peat 


\section{Core: TS97-1}

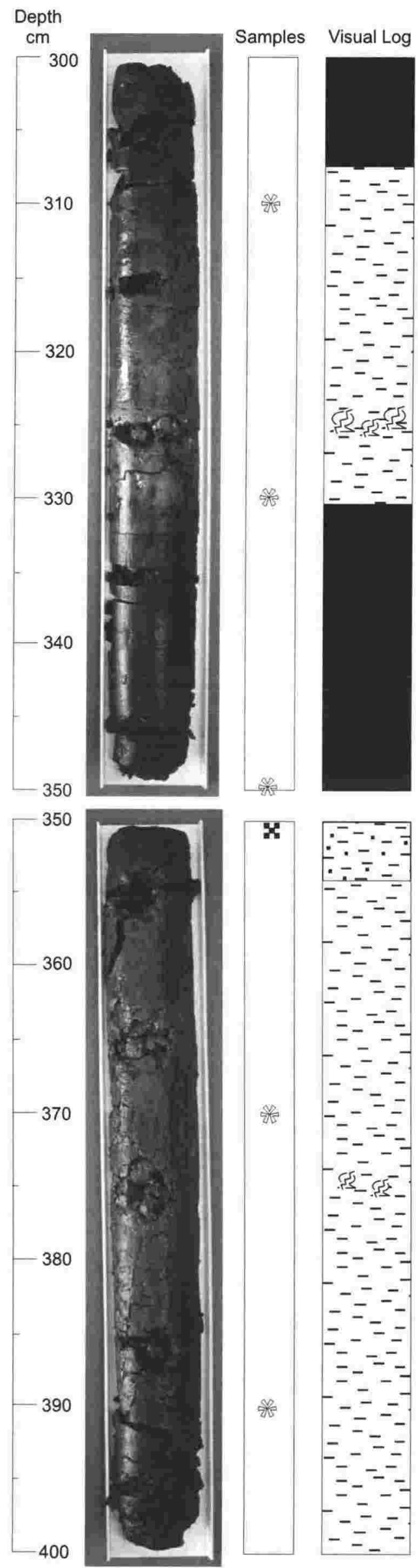

Description

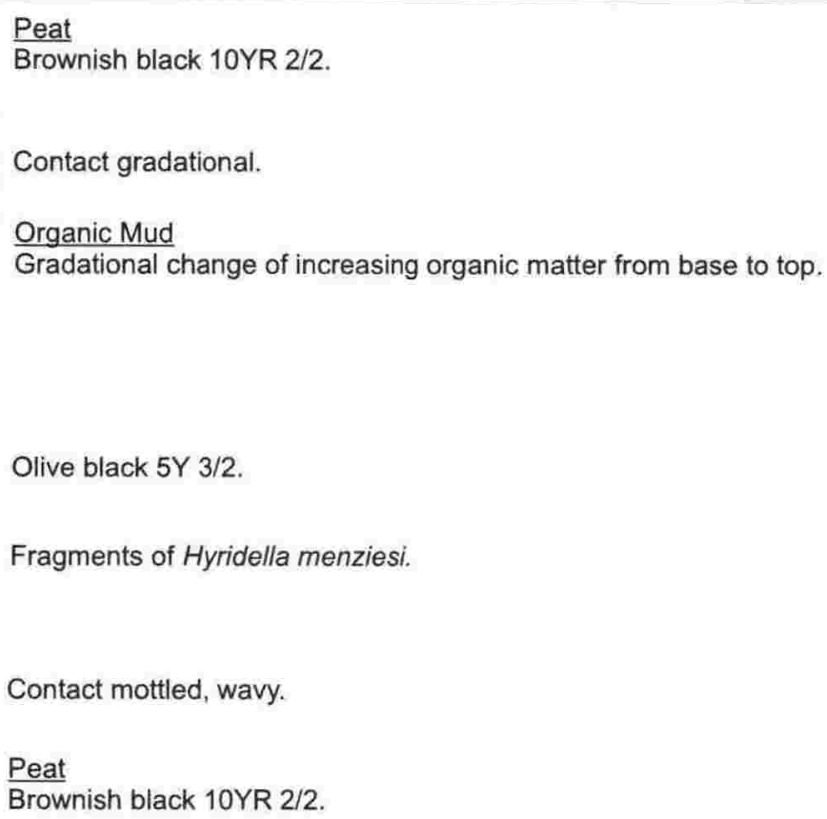

\section{Organic Mud}

Brownish black as above. Gradational change of decreasing organic material from base to top. 


\section{Core: TS97-1}

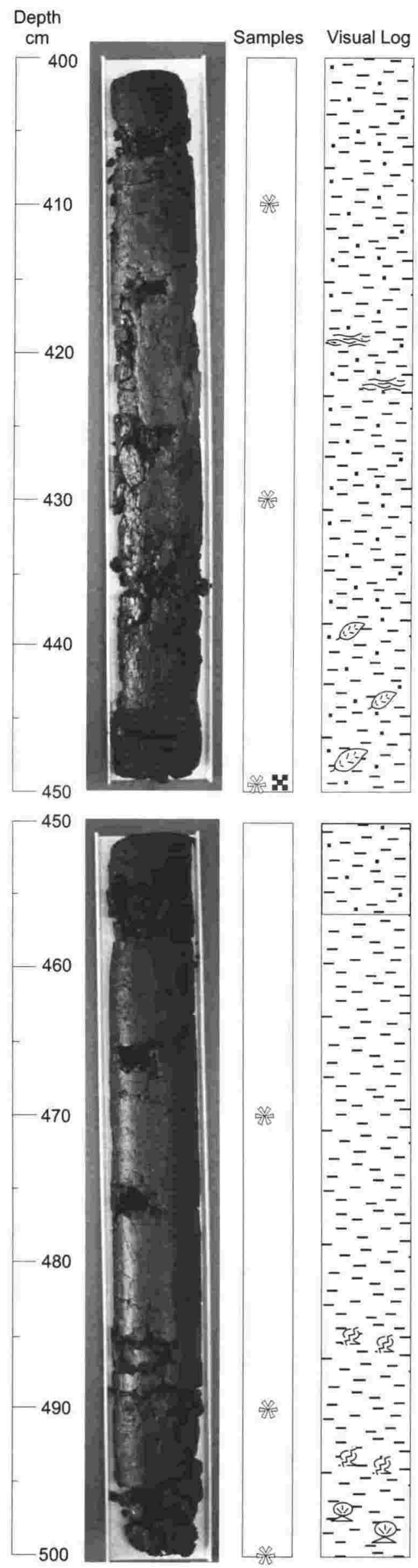

Description

\section{Organic Sand-poor Mud}

Brownish black 10YR 2/2.

Brownish black 2.5 Y $3 / 2$.

Mottles of lighter mud.

Numerous pieces of organic material

Organic Sand-poor Mud

Brownish black.

Contact gradational.

Organic Mud

Brownish black 10YR 2/2. Uniform. 


\section{Core: TS97-1}

\section{Depth}

$\mathrm{cm}$

- 500

Samples Visual Log

Description

\begin{tabular}{|c|c|c|}
\hline-510 & 糈 & (53- \\
\hline-520 & और & - \\
\hline-530 & 艛 & $-\overline{-}=$ \\
\hline-540 & 兟 & $=$ \\
\hline-550 & $x$ & \\
\hline
\end{tabular}

\section{Organic Mud}

Olive black 5 Y $3 / 1$.

Olive black $10 \mathrm{Y} 3 / 1$. 


\section{Core: TS98-2}

Depth

$\mathrm{cm}$

- 0

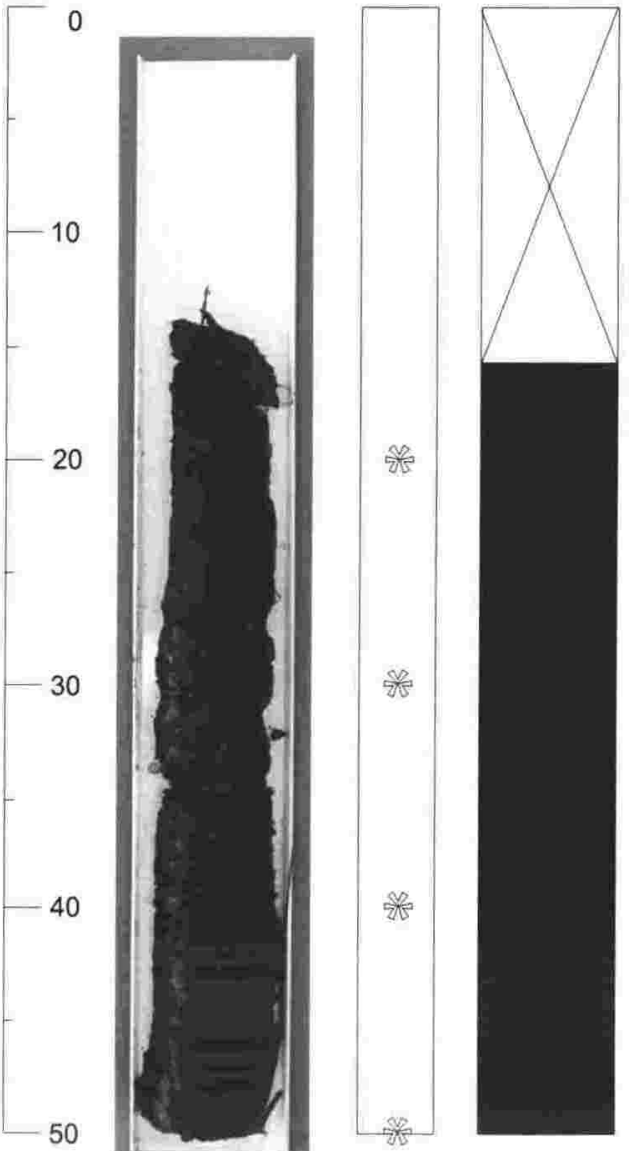

$-50$

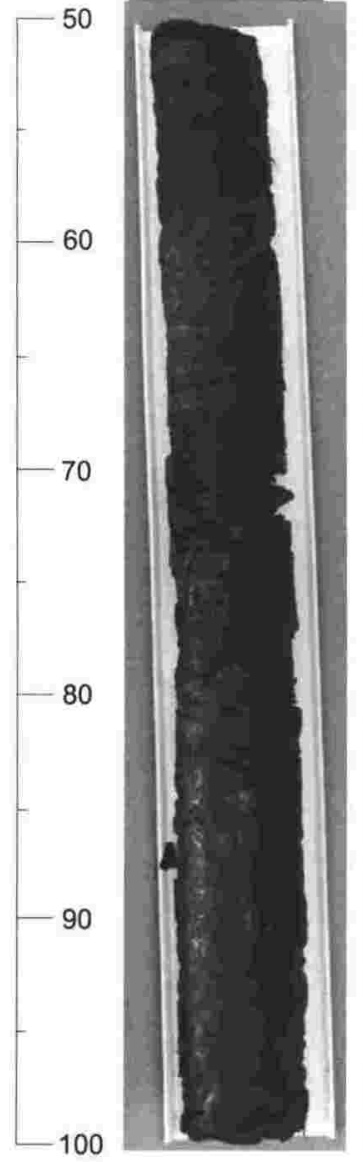

Samples

Visual Log

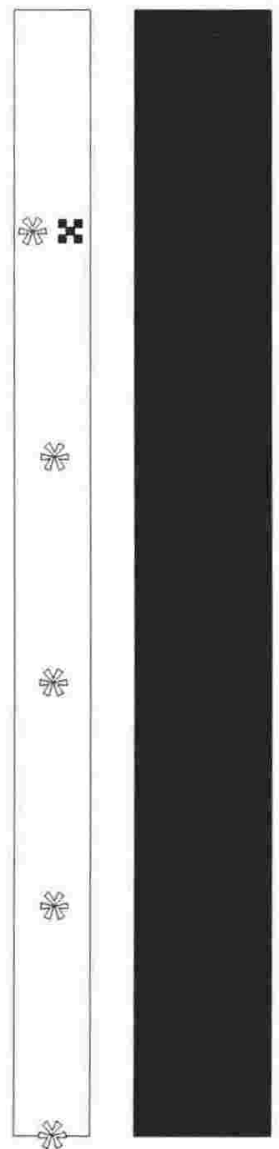

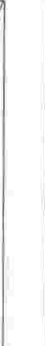

Brownish black 10YR 2/2. No silt, high clay content. Many modern

\section{Peat} roots and reed material.

Black 10YR 1.7/1. Crumbly texture.

Description

Too wet and with too many roots to collect

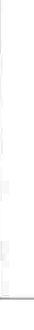

Brownish black 10YR 2/2. High clay content.

Black 10YR 2/1. Fibrous peat with small wood, reed and other organic fragments. 


\section{Core: TS98-2}

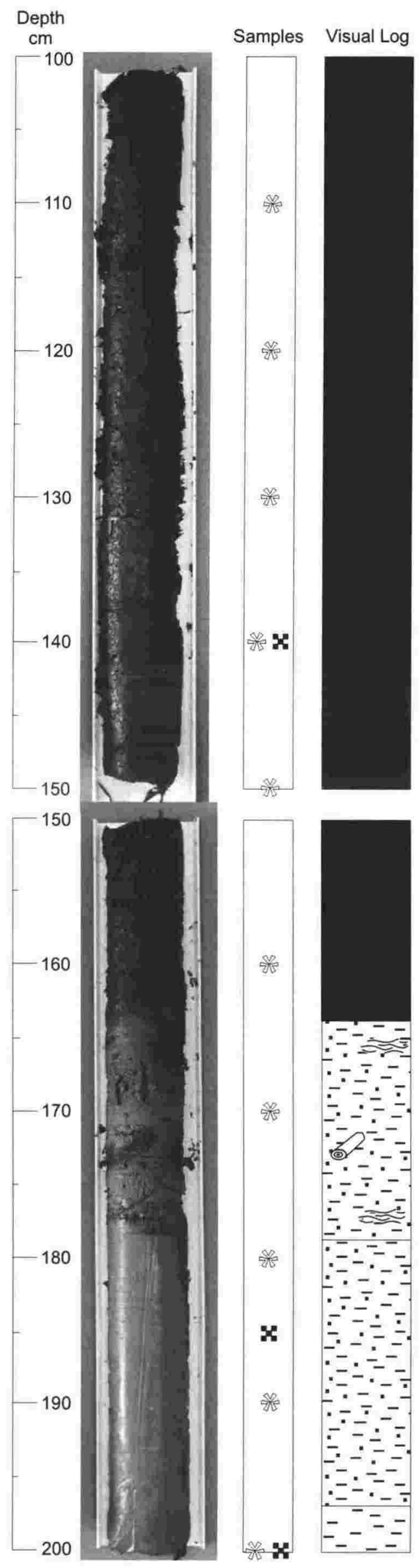

\section{Description}

Peat

Black 10YR 1.7/1. Fibrous peat with numerous small wood, reed and other organic fragments.

Peat

Black 10YR 1.7/1. Very low mud content, mainly wood fragments and fibrous material.

Contact wavy (over $\sim 1 \mathrm{~cm}$ )

\section{Sand-rich mud}

Olive brown $2.5 Y 4 / 3$. Bands of organic matter and wood fragments between 164 \& $179 \mathrm{~cm}$. Uniform, brown mud below this interval.

Contact gradational (over $\sim 3 \mathrm{~cm}$ ).

Mud 


\section{Core: TS98-2}

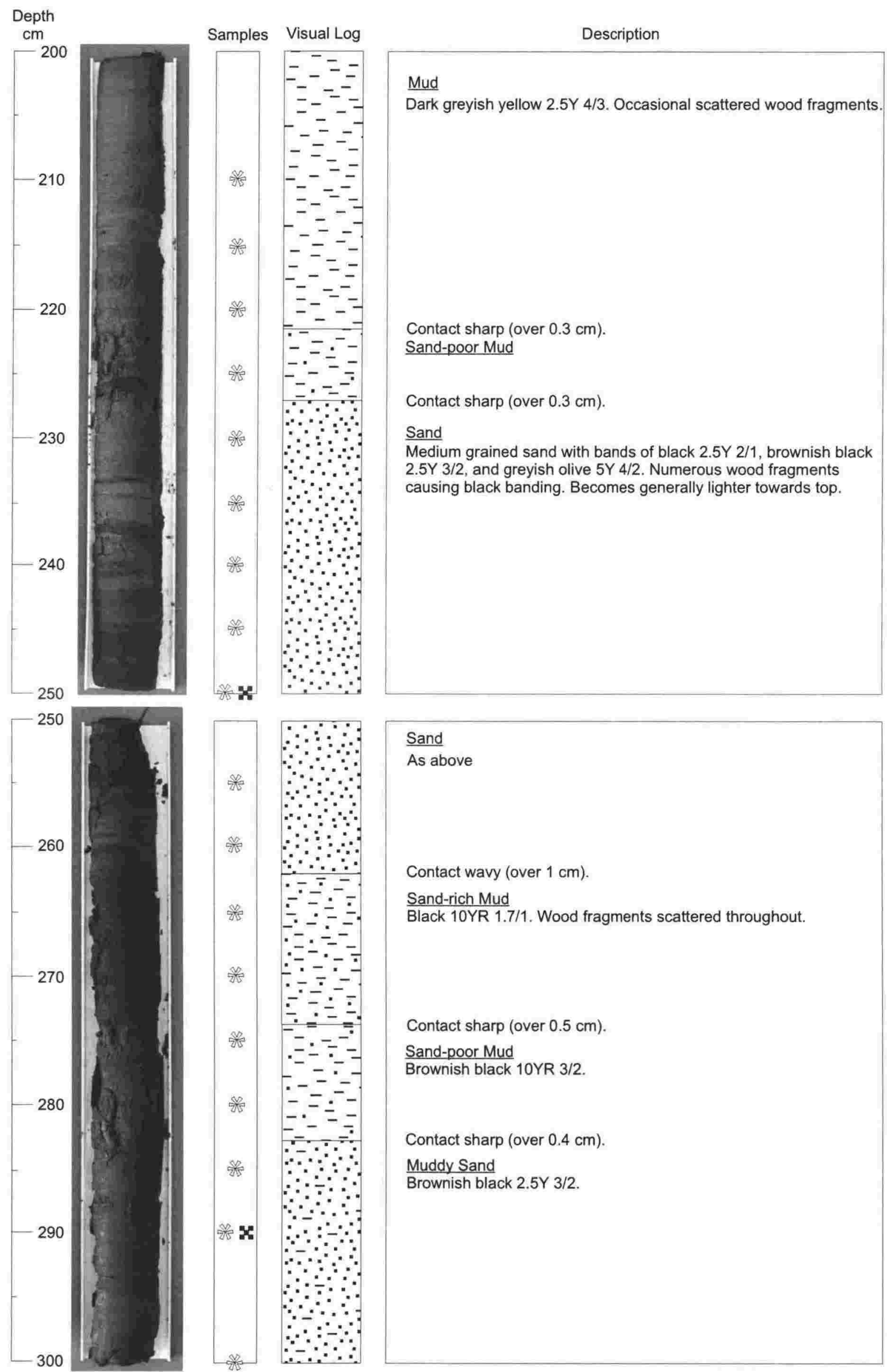




\section{Core: TS98-2}
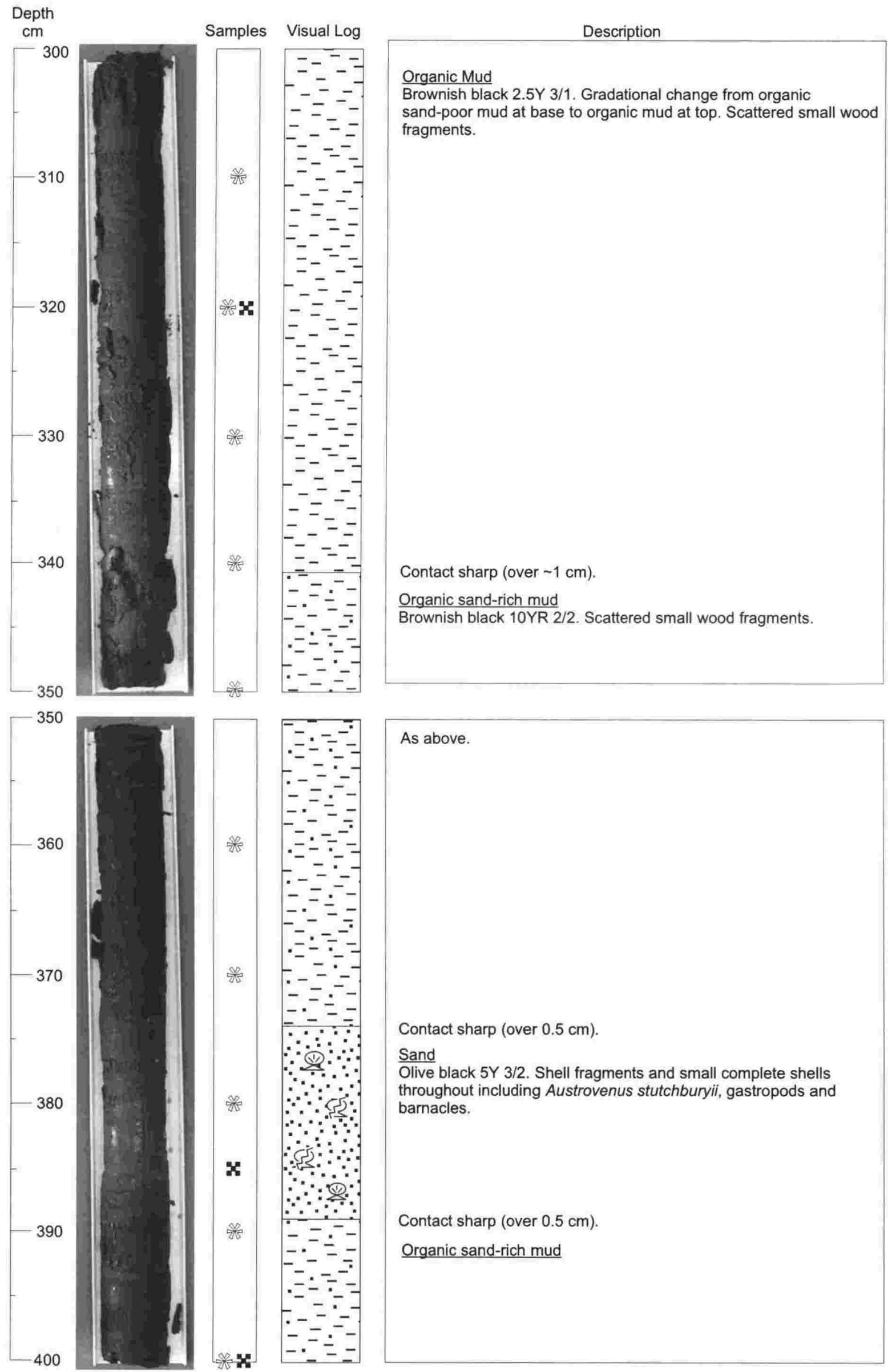

As above.

Contact sharp (over $0.5 \mathrm{~cm}$ ).

Sand

Olive black $5 Y 3 / 2$. Shell fragments and small complete shells throughout including Austrovenus stutchburyii, gastropods and barnacles.

Contact sharp (over $0.5 \mathrm{~cm}$ ).

Organic sand-rich mud 


\section{Core: TS98-2}

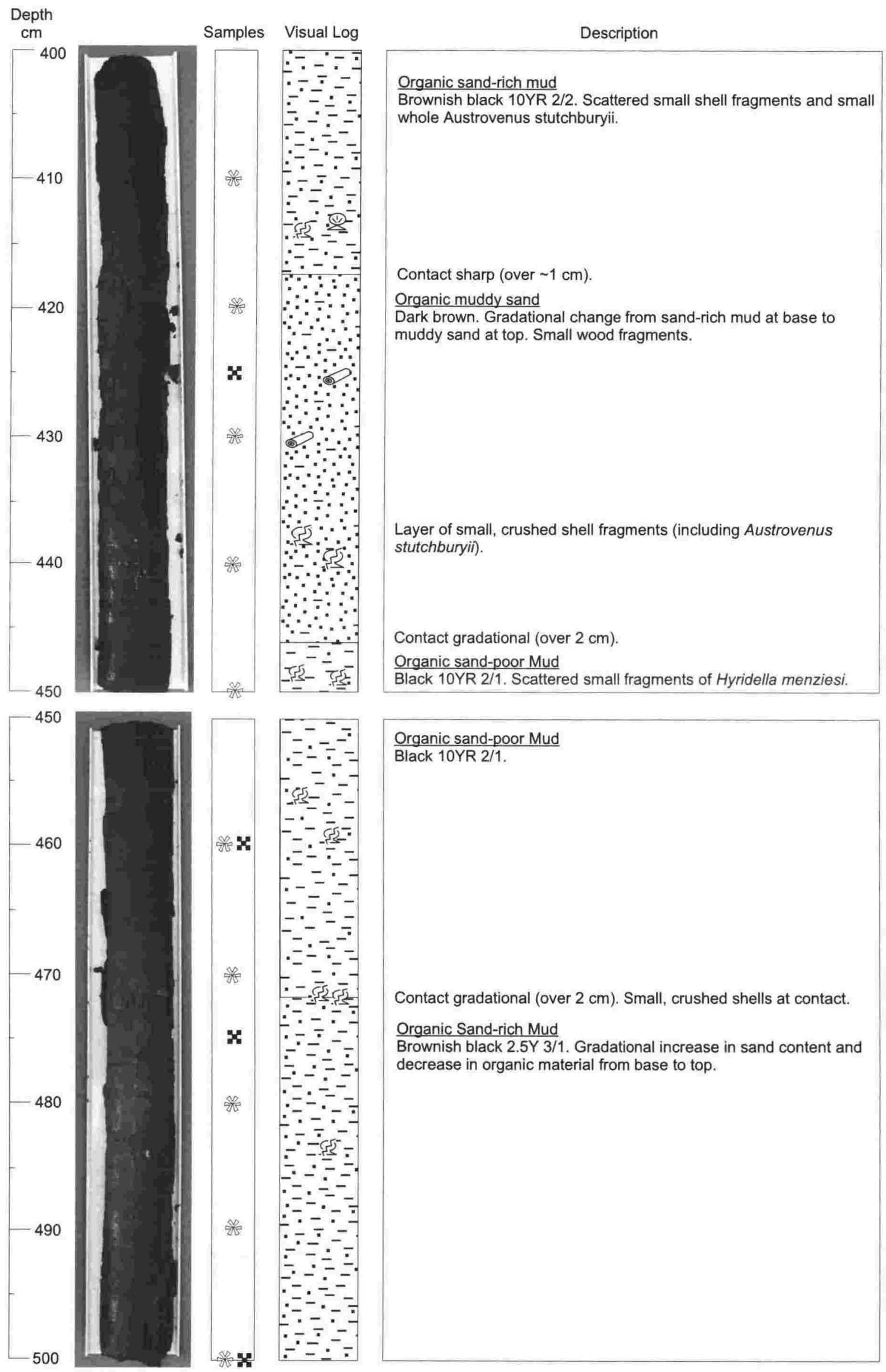




\section{Core: OL97-3}

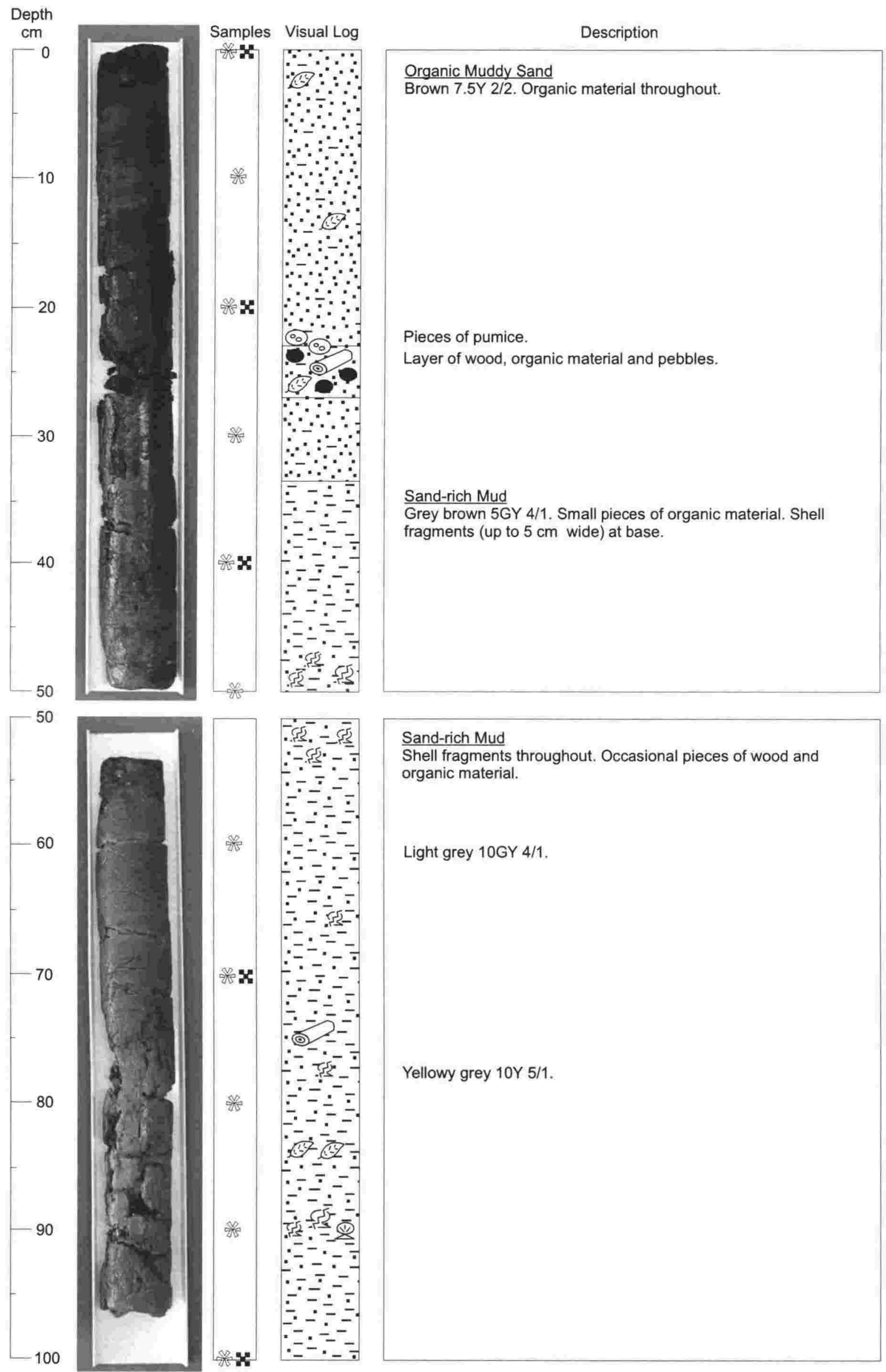




\section{Core: OL97-3}

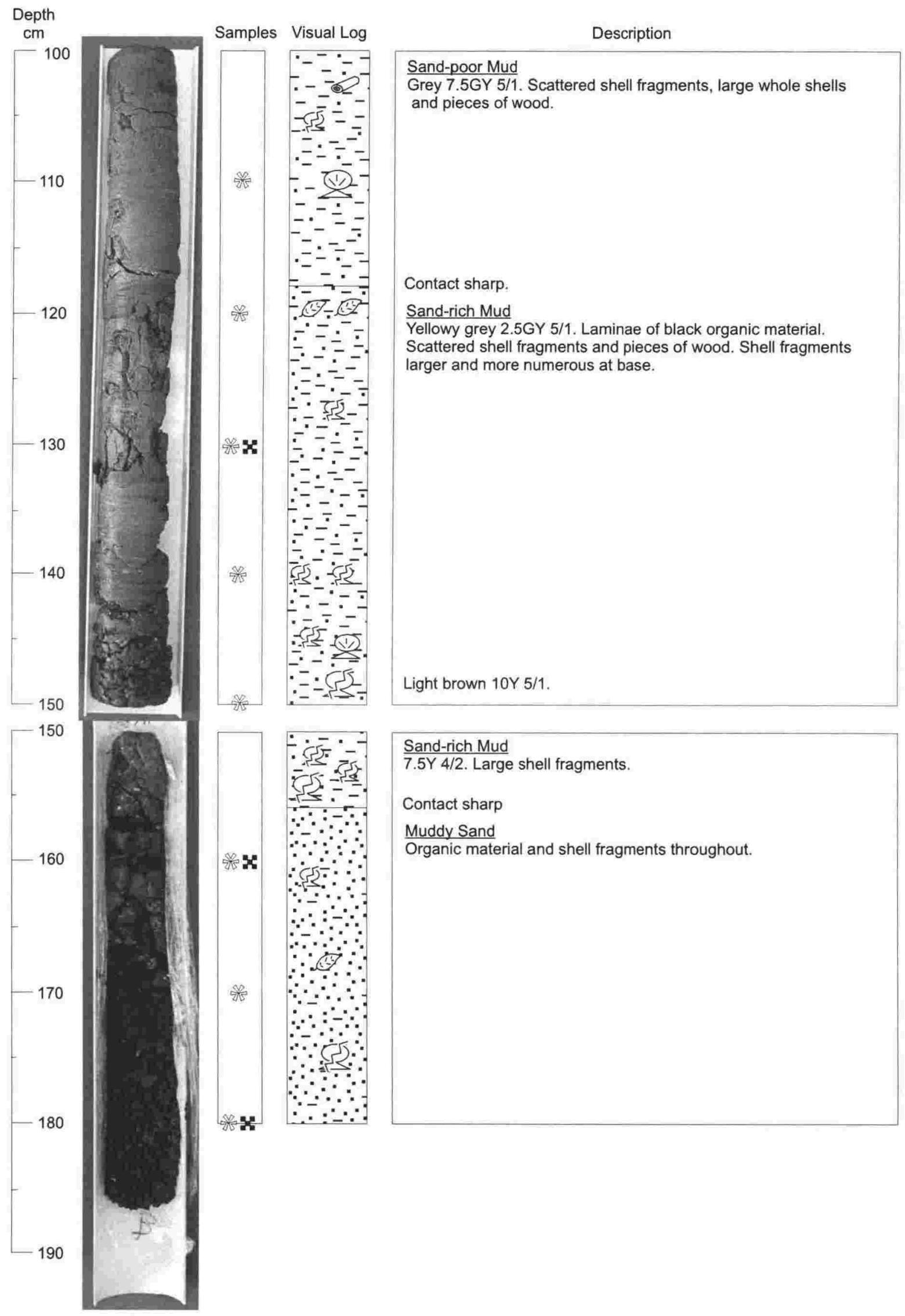




\section{Core: OL97-5}

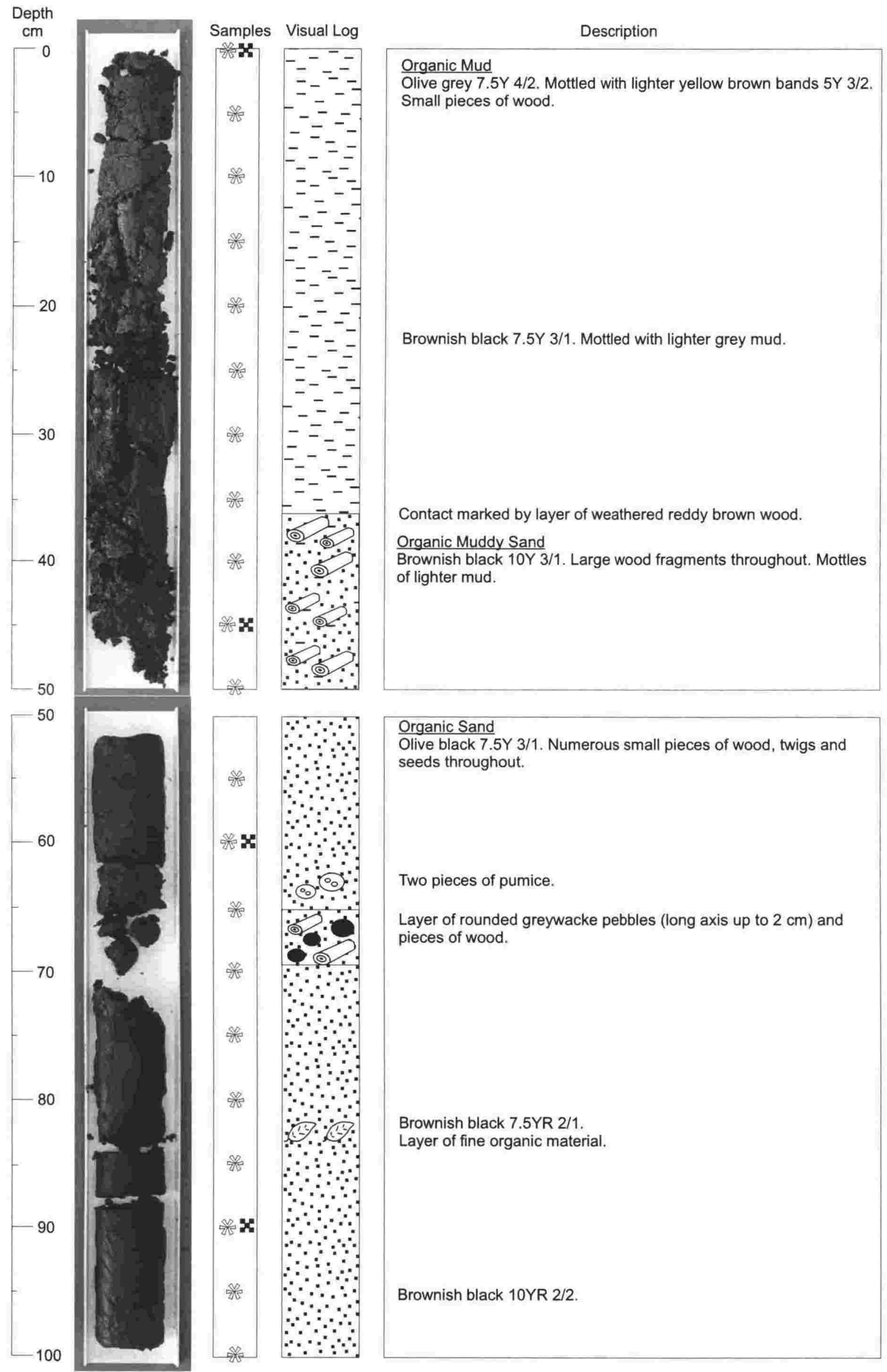




\section{Core: OL97-5}

Depth

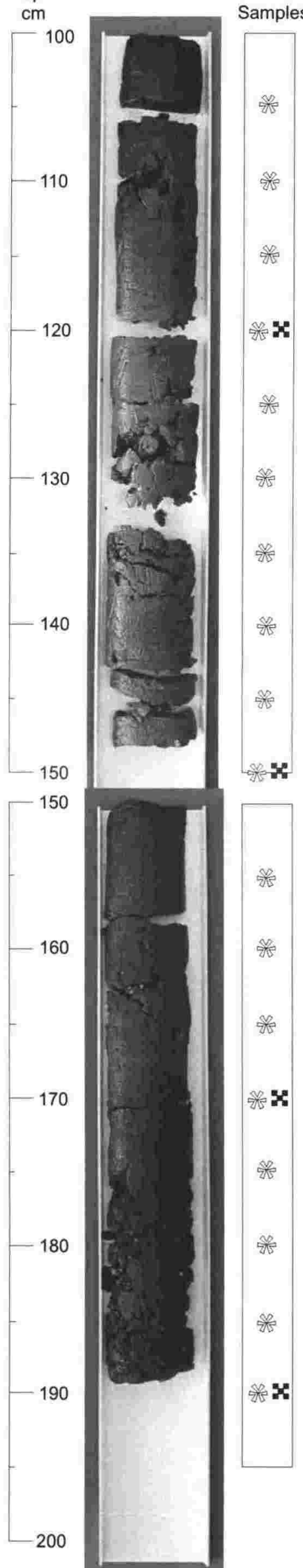

\section{Description}

Organic Muddy Sand

Gradational change from Grey Brown 7.5Y $3 / 2$ above pebble layer to Brown $7.5 Y 2 / 2$ at top.

Layer of pebbles and pieces of wood.

\section{Muddy Sand}

Yellowy Grey 5GY 4/1. Scattered small wood fragments.

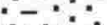

$\because \because \because \because$

$\therefore \therefore$

$\therefore \because \because$

$\because \because \because \therefore$

$\therefore \because \because$

$\therefore \div \therefore$

$\therefore \therefore \therefore$

$\therefore: \therefore$

险:

$\therefore \therefore \therefore$

$\because \sqrt{5}, \therefore$

$\therefore \div$

$\because \because$

$\therefore \therefore:$

$\therefore \because$

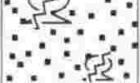

$\therefore \therefore \therefore$

Layer of reed and small shell fragments.

\section{Sand}

Grey $10 \mathrm{GY} 4 / 1$. Small shell fragments (mainly Austrovenus stutchburyii) scattered throughout.

\section{Sand}

Grey Brown 5GY 4/1. Numerous shell fragments and small pebbles throughout. Sand fines gradually and shell fragments decrease in size from base to top of section.

Brown $7.5 \mathrm{Y} 3 / 2$ 


\section{Core: KP99-1\&2}

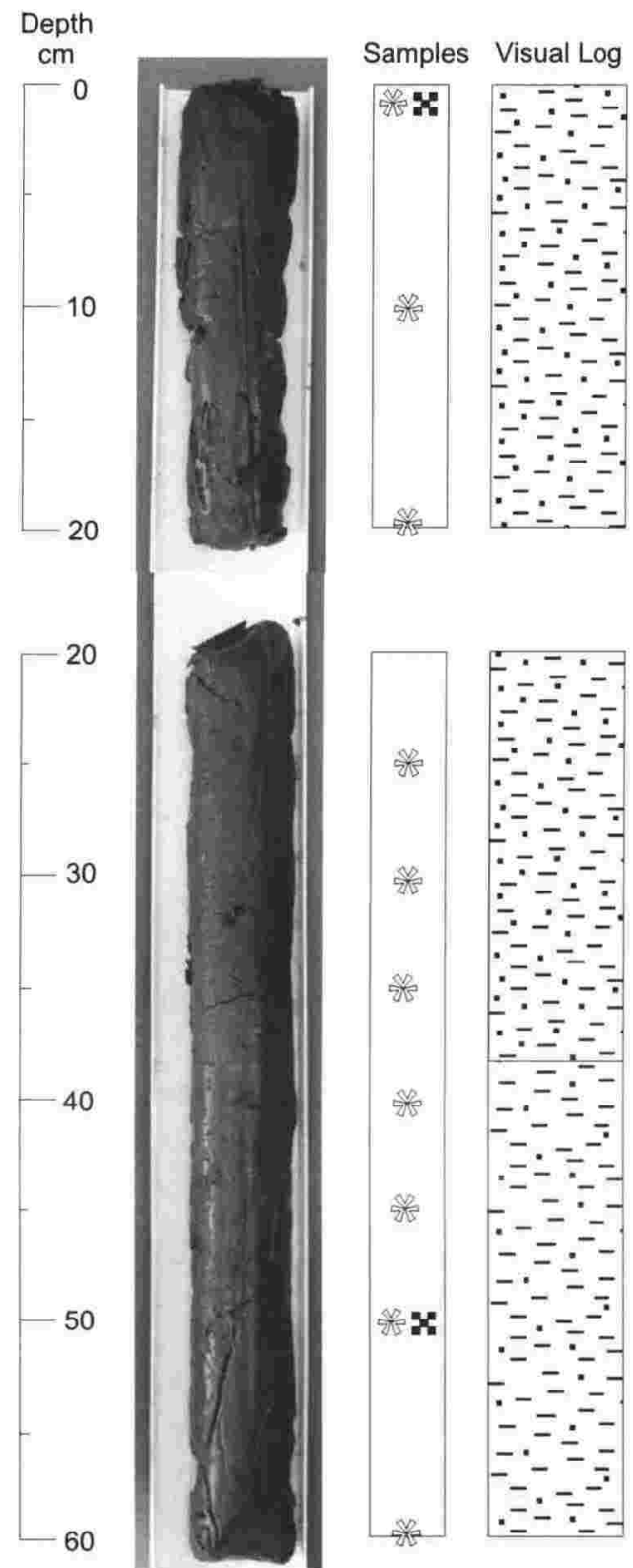

Description

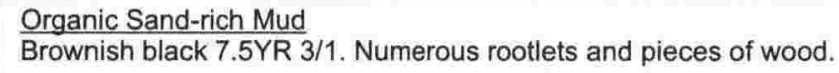

As above except fewer roots.

Contact sharp.

Organic Sand-poor Mud

Yellowish grey 2.5 Y $4 / 1$. 


\section{Core: KP99-1\&2}

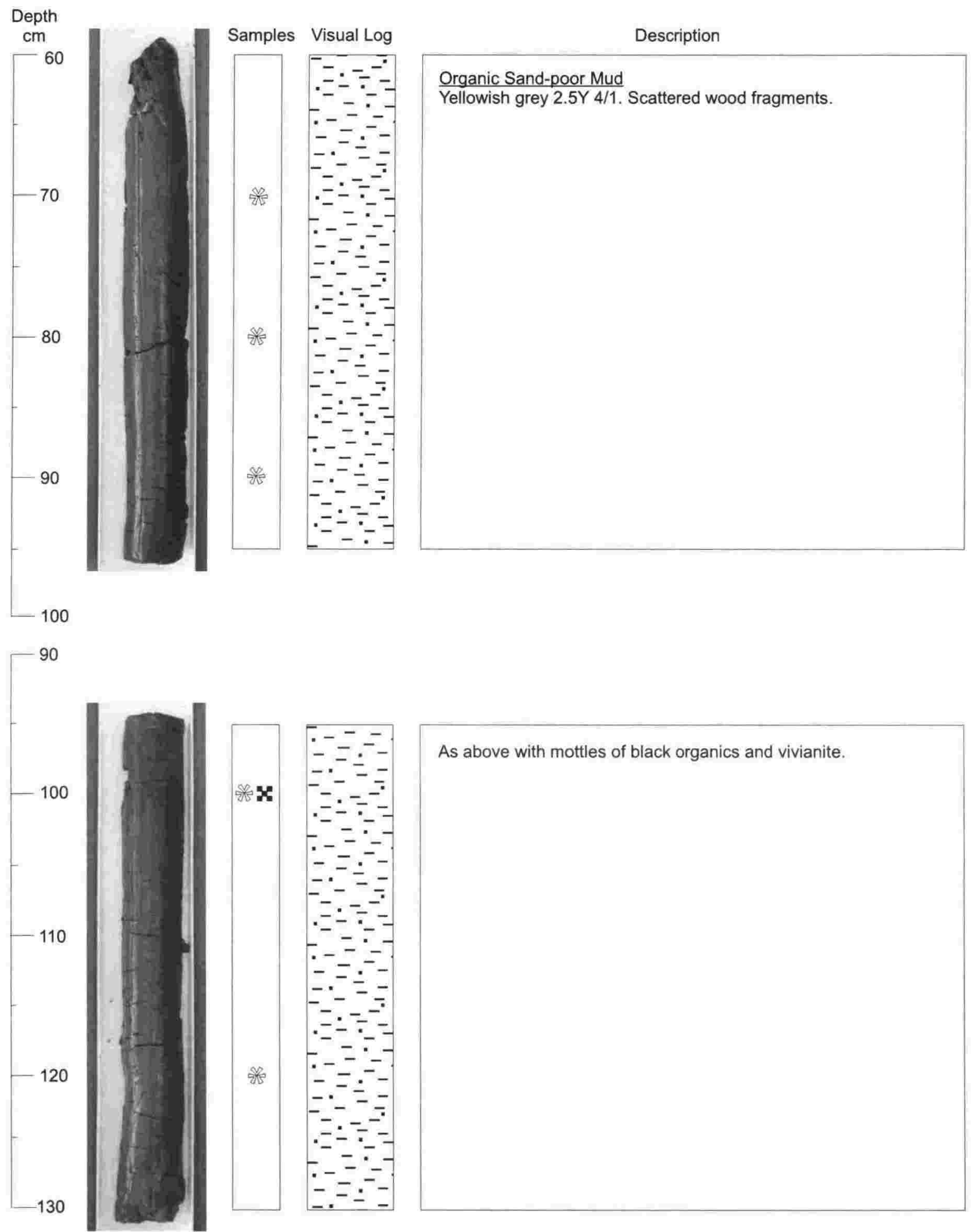


Core: KP99-1\&2

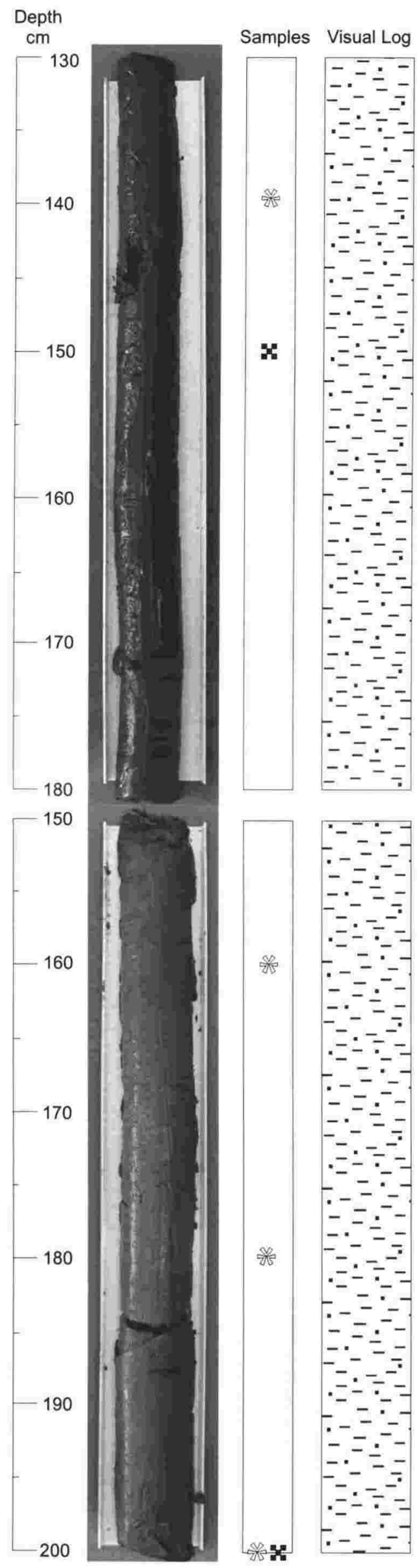

Description

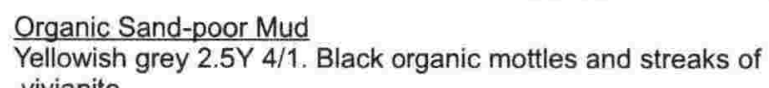

vivianite.

Overlap between KP99-1 (piston-cored) and KP99-2 (hand-augured) sections.

As above. 


\section{Core: KP99-1\&2}

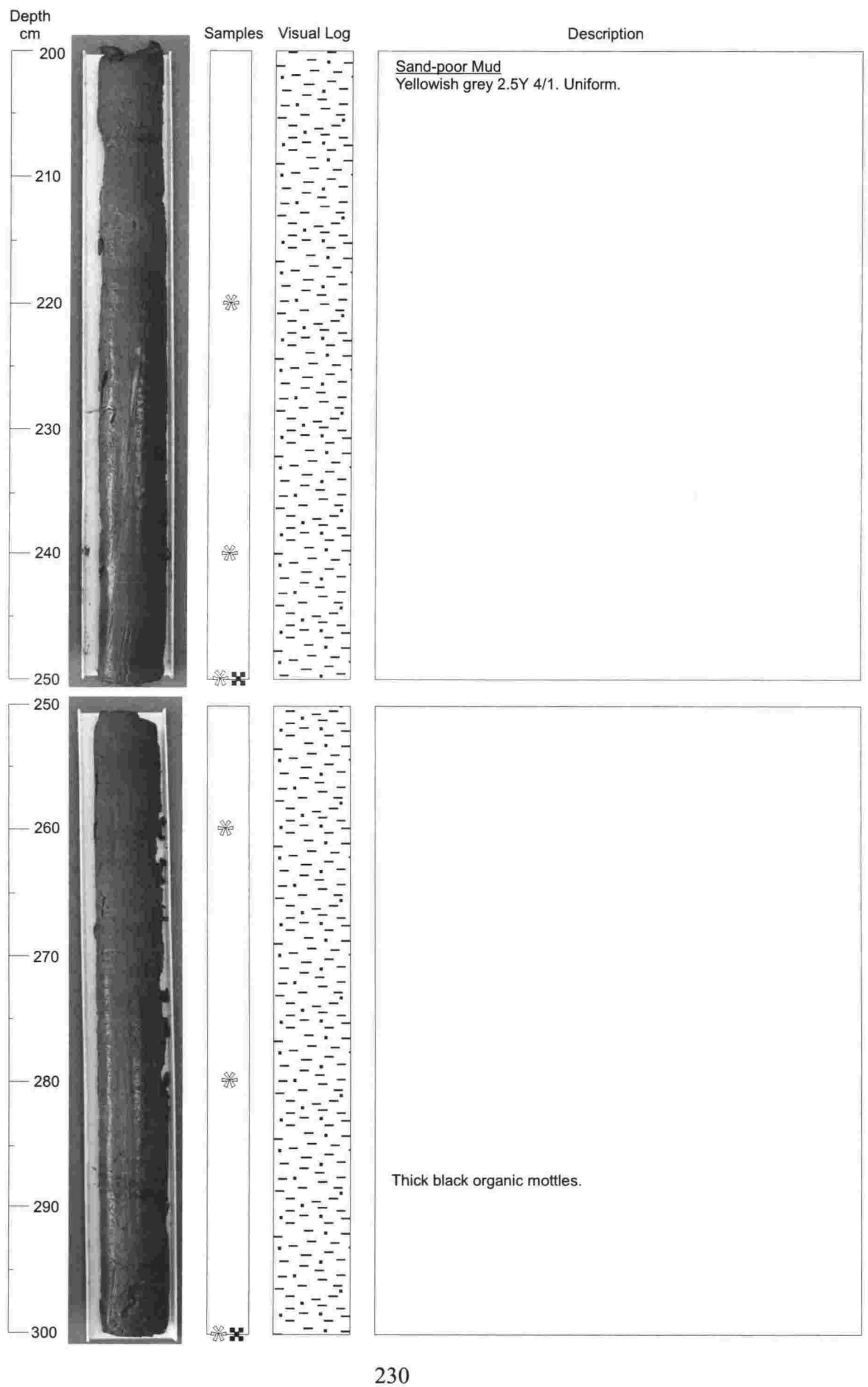




\section{Core: KP99-1\&2}

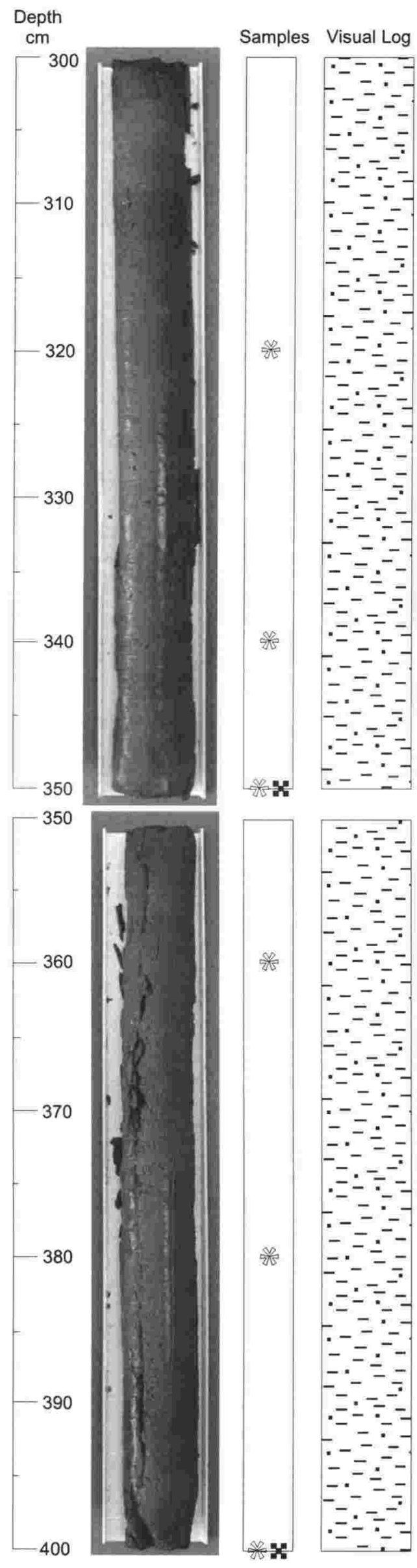

Description

\section{Sand-poor Mud}

Dark yellowish grey $2.5 Y 4 / 2$. Uniform.

As above. 


\section{Core: KP99-1\&2}

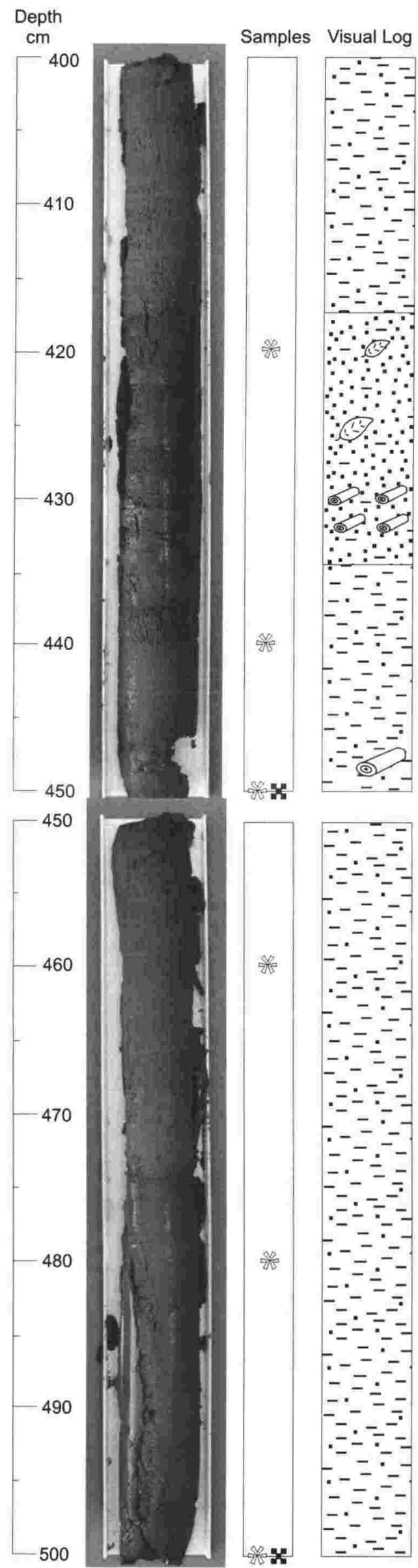

Description

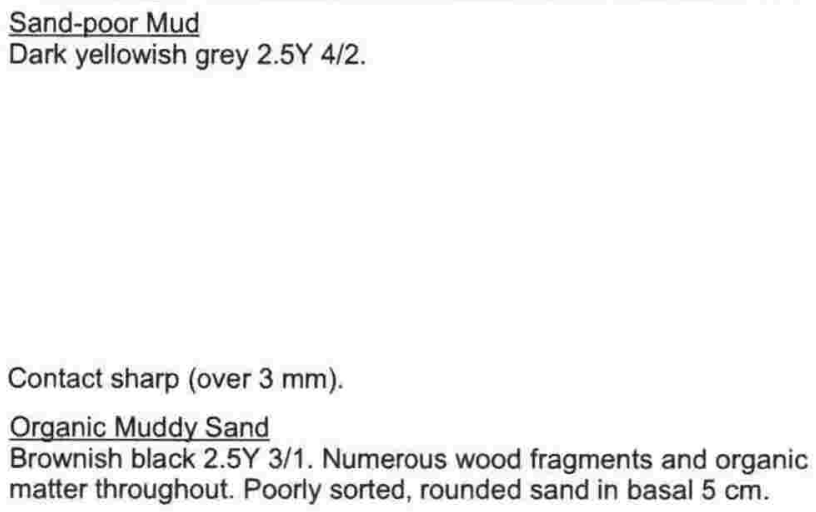

Brownish black 2.5Y $3 / 1$. Numerous wood fragments and organic matter throughout. Poorly sorted, rounded sand in basal $5 \mathrm{~cm}$.

Two distinct layers of wood fragments.

Contact wavy (over $1 \mathrm{~cm}$ ).

\section{Sand-poor Mud}

Dark yellowish grey $2.5 Y 4 / 2$.

Large piece of wood

As above. 


\section{Core: KP99-1\&2}

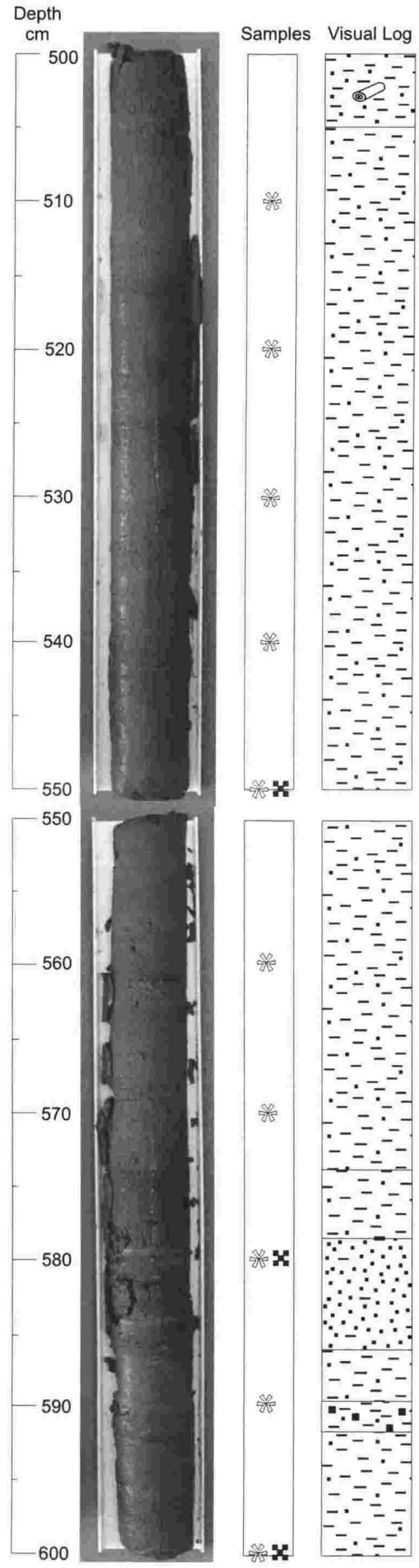

\section{Description}

\section{Sand-rich Mud}

Coarse, weathered sand grains and wood framents throughout.

Contact sharp (over $5 \mathrm{~mm}$ ).

\section{Sand-poor Mud}

Dark yellowish grey $2.5 Y$ 4/2. Uniform.

As above.

Contact sharp (over $3 \mathrm{~mm}$ ).

Organic Sand-poor Mud

Olive black 5 Y $3 / 2$.

Contact wavy (over $1 \mathrm{~cm}$ ).

Muddy Sand

Angular, poorly sorted sand with organic material and vivianite.

Two light brown bands across sand layer.

Contact sharp (over $5 \mathrm{~mm}$ ).

Gravelly Mud

Angular, poorly sorted gravel within mud.

\section{Sand-poor Mud}

Dark yellowish grey 2.5 Y $4 / 2$. Uniform. 


\section{Core: KP99-1\&2}

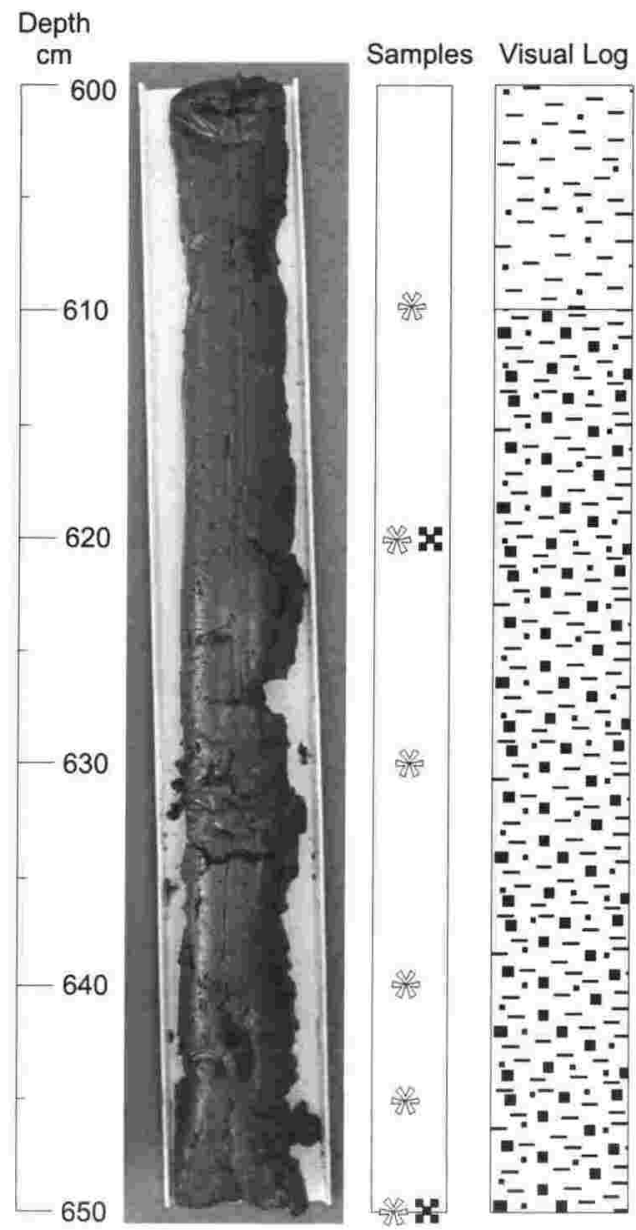

Description

$\frac{\text { Sand-poor Mud }}{\text { As above. }}$
Contact gradational.

Gravelly Mud

Brownish black 2.5Y3/2. Angular, weathered greywacke gravel

within mud. 


\section{A P P E N D IX II}

\section{Diatom Species List}

Item 1: Species List.

Slide Reference:

This contains the sample, and slide co-ordinates where a species was first identified. Samples beginning with ' $M$ ' are modern samples. Sample codes are the modern site codes (see Appendix V) and one of the following:

DA death assemblage

ES epipsammic/epipelic

EP epiphytic

EL epilithic

Samples not beginning with ' $M$ ' are core samples - the code consists of the core name and sample depth.

Abbreviations used for references:

$\begin{array}{ll}\text { K \& L-B } & \text { Krammer \& Lange-Bertalot } \\ \text { W, L-B \& M } & \text { Witkowski, Lange-Bertalot \& Metzeltin } \\ \text { R, C, \& M } & \text { Round, Crawford \& Mann } \\ \text { R \& B } & \text { Round \& Bukhtiyarova } \\ \text { B \& R } & \text { Bukhtiyarova \& Round } \\ \text { P \& R } & \text { Patrick \& Reimer } \\ \text { L-B } & \text { Lange-Bertalot }\end{array}$

Item 2: Codes used for species' names in Chapter Three. 


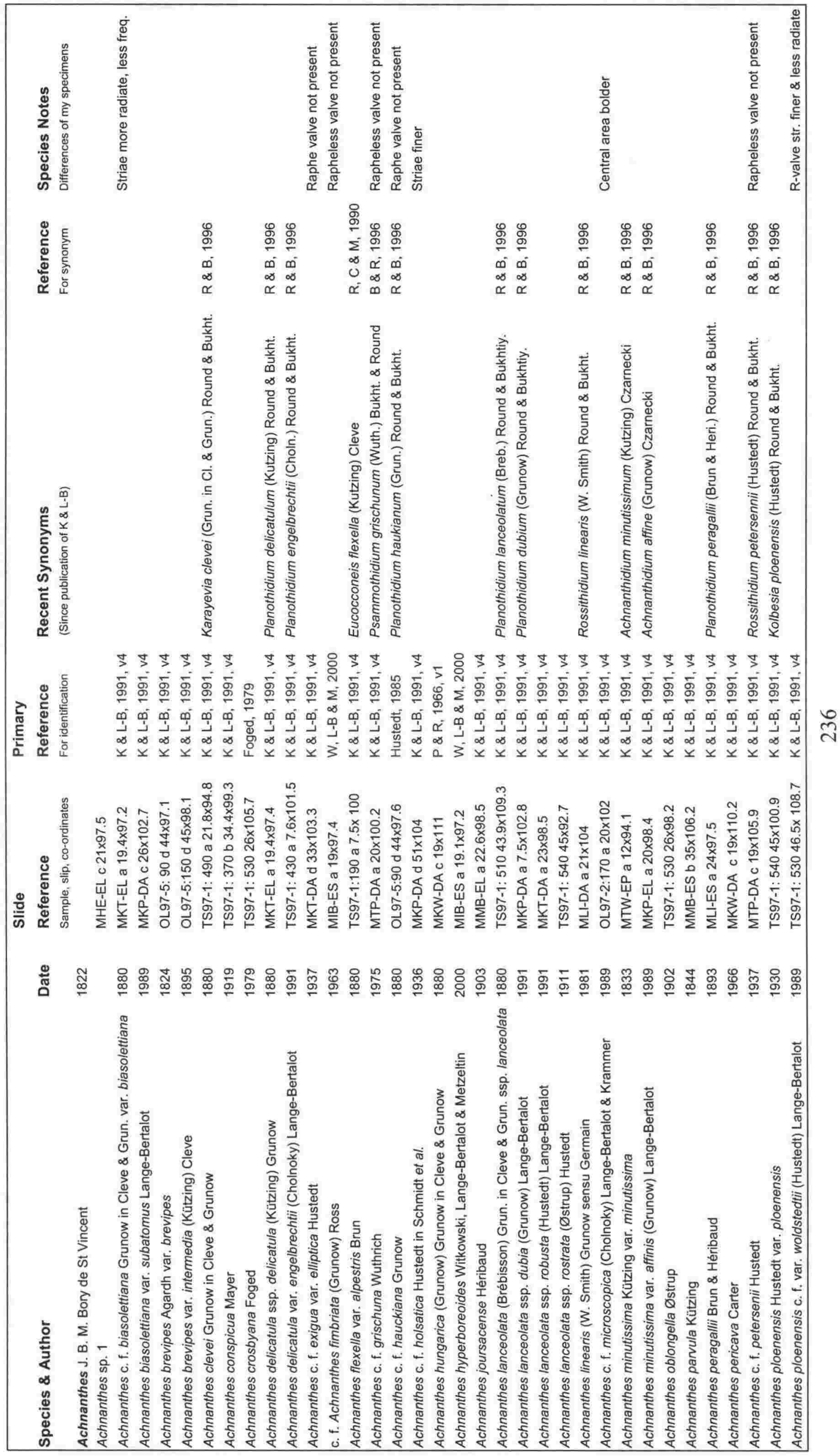




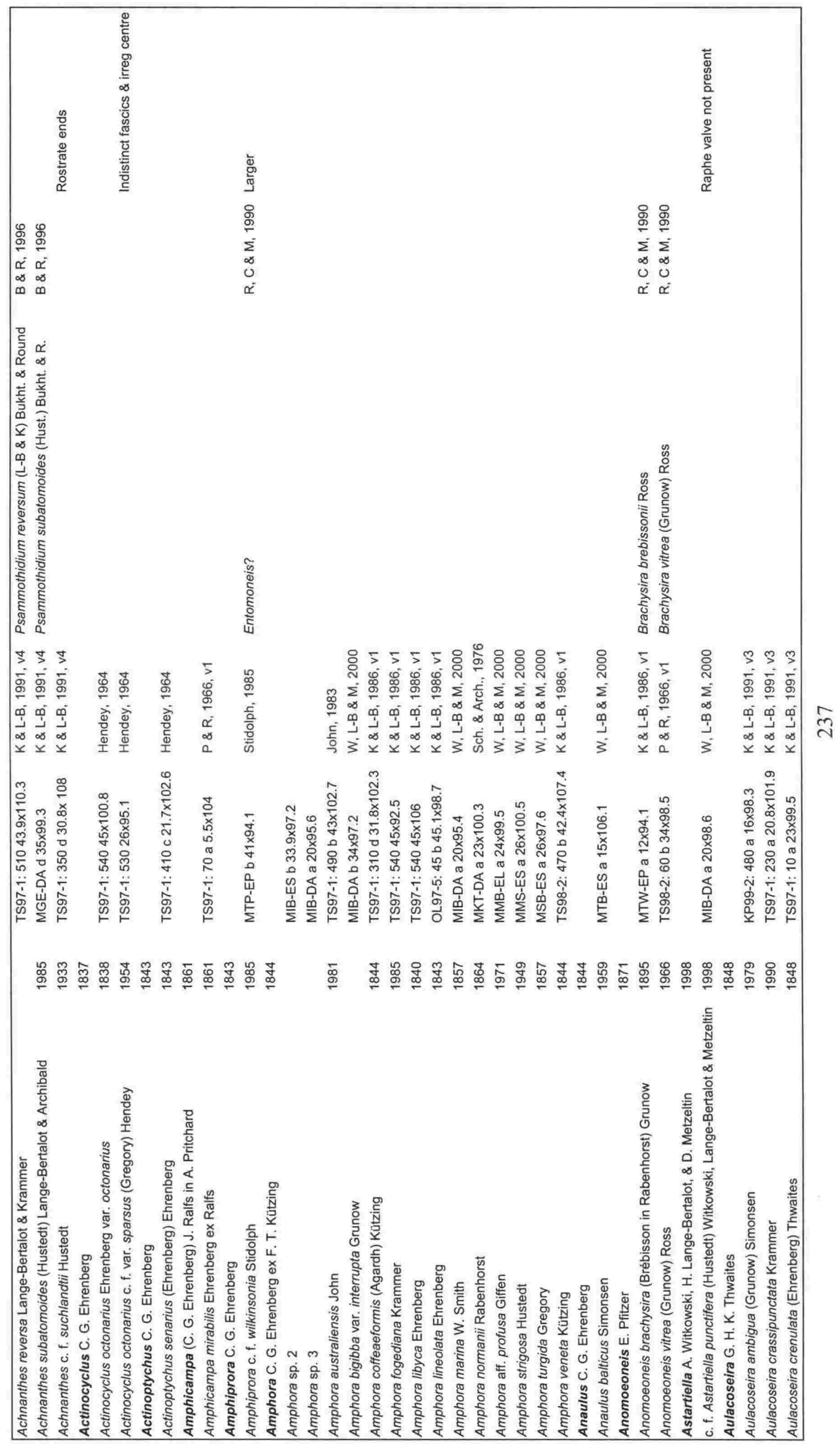




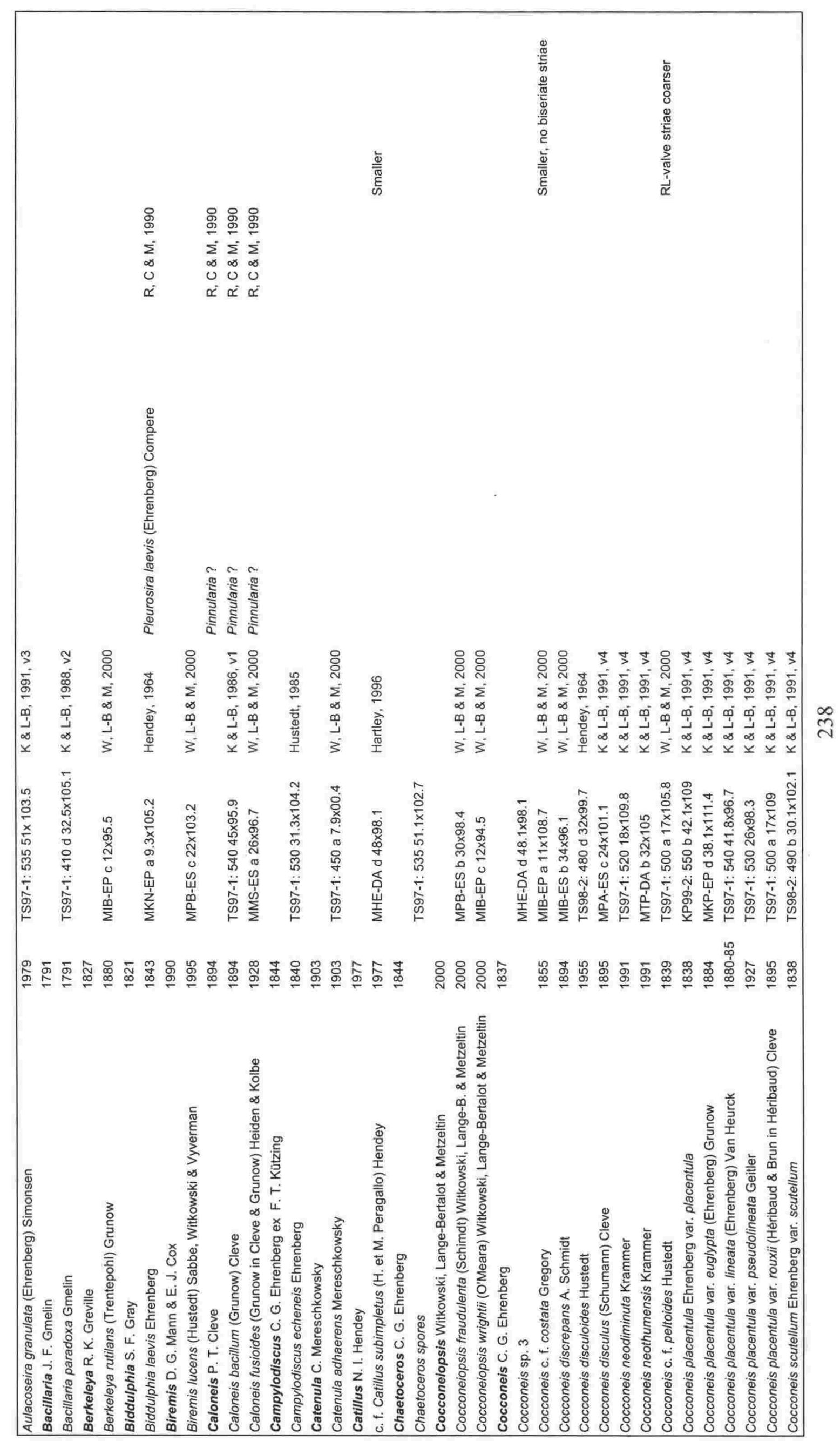



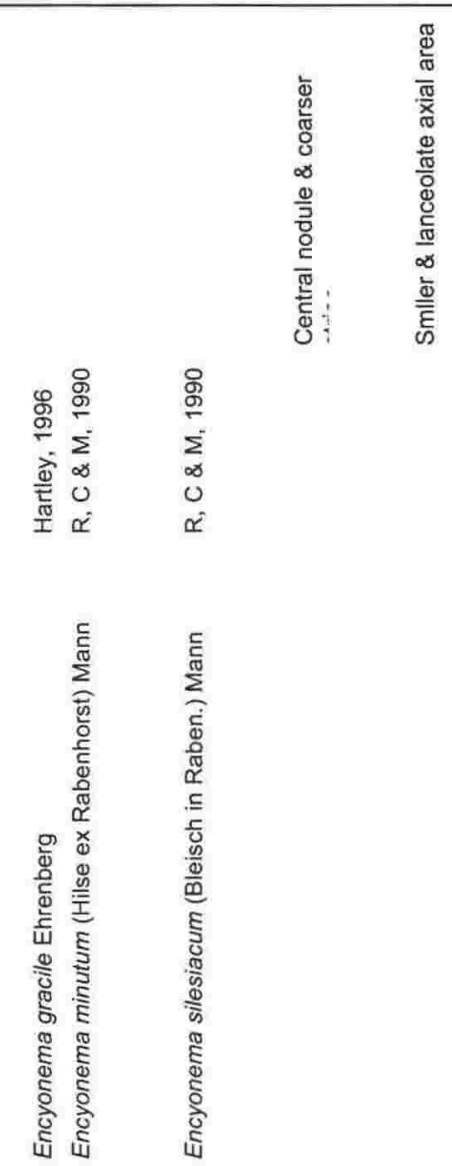

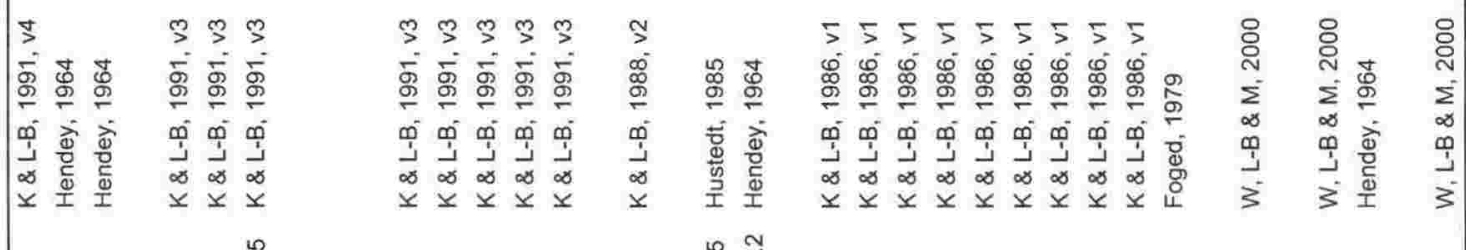

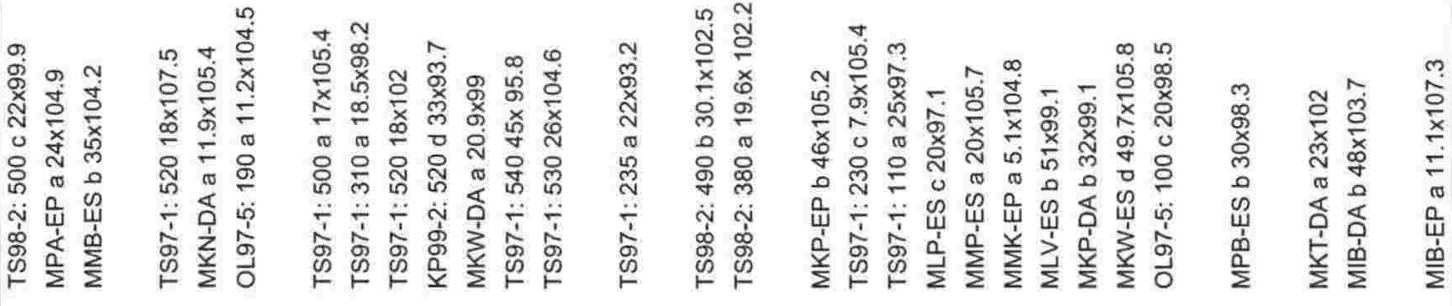

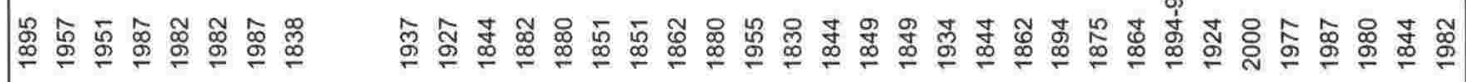

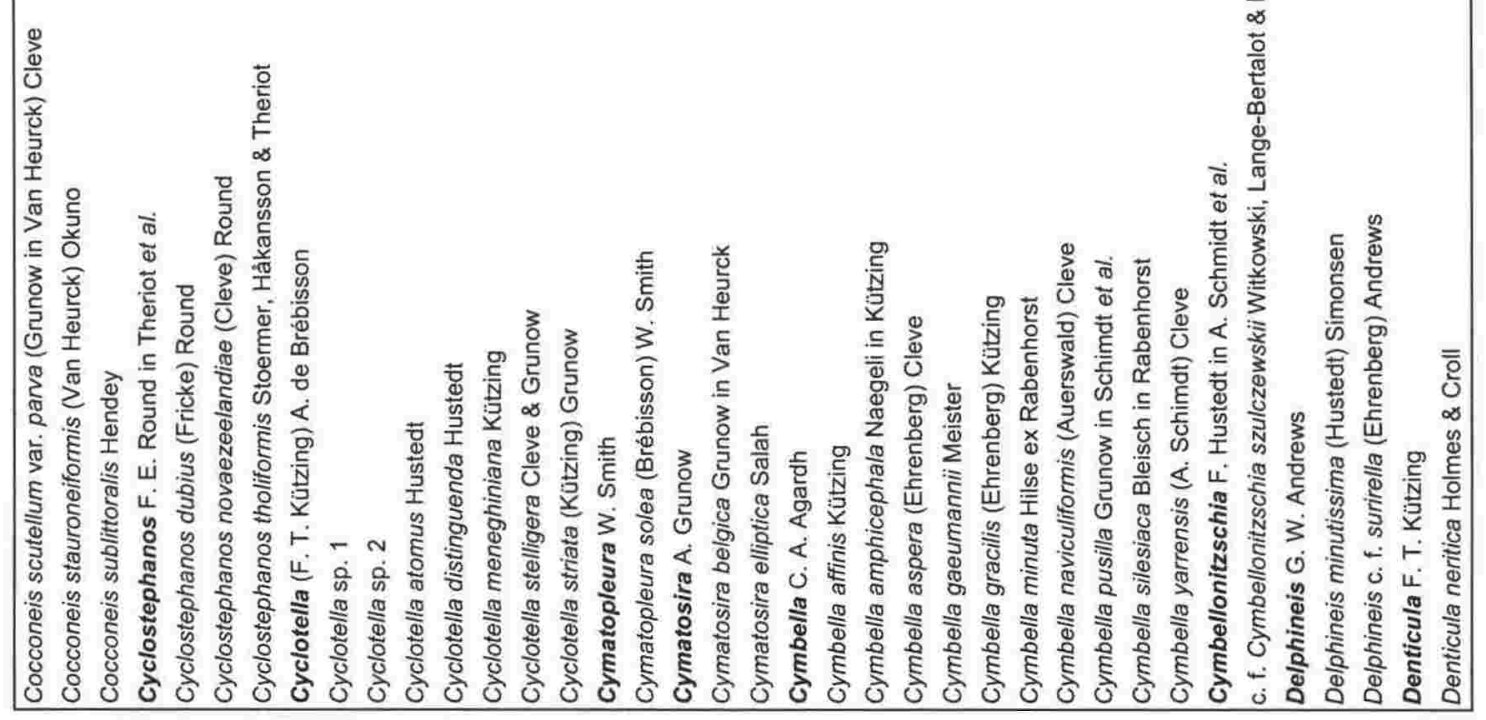




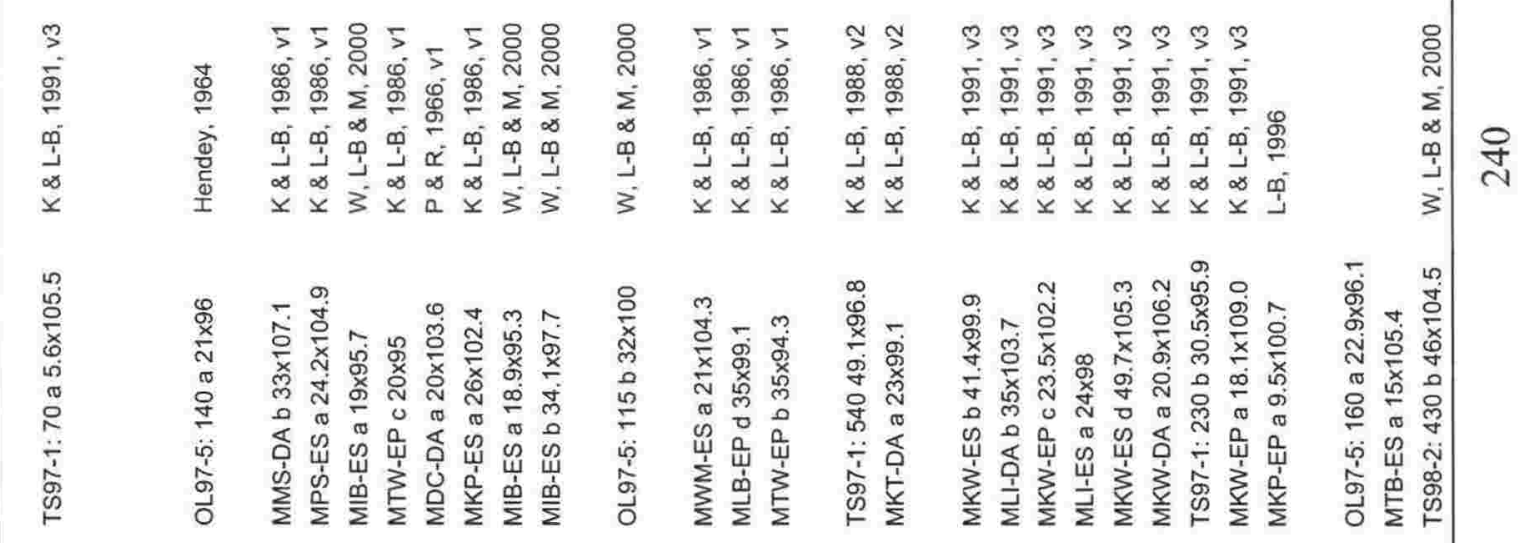

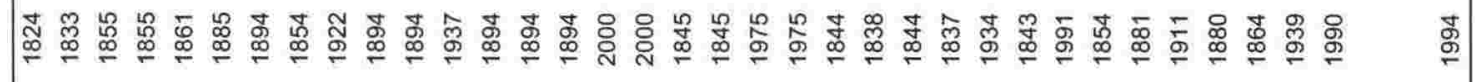

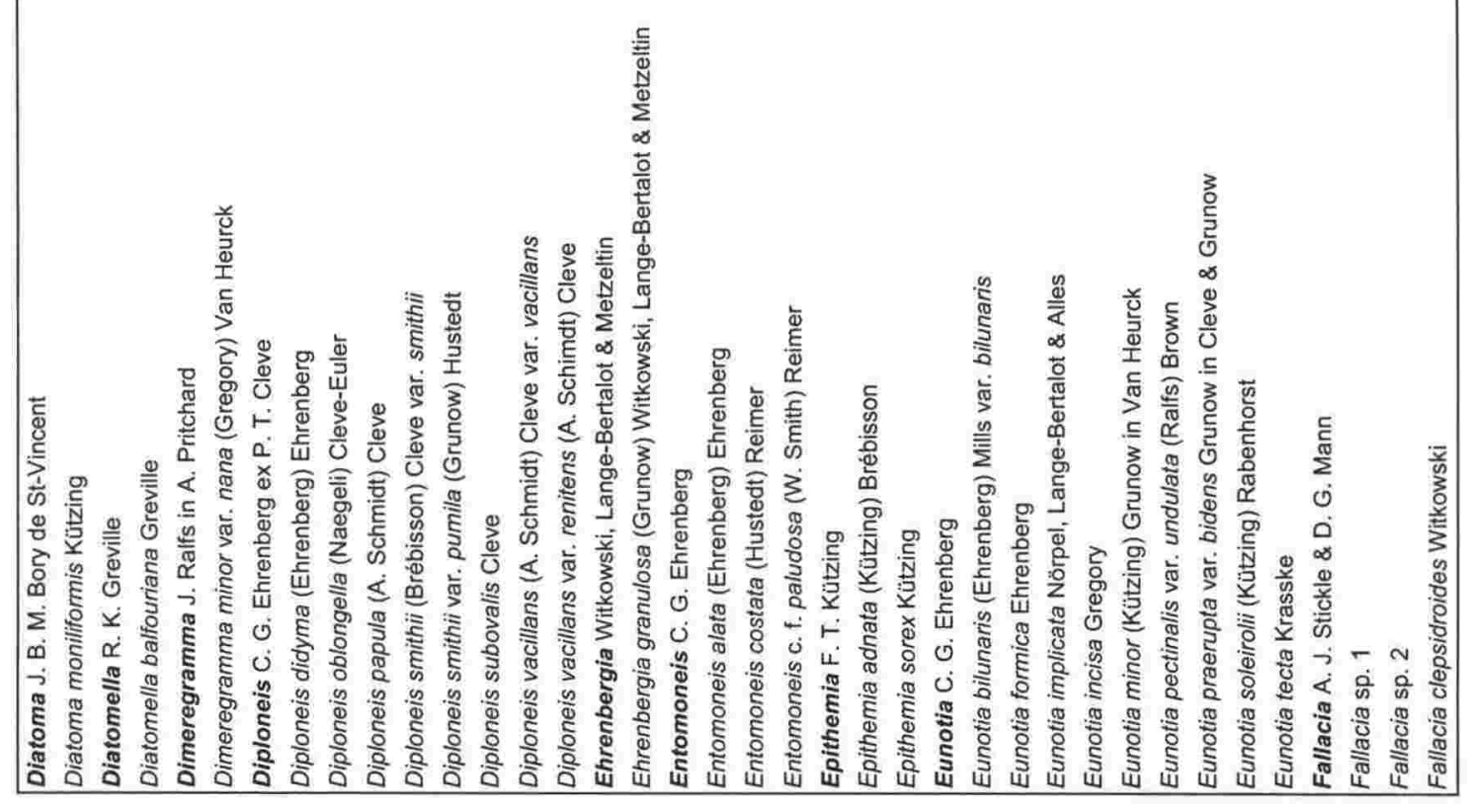




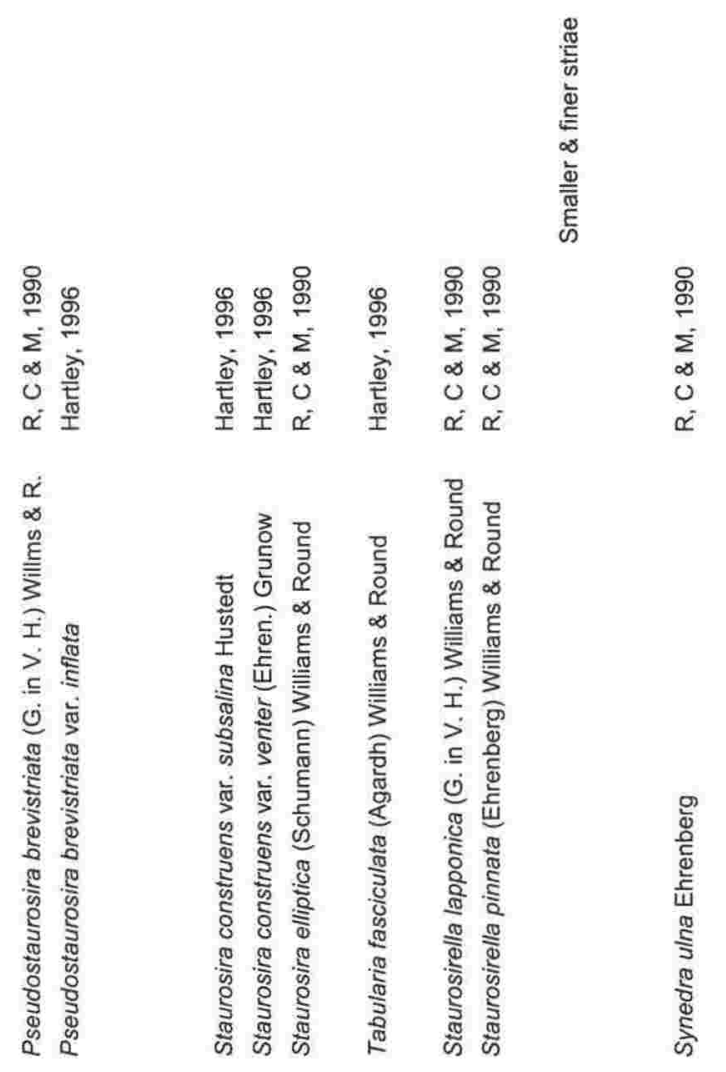

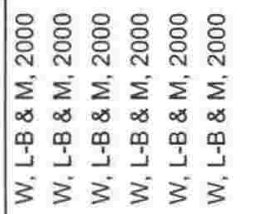

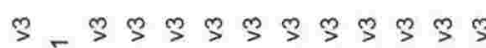

$\stackrel{-4}{3} \stackrel{-1}{m}$

5555555555

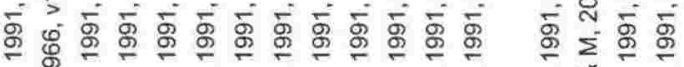

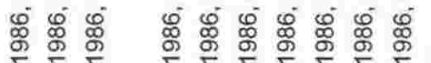

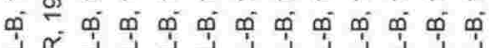

co

का m

$\dot{\infty} \underset{\infty}{\dot{L}} \dot{\vec{d}}$

का कृ का की की

×

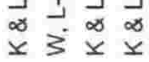

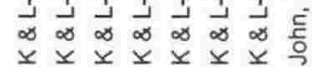

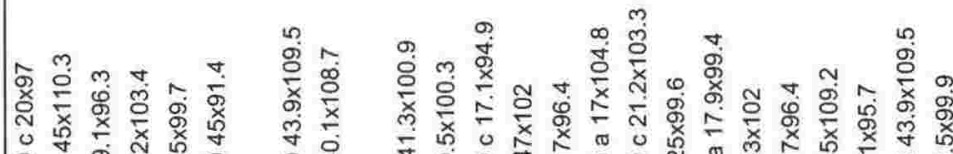

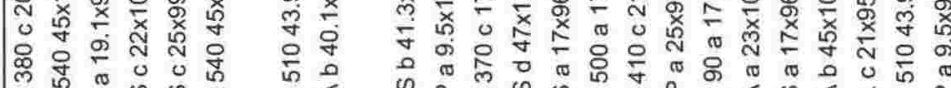

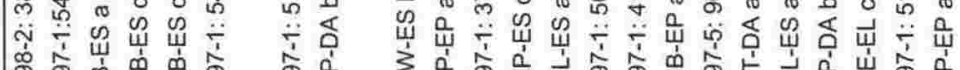

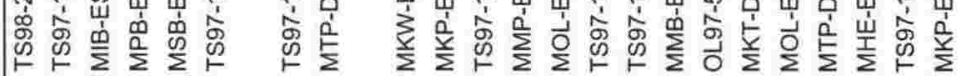

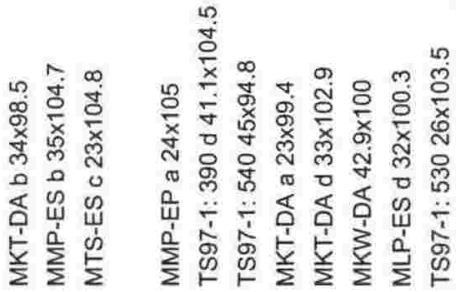

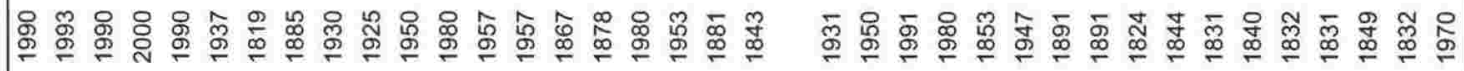

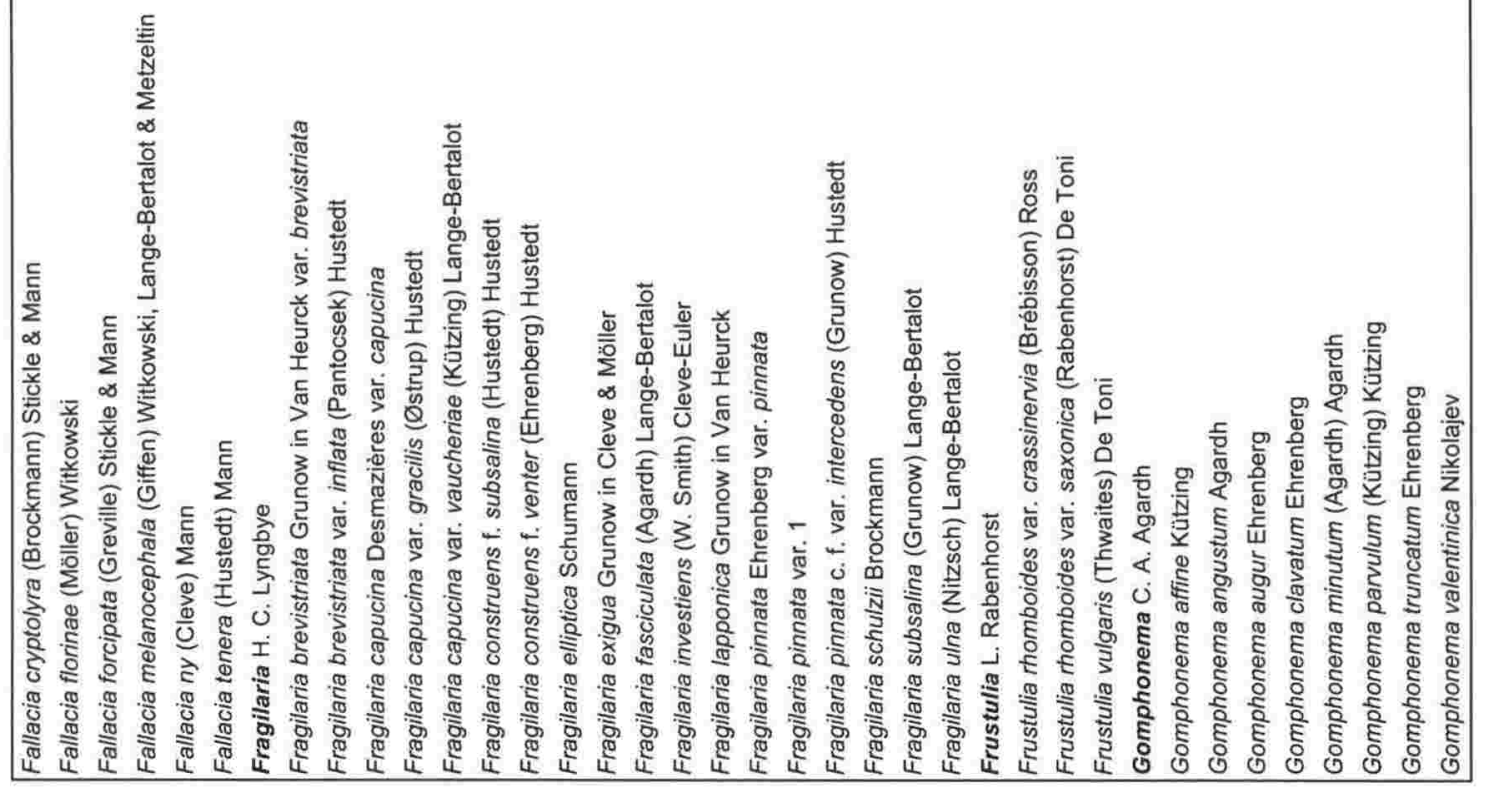




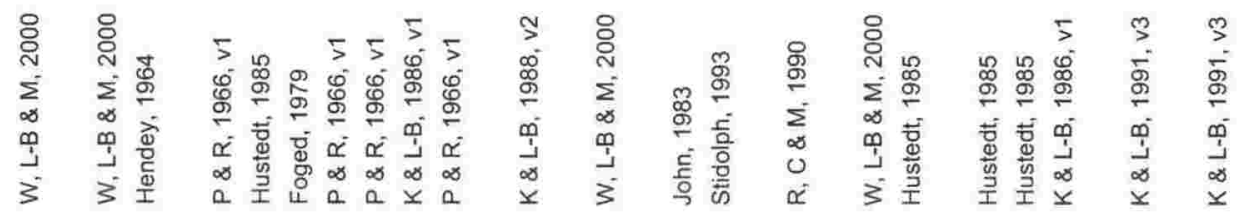

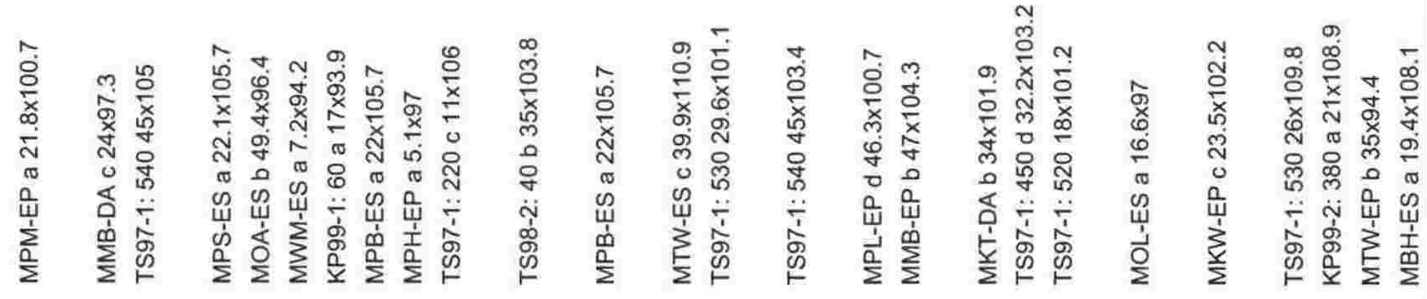

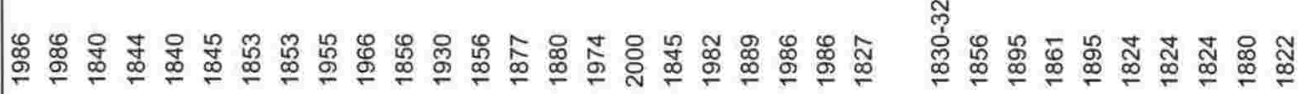

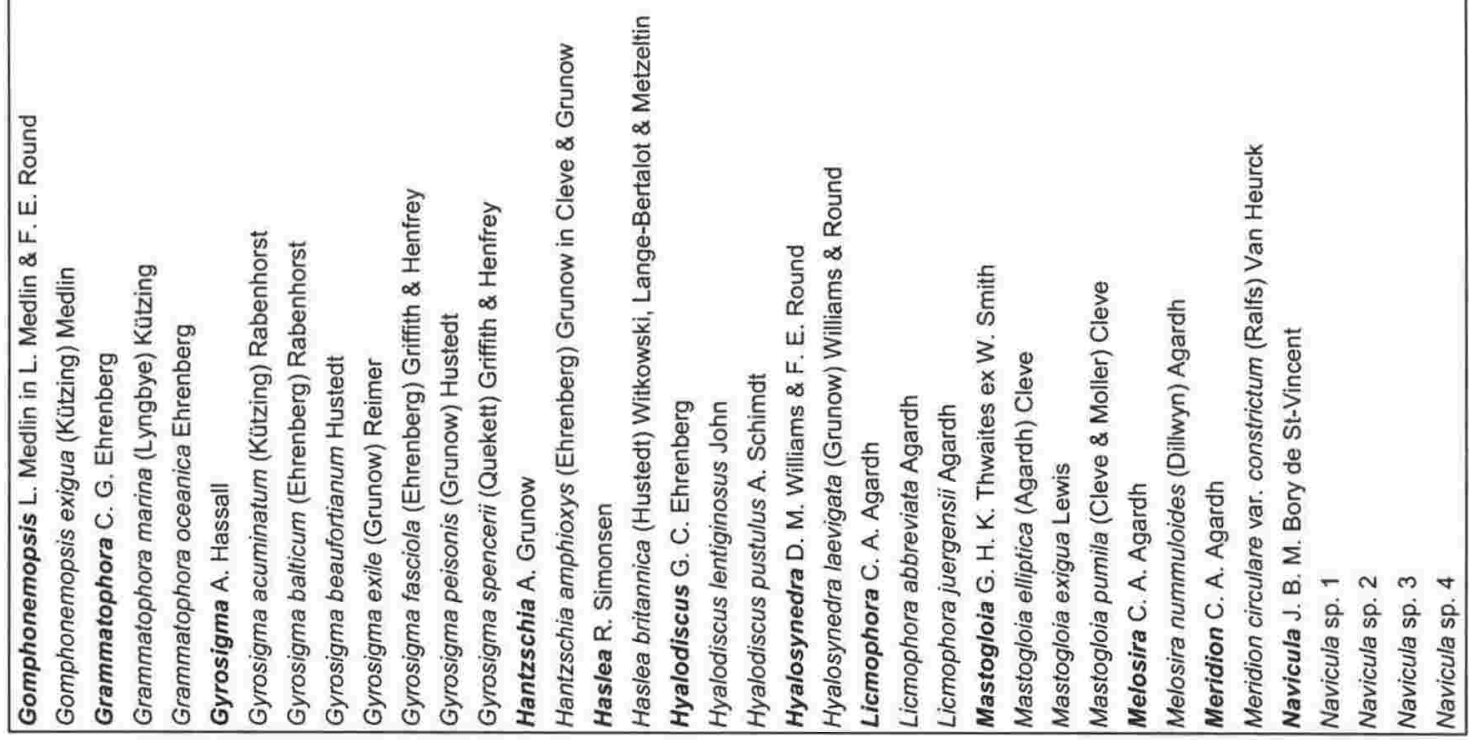




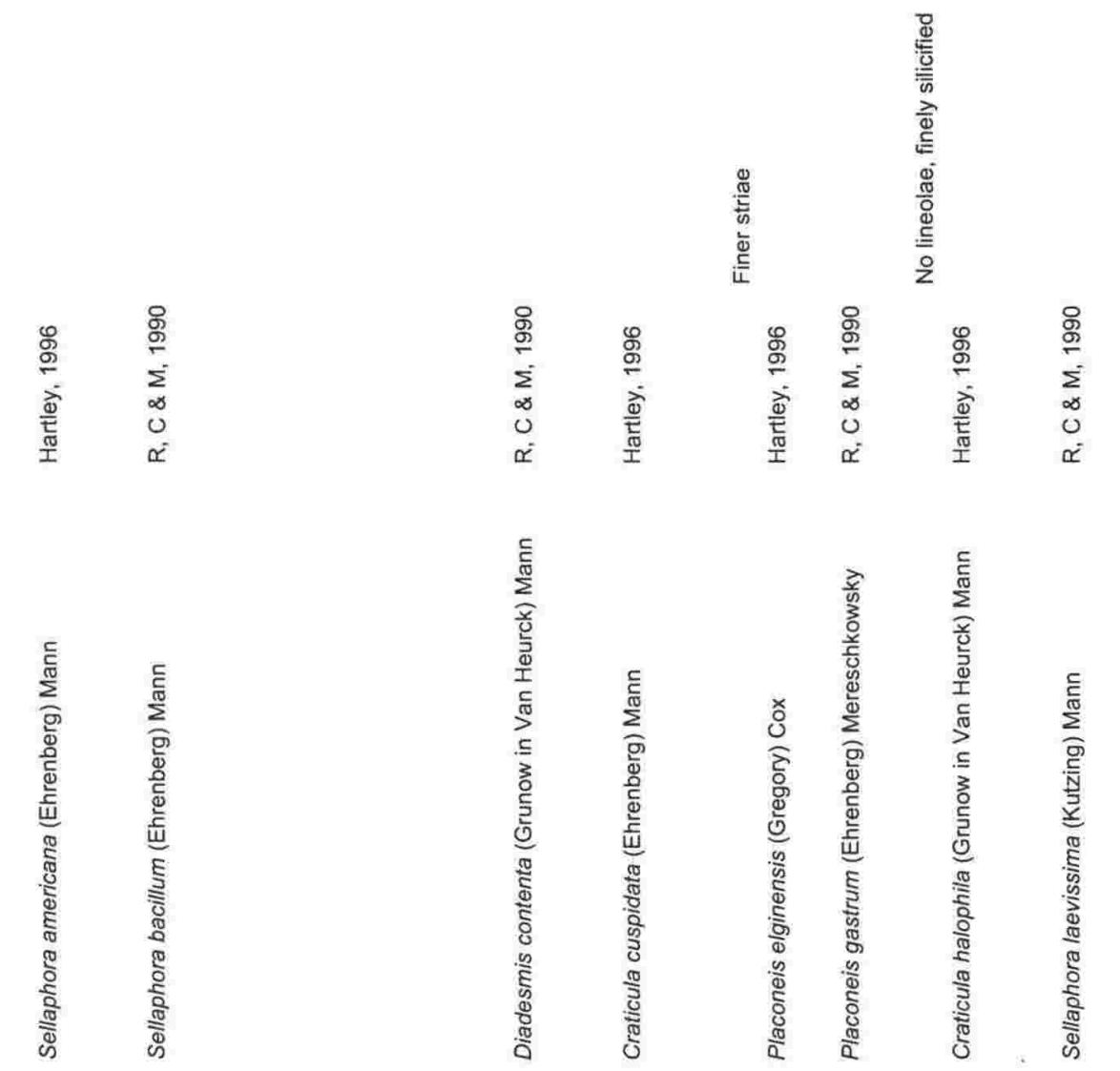

5555555555555555555555555555555

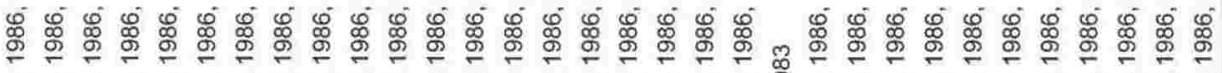
के po

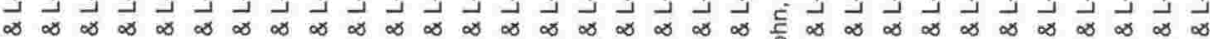
$\stackrel{\text { ? }}{\mathrm{N}}$

m.

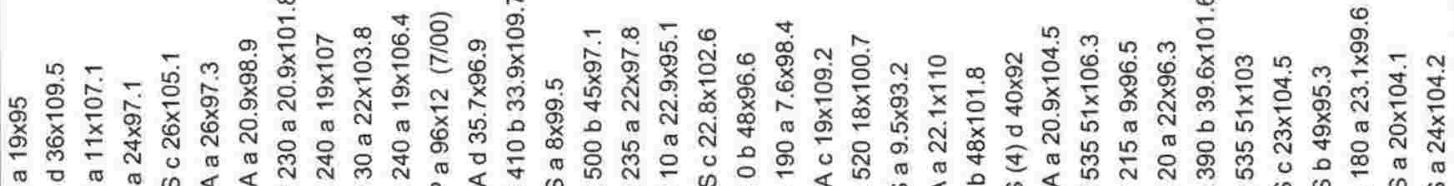
出

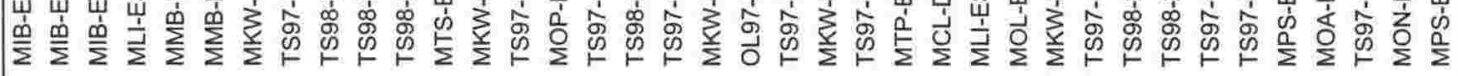

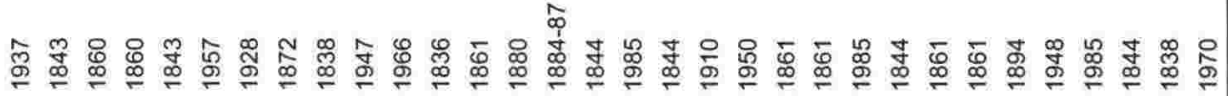

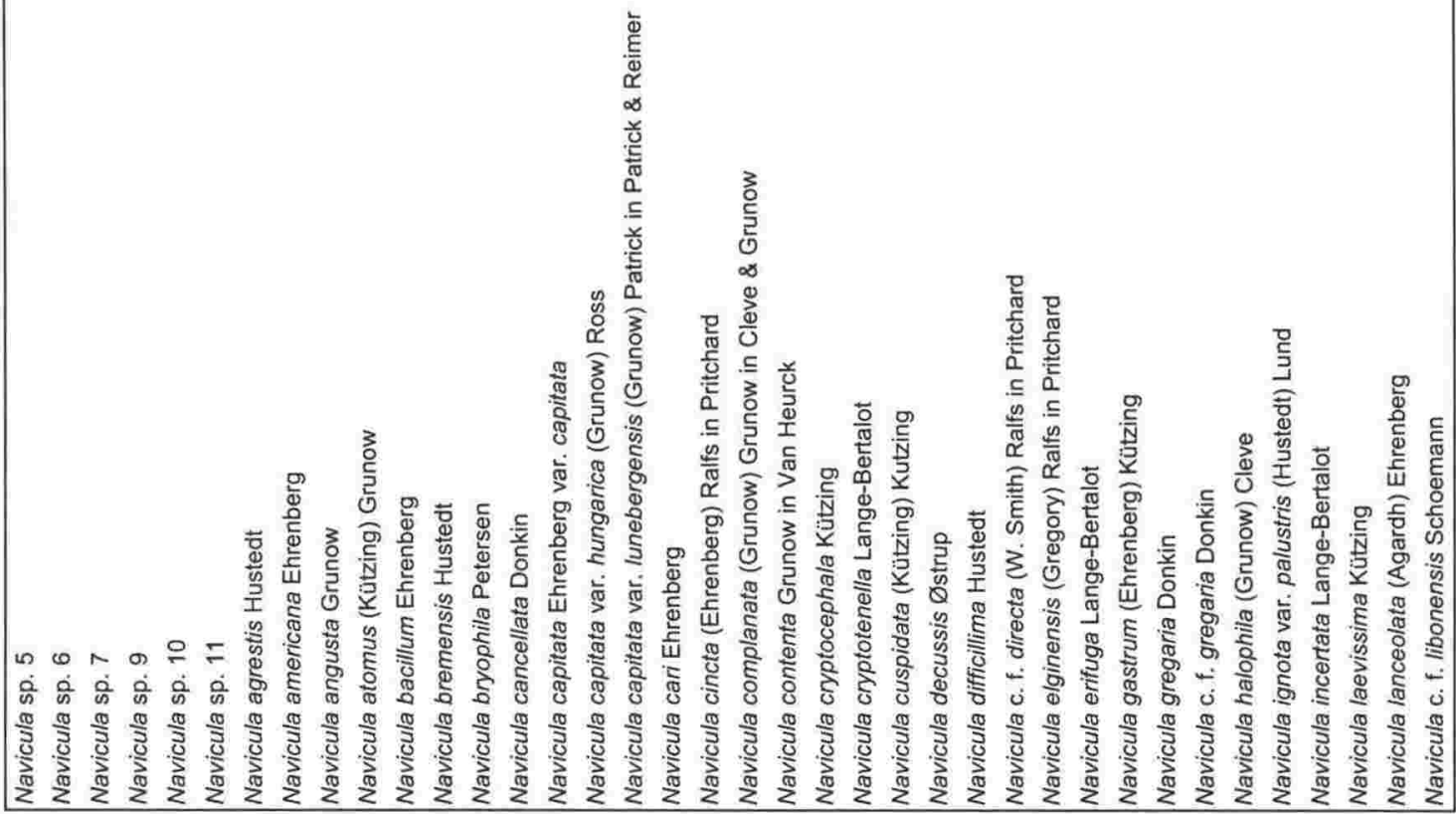

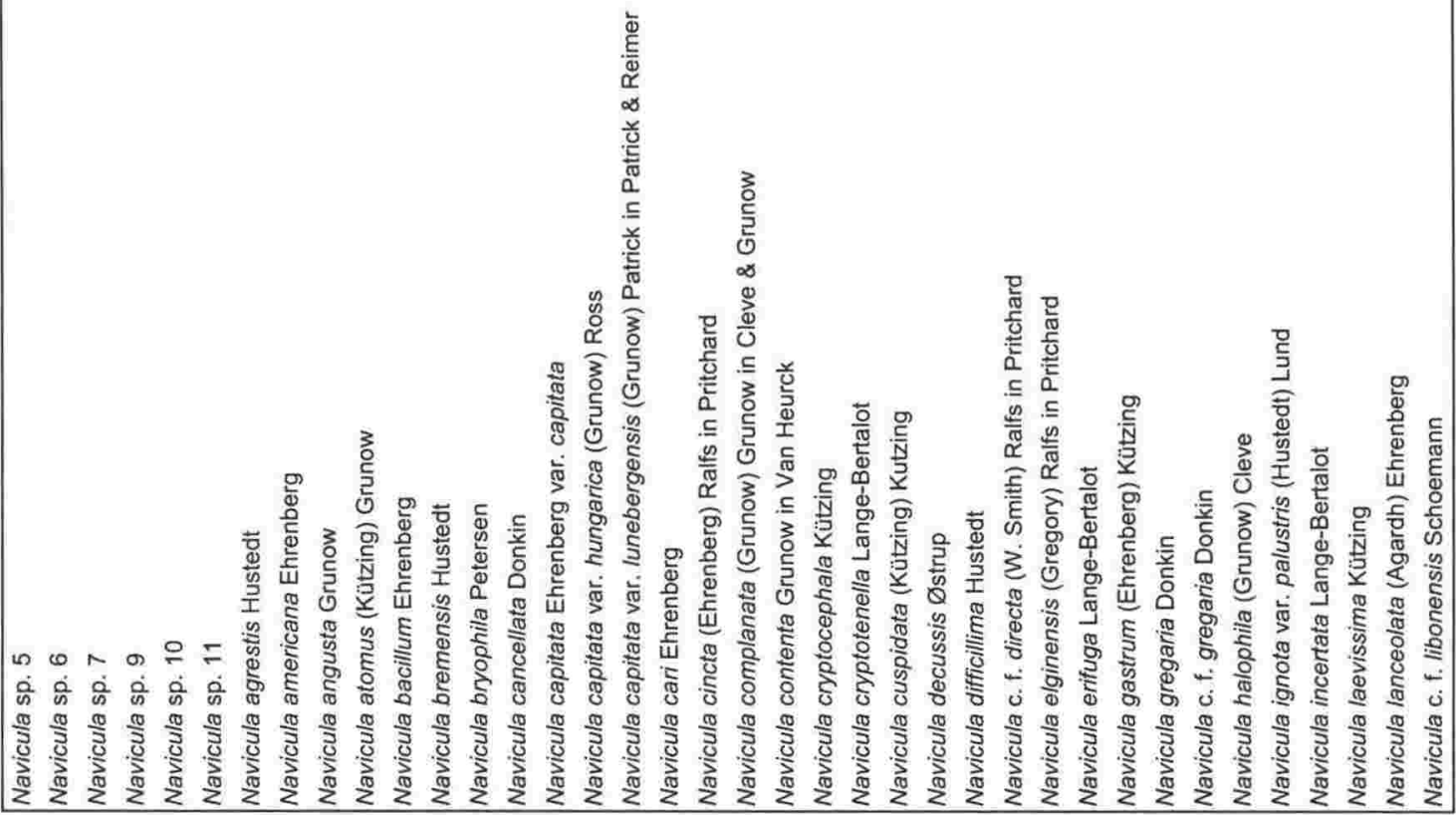

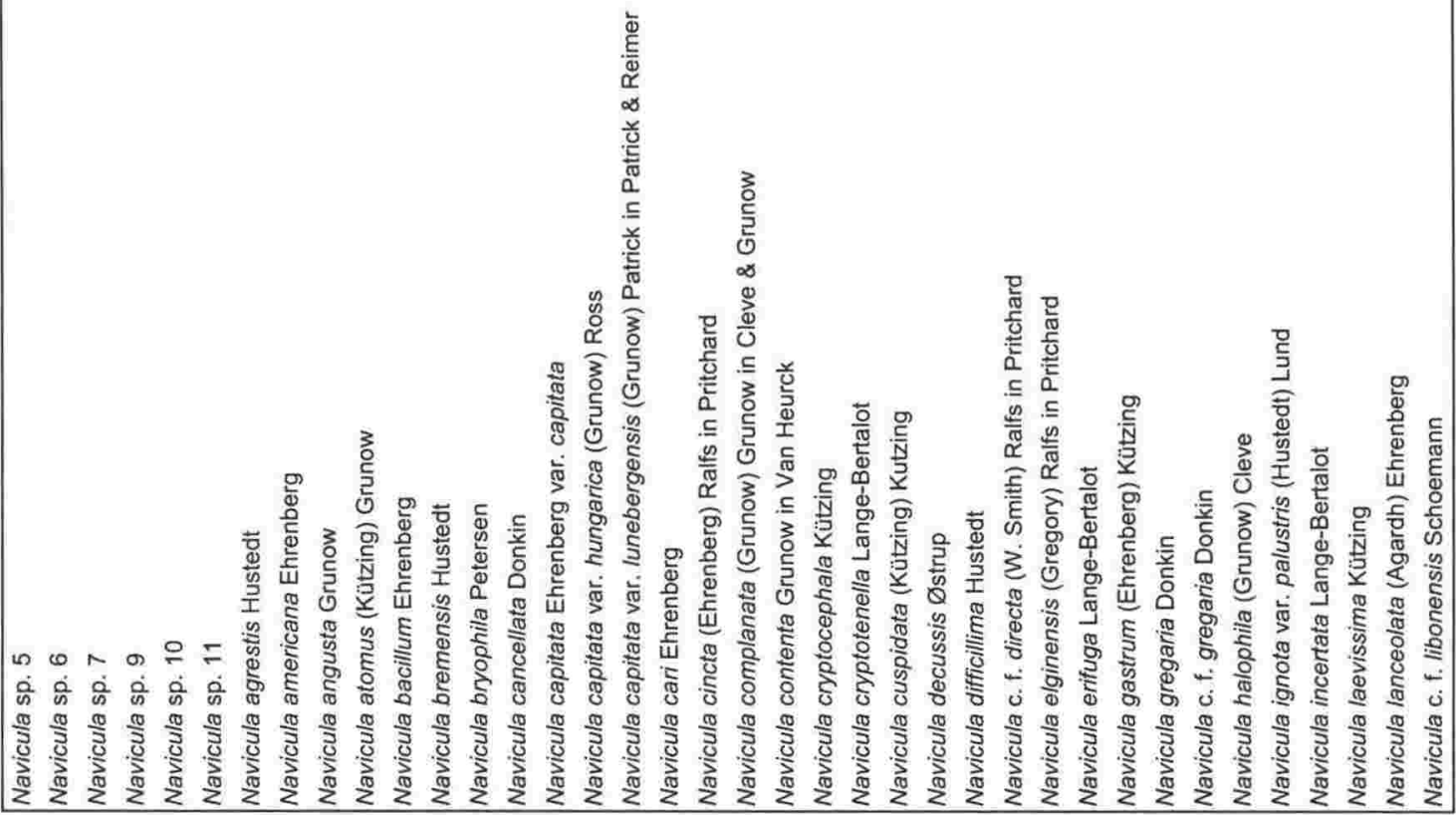

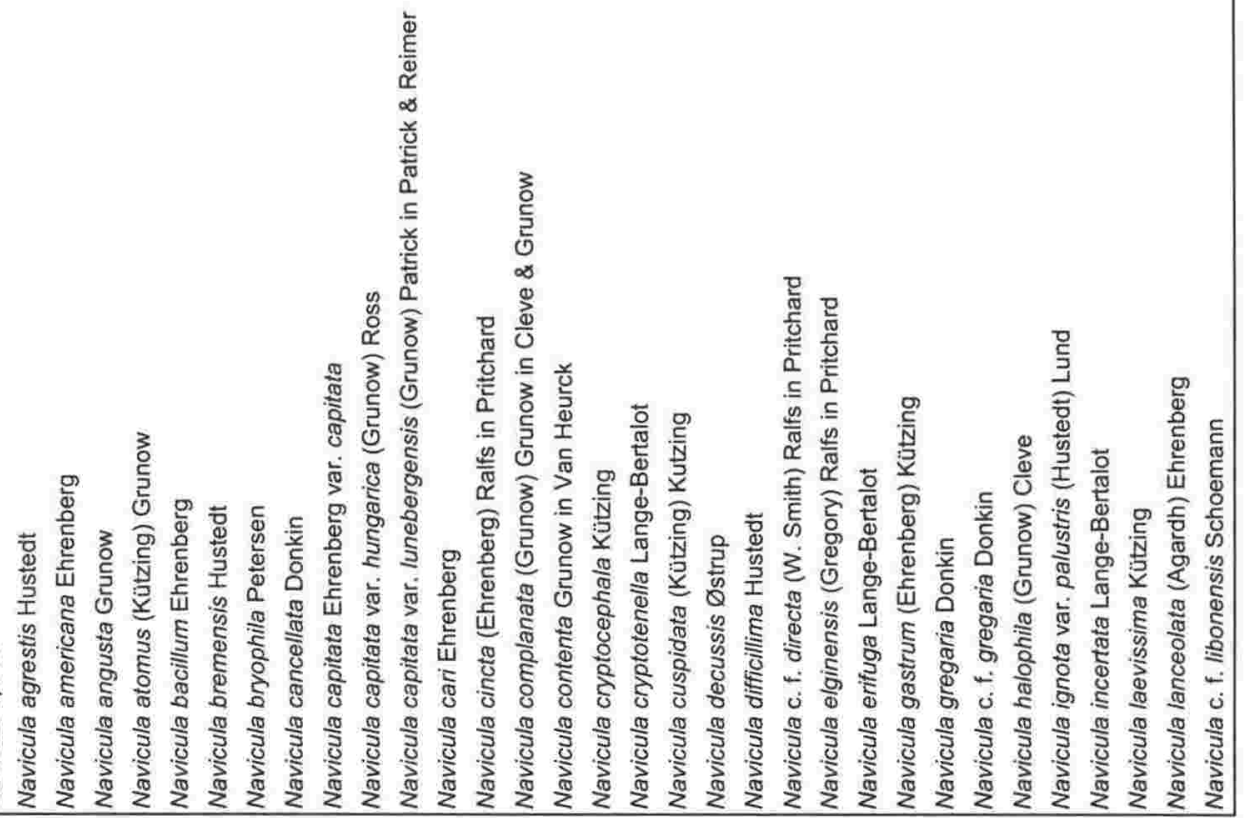



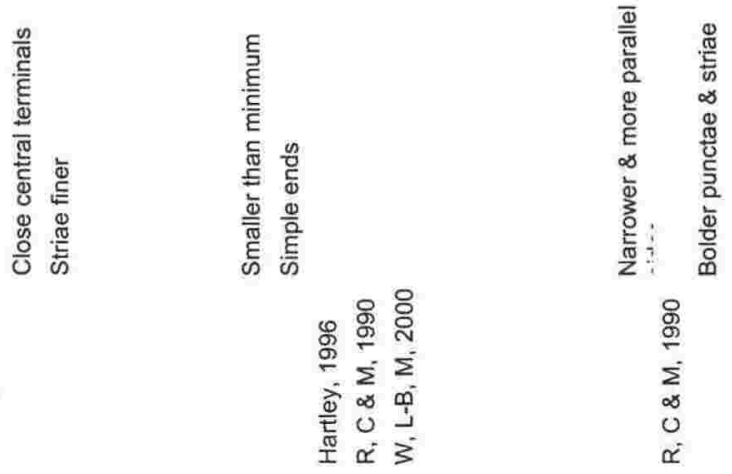

$$
\begin{aligned}
& 8 \\
& \stackrel{8}{\circ} \\
& \sum \\
& \infty \\
& 0 \\
& x
\end{aligned}
$$

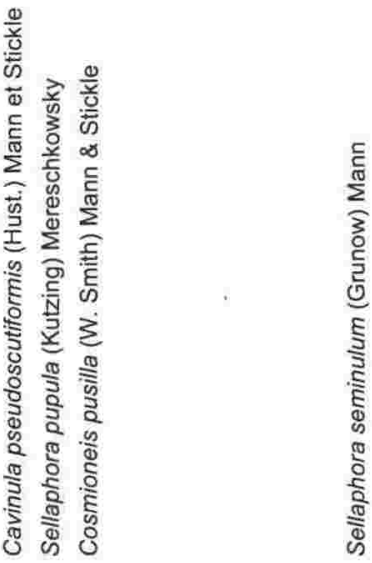

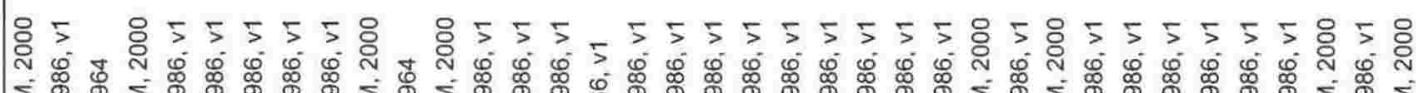

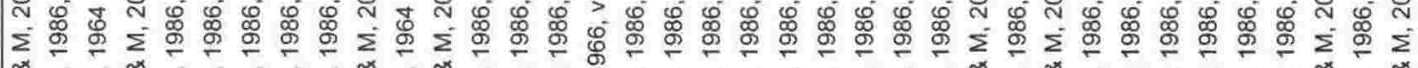

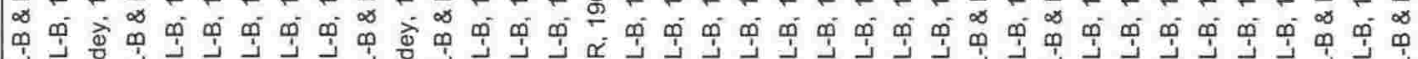

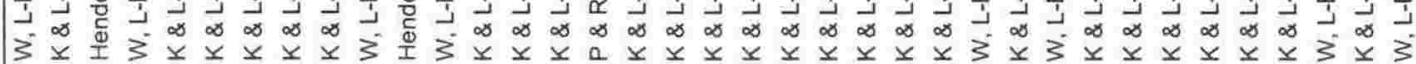

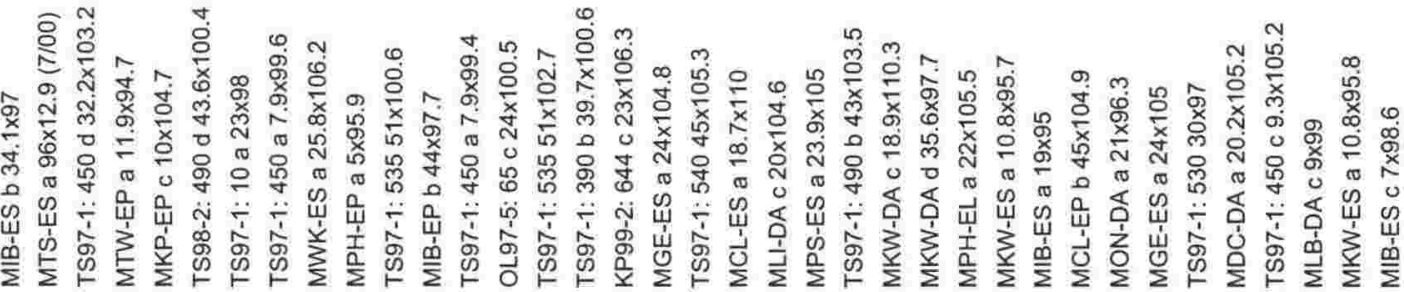

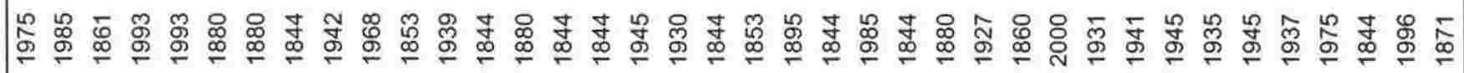

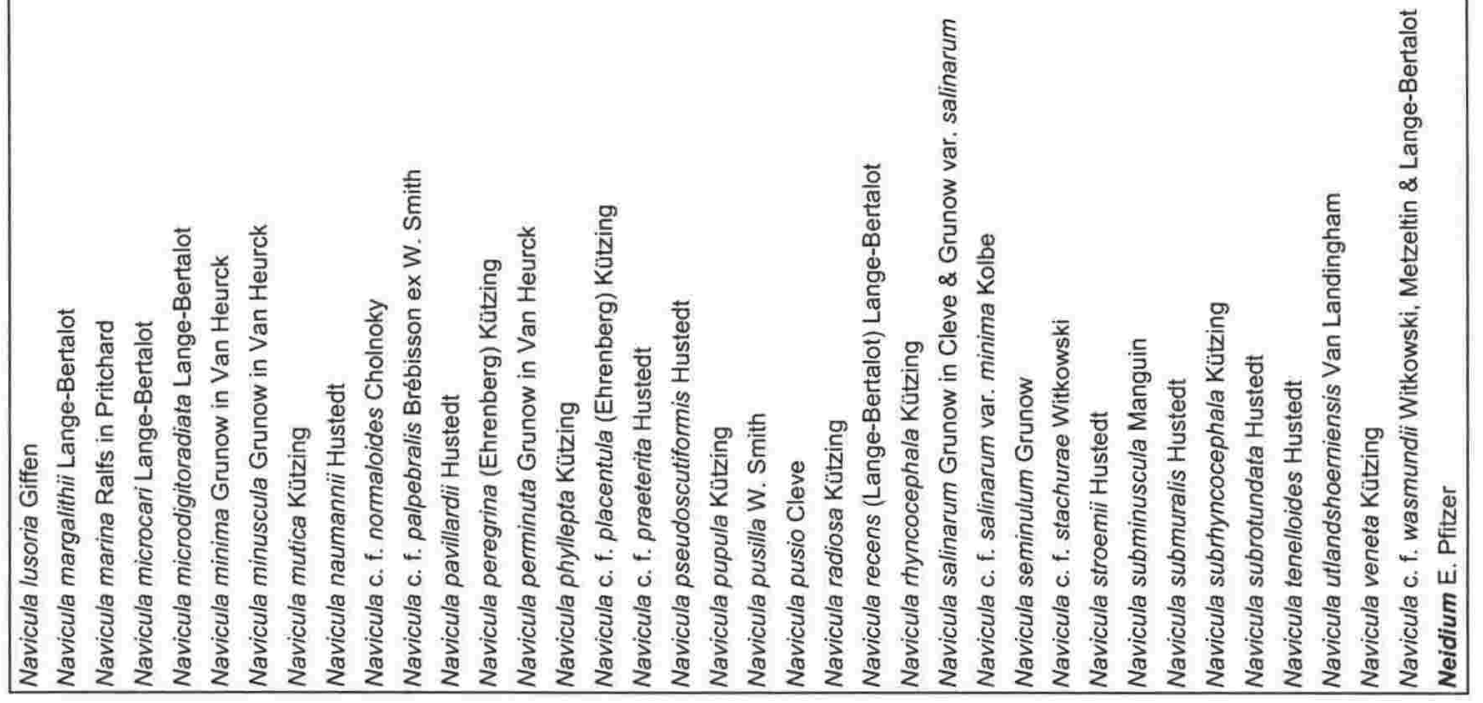




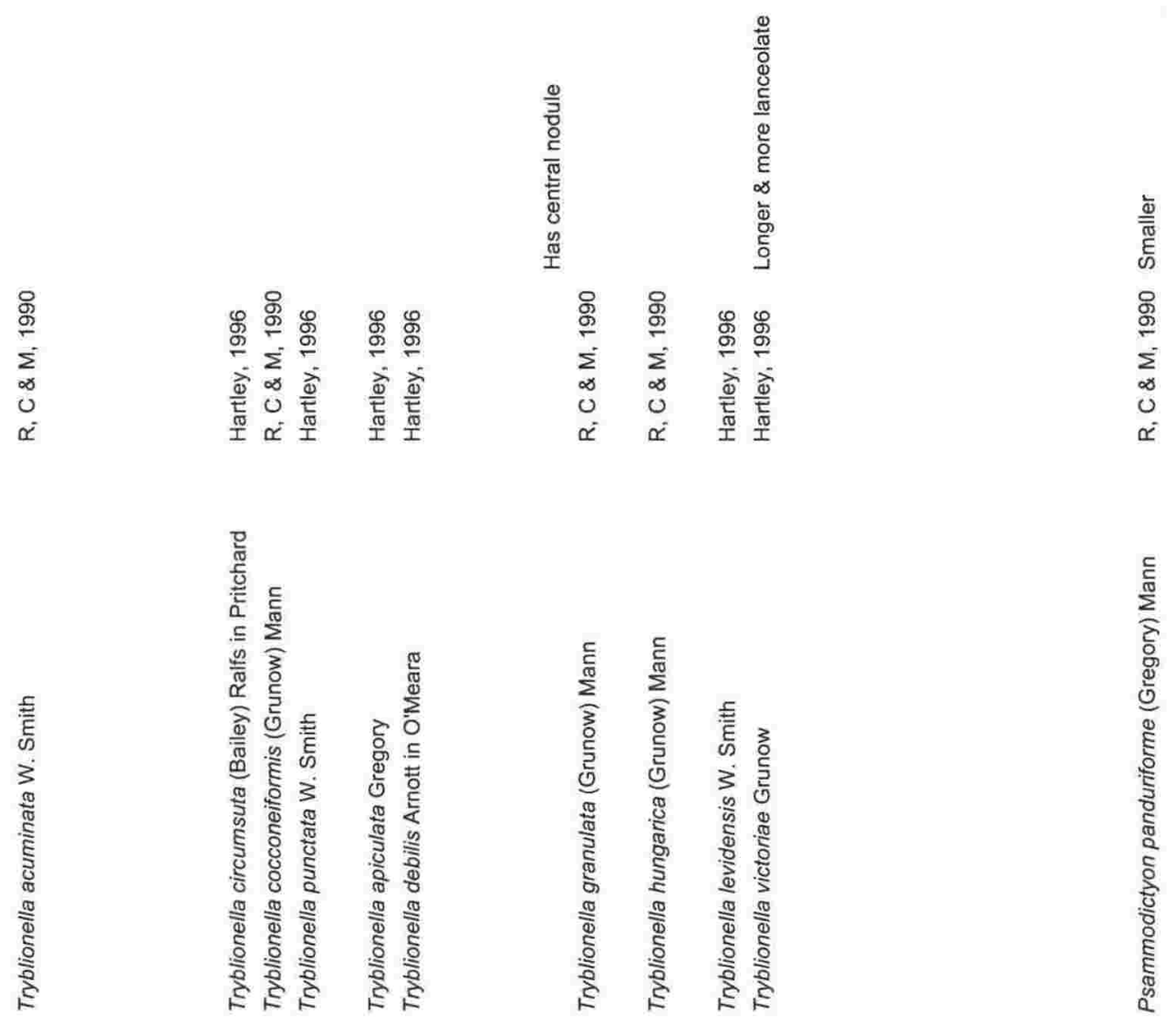

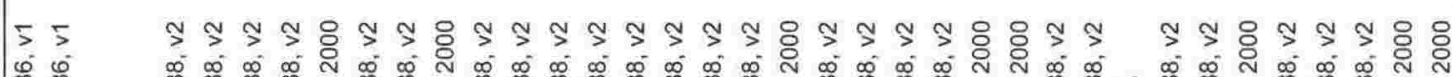

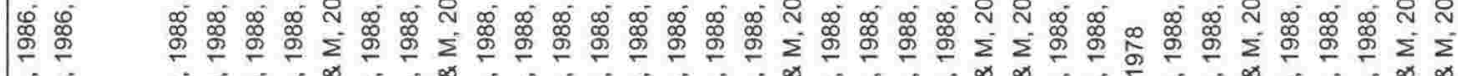
范

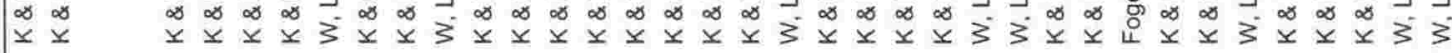

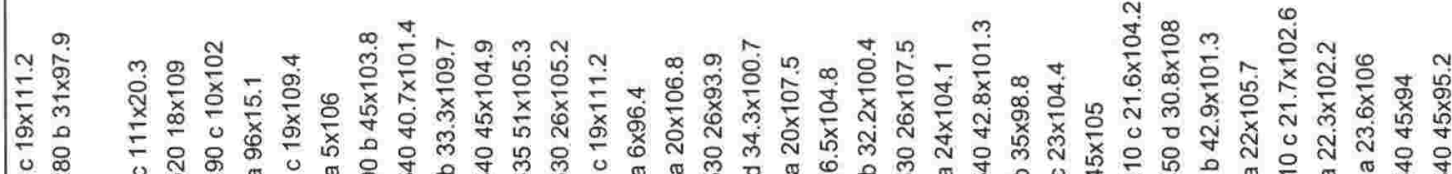

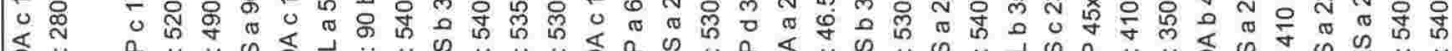

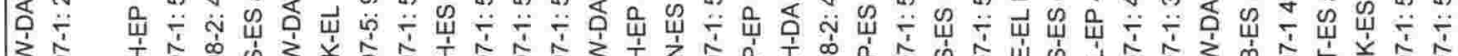

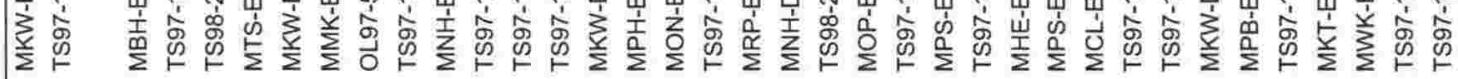

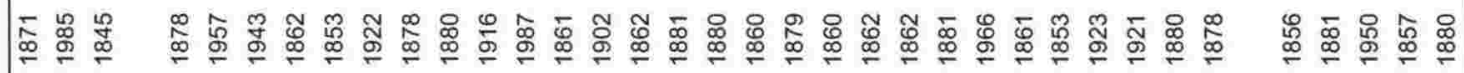

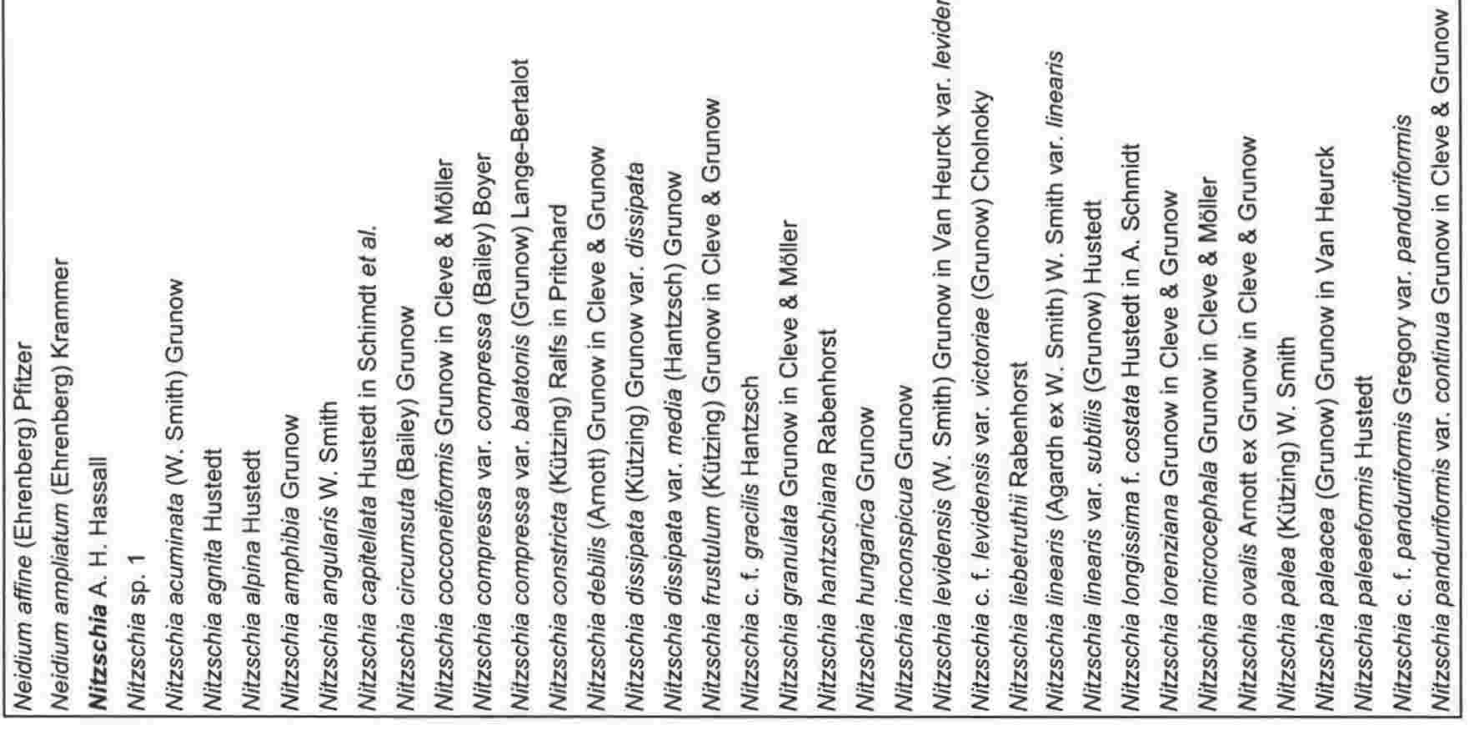




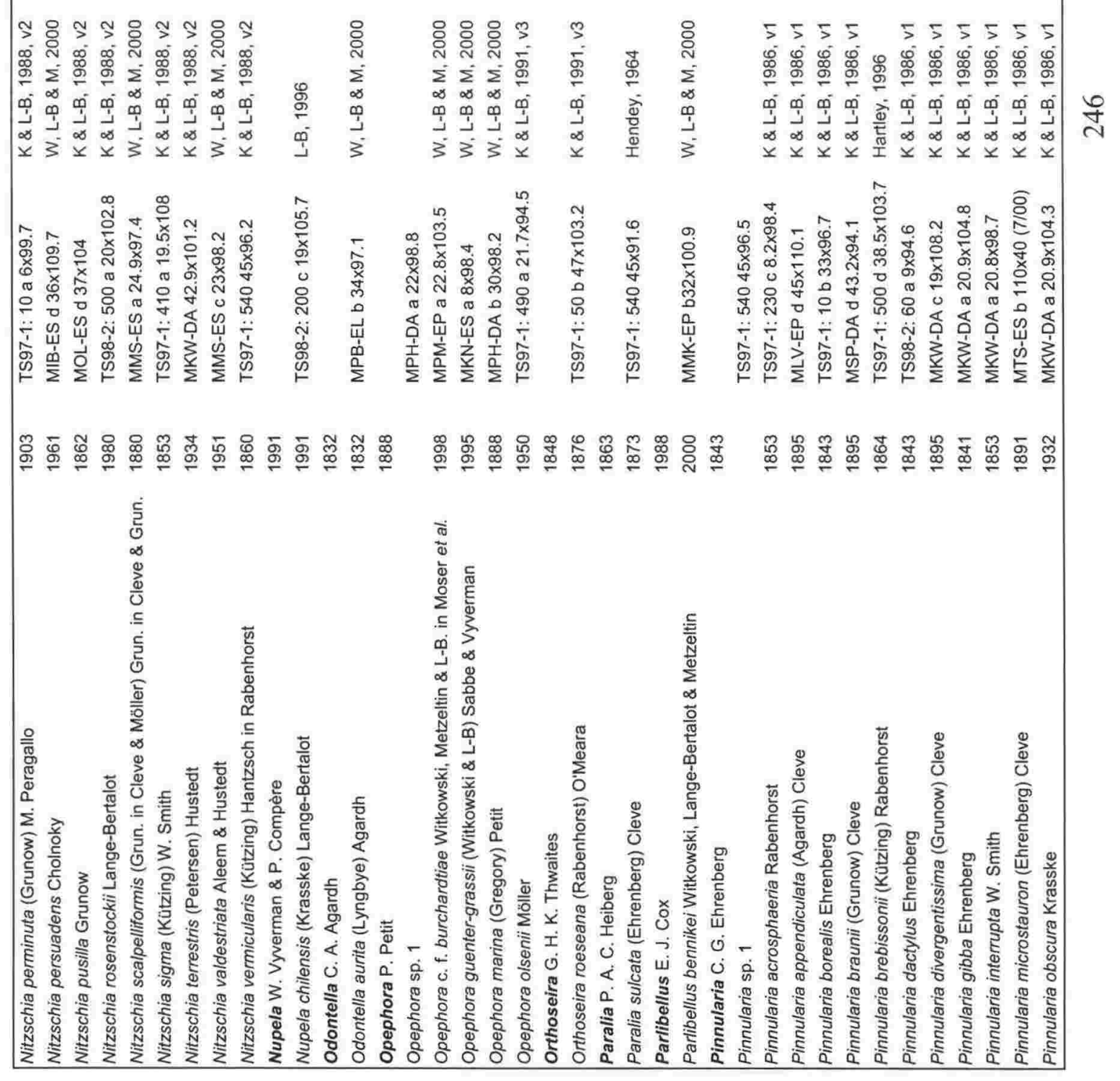




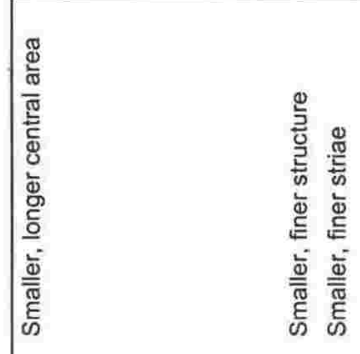

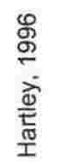

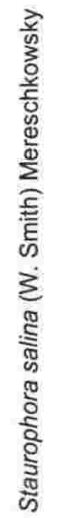

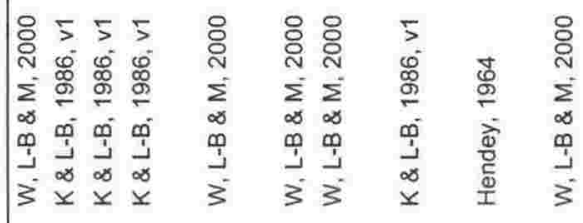

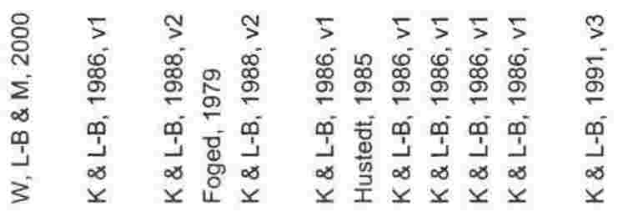

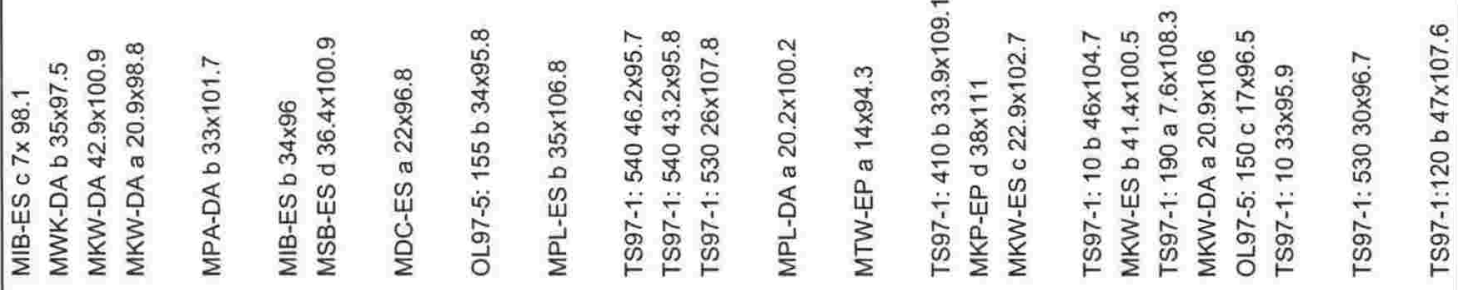

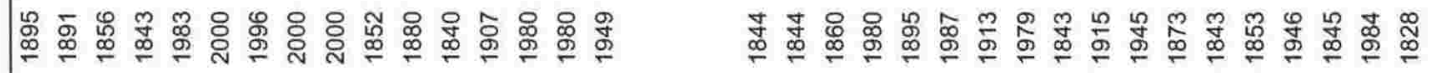

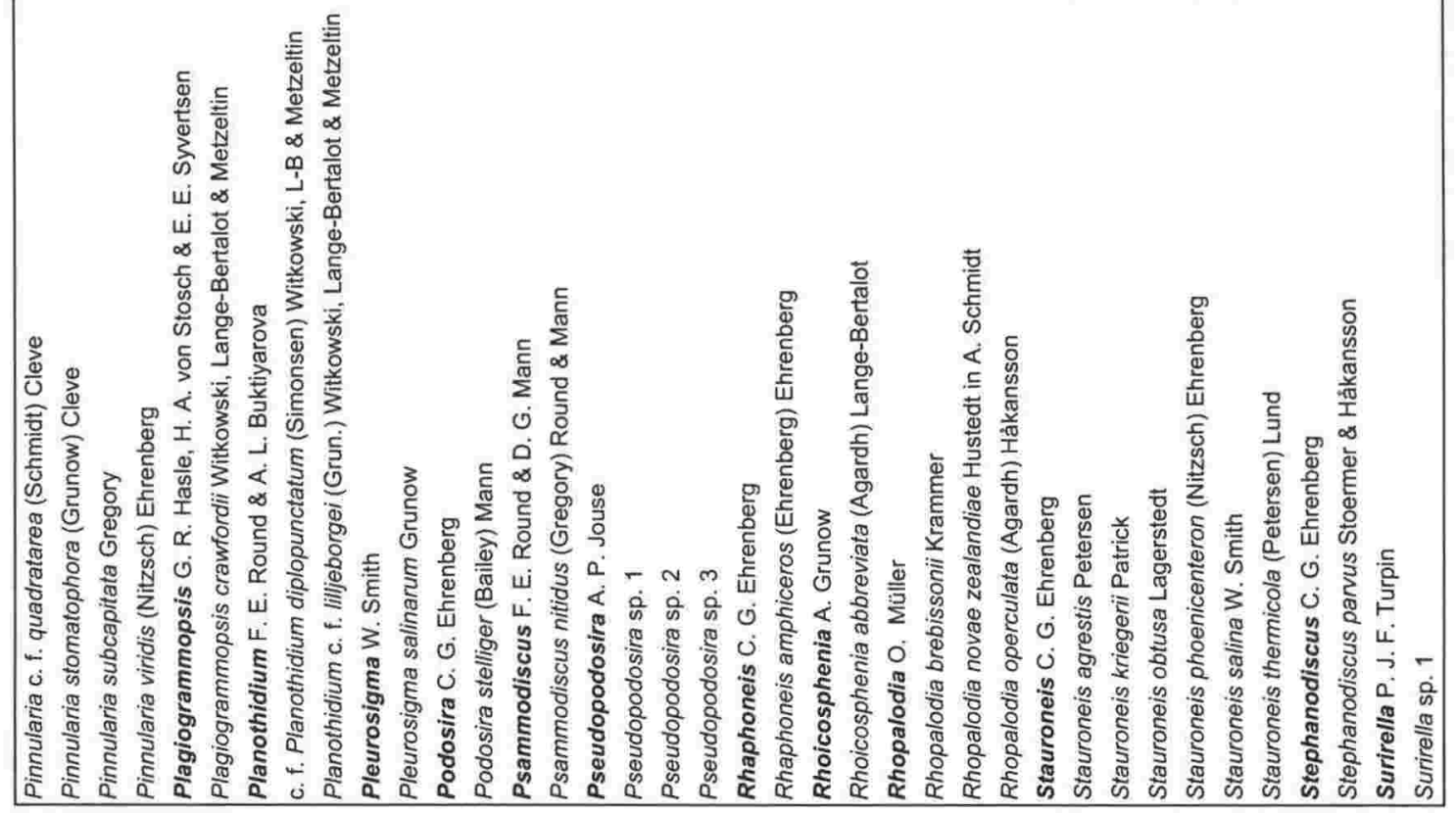




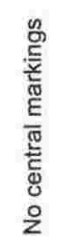

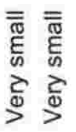

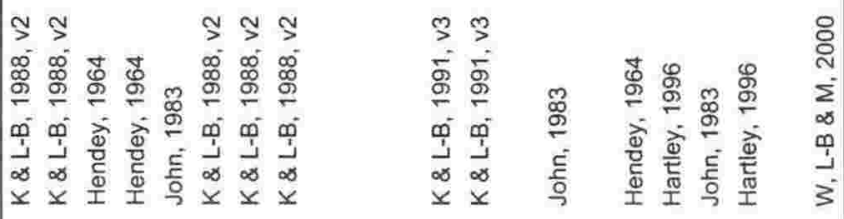

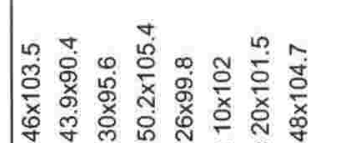

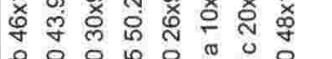

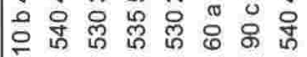

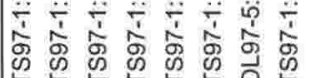

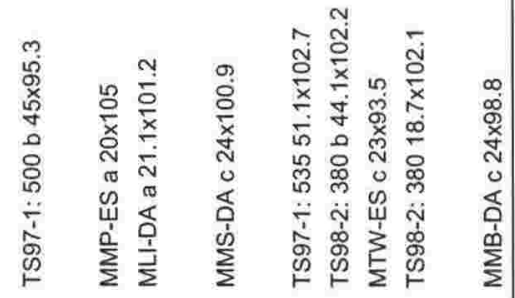

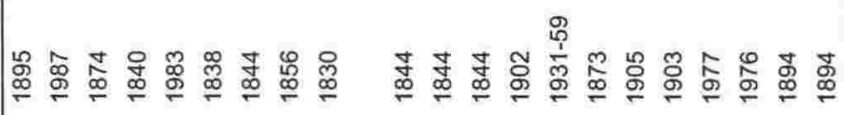

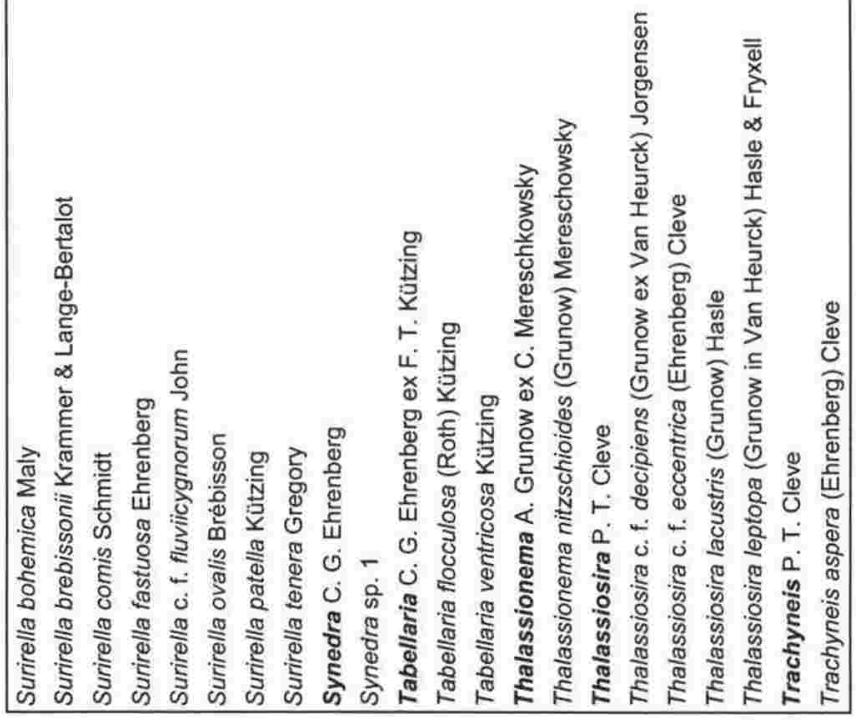




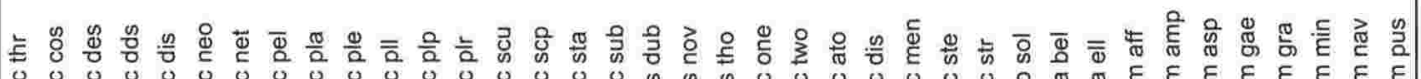

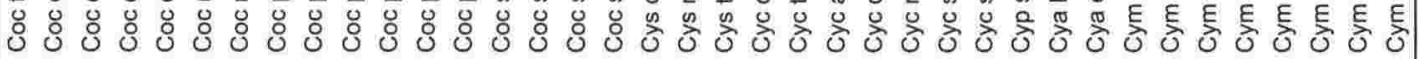

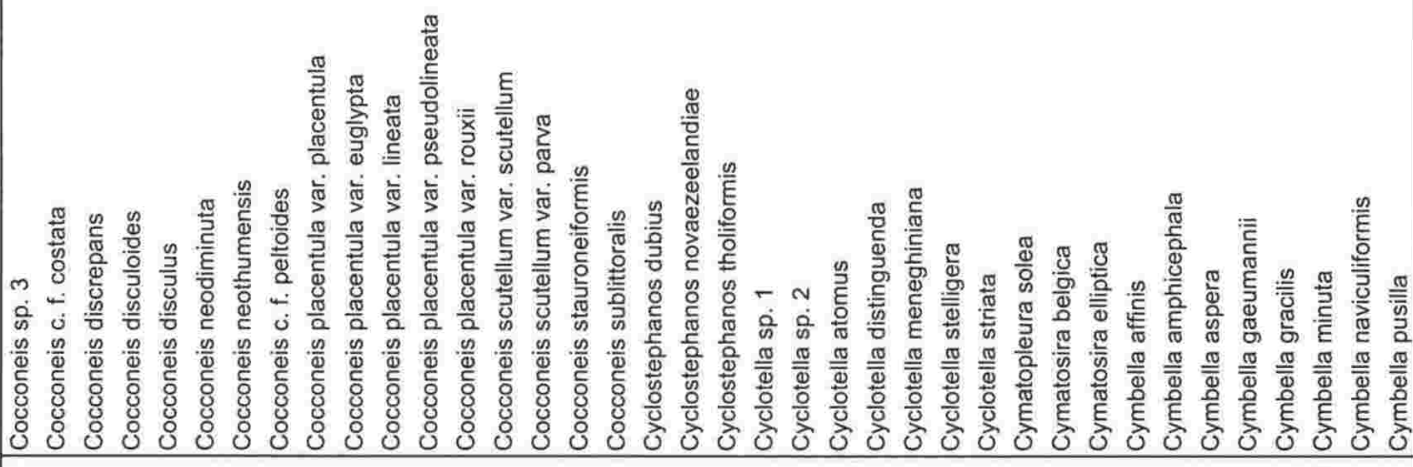

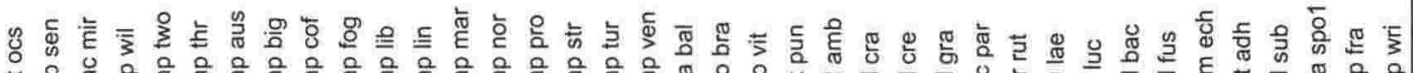

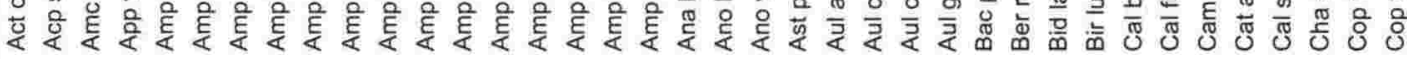

氙
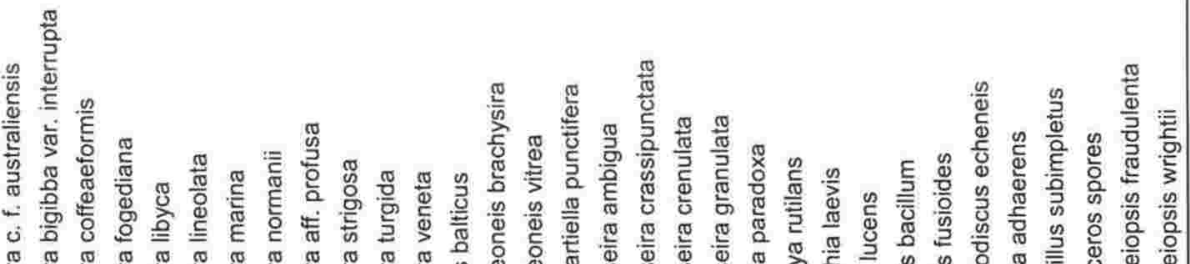

交

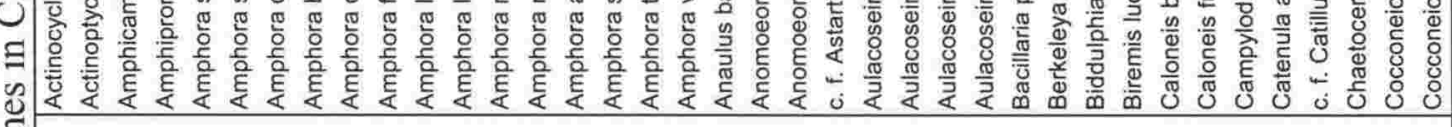

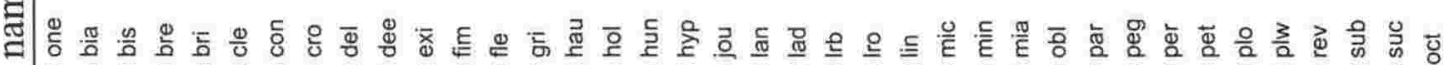

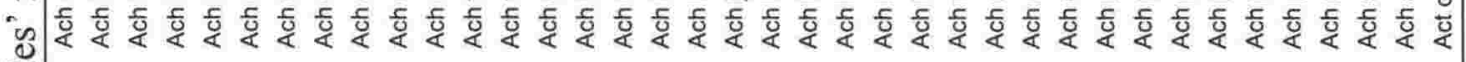

营

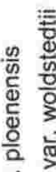

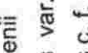

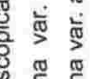




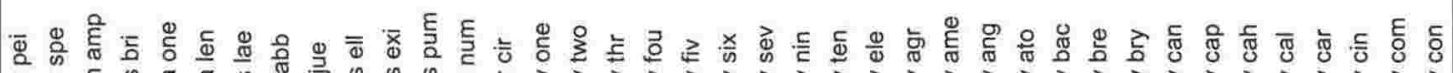

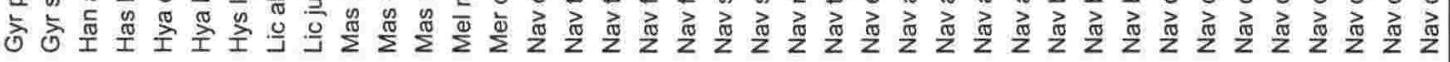

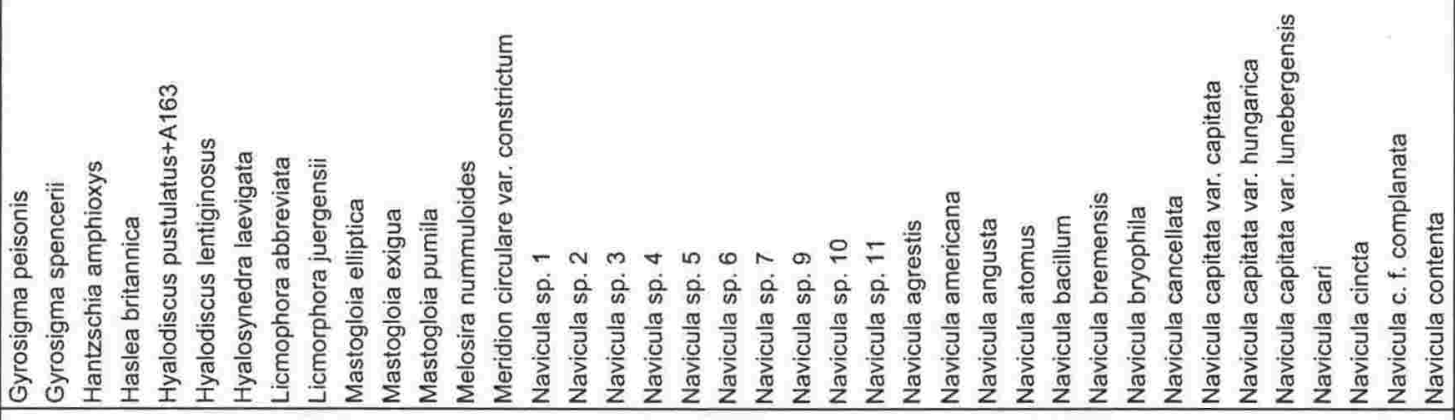

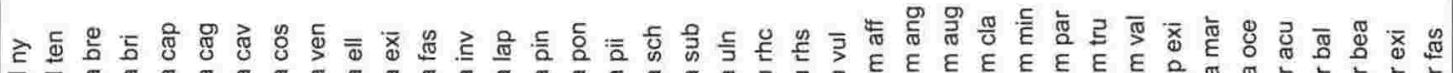

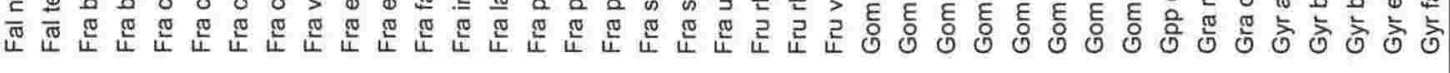

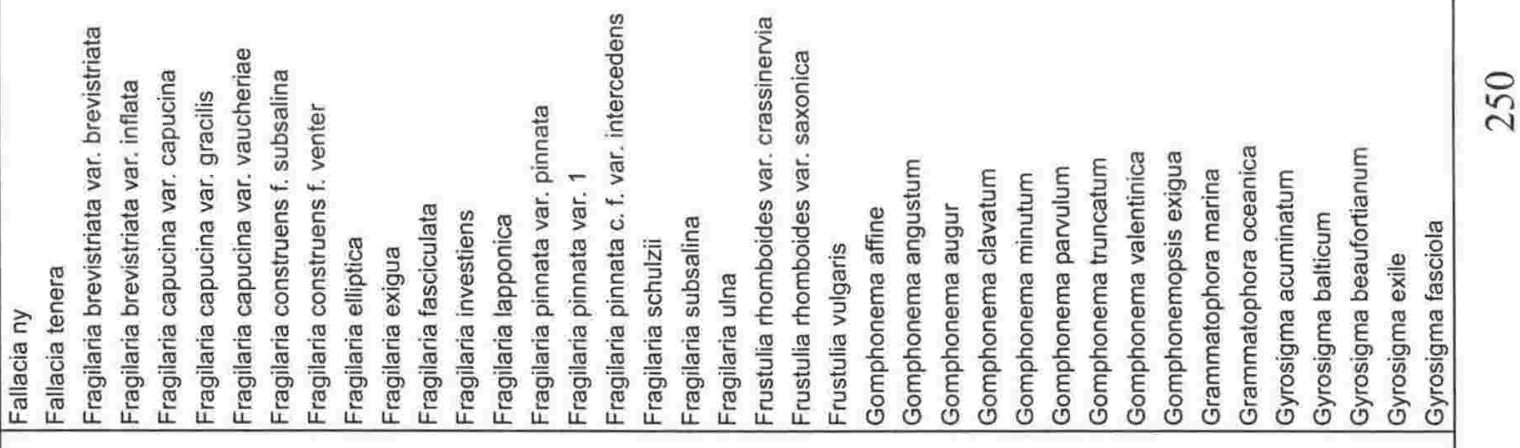

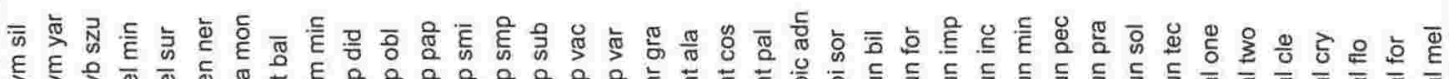

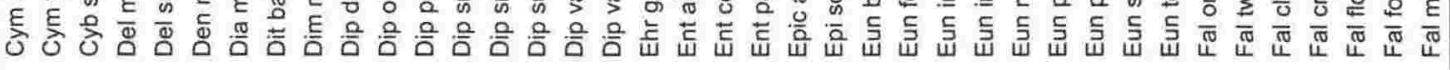

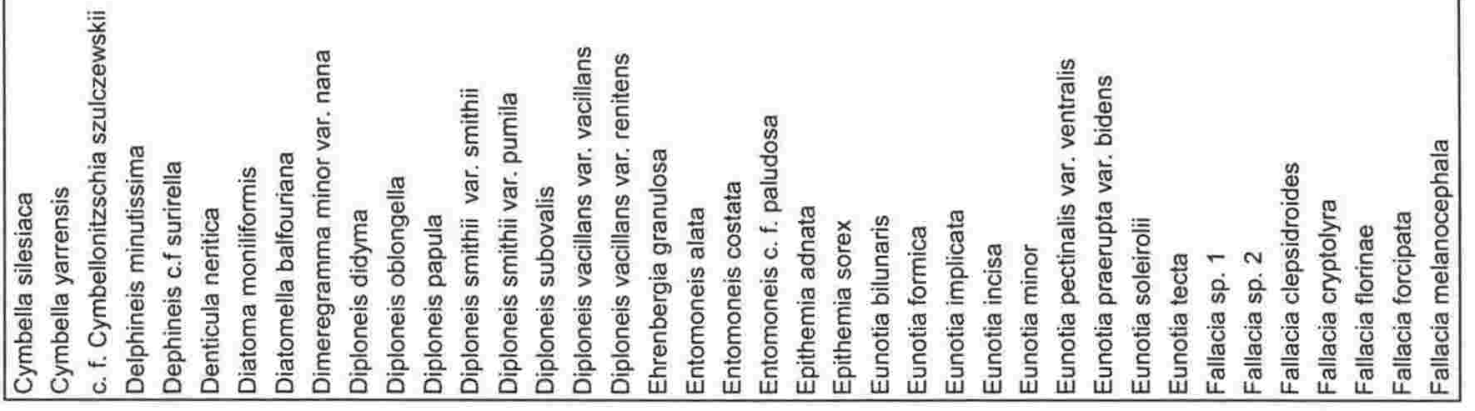




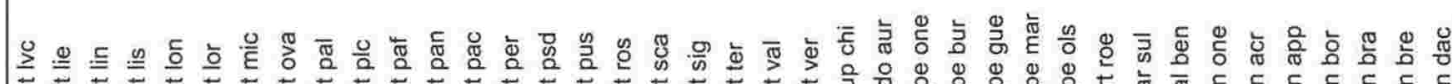

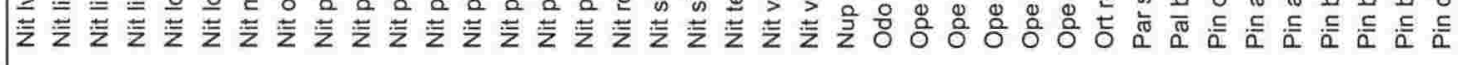

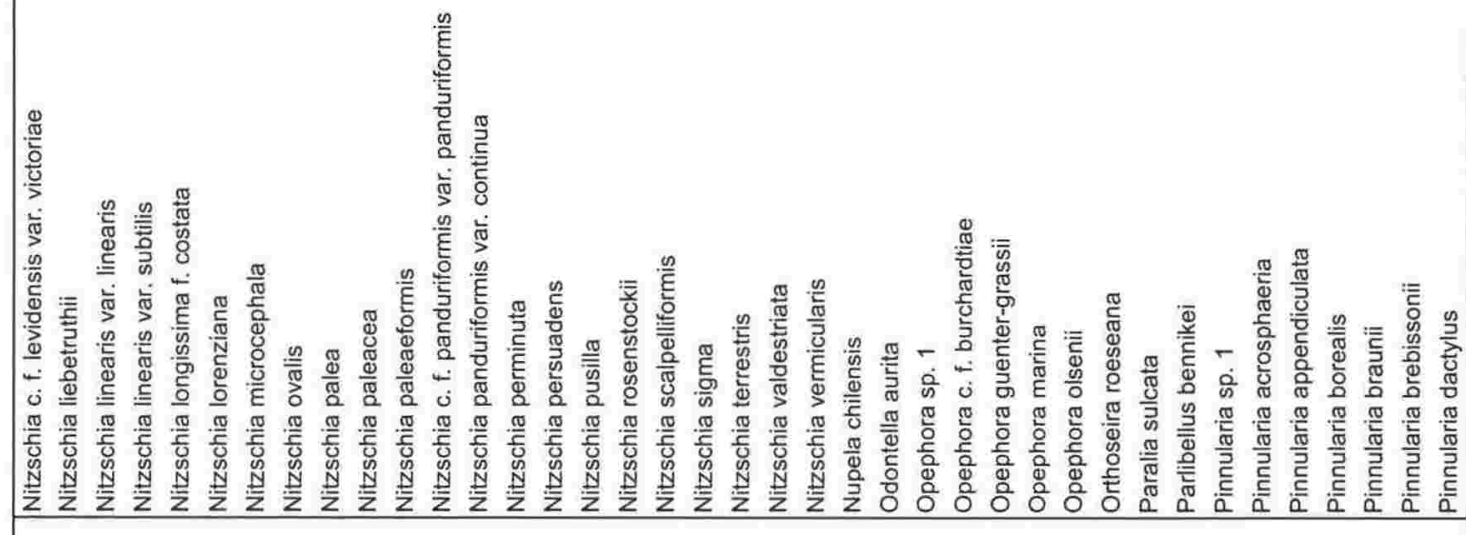

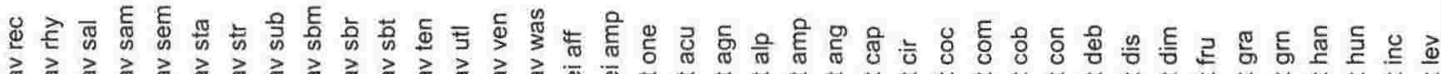

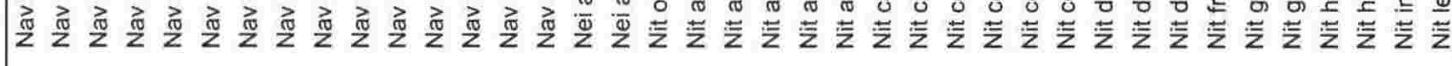

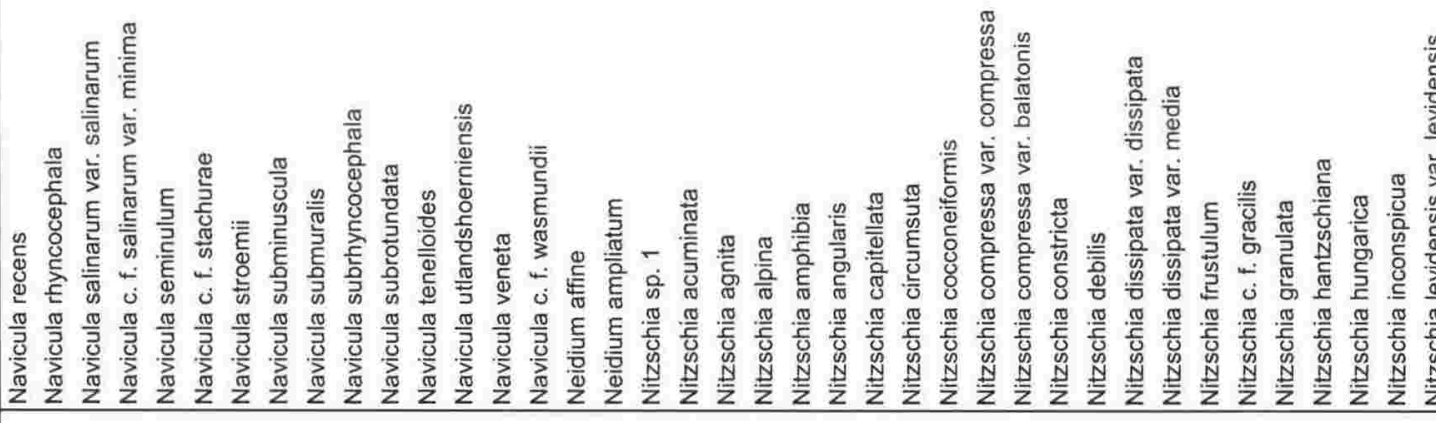

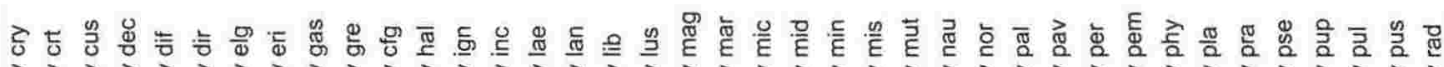

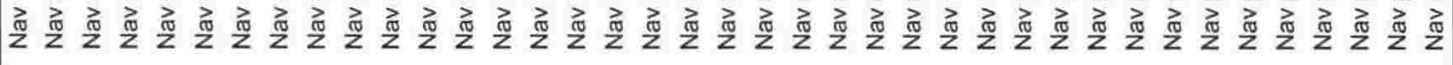

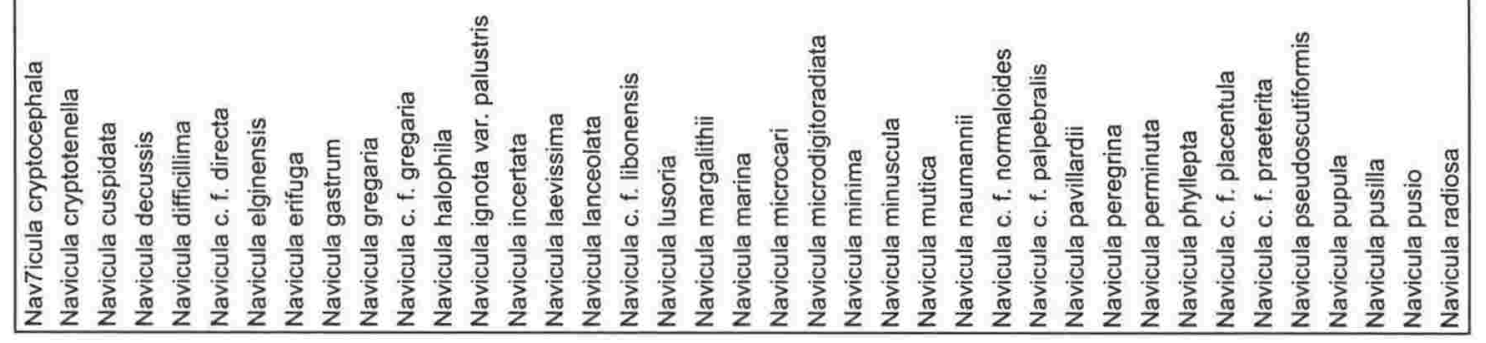




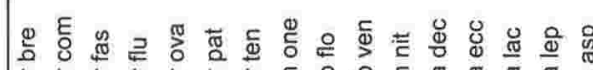

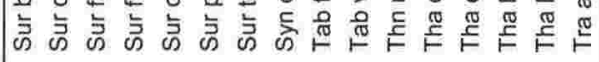

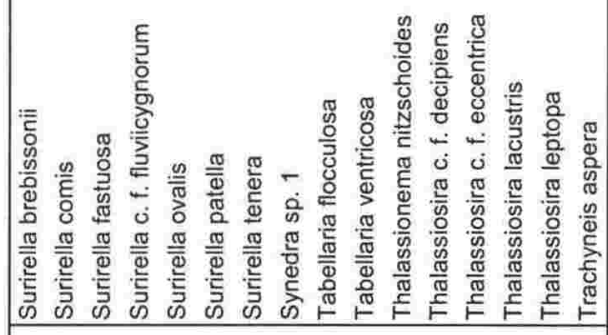

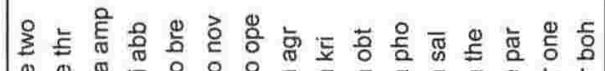

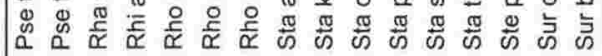

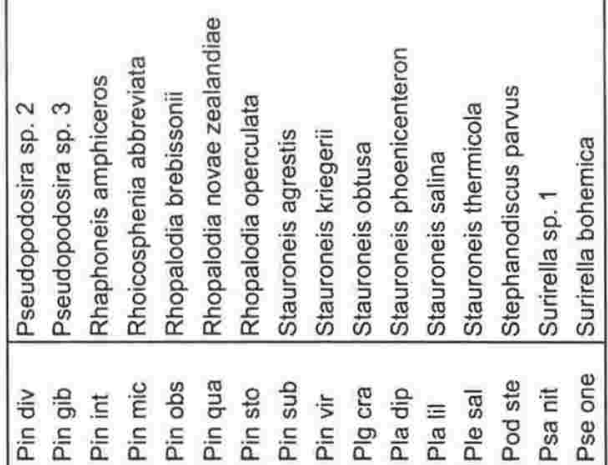

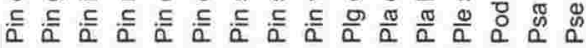

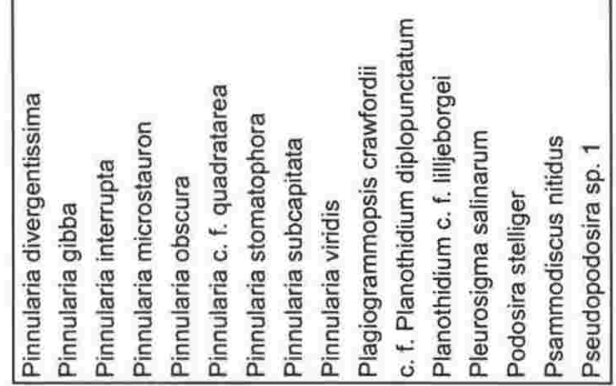




\section{A P P E N D IX III}

\section{Environmental Preferences of Diatom Species}

Item 1: Table of Environmental Preferences of Diatom Species

Key to Table:

1. Species' names (see Appendix II for full names and authors).

2. Published habitat and salinity preferences for species showing categories used for paleoecological interpretation in Chapters Four, Five and Six, and the source of information. Abbreviations used for references:
$\mathrm{R}, \mathrm{C}, \& \mathrm{M}$
Round, Crawford \& Mann
$\mathrm{VD}, \mathrm{M} \& \mathrm{~S}$
Van Dam, Mertens \& Sinkeldam
$\mathrm{V} \& \mathrm{~d} \mathrm{~W}$
Vos \& de Wolf
$\mathrm{Z} \& \mathrm{H}$
Zong \& Horton
W, L-B \& M
Witkowski, Lange-Bertalot \& Metzeltin
$\mathrm{K} \& \mathrm{~L}-\mathrm{B}$
Krammer \& Lange-Bertalot
$\mathrm{P} \& \mathrm{R}$
Patrick \& Reimer

3. Comparison between published salinity preferences and salinity optima derived from the calibration set. Preferences were compared using the salinity category definitions in Table 2.4 (Chapter Two).

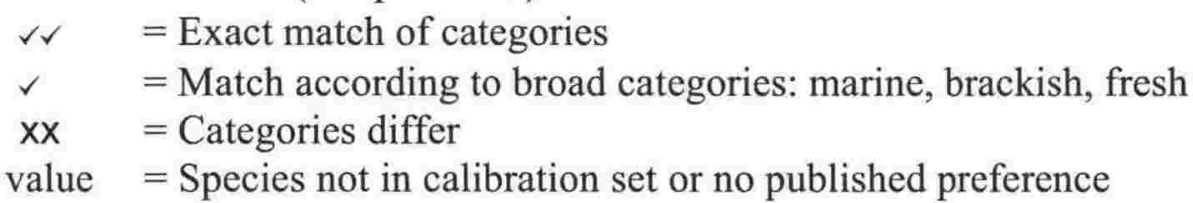

4. Salinity preferences (jackknifed optima and tolerance) derived from weighted averaging of the calibration set described in Chapter Three.

5. Occurrences of species in the calibration set and presence in the fossil dataset. The effective number of occurrences (N2) should be considered before using salinity preferences derived from the calibration set. 


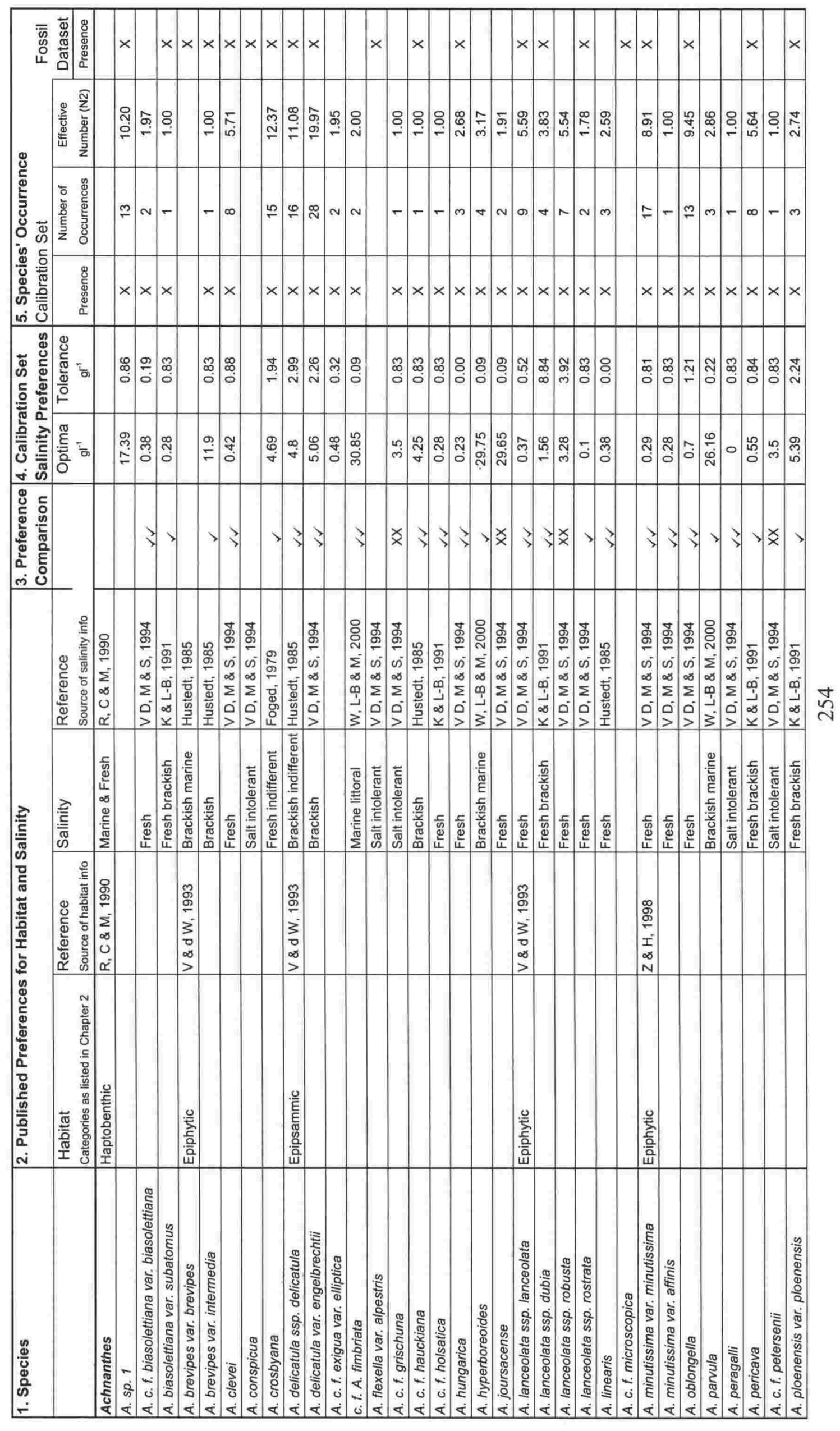




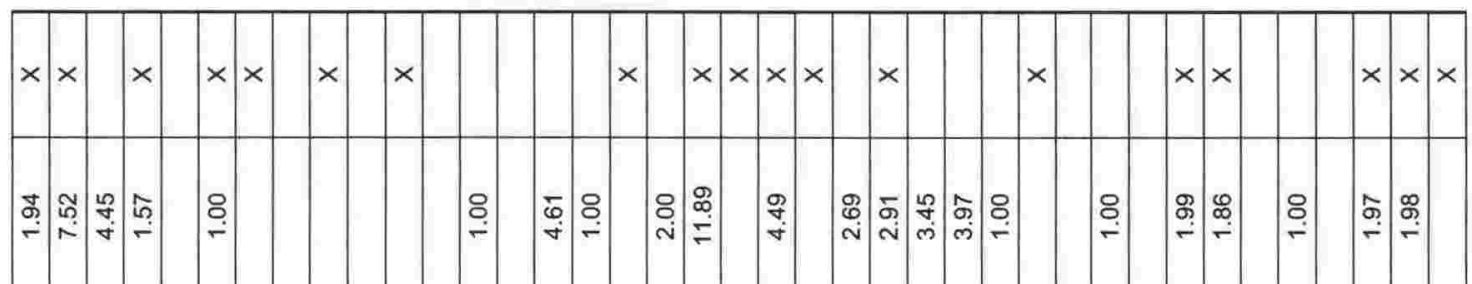

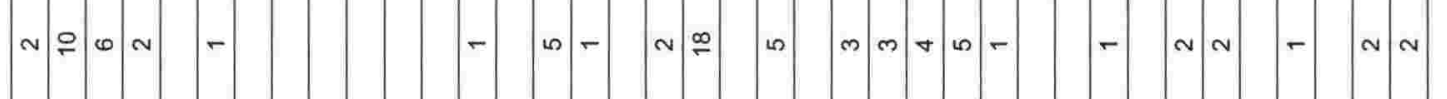

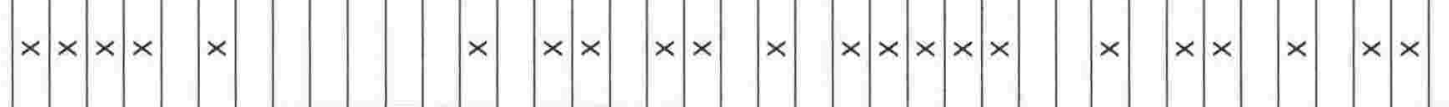

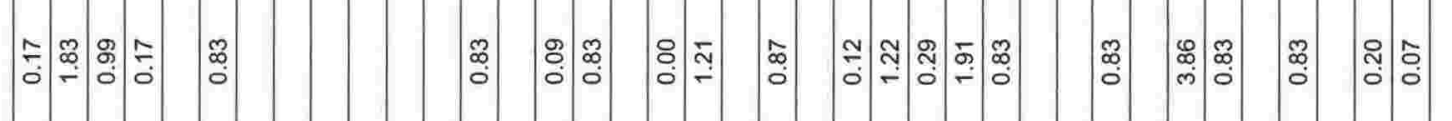

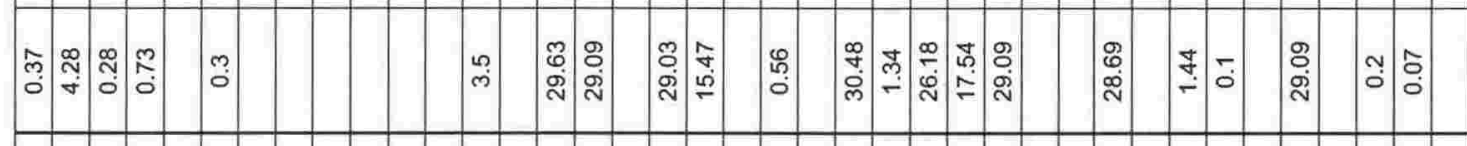

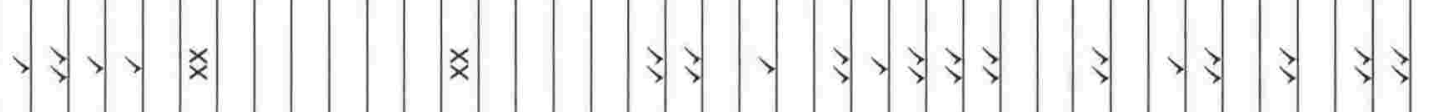

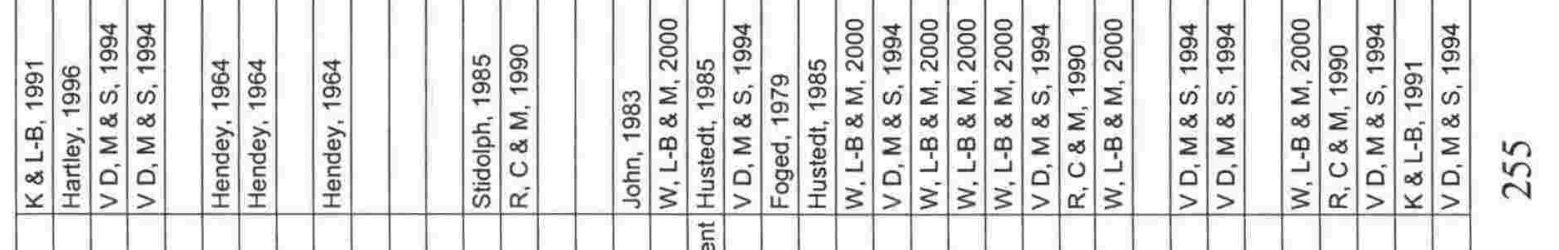

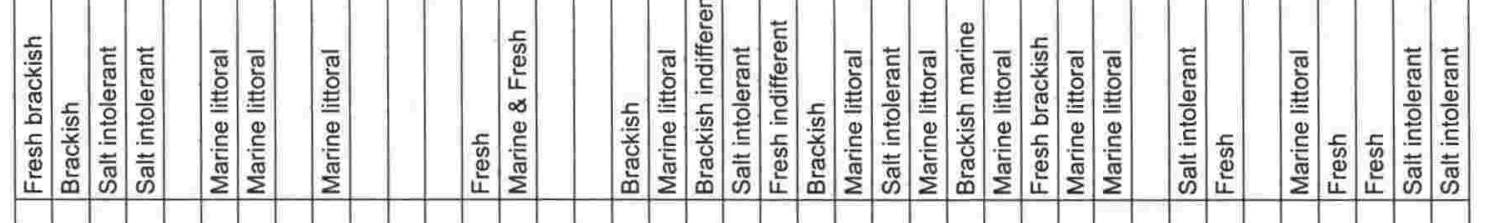

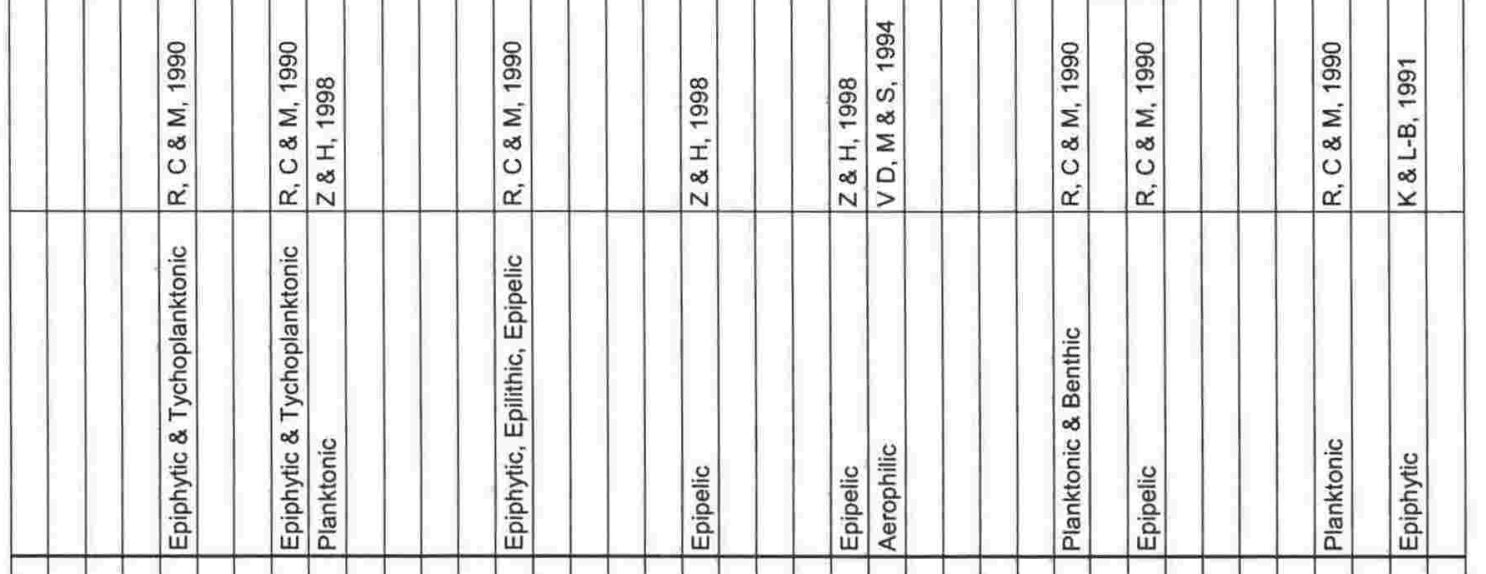

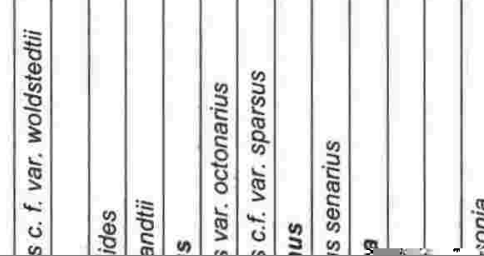




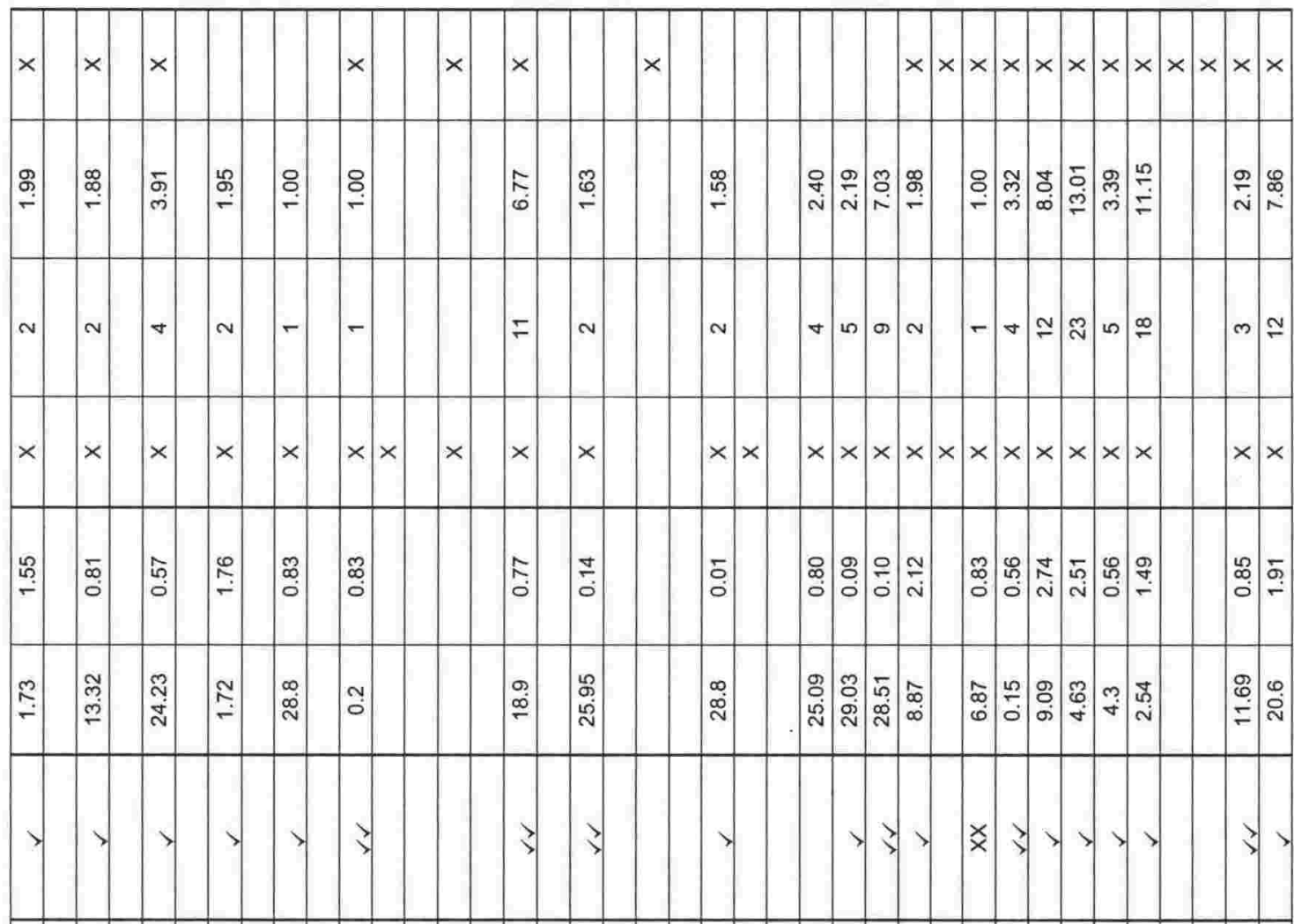

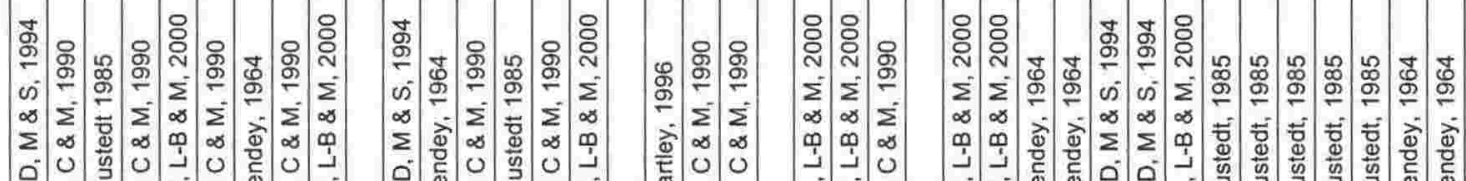

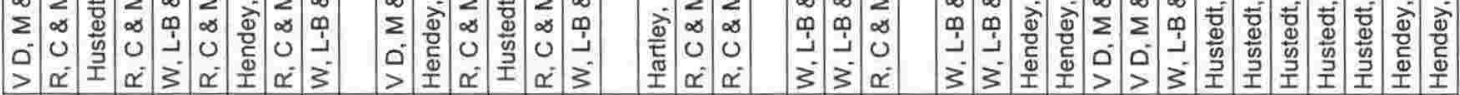

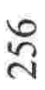

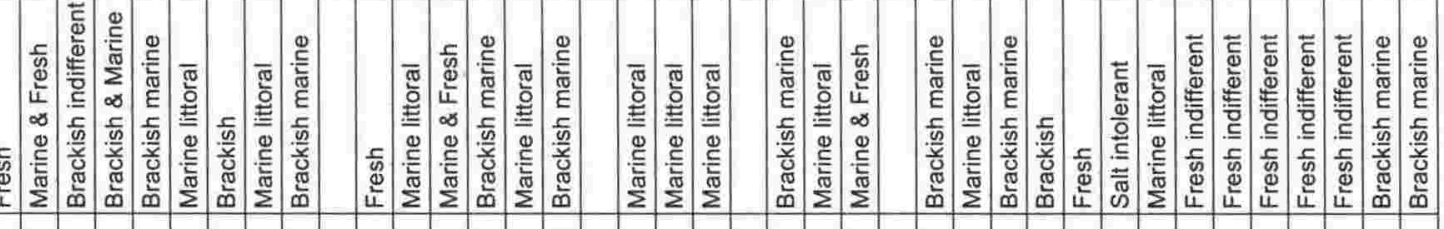

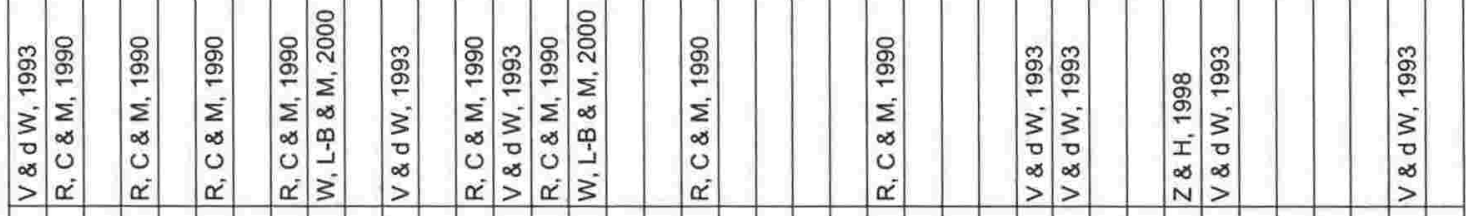

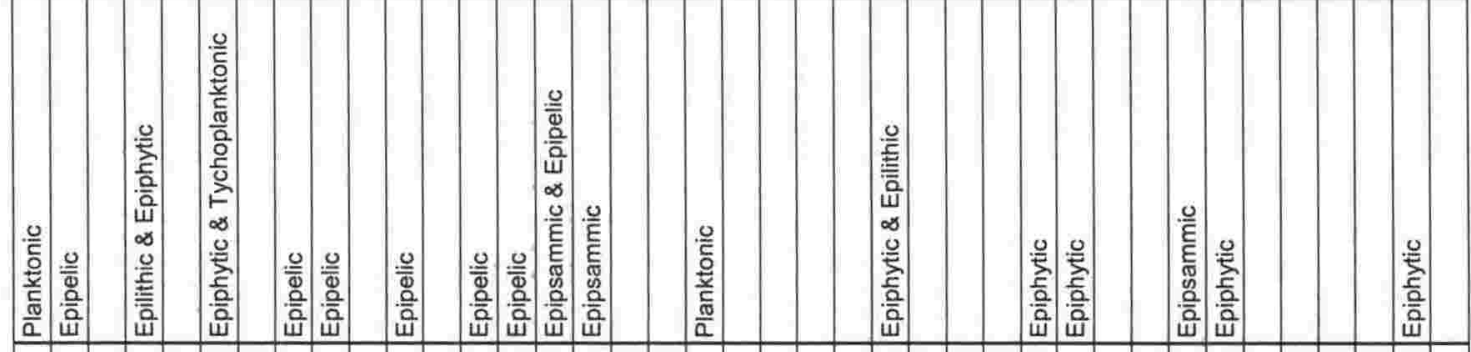

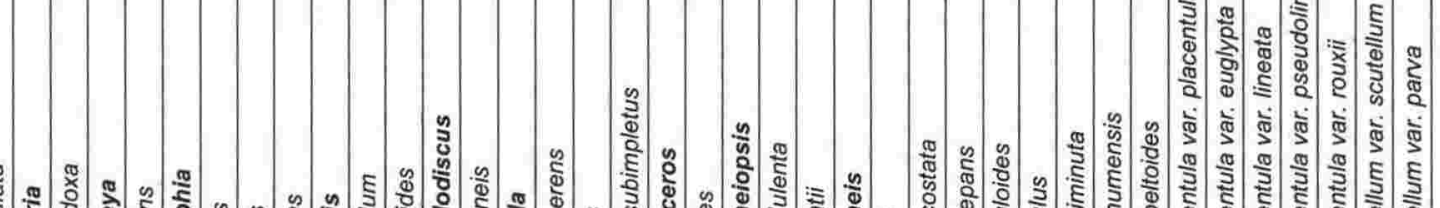

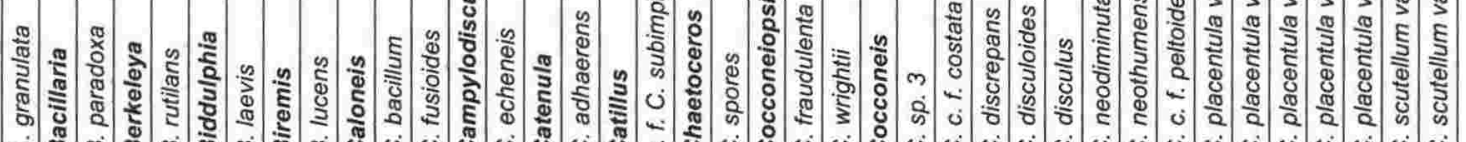

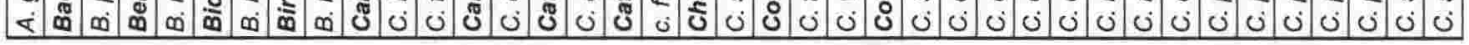




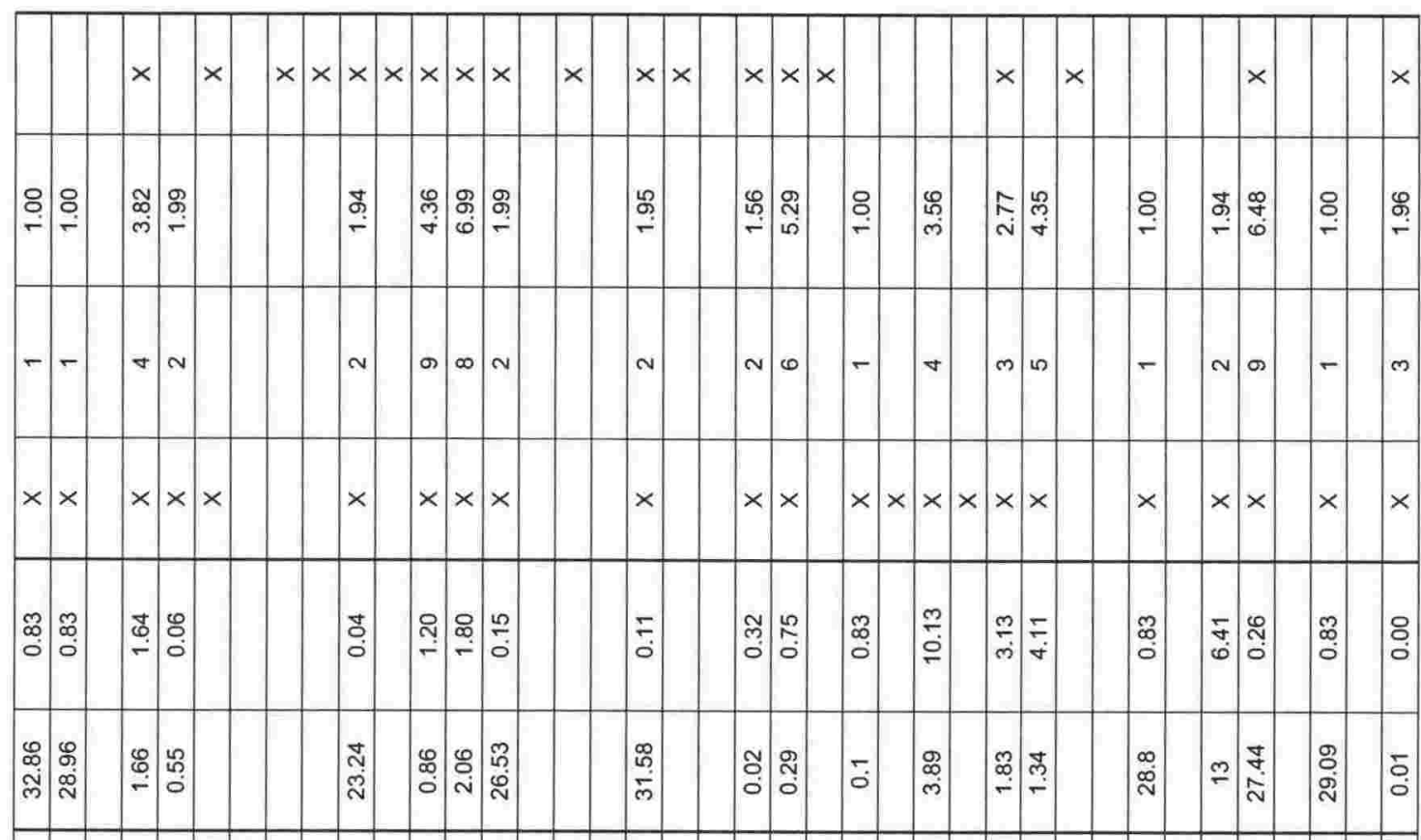

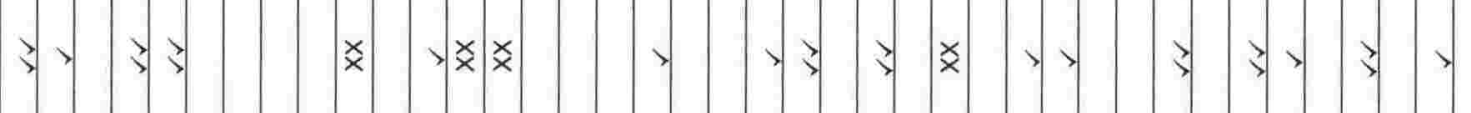

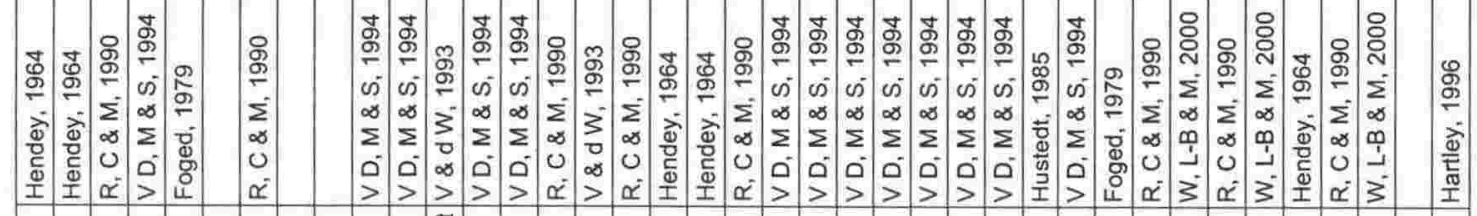

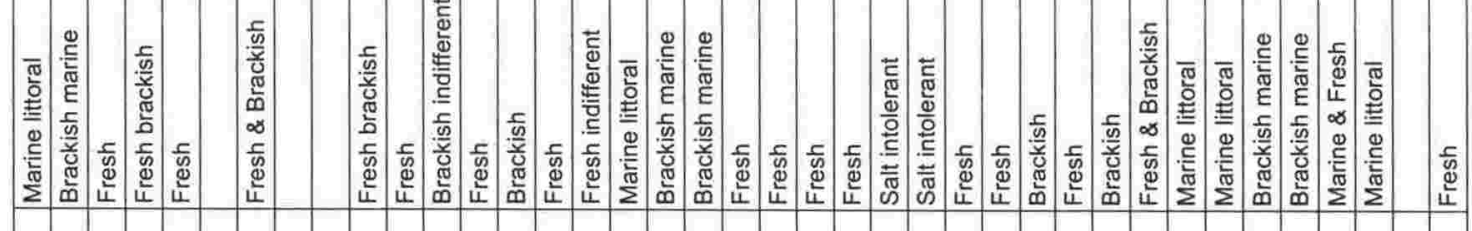

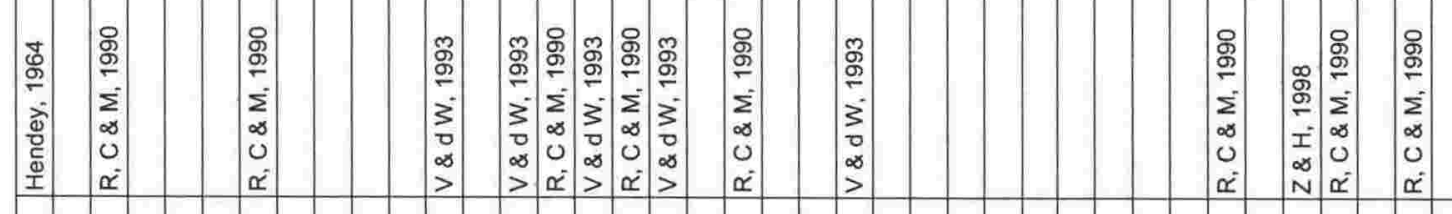

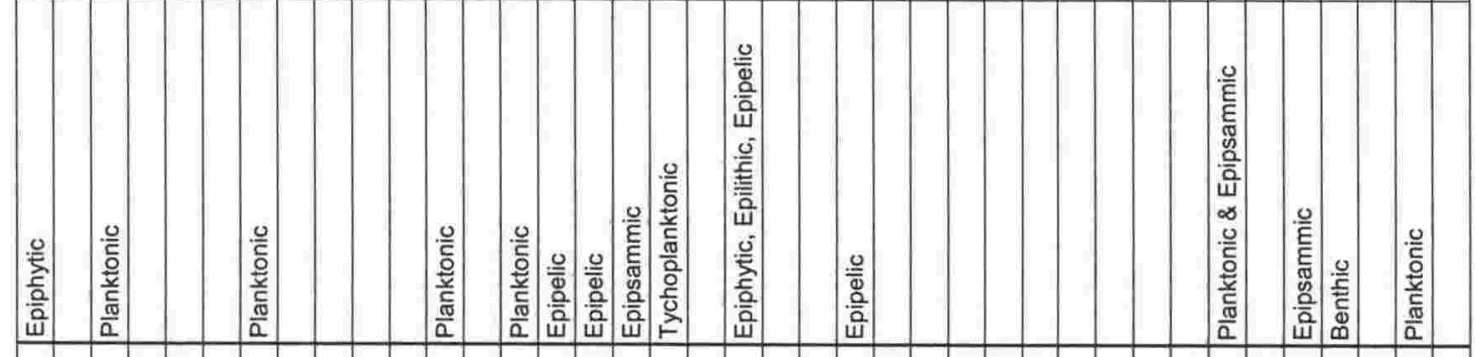

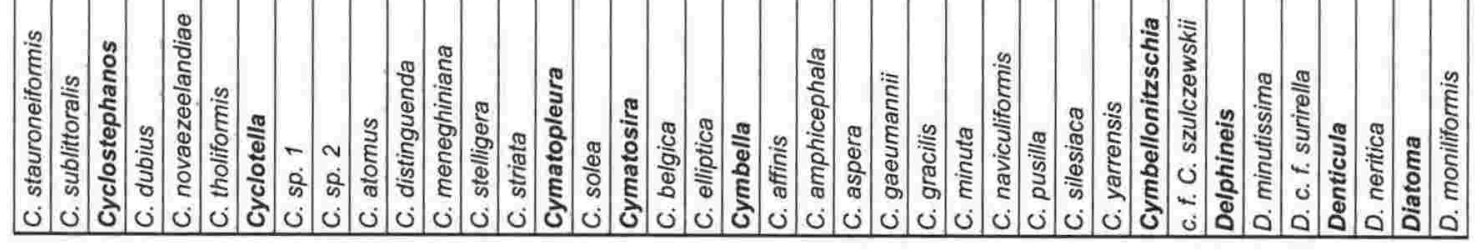




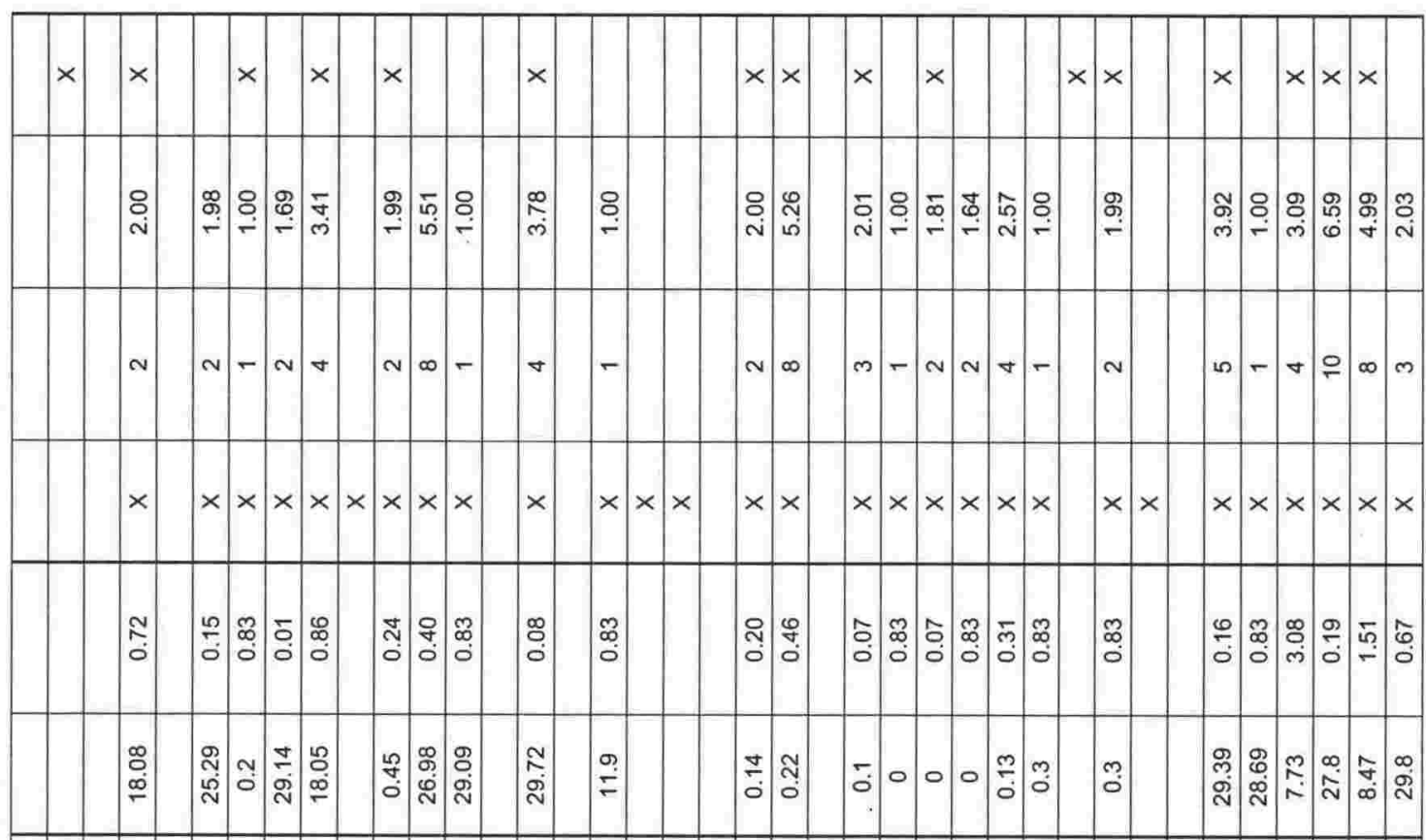

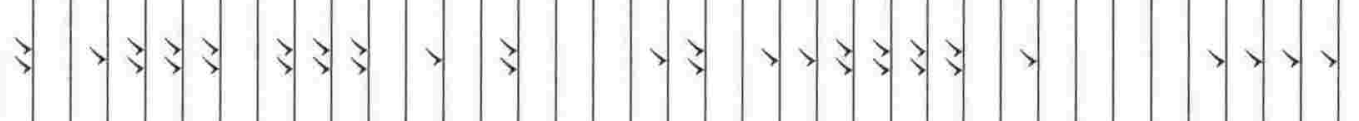

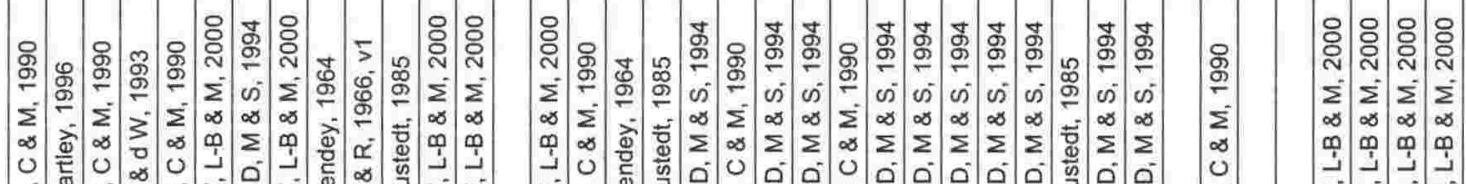

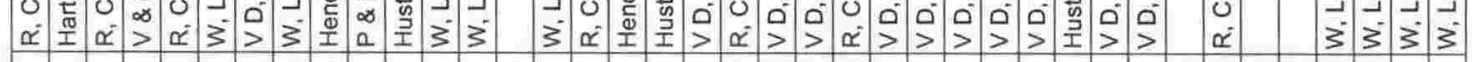

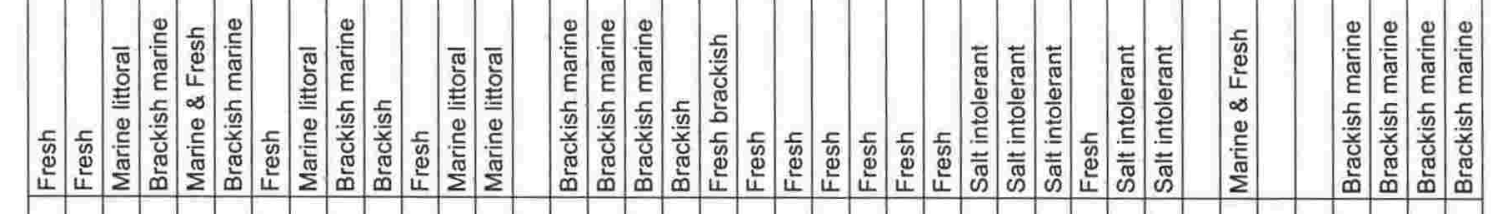

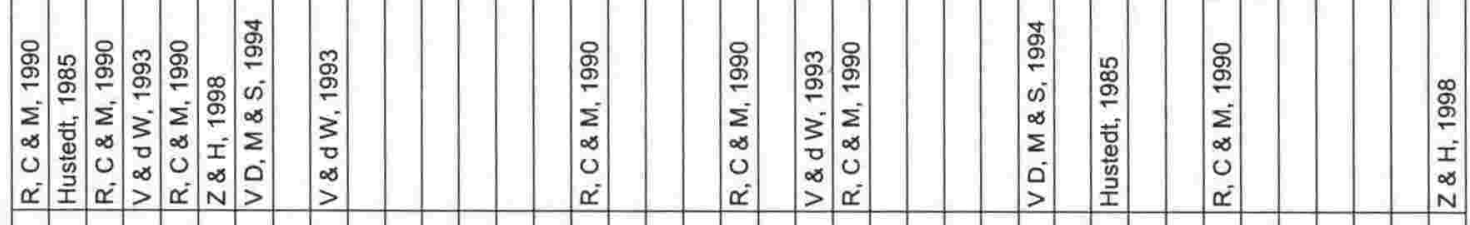

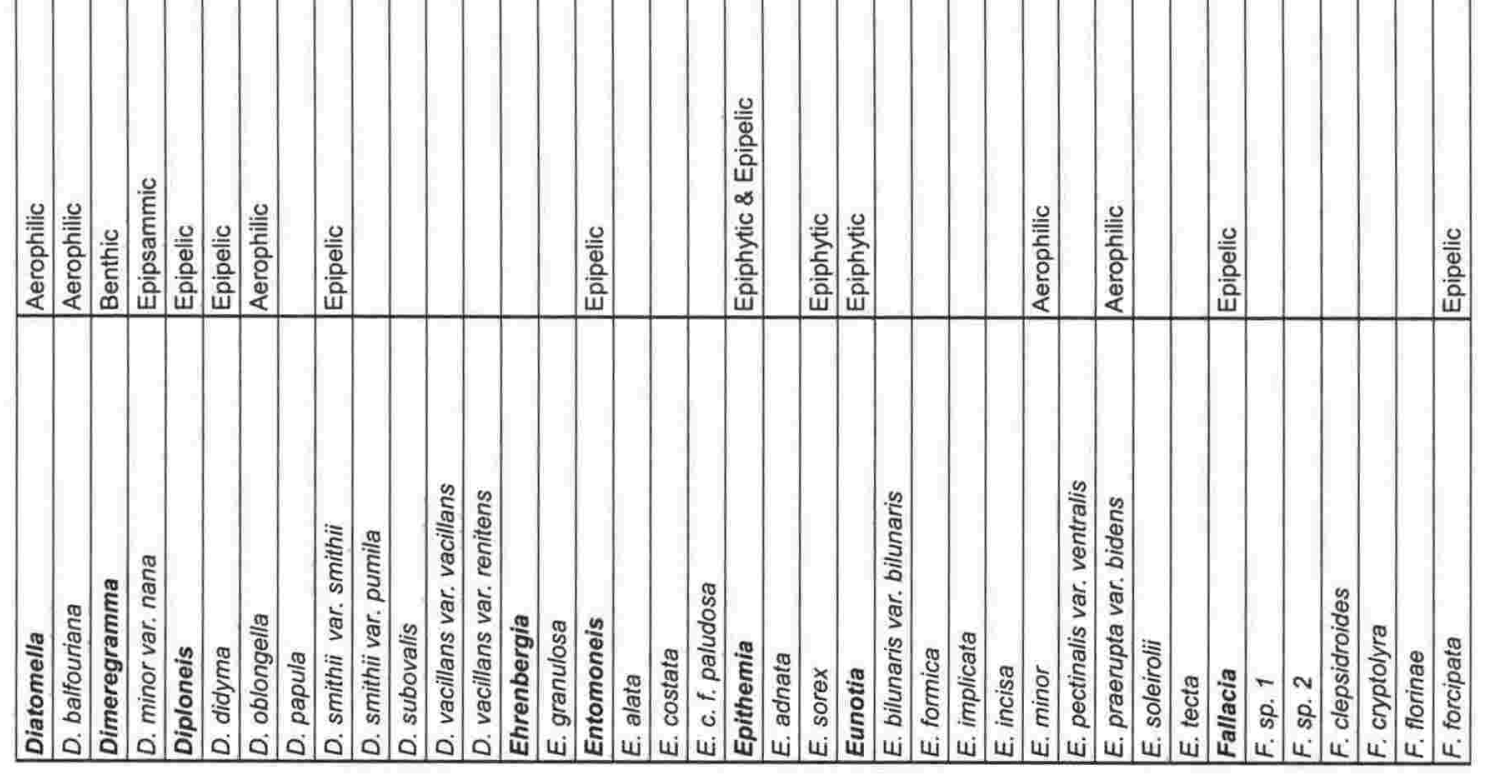




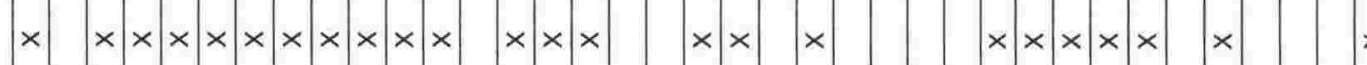

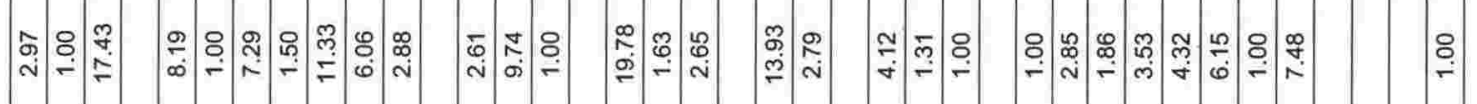

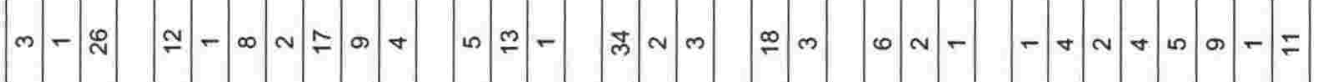

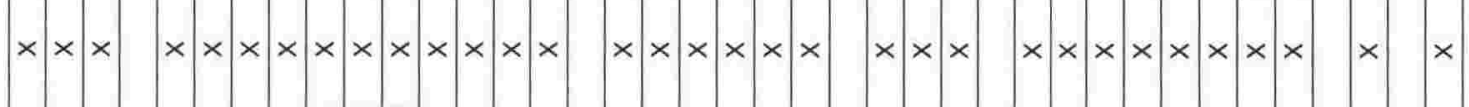

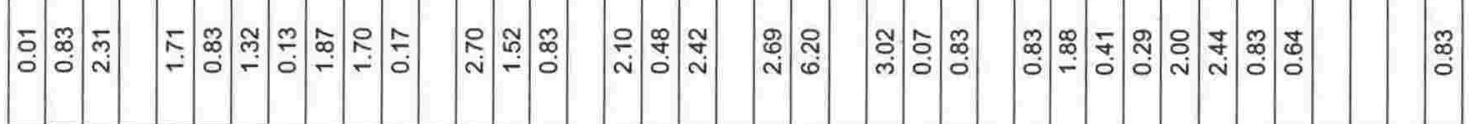

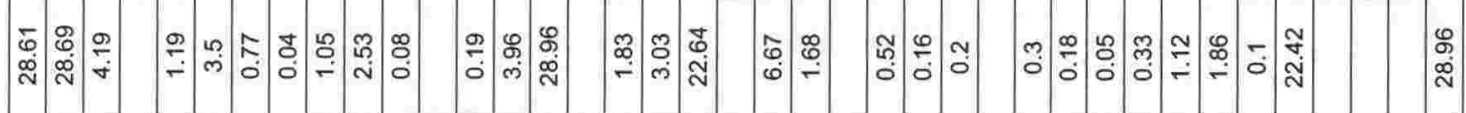

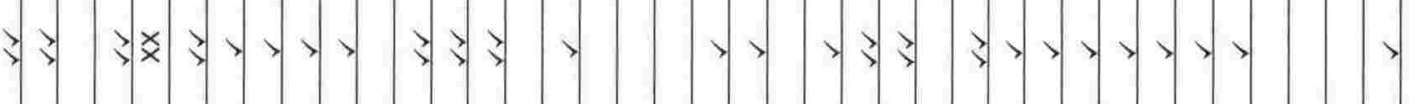

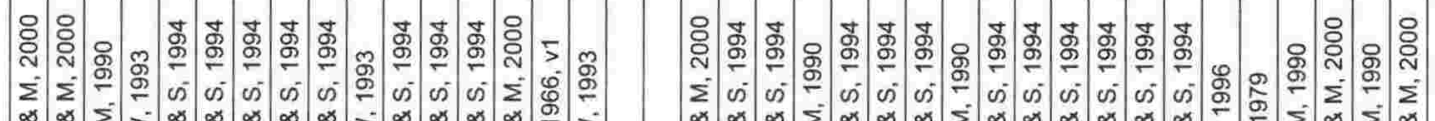

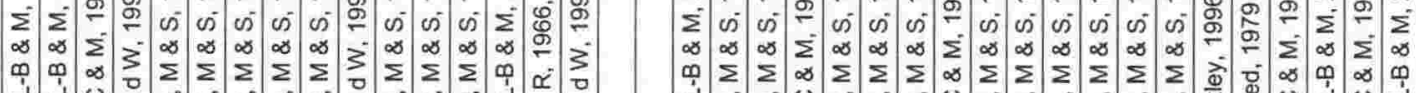

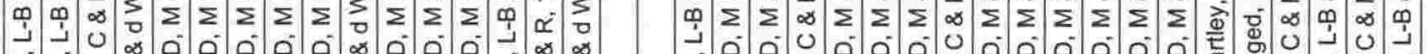

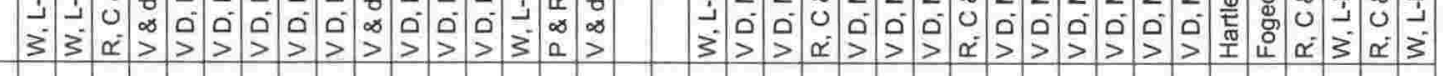

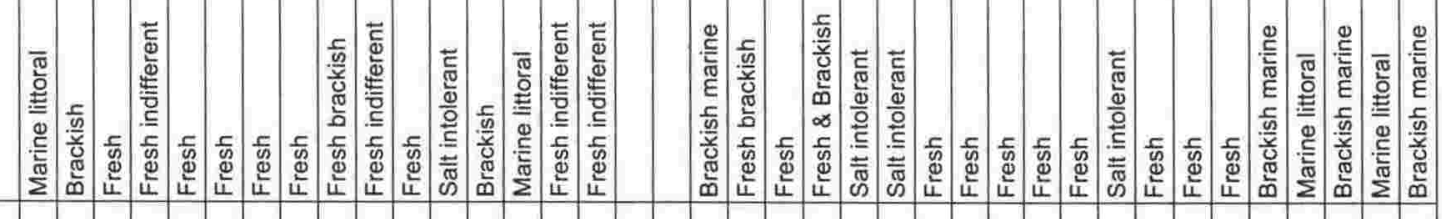




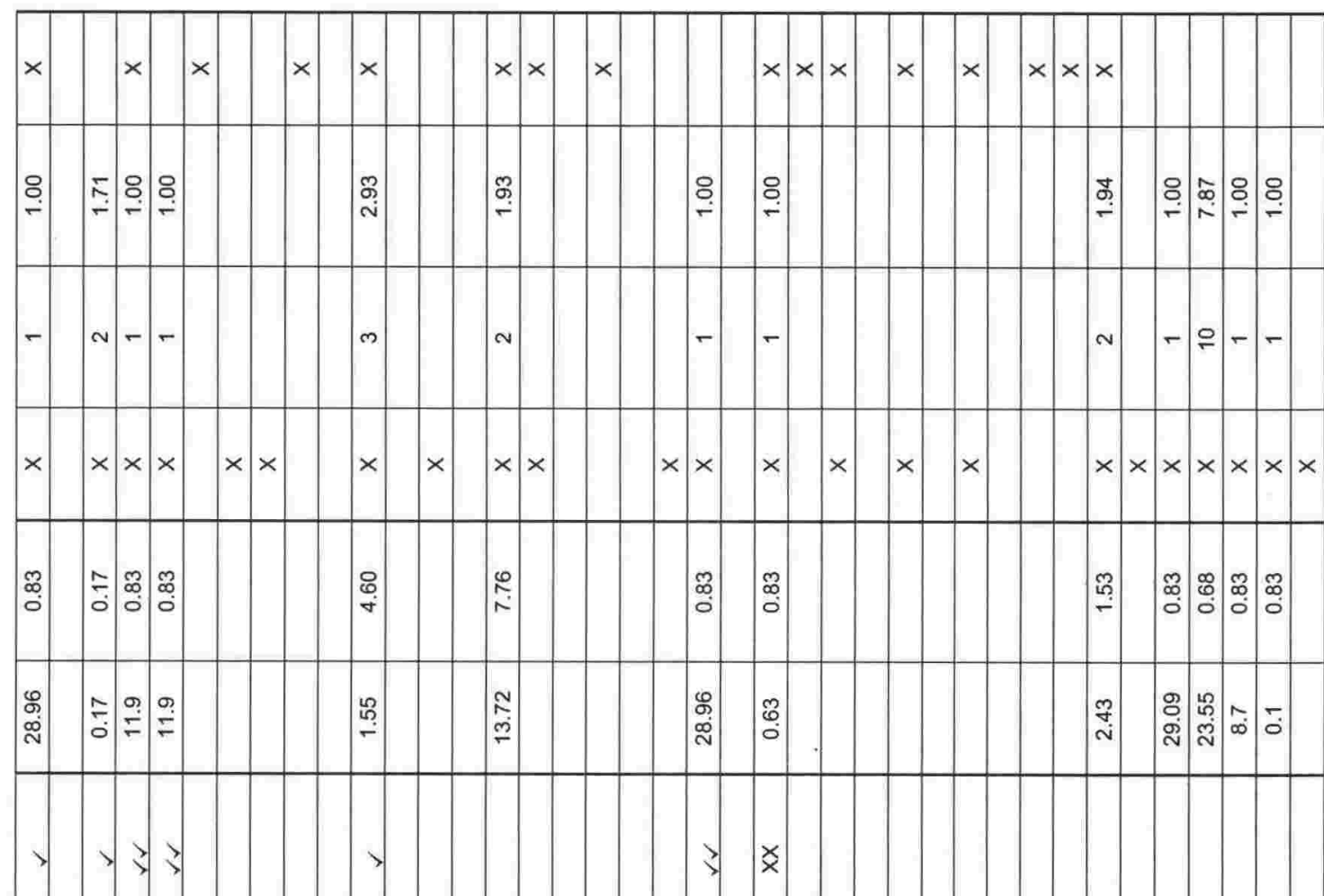

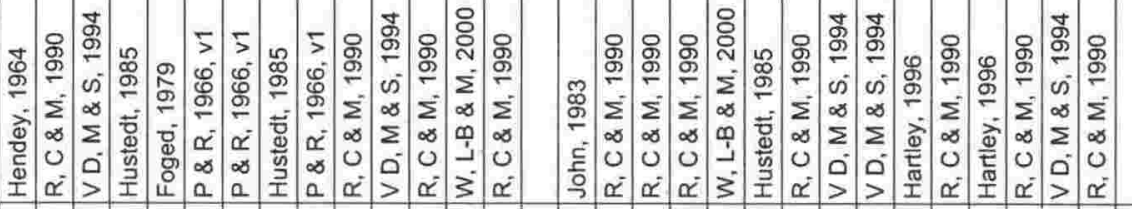

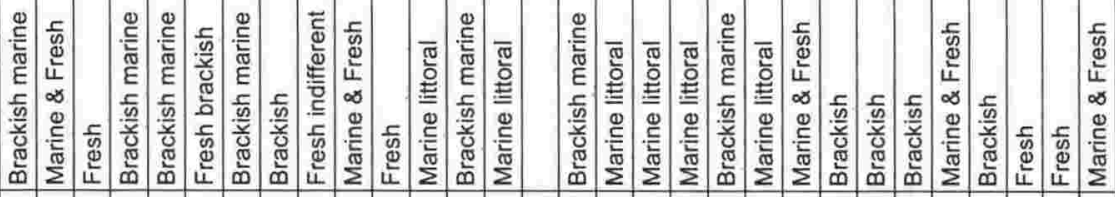

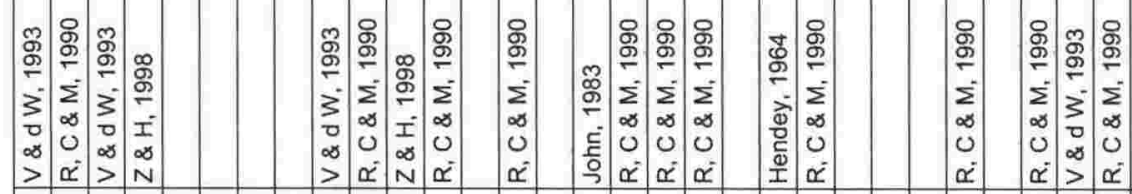

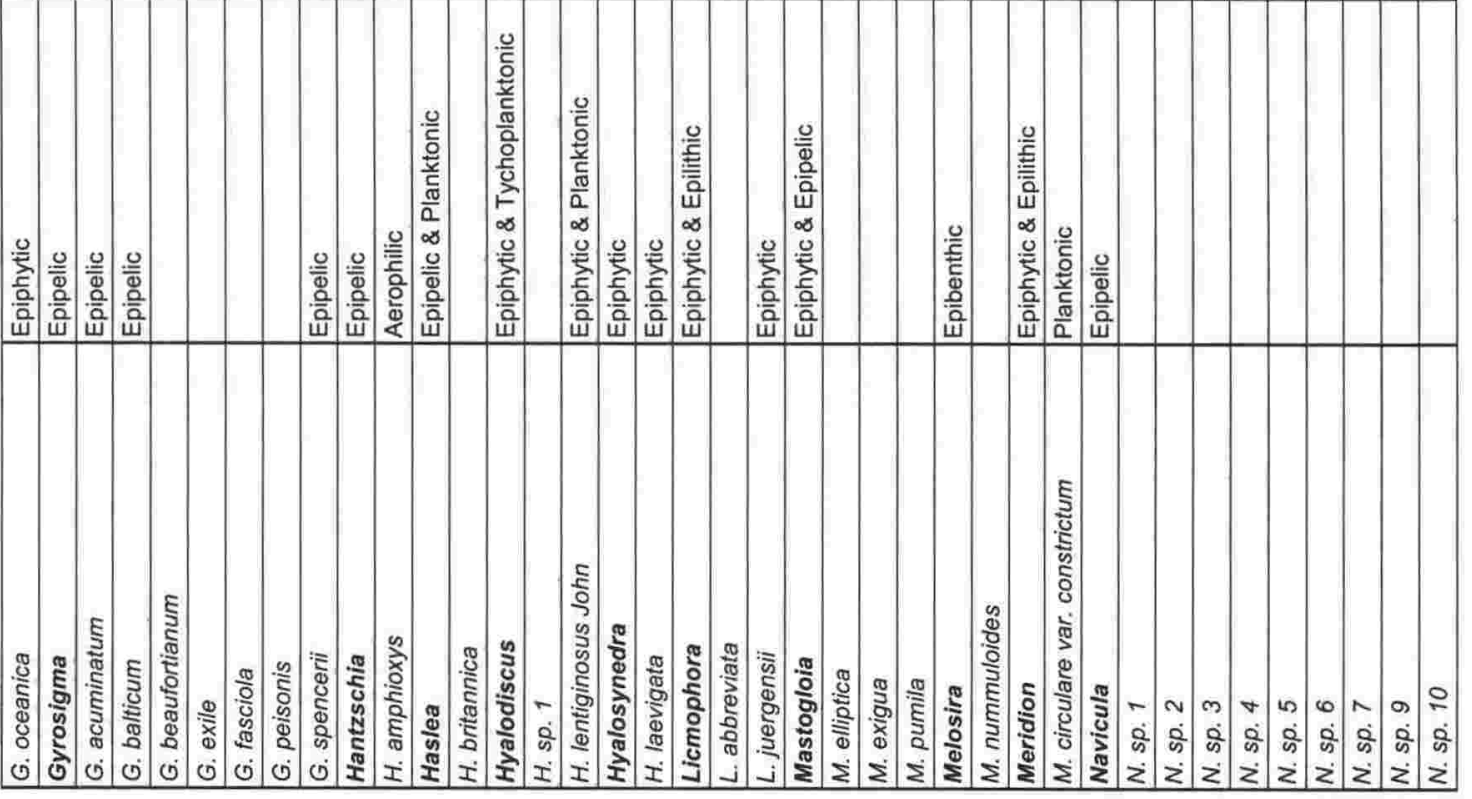




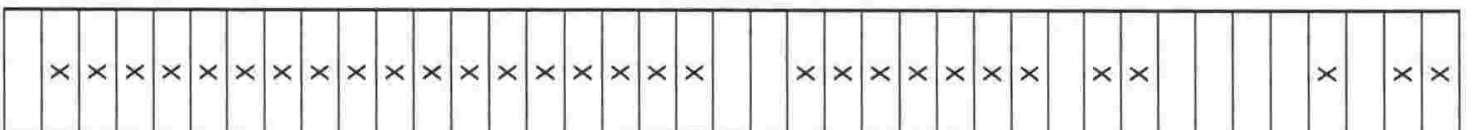

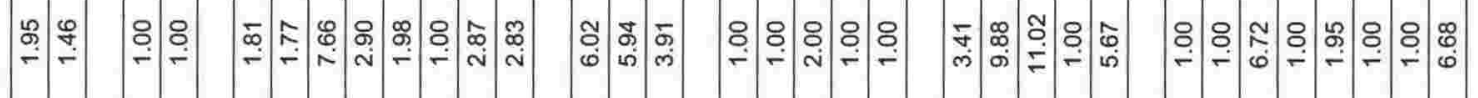

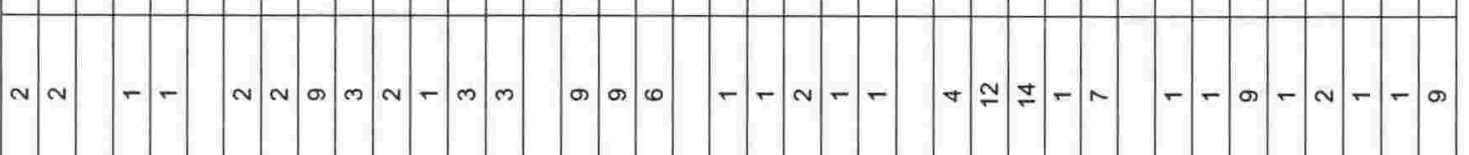

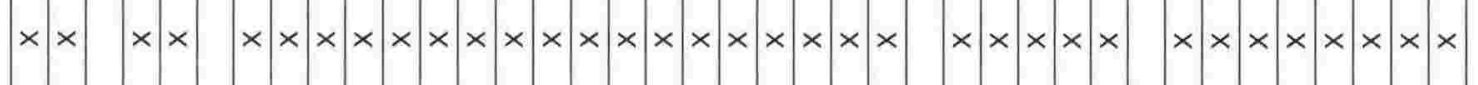

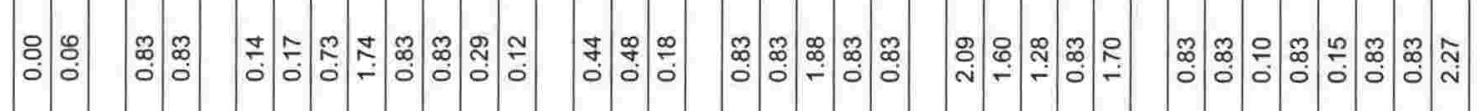

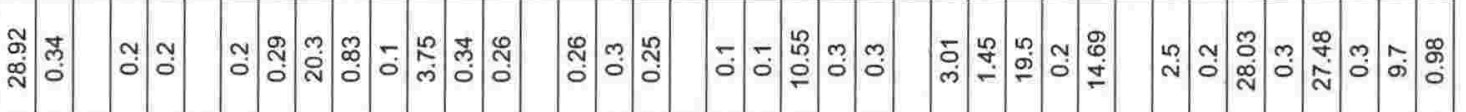

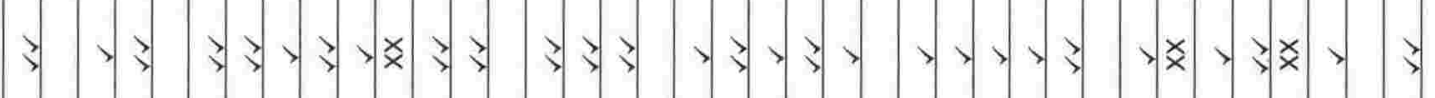

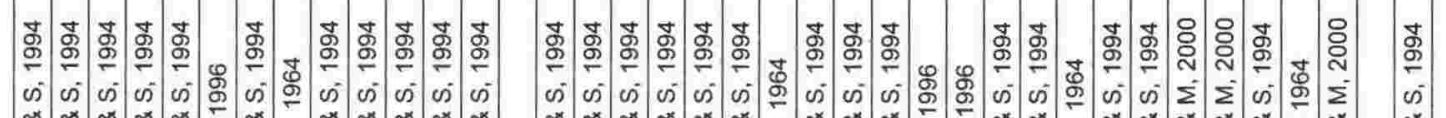

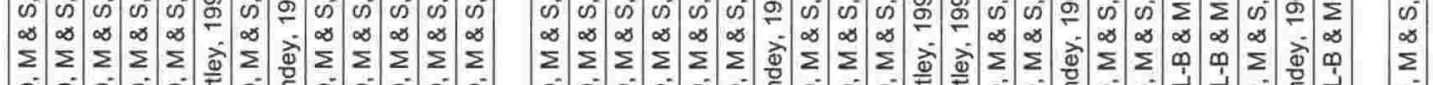

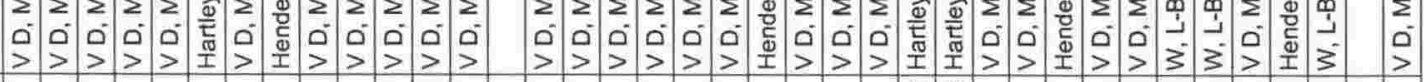

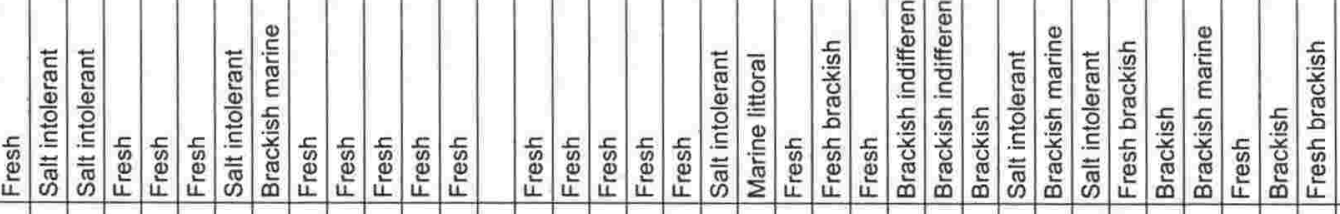

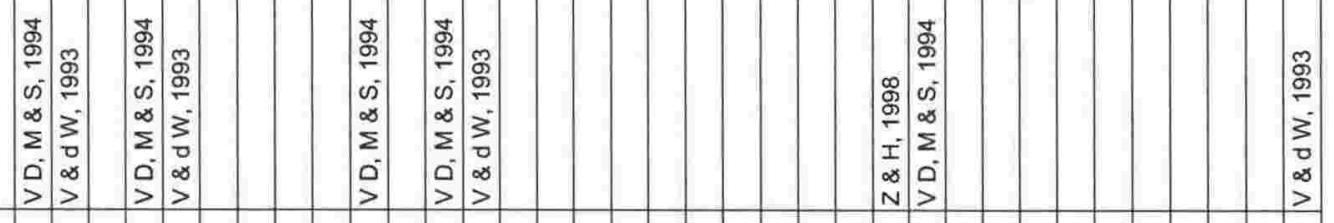

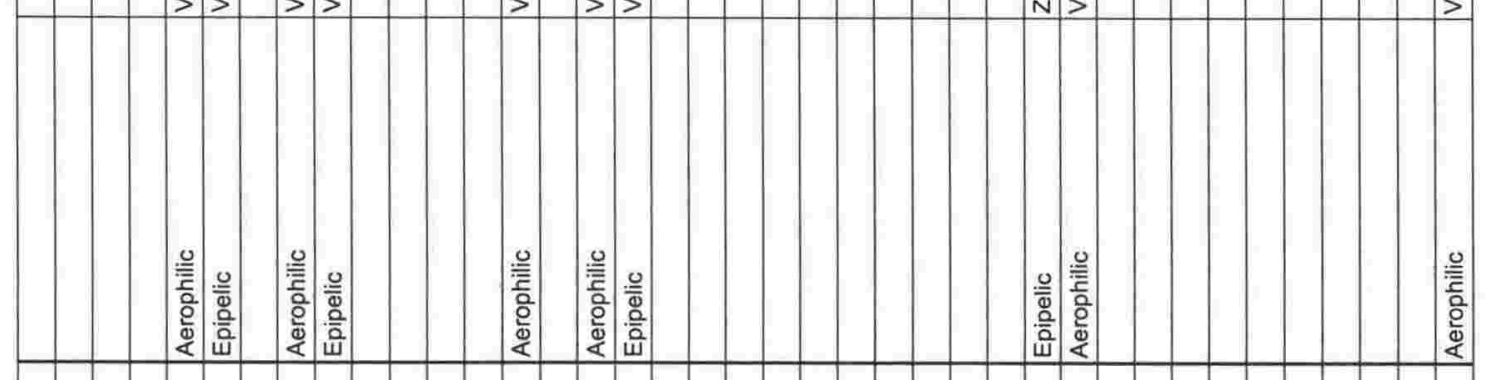

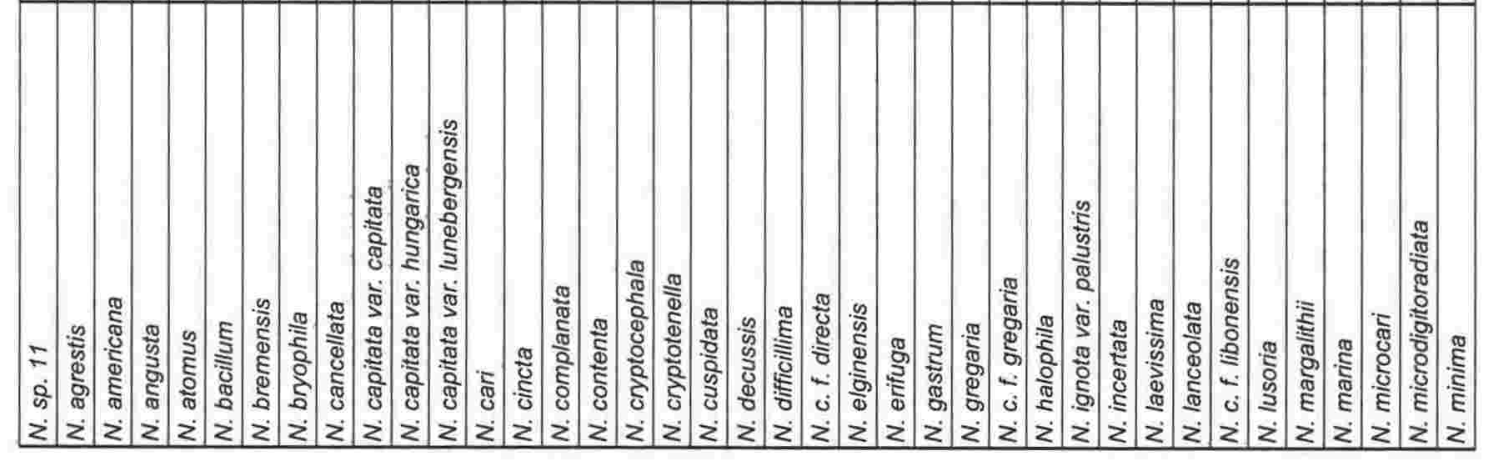




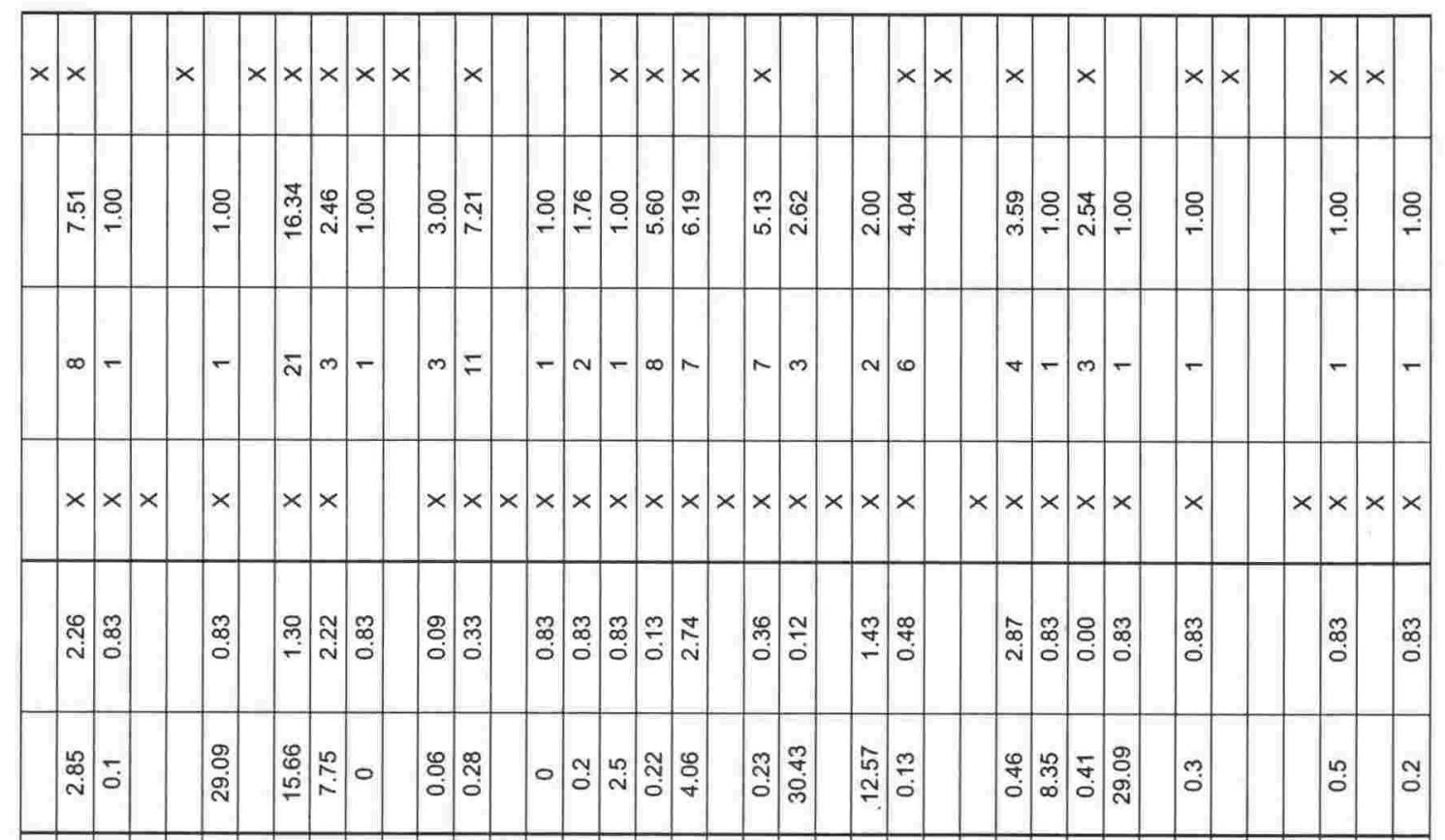

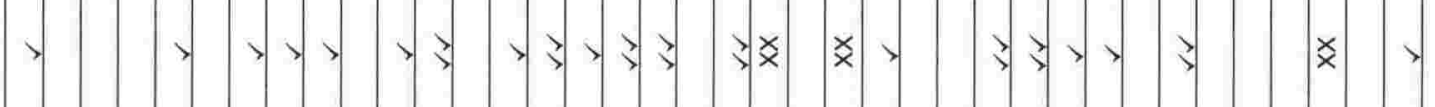

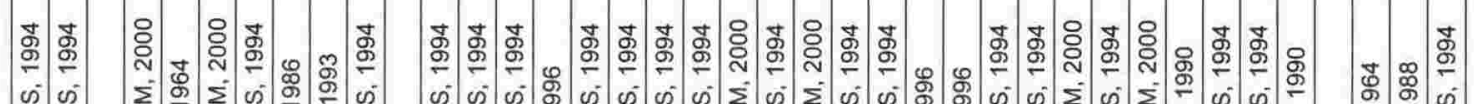

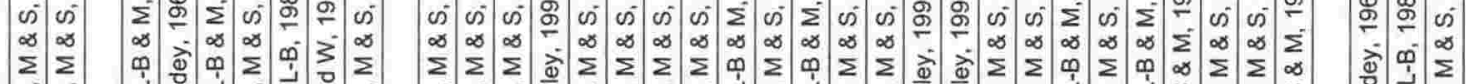

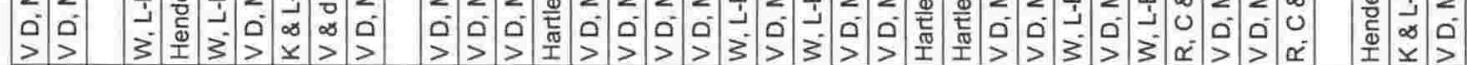

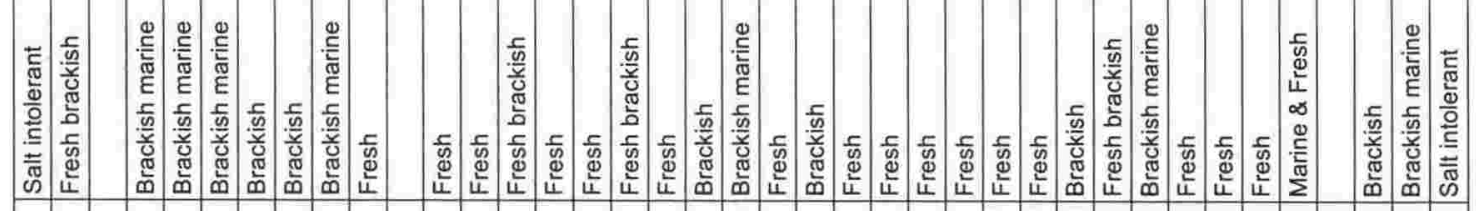

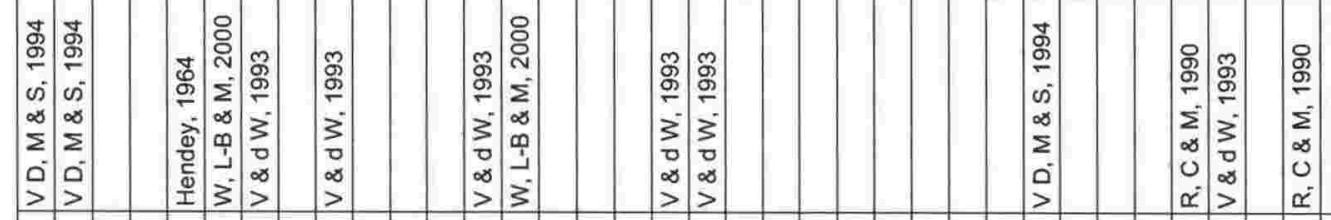

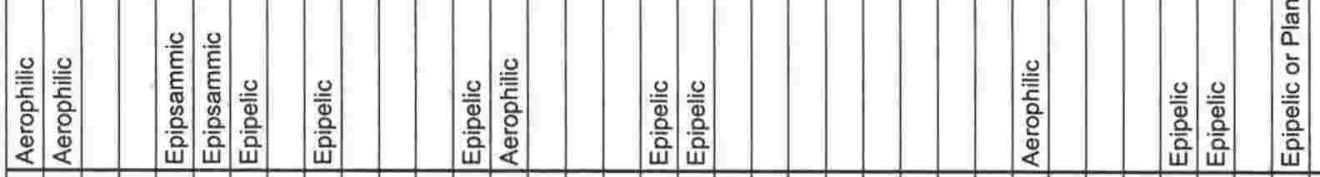

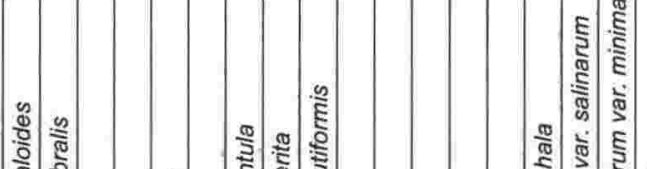

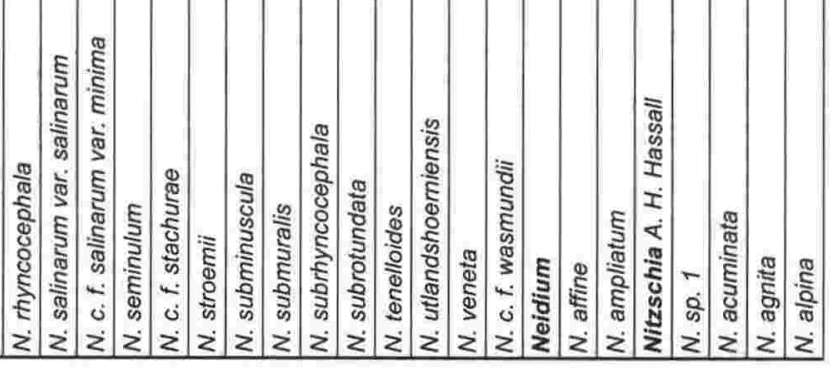




\begin{tabular}{|c|c|c|c|c|c|c|c|c|c|c|c|c|c|c|c|c|c|c|c|c|c|c|}
\hline$\times$ & $x$ & $\times$ & $\times$ & $x$ & $\times$ & $x$ & $\times$ & $\times$ & $\times$ & $\times$ & $\times$ & $\times$ & $\times$ & $\times$ & $\times$ & $\times$ & $\times$ & $\times$ & $\times$ & $\times$ & $\times$ & $\times$ \\
\hline
\end{tabular}

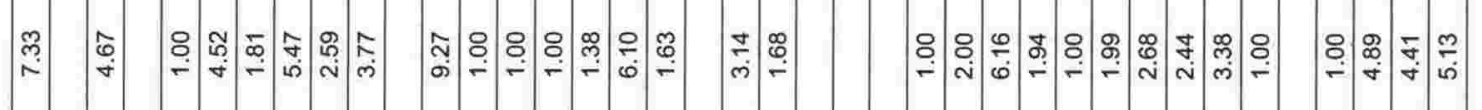

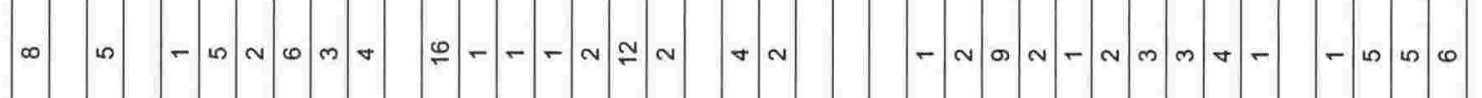

$x \times x \times x \times x \times x \times x \times x \times x \times x \times x \times x \times x \times x \times x \times x \times x \times x \times x$

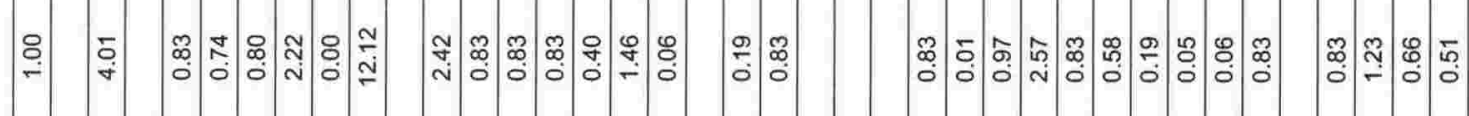

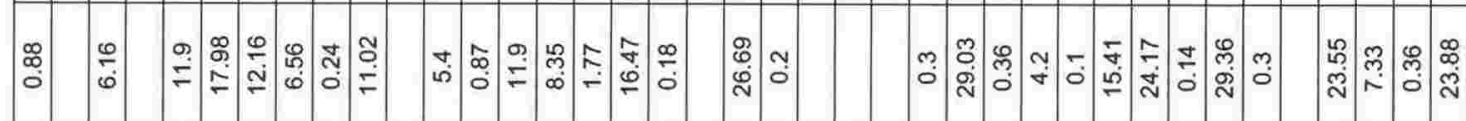

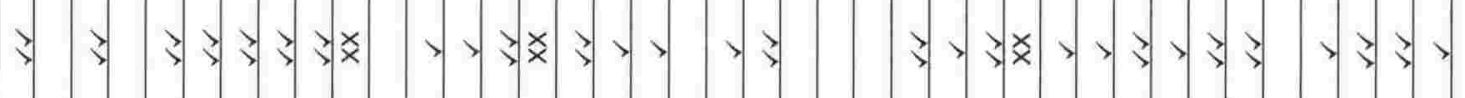

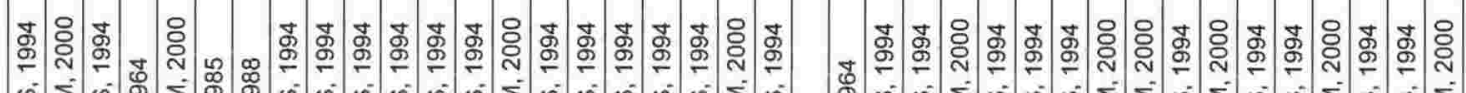

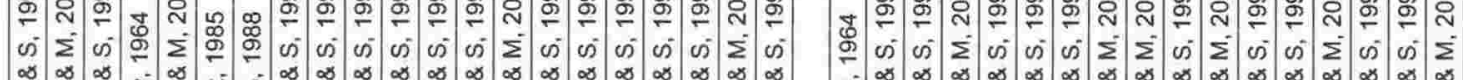

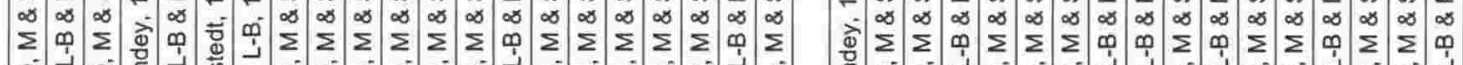

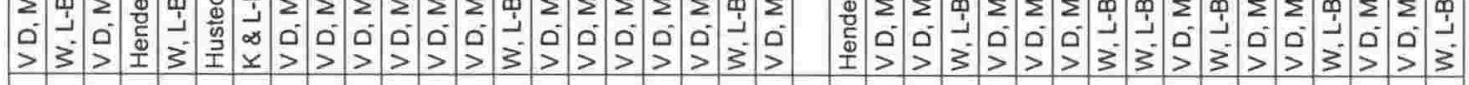

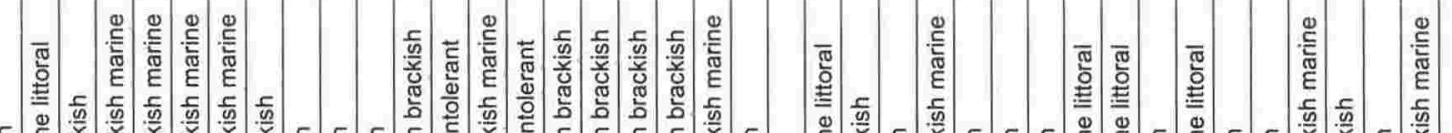

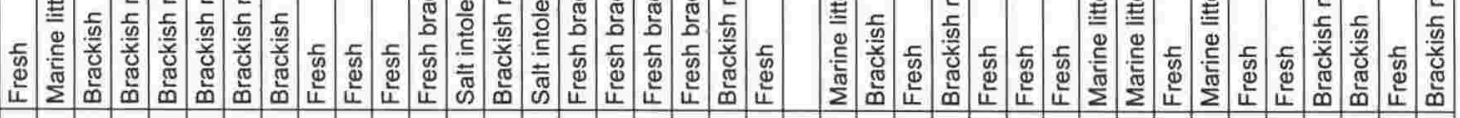

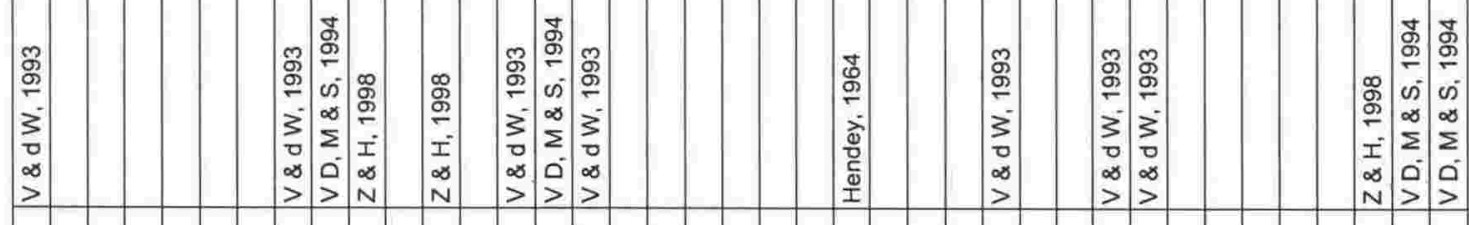

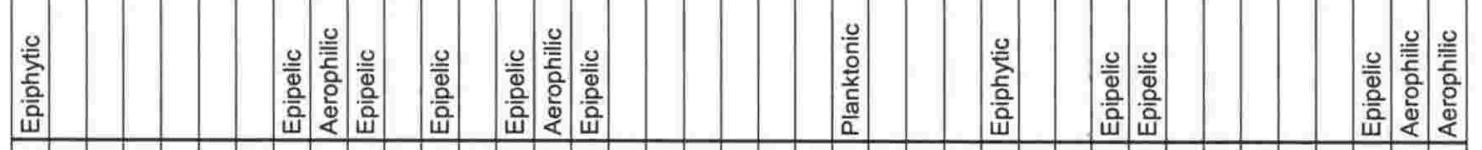

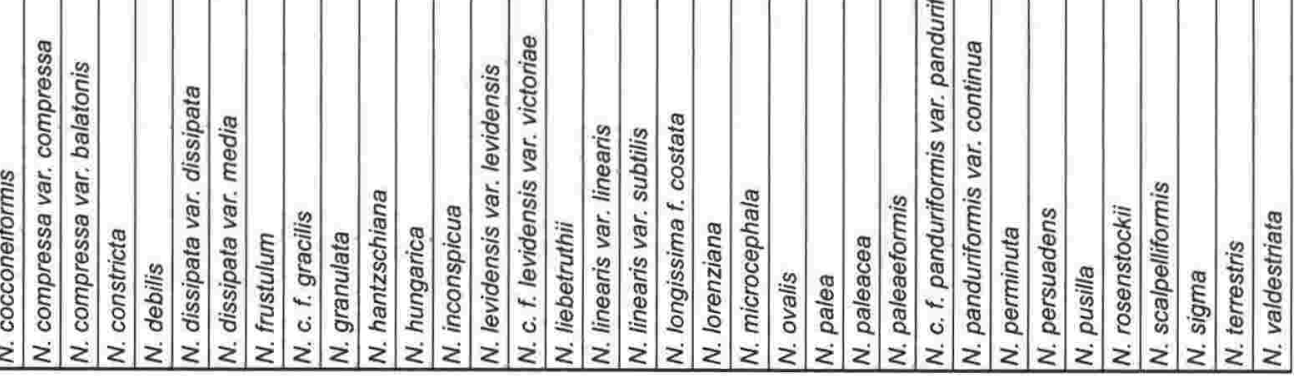




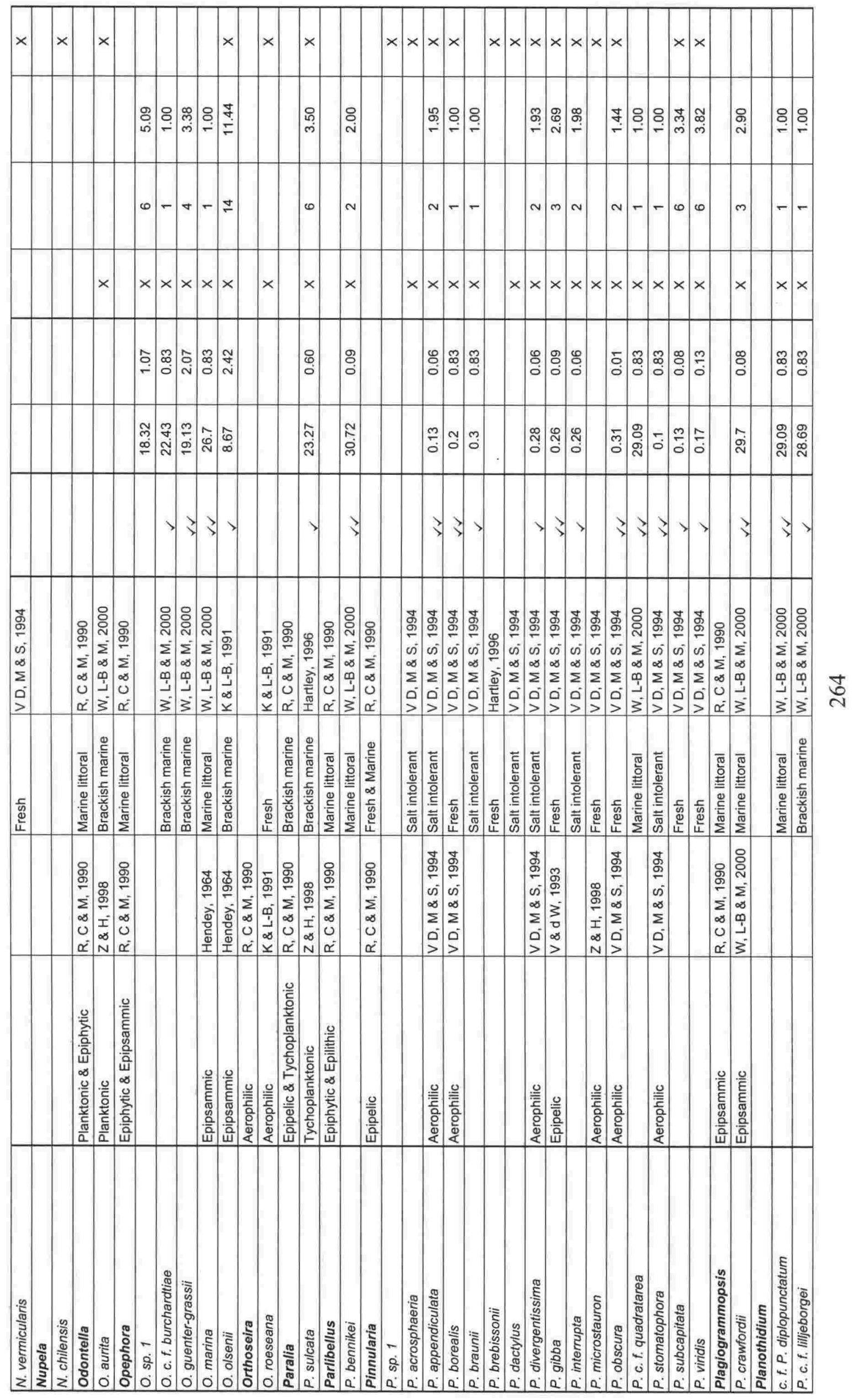




\begin{tabular}{|c|c|c|c|c|c|c|c|c|c|c|c|c|c|c|c|c|c|c|c|c|c|c|c|c|}
\hline$\times$ & $x$ & & $x$ & $\times$ & $x$ & & $x$ & $x$ & $x$ & & $\times$ & $x$ & $\times \times$ & $x \times$ & $<\times$ & $\times$ & $\times$ & $\times>$ & $\times \times$ & $\times$ & $\times$ & $\times \times$ & $<x$ & $x$ \\
\hline & & 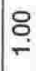 & 丞 & & $\stackrel{\substack{\infty \\
\forall}}{+}$ & $\begin{array}{l}\mathbb{N} \\
\stackrel{\sim}{*}\end{array}$ & $\begin{array}{l}\stackrel{\infty}{0}_{0} \\
\stackrel{0}{\circ}\end{array}$ & $\begin{array}{l}\tilde{\omega} \\
\text { ల్ }\end{array}$ & 足 & & & 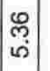 & & హి & $\stackrel{\widehat{S}}{=}$ & & & & $\begin{array}{l}\stackrel{a}{a} \\
\text { N. }\end{array}$ & & & & & $\begin{array}{l}\text { ठ } \\
\text { in }\end{array}$ \\
\hline$\sim$ & & - & o & & in & $\omega$ & ? & $\nabla$ & - & & & $\omega$ & N & N & $-m$ & & & & $m-$ & & & & & 0 \\
\hline$\times$ & & $x$ & $x$ & $\times$ & $x$ & $x$ & $\times$ & $x$ & $x$ & $x$ & $x$ & $\times$ & $\times$ & $x \mid \times$ & $x \times$ & & & $x>$ & $\times \times$ & & & & $\times$ & $\times$ \\
\hline 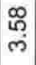 & & ס̊ & ले & & $\overline{\tilde{N}}$ & $\frac{0}{\circ}$ & $\stackrel{్}{\mathrm{G}}$ & \begin{tabular}{|l|}
8 \\
0 \\
\end{tabular} & \begin{tabular}{l}
$\mathscr{0}$ \\
\hdashline \\
0 \\
0
\end{tabular} & & & 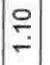 & & $+\infty$ & : & & & & ले & & & & & 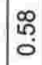 \\
\hline$\frac{\mathfrak{N}}{\circ}$ & & $\stackrel{\text { ? }}{=}$ & 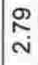 & & & $\begin{array}{l}\mathscr{L}_{0} \\
\text { i. }\end{array}$ & $\underset{\mathrm{i}}{\tau}$ & $\stackrel{m}{m}$ & $\begin{array}{l}\mathscr{0} \\
0 \\
0\end{array}$ & & & $\begin{array}{l}\tilde{0} \\
\tilde{0}\end{array}$ & $\mathbb{8}$ & $\begin{array}{c}8 \\
0 \\
0\end{array}$ & 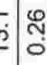 & & & & 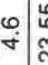 & $\begin{array}{l}? \\
\text { ?े }\end{array}$ & & & & $\begin{array}{l}\text { ্ָ } \\
\text { N }\end{array}$ \\
\hline
\end{tabular}

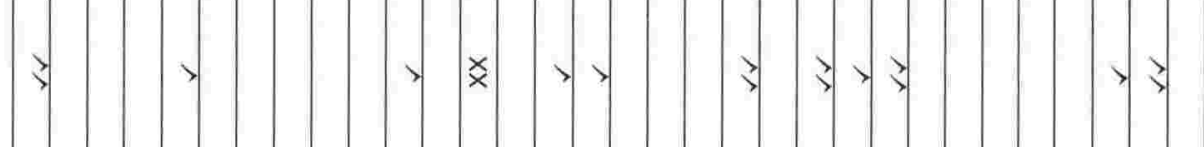

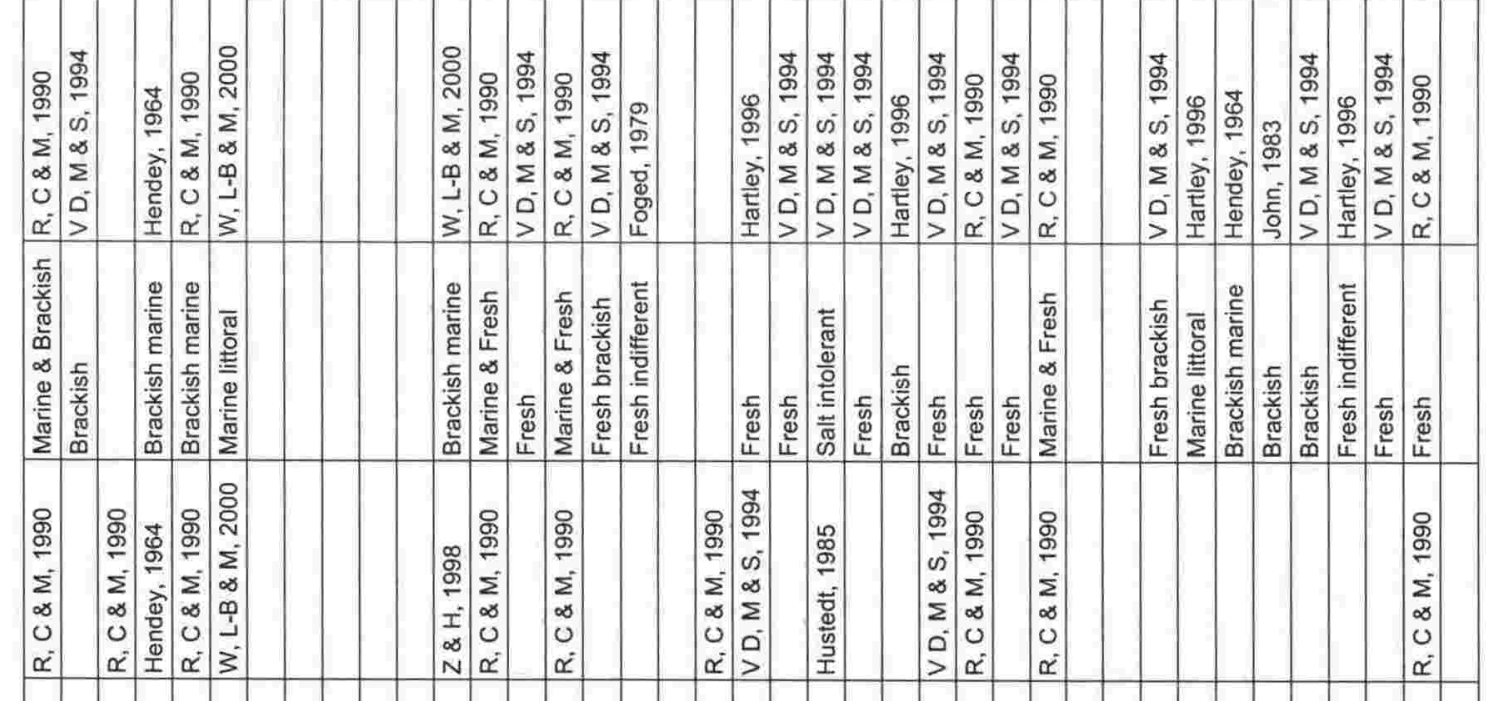

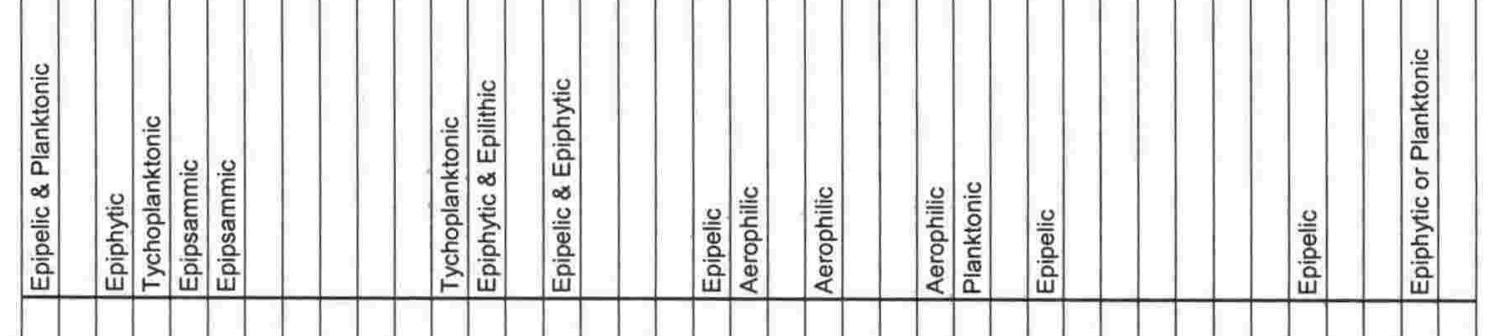

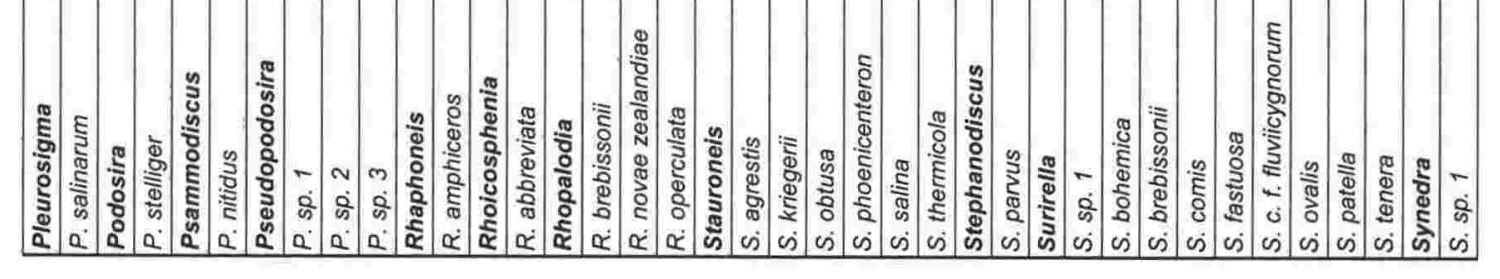




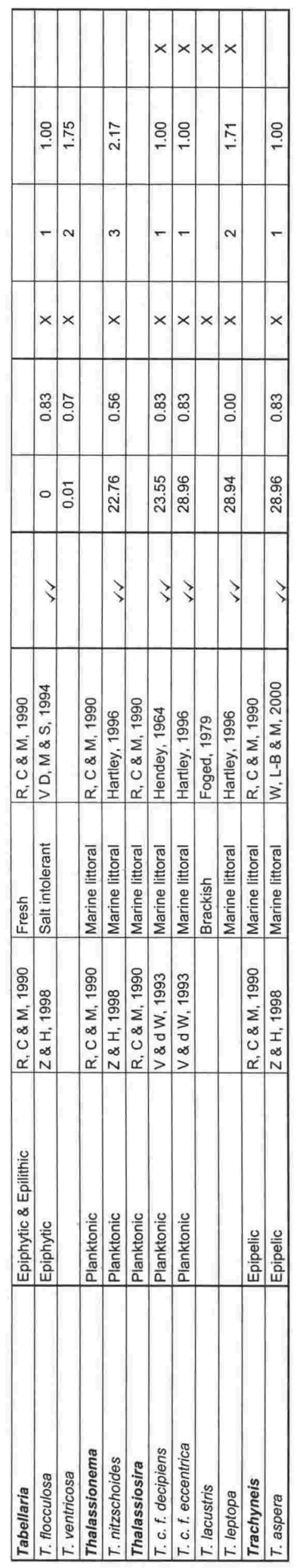

¿ 


\section{A P PENDIX IV}

\section{Plates of Diatom Species}

Abbreviations used:

$\begin{array}{ll}\text { LM } & \text { Light Microscope } \\ \text { SEM } & \text { Scanning Electron Microscope }\end{array}$

Magnifications are listed at the top of each plate. 


\section{Plate 1}

LM: Fig. 1 x800; Fig. 3-6 and 8-11 x1600.

SEM: Fig. $2 \times 500$; Fig. $7 \times 3700$.

Fig. 1. Actinocyclus octonarius Ehrenberg

Fig. 2. Actinocyclus octonarius Ehrenberg

Fig. 3. Actinoptychus senarius (Ehrenberg) Ehrenberg

Fig. 4. Aulacoseira ambigua (Grunow) Simonsen

Fig. 5. Aulacoseira ambigua (Grunow) Simonsen

Fig. 6. Aulacoseira crassipunctata Krammer

Fig. 7. Aulacoseira crenulata (Ehrenberg) Thwaites

Fig. 8. Aulacoseira crenulata (Ehrenberg) Thwaites

Fig. 9. Aulacoseira crenulata (Ehrenberg) Thwaites

Fig. 10. Aulacoseira granulata (Ehrenberg) Simonsen

Fig. 11. Aulacoseira granulata (Ehrenberg) Simonsen 

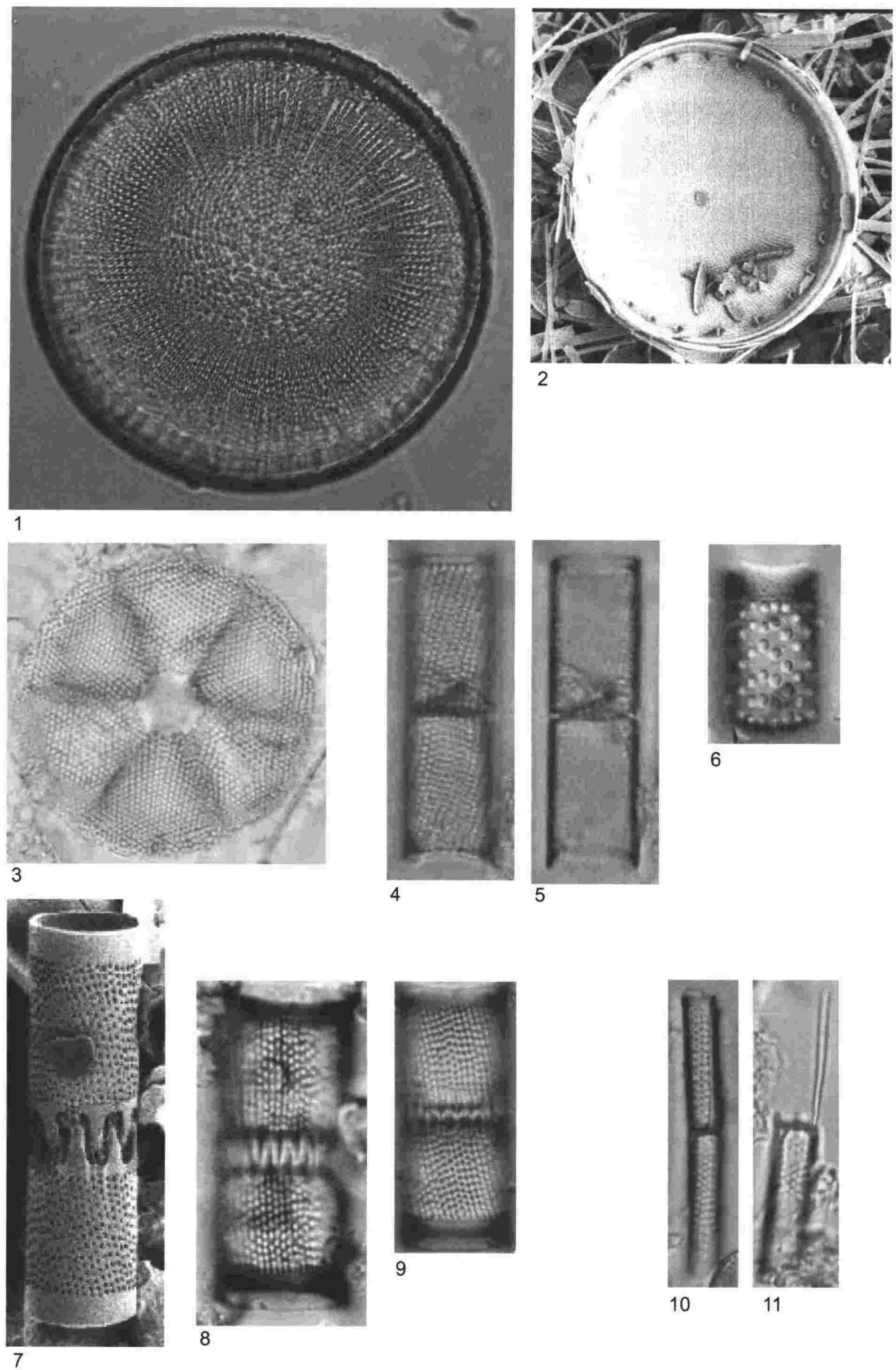

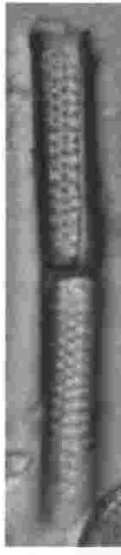

10

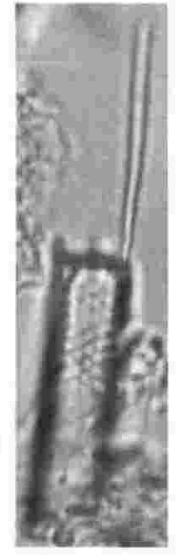

11 


\section{Plate 2}

LM: Fig. 1-12 and 14-17 x1600

SEM: Fig. $13 \times 4000$

Fig. 1. Cyclostephanos dubius (Fricke) Round

Fig. 2. Cyclostephanos novaezeelandiae (Cleve) Round

Fig. 3. Cyclostephanos novaezeelandiae (Cleve) Round

Fig. 4. Cyclotella sp. 1

Fig. 5. Cyclotella sp. 2

Fig. 6. Cyclotella atomus Hustedt

Fig. 7. Cyclotella distinguenda Hustedt

Fig. 8. Cyclotella meneghiniana Kützing

Fig. 9. Cyclotella stelligera Cleve \& Grunow

Fig. 10. Ehrenbergia granulosa (Grunow) Witkowski, Lange-Bertalot \& Metzeltin

Fig. 11. Melosira nummuloides (Dillwyn) Agardh

Fig. 12. Melosira nummuloides (Dillwyn) Agardh

Fig. 13. Paralia sulcata (Ehrenberg) Cleve

Fig. 14. Paralia sulcata (Ehrenberg) Cleve

Fig. 15. Paralia sulcata (Ehrenberg) Cleve

Fig. 16. Paralia sulcata (Ehrenberg) Cleve

Fig. 17. Chaetoceros spore 


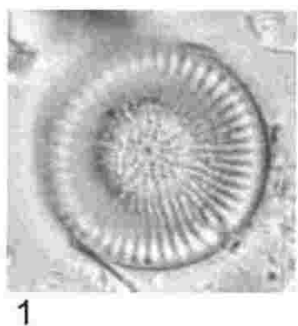

1

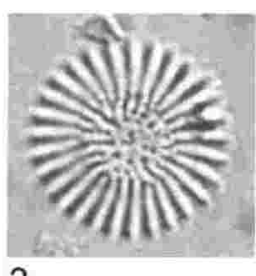

2

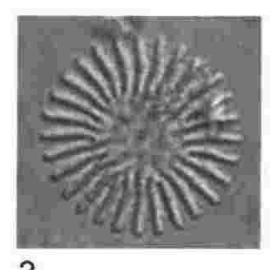

3

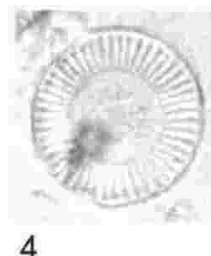

4

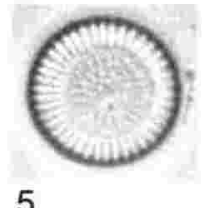

5
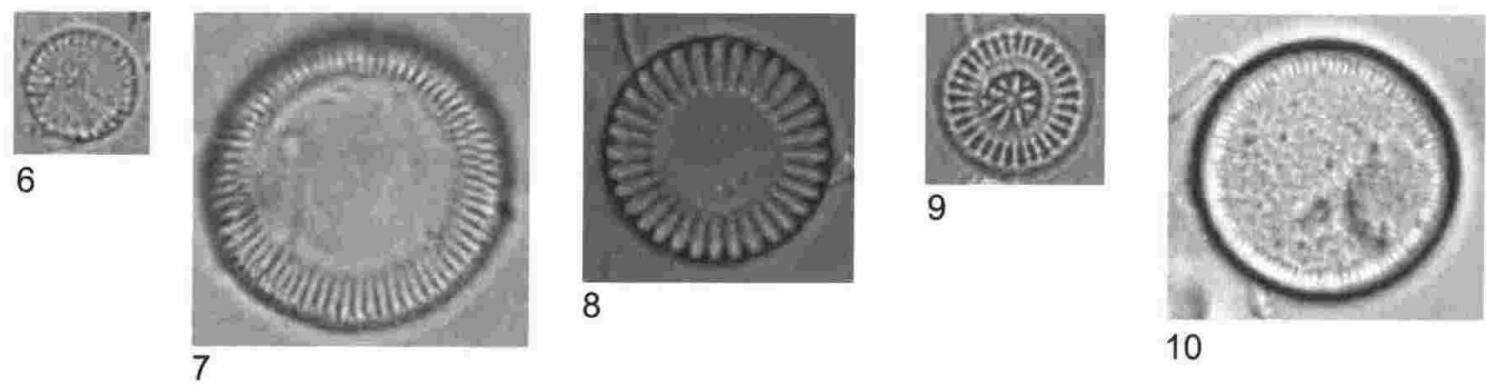

10
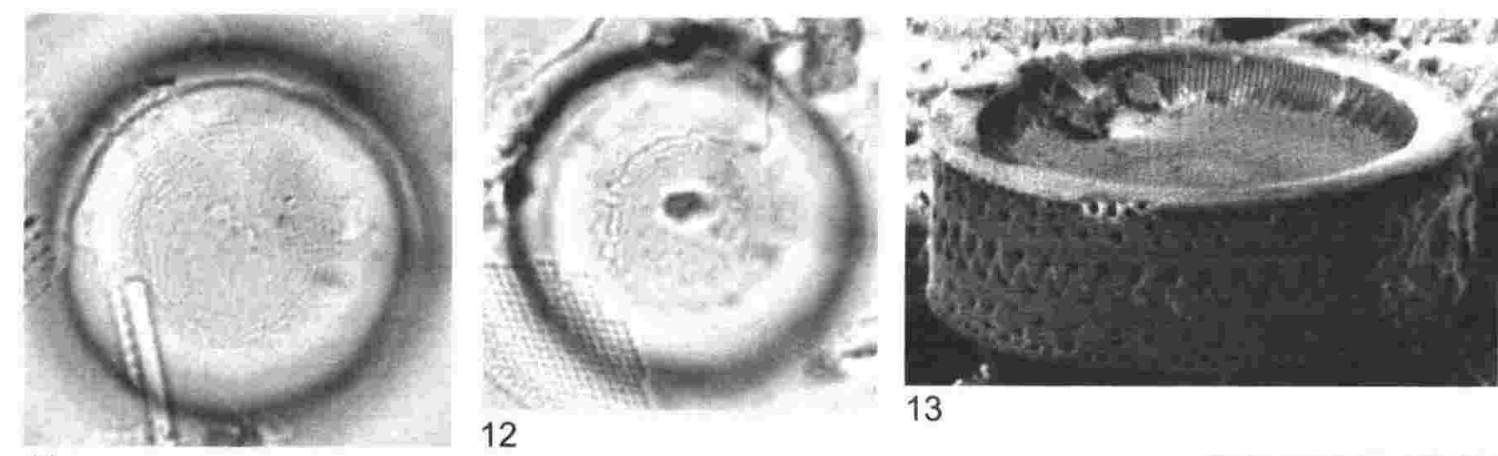

11

\section{2}

13
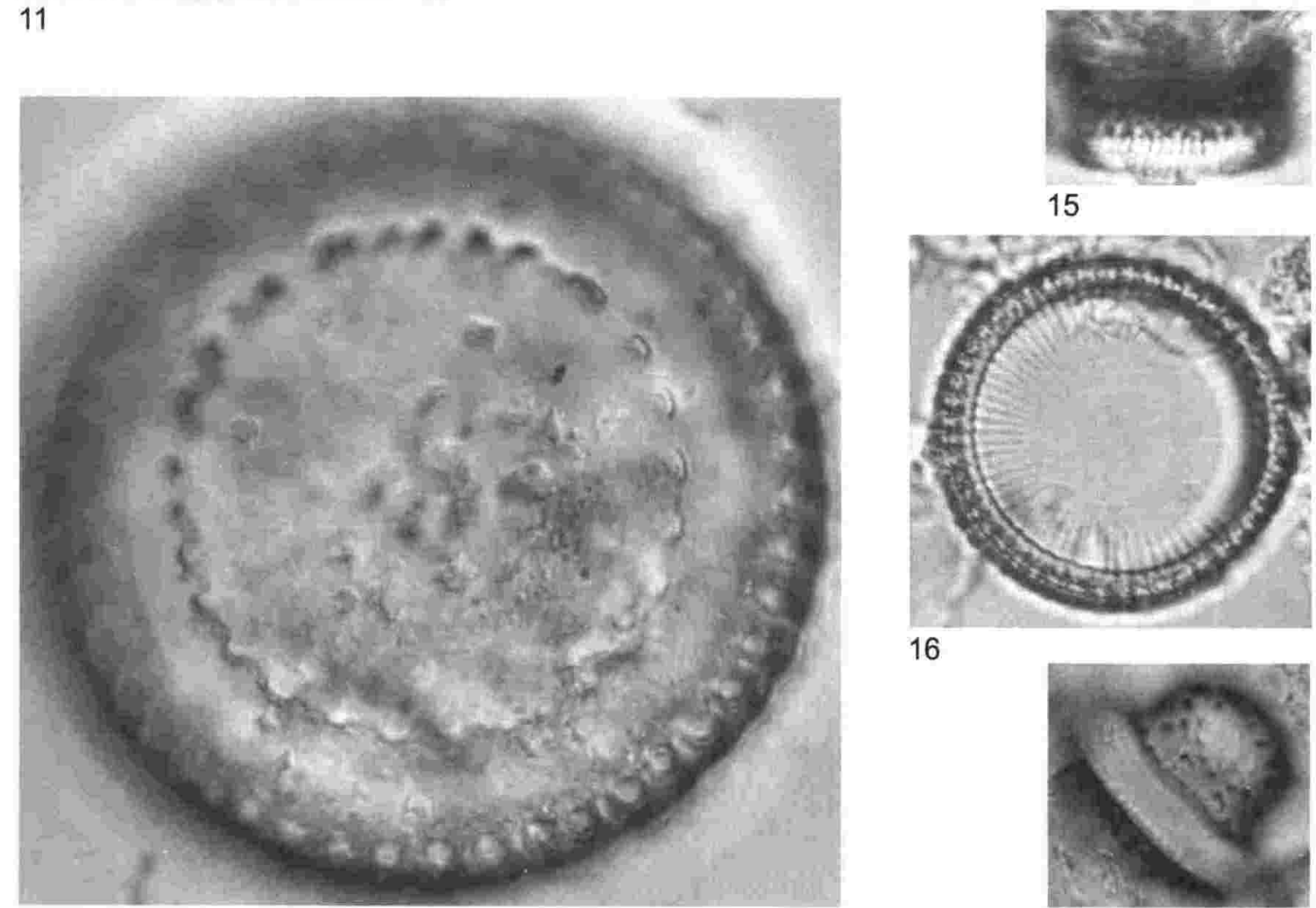

15

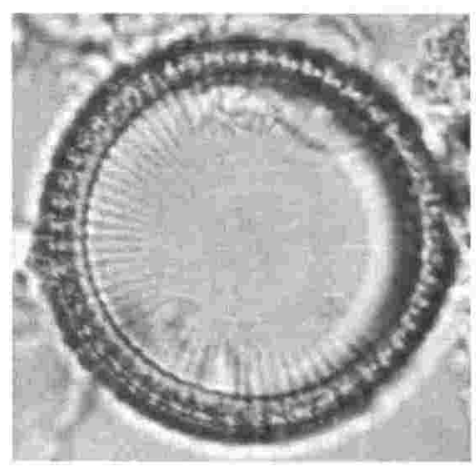

16

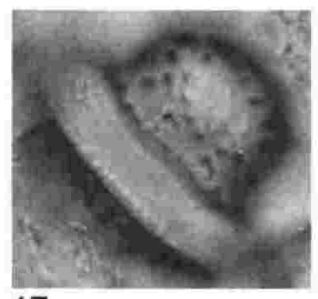




\section{Plate 3}

LM: Fig. $2 \times 500$; Fig. $7 \times 1000$

SEM: Fig. 1 x2000; Fig. 3 x 1300; Fig. 4 x1000; Fig. 5 x1200; Fig. 6 ×1400

Fig. 1.. Hyalodiscus pustulatus A. Schmidt

Fig. 2. Hyalodiscus pustulatus A. Schmidt

Fig. 3. Hyalodiscus pustulatus A. Schmidt

Fig. 4. Hyalodiscus pustulatus A. Schmidt

Fig. 5. Hyalodiscus pustulatus A. Schmidt

Fig. 6. Hyalodiscus lentiginosus John

Fig. 7. Hyalodiscus lentiginosus John 

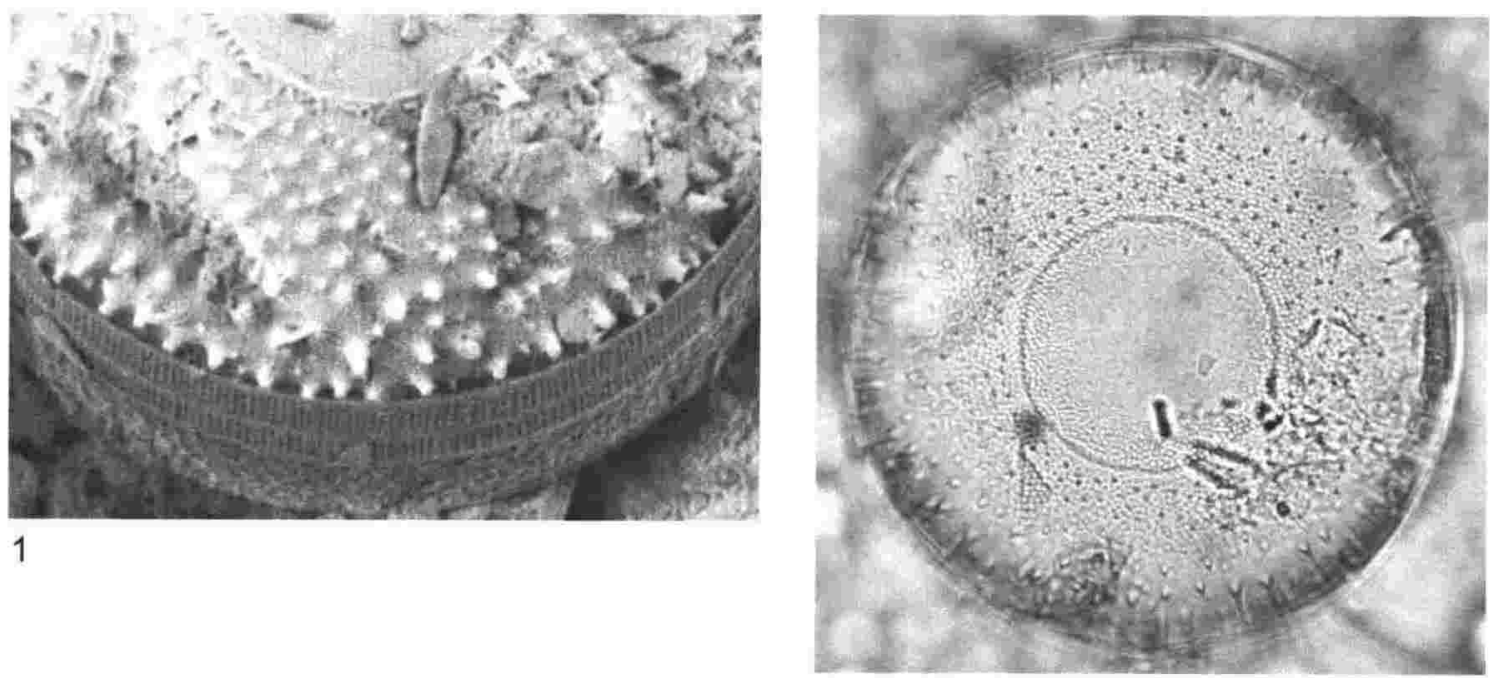

2
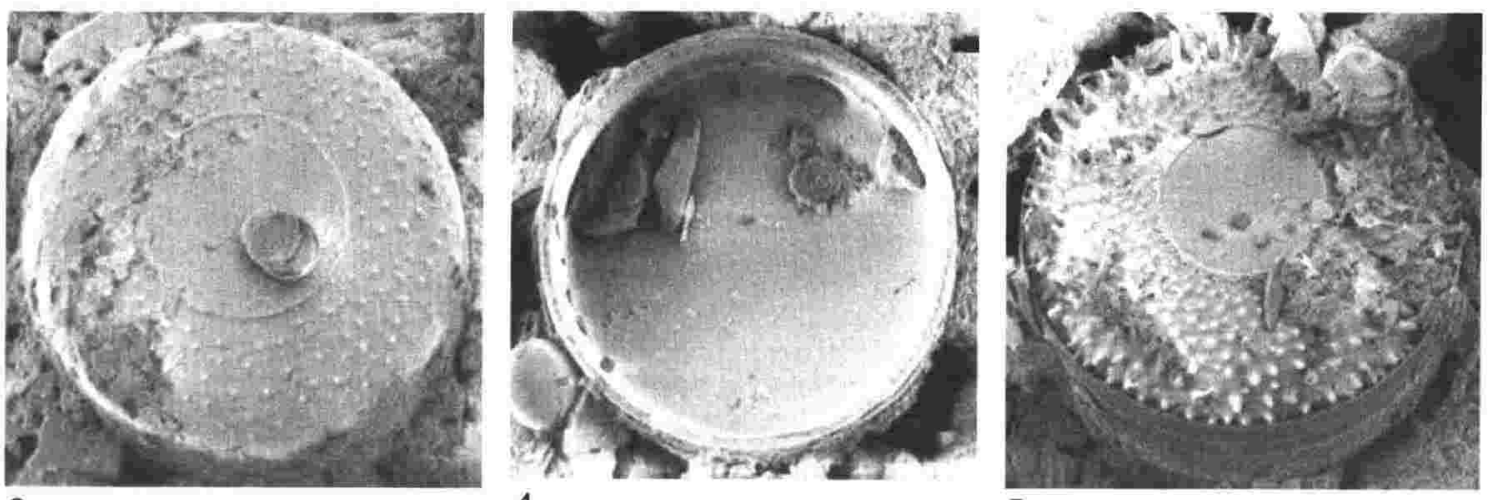

3

4

5
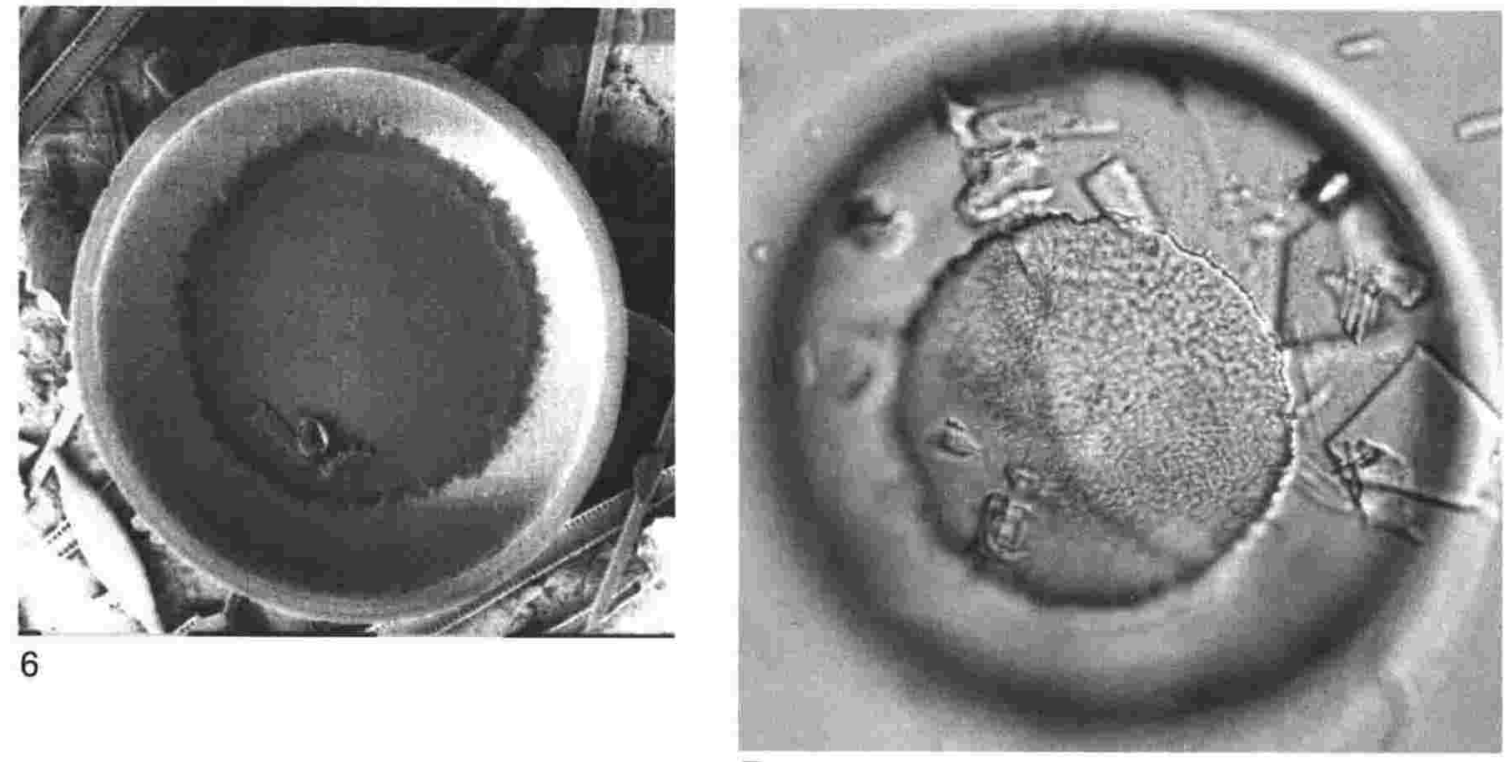

7 


\section{Plate 4}

LM: Fig. 1, 2, 5-12 x1600

SEM: Fig. 3 x6700; Fig. 4 x6800

Fig. 1. Podosira stelliger (Bailey) Mann

Fig. 2. Psammodiscus nitidus (Gregory) Round \& Mann

Fig. 3. Pseudopodosira sp.1

Fig. 4. Pseudopodosira sp.1

Fig. 5. Pseudopodosira sp.1

Fig. 6. Pseudopodosira sp.1

Fig. 7. Pseudopodosira sp.1

Fig. 8. Pseudopodosira sp.2

Fig. 9. Pseudopodosira sp.2

Fig. 10. Pseudopodosira sp.2

Fig. 11. Thalassiosira lacustris (Grunow) Hasle

Fig. 12. Thalassiosira leptopa (Grunow in Van Heurck) Hasle \& Fryxell 

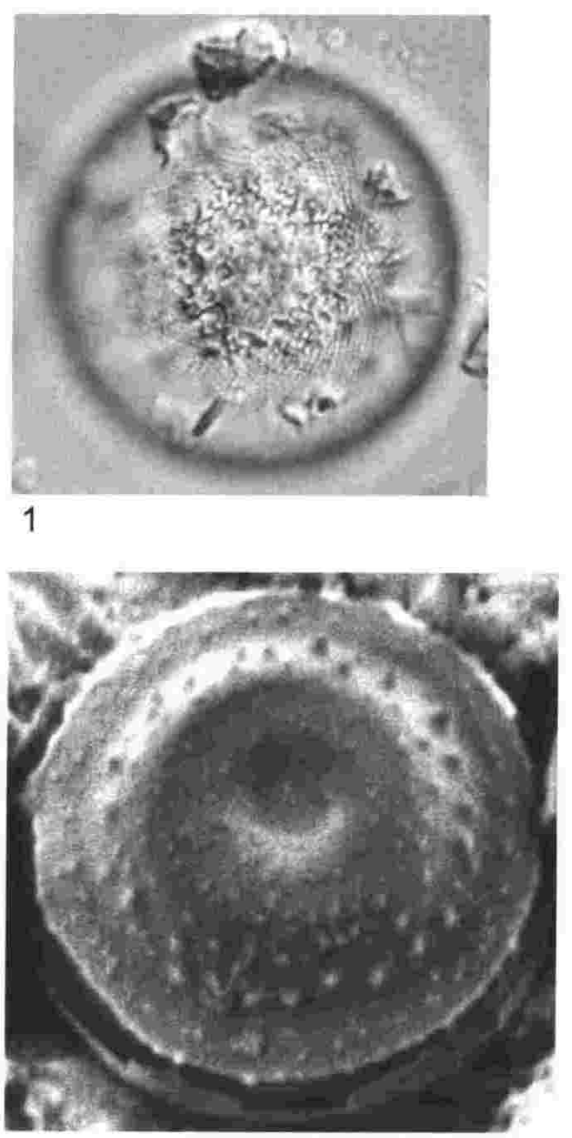

3

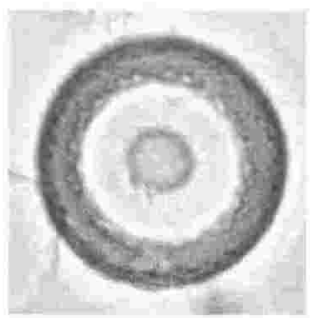

5

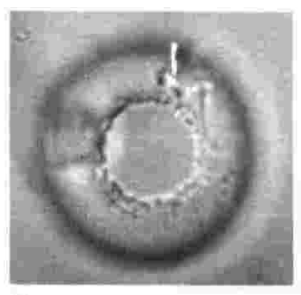

8

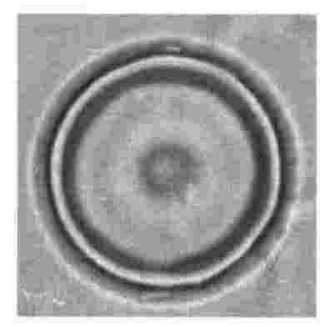

6

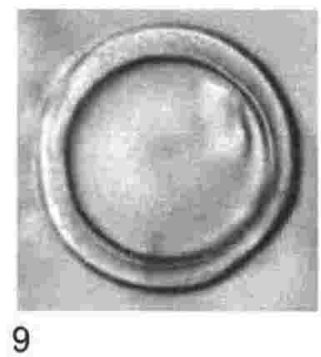

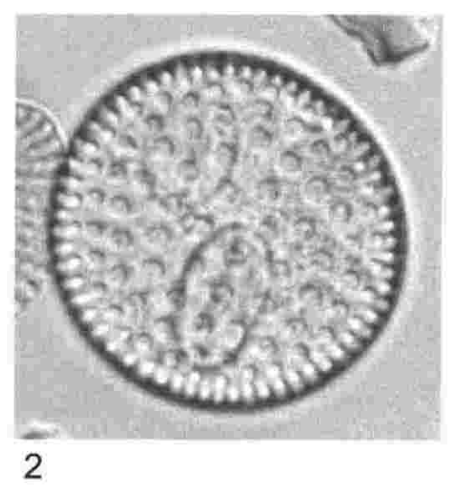

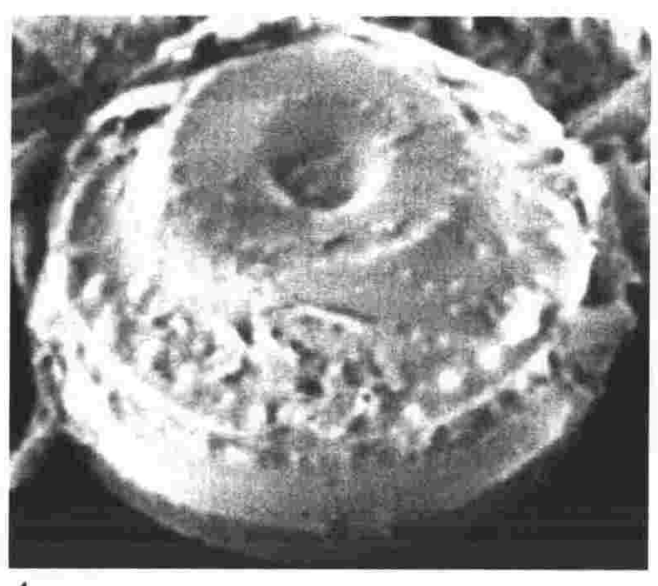

4

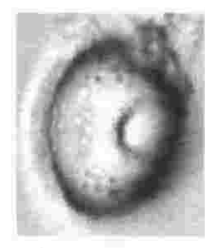

7

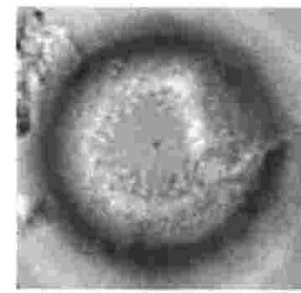

10

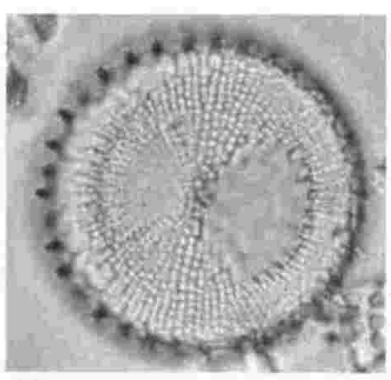

11

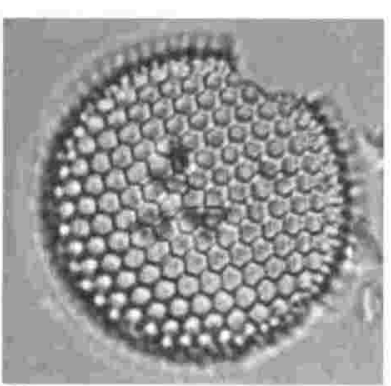

12 


\section{Plate 5}

LM: Fig. 1 x1000; Fig. 4 x500; Fig. 2, 3 and 5-13 x1600

Fig. 1. Biddulphia laevis Ehrenberg

Fig. 2. Biddulphia laevis Ehrenberg

Fig. 3. Odontella aurita (Lyngbye) Agardh

Fig. 4. Campylodiscus echeneis Ehrenberg

Fig. 5. Anaulus balticus Simonsen

Fig. 6. Cymatosira belgica Grunow in Van Heurck

Fig. 7. Cymatosira elliptica Salah

Fig. 8. Delphineis c. f. surirella (Ehrenberg) Andrews

Fig. 9. Delphineis c. f. surirella (Ehrenberg) Andrews

Fig. 10. Diatoma moniliformis Kützing

Fig. 11. Dimeregramma minor var. nana (Gregory) Van Heurck

Fig. 12. Dimeregramma minor var. nana (Gregory) Van Heurck 

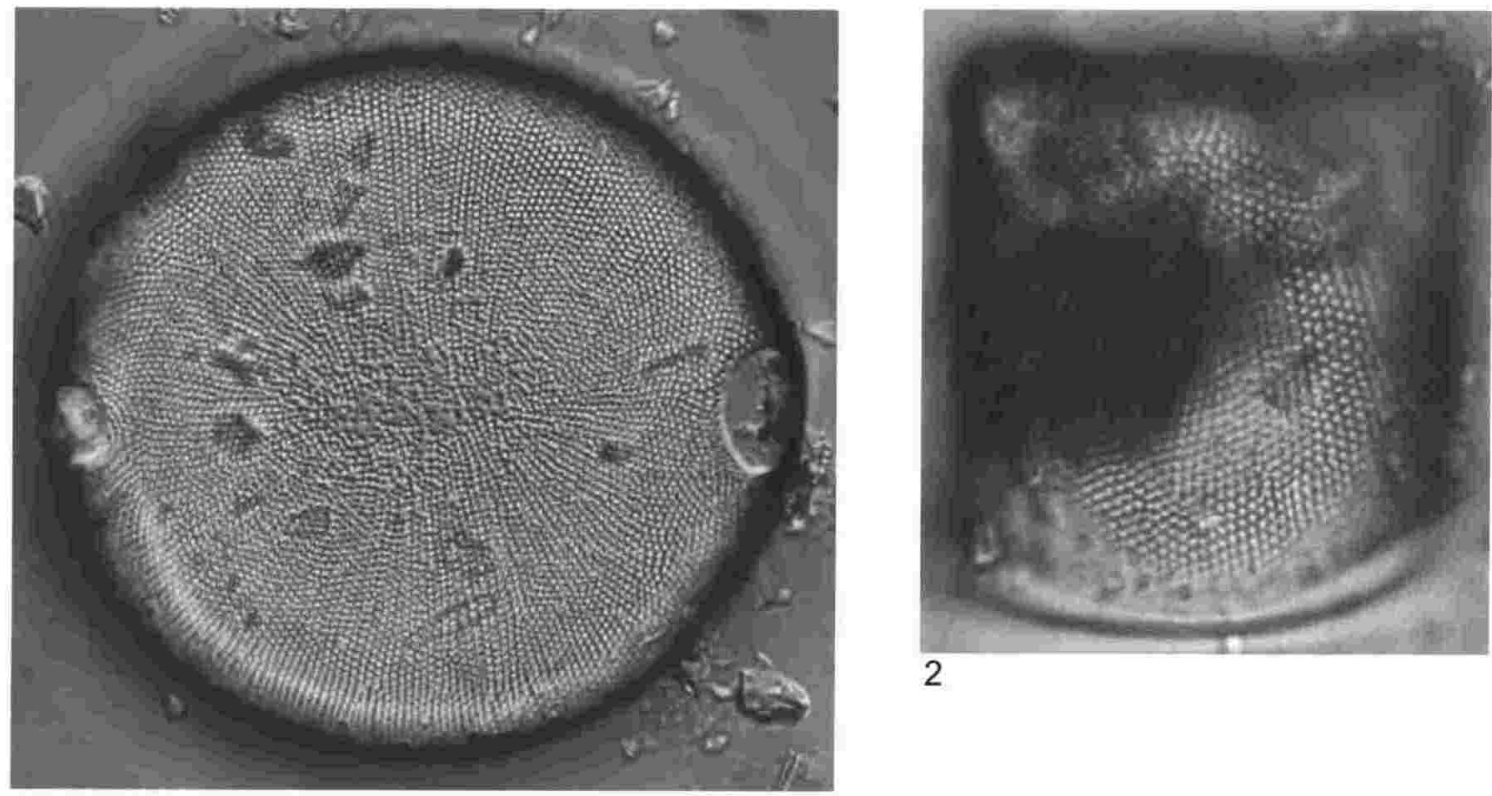

1

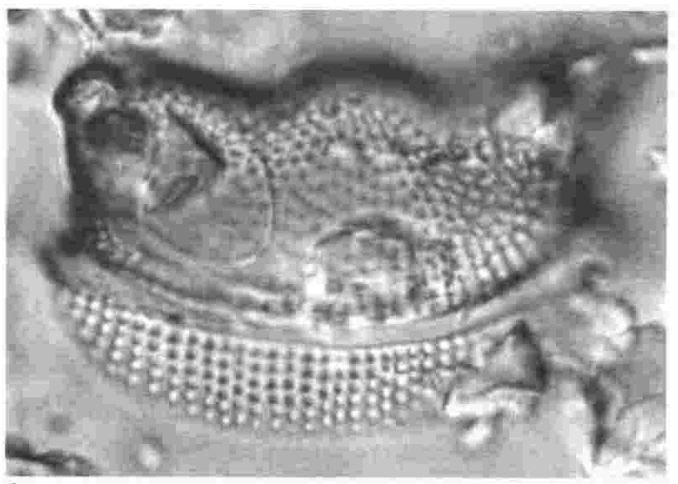

3
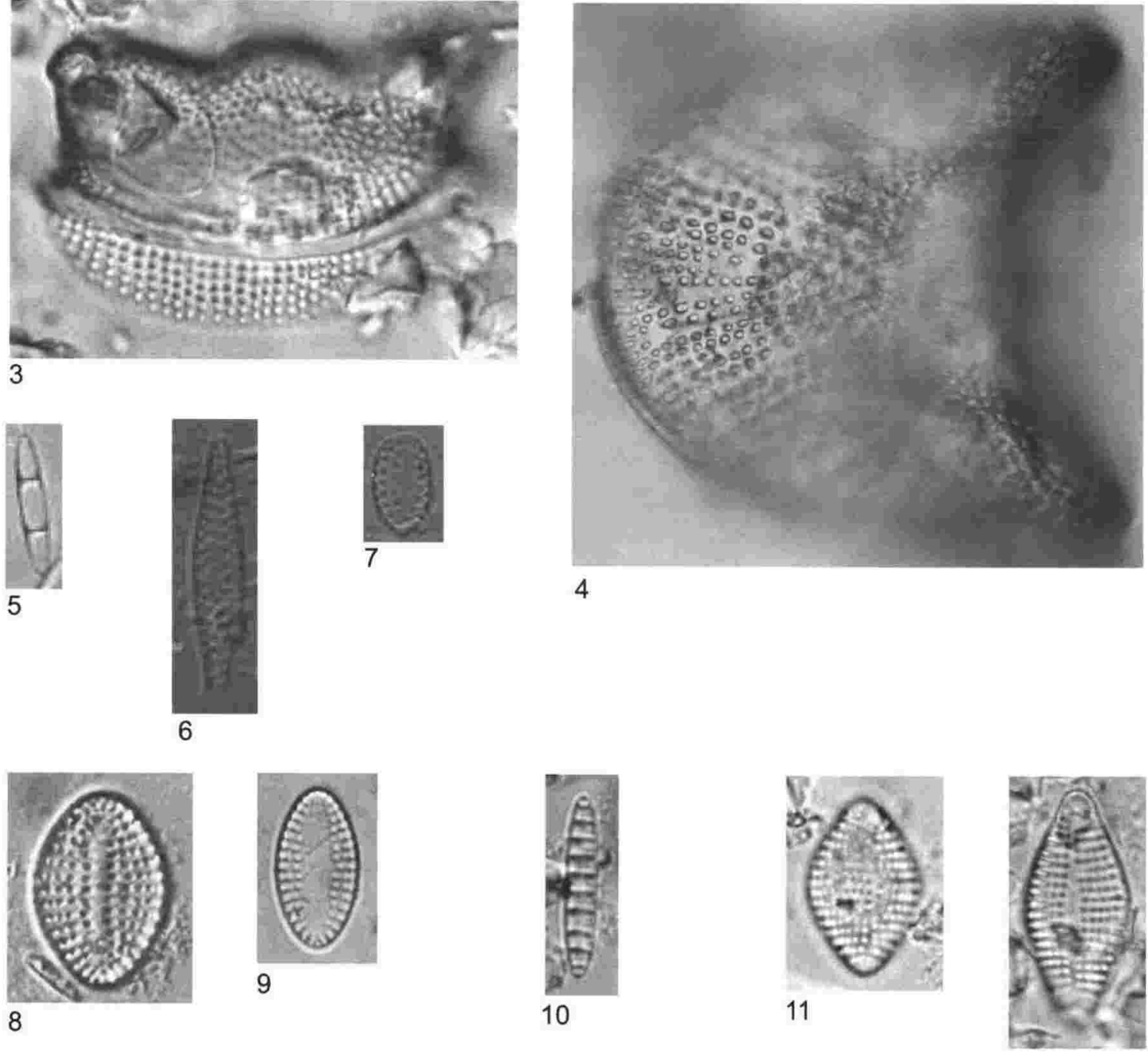


\section{Plate 6}

LM: Fig. 1-8, 10-14 and 16-21 x1600; Fig. 9 x800; Fig. 15 x1000

Fig. 1. Fragilaria brevistriata Grunow in Van Heurck var. brevistriata

Fig. 2. Fragilaria brevistriata var. inflata (Pantocsek) Hustedt

Fig. 3. Fragilaria elliptica Schumann

Fig. 4. Fragilaria capucina Desmazières var. capucina

Fig. 5. Fragilaria capucina var. gracilis (Østrup) Hustedt

Fig. 6. Fragilaria capucina var. vaucheriae (Kützing) Lange-Bertalot

Fig. 7. Fragilaria construens f. venter (Ehrenberg) Hustedt

Fig. 8. Fragilaria construens f. subsalina (Hustedt) Hustedt

Fig. 9. Fragilaria fasciculata (Agardh) Lange-Bertalot

Fig. 10. Fragilaria fasciculata (Agardh) Lange-Bertalot

Fig. 11. Fragilaria investiens (W. Smith) Cleve-Euler

Fig. 12. Fragilaria lapponica Grunow in Van Heurck

Fig. 13. Fragilaria exigua Grunow in Cleve and Möller

Fig. 14. Fragilaria schulzii Brockmann

Fig. 15. Fragilaria ulna (Nitzsch) Lange-Bertalot

Fig. 16. Fragilaria pinnata Ehrenberg var. pinnata

Fig. 17. Fragilaria pinnata Ehrenberg var. pinnata

Fig. 18. Fragilaria pinnata Ehrenberg var. pinnata

Fig. 19. Fragilaria pinnata var. 1

Fig. 20. Fragilaria pinnata c. f. var. intercedens (Grunow) Hustedt

Fig. 21. Fragilaria subsalina (Grunow Lange-Bertalot) 

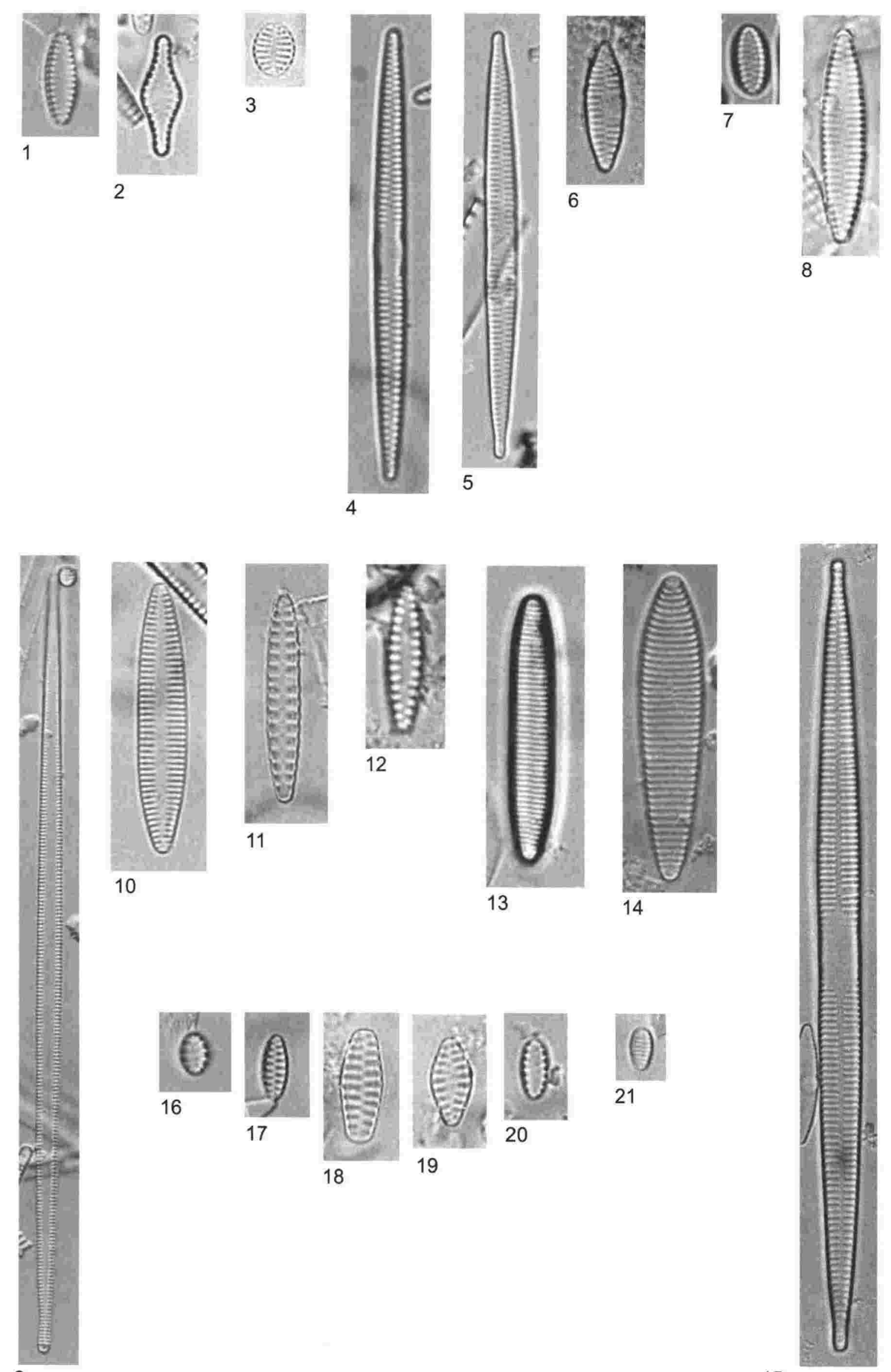


\section{Plate 7}

LM: Fig. 1, 2, 4-18 x1600; Fig. 3 x640

Fig. 1. Grammatophora marina (Lyngbye) Kützing

Fig. 2. Grammatophora marina (Lyngbye) Kützing

Fig. 3. Grammatophora oceanica Ehrenberg

Fig. 4. Grammatophora oceanica Ehrenberg

Fig. 5. Licmophora abbreviata Agardh

Fig. 6. Licmophora juergensii Agardh

Fig. 7. Meridion circulare var. constrictum (Ralfs) Van Heurck

Fig. 8. Opephora c. f. burchardtiae Witkowski, Metzeltin \& Lange-Bertalot in Moser et al.

Fig. 9. Opephora guenter-grassii (Witkowski \& Lange-Bertalot) Sabbe \& Vyverman

Fig. 10. Opephora olsenii Möller

Fig. 11. Opephora olsenii Möller

Fig. 12. Opephora marina (Gregory) Petit

Fig. 13. Opephora sp. 1

Fig. 14. Tabellaria flocculosa (Roth) Kützing

Fig. 15. Tabellaria ventricosa Kützing

Fig. 16. Thalassionema nitzschioides (Grunow) Mereschowsky

Fig. 17. Rhaphoneis amphiceros (Ehrenberg) Ehrenberg

Fig. 18. Plagiogrammopsis crawfordii Witkowski, Lange-Bertalot \& Metzeltin 

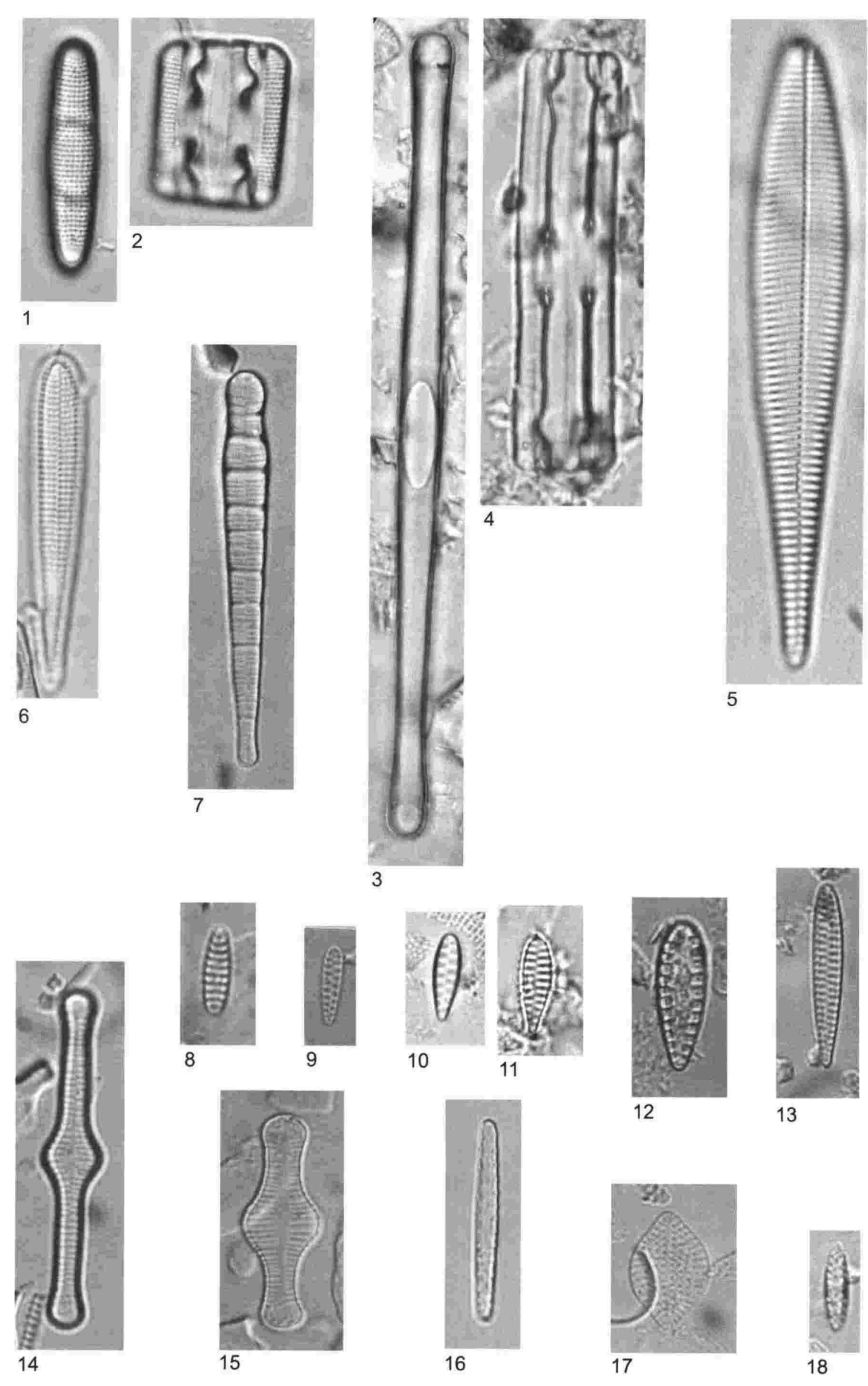


\section{Plate 8}

LM: Fig. 1 x800; Fig. 4-9 x1600

SEM: Fig. $2 \times 4700$; Fig. $3 \times 2700$

Fig. 1. Synedra sp. 1

Fig. 2. Synedra sp. 1

Fig. 3. Synedra sp. 1

Fig. 4. Eunotia bilunaris (Ehrenberg) Mills var. bilunaris

Fig. 5. Eunotia incisa Gregory

Fig. 6. Eunotia tecta Krasske

Fig. 7. Eunotia soleirolii (Kützing) Rabenhorst

Fig. 8. Eunotia formica Ehrenberg

Fig. 9. Eunotia pectinalis var. undulata (Ralfs) Brown 


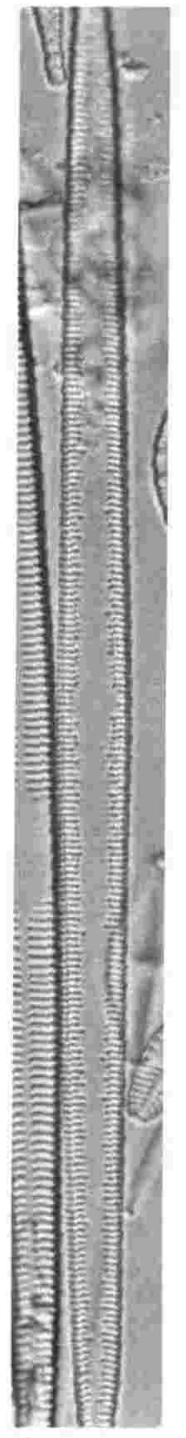

1
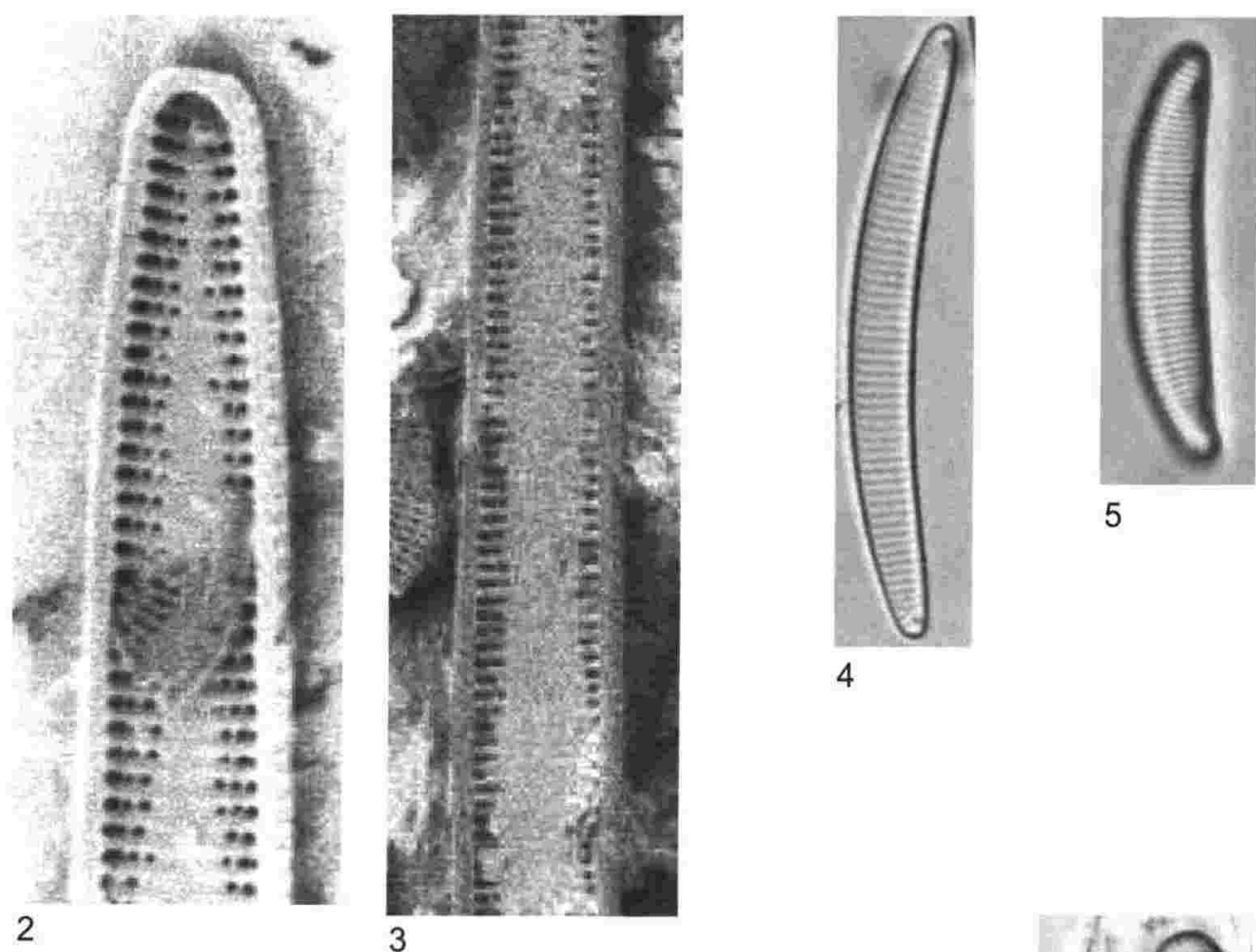

5

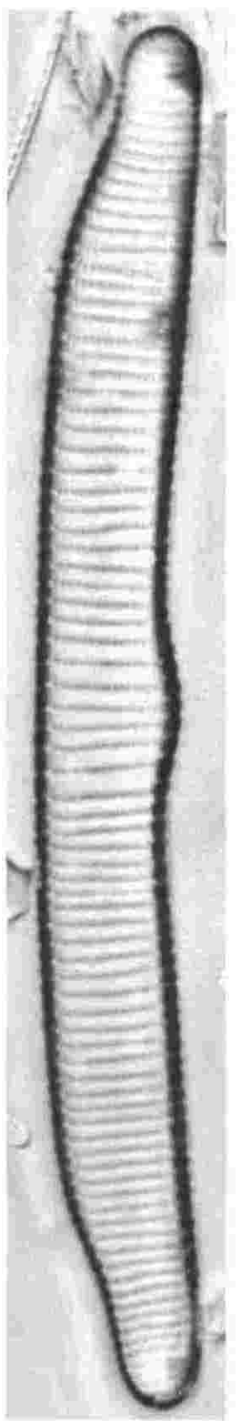

9
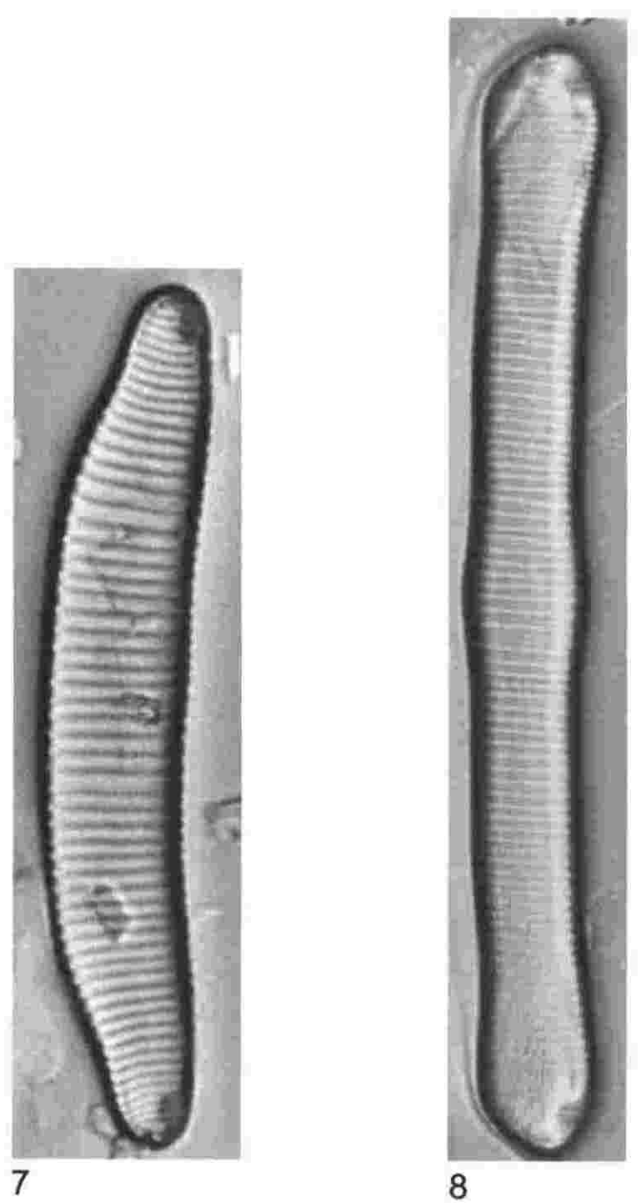


\section{Plate 9}

LM: x1600

Fig. 1. Cocconeiopsis wrightii (O’Meara) Witkowski, Lange-Bertalot \& Metzeltin

Fig. 2. Cocconeis c. f. costata Gregory

Fig. 3. Cocconeis c. f. costata Gregory

Fig. 4. Cocconeis discrepans Schmidt

Fig. 5. Cocconeis neothumensis Krammer

Fig. 6. Cocconeis neothumensis Krammer

Fig. 7. Cocconeis c. f. peltoides Hustedt

Fig. 8. Cocconeis c. f. peltoides Hustedt

Fig. 9. Cocconeis c. f. peltoides Hustedt

Fig. 10. Cocconeis sp. 3

Fig. 11. Cocconeis placentula var. euglypta (Ehrenberg) Grunow

Fig. 12. Cocconeis placentula Ehrenberg var. placentula

Fig. 13. Cocconeis placentula Ehrenberg var. placentula

Fig. 14. Cocconeis placentula var. lineata (Ehrenberg) Van Heurck

Fig. 15. Cocconeis placentula var. lineata (Ehrenberg) Van Heurck

Fig. 16. Cocconeis scutellum Ehrenberg var. scutellum

Fig. 17. Cocconeis scutellum Ehrenberg var. scutellum

Fig. 18. Cocconeis scutellum var. parva (Grunow in Van Heurck) Cleve

Fig. 19. Cocconeis scutellum var. parva (Grunow in Van Heurck) Cleve

Fig. 20. Cocconeis stauroneiformis (Van Heurck) Okuno

Fig. 21. Cocconeis stauroneiformis (Van Heurck) Okuno

Fig. 22. Cocconeis sublittoralis Hendey

Fig. 23. Cocconeis sublittoralis Hendey 

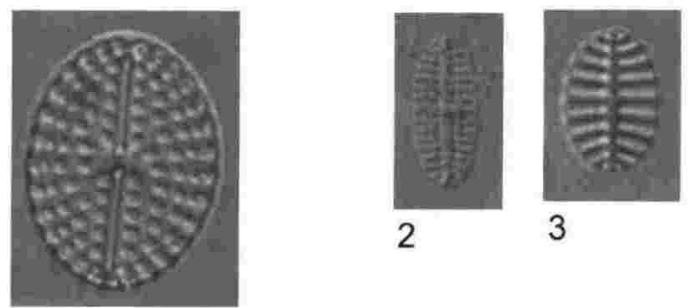

3

1

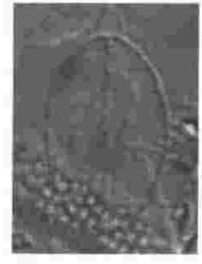

7
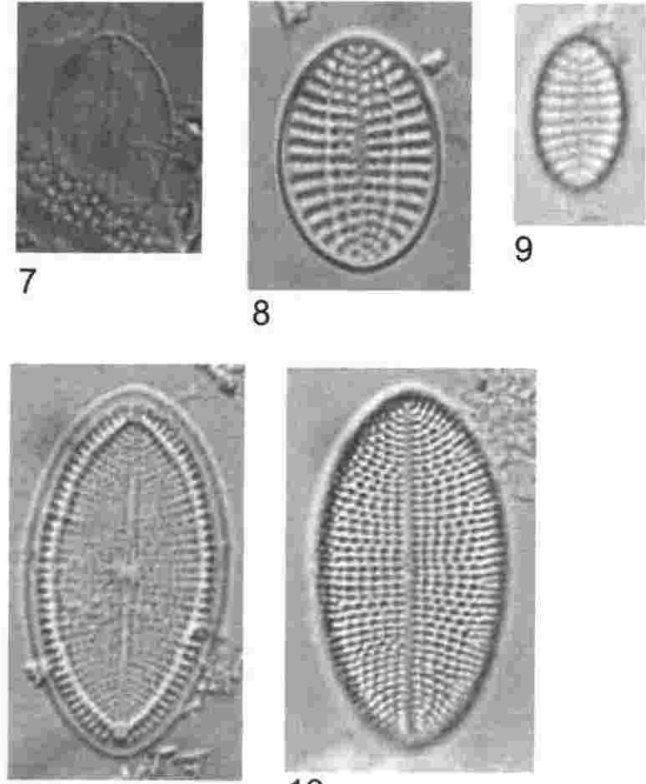

12
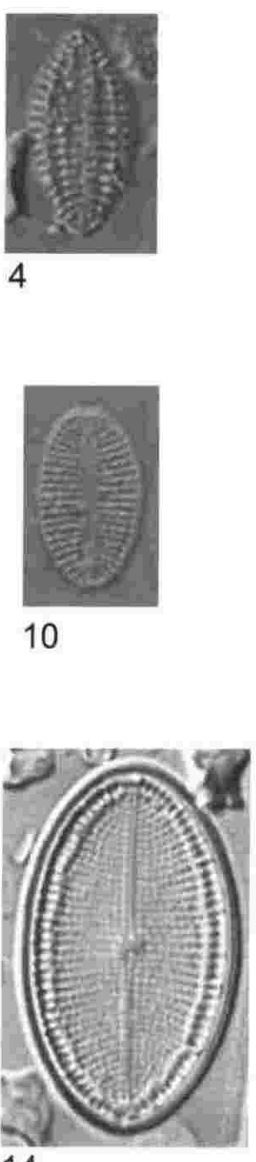

14
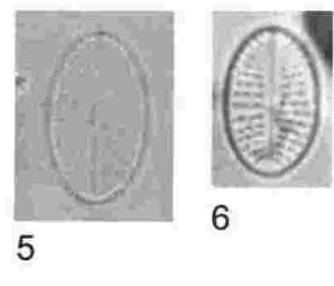

6

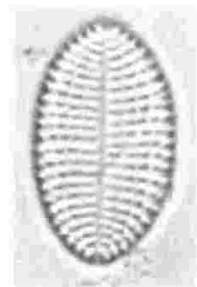

11
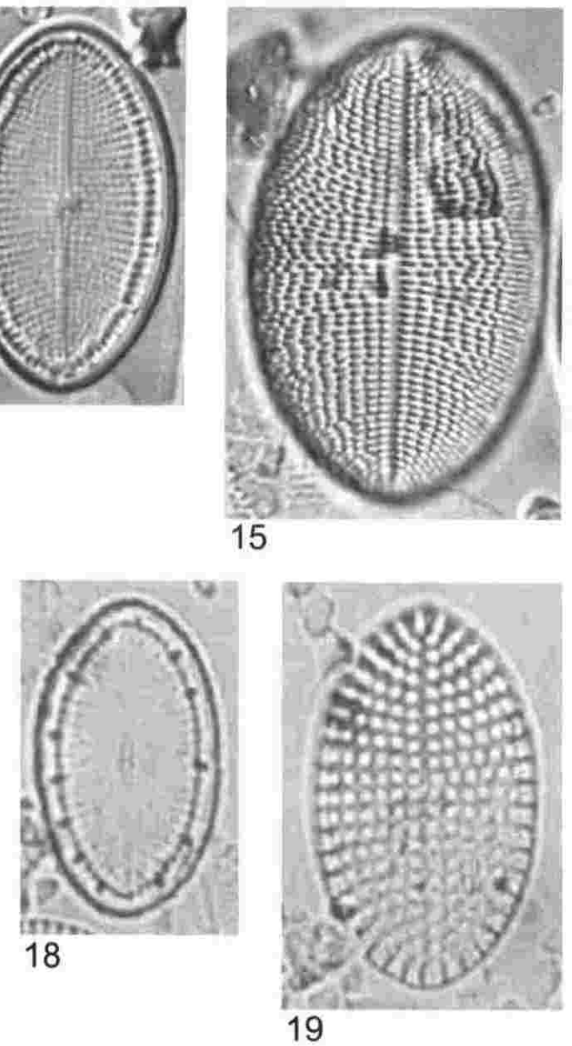

17

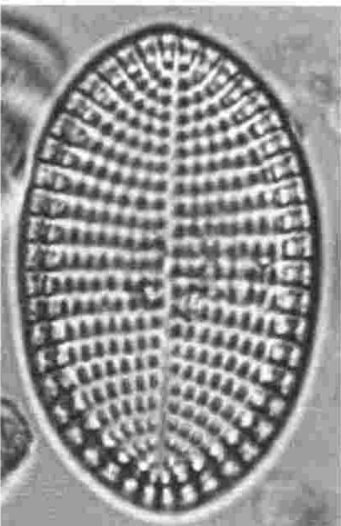

16

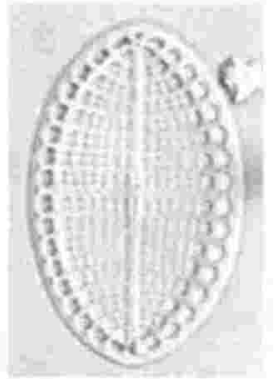

20

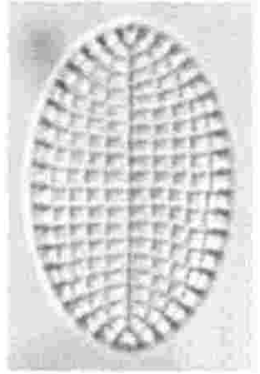

21

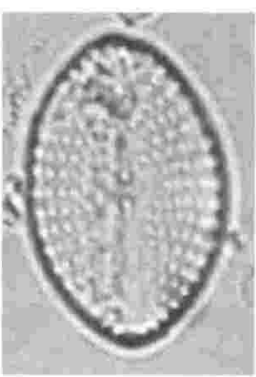

22

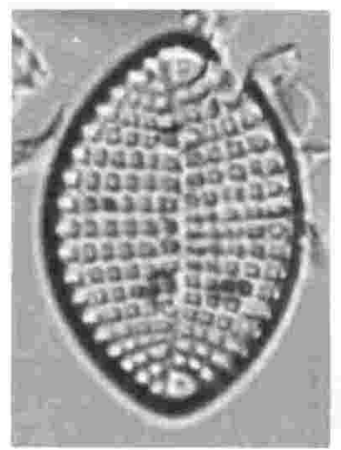

23 


\section{Plate 10}

LM: $x 1600$

Fig. 1. Achnanthes brevipes var. intermedia (Kützing) Cleve

Fig. 2. Achnanthes brevipes var. intermedia (Kützing) Cleve

Fig. 3. Achnanthes brevipes var. intermedia (Kützing) Cleve

Fig. 4. Achnanthes clevei Grunow in Cleve \& Grunow

Fig. 5. Achnanthes clevei Grunow in Cleve \& Grunow

Fig. 6. Achnanthes crosbyana Foged

Fig. 7. Achnanthes crosbyana Foged

Fig. 8. Achnanthes delicatula ssp. delicatula (Kützing) Grunow

Fig. 9. Achnanthes delicatula ssp. delicatula (Kützing) Grunow

Fig. 10. Achnanthes delicatula var. engelbrechtii (Cholnoky) Lange-Bertalot

Fig. 11. Achnanthes delicatula var. engelbrechtii (Cholnoky) Lange-Bertalot

Fig. 12. Achnanthes hungarica (Grunow) Grunow in Cleve \& Grunow

Fig. 13. Achnanthes hungarica (Grunow) Grunow in Cleve \& Grunow

Fig. 14. Achnanthes hyperboreoides Witkowski, Lange-Bertalot \& Metzeltin

Fig. 15. Achnanthes lanceolata (Brébisson) Grunow in Cleve \& Grunow ssp. lanceolata

Fig. 16. Achnanthes lanceolata (Brébisson) Grunow in Cleve \& Grunow ssp. lanceolata

Fig. 17. Achnanthes lanceolata ssp. dubia (Grunow) Lange-Bertalot

Fig. 18. Achnanthes lanceolata ssp. dubia (Grunow) Lange-Bertalot

Fig. 19. Achnanthes lanceolata ssp. robusta (Hustedt) Lange-Bertalot

Fig. 20. Achnanthes lanceolata ssp. rostrata (Østrup) Hustedt

Fig. 21. Achnanthes lanceolata ssp. rostrata (Østrup) Hustedt

Fig. 22. Achnanthes linearis (W. Smith) Grunow

Fig. 23. Achnanthes minutissima Kützing var. minutissima

Fig. 24. Achnanthes minutissima Kützing var. minutissima

Fig. 25. Achnanthes oblongella Østrup

Fig. 26. Achnanthes oblongella Østrup 

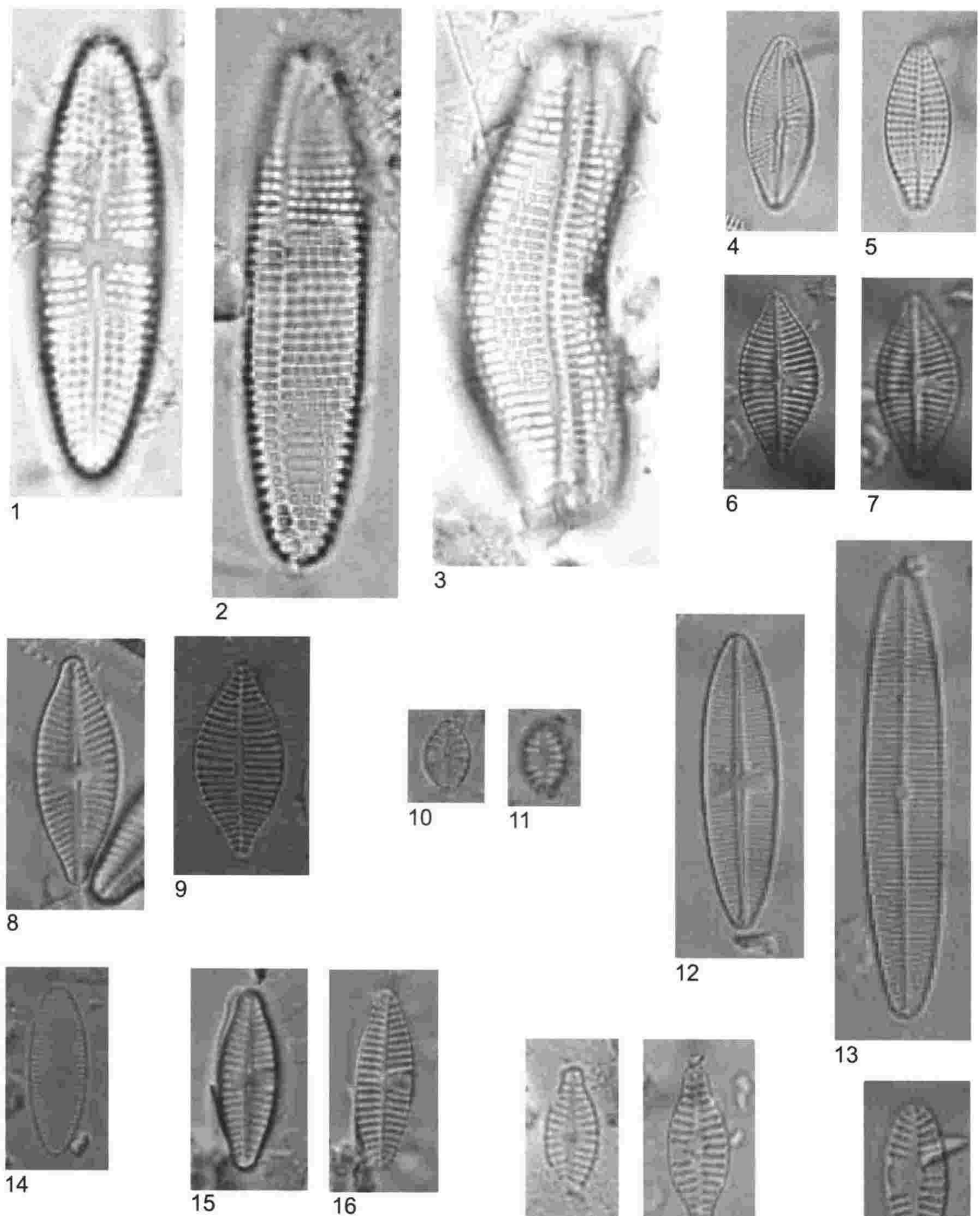

8

9

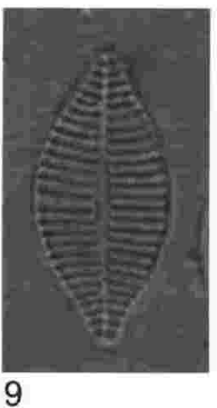




\section{Plate 11}

LM: $x 1600$

Fig. 1. Achnanthes parvula Kützing

Fig. 2. Achnanthes pericava Carter

Fig. 3. Achnanthes pericava Carter

Fig. 4. Achnanthes ploenensis Hustedt var. ploenensis

Fig. 5. Achnanthes ploenensis Hustedt var. ploenensis

Fig. 6. Achnanthes ploenensis c. f. var. woldstedtii (Hustedt) Lange-Bertalot

Fig. 7. Achnanthes ploenensis c. f. var. woldstedtii (Hustedt) Lange-Bertalot

Fig. 8. Achnanthes reversa Lange-Bertalot and Krammer

Fig. 9. Achnanthes reversa Lange-Bertalot and Krammer

Fig. 10. Achnanthes subatomoides (Hustedt) Lange-Bertalot \& Archibald

Fig. 11. Achnanthes subatomoides (Hustedt) Lange-Bertalot \& Archibald

Fig. 12. Achnanthes c. f. suchlandtii Hustedt

Fig. 13. Achnanthes c. f. suchlandtii Hustedt

Fig. 14. Achnanthes sp. 1

Fig. 15. c. f. Planothidium diplopunctatum (Simonsen) Witkowski, L-B \& Metzeltin

Fig. 16. Planothidium c. f. lilljeborgei (Grunow) Witkowski, Lange-Bertalot \& Metzeltin

Fig. 17. Denticula neritica Holmes \& Croll

Fig. 18. Epithemia adnata (Kützing) Brébisson

Fig. 19. Epithemia adnata (Kützing) Brébisson

Fig. 20. Epithemia sorex Kützing

Fig. 21. Rhopalodia brebissonii Krammer 

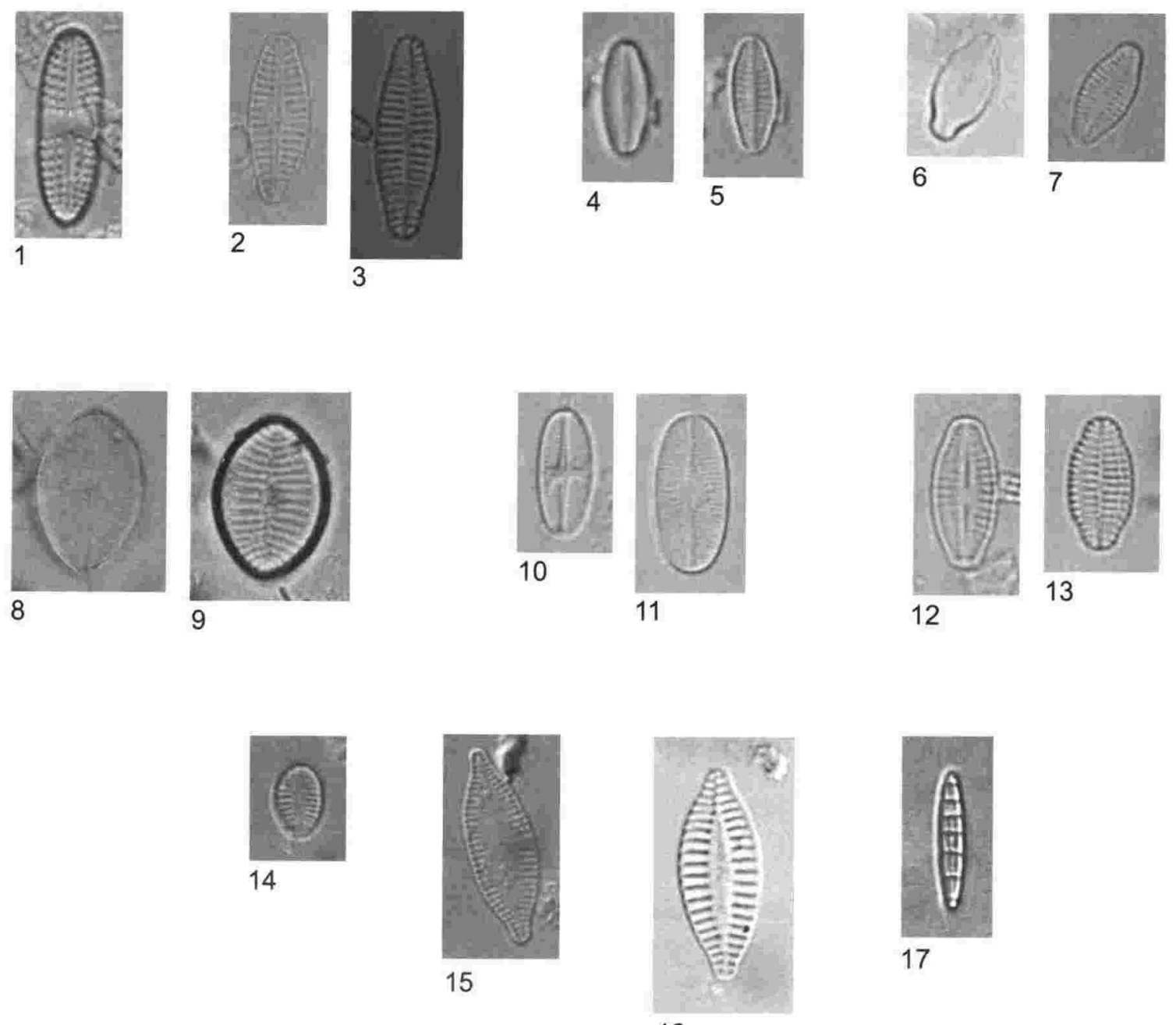

15

16

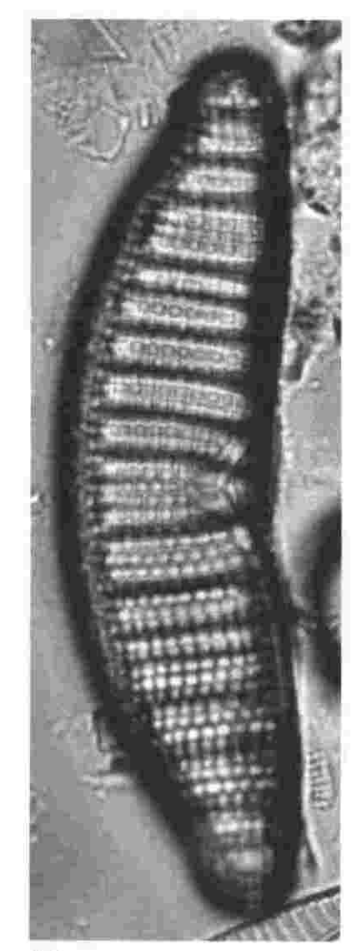

18

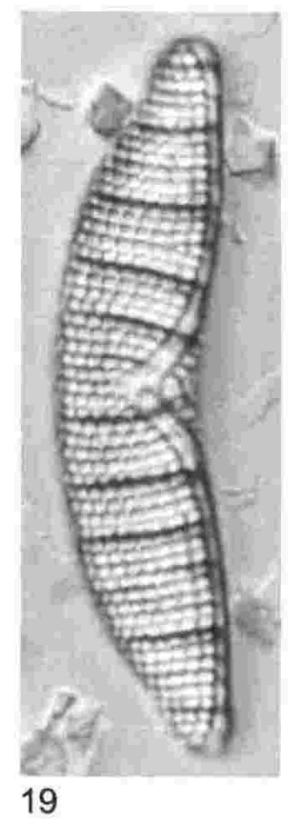

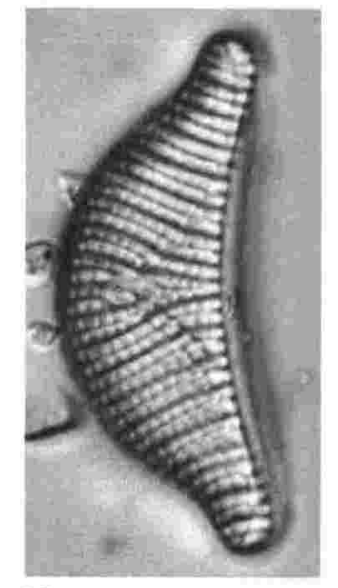

20

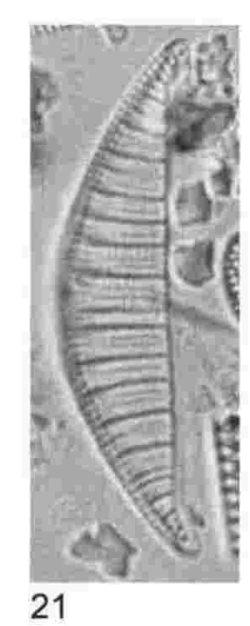




\section{Plate 12}

LM: $\times 1600$

Fig. 1. Amphiprora c. f. wilkinsonia Stidolph

Fig. 2. Entomoneis alata (Ehrenberg) Ehrenberg

Fig. 3. Entomoneis alata (Ehrenberg) Ehrenberg

Fig. 4. Entomoneis costata (Hustedt) Reimer

Fig. 5. Amphora bigibba var. interrupta Grunow

Fig. 6. Amphora coffeaeformis (Agardh) Kützing

Fig. 7. Amphora libyca Ehrenberg

Fig. 8. Amphora normanii Rabenhorst

Fig. 9. Amphora aff. profusa Giffen

Fig. 10. Amphora strigosa Hustedt

Frig. 111. Amripriora turgida Gregory

Fig. 12. Amphora turgida Gregory

Fig. 13. Amphora sp. 2

Fig. 14. Catenula adhaerens Mereschkowsky 

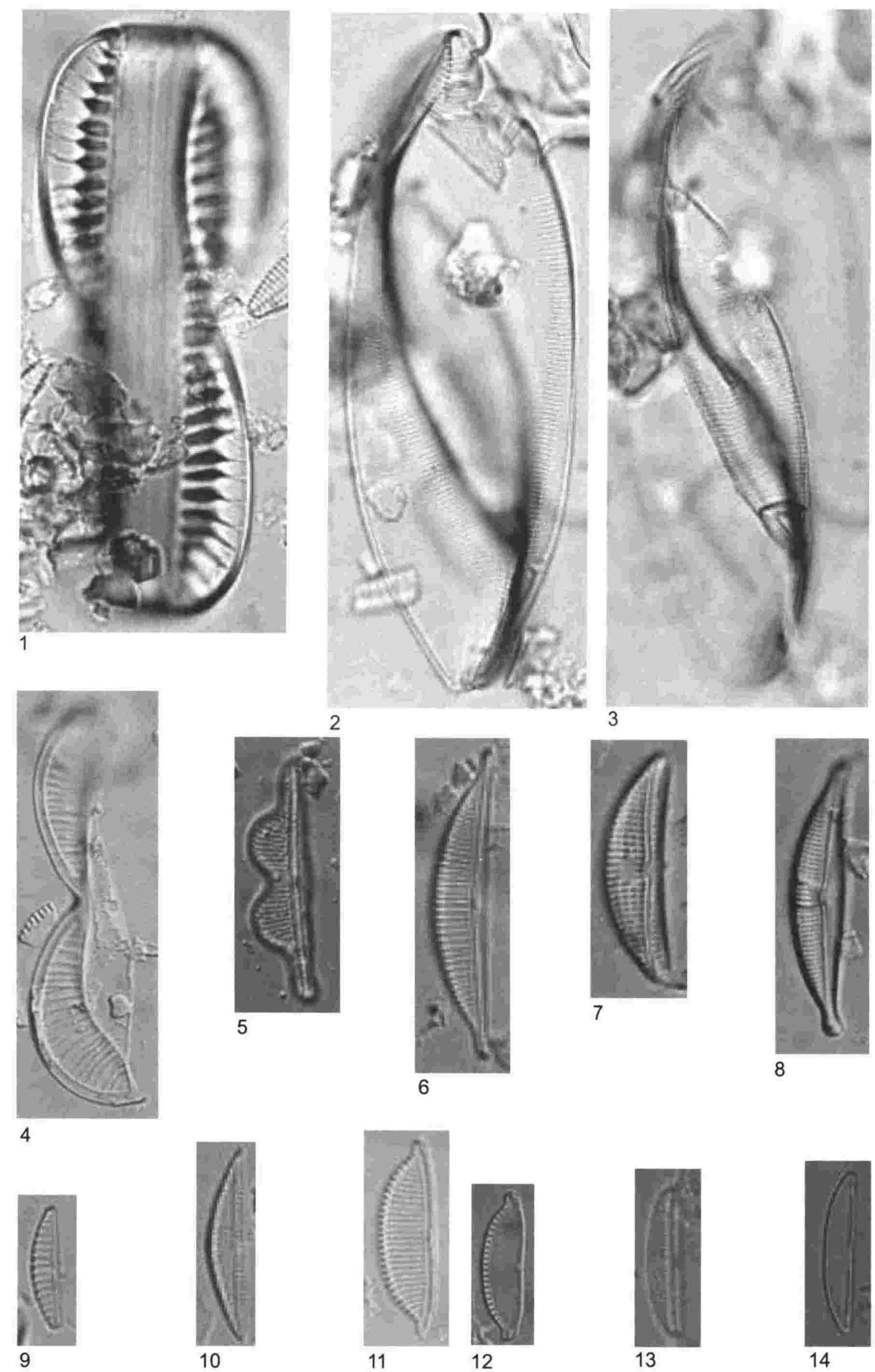


\section{Plate 13}

LM: Fig. 3-12 and 14-16 x1600; Fig. 13 x640

SEM: Fig. 1 x4400; Fig. 2 x4600

Fig. 1. Anomoeoneis brachysira (Brébisson in Rabenhorst) Grunow

Fig. 2. Anomoeoneis brachysira (Brébisson in Rabenhorst) Grunow

Fig. 3. Anomoeoneis brachysira (Brébisson in Rabenhorst) Grunow

Fig. 4. Anomoeoneis vitrea (Grunow) Ross

Fig. 5. Berkeleya rutilans (Trentepohl) Grunow

Fig. 6. Biremus lucens (Hustedt) Sabbe, Witkowski \& Vyverman

Fig. 7. Cymbella amphicephala Naegeli in Kützing

Fig. 8. Cymbella gaeumannii Meister

Fig. 9. Cymbella minuta Hilse ex Rabenhorst

Fig. 10. Cymbella gracilis (Ehrenberg) Kützing

Fig. 11. Cymbella pusilla Grunow in Schmidt et al.

Fig. 12. Cymbella silesiaca Bleisch in Rabenhorst

Fig. 13. Cymbella aspera (Ehrenberg) Cleve

Fig. 14. Cymbella affinis Kützing

Fig. 15. Cymbella naviculiformis (Auerswald) Cleve

Fig. 16. Cymbella yarrensis (A. Schimdt) Cleve 

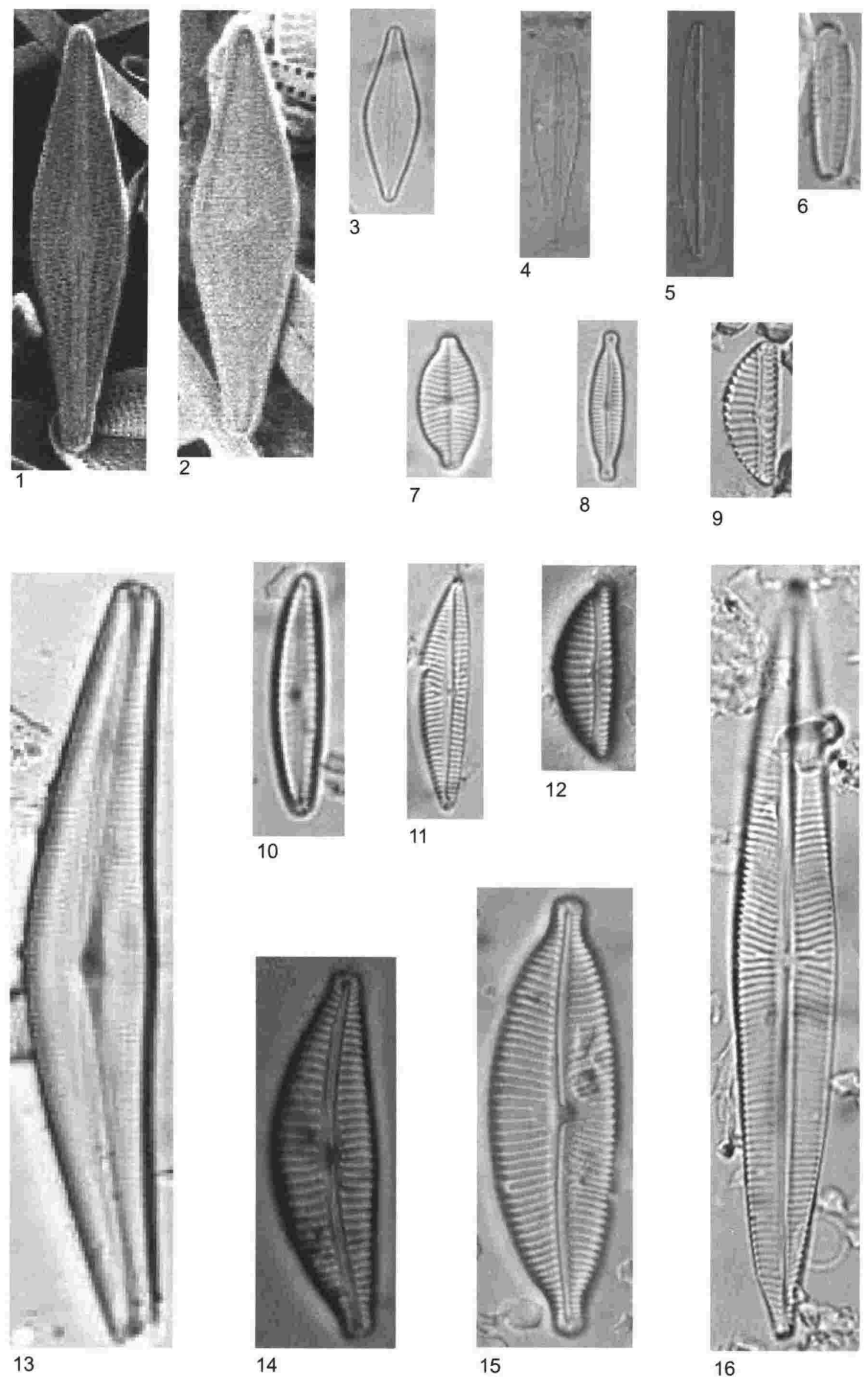


\section{Plate 14}

LM: Fig. $1-15$ and $18,19 \times 1600$

SEM: Fig. 16 and $17 \times 4000$

Fig. 1. Diploneis didyma (Ehrenberg) Ehrenberg

Fig. 2. Diploneis smithii (Brébisson) Cleve

Fig. 3. Diploneis subovalis Cleve

Fig. 4. Diploneis oblongella (Naegeli) Cleve-Euler

Fig. 5. Diploneis vacillans (Schmidt) Cleve

Fig. 6. Diploneis vacillans (Schmidt) Cleve

Fig. 7. Fallacia tenera (Hustedt) Mann

Fig. 8. Fallacia tenera (Hustedt) Mann

Fig. 9. Fallacia clepsidroides Witkowski

Fig. 10. Fallacia clepsidroides Witkowski

Fig. 11. Fallacia florinae (Möller) Witkowski

Fig. 12. Fallacia melanocephala (Giffen) Witkowski, Lange-Bertalot and Metzeltin

Fig. 13. Fallacia cryptolyra (Brockmann) Stickle and Mann

Fig. 14. Fallacia forcipata (Greville) Stickle and Mann

Fig. 15. Fallacia ny (Cleve) Mann

Fig. 16. Fallacia sp. 1

Fig. 17. Fallacia sp. 1

Fig. 18. Fallacia sp. 1

Fig. 19. Fallacia sp. 2 

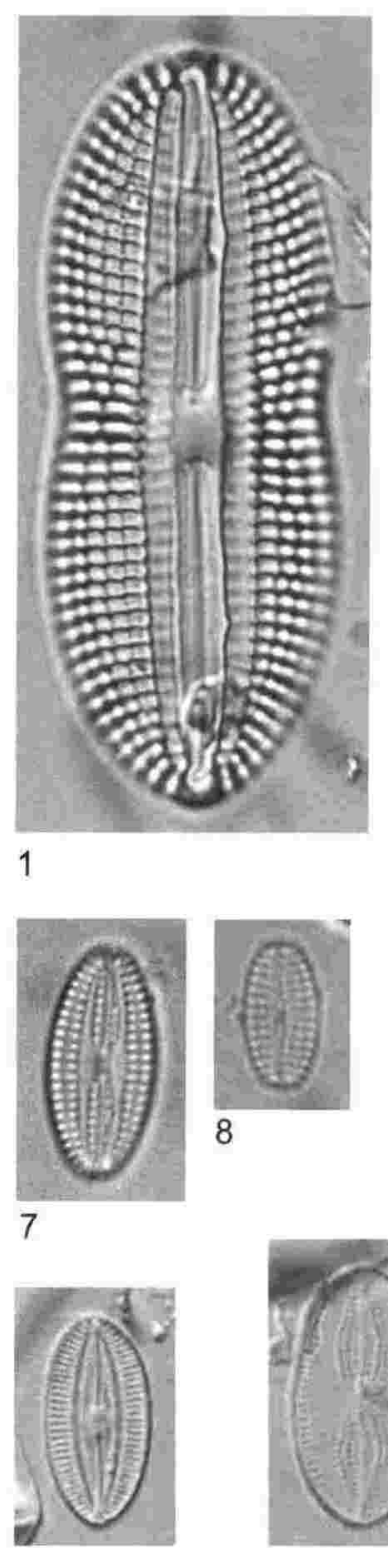

11

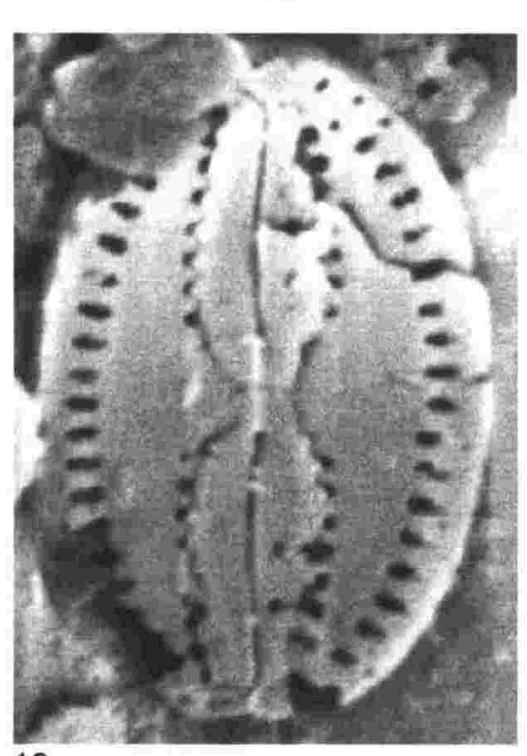

2

9
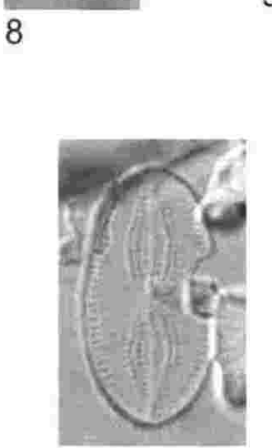

12
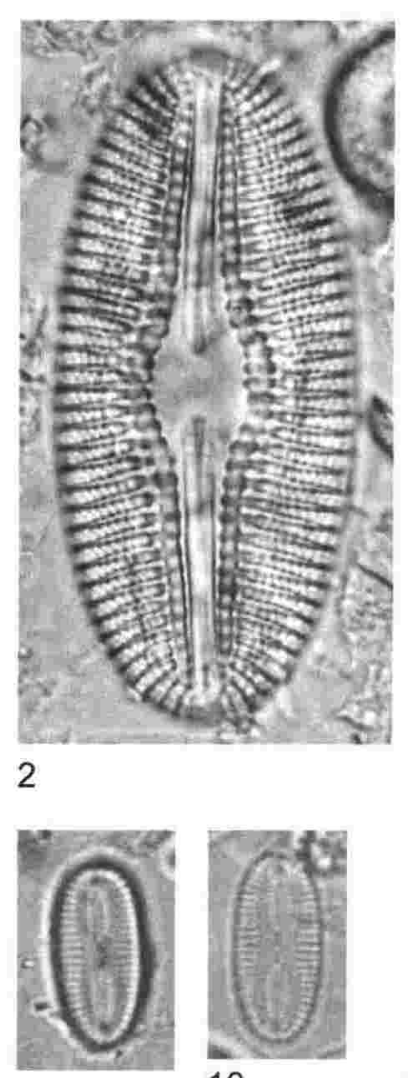

13

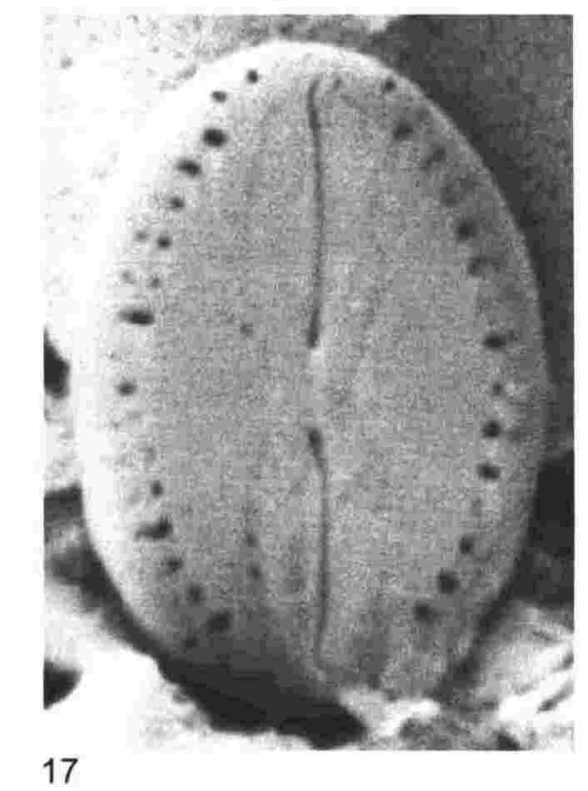

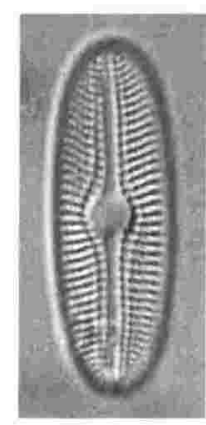

4

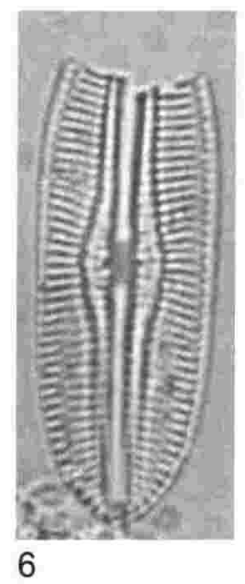

5
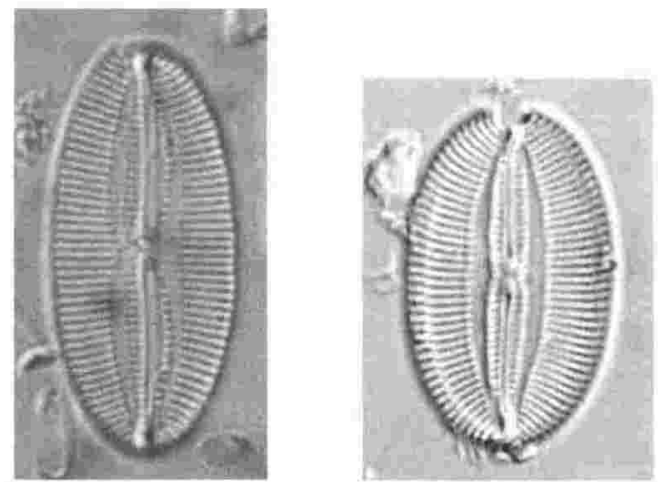

15

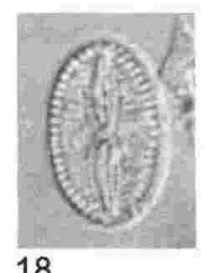

18

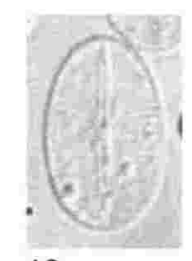

19 


\section{Plate 15}

LM: x1600

Fig. 1. Frustulia rhomboides var. crassinervia (Brébisson) Ross

Fig. 2. Frustulia rhomboides var. saxonica (Rabenhorst) De Toni

Fig. 3. Frustulia vulgaris (Thwaites) De Toni

Fig. 4. Gomphonema clavatum Ehrenberg

Fig. 5. Gomphonema affine Kützing

Fig. 6. Gomphonema truncatum Ehrenberg

Fig. 7. Gomphonema angustum Agardh

Fig. 8. Gomphonema minutum (Agardh) Agardh

Fig. 9. Gomphonema minutum (Agardh) Agardh

Fig. 10. Gomphonema parvulum (Kützing) Kützing

Fig. 11. Gomphonema valentinica Nikolajev

Fig. 12. Gomphonema valentinica Nikolajev

Fig. 13. Gomphonemopsis exigua (Kützing) Medlin

Fig. 14. Rhoicosphenia abbreviata (Agardh) Lange-Bertalot

Fig. 15. Rhoicosphenia abbreviata (Agardh) Lange-Bertalot

Fig. 16. Neidium ampliatum (Ehrenberg) Krammer

Fig. 17. Neidium affine (Ehrenberg) Pfitzer 

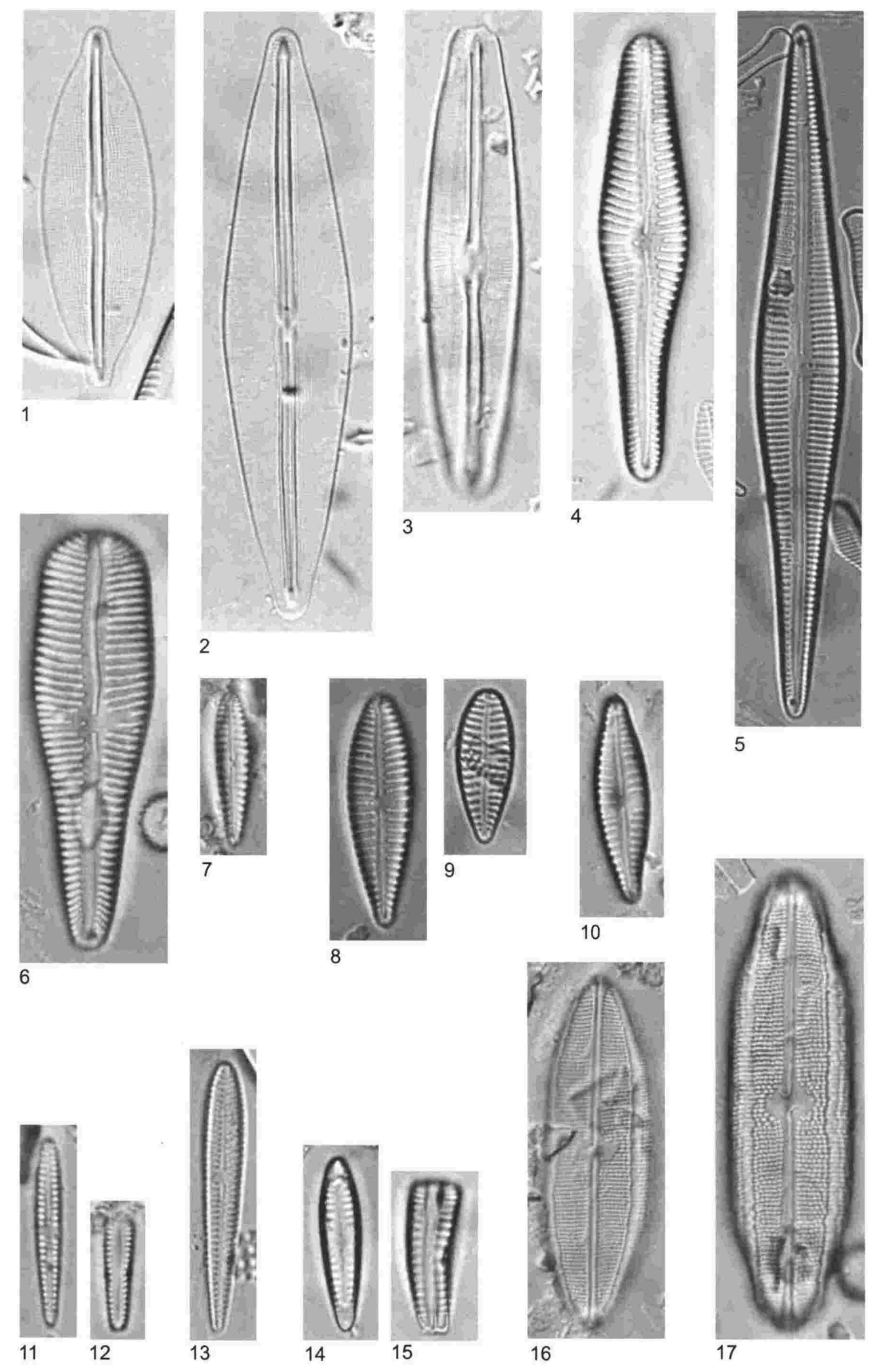


\section{Plate 16}

LM: Fig. 1 and 4 x1000; Fig. 2 x400; Fig. 3, 6 and 7 x1600; Fig. 5 ×1250

Fig. 1. Gyrosigma acuminatum (Kützing) Rabenhorst

Fig. 2. Gyrosigma balticum (Ehrenberg) Rabenhorst

Fig. 3. Gyrosigma fasciola (Ehrenberg) Griffith \& Henfrey

Fig. 4. Pleurosigma salinarum Grunow

Fig. 5. Gyrosigma peisonis (Grunow) Hustedt

Fig. 6. Gyrosigma beaufortianum Hustedt

Fig. 7. Gyrosigma balticum (Ehrenberg) Rabenhorst 

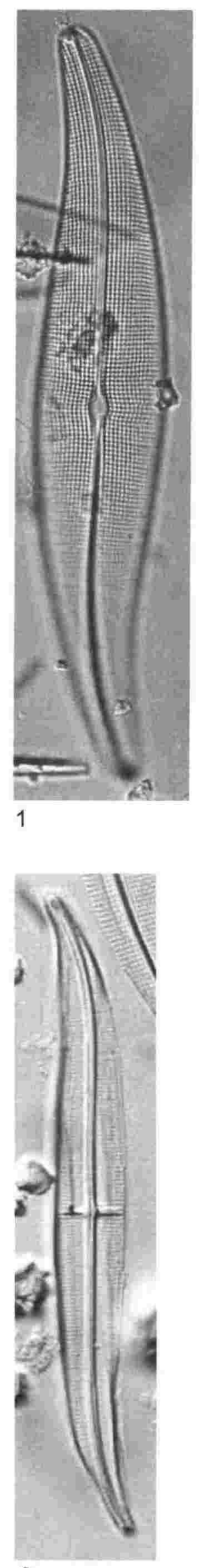

6
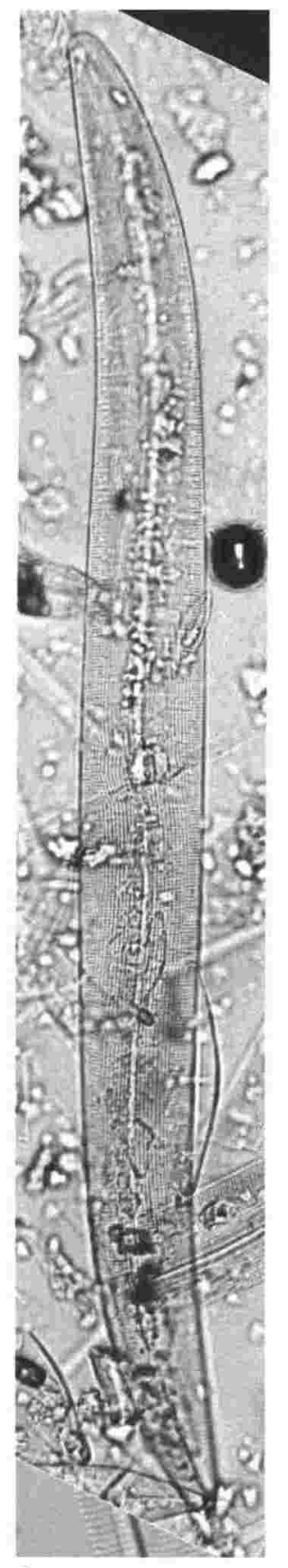

2
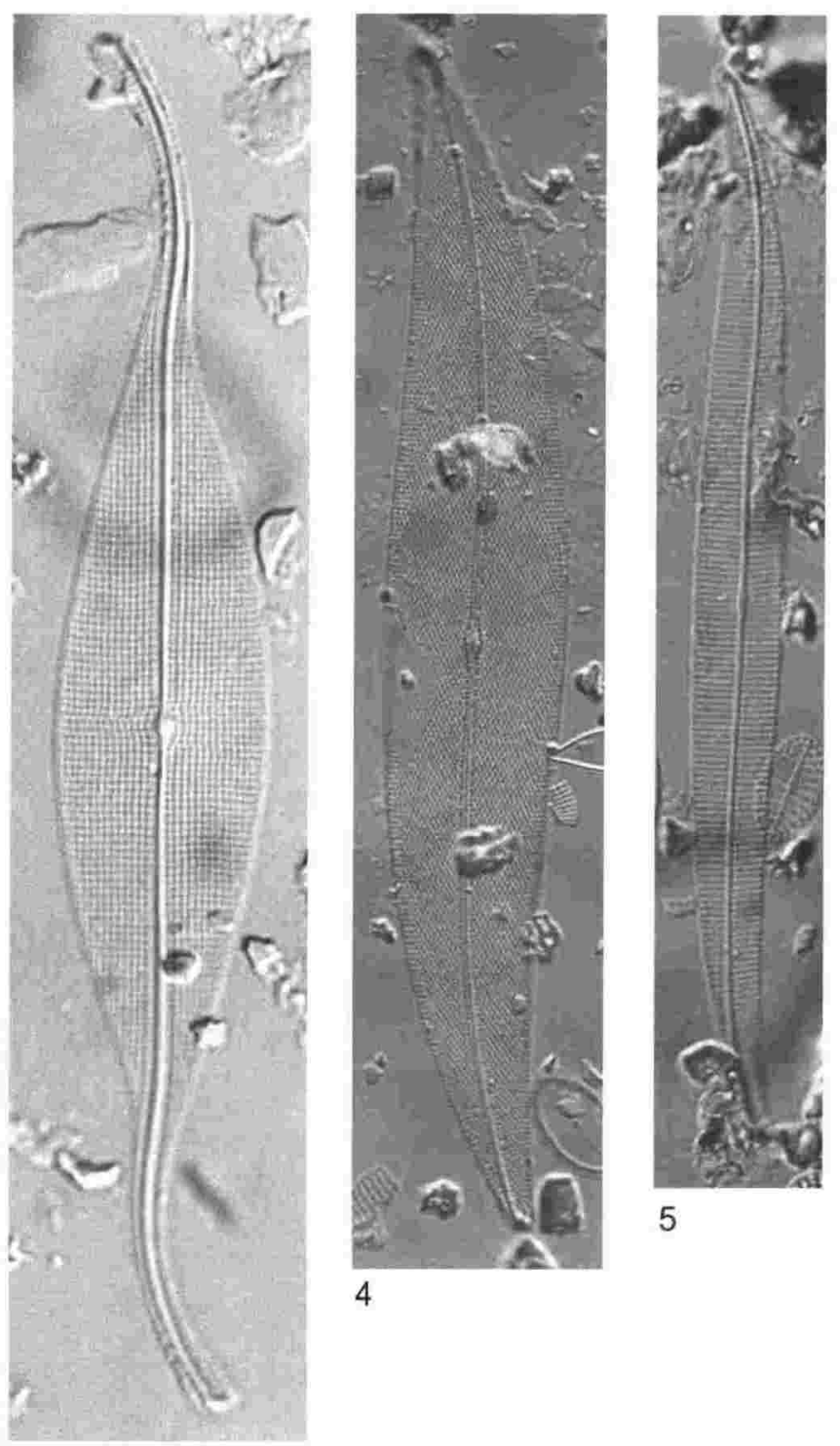

3

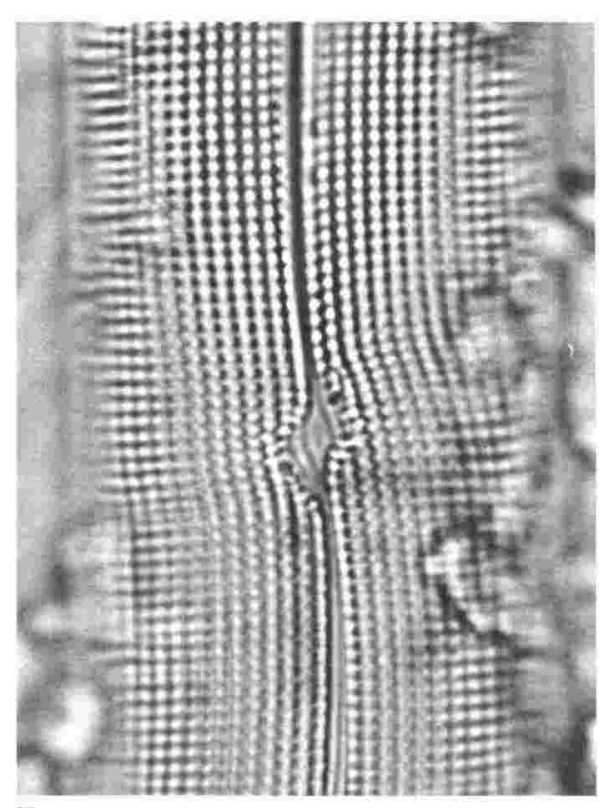

7 


\section{Plate 17}

LM: Fig. 3-21 x1600

SEM: Fig. 1 and $2 \times 2300$

Fig. 1. Mastogloia elliptica (Agardh) Cleve

Fig. 2. Mastogloia elliptica (Agardh) Cleve

Fig. 3. Mastogloia elliptica (Agardh) Cleve

Fig. 4. Mastogloia elliptica (Agardh) Cleve

Fig. 5. Mastogloia pumila (Cleve \& Möller) Cleve

Fig. 6. Mastogloia pumila (Cleve \& Möller) Cleve

Fig. 7. Navicula sp. 3

Fig. 8. Navicula sp. 5

Fig. 9. Navicula sp. 6

Fig. 10. Navicula cancellata Donkin

Fig. 11. Navicula sp. 2

Fig. 12. Navicula sp. 7

Fig. 13. Navicula sp. 9

Fig. 14. Navicula seminulum Grunow

Fig. 15. Navicula seminulum Grunow

Fig. 16. Navicula americana Ehrenberg

Fig. 17. Navicula laevissima Kützing

Fig. 18. Navicula bacillum Ehrenberg

Fig. 19. Navicula pupula Kützing

Fig. 20. Navicula sp. 10

Fig. 21. Navicula sp. 11 


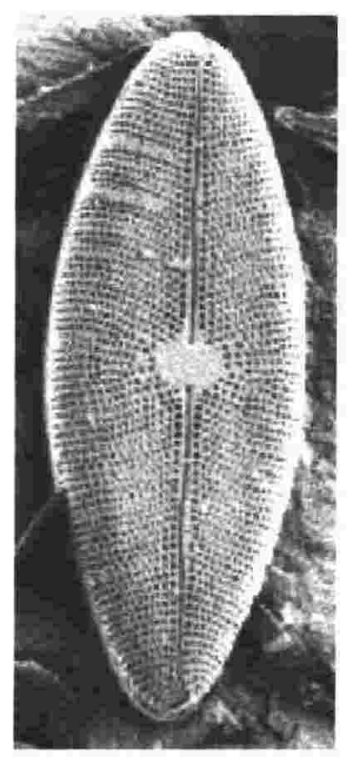

1

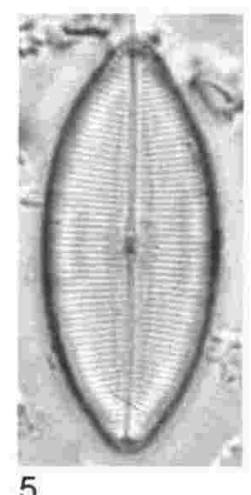

5

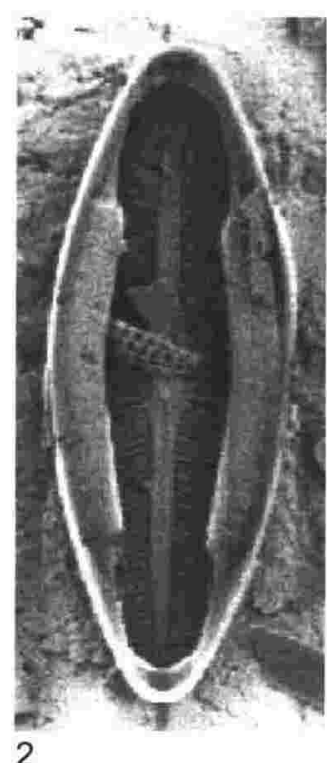

2
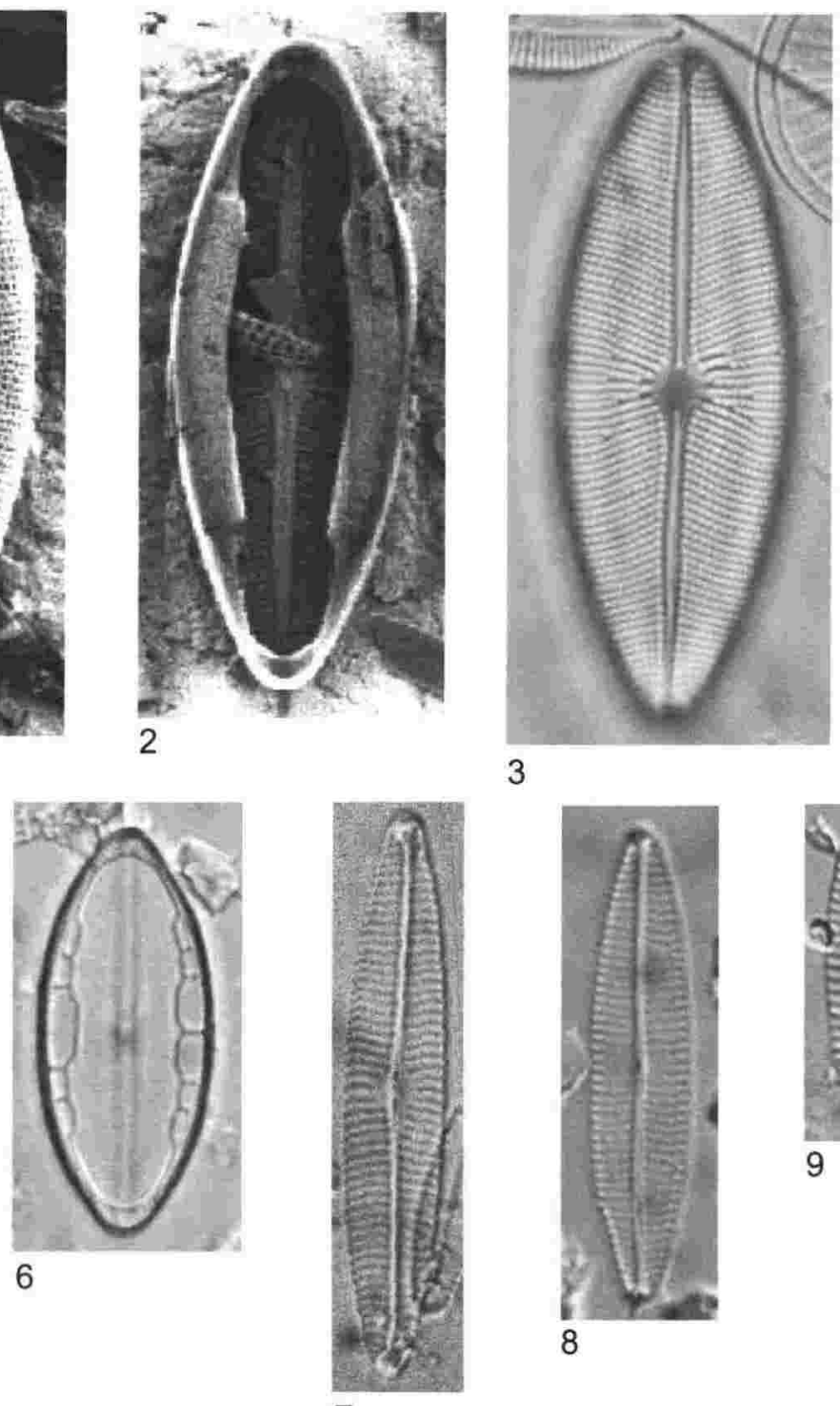

3
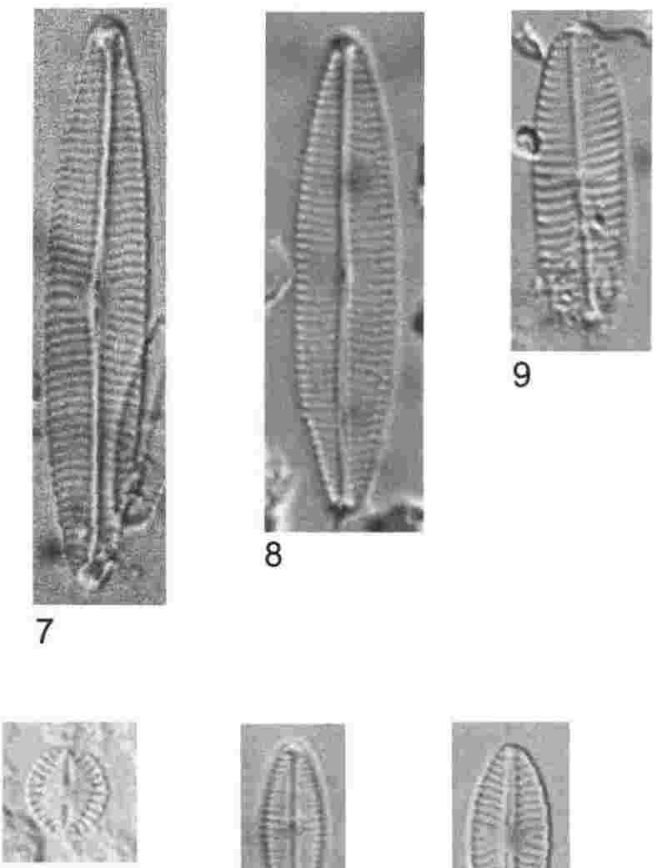

11

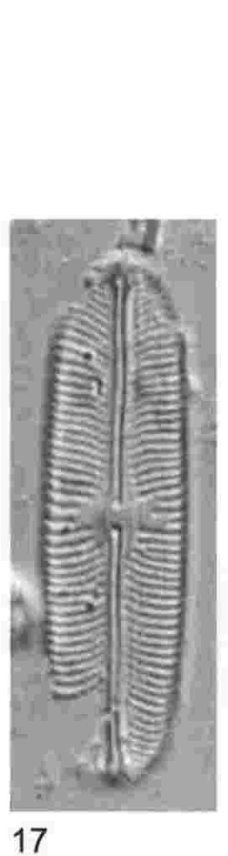

17
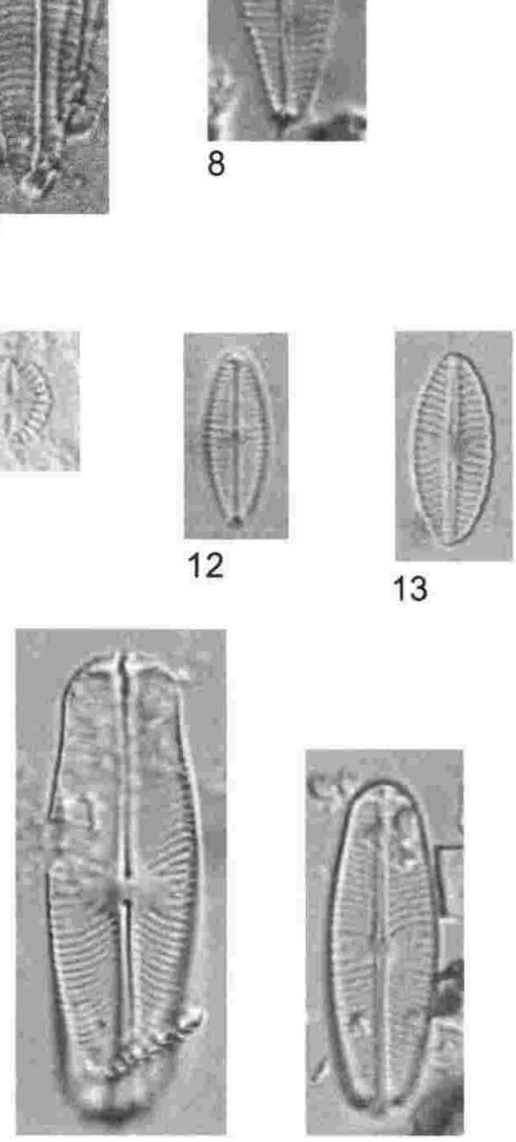

18

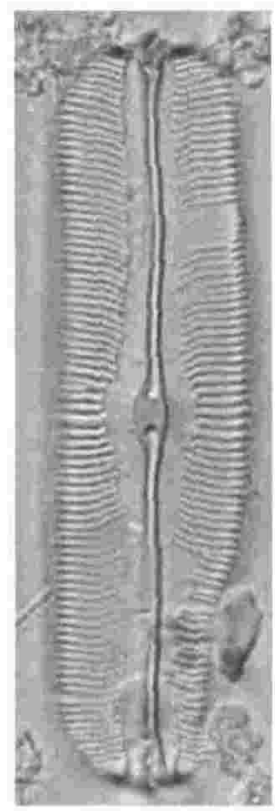

16
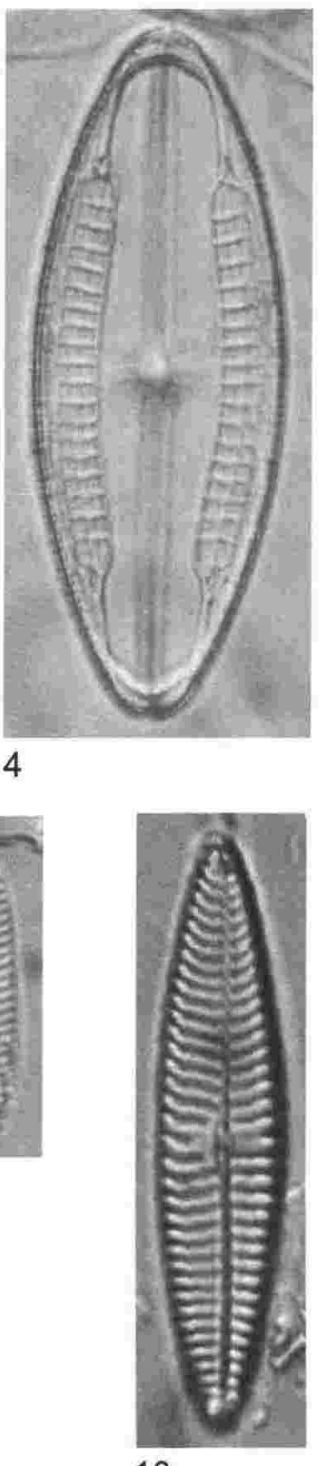

10
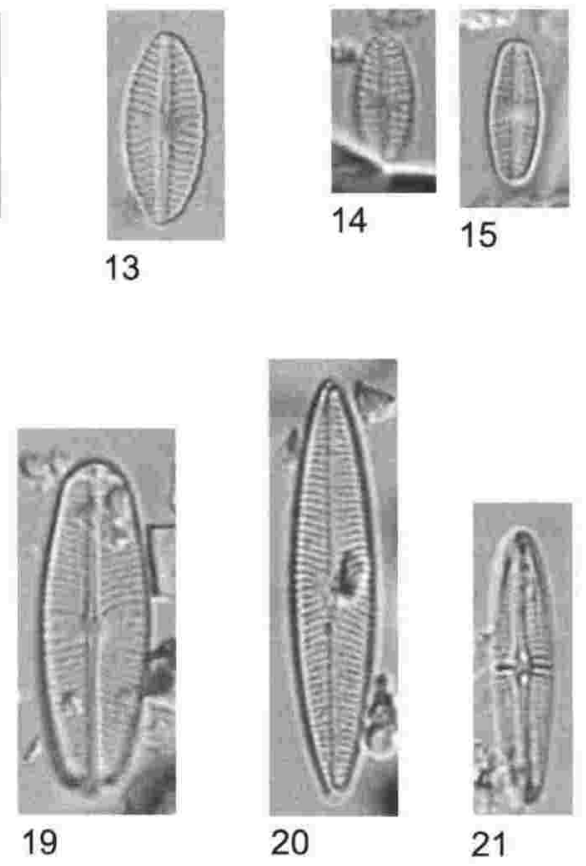


\section{Plate 18}

LM: $\times 1600$

Fig. 1. Navicula bryophila Petersen

Fig. 2. Navicula capitata Ehrenberg var. capitata

Fig. 3. Navicula capitata var. hungarica (Grunow) Ross

Fig. 4. Navicula capitata var. lunebergensis (Grunow) Patrick in Patrick \& Reimer

Fig. 5. Navicula contenta Grunow in Van Heurck

Fig. 6. Navicula cryptocephala Kützing

Fig. 7. Navicula cryptotenella Lange-Bertalot

Fig. 8. Navicula erifuga Lange-Bertalot

Fig. 9. Navicula cuspidata (Kützing) Kützing

Fig. 10. Navicula c. f. directa (W. Smith) Ralfs in Pritchard

Fig. 11. Navicula c. f. placentula (Ehrenberg) Kützing

Fig. 12. Navicula gastrum (Ehrenberg) Kützing

Fig. 13. Navicula elginensis (Gregory) Ralfs in Pritchard

Fig. 14. Navicula decussis Østrup

Fig. 15. Navicula phyllepta Kützing

Fig. 16. Navicula agrestis Hustedt

Fig. 17. Navicula atomus (Kützing) Grunow

Fig. 18. Navicula minima Grunow in Van Heurck

Fig. 19. Navicula c. f. gregaria Donkin

Fig. 20. Navicula gregaria Donkin

Fig. 21. Navicula gregaria Donkin

Fig. 22. Navicula halophila (Grunow) Cleve

Fig. 23. Navicula ignota var. palustris (Hustedt) Lund

Fig. 24. Navicula incertata Lange-Bertalot

Fig. 25. Navicula incertata Lange-Bertalot 

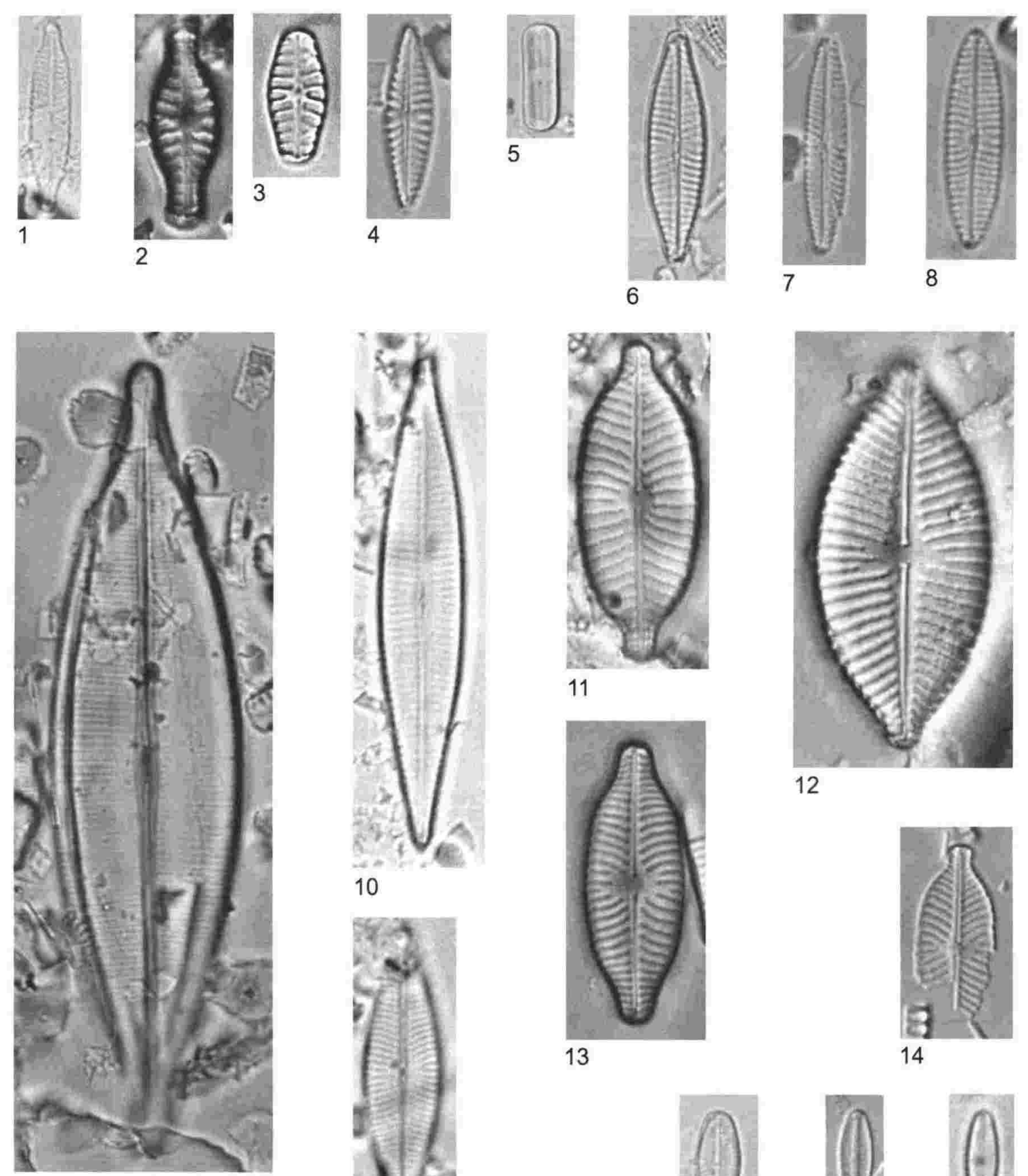

9
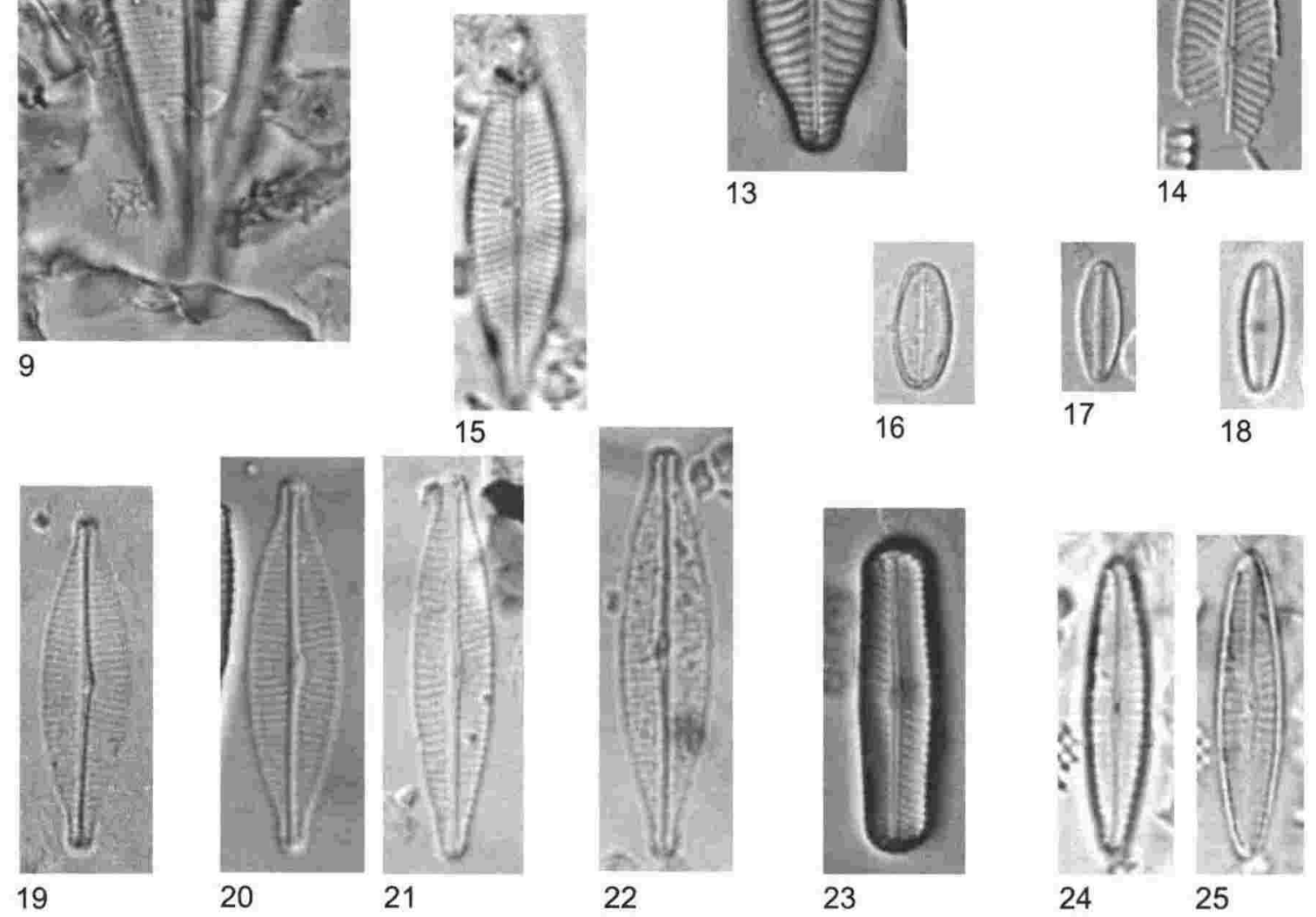


\section{Plate 19}

LM: $x 1600$

Fig. 1. Navicula angusta Grunow

Fig. 2. Navicula lanceolata (Agardh) Ehrenberg

Fig. 3. Navicula peregrina (Ehrenberg) Kützing

Fig. 4. Navicula radiosa Kützing

Fig. 5. Navicula rhyncocephala Kützing

Fig. 6. Navicula c. f. libonensis Schoemann

Fig. 7. Navicula margalithii Lange-Bertalot

Fig. 8. Navicula cari Ehrenberg

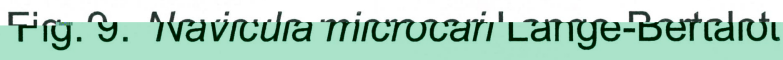

Fig. 10. Navicula microdigitoradiata Lange-Bertalot

Fig. 11. Navicula recens (Lange-Bertalot) Lange-Bertalot

Fig. 12. Navicula c. f. normaloides Cholonoky

Fig. 13. Navicula lusoria Giffen

Fig. 14. Navicula mutica Kützing

Fig. 15. Navicula perminuta Grunow in Van Heurck

Fig. 16. Navicula perminuta Grunow in Van Heurck

Fig. 17. Navicula naumannii Hustedt

Fig. 18. Navicula marina Ralfs in Pritchard

Fig. 19. Navicula pusilla W. Smith 

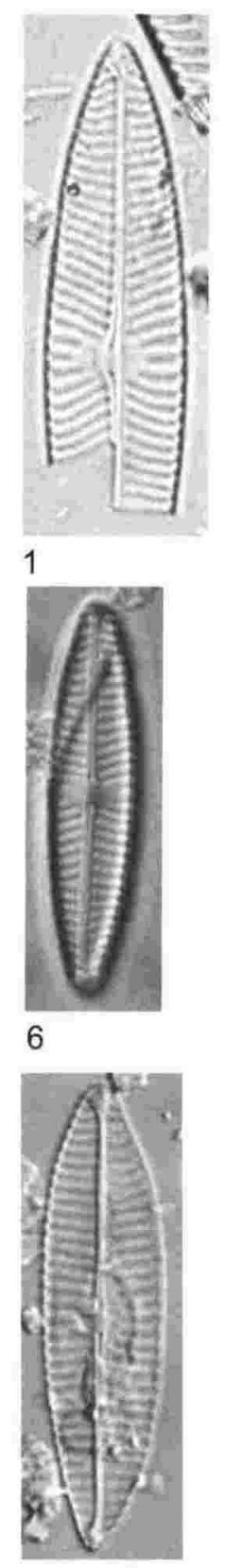

7

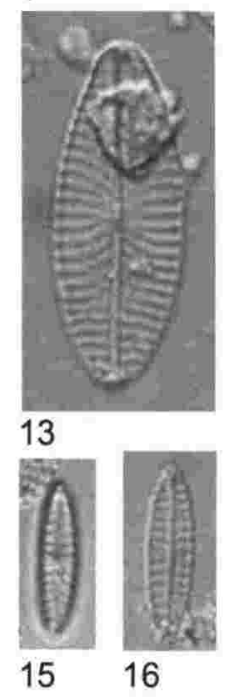

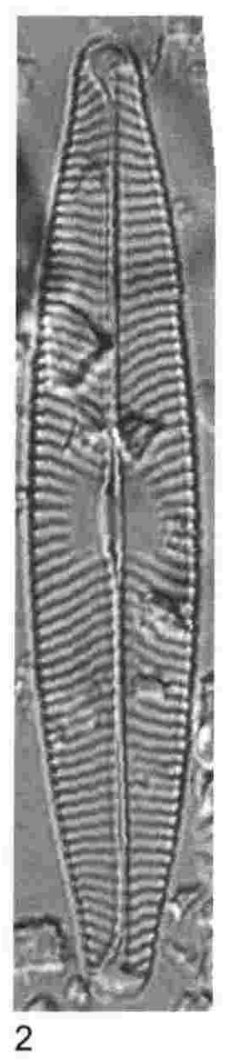
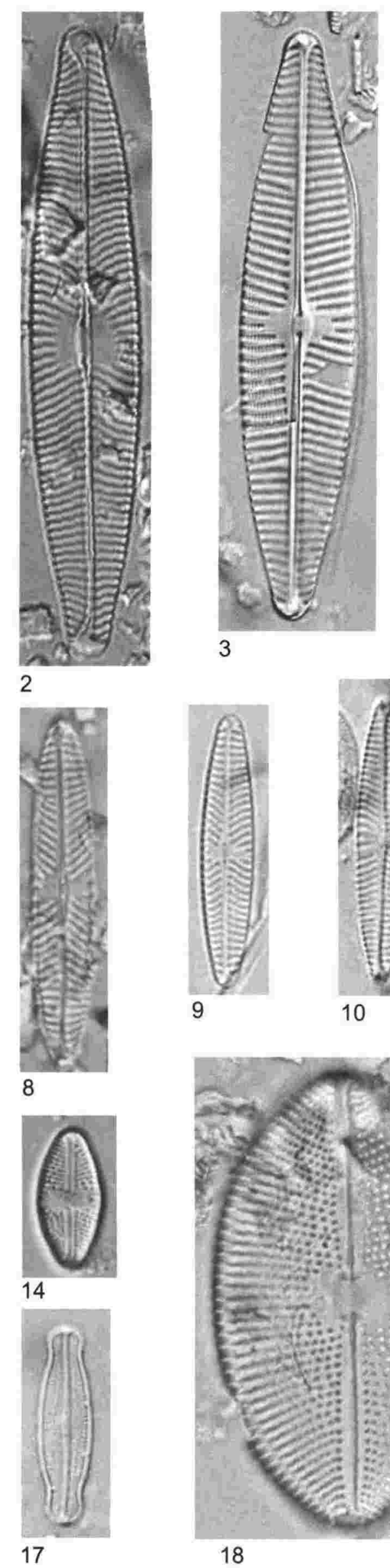
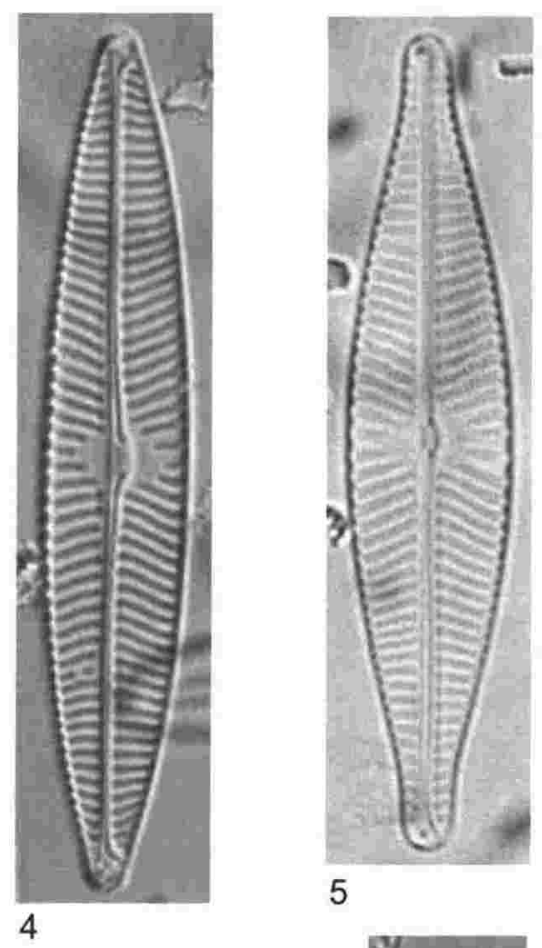

5

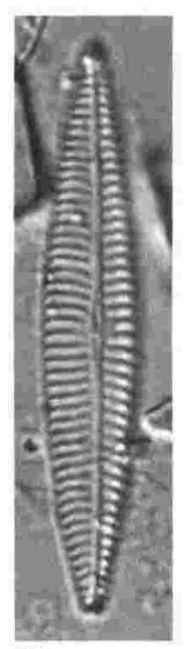

11

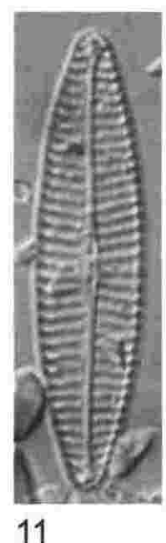

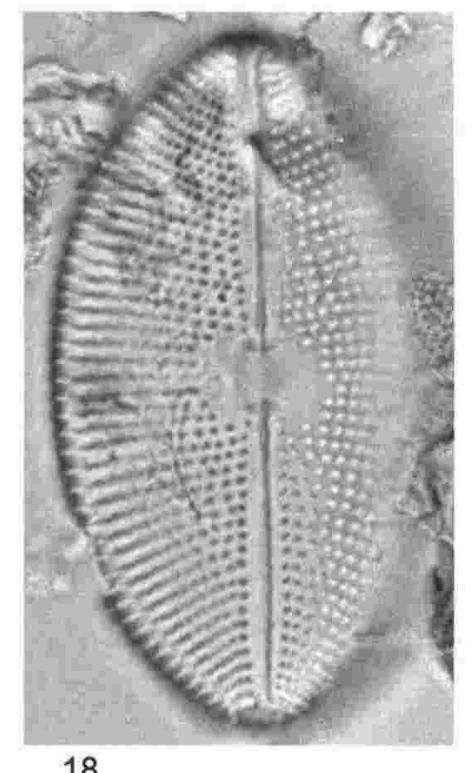

18
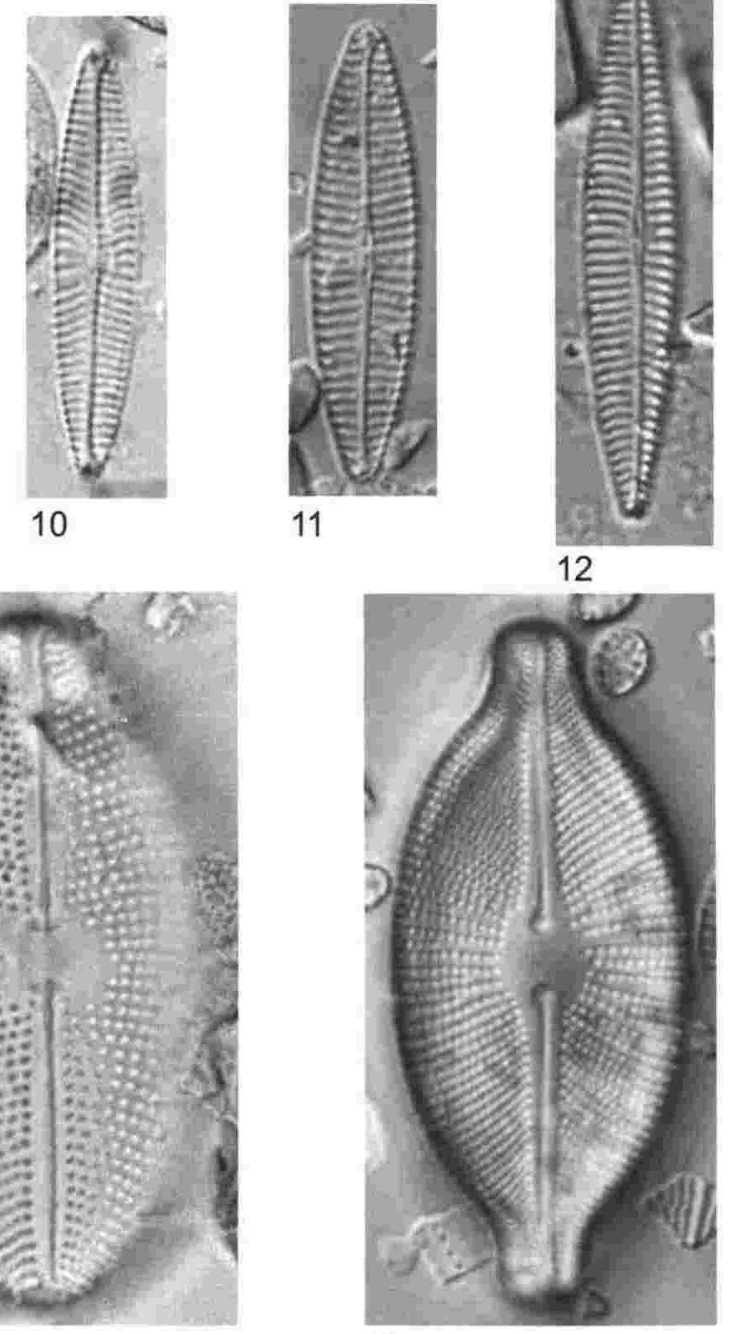

19 


\section{Plate 20}

LM: $x 1600$

Fig. 1. Navicula salinarum Grunow in Cleve \& Grunow var. salinarum

Fig. 2. Navicula c. f. salinarum var. minima Kolbe

Fig. 3. Navicula c. f. stachurae Witkowski

Fig. 4. Navicula submuralis Hustedt

Fig. 5. Navicula tenelloides Hustedt

Fig. 6. Navicula utlandshoerniensis Van Landingham

Fig. 7. Navicula veneta Kützing

Fig. 8. Navicula pseudoscutiformis Hustedt

Fig. 9. Parlibellus bennikei Witkowski, Lange-Bertalot \& Metzeltin

Fig. 10. Stauroneis agrestis Petersen

Fig. 11. Stauroneis kriegerii Patrick

Fig. 12. Stauroneis thermicola (Petersen) Lund

Fig. 13. Stauroneis thermicola (Petersen) Lund

Fig. 14. Stauroneis obtusa Lagerstedt

Fig. 15. Stauroneis phoenicenteron (Nitzsch) Ehrenberg

Fig. 16. Stauroneis salina W. Smith

Fig. 17. Caloneis fusioides (Grunow in Cleve and Grunow) Heiden \& Kolbe

Fig. 18. Trachyneis aspera (Ehrenberg) Cleve 

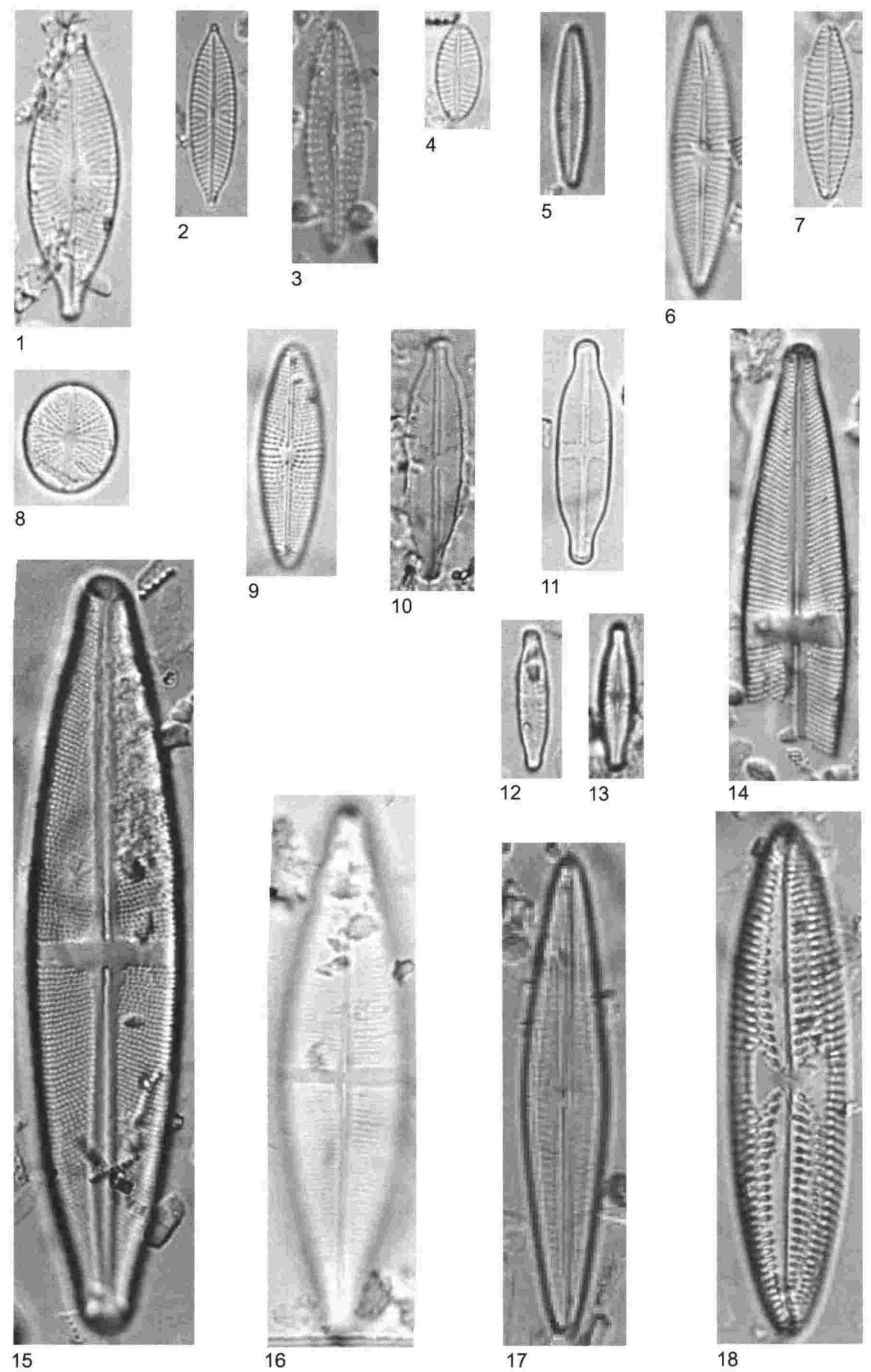


\section{Plate 21}

LM: Fig. 1-11 and 14 x1600; Fig. 12 x800; Fig. 13 x1000

Fig. 1. Pinnularia borealis Ehrenberg

Fig. 2. Pinnularia interrupta W. Smith

Fig. 3. Pinnularia subcapitata Gregory

Fig. 4. Pinnularia subcapitata Gregory

Fig. 5. Pinnularia appendiculata (Agardh) Cleve

Fig. 6. Pinnularia divergentissima (Grunow) Cleve

Fig. 7. Pinnularia obscura Krasske

Fig. 8. Pinnularia braunii (Grunow) Cleve

Fig. 9. Pinnularia brebissonii (Kützing) Rabenhorst

Fig. 10. Pinnularia acrosphaeria Rabenhorst

Fig. 11. Pinnularia microstauron (Ehrenberg) Cleve

Fig. 12. Pinnularia stomatophora (Grunow) Cleve

Fig. 13. Pinnularia viridis (Nitzsch) Ehrenberg

Fig. 14. Pinnularia gibba Ehrenberg 


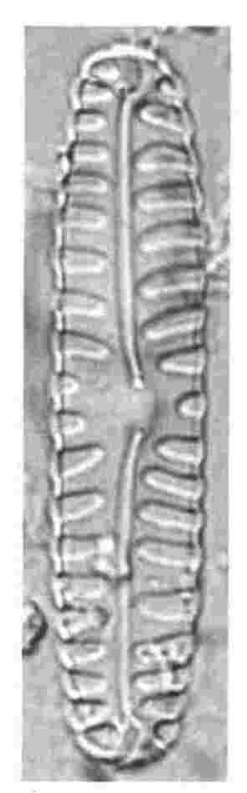

1

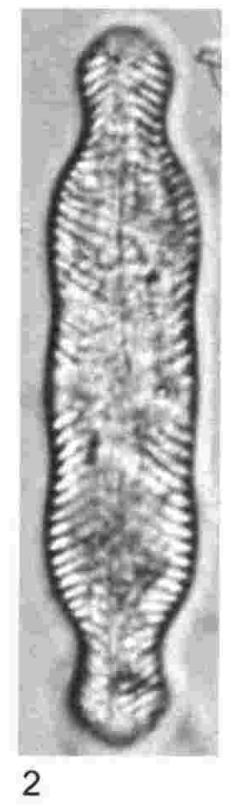

3

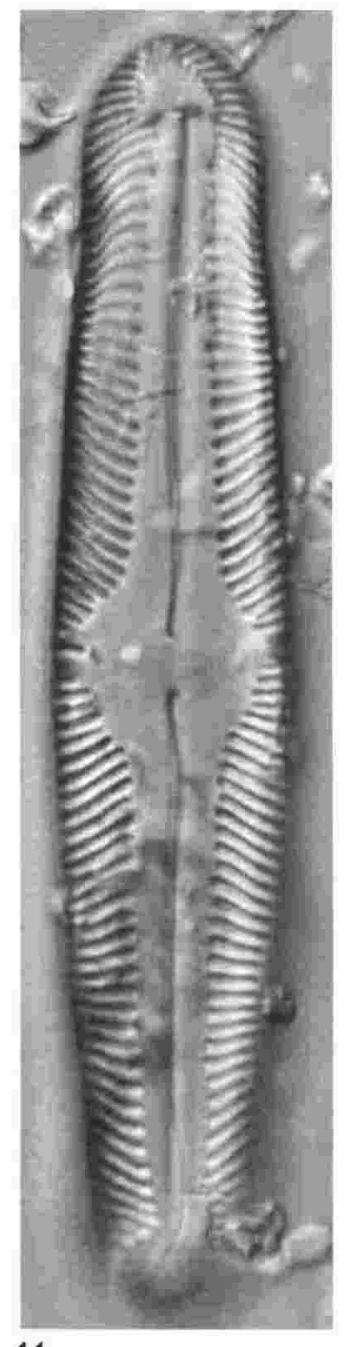

11
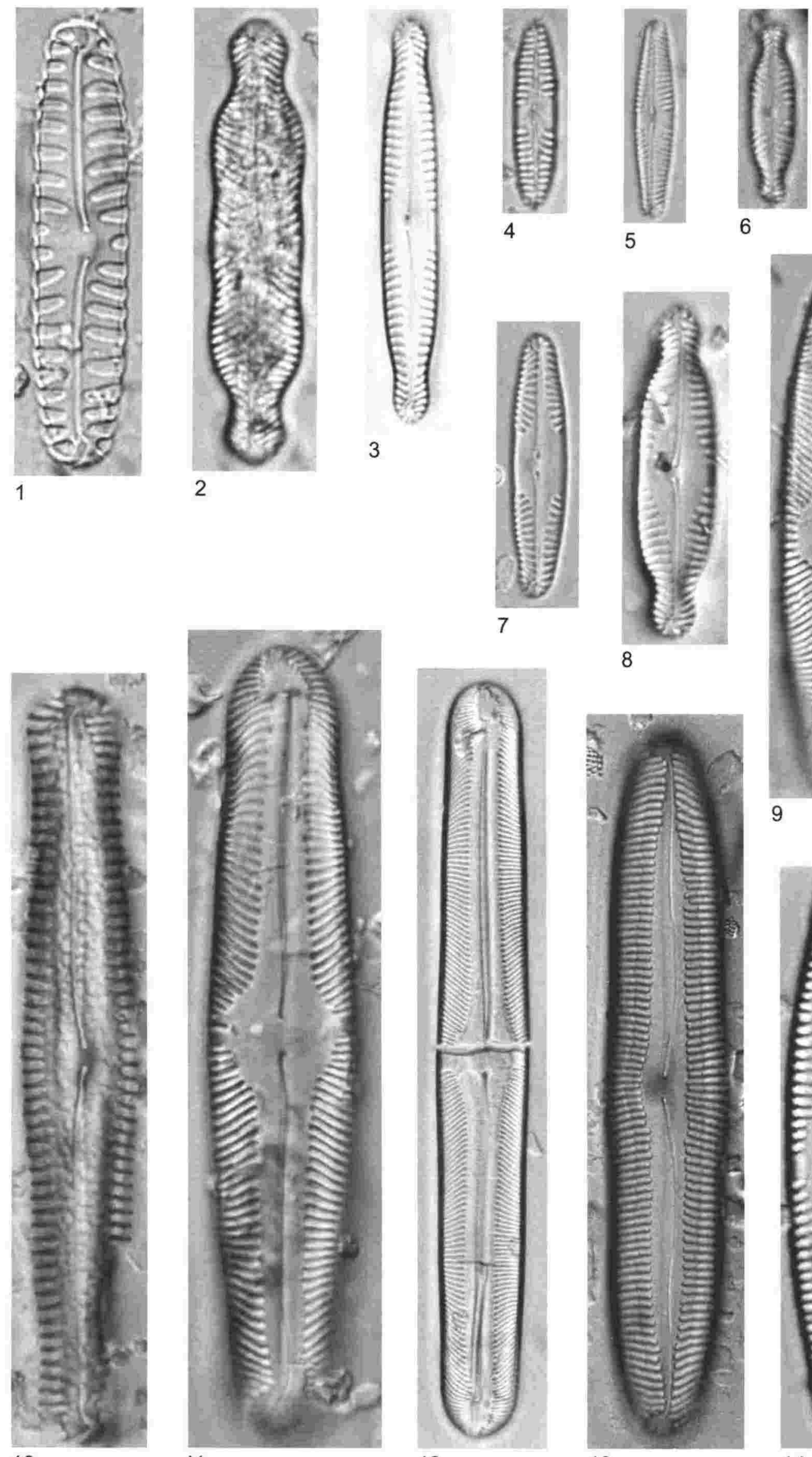

12
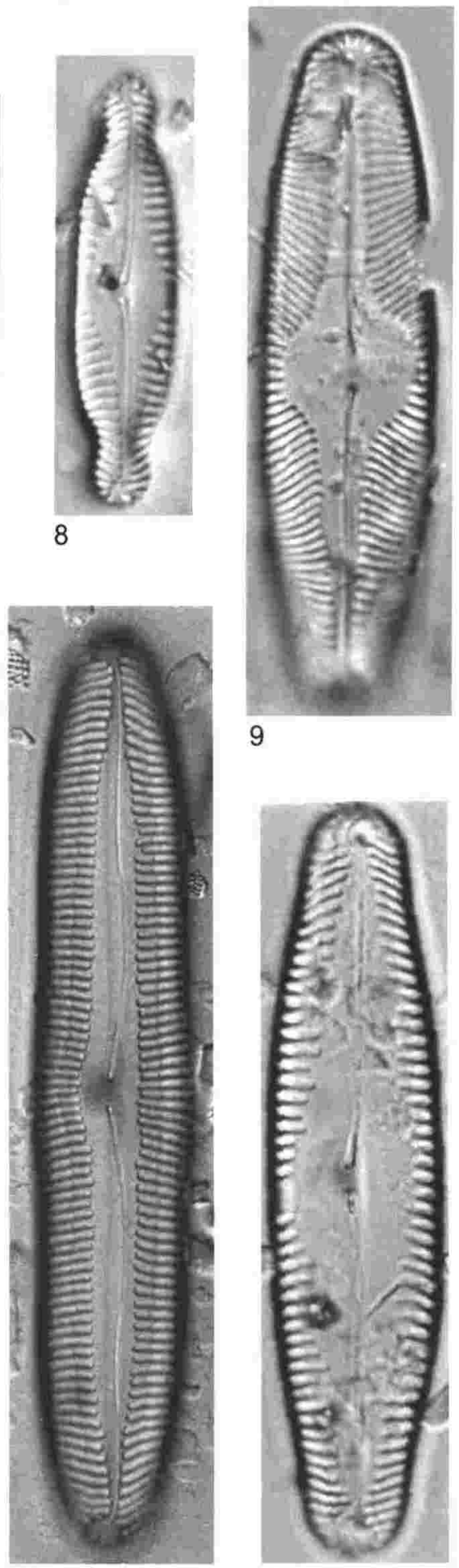

9

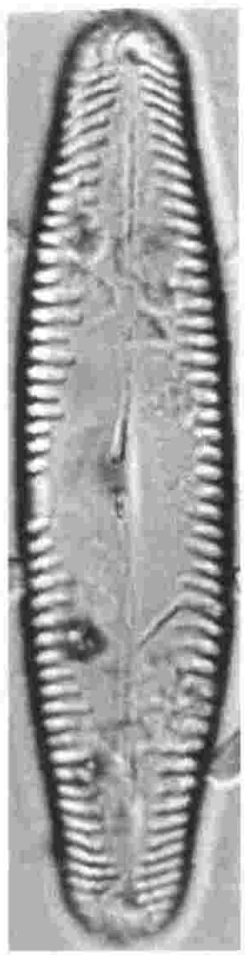

14 


\section{Plate 22}

LM: Fig. 1, 2, 5, 6, 8-12 x1600; Fig. 3 x640; Fig. 4 ×1000

SEM: Fig. $7 \times 4500$

Fig. 1. Nitzschia acuminata (W. Smith) Grunow

Fig. 2. Nitzschia hungarica Grunow

Fig. 3. Nitzschia circumsuta (Bailey) Grunow

Fig. 4. Nitzschia c. f. levidensis var. victoriae (Grunow) Cholnoky

Fig. 5. Nitzschia levidensis (W. Smith) Grunow in Van Heurck var. levidensis

Fig. 6. Nitzschia cocconeiformis Grunow in Cleve \& Möller

Fig. 7. Nitzschia debilis (Arnott) Grunow in Cleve \& Grunow

Fig. 8. Nitzschia compressa (Bailey) Boyer var. compressa

Fig. 9. Nitzschia constricta (Kützing) Ralfs in Pritchard

Fig. 10. Nitzschia granulata Grunow in Cleve \& Möller

Fig. 11. Nitzschia compressa var. balatonis (Grunow) Lange-Bertalot

Fig. 12. Nitzschia debilis (Arnott) Grunow in Cleve \& Grunow 


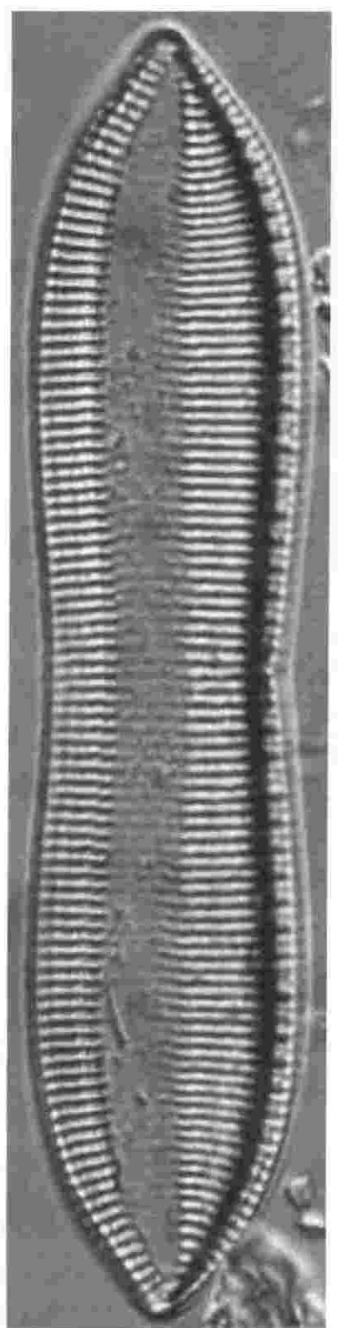

1

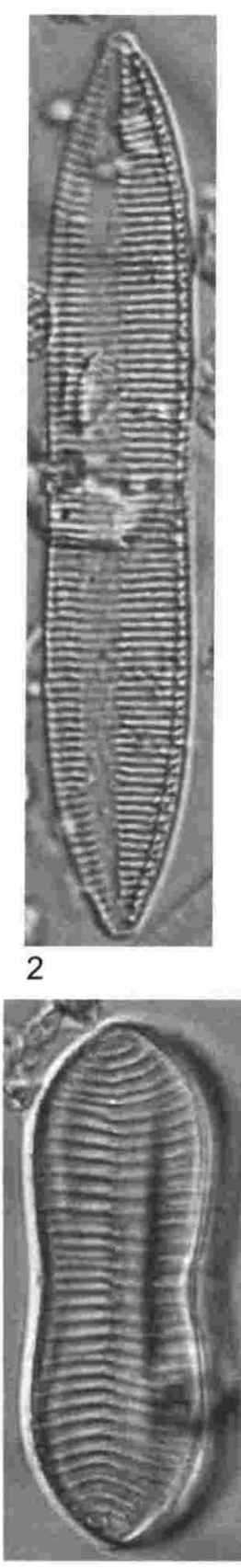

5

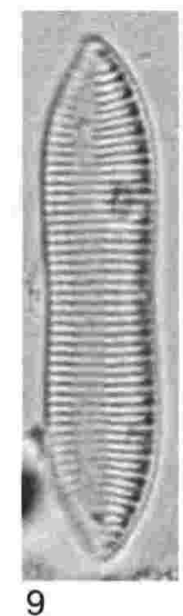

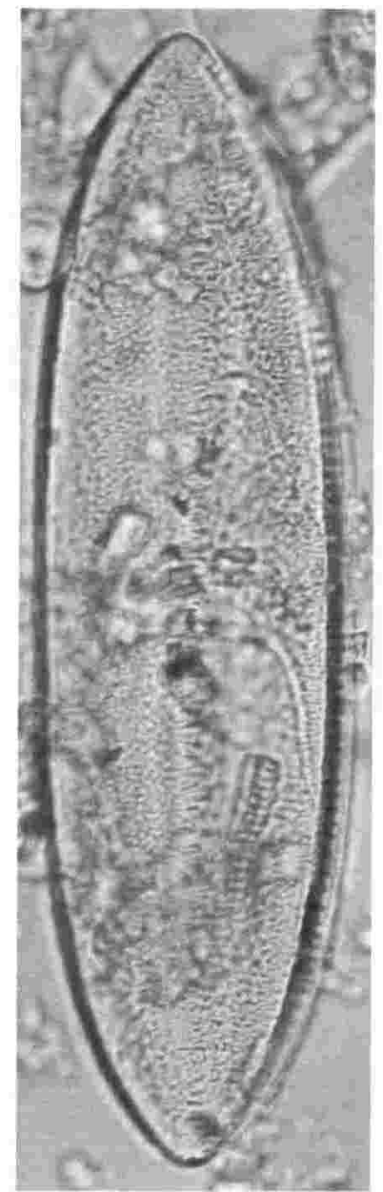

3
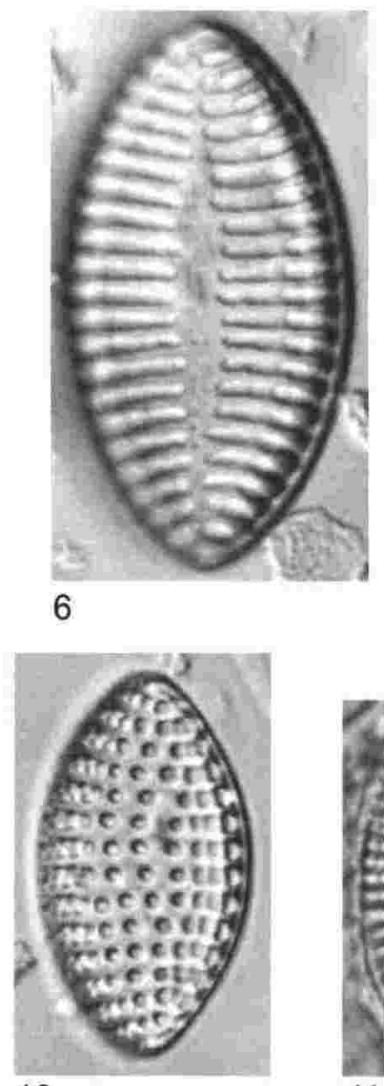

10

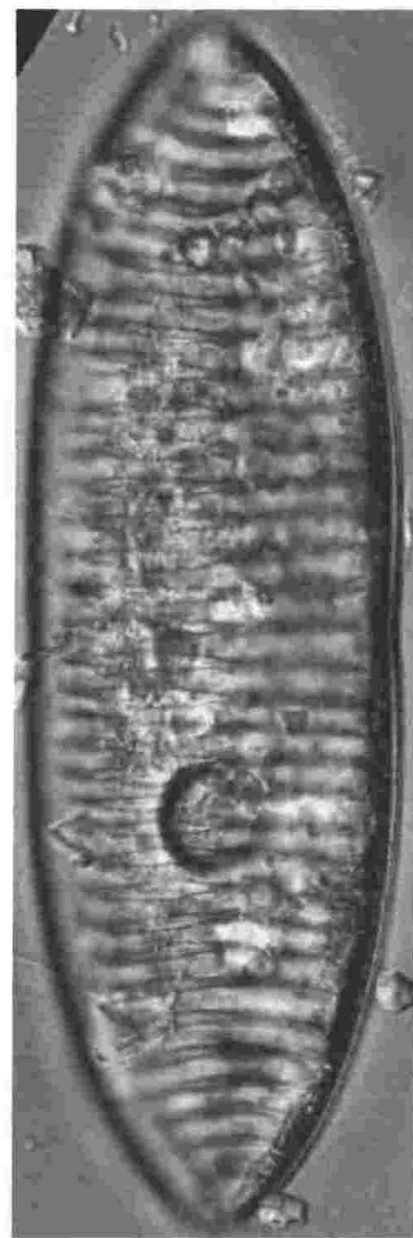

4
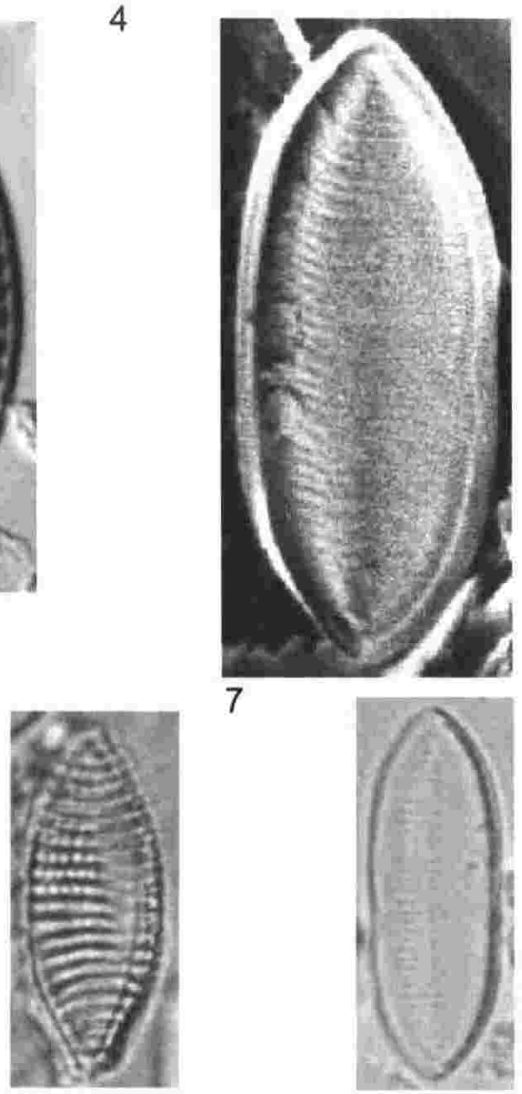

12 


\section{Plate 23}

LM: Fig. 1-19, 22, 24-26 x1600; Fig. 20 ×1000; 21 ×800

SEM: Fig. $23 \times 2700$

Fig. 1. Nitzschia dissipata (Kützing) Grunow var. dissipata

Fig. 2. Nitzschia dissipata var. media (Hantzsch) Grunow

Fig. 3. Nitzschia amphibia Grunow

Fig. 4. Nitzschia c. f. panduriformis Gregory var. panduriformis

Fig. 5. Nitzschia c. f. panduriformis Gregory var. panduriformis

Fig. 6. Nitzschia panduriformis var. continua Grunow in Cleve \& Grunow

Fig. 7. Nitzschia persuadens Cholnoky

Fig. 8. Nitzschia valdestriata Aleem \& Hustedt

Fig. 9. Nitzschia pusilla Grunow

Fig. 10. Nitzschia agnita Hustedt

Fig. 11. Nitzschia paleacea (Grunow) Grunow in Van Heurck

Fig. 12. Nitzschia palea (Kützing) W. Smith

Fig. 13. Nitzschia paleaeformis Hustedt

Fig. 14. Nitzschia perminuta (Grunow) M. Peragallo

Fig. 15. Nitzschia microcephala Grunow in Cleve \& Möller

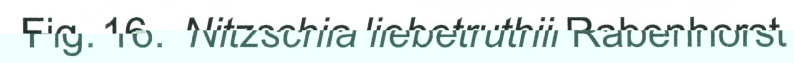

Fig. 17. Nitzschia frustulum (Kützing) Grunow in Cleve \& Grunow

Fig. 18. Nitzschia inconspicua Grunow

Fig. 19. Nitzschia c. f. gracilis Hantzsch

Fig. 20. Nitzschia linearis (Agardh ex W. Smith) W. Smith

Fig. 21. Nitzschia scalpelliformis (Grunow in Cleve \& Möller) Grunow in Cleve \& Grunow

Fig. 22. Nitzschia sigma (Kützing) W. Smith

Fig. 23. Nitzschia terrestris (Petersen) Hustedt

Fig. 24. Nitzschia terrestris (Petersen) Hustedt

Fig. 25. Nitzschia capitellata Hustedt in Schimdt et al.

Fig. 26. Hantzschia amphioxys (Ehrenberg) Grunow in Cleve \& Grunow 

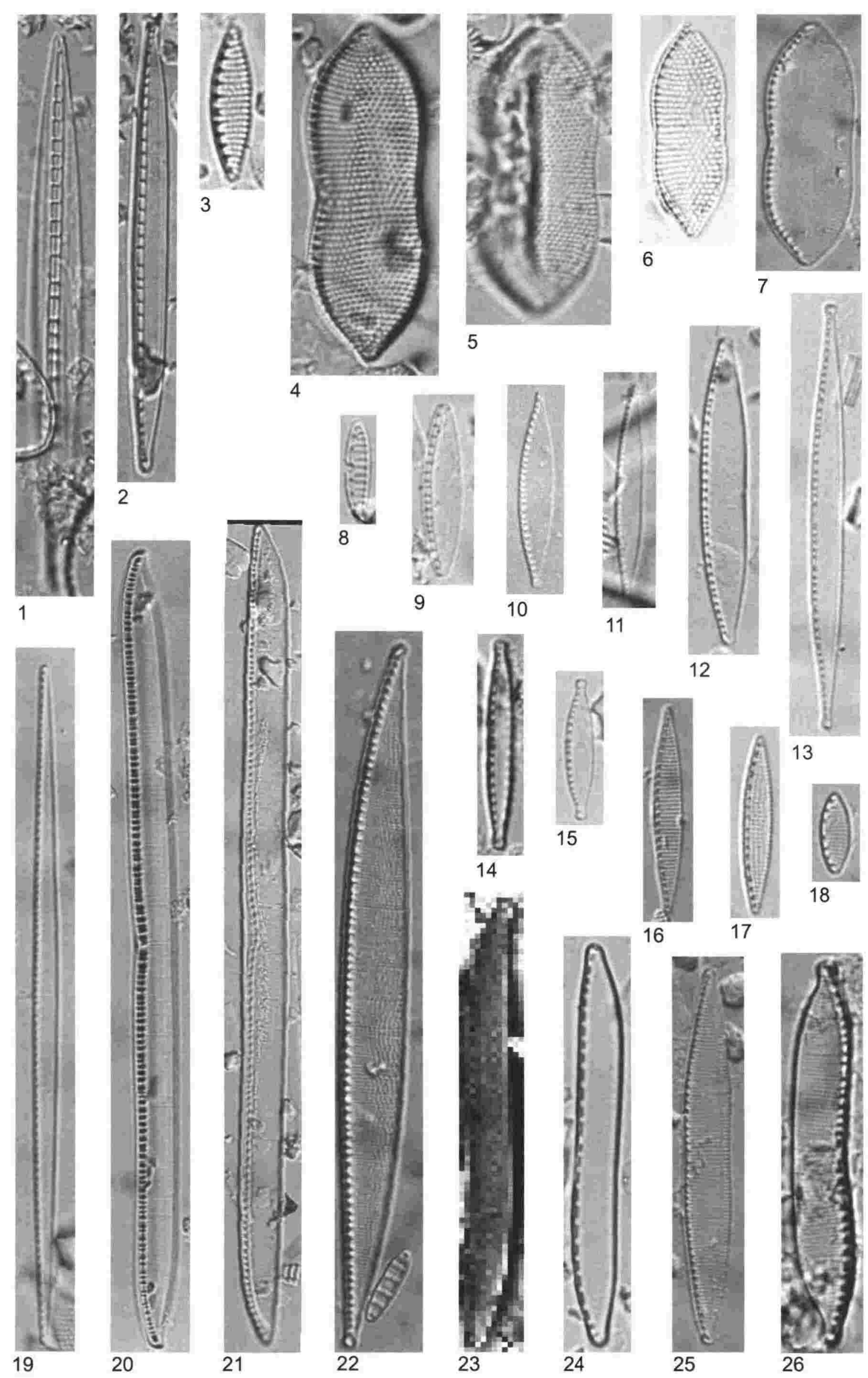

13
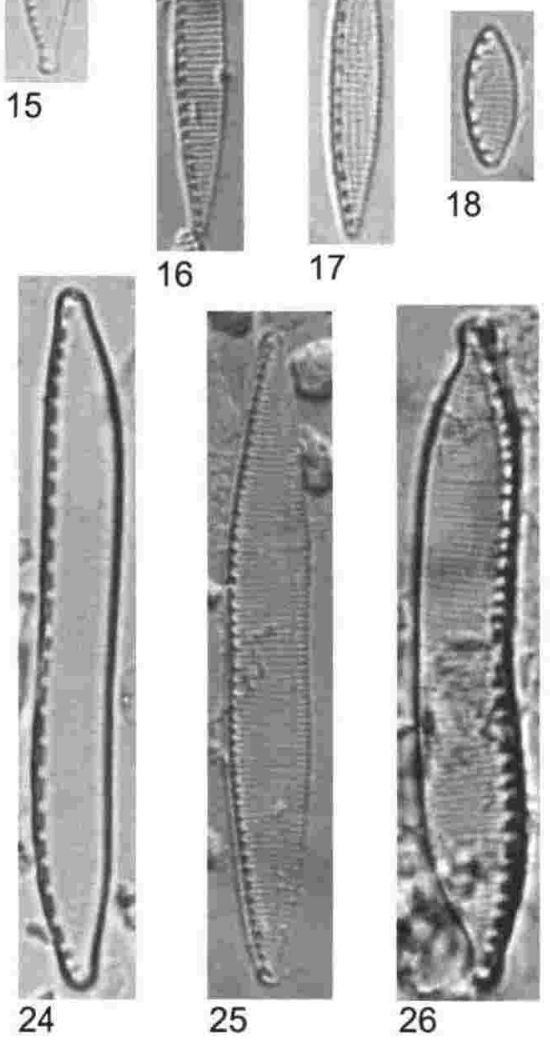


\section{Plate 24}

LM: Fig. 1-5 x1600; Fig. 6 ×1250; Fig. 7 ×1450; Fig. 8 ×1150

Fig. 1. Surirella bohemica Maly

Fig. 2. Surirella fastuosa Ehrenberg

Fig. 3. Surirella c. f. fluviicygnorum John

Fig. 4. Surirella brebissonii Krammer \& Lange-Bertalot

Fig. 5. Surirella comis Schimdt

Fig. 6. Surirella ovalis Brébisson

Fig. 7. Surirella patella Kützing

Fig. 8. Surirella tenera Gregory 

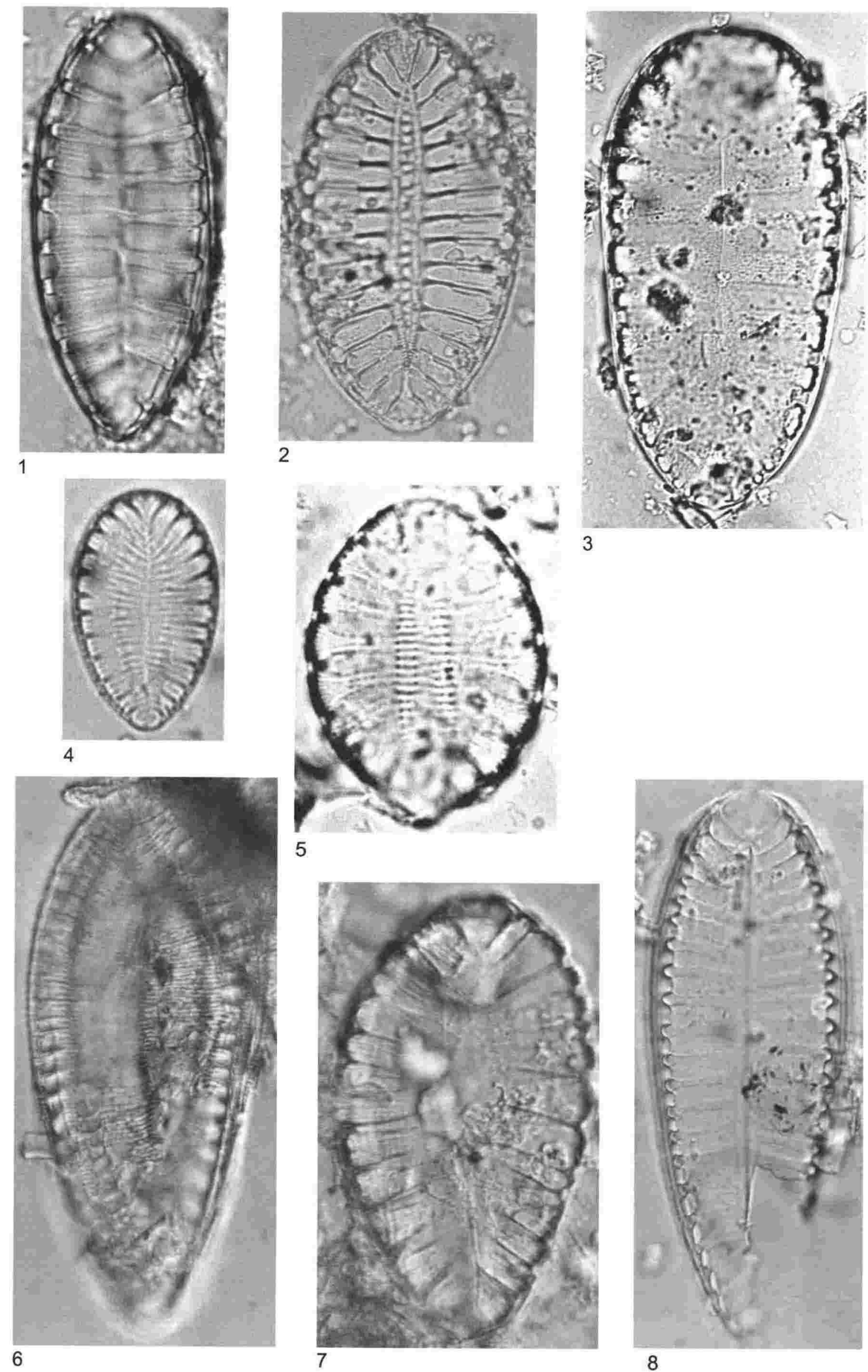


\section{A P P E N D I X V \\ Environmental Variables for Calibration Set Sites}

Table 1: Location and Catchment Characteristics of Calibration Set Sites.

Table 2: $\quad$ A. Environmental Variable Codes and Units for Continuous and Nominal Variables.

B. Environmental Variable Codes and Units for Ordinal Variables. Calibration Set Sites.

Table 3: A. Values of Continuous Environmental Variables of Calibration Set Sites.

B. Values of Nominal and Ordinal Environmental Variables of

Table 4: Repeat Measurement Data for Calibration Set Sites - Salinity, Conductivity, pH, Water Temperature and Water Depth.

Table 5: Grainsize Data for Calibration Set Samples. 
Table 1. Location and Catchment Characteristics of Calibration Set Sites.

\begin{tabular}{|c|c|c|c|c|c|}
\hline NAME & CODE & $\begin{array}{l}\text { GRID } \\
\text { REFERENCE } \\
\text { (NZMS-260) }\end{array}$ & $\begin{array}{l}\text { AREA } \\
\text { Hectares }\end{array}$ & $\begin{array}{l}\text { CATCHMENT } \\
\text { AREA } \\
\text { Hectares }\end{array}$ & $\begin{array}{l}\text { CATCHMENT } \\
\text { RELIEF } \\
\text { Metres }\end{array}$ \\
\hline Bird Hide Lagoon & $\mathrm{BH}$ & R27 708099 & 1 & 57 & 90 \\
\hline Brooklands Lagoon & BL & M35 863533 & 128 & 470 & 10 \\
\hline Coopers Lagoon & $\mathrm{CL}$ & M37 541031 & 36 & 905 & 10 \\
\hline Duck Creek Wetland & DC & R27 696093 & 1 & 785 & 410 \\
\hline Lake George & GE & D46 148158 & 90 & 1484 & 450 \\
\hline Haldane Estuary & $\mathrm{HE}$ & F47 059876 & 194 & 7391 & 399 \\
\hline Island Bay & IB & R27 580829 & 30 & 340 & 178 \\
\hline Kiriwai Lagoon & $\mathrm{KL}$ & R28 861783 & 5 & 93 & 60 \\
\hline Kaituna Lagoon & $\mathrm{KN}$ & M36 829124 & 585 & 6747 & 918 \\
\hline Lake Kohangapiripiri & KP & R27 654809 & 11 & 343 & 250 \\
\hline Kaikorai Stream Mouth & KS & 144078727 & 28 & 4440 & 472 \\
\hline Lake Kohangatera & KT & R27 659798 & 22 & 2035 & 370 \\
\hline Kohangapiripiri Wetland & KW & R27 657814 & 11 & 343 & 250 \\
\hline Lake Brunton & LB & F47 947870 & 24 & 1239 & 128 \\
\hline Lake lanthe & LI & I34 172914 & 456 & 1320 & 790 \\
\hline Lake Pounui & LP & R27 874826 & 43 & 630 & 465 \\
\hline Lake Vincent & LV & F47 904933 & 16 & 298 & 65 \\
\hline Lake Waihola & LW & H45 847609 & 581 & 11875 & 380 \\
\hline Massey Bay & MB & R27 627895 & 8 & 10 & 80 \\
\hline Motukaraka Point & MK & R27 696106 & 450 & 10000 & 555 \\
\hline Lake Mahinapua & MP & J33 384213 & 389 & 6160 & 165 \\
\hline Mahau Sound & MS & P27 846976 & 33 & 27 & 120 \\
\hline Nelson Haven & $\mathrm{NH}$ & O27 374992 & 1233 & 12632 & 1168 \\
\hline Okupe Arm & OA & R25 742403 & 8 & 80 & 206 \\
\hline Okupe Lagoon & $\mathrm{OL}$ & R25 741405 & 8 & 80 & 206 \\
\hline Lake Onoke & ON & R28 892769 & 592 & 10740 & 860 \\
\hline Orore Point & OP & $J 42437528$ & 8 & 1807 & 132 \\
\hline Point Arthur Bay & PA & R27 677870 & 11 & 34 & 178 \\
\hline Paramata Bridge & PB & R27 671099 & 450 & 10000 & 555 \\
\hline Porirua Harbour & $\mathrm{PH}$ & R27 645082 & 262 & 6212 & 458 \\
\hline Plimmerton South Beach & PL & R26 667114 & 65 & 1070 & 247 \\
\hline Pauatahanui Mudflats & PM & R27 706099 & 450 & 10000 & 555 \\
\hline Paraparaumu Lagoon & PR & R26 785347 & 3 & 43 & 6 \\
\hline Papaitonga Swamp & PS & S25 987596 & 9 & 75 & 30 \\
\hline Pauatahanui Inlet & PT & R26 679111 & 450 & 10000 & 555 \\
\hline Paraparaumu Wetland & PW & R27 785345 & 2 & 43 & 5 \\
\hline Rangitata Mouth Wetland & $\mathrm{RM}$ & K38 901673 & 2 & 34 & 5 \\
\hline Rotary Pond & $\mathrm{RP}$ & R27 794352 & 3 & 45 & 8 \\
\hline Shipwreck Bay & SB & F47 943866 & 480 & 1239 & 129 \\
\hline Stuart Pond & SP & O27 445052 & 1 & 5 & 120 \\
\hline Titahi Bay & TB & R27 642098 & 20 & 103 & 100 \\
\hline Tomahawk Lagoon & $\mathrm{TH}$ & 144199756 & 9 & 173 & 255 \\
\hline Tsunami Lagoon & $\mathrm{TL}$ & R28 895766 & 2 & 339 & 78 \\
\hline Te Paeroa Lagoon & TP & X19011294 & 62 & 196 & 40 \\
\hline Taupo Swamp & TS & R26 675129 & 25 & 821 & 240 \\
\hline Kohangatera Wetland & TW & R27 660799 & 22 & 2035 & 370 \\
\hline Lake Wilkie & WK & G47 368971 & 2 & 9 & 20 \\
\hline Waitarakao Lagoon & WL & K39 708482 & 21 & 4646 & 260 \\
\hline Whakamahi Lagoon & WM & X19 907284 & 4 & 110 & 60 \\
\hline Wainono Lagoon & WN & J40 629101 & 359 & 10690 & 1080 \\
\hline
\end{tabular}


Table 2.

A: Environmental Variable Codes and Units for Continuous and Nominal Variables.

\begin{tabular}{|lll|}
\hline ENVIRONMENTAL VARIABLE & CODE & UNIT / CATEGORY \\
\hline Continuous variables: & & \\
Distance from sea (via opening if present) & DIS SEA & Metres \\
Proximity to nearest fresh water inflow & PROXFI & Metres \\
Water depth (where sample collected) & DEPTH & Metres \\
Water Temperature & TEMP & Degrees Celsius \\
Salinity & SAL & Grams per litre \\
Conductivity & COND & Millisiemens per centimetre \\
pH & pH & pH units \\
Sand (>60 microns) & SAND & Percent \\
Mud (<60 microns) & MUD & Percent \\
Organic matter & ORGAN & Percent \\
Carbonate & CARB & Percent \\
Air Temperature (annual for 1998) & AIR TEM & Degrees celcius \\
Precipitation (annual for 1998) & PRECIP & Millimetres \\
Nominal variables: & & \\
Catchment cover (dominant type) & BUSH & 5 Native Bush \\
COVER & FOREST & 4 Exotic forest \\
& SCRUB & 3 Scrub \\
& PASTURE & 2 Pasture \\
& URBAN & 1 Urban \\
Catchment bedrock (dominant type) & FELSIC & 7 Felsic intrusives \\
BEDRK & MAFIC & 6 Mafic intrusives \\
& MAFINT & 5 Mafic / intermediate volcanics \\
& SCHIST & 4 Quartzo-feldspathic schist \\
& TERT & 3 Tertiary \& Mesozoic sandstone, mudstone, conglomerate \\
Rock surfaces / Pebbles & QUAT & 2 Quaternary sands, gravels, tills \\
& HOLO & 1 Holocene coastal sediments \\
& PEBB & 1 Present \\
& & 0 Absent \\
\hline
\end{tabular}


Table 2 .

B: Environmental Variable Codes and Units for Ordinal Variables.

\begin{tabular}{|c|c|c|c|c|c|}
\hline ENVIRONMENTAL VARIABLE & CODE & \multicolumn{4}{|c|}{ CATEGORY } \\
\hline \multicolumn{6}{|l|}{ Ordinal variables: } \\
\hline \multirow[t]{10}{*}{ Water body type } & \multirow[t]{10}{*}{$\mathrm{H} 2 \mathrm{O} \mathrm{BOD}$} & \multicolumn{4}{|c|}{10 Open bay } \\
\hline & & \multicolumn{4}{|c|}{9 Sheltered embayment } \\
\hline & & \multicolumn{4}{|c|}{8 Inlet } \\
\hline & & \multicolumn{4}{|c|}{7 Predominantly open lagoon } \\
\hline & & \multicolumn{4}{|c|}{6 Predominantly closed lagoon } \\
\hline & & \multicolumn{4}{|c|}{5 Permanently closed lagoon } \\
\hline & & \multicolumn{4}{|c|}{4 Coastal lake } \\
\hline & & \multicolumn{4}{|c|}{3 Lake } \\
\hline & & \multicolumn{4}{|c|}{2 Coastal wetland } \\
\hline & & \multicolumn{4}{|c|}{1 Wetland } \\
\hline \multirow[t]{9}{*}{ Exposure to salt } & \multirow[t]{9}{*}{ EXPOS } & \multicolumn{4}{|c|}{9 Continuous open connection with sea } \\
\hline & & \multicolumn{4}{|c|}{8 Daily connection with sea (tidal) } \\
\hline & & \multicolumn{4}{|c|}{7 Daily connection with inlet (tidal) } \\
\hline & & \multicolumn{4}{|c|}{6 Seasonal / annual influx from sea } \\
\hline & & \multicolumn{4}{|c|}{5 Seasonal / annual influx from inlet / lagoon } \\
\hline & & & Seepage through barrie & $\mathrm{r} / \mathrm{g}$ & roundwater \\
\hline & & & Windblown only - freque & & \\
\hline & & & Windblown only - occas & iona & \\
\hline & & 1 & No exposure & & 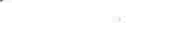 \\
\hline Size of nearest fresh water inflow & SIZE FI & 4 & River & & \\
\hline & & & Stream & & \\
\hline & & & Creek & & \\
\hline [1] & & & Runoff only & & \\
\hline Salinity Range ( $\max$ - min of measurements) & RANGE & 1 & $<0.1 \mathrm{gl}^{-1}$ & & \\
\hline & & & $0.1-1 \mathrm{gl}^{-1}$ & & \\
\hline & & & $1-5 g^{-1}$ & & \\
\hline & & & $5-10 \mathrm{gl}^{-1}$ & & \\
\hline & & & $10-15 \mathrm{gl}^{-1}$ & & \\
\hline & & & $15-20 \mathrm{gl}^{-1}$ & & \\
\hline & & 7 & $20-25 \mathrm{gl}^{-1}$ & & \\
\hline Height above mean sea level & ELEVTN & 1 & $<0$ metres above mear & sea & level \\
\hline & & 2 & $0-2.5 \mathrm{~m}$ & & \\
\hline & & & $2.5-5 m$ & & \\
\hline & & & $5-10 m$ & & \\
\hline & & & $10-15 \mathrm{~m}$ & & \\
\hline & & 6 & $>15 \mathrm{~m}$ & & \\
\hline Shading & SHADE & 4 & Shaded for most of day & & \\
\hline & & & Shaded part of day by & ills & and plants \\
\hline & & & Shaded part of day by $\mathrm{r}$ & ills & r plants \\
\hline & & 1 & Exposed throughout da & & \\
\hline Aquatic plant growth & PLANT & 4 & Abundant & 2 & Present \\
\hline & & 3 & Frequent & 1 & Occasional \\
\hline Flow & FLOW & 6 & Continuously fast & 3 & Periodic \\
\hline & & 5 & Periodically fast & 2 & Slow \\
\hline & & 4 & Continuous & 1 & None \\
\hline Exposure to air & EXPO & 5 & Daily & & \\
\hline & & 4 & Monthly & 2 & Occasionally \\
\hline & & 3 & Seasonally / Annually & 1 & Never \\
\hline
\end{tabular}


Table 3.

A: Values of Continuous Environmental Variables of Calibration Set Sites.

See Table 1 for site codes and Table 2 for environmental variable codes.

\begin{tabular}{|c|c|c|c|c|c|c|c|c|c|c|c|c|c|}
\hline SITE & DIS SEA & PROXFI & DEPTH & TEMP & SAL & COND & $\mathrm{pH}$ & SAND & MUD & ORGAN & CARB & AIR TEM & PRECIP \\
\hline $\mathrm{BH}$ & 5300 & 100 & 0.04 & 18.79 & 18.54 & 28.16 & 8.17 & 63.21 & 13.82 & 15.83 & 6.57 & 14.4 & 1073.6 \\
\hline $\mathrm{BL}$ & 4600 & 20 & 0.03 & 30.35 & 22.05 & 31.9 & 7.85 & 0.00 & 38.32 & 40.50 & 18.26 & 12.31 & 534.7 \\
\hline $\mathrm{CL}$ & 150 & 350 & 0.4 & 23.2 & 0.1 & 0.126 & 8.07 & 83.95 & 1.71 & 11.40 & 2.93 & 12.31 & 726.8 \\
\hline DC & 4500 & 10 & 0.02 & 17.7 & 12.7 & 19.86 & 7.67 & 0.99 & 15.07 & 69.04 & 14.40 & 14.4 & 1073.6 \\
\hline GE & 1900 & 25 & 0.02 & 25.35 & 0.1 & 0.271 & 8.16 & 82.05 & 11.65 & 2.88 & 2.91 & 10.54 & 1165.2 \\
\hline $\mathrm{HE}$ & 1450 & 350 & 0.45 & 18.9 & 26.98 & 38 & 7.98 & 91.28 & 3.39 & 0.00 & 5.57 & 10.54 & 1346.6 \\
\hline IB & 0 & 50 & 0.6 & 14.91 & 29.09 & 43.66 & 8.01 & 89.90 & 0.10 & 0.18 & 9.88 & 14.4 & 985 \\
\hline $\mathrm{KL}$ & 3300 & 300 & 0.49 & 14.23 & 4.63 & 7.77 & 7.62 & 2.64 & 68.88 & 23.41 & 6.27 & 13.81 & 994.9 \\
\hline $\mathrm{KN}$ & 3800 & 600 & 0.03 & 20.7 & 0.55 & 0.977 & 6.17 & 38.54 & 52.12 & 3.11 & 4.73 & 12.31 & 534.7 \\
\hline KP & 300 & 350 & 0.45 & 15.45 & 0.28 & 0.517 & 8.14 & 75.94 & 2.59 & 19.13 & 2.25 & 14.4 & 985 \\
\hline KS & 1000 & 1500 & 0.3 & 25.2 & 13.1 & 19.76 & 8.02 & 72.25 & 19.17 & 3.69 & 4.17 & 11 & 588.6 \\
\hline KT & 200 & 350 & 0.27 & 14.1 & 0.63 & 1.11 & 7.75 & 85.22 & 2.33 & 10.77 & 1.69 & 14.4 & 985 \\
\hline KW & 900 & 0 & 0.1 & 20.2 & 0.3 & 0.546 & 6.95 & 2.73 & 20.42 & 66.94 & 9.85 & 14.4 & 985 \\
\hline LB & 1300 & 400 & 0.06 & 25 & 8.35 & 13.05 & 8.03 & 32.70 & 18.62 & 40.47 & 7.71 & 10.54 & 1346.6 \\
\hline LI & 8000 & 20 & 0.4 & 22.95 & 0 & 0.033 & 7.23 & 77.53 & 8.69 & 7.84 & 5.21 & 12.31 & 2988.1 \\
\hline LP & 4800 & 1000 & 0.45 & 13.78 & 0.1 & 0.171 & 8.99 & 89.34 & 7.28 & 0.54 & 2.78 & 13.81 & 994.9 \\
\hline LV & 925 & 10 & 0.2 & 23.3 & 0.1 & 0.279 & 8.12 & 94.76 & 2.83 & 0.40 & 1.78 & 10.54 & 755.2 \\
\hline LW & 1500 & 850 & 0.4 & 28.55 & 3.75 & 6.22 & 8.62 & 81.43 & 8.29 & 5.55 & 3.73 & 11 & 660.9 \\
\hline MB & 0 & 5 & 0.6 & 17.73 & 28.96 & 42.78 & 8.06 & 73.31 & 3.88 & 6.13 & 16.97 & 14.4 & 985 \\
\hline MK & 4200 & 650 & 0.04 & 19.6 & 22.35 & 35.71 & 7.92 & 51.17 & 29.91 & 10.88 & 7.13 & 14.4 & 1073.6 \\
\hline MP & 1150 & 1100 & 0.4 & 23.2 & 0 & 0.046 & 7.75 & 78.71 & 8.63 & 9.30 & 2.05 & 12.31 & 2988.1 \\
\hline MS & 3000 & 80 & 0.6 & 19.76 & 23.55 & 33.6 & 8.09 & 72.52 & 11.21 & 6.10 & 9.93 & 13.5 & 1506 \\
\hline $\mathrm{NH}$ & 8000 & 200 & 0.4 & 10.95 & 11.9 & 20.55 & 7.73 & 75.84 & 8.85 & 5.87 & 9.07 & 13.41 & 1011.3 \\
\hline OA & 150 & 650 & 0.03 & 19.5 & 26 & 40.1 & 8.42 & 0.00 & 56.28 & 33.57 & 9.31 & 14.4 & 1326.8 \\
\hline $\mathrm{OL}$ & 350 & 150 & 0.04 & 19.6 & 21.85 & 29.8 & 8.89 & 60.29 & 21.52 & 12.38 & 5.33 & 14.4 & 1326.8 \\
\hline ON & 400 & 600 & 0.33 & 11.26 & 6.87 & 11.48 & 8.48 & 89.08 & 0.21 & 7.50 & 2.98 & 13.81 & 994.9 \\
\hline OP & 350 & 100 & 0.1 & 23.95 & 2.5 & 4.28 & 9.05 & 85.56 & 2.46 & 0.48 & 11.33 & 11.16 & 367.5 \\
\hline PA & 0 & 20 & 0.7 & 18.94 & 32.86 & 45.18 & 8.05 & 94.36 & 0.09 & 0.09 & 5.59 & 14.4 & 985 \\
\hline PB & 0 & 1100 & 0.4 & 17.97 & 28.8 & 42.64 & 8.06 & 78.41 & 9.25 & 4.26 & 8.32 & 14.4 & 1073.6 \\
\hline $\mathrm{PH}$ & 3800 & 600 & 0.35 & 19.2 & 26.7 & 41.1 & 8.11 & 78.60 & 1.35 & 10.96 & 7.99 & 14.4 & 1073.6 \\
\hline PL & 0 & 20 & 0.4 & 16.26 & 29.28 & 40.72 & 8.13 & 78.96 & 0.80 & 2.42 & 17.84 & 14.4 & 1073.6 \\
\hline PM & 5200 & 150 & 0.15 & 17.89 & 22.43 & 33.4 & 8.21 & 80.87 & 2.37 & 6.70 & 9.86 & 14.4 & 1073.6 \\
\hline PR & 350 & 20 & 0.1 & 19.83 & 4.08 & 6.46 & 8.15 & 64.51 & 18.88 & 7.30 & 9.27 & 14.4 & 1244.1 \\
\hline PS & 5900 & 40 & 0.01 & 21.95 & 0.2 & 0.341 & 7.09 & 0.00 & 19.75 & 69.36 & 10.42 & 13.87 & 964.5 \\
\hline PT & 3000 & 30 & 0.7 & 18.39 & 28.28 & 42.13 & 7.98 & 56.27 & 18.30 & 14.00 & 10.81 & 14.4 & 1073.6 \\
\hline PW & 450 & 15 & 0.05 & 20.35 & 8.7 & 12.99 & 7.9 & 88.44 & 4.27 & 1.36 & 6.03 & 14.4 & 1244.1 \\
\hline RM & 50 & 150 & 0.4 & 18.4 & 0.1 & 0.122 & 6.17 & 0.00 & 61.03 & 38.10 & 0.00 & 11.64 & 379.9 \\
\hline RP & 450 & 100 & 0.18 & 22.5 & 0.87 & 1.582 & 9.5 & 89.43 & 2.96 & 0.61 & 6.93 & 14.4 & 1244.1 \\
\hline SB & 0 & 1300 & 0.3 & 18.6 & 30 & 41.7 & 8.04 & 90.47 & 0.13 & 0.03 & 9.32 & 10.54 & 1346.6 \\
\hline SP & 50 & 40 & 0.3 & 11.65 & 0.3 & 0.589 & 6.61 & 3.63 & 77.49 & 13.40 & 5.48 & 13.41 & 1011.3 \\
\hline TB & 0 & 100 & 0.63 & 17.47 & 28.69 & 42.49 & 8.05 & 79.71 & 0.33 & 8.01 & 11.38 & 14.4 & 1073.6 \\
\hline TH & 1300 & 250 & 0.5 & 21.65 & 0.5 & 0.865 & 7.7 & 31.36 & 18.73 & 41.43 & 8.36 & 11 & 588.6 \\
\hline TL & 500 & 20 & 0.6 & 15.3 & 4.25 & 7.298 & 7.76 & 65.51 & 27.22 & 3.32 & 3.47 & 13.81 & 994.9 \\
\hline TP & 550 & 1250 & 0.03 & 18.95 & 3.5 & 6.51 & 5.25 & 32.10 & 51.37 & 11.49 & 4.19 & 15.19 & 1287.7 \\
\hline TS & 1200 & 2 & 0.04 & 15.65 & 0.2 & 0.383 & 6.78 & 0.00 & 9.34 & 84.66 & 5.90 & 14.4 & 1073.6 \\
\hline TW & 350 & 200 & 0.5 & 12.75 & 0.3 & 0.521 & 7.17 & 6.75 & 9.05 & 83.30 & 0.72 & 14.4 & 985 \\
\hline WK & 300 & 75 & 0.08 & 27.45 & 0.1 & 0.251 & 5.08 & 0.00 & 0.70 & 98.06 & 1.21 & 10.54 & 1346.6 \\
\hline WL & 320 & 250 & 0.03 & 22.5 & 0.1 & 0.146 & 8.95 & 65.24 & 29.21 & 2.10 & 2.88 & 11.64 & 413.7 \\
\hline WM & 1800 & 200 & 0.06 & 21.15 & 12.95 & 21.58 & 8.61 & 67.90 & 14.94 & 6.79 & 10.15 & 15.19 & 1287.7 \\
\hline WN & 150 & 500 & 0.03 & 19.4 & 1.55 & 2.655 & 8.16 & 23.77 & 60.49 & 8.78 & 5.94 & 11.16 & 378.9 \\
\hline
\end{tabular}


Table 3.

B: Values of Nominal and Ordinal Environmental Variables of Calibration Set Sites. See Table 1 for site codes and Table 2 for environmental variable codes.

\begin{tabular}{|c|c|c|c|c|c|c|c|c|c|c|c|c|}
\hline SITE & COVER & BEDRK & PEBB & $\mathrm{H} 2 \mathrm{O} \mathrm{BOD}$ & EXPOS & SIZE FI & RANGE & ELEVTN & SHADE & PLANT & FLOW & EXPO \\
\hline $\mathrm{BH}$ & 2 & 3 & 0 & 7 & 7 & 2 & 7 & 2 & 1 & 2 & 2 & 4 \\
\hline$B L$ & 3 & 1 & 0 & 8 & 8 & 2 & 4 & 2 & 1 & 2 & 2 & 3 \\
\hline $\mathrm{CL}$ & 2 & 1 & 0 & 5 & 4 & 2 & 1 & 2 & 2 & 3 & 3 & 2 \\
\hline$D C$ & 2 & 3 & 0 & 2 & 7 & 2 & 5 & 2 & 3 & 4 & 1 & 2 \\
\hline GE & 3 & 6 & 0 & 4 & 2 & 2 & 1 & 4 & 1 & 3 & 3 & 2 \\
\hline $\mathrm{HE}$ & 5 & 3 & 1 & 8 & 8 & 2 & 4 & 1 & 1 & 2 & 4 & 3 \\
\hline IB & 1 & 3 & 1 & 10 & 9 & 1 & 4 & 1 & 2 & 2 & 6 & 4 \\
\hline$K L$ & 2 & 2 & 1 & 6 & 5 & 2 & 4 & 2 & 2 & 3 & 3 & 2 \\
\hline $\mathrm{KN}$ & 2 & 5 & 0 & 6 & 5 & 3 & 2 & 2 & 1 & 1 & 2 & 2 \\
\hline $\mathrm{KP}$ & 2 & 3 & 1 & 5 & 4 & 2 & 1 & 2 & 3 & 4 & 3 & 1 \\
\hline KS & 1 & 3 & 1 & 7 & 8 & 3 & 3 & 2 & 1 & 2 & 3 & 3 \\
\hline $\mathrm{KT}$ & 3 & 3 & 1 & 6 & 6 & 2 & 2 & 2 & 2 & 2 & 3 & 2 \\
\hline KW & 2 & 3 & 0 & 1 & 2 & 2 & 1 & 3 & 3 & 4 & 1 & 1 \\
\hline LBB & 2 & 2 & 0 & 7 & 6 & 2 & 2 & 2 & 2 & 3 & 3 & 2 \\
\hline LI & 5 & 2 & 1 & 3 & 1 & 2 & 1 & 6 & 3 & 4 & 3 & 1 \\
\hline L.P & 5 & 2 & 0 & 3 & 1 & 2 & 1 & 6 & 3 & 4 & 3 & 1 \\
\hline LV & 2 & 2 & 0 & 4 & 2 & 2 & 1 & 4 & 3 & 3 & 3 & 2 \\
\hline LW & 2 & 4 & 1 & 6 & 5 & 2 & 3 & 6 & 2 & 3 & 3 & 1 \\
\hline MB & 4 & 3 & 1 & 9 & 9 & 1 & 3 & 1 & 2 & 2 & 5 & 4 \\
\hline MK & 2 & 3 & 1 & 8 & 8 & 3 & 7 & 2 & 1 & 2 & 3 & 5 \\
\hline MP & 5 & 2 & 1 & 4 & 2 & 2 & 1 & 2 & 3 & 4 & 3 & 1 \\
\hline MS & 5 & 3 & 1 & 8 & 8 & 2 & 3 & 1 & 2 & 1 & 4 & 3 \\
\hline $\mathrm{NH}$ & 5 & 5 & 1 & 8 & 8 & 2 & 3 & 2 & 2 & 1 & 3 & 5 \\
\hline$O A$ & 3 & 3 & 0 & 5 & 4 & 1 & 4 & 2 & 1 & 2 & 2 & 3 \\
\hline OL & 3 & 3 & 0 & 5 & 4 & 1 & 3 & 2 & 2 & 3 & 2 & 1 \\
\hline ON & 2 & 2 & 1 & 7 & 6 & 2 & 7 & 2 & 2 & 1 & 5 & 3 \\
\hline OP & 2 & 2 & 0 & 6 & 6 & 2 & 3 & 2 & 2 & 4 & 2 & 2 \\
\hline PA & 3 & 3 & 1 & 9 & 9 & 1 & 6 & 1 & 2 & 2 & 6 & 4 \\
\hline PB & 2 & 3 & 1 & 9 & 9 & 2 & 4 & 1 & 2 & 2 & 5 & 3 \\
\hline $\mathrm{PH}$ & 1 & 3 & 1 & 8 & 8 & 2 & 4 & 1 & 2 & 3 & 3 & 4 \\
\hline PL & 2 & 3 & 1 & 10 & 9 & 1 & 6 & 1 & 2 & 1 & 5 & 4 \\
\hline PM & 2 & 3 & 0 & 8 & 8 & 3 & 7 & 2 & 1 & 2 & 3 & 5 \\
\hline PR & 1 & 1 & 0 & 6 & 6 & 2 & 4 & 2 & 1 & 2 & 2 & 3 \\
\hline PS & 5 & 1 & 0 & 1 & 1 & 2 & 1 & 5 & 4 & 4 & 1 & 2 \\
\hline PT & 2 & 3 & 1 & 8 & 8 & 2 & 4 & 1 & 2 & 2 & 3 & 4 \\
\hline PW & 1 & 1 & 0 & 2 & 5 & 2 & 7 & 2 & 3 & 4 & 1 & 2 \\
\hline RM & 2 & 1 & 0 & 5 & 4 & 2 & 1 & 2 & 3 & 4 & 1 & 1 \\
\hline RP & 1 & 1 & 1 & 6 & 5 & 2 & 2 & 2 & 2 & 3 & 3 & 1 \\
\hline SB & 2 & 2 & 1 & 10 & 9 & 3 & 4 & 1 & 1 & 2 & 6 & 5 \\
\hline SP & 2 & 7 & 1 & 5 & 3 & 1 & 1 & 2 & 2 & 3 & 2 & 2 \\
\hline TB & 1 & 2 & 0 & 10 & 9 & 2 & 4 & 1 & 2 & 1 & 5 & 4 \\
\hline TH & 3 & 5 & 1 & 6 & 5 & 2 & 3 & 4 & 2 & 2 & 2 & 1 \\
\hline TL & 2 & 2 & 1 & 6 & 5 & 2 & 5 & 2 & 2 & 2 & 3 & 2 \\
\hline TP & 2 & 1 & 0 & 6 & 5 & 2 & 2 & 2 & 2 & 3 & 2 & 2 \\
\hline TS & 2 & 3 & 0 & 1 & 2 & 2 & 1 & 3 & 3 & 4 & 1 & 2 \\
\hline TW & 3 & 3 & 0 & 5 & 4 & 2 & 1 & 2 & 3 & 4 & 3 & 1 \\
\hline WK & 5 & 1 & 0 & 1 & 1 & 2 & 1 & 6 & 3 & 4 & 2 & 1 \\
\hline WL & 2 & 2 & 0 & 5 & 4 & 2 & 1 & 2 & 1 & 2 & 3 & 2 \\
\hline WM & 2 & 1 & 0 & 7 & 7 & 2 & 4 & 2 & 1 & 2 & 2 & 3 \\
\hline WN & 2 & 2 & 0 & 5 & 4 & 3 & 2 & 2 & 1 & 2 & 3 & 2 \\
\hline
\end{tabular}


Table 4. Repeat Measurement Data for Calibration Set Sites - Salinity (sal), Conductivity (cond), $\mathrm{pH}$, Water Temperature (temp) and Water Depth.

Values below each site are the averages used in the calibration set.

See Table 1 for site codes.

\begin{tabular}{|c|c|c|c|c|c|c|c|c|c|}
\hline SITE & SAL & COND & $\mathrm{pH}$ & TEMP & DEPTH & TIME & DATE & TIDE & SEASON \\
\hline $\mathrm{BH}$ & 9 & 15.65 & 7.68 & & 0.03 & $4.15 \mathrm{pm}$ & $19 / 10 / 97$ & Outgoing & Spring \\
\hline $\mathrm{BH}$ & 3.5 & 6.7 & & & 0.02 & $4.40 \mathrm{pm}$ & $7 / 8 / 98$ & Low & Winter \\
\hline $\mathrm{BH}$ & 9.6 & 16.62 & 8.55 & 15 & & $1.30 \mathrm{pm}$ & $9 / 8 / 98$ & Outgoing & Winter \\
\hline $\mathrm{BH}$ & 20.8 & 33.8 & & 14.3 & 0.04 & $10.45 \mathrm{am}$ & $12 / 8 / 98$ & Incoming & Winter \\
\hline $\mathrm{BH}$ & 20.7 & 33.8 & & 16 & 0.05 & $1.15 \mathrm{pm}$ & $12 / 8 / 98$ & High & Winter \\
\hline $\mathrm{BH}$ & 25.9 & 36.7 & 8.13 & 21.6 & & $11.15 \mathrm{am}$ & $22 / 1 / 99$ & Incoming & Summer \\
\hline $\mathrm{BH}$ & 26 & 36.7 & 8.16 & 24 & 0.06 & $2.05 \mathrm{pm}$ & $22 / 1 / 99$ & High & Summer \\
\hline $\mathrm{BH}$ & 26.2 & 36.9 & 8.32 & 20.8 & & $4.45 \mathrm{pm}$ & $22 / 1 / 99$ & Outgoing & Summer \\
\hline \multirow[t]{2}{*}{$\mathrm{BH}$} & 25.2 & 36.6 & & 19.8 & 0.03 & $8.45 \mathrm{pm}$ & $22 / 1 / 99$ & Low & Summer \\
\hline & 18.54 & 28.16 & 8.17 & 18.79 & 0.04 & & & & \\
\hline$B L$ & 22 & 31.9 & 7.84 & 30.7 & 0.03 & $12.15 \mathrm{pm}$ & $9 / 2 / 99$ & High & Summer \\
\hline \multirow[t]{2}{*}{$B L$} & 22.1 & 31.9 & 7.86 & 34 & 0.03 & $12.50 \mathrm{pm}$ & $9 / 2 / 99$ & High & Summer \\
\hline & 22.05 & 31.9 & 7.85 & 32.35 & 0.03 & & & & \\
\hline CL & 0.1 & 0.123 & 8.04 & 23.4 & 0.4 & $3.20 \mathrm{pm}$ & 9/2/99 & NA & Summer \\
\hline \multirow[t]{2}{*}{$\mathrm{CL}$} & 0.1 & 0.129 & 8.09 & 23 & 0.4 & $3.50 \mathrm{pm}$ & $9 / 2 / 99$ & NA & Summer \\
\hline & 0.1 & 0.126 & 8.07 & 23.2 & 0.4 & & & & \\
\hline DC & 3.2 & 6.04 & 6.78 & 12.3 & 0.02 & $11.30 \mathrm{am}$ & $7 / 8 / 98$ & High & Winter \\
\hline DC & 13.2 & 22.6 & & 12.6 & & $11.15 \mathrm{am}$ & $12 / 8 / 98$ & Incoming & Winter \\
\hline DC & 10 & 17.57 & 6.7 & 12.2 & 0.02 & $3.10 \mathrm{pm}$ & $12 / 8 / 98$ & Outgoing & Winter \\
\hline DC & 15.4 & 22.8 & 7.03 & 20.8 & & $10.40 \mathrm{am}$ & $22 / 1 / 99$ & Incoming & Summer \\
\hline DC & 15.5 & 23.1 & 8.75 & 24.7 & 0.03 & $2.30 \mathrm{pm}$ & $22 / 1 / 99$ & High & Summer \\
\hline DC & 16 & 23.7 & 9.11 & 21.8 & & $5.10 \mathrm{pm}$ & $22 / 1 / 99$ & Outgoing & Summer \\
\hline \multirow[t]{2}{*}{ DC } & 15.6 & 23.2 & 7.65 & 19.5 & 0.02 & $8.30 \mathrm{pm}$ & $22 / 1 / 99$ & Low & Summer \\
\hline & 12.7 & 19,86 & 7.67 & 17.7 & 0.02 & & & & \\
\hline GE & 0.1 & 0.274 & 8.18 & 23.7 & 0.02 & $12.00 \mathrm{pm}$ & $16 / 2 / 99$ & NA & Summer \\
\hline \multirow[t]{2}{*}{ GE } & 0.1 & 0.268 & 8.13 & 27 & 0.02 & $12.15 \mathrm{pm}$ & $16 / 2 / 99$ & NA & Summer \\
\hline & 0.1 & 0.271 & 8.16 & 25.35 & 0.02 & & & & \\
\hline $\mathrm{HE}$ & 22.4 & 32.4 & 7.9 & 19.5 & 0.4 & $11.45 \mathrm{am}$ & $15 / 2 / 99$ & Incoming & Summer \\
\hline HE & 25.8 & 36.5 & 7.97 & 18.5 & & $12.10 \mathrm{pm}$ & $15 / 2 / 99$ & Incoming & Summer \\
\hline $\mathrm{HE}$ & 29.8 & 41.6 & 8.03 & 18.2 & 0.5 & 1.15 pm & $15 / 2 / 99$ & High & Summer \\
\hline \multirow[t]{2}{*}{$\mathrm{HE}$} & 29.9 & 41.5 & 8.02 & 19.4 & & $1.45 \mathrm{pm}$ & $15 / 2 / 99$ & High & Summer \\
\hline & 26.98 & 38 & 7.98 & 18.9 & 0.45 & & & & \\
\hline IB & 29.6 & 47.3 & 8.1 & 12 & 0.3 & $9.25 \mathrm{am}$ & $5 / 8 / 98$ & Low & Winter \\
\hline IB & 30.1 & 47.8 & & 12.3 & & $9.30 \mathrm{am}$ & $10 / 8 / 98$ & Outgoing & Winter \\
\hline IB & 29.5 & 47.1 & & 13.7 & & $3.45 \mathrm{pm}$ & $10 / 8 / 98$ & Incoming & Winter \\
\hline IB & 29.7 & 46.7 & & 13.5 & 0.8 & $6.30 \mathrm{pm}$ & $10 / 8 / 98$ & High & Winter \\
\hline IB & 24.7 & 35.5 & 7.95 & 15.8 & 0.4 & $8.40 \mathrm{am}$ & $27 / 1 / 99$ & Low & Summer \\
\hline IB & 29.4 & 41.2 & & 16.6 & & $11.30 \mathrm{am}$ & $27 / 1 / 99$ & Incoming & Summer \\
\hline IB & 29.5 & 41.4 & 8.01 & 17.3 & 0.9 & $2.20 \mathrm{pm}$ & $27 / 1 / 99$ & High & Summer \\
\hline \multirow[t]{2}{*}{ IB } & 30.2 & 42.3 & 7.96 & 18.1 & & $4.25 \mathrm{pm}$ & $27 / 1 / 99$ & Outgoing & Summer \\
\hline & 29.09 & 43.66 & 8.01 & 14.91 & 0.6 & & & & \\
\hline$K L$ & 1.3 & 2.53 & 7.07 & 9.7 & 0.8 & $10.15 \mathrm{am}$ & $19 / 8 / 98$ & Incoming & Winter \\
\hline $\mathrm{KL}$ & 6.9 & 12.09 & & 22.3 & 0.05 & $5.45 \mathrm{pm}$ & $15 / 11 / 98$ & Outgoing & Spring \\
\hline $\mathrm{KL}$ & 3.8 & 6.21 & 7.63 & 12.7 & 0.8 & $5.00 \mathrm{pm}$ & $18 / 4 / 99$ & Incoming & Autumn \\
\hline \multirow[t]{2}{*}{$\mathrm{KL}$} & 6.5 & 10.24 & 8.17 & 12.2 & 0.3 & $12.15 \mathrm{pm}$ & $22 / 8 / 99$ & High & Winter \\
\hline & 4.63 & 7.77 & 7.62 & 14.23 & 0.49 & & & & \\
\hline
\end{tabular}




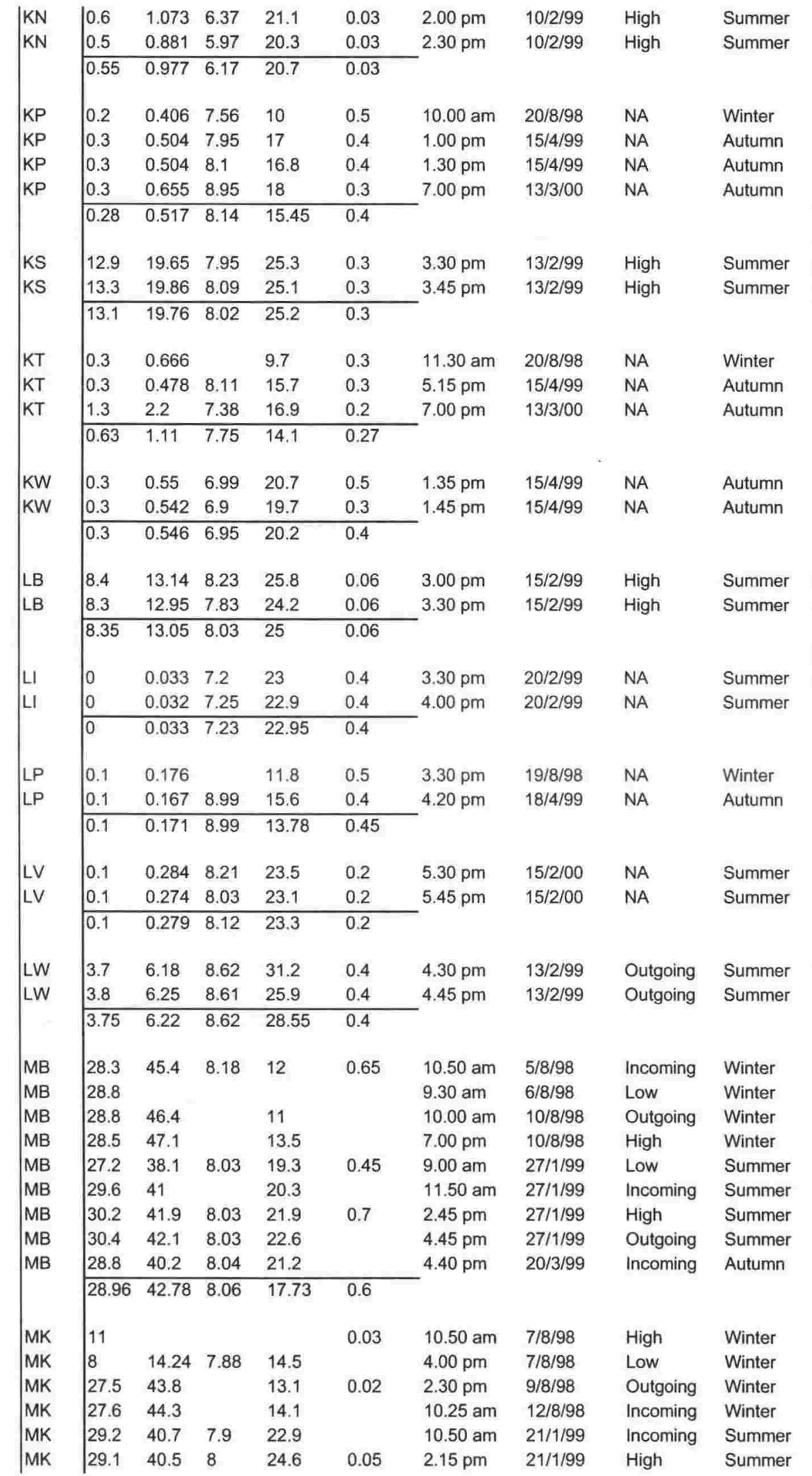




\begin{tabular}{|c|c|c|c|c|c|c|c|c|c|}
\hline MK & 23.4 & 33.4 & & 25.3 & 0.04 & $5.05 \mathrm{pm}$ & $21 / 1 / 99$ & Outgoing & Summer \\
\hline \multirow[t]{2}{*}{ MK } & 23 & 33.1 & 7.91 & 22.7 & & $7.30 \mathrm{pm}$ & 21/1/99 & Low & Summer \\
\hline & 22.35 & 35.71 & 7.92 & 19.6 & 0.04 & & & & \\
\hline MP & 0 & 0.045 & 7.67 & 24.3 & 0.4 & $5.40 \mathrm{pm}$ & $20 / 2 / 99$ & NA & Summer \\
\hline \multirow[t]{2}{*}{ MP } & 0 & 0.046 & 7.82 & 22.1 & 0.4 & $6.00 \mathrm{pm}$ & $20 / 2 / 99$ & NA & Summer \\
\hline & 0 & 0.046 & 7.75 & 23.2 & 0.4 & & & & \\
\hline MS & 21.7 & 31 & 8.06 & 16.6 & 0.4 & $8.45 \mathrm{pm}$ & $25 / 12 / 98$ & Low & Summer \\
\hline MS & 21.9 & 31.5 & & 18.7 & 0.8 & $11.55 \mathrm{am}$ & $26 / 12 / 98$ & Incoming & Summer \\
\hline MS & 25.5 & 36 & 8.12 & 19.4 & & $7.10 \mathrm{pm}$ & $26 / 12 / 98$ & Outgoing & Summer \\
\hline \multirow[t]{2}{*}{ MS } & 25.1 & 35.9 & & 24.4 & & $12.00 \mathrm{pm}$ & $27 / 12 / 98$ & Low & Summer \\
\hline & 23.55 & 33.6 & 8.09 & 19.76 & 0.6 & & & & \\
\hline $\mathrm{NH}$ & 11.8 & 20.1 & & 11.6 & 0.35 & $11.30 \mathrm{am}$ & $7 / 8 / 98$ & Outgoing & Winter \\
\hline \multirow[t]{2}{*}{$\mathrm{NH}$} & 12 & 21 & 7.73 & 10.3 & 0.45 & $10.00 \mathrm{am}$ & $8 / 9 / 98$ & Incoming & Spring \\
\hline & 11.9 & 20.55 & 7.73 & 10.95 & 0.4 & & & & \\
\hline OA & 23.3 & & & 20.3 & 0.03 & $9.45 \mathrm{am}$ & $18 / 7 / 97$ & NA & Winter \\
\hline \multirow[t]{2}{*}{$O A$} & 28.7 & 40.1 & 8.42 & 18.7 & 0.03 & $11.00 \mathrm{am}$ & $30 / 10 / 99$ & NA & Spring \\
\hline & 26 & 40.1 & 8.42 & 19.5 & 0.03 & & & & \\
\hline $\mathrm{OL}$ & 23 & & & 20.4 & 0.03 & $9.30 \mathrm{am}$ & $18 / 7 / 97$ & NA & Winter \\
\hline \multirow[t]{2}{*}{$\mathrm{OL}$} & 20.7 & 29.8 & 8.89 & 18.8 & 0.05 & $1.00 \mathrm{pm}$ & $30 / 10 / 99$ & NA & Spring \\
\hline & 21.85 & 29.8 & 8.89 & 19.6 & 0.04 & & & & \\
\hline ON & 14.7 & 25.2 & & 10.8 & 0.4 & $5.40 \mathrm{pm}$ & $18 / 8 / 98$ & Outgoing & Winter \\
\hline ON & 1.4 & 2.73 & & 8.6 & & $8.50 \mathrm{am}$ & $19 / 8 / 98$ & Low & Winter \\
\hline ON & 1.3 & 2.63 & 7.47 & 10 & 0.5 & $11.50 \mathrm{am}$ & $19 / 8 / 98$ & Incoming & Winter \\
\hline ON & 1.3 & 2.67 & & 10.1 & & $5.00 \mathrm{pm}$ & $19 / 8 / 98$ & Outgoing & Winter \\
\hline ON & 27.1 & 43.2 & & 16.8 & 0 & $4.10 \mathrm{pm}$ & $15 / 11 / 98$ & High & Spring \\
\hline ON & 2.2 & 3.75 & 7.63 & 12.7 & 0.4 & $5.40 \mathrm{pm}$ & $18 / 4 / 99$ & Incoming & Autumn \\
\hline \multirow[t]{2}{*}{ ON } & 0.1 & 0.18 & 10.35 & 9.8 & & $11.15 \mathrm{am}$ & $22 / 8 / 99$ & Incoming & Winter \\
\hline & 6.87 & 11.48 & 8.48 & 11.26 & 0.33 & & & & \\
\hline OP & 2.5 & 4.3 & 9.16 & 22.4 & 0.1 & $1.30 \mathrm{pm}$ & $12 / 2 / 99$ & High & Summer \\
\hline \multirow[t]{2}{*}{ OP } & 2.5 & 4.26 & 8.94 & 25.5 & 0.1 & $2.00 \mathrm{pm}$ & $12 / 2 / 99$ & High & Summer \\
\hline & 2.5 & 4.28 & 9.05 & 23.95 & 0.1 & & & & \\
\hline PA & 30.1 & 42.2 & 8.02 & 21.6 & 0.8 & $5.30 \mathrm{pm}$ & $20 / 3 / 99$ & Incoming & Autumn \\
\hline PA & 30.6 & 42.2 & 8.03 & 21.1 & & $5.50 \mathrm{pm}$ & $20 / 3 / 99$ & Incoming & Autumn \\
\hline PA & 26 & 36.6 & 8.04 & 12.1 & 0.4 & $7.00 \mathrm{pm}$ & $27 / 6 / 99$ & Outgoing & Winter \\
\hline PA & 38.9 & 52.6 & & 21.5 & & $8.45 p m$ & $9 / 12 / 99$ & Outgoing & Summer \\
\hline \multirow[t]{2}{*}{ PA } & 38.7 & 52.3 & 8.11 & 18.4 & 0.9 & $7.30 \mathrm{pm}$ & $13 / 3 / 00$ & High & Autumn \\
\hline & 32.86 & 45.18 & 8.05 & 18.94 & 0.7 & & & & \\
\hline PB & 28.5 & 45.5 & 8.3 & & 0.5 & $1.00 \mathrm{pm}$ & $19 / 10 / 97$ & High & Spring \\
\hline PB & 25.2 & 40.8 & 8.07 & 11.9 & 0.3 & $3.30 \mathrm{pm}$ & $6 / 8 / 98$ & Low & Winter \\
\hline PB & 28.5 & & & & & $10.00 \mathrm{am}$ & $7 / 8 / 98$ & High & Winter \\
\hline PB & 28.5 & & & & & $3.30 \mathrm{pm}$ & $9 / 8 / 98$ & Outgoing & Winter \\
\hline PB & 28.7 & 45.8 & & 14.6 & 0.4 & $9.50 \mathrm{am}$ & $12 / 8 / 98$ & Incoming & Winter \\
\hline PB & 29.1 & 40.5 & 7.99 & 20.4 & & $10.20 \mathrm{am}$ & $21 / 1 / 99$ & Incoming & Summer \\
\hline PB & 29.6 & 41.1 & 7.95 & 20.2 & 0.6 & $1.35 \mathrm{pm}$ & $21 / 1 / 99$ & High & Summer \\
\hline PB & 30.3 & 42 & & 21.1 & & $4.35 \mathrm{pm}$ & $21 / 1 / 99$ & Outgoing & Summer \\
\hline \multirow[t]{2}{*}{ PB } & 30.8 & 42.8 & 8.01 & 19.6 & 0.2 & $8.10 \mathrm{pm}$ & $21 / 1 / 99$ & Low & Summer \\
\hline & 28.8 & 42.64 & 8.06 & 17.97 & 0.4 & & & & \\
\hline $\mathrm{PH}$ & 24.5 & 39.9 & 8.11 & 12.5 & 0.2 & $3.40 \mathrm{pm}$ & $5 / 8 / 98$ & Low & Winter \\
\hline $\mathrm{PH}$ & 24.3 & & & & & $6.00 \mathrm{pm}$ & $5 / 8 / 98$ & Incoming & Winter \\
\hline $\mathrm{PH}$ & 25.7 & & & & & $2.00 \mathrm{pm}$ & $6 / 8 / 98$ & Outgoing & Winter \\
\hline
\end{tabular}




\begin{tabular}{|c|c|c|c|c|c|c|c|c|c|}
\hline $\mathrm{PH}$ & 21.9 & & & & 0.5 & $9.30 \mathrm{am}$ & $7 / 8 / 98$ & High & Winter \\
\hline $\mathrm{PH}$ & 28.6 & 45.2 & & 14.5 & & $9.30 \mathrm{am}$ & $12 / 8 / 98$ & Incoming & Winter \\
\hline PH & 27.7 & 38.9 & 8.08 & 22.5 & 0.3 & $10.00 \mathrm{am}$ & $21 / 1 / 99$ & Incoming & Summer \\
\hline $\mathrm{PH}$ & 27.8 & 39.2 & 8.05 & 23.5 & & $1.15 \mathrm{pm}$ & $21 / 1 / 99$ & High & Summer \\
\hline $\mathrm{PH}$ & 29.5 & 41 & & 23.2 & 0.4 & $4.15 \mathrm{pm}$ & $21 / 1 / 99$ & Outgoing & Summer \\
\hline \multirow[t]{2}{*}{$\mathrm{PH}$} & 30.3 & 42.4 & 8.2 & 19 & & $8.30 \mathrm{pm}$ & $21 / 1 / 99$ & Low & Summer \\
\hline & 26.7 & 41.1 & 8.11 & 19.2 & 0.35 & & & & \\
\hline PL & 25.4 & 35.6 & 8.16 & 17.2 & 0.4 & $5.10 \mathrm{pm}$ & $27 / 4 / 99$ & Incoming & Autumn \\
\hline PL & 27.9 & 39 & 8.21 & 16 & 0.4 & $5.40 \mathrm{pm}$ & $27 / 4 / 99$ & Incoming & Autumn \\
\hline PL & 29.2 & 40.9 & 8.03 & 14.3 & 0.8 & $7.00 \mathrm{pm}$ & $10 / 5 / 99$ & High & Autumn \\
\hline PL & 24 & 34.4 & 8.1 & 12.5 & 0.1 & $3.30 \mathrm{pm}$ & $27 / 6 / 99$ & Low & Winter \\
\hline \multirow[t]{2}{*}{ PL } & 39.9 & 53.7 & & 21.3 & 0.3 & $8.00 \mathrm{pm}$ & $9 / 12 / 99$ & Outgoing & Summer \\
\hline & 29.28 & 40.72 & 8.13 & 16.26 & 0.4 & & & & \\
\hline PM & 13.8 & 23.3 & 7.92 & & 0.1 & $4.30 \mathrm{pm}$ & $19 / 10 / 97$ & Outgoing & Spring \\
\hline PM & 6.1 & 11.09 & 8.28 & 12.5 & 0.03 & $1.50 \mathrm{pm}$ & 9/8/98 & Outgoing & Winter \\
\hline PM & 17.2 & 29 & & 13.6 & & $10.50 \mathrm{am}$ & $12 / 8 / 98$ & Incoming & Winter \\
\hline PM & 23.6 & 38 & 7.99 & 14.5 & 0.4 & $1.30 \mathrm{pm}$ & $12 / 8 / 98$. & High & Winter \\
\hline PM & 30.5 & 42.5 & 8.55 & 22.8 & 0.2 & $11.10 \mathrm{am}$ & 22/1/99 & Incoming & Summer \\
\hline PM & 29.3 & 41.2 & 8.3 & 22.7 & & $2.00 \mathrm{pm}$ & 22/1/99 & High & Summer \\
\hline PM & 29.5 & 41.1 & 8.21 & 19.9 & & $4.40 \mathrm{pm}$ & $22 / 1 / 99$ & Outgoing & Summer \\
\hline \multirow[t]{2}{*}{ PM } & 29.4 & 41 & & 19.2 & 0.02 & $8.50 \mathrm{pm}$ & $22 / 1 / 99$ & Low & Summer \\
\hline & 22.43 & 33.4 & 8.21 & 17.89 & 0.15 & & & & \\
\hline PR & 1.8 & 3.05 & 8.86 & 29 & 0.05 & $1.40 \mathrm{pm}$ & $24 / 1 / 99$ & Incoming & Summer \\
\hline PR & 7.7 & 12 & 7.58 & 20.3 & & $8.00 \mathrm{pm}$ & 20/3/99 & Incoming & Autumn \\
\hline PR & 6.3 & 9.9 & 8.49 & 11.3 & 0.06 & $4.20 \mathrm{pm}$ & $27 / 6 / 99$ & Incoming & Winter \\
\hline \multirow[t]{2}{*}{ PR } & 0.5 & 0.868 & 7.65 & 18.7 & 0.15 & $3.30 \mathrm{pm}$ & $30 / 10 / 99$ & High & Spring \\
\hline & 4.08 & 6.46 & 8.15 & 19.83 & 0.09 & & & & \\
\hline \multirow[t]{3}{*}{ PS } & 0.2 & 0.343 & 7.11 & 21.9 & 0.01 & $1.50 \mathrm{pm}$ & $25 / 1 / 99$ & NA & Summer \\
\hline & 0.2 & 0.339 & 7.06 & 22 & 0.01 & $2.30 \mathrm{pm}$ & $25 / 1 / 99$ & NA & Summer \\
\hline & 0.2 & 0.341 & 7.09 & 21.95 & 0.01 & & & & \\
\hline PT & 26.2 & 41.9 & 8.24 & & 1.3 & $12.30 \mathrm{pm}$ & $19 / 10 / 97$ & High & Spring \\
\hline PT & 26.1 & 42.3 & 8.23 & 11.9 & 0.5 & $4.50 \mathrm{pm}$ & $6 / 8 / 98$ & Low & Winter \\
\hline PT & 24.1 & & & & & $10.20 \mathrm{am}$ & $7 / 8 / 98$ & High & Winter \\
\hline PT & 28.9 & & & & & $3.00 \mathrm{pm}$ & $9 / 8 / 98$ & Outgoing & Winter \\
\hline PT & 28.7 & 45.2 & & 14.7 & & $10.05 \mathrm{am}$ & $12 / 8 / 98$ & Incoming & Winter \\
\hline PT & 29.2 & 40.5 & 7.9 & 20.3 & & $10.35 \mathrm{am}$ & $21 / 1 / 99$ & Incoming & Summer \\
\hline PT & 29.3 & 41.1 & 7.94 & 22.4 & & $1.50 \mathrm{pm}$ & $21 / 1 / 99$ & High & Summer \\
\hline PT & 30.2 & 42.2 & & 22.8 & 0.6 & $4.55 \mathrm{pm}$ & 21/1/99 & Outgoing & Summer \\
\hline PT & 31 & 43.1 & 7.9 & 20.1 & & $7.45 \mathrm{pm}$ & $21 / 1 / 99$ & Low & Summer \\
\hline \multirow[t]{2}{*}{ PT } & 29.1 & 40.7 & 7.65 & 16.5 & 0.4 & $6.10 \mathrm{pm}$ & $27 / 4 / 99$ & Incoming & Autumn \\
\hline & 28.28 & 42.13 & 7.98 & 18.39 & 0.7 & & & & \\
\hline PW & 7.6 & 11.98 & 8.8 & 33.1 & 0.01 & $3.15 \mathrm{pm}$ & $24 / 1 / 99$ & High & Summer \\
\hline PW & 23.2 & 33.2 & 7.66 & 20.3 & & $8.15 \mathrm{pm}$ & $20 / 3 / 99$ & Incoming & Autumn \\
\hline PW & 2.6 & 4.42 & 7.55 & 9.1 & 0.03 & $4.30 \mathrm{pm}$ & $27 / 6 / 99$ & Incoming & Winter \\
\hline \multirow[t]{2}{*}{ PW } & 1.4 & 2.37 & 7.59 & 18.9 & 0.1 & $3.45 \mathrm{pm}$ & $30 / 10 / 99$ & High & Spring \\
\hline & 8.7 & 12.99 & 7.9 & 20.35 & 0.05 & & & & \\
\hline RM & 0.1 & 0.121 & 5.87 & 18.3 & 0.4 & $1.30 \mathrm{pm}$ & $11 / 2 / 99$ & NA & Summer \\
\hline \multirow[t]{2}{*}{ RM } & 0.1 & 0.123 & 6.46 & 18.4 & 0.4 & $2.00 \mathrm{pm}$ & $11 / 2 / 99$ & NA & Summer \\
\hline & 0.1 & 0.122 & 6.17 & 18.4 & 0.4 & & & & \\
\hline RP & 0.8 & 1.44 & 9.4 & 26.1 & 0.15 & $4.50 \mathrm{pm}$ & $24 / 1 / 99$ & NA & Summer \\
\hline RP & 0.9 & 1.63 & 9.51 & 22.7 & & $7.30 \mathrm{pm}$ & $20 / 3 / 99$ & NA & Autumn \\
\hline \multirow[t]{2}{*}{$\mathrm{RP}$} & 0.9 & 1.676 & 9.6 & 18.7 & 0.2 & $4.00 \mathrm{pm}$ & $30 / 10 / 99$ & NA & Spring \\
\hline & 0.87 & 1.582 & 9.5 & 22.5 & 0.18 & & & & \\
\hline
\end{tabular}




\begin{tabular}{|c|c|c|c|c|c|c|c|c|c|}
\hline SB & 30.6 & 42.6 & 8.03 & 18.6 & 0.3 & $4.00 \mathrm{pm}$ & $15 / 2 / 99$ & Outgoing & Summer \\
\hline \multirow[t]{2}{*}{ SB } & 29.4 & 40.8 & 8.05 & 18.6 & 0.3 & $4.30 \mathrm{pm}$ & $15 / 2 / 99$ & Outgoing & Summer \\
\hline & 30 & 41.7 & 8.04 & 18.6 & 0.3 & & & & \\
\hline \multirow[t]{3}{*}{ SP } & 0.3 & 0.583 & 6.57 & 11.6 & 0.3 & $4.40 \mathrm{pm}$ & $7 / 9 / 98$ & NA & Spring \\
\hline & 0.3 & 0.594 & 6.64 & 11.7 & 0.3 & $5.10 \mathrm{pm}$ & $7 / 9 / 98$ & NA & Spring \\
\hline & 0.3 & 0.589 & 6.61 & 11.65 & 0.3 & & & & \\
\hline TB & 29.1 & 46.5 & 8.19 & 14 & 0.4 & $2.50 \mathrm{pm}$ & $5 / 8 / 98$ & Low & Winter \\
\hline TB & 28.9 & & & & 1.2 & $9.10 \mathrm{am}$ & $7 / 8 / 98$ & High & Winter \\
\hline TB & 29.3 & 46.7 & & 13.7 & & $9.15 \mathrm{am}$ & $12 / 8 / 98$ & Incoming & Winter \\
\hline TB & 29.4 & 46.8 & & 14.1 & & $4.00 \mathrm{pm}$ & $12 / 8 / 98$ & Outgoing & Winter \\
\hline TB & 24.1 & 33.7 & 8 & 19.1 & 0.5 & $9.45 \mathrm{am}$ & $21 / 1 / 99$ & Incoming & Summer \\
\hline TB & 27.5 & 38.5 & 7.95 & 23.1 & 0.4 & $1.05 \mathrm{pm}$ & $21 / 1 / 99$ & High & Summer \\
\hline TB & 30.3 & 42.3 & & 21.2 & & $4.00 \mathrm{pm}$ & $21 / 1 / 99$ & Outgoing & Summer \\
\hline \multirow[t]{2}{*}{ TB } & 30.9 & 42.9 & & 17.1 & & $8.30 \mathrm{pm}$ & 21/1/99 & Low & Summer \\
\hline & 28.69 & 42.49 & 8.05 & 17.47 & 0.63 & & & & \\
\hline TH & 0.5 & 0.871 & 7.74 & 20.9 & 0.5 & $11.30 \mathrm{am}$ & $13 / 2 / 99$ & Incoming & Summer \\
\hline \multirow[t]{2}{*}{ TH } & 0.5 & 0.859 & 7.66 & 22.4 & 0.5 & $12.00 \mathrm{pm}$ & $13 / 2 / 99$ & Incoming & Summer \\
\hline & 0.5 & 0.865 & 7.7 & 21.65 & 0.5 & & & & \\
\hline TL & 13.4 & 22.9 & & 11.9 & 0.85 & $5.10 \mathrm{pm}$ & 19/8/98 & Outgoing & Winter \\
\hline TL & 0.6 & 1.25 & & 24.4 & 0.1 & $4.45 \mathrm{pm}$ & $15 / 11 / 98$ & Outgoing & Spring \\
\hline TL & 2.8 & 4.72 & 7.62 & 12.2 & 0.85 & $5.50 \mathrm{pm}$ & $18 / 4 / 99$ & Incoming & Autumn \\
\hline \multirow[t]{2}{*}{ TL } & 0.2 & 0.32 & 7.9 & 12.7 & 0.6 & $11.30 \mathrm{am}$ & $22 / 8 / 99$ & Incoming & Winter \\
\hline & 4.25 & 7.298 & 7.76 & 15.3 & 0.6 & & & & \\
\hline TP & 3.3 & 6.14 & & 18 & 0.03 & $3.30 \mathrm{pm}$ & $20 / 10 / 98$ & NA & Spring \\
\hline \multirow[t]{2}{*}{ TP } & 3.7 & 6.88 & 5.25 & 19.9 & 0.03 & $4.20 \mathrm{pm}$ & $22 / 10 / 98$ & NA & Spring \\
\hline & 3.5 & 6.51 & 5.25 & 18.95 & 0.03 & & & & \\
\hline TS & 0.2 & 0.363 & 6.85 & & 0.06 & $2.00 \mathrm{pm}$ & $4 / 11 / 97$ & NA & Spring \\
\hline TS & 0.2 & 0.392 & 6.66 & 11.1 & 0.01 & $2.45 \mathrm{pm}$ & $6 / 8 / 98$ & NA & Winter \\
\hline \multirow[t]{2}{*}{ TS } & 0.2 & 0.393 & 6.84 & 20.2 & & $11.00 \mathrm{am}$ & $21 / 1 / 99$ & NA & Summer \\
\hline & 0.2 & 0.383 & 6.78 & 15.65 & 0.04 & & & & \\
\hline TW & 0.3 & 0.56 & 6.75 & 9.6 & 0.6 & $11.15 \mathrm{am}$ & 20/8/98 & NA & Winter \\
\hline \multirow[t]{2}{*}{ TW } & 0.3 & 0.482 & 7.59 & 15.9 & 0.4 & $5.30 \mathrm{pm}$ & $15 / 4 / 99$ & NA & Autumn \\
\hline & 0.3 & 0.521 & 7.17 & 12.75 & 0.5 & & & & \\
\hline WK & 0.1 & 0.256 & 5.11 & 27.9 & 0.08 & $6.35 \mathrm{pm}$ & $14 / 2 / 99$ & NA & Summer \\
\hline \multirow[t]{2}{*}{ WK } & 0.1 & 0.245 & 5.04 & 27 & 0.08 & $7.00 \mathrm{pm}$ & $14 / 2 / 99$ & NA & Summer \\
\hline & 0.1 & 0.251 & 5.08 & 27.45 & 0.08 & & & & \\
\hline WL & 0.1 & 0.145 & 8.95 & 23 & 0.03 & $5.30 \mathrm{pm}$ & $11 / 2 / 99$ & NA & Summer \\
\hline \multirow[t]{2}{*}{ WL } & 0.1 & 0.146 & & 22 & 0.03 & $5.45 \mathrm{pm}$ & $11 / 2 / 99$ & NA & Summer \\
\hline & 0.1 & 0.146 & 8.95 & 22.5 & 0.03 & & & & \\
\hline WM & 8.5 & 14.65 & & 22.5 & 0.08 & $10.30 \mathrm{am}$ & $21 / 10 / 98$ & Outgoing & Spring \\
\hline \multirow[t]{2}{*}{ WM } & 17.4 & 28.5 & 8.61 & 19.8 & 0,04 & $1.00 \mathrm{pm}$ & $22 / 10 / 98$ & Low & Spring \\
\hline & 12.95 & 21.58 & 8.61 & 21.15 & 0.06 & & & & \\
\hline WN & 1.4 & 2.41 & 8.15 & 20.4 & 0.03 & $7.40 \mathrm{pm}$ & $11 / 2 / 99$ & NA & Summer \\
\hline \multirow[t]{2}{*}{ WN } & 1.7 & 2.9 & 8.16 & 18.4 & 0.03 & $8.00 \mathrm{pm}$ & $11 / 2 / 99$ & NA & Summer \\
\hline & 1.55 & 2.655 & 8.16 & 19.4 & 0.03 & & & & \\
\hline
\end{tabular}


Table 5. Grainsize Data for Calibration Set Samples.

See Table 1 for site codes.

\begin{tabular}{|c|c|c|c|c|c|c|c|c|c|c|c|c|c|}
\hline \multirow[t]{2}{*}{ SITES } & \multicolumn{8}{|c|}{ WEIGHTS (g) } & \multicolumn{5}{|c|}{ PERCENTAGES } \\
\hline & WET SED & DRY SED & ORGS & CARB & CRSE & FINE & TOTAL & LOSS & SAND & MUD & ORGS & CARB & LOSS \\
\hline $\mathrm{BH}$ & 42.67 & 29.38 & 4.65 & 1.93 & 18.57 & 4.06 & 29.21 & 0.17 & 63.21 & 13.82 & 15.83 & 6.57 & 0.58 \\
\hline BL. & 45.10 & 28.47 & 11.53 & 5.20 & 0.00 & 10.91 & 27.64 & 0.83 & 0.00 & 38.32 & 40.50 & 18.26 & 2.92 \\
\hline $\mathrm{CL}$ & 81.05 & 62.54 & 7.13 & 1.83 & 52.50 & 1.07 & 62.53 & 0.01 & 83.95 & 1.71 & 11.40 & 2.93 & 0.02 \\
\hline DC & 33.10 & 6.04 & 4.17 & 0.87 & 0.06 & 0.91 & 6.01 & 0.03 & 0.99 & 15.07 & 69.04 & 14.40 & 0.50 \\
\hline GE & 67.42 & 40.61 & 1.17 & 1.18 & 33.32 & 4.73 & 40.40 & 0.21 & 82.05 & 11.65 & 2.88 & 2.91 & 0.52 \\
\hline $\mathrm{HE}$ & 70.72 & 52.28 & 0.00 & 2.91 & 47.72 & 1.77 & 52.40 & -0.12 & 91.28 & 3.39 & 0.00 & 5.57 & -0.23 \\
\hline IB & 61.94 & 50.39 & 0.09 & 4.98 & 45.30 & 0.05 & 50.42 & -0.03 & 89.90 & 0.10 & 0.18 & 9.88 & -0.06 \\
\hline KL & 25.49 & 13.24 & 3.10 & 0.83 & 0.35 & 9.12 & 13.40 & -0.16 & 2.64 & 68.88 & 23.41 & 6.27 & -1.21 \\
\hline KN & 39.61 & 24.08 & 0.75 & 1.14 & 9.28 & 12.55 & 23.72 & 0.36 & 38.54 & 52.12 & 3.11 & 4.73 & 1.50 \\
\hline KP & 57.93 & 43.18 & 8.26 & 0.97 & 32.79 & 1.12 & 43.14 & 0.04 & 75.94 & 2.59 & 19.13 & 2.25 & 0.09 \\
\hline KS & 46.50 & 31.14 & 1.15 & 1.30 & 22.50 & 5.97 & 30.92 & 0.22 & 72.25 & 19.17 & 3.69 & 4.17 & 0.71 \\
\hline KT & 78.41 & 59.68 & 6.43 & 1.01 & 50.86 & 1.39 & 59.69 & -0.01 & 85.22 & 2.33 & 10.77 & 1.69 & -0.02 \\
\hline KW & 51.14 & 32.18 & 21.54 & 3.17 & 0.88 & 6.57 & 32.16 & 0.02 & 2.73 & 20.42 & 66.94 & 9.85 & 0.06 \\
\hline LB & 58.57 & 36.30 & 14.69 & 2.80 & 11.87 & 6.76 & 36.12 & 0.18 & 32.70 & 18.62 & 40.47 & 7.71 & 0.50 \\
\hline LI & 66.04 & 35.33 & 2.77 & 1.84 & 27.39 & 3.07 & 35.07 & 0.26 & 77.53 & 8.69 & 7.84 & 5.21 & 0.74 \\
\hline LP & 67.73 & 46.71 & 0.25 & 1.30 & 41.73 & 3.40 & 46.68 & 0.03 & 89.34 & 7.28 & 0.54 & 2.78 & 0.06 \\
\hline LV & 75.65 & 52.67 & 0.21 & 0.94 & 49.91 & 1.49 & 52.55 & 0.12 & 94.76 & 2.83 & 0.40 & 1.78 & 0.23 \\
\hline LW & 70.45 & 62.21 & 3.45 & 2.32 & 50.66 & 5.16 & 61.59 & 0.62 & 81.43 & 8.29 & 5.55 & 3.73 & 1.00 \\
\hline MB & 65.46 & 54.14 & 3.32 & 9.19 & 39.69 & 2.10 & 54.30 & -0.16 & 73.31 & 3.88 & 6.13 & 16.97 & -0.30 \\
\hline MK & 40.26 & 28.32 & 3.08 & 2.02 & 14.49 & 8.47 & 28.06 & 0.26 & 51.17 & 29.91 & 10.88 & 7.13 & 0.92 \\
\hline MP & 58.24 & 45.29 & 4.21 & 0.93 & 35.65 & 3.91 & 44.70 & 0.59 & 78.71 & 8.63 & 9.30 & 2.05 & 1.30 \\
\hline MS & 64.48 & 52.98 & 3.23 & 5.26 & 38.42 & 5.94 & 52.85 & 0.13 & 72.52 & 11.21 & 6.10 & 9.93 & 0.25 \\
\hline $\mathrm{NH}$ & 46.42 & 32.53 & 1.91 & 2.95 & 24.67 & 2.88 & 32.41 & 0.12 & 75.84 & 8.85 & 5.87 & 9.07 & 0.37 \\
\hline$O A$ & 20.17 & 7.09 & 2.38 & 0.66 & 0.00 & 3.99 & 7.03 & 0.06 & 0.00 & 56.28 & 33.57 & 9.31 & 0.85 \\
\hline $\mathrm{OL}$ & 19.29 & 10.50 & 1.30 & 0.56 & 6.33 & 2.26 & 10.45 & 0.05 & 60.29 & 21.52 & 12.38 & 5.33 & 0.48 \\
\hline ON & 91.41 & 66.37 & 4.98 & 1.98 & 59.12 & 0.14 & 66.22 & 0.15 & 89.08 & 0.21 & 7.50 & 2.98 & 0.23 \\
\hline OP & 56.48 & 43.96 & 0.21 & 4.98 & 37.61 & 1.08 & 43.88 & 0.08 & 85.56 & 2.46 & 0.48 & 11.33 & 0.18 \\
\hline PA & 83.40 & 76.00 & 0.07 & 4.25 & 71.71 & 0.07 & 76.10 & -0.10 & 94.36 & 0.09 & 0.09 & 5.59 & -0.13 \\
\hline PB & 61.62 & 49.97 & 2.13 & 4.16 & 39.18 & 4.62 & 50.09 & -0.12 & 78.41 & 9.25 & 4.26 & 8.32 & -0.24 \\
\hline $\mathrm{PH}$ & 59.82 & 48.83 & 5.35 & 3.90 & 38.38 & 0.66 & 48.29 & 0.54 & 78.60 & 1.35 & 10.96 & 7.99 & 1.11 \\
\hline PL & 58.52 & 42.49 & 1.03 & 7.58 & 33.55 & 0.34 & 42.50 & -0.01 & 78.96 & 0.80 & 2.42 & 17.84 & -0.02 \\
\hline PM & 45.27 & 35.08 & 2.35 & 3.46 & 28.37 & 0.83 & 35.01 & 0.07 & 80.87 & 2.37 & 6.70 & 9.86 & 0.20 \\
\hline PR & 43.15 & 24.26 & 1.77 & 2.25 & 15.65 & 4.58 & 24.25 & 0.01 & 64.51 & 18.88 & 7.30 & 9.27 & 0.04 \\
\hline PS & 30.53 & 6.43 & 4.46 & 0.67 & 0.00 & 1.27 & 6.40 & 0.03 & 0.00 & 19.75 & 69.36 & 10.42 & 0.47 \\
\hline PT & 42.62 & 31.92 & 4.47 & 3.45 & 17.96 & 5.84 & 31.72 & 0.20 & 56.27 & 18.30 & 14.00 & 10.81 & 0.63 \\
\hline PW & 30.77 & 19.90 & 0.27 & 1.20 & 17.60 & 0.85 & 19.92 & -0.02 & 88.44 & 4.27 & 1.36 & 6.03 & -0.10 \\
\hline $\mathrm{RM}$ & 46.99 & 19.63 & 7.48 & 0.00 & 0.00 & 11.98 & 19.46 & 0.17 & 0.00 & 61.03 & 38.10 & 0.00 & 0.87 \\
\hline RP & 38.58 & 26.39 & 0.16 & 1.83 & 23.60 & 0.78 & 26.37 & 0.02 & 89.43 & 2.96 & 0.61 & 6.93 & 0.08 \\
\hline SB & 72.34 & 62.76 & 0.02 & 5.85 & 56.78 & 0.08 & 62.73 & 0.03 & 90.47 & 0.13 & 0.03 & 9.32 & 0.05 \\
\hline SP & NA & NA & NA & NA & NA & NA & NA & NA & 3.63 & 77.49 & 13.40 & 5.48 & NA \\
\hline TB & 60.32 & 48.93 & 3.92 & 5.57 & 39.00 & 0.16 & 48.65 & 0.28 & 79.71 & 0.33 & 8.01 & 11.38 & 0.57 \\
\hline TH & 44.32 & 26.21 & 10.86 & 2.19 & 8.22 & 4.91 & 26.18 & 0.03 & 31.36 & 18.73 & 41.43 & 8.36 & 0.11 \\
\hline TL & 52.56 & 40.01 & 1.33 & 1.39 & 26.21 & 10.89 & 39.82 & 0.19 & 65.51 & 27.22 & 3.32 & 3.47 & 0.47 \\
\hline TP & 51.97 & 39.85 & 4.58 & 1.67 & 12.79 & 20.47 & 39.51 & 0.34 & 32.10 & 51.37 & 11.49 & 4.19 & 0.85 \\
\hline TS & 32.61 & 10.17 & 8.61 & 0.60 & 0.00 & 0.95 & 10.16 & 0.01 & 0.00 & 9.34 & 84.66 & 5.90 & 0.10 \\
\hline TW & 58.84 & 44.31 & 36.91 & 0.32 & 2.99 & 4.01 & 44.23 & 0.08 & 6.75 & 9.05 & 83.30 & 0.72 & 0.18 \\
\hline WK & 91.82 & 51.12 & 50.13 & 0.62 & 0.00 & 0.36 & 51.11 & 0.01 & 0.00 & 0.70 & 98.06 & 1.21 & 0.02 \\
\hline$W L$ & 41.23 & 22.90 & 0.48 & 0.66 & 14.94 & 6.69 & 22.77 & 0.13 & 65.24 & 29.21 & 2.10 & 2.88 & 0.57 \\
\hline WM & 44.59 & 30.06 & 2.04 & 3.05 & 20.41 & 4.49 & 29.99 & 0.07 & 67.90 & 14.94 & 6.79 & 10.15 & 0.23 \\
\hline WN & 34.80 & 18.68 & 1.64 & 1.11 & 4.44 & 11.30 & 18.49 & 0.19 & 23.77 & 60.49 & 8.78 & 5.94 & 1.02 \\
\hline
\end{tabular}

